\title{
Synthesis, Properties, and Coordination Chemistry of $t$-Bu-Xantphos Ligands
}

by

Melanie Ruth Maria Nelson

A thesis

submitted to the Victoria University of Wellington

in fulfilment of the

requirements for the degree of

Doctor of Philosophy

in Chemistry.

Victoria University of Wellington

2015 



\begin{abstract}
This thesis provides an account of research into a group of diphosphine ligands with a rigid xanthene backbone and tert-butyl substituents on the phosphorus atoms. The three ligands have different groups in the bridgehead position of the backbone $\left(\mathrm{CMe}_{2}, \mathrm{SiMe}_{2}\right.$, or S) which change the natural (calculated) bite-angle of the ligand. The coordination chemistry of these $t$-Bu-xantphos ligands with late-transition metals has been investigated with a focus on metal complexes that may form in catalytic reactions.

The three $t$-Bu-xantphos ligands were synthesised by lithiation of the backbone using sec-butyllithium/TMEDA and treatment with $\mathrm{P}^{\mathrm{t}} \mathrm{Bu}_{2} \mathrm{Cl}$. The natural biteangles of the Ph-xantphos (111.89-114.18 $)$ and $t$-Bu-xantphos (126.80-127.56 $\left.{ }^{\circ}\right)$ ligands were calculated using DFT. The bite-angle of the $t$-Bu-xantphos ligands is larger due to the increased steric bulk of the tert-butyl substituents. The electronic properties of the $t$-Bu-xantphos ligands were also investigated by synthesis of their phosphine selenides. The values of ${ }^{1} J_{\text {PSe }}(689.1-698.5 \mathrm{~Hz})$ indicate that the $t$-Bu-xantphos ligands have a higher basicity than $\mathrm{Ph}$-xantphos between $\mathrm{PPh}_{2} \mathrm{Me}$ and $\mathrm{PMe}_{3}$.
\end{abstract}

The silver complexes, $[\mathrm{Ag}(t-\mathrm{Bu}$-xantphos $) \mathrm{Cl}]$ and $[\mathrm{Ag}(t-\mathrm{Bu}$-xantphos $)] \mathrm{BF}_{4}$ were synthesised with the $t$-Bu-xantphos ligands. In contrast to systems with phenyl phosphines, all species were monomeric. [Rh(t-Bu-xantphos $) \mathrm{Cl}]$ complexes were synthesised, which reacted with $\mathrm{H}_{2}$, forming $\left[\mathrm{Rh}\left(t\right.\right.$-Bu-xantphos- $\left.\left.\kappa P, O, P^{\prime}\right) \mathrm{Cl}(\mathrm{H})_{2}\right]$ complexes, and with $\mathrm{CO}$, forming $\left[\mathrm{Rh}(t-\mathrm{Bu}\right.$-xantphos $\left.)(\mathrm{CO})_{2} \mathrm{Cl}\right]$ complexes. The [ $\mathrm{Rh}(t$-Bu-xantphos $) \mathrm{Cl}]$ species are air-sensitive readily forming [ $\mathrm{Rh}(t$-Bu-xantphos $\left.) \mathrm{Cl}\left(\eta^{2}-\mathrm{O}_{2}\right)\right]$ complexes. The crystal structure of $\left[\mathrm{Rh}(t\right.$-Bu-xantphos $\left.) \mathrm{Cl}\left(\eta^{2}-\mathrm{O}_{2}\right)\right]$, contained $15 \%$ of the dioxygen sites replaced with an oxo ligand. This is the first crystallographic evidence of a rhodium(III) oxo complex, and only the third rhodium oxo species reported.

The coordination chemistry of the ligands with platinum(0) and palladium( 0 ) showed some differences. [Pt(t-Bu-xantphos $\left.)\left(\mathrm{C}_{2} \mathrm{H}_{4}\right)\right]$ complexes were synthe- 
sised for all three ligands. However, reaction with $\left[\mathrm{Pt}(\mathrm{nb})_{3}\right]$ produced a mixture of [Pt(t-Bu-xantphos)] and [Pt(t-Bu-xantphos)(nb)] for $t$-Bu-sixantphos and $t$-Buthixantphos. Although few examples of isolable $[\mathrm{Pt}(\mathrm{PP})]$ complexes with diphosphines have been reported [Pt $(t-B u$-thixantphos $)]$ was isolated by removal of the norbornene. $t$-Bu-Xantphos formed small amounts of [Pt $(t-B u-x a n t p h o s)]$ initially, which progressed to $[\mathrm{Pt}(t-\mathrm{Bu}$-xantphos) $\mathrm{H}] \mathrm{X}$. The analogous reactions with $\left[\mathrm{Pd}(\mathrm{nb})_{3}\right]$ gave $[\mathrm{Pd}(t-\mathrm{Bu}-x a n t p h o s)]$ and $[\mathrm{Pd}(t-\mathrm{Bu}$-xantphos $)(\mathrm{nb})]$ complexes in all cases. $\left[\mathrm{Pt}(t-\mathrm{Bu}\right.$-thixantphos $\left.)\left(\mathrm{C}_{2} \mathrm{H}_{4}\right)\right]$ and $[\mathrm{M}(t$-Bu-thixantphos $)](\mathrm{M}=\mathrm{Pd}, \mathrm{Pt})$ react with oxygen forming $\left[\mathrm{Pt}(t-\mathrm{Bu}\right.$-thixantphos $\left.)\left(\eta^{2}-\mathrm{O}_{2}\right)\right]$, which reacts with $\mathrm{CO}$ to give

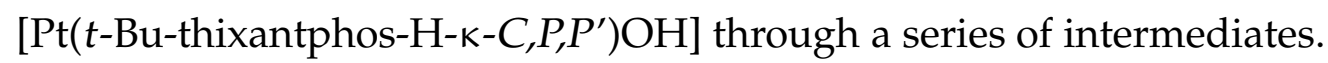

$\left[\mathrm{M}(t\right.$-Bu-xantphos $\left.) \mathrm{Cl}_{2}\right](\mathrm{M}=\mathrm{Pd}, \mathrm{Pt})$ complexes were synthesised, showing exclusive trans coordination of the diphosphine ligands. The $\mathrm{X}$-ray crystal structure of $\left[\mathrm{Pt}(t\right.$-Bu-thixantphos $\left.) \mathrm{Cl}_{2}\right]$ has a bite-angle of $151.722(15)^{\circ}$. This is the first $\left[\mathrm{PtCl}_{2}(\mathrm{PP})\right]$ complex with a bite-angle between 114 and $171^{\circ}$. In polar solvents a chloride ligand dissociates from the $\left[\mathrm{Pt}(t-\mathrm{Bu}\right.$-xantphos $\left.) \mathrm{Cl}_{2}\right]$ complexes producing $\left[\mathrm{Pt}\left(t-\mathrm{Bu} \text {-xantphos- } \mathrm{k} P, \mathrm{O}, \mathrm{P}^{\prime}\right) \mathrm{Cl}\right]^{+}$. The analogous $[\mathrm{Pd}(t$-Bu-xantphos$\left.\left.\kappa P, O, P^{\prime}\right) C l\right]^{+}$complexes were formed by reaction of the dichlorides complexes

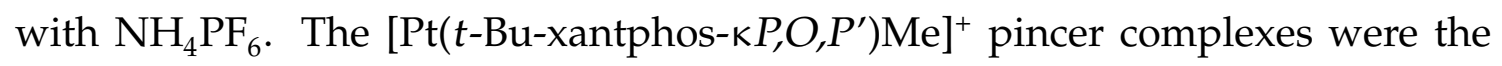
only product from reaction with $\left[\mathrm{Pt}\left(\mathrm{C}_{6} \mathrm{H}_{10}\right) \mathrm{ClMe}\right]$, with the stronger trans influence of the methyl ligand promoting loss of the chloride. The formation of the pincer complexes was further explored using DFT.

The values of $J_{\mathrm{PtC}}$ for the methyl carbons in the $\left[\mathrm{Pt}\left(t-\mathrm{Bu} \text {-xantphos- } \kappa P, O, P^{\prime}\right) \mathrm{Me}\right]^{+}$ complexes, and $J_{\mathrm{RhH}}$ for the hydride trans to the oxygen atom in the $[\mathrm{Rh}(t-\mathrm{Bu}-$ xantphos- $\left.\left.\kappa P, O, P^{\prime}\right) \mathrm{Cl}(\mathrm{H})_{2}\right]$ complexes were largest for $t$-Bu-sixantphos, then $t$-Buthixantphos, then $t$-Bu-xantphos. The trans influence of the $t$-Bu-xantphos oxygen donor follows the trend $t$-Bu-sixantphos $<t$-Bu-thixantphos $<t$-Bu-xantphos. 


\section{Acknowledgements}

As always there are numerous people that have helped me over the years. My supervisor, Prof. John Spencer, thank you for your guidance, advice and support, even coming in to University after a major earthquake to rescue our reaction. Dr. Matthias Lein, thank you for being an excellent supervisor while John was on research and study leave and for all of your assistance with DFT. Thanks to both of you for reading through various sections while on holiday.

The past and present members of the JLS research group: Almas, Brad, Chris, David, Kathryn, Lia, Rosie, Sarah and Teresa. Thank you for getting excited with me over the minutiae of NMR spectra, and for all of the good times and commiserations that we've shared. The other postgraduate students in SCPS, particularly my office mates in AM204, thanks for your friendship over the years.

The general staff of SCPS are amazingly supportive towards the research in the school and many things would be impossible without them. Particular thanks to the lab technicians Teresa, Jamie-Ann and Jackie, for letting me borrow glassware and other equipment. Thanks to Peter Northcote, Ian Vorster and John Ryan for helping with NMR. I am grateful to Rob Keyzers and Yinrong Lu for help with mass spectrometry and to Jan Wikaira, Christ Fitchett and Matt Polson at the University of Canterbury for X-ray crystallography.

Thank you to the members of Disarray, and all of my non-chemistry friends for giving me something else to think about when I just needed a break. My family have been extremely supportive and caring, as they always are. Finally to my husband, Stephen, these last 10 years have been wonderful, thank you for all of your love and understanding especially over these last few months.

I would also like to acknowledge Victoria University of Wellington for funding through the $\mathrm{PhD}$, submission, and Curtis-Gordon scholarships. 


\section{Contents}

Contents $\quad$ v

\begin{tabular}{|l|l} 
List of Figures & ix
\end{tabular}

List of Schemes $\quad x$

List of Tables $\quad$ xiii

Acronyms $\quad$ XV

1 Introduction 1

1.1 Tertiary Phosphine Ligands . . . . . . . . . . . . . . . . 1

1.1.1 Electronic and Steric Properties . . . . . . . . . . . . . . 2

1.1.2 Diphosphine Ligands . . . . . . . . . . . . . . . 3

1.1.3 Wide Bite-Angle Diphosphine Ligands . . . . . . . . . 6

1.2 Pincer Ligands . . . . . . . . . . . . . . . . . . . 6

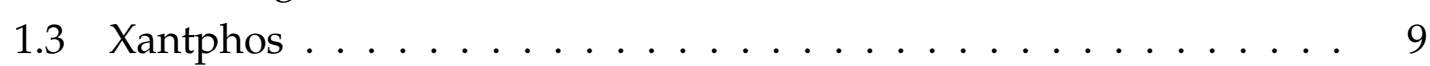

1.3.1 Alkvl-Substituted Xantphos Ligands . . . . . . . . . . . . . . 12

$1.3 .2 t$-Bu-Xantphos . . . . . . . . . . . . 16

1.4 Research Objectives . . . . . . . . . . . . . . . . . . 20

2 Ligand Synthesis and Properties 23

2.1 Ligand Svnthesis . . . . . . . . . . . . . . . . . . . . . . . . . . . . . . . . . . . . . . . . . .

2.2 Bite-Angle Calculations . . . . . . . . . . . . . . . 29

2.3 Basicity . . . . . . . . . . . . . . . . . . 33

2.4 Selenides . . . . . . . . . . . . . . . . . 38

2.5 summary . . . . . . . . . . . . . . . . . . . 45

3 Coordination Complexes with Silver 47 
3.1 Silver Chloride Complexes . . . . . . . . . . . . . . . . . . . . . . 49

3.2 Reactions with Silver Tetrafluoroborate . . . . . . . . . . . . . 56

3.2.1 Reactions with $\mathrm{LiCCPh} \ldots \ldots$. . . . . . . . . . 60

3.3 summary . . . . . . . . . . . . . . . . . 61

4 Coordination Complexes with Rhodium 63

4.1 Svnthesis of $\left[\mathrm{Rh}\left(\mathrm{k} P, \mathrm{O}, \mathrm{P}^{\prime}\right) \mathrm{Cl}\right]$ Complexes $\ldots \ldots \ldots \ldots$. . . . . . . 65

4.2 Reaction with Hydrogen . . . . . . . . . . . . . . . . . . . . . . . . . . . . 71

4.3 Rhodium Carbonvl Complexes . . . . . . . . . . . . . . . . . . . . . . . . 88

4.4 Dioxygen and Oxo Complexes . . . . . . . . . . . . . . 87

4.5 summary . . . . . . . . . . . . . . . . . . . 98

5 Coordination Complexes with Platinum(0) and Palladium(0) 101

5.1 Reactions of Ph-thixantphos with Platinum(0) Precursors ........................ 103

5.2 Reaction of $t$-Bu-xantphos Ligands with $\left[\mathrm{Pt}(\mathrm{nb})_{3}\right] \ldots . . . . . . . .112$

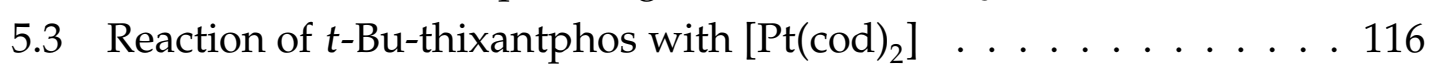

5.4 Reaction of $t$-Bu-xantphos Ligands and $\left[\mathrm{Pt}_{2}\left(\mathrm{C}_{2} \mathrm{H}_{4}\right)_{3}\right] \quad \ldots . . . . . .116$

5.5 Formation of Platinum Dioxygen Complexes . . . . . . . . . . . . . 119

5.6 Reactions of $\left[\mathrm{Pt}(t-\mathrm{Bu}\right.$-thixantphos $\left.)\left(n^{2}-\mathrm{O}_{2}\right)\right] \ldots \ldots$

5.7 Reactions with Palladium(0) Precursors . . . . . . . . . . . . . . 130

5.7.1 Reaction of $[\mathrm{Pd}(t$-Bu-thixantphos)] with Oxygen . . . . . . . 132

5.8 Computational Results . . . . . . . . . . . . . . . . . . . . 133

5.9 summary . . . . . . . . . . . . . . . . . . . . . 135

6 Coordination Complexes with Platinum(II) and Palladium(II) 141

6.1 Reactions with Platinum Dichloride Starting Materials . . . . . . 144

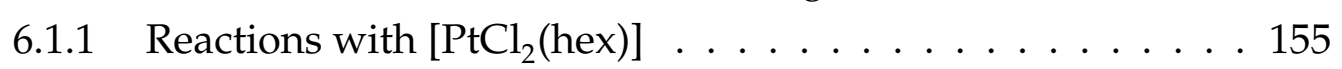

6.2 Reactions with $\left[\mathrm{Pd}(\mathrm{cod}) \mathrm{Cl}_{2}\right] \ldots \ldots \ldots \ldots 16 \ldots \ldots$

6.2.1 Formation of $\left[\mathrm{Pd}\left(t-\mathrm{Bu}-\mathrm{xantphos}-\mathrm{K} P, \mathrm{O}, \mathrm{P}^{\prime}\right) \mathrm{Cl}\right] \mathrm{PF}_{6} \mathrm{Complexes}_{163}$

6.3 Computational Results . . . . . . . . . . . . . . . . . . . . . 164

6.4 Reactions with Platinum Dimethyl Starting Materials . . . . . . . 166

6.5 Reactions with $[\mathrm{PtCl}($ hex)Mel . . . . . . . . . . . . . . . 168

6.5 .1 Computational Results . . . . . . . . . . . . . . . . 174

6.6 Summary . . . . . . . . . . . . . . . . . . . 178

\begin{tabular}{ll|l}
7 & Conclusion & 181
\end{tabular} 
8 Experimental 185

8.1 General Procedures . . . . . . . . . . . . . . . . . . . . . 185

8.2 Ligands and Non-Transition Metal Derivatives . . . . . . . . . . . . 186

8.3 Silver Complexes . . . . . . . . . . . . . . . . . . . . . . . . 192

8.4 Rhodium Complexes . . . . . . . . . . . . . . . . . . 196

8.5 Platinum Complexes . . . . . . . . . . . . . . . . . . . . . . 204

8.6 Palladium Complexes . . . . . . . . . . . . . . . . 221

$\begin{array}{lr}\text { Bibliography } & 227\end{array}$ 


\section{List of Figures}

1.1 Selection of diphosphine ligands . . . . . . . . . . . . . 4

1.2 The bite angle ...................... 5

1.3 Wide bite-angle diphosphine ligands . . . . . . . . . . . . 6

1.4 Trans-spanning diphosphine ligands . . . . . . . . . . . . 7

1.5 Naming of pincer ligands . . . . . . . . . . . . 7

1.6 First reported pincer ligands . . . . . . . . . . . . . . 8

1.7 General representation of pincer complexes . . . . . . . . . . . . 8

1.8 Selection of xantphos derivatives . . . . . . . . . . . . . . 10

2.1 Comparison of the natural and crystallographic bite-angles . . . . . 30

$2.2{ }^{1} \mathrm{H}$ NMR spectra for the $[(t \text {-Bu-xantphos }) \mathrm{H}]^{+}$ions $\ldots . . . . . . .35$

2.3 VT-NMR for $[(t \text {-Bu-thixantphos }) \mathrm{H}]^{+} \ldots \ldots \ldots \ldots$

$2.4 \mathrm{VT}^{1} \mathrm{H}$-coupled ${ }^{31} \mathrm{P}$ NMR for $[(t \text {-Bu-thixantphos }) \mathrm{H}]^{+} \ldots \ldots$. . . . . 38

2.5 X-ray crystal structure of $[(t-B u$-thixantphos $\left.) \mathrm{H}] \mathrm{CPh}\left(\mathrm{SO}_{2} \mathrm{CF}_{3}\right)_{2}\right] \ldots 39$

$3.1 \quad 1: 1$ silver diphosphine complexes . . . . . . . . . . . . . . . . . . 48

3.2 Silver $\mathrm{Ph}$-xantphos complexes . . . . . . . . . . . . . . . . . 48

3.3 X-rav crystal structure of $\mathrm{AgBr}(\mathrm{Ph}$-xantphos) . . . . . . . . . . . . . . 49

3.4 X-ray crystal structure of $\mathrm{Ag}(\mathrm{Ph}$-xantphos)(HpyS)Br . . . . . . . . . 50

3.5 NMR spectra for $[\mathrm{Ag}(t$-Bu-sixantphos $) \mathrm{Cl}] \ldots \ldots$. . . . . . . . . 51

3.6 X-rav crystal structure of $[\mathrm{Ag}(t$-Bu-thixantphos $) \mathrm{Cl}] \ldots \ldots$

3.7 X-rav crystal structure of $[\mathrm{Ag}(t$-Bu-thixantphos $) \mathrm{Cll}$, side view $\ldots .54$

3.8 Silver complexes with free coordination sites and $\mathrm{BF}_{4}{ }^{-}$counterions $\quad 57$

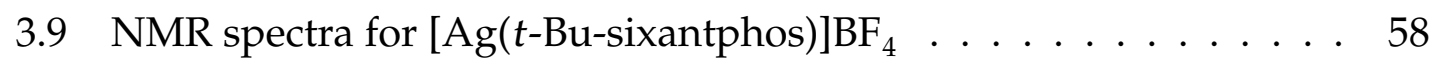

4.1 Chiral phosphine ligands used in asymmetric catalysis . . . . . . . 64

4.2 Complexes from $\left[\mathrm{Rh}(\mathrm{coe})_{2} \mathrm{Cl}_{2}\right.$ and phosphine ligands . . . . . . . . 67

$4.3{ }^{31} \mathrm{P}$ and ${ }^{1} \mathrm{H}$ NMR spectra for $[\mathrm{Rh}(t$-Bu-xantphos $) \mathrm{Cl}] \ldots$. . . . . . . 68 


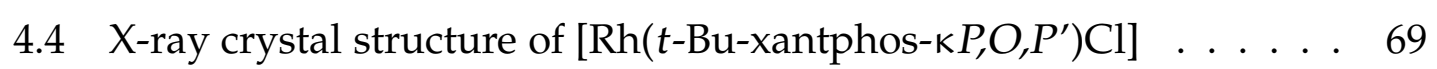

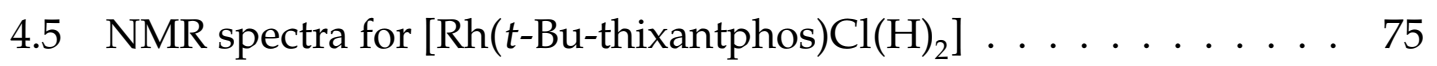

4.6 NMR spectra of $[\mathrm{Rh}(t$-Bu-sixantphos $) \mathrm{Cl}]$ with $\mathrm{CO} \ldots . . . . . .881$

4.7 Possible products from reaction of $\left[\mathrm{Rh}\left(t-\mathrm{Bu}\right.\right.$-xantphos-k $\left.\left.P, O, P^{\prime}\right) \mathrm{Cl}\right]$ complexes with $\mathrm{CO} \ldots \ldots . \ldots . \ldots . \ldots 83$

4.8 X-rav crystal structure of $\left[\mathrm{Rh}(t\right.$-Bu-xantphos $\left.) \mathrm{Cl}\left(\eta^{2}-\mathrm{O}_{2}\right)\right] \ldots . . . .90$

4.9 X-ray crystal structure of $\left[\mathrm{Rh}\left(t\right.\right.$-Bu-xantphos-k $\left.\left.P, O, P^{\prime}\right) \mathrm{Cl}(\mathrm{O})\right] \ldots 92$

4.10 Rhodium dioxygen complexes with shorter Rh-O bonds than $[\mathrm{Rh}(t-$ Bu-xantphos-kP,O, $\left.P^{\prime}\right)\left(\eta^{2}-\mathrm{O}_{2}\right) \mathrm{Cl} \ldots \ldots . . . \ldots 93$

4.11 A rhodium oxo crystal structure . . . . . . . . . . . . . . . . . . 95

4.12 Previously reported late transition metal oxo complexes . . . . . . . 96

5.1 X-rav crystal structure of $\left[\mathrm{Pt}(\mathrm{Ph} \text {-thixantphos })_{2}\right]$. . . . . . . . . . . 105

5.2 X-rav crystal structure of $\left[\mathrm{Pt}(\mathrm{Ph} \text {-thixantphos })_{2}\right] \ldots 105$

5.3 X-rav crystal structures of $\left[\mathrm{Pt}(\text { Et-xantphos })_{2}\right]$ and $\left[\mathrm{Pd}(\mathrm{Ph} \text {-xantphos })_{2}\right] 108$

5.4 Low temperature ${ }^{31} \mathrm{P}$ NMR spectra of $\left[\mathrm{Pt}(\mathrm{Ph} \text {-thixantphos })_{2}\right]$. . . . . 109

5.5 Low temperature ${ }^{1} \mathrm{H}$ NMR spectra of $\left[\mathrm{Pt}(\mathrm{Ph} \text {-thixantphos })_{2}\right]$. . . . . 110

5.6 X-ray crystal structure of $\left[\mathrm{Pt}(t-\mathrm{Bu}\right.$-thixantphos $\left.\left.)\left(\eta^{2}-\mathrm{O}_{2}\right)\right] \cdot 2 \mathrm{C}_{6} \mathrm{D}_{6}\right] \ldots 122$

5.7 X-ray crystal structure of $\left[\mathrm{Pt}(t\right.$-Bu-thixantphos $\left.)\left(\eta^{2}-\mathrm{O}_{2}\right)\right] \cdot 2 \mathrm{C}_{6} \mathrm{D}_{6}$ side view . . . . . . . . . . . . . . . . . . 123

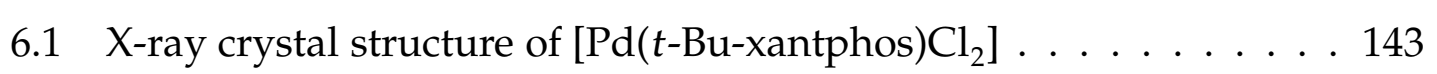

6.2 Previously reported palladium complex of $t$-Bu-xantphos and Phxantphos ........................ . . 143

6.3 X-rav crystal structure of $\left[\mathrm{Pt}(t\right.$-Bu-thixantphos $\left.) \mathrm{Cl}_{2}\right] \ldots . . . . . . .146$

6.4 X-ray crystal structure of $\left[\mathrm{Pt}(t\right.$-Bu-thixantphos $\left.) \mathrm{Cl}_{2}\right]$, side view $\ldots 146$

6.5 Distribution of the P-Pt-P angles in $\left[\mathrm{PtCl}_{2}\left(\mathrm{P}_{2}\right)\right]$ complexes . . . . . . 149

6.6 X-ray crystal structures of dichloridoplatinum complexes with transspanning diphosphine ligands . . . . . . . . . . . . . . 150

6.7 X-ray crystal structures of complexes of the type [ $\mathrm{PtCl}_{2}$ (xantphos)] 151

6.8 Side-on view of X-ray crystal structures of complexes of the type $\left[\mathrm{PtCl}_{2}\right.$ (xantphos) $\ldots \ldots \ldots$. . . . . . . . . . . 153

6.9 Energy change for conversion from $\left[\mathrm{M}(t-\mathrm{Bu}-\mathrm{xantphos}) \mathrm{Cl}_{2}\right]$ to $[\mathrm{M}(t-$

Bu-xantphos) $\mathrm{CllCl}(\mathrm{M}=\mathrm{Pd}, \mathrm{Pt}) \ldots \ldots . \ldots . \ldots . . \ldots 165$

$6.10 X$-ray crystal structures of $\left[\mathrm{PtMe}\left(\mathrm{P}^{\mathrm{i}} \mathrm{Pr}_{3}\right)_{2}\right]^{+}$and $\left[\mathrm{PdMe}\left(\mathrm{P}^{\mathrm{t}} \mathrm{Bu}_{3}\right)_{2}\right]^{+} \ldots 173$ 


\section{List of Schemes}

1.1 Chloride abstraction from [PdClMe(xantphos)] . . . . . . . . . . 13

1.2 Reactions of $[\operatorname{Rh}(i-\operatorname{Pr}$-xantphos $)] \ldots \ldots \ldots \ldots . \ldots . \ldots 15$

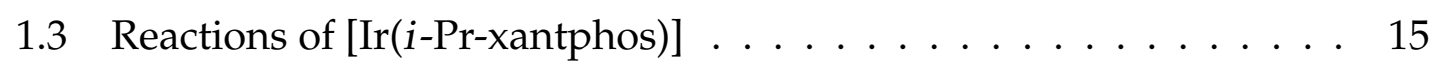

1.4 Reactions of $\mathrm{Rh}$ and $\operatorname{Ir} i$-Pr-xantphos complexes with silanes . . . . 17

2.1 Literature synthesis of $t$-Bu-xantphos . . . . . . . . . . . . . 24

2.2 Ligand svnthesis . . . . . . . . . . . . . . . . . . . 25

2.3 Reaction of n-butyllithium with diethyl ethen . . . . . . . . . . 26

2.4 Influence of methvl groups on the synthesis of $t$-Bu-thixantphos . . 27

2.5 Protonation of the ligands using a strong acid . . . . . . . . . 34

2.6 Selenation of $t$-Bu-xantphos ligands . . . . . . . . . . . 42

3.1 Synthesis of $[\mathrm{Ag}(t$-Bu-xantphos $) \mathrm{Cl}]$ complexes $\ldots \ldots \ldots \ldots . . .52$

3.2 Inversion of the xantphos backbone in $[\mathrm{Ag}(t-\mathrm{Bu}$-xantphos $) \mathrm{Cl}] \ldots 56$

3.3 Synthesis of $[\mathrm{Ag}(t-\mathrm{Bu}-\mathrm{xantphos})] \mathrm{BF}_{4}$ complexes $\ldots \ldots \ldots \ldots . . . \quad 59$

4.1 Asvmmetric catalvtic hydrogenation developed by Knowles . . . . 64

4.2 Reaction of $\left[\mathrm{Rh}\left(\mathrm{Coe}_{2} \mathrm{Cl}_{2}\right.\right.$ and $t$-Bu-xantphos ligands . . . . . . 68

4.3 Catalytic cycle for homogeneous hydrogenation . . . . . . . . 72

4.4 Catalytic cycle for homogeneous hydroformylation . . . . . . 73

4.5 Reaction of $[\mathrm{Rh}(t$-Bu-xantphos $) \mathrm{Cl}]$ with hydrogen $\ldots \ldots \ldots . . .74$

4.6 Reaction of $[\mathrm{Rh}(t$-Bu-xantphos $) \mathrm{Cl}]$ with carbon monoxide . . . . . 86

4.7 Reaction of $[\mathrm{Rh}(t$-Bu-xantphos $) \mathrm{Cl}]$ complexes with oxvgen . . . . . 89

4.8 Reaction of $[\mathrm{Rh}(t$-Bu-xantphos $) \mathrm{Cl}]$ with trimethylamine oxide . . . 98

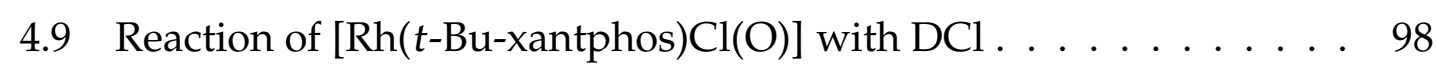

5.1 Reaction of $\mathrm{Ph}$-thixantphos with tris(norbornene)platinum . . . . 104

5.2 Reaction between $\mathrm{tBu}$-thixantphos and $\left[\mathrm{Pt}(\mathrm{nb})_{3}\right] \ldots \ldots . . . . .113$

5.3 Reaction between $t$-Bu-xantphos and $\left[\mathrm{Pt}(\mathrm{nb})_{3}\right] \ldots \ldots . . . .114$ 
5.4 Reaction between $t$-Bu-thixantphos and $\left[\mathrm{Pt}\left(\mathrm{C}_{2} \mathrm{H}_{4}\right)_{3}\right] \ldots 117$

5.5 Reaction of [Pt(alkene)( $t$-Bu-thixantphos)] and [Pt(t-Bu-thixantphos)] with air . . . . . . . . . . . . . . . . . . . . . . . 119

5.6 Reaction between $\mathrm{Pt}(t$-Bu-thixantphos $\left.)\left(\eta^{2}-\mathrm{O}_{2}\right)\right]$ and $\mathrm{CO} \ldots 129$

5.7 Synthesis of $[\mathrm{Pd}(t$-Bu-xantphos $)] \ldots$. . . . . . . . . . . . . 132

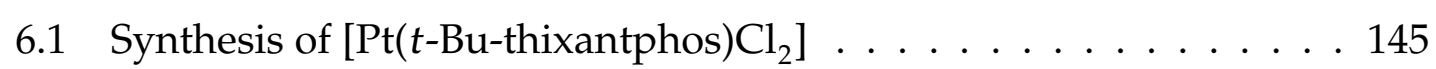

6.2 Equilibrium between $\left[\mathrm{Pt}(t\right.$-Bu-thixantphos $\left.) \mathrm{Cl}_{2}\right]$ and $\mathrm{Pt}(t$-Bu-thixant-

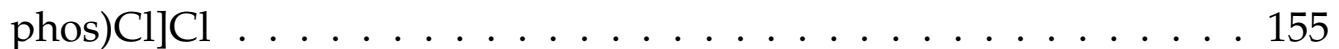

6.3 Synthesis of $\left[\mathrm{Pd}(t-\mathrm{Bu}-\mathrm{xantphos}) \mathrm{Cl}_{2}\right] \ldots \ldots . . \ldots . . \ldots 161$

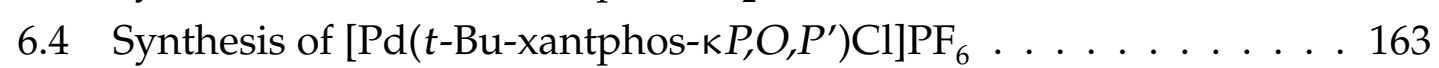

6.5 Attempted reaction of $\left[\mathrm{Pt}(\right.$ hex $\left.) \mathrm{Me}_{2}\right]$ and $t$-Bu-thixantphos . . . . . 167

6.6 Proposed reaction between $\left[\mathrm{Pt}(\mathrm{hex}) \mathrm{Me}_{2}\right]$ and a diphosphine ligand 168

6.7 Reaction between $[\mathrm{PtCl}($ hex $) \mathrm{Me}]$ and $t$-Bu-xantphos ligands $\ldots . .169$

6.8 Equilibrium between $[\mathrm{Pt}(t$-Bu-xantphos $) \mathrm{Me}]$ and $[\mathrm{Pt}(t$-Bu-xantphos$\left.\left.\kappa P, O, P^{\prime}\right) \mathrm{Me}\right] \ldots \ldots \ldots . \ldots \ldots$. . . . . . . . . . . . . . . . . . . . . . . 


\section{List of Tables}

2.1 Selected NMR data for $t$-Bu-xantphos ligands . . . . . . . . . . . 28

2.2 Crystallographic and natural bite-angles for diphosphine ligands . 31

2.3 Natural bite-angles of xantphos ligands . . . . . . . . . . . . 32

2.4 Selected NMR data for $\left.[(t-B u-x a n t p h o s) H] C H\left(\mathrm{SO}_{2} \mathrm{CF}_{3}\right)_{2}\right] \ldots . . \quad \ldots 34$

2.5 Selected bond distances $(\AA)$ and angles $\left(^{\circ}\right)$ of $2[(t$-Bu-thixantphos $) \mathrm{H}]$

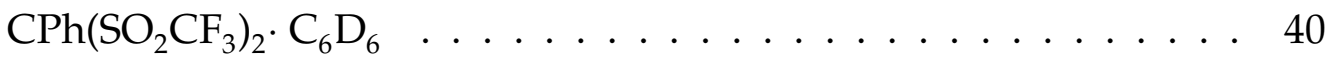

2.6 Crystallographic data of $2[t$-Bu-thixantphos $\left.(\mathrm{H})] \mathrm{CPh}\left(\mathrm{SO}_{2} \mathrm{CF}_{3}\right)_{2} \cdot \mathrm{C}_{6} \mathrm{D}_{6}\right] 41$

2.7 Selected ${ }^{31} \mathrm{P}$ NMR data for $t$-Bu-xantphos selenides . . . . . . . . 44

3.1 Selected NMR data of $[\mathrm{Ag}(t-\mathrm{Bu}$-xantphos $) \mathrm{Cl}$ complexes $\ldots . . .52$

3.2 Selected bond distances and angles of $[\mathrm{Ag}(t-\mathrm{Bu}$-thixantphos $) \mathrm{Cl}] \ldots 54$

3.3 Crystallographic data for $[\mathrm{Ag}(t-B u-$ thixantphos $) \mathrm{Cl}] \ldots \ldots \ldots$

3.4 Selected NMR data of $[\mathrm{Ag}(t-\mathrm{Bu}$-xantphos $)] \mathrm{BF}_{4}$ complexes $\ldots . . . .59$

4.1 Selected NMR data of $[\mathrm{Rh}(t-\mathrm{Bu}$-xantphos $) \mathrm{Cl}]$ complexes $\ldots . .69$

4.2 Chemical shift and coupling of the O-ipso carbon in the free ligand, $[\mathrm{Ag}(t-\mathrm{Bu}$-xantphos $) \mathrm{Cl}]$ and $[\mathrm{Rh}(t-\mathrm{Bu}$-xantphos $) \mathrm{Cl}]$ complexes $\quad 70$

$4.3 \quad$ Hvdride ${ }^{1} \mathrm{H} N M R$ data for $\left[\mathrm{Rh}\left(t-\mathrm{Bu}\right.\right.$-xantphos- $\left.\left.\mathrm{K} P, \mathrm{O}, P^{\prime}\right) \mathrm{Cl}(\mathrm{H})_{2}\right] \ldots 76$

4.4 Selected NMR data of [Rh(t-Bu-xantphos)(CO$\left.)_{2} \mathrm{Cl}\right]$ complexes $\ldots 80$

4.5 Chemical shift and coupling for the O-ipso carbon in t-Bu-xantphos, $\left[\mathrm{Rh}\left(t\right.\right.$-Bu-xantphos-k $\left.\left.P, \mathrm{O}, P^{\prime}\right) \mathrm{Cl}\right]$ and carbonyl complexes . . . 84

4.6 Selected NMR data for $\left[\mathrm{Rh}\left(t-\mathrm{Bu}\right.\right.$-xantphos- $\left.\left.\mathrm{K} P, \mathrm{O}, P^{\prime}\right) \mathrm{Cl}\left(\eta^{2}-\mathrm{O}_{2}\right)\right] \ldots 88$

4.7 Crystallographic data and structure refinement of $[\mathrm{Rh}(t-\mathrm{Bu}-\mathrm{xant}-$ $\left.\operatorname{phos}) \mathrm{Cl}\left(\eta^{2}-\mathrm{O}_{2}\right)\right] \ldots \ldots \ldots \ldots \ldots \ldots \ldots \ldots$

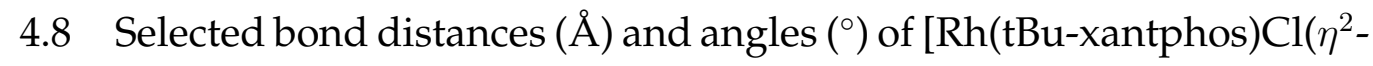

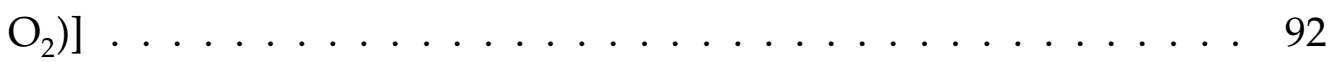

5.1 Selected bond distances $(\AA)$ and angles $\left({ }^{\circ}\right)$ of $\left[\mathrm{Pt}(\mathrm{Ph} \text {-thixantphos })_{2}\right] \quad 106$ 
5.2 Crystallographic data and refinement of $\left[\mathrm{Pt}(\mathrm{Ph} \text {-thixantphos })_{2}\right] \ldots 107$

$5.3{ }^{31} \mathrm{P} N M R$ data for $[\mathrm{Pt}(t$-Bu-xantphos $)]$ complexes . . . . . . . . . . 115

$5.4{ }^{31} \mathrm{P} N M R$ data for $\left[\mathrm{Pt}(t\right.$-Bu-xantphos $\left.)\left(\mathrm{C}_{2} \mathrm{H}_{4}\right)\right]$ complexes . . . . . . . 118

5.5 Selected bond distances $(\AA)$ and angles $\left(^{\circ}\right)$ of $\left[\mathrm{Pt}(t\right.$-Bu-thixantphos $)\left(\eta^{2}\right.$ -

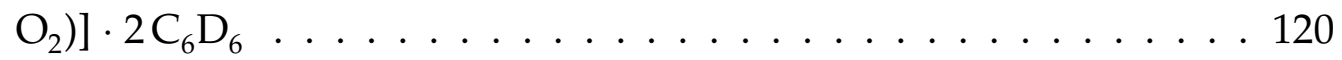

5.6 Crystallographic data and structure refinement of [ $\mathrm{Pt}(t-\mathrm{Bu}$-thixantphos $\left.)\left(\eta^{2}-\mathrm{O}_{2}\right)\right] \cdot 2 \mathrm{C}_{6} \mathrm{D}_{6} \ldots \ldots \ldots \ldots$. . . . . . . . . . 121

5.7 Selected bond distances and angles of $\left[\mathrm{Pt}(\mathrm{PP})\left(\eta^{2}-\mathrm{O}_{2}\right)\right]$

5.8 Calculated bond lengths and angles for $[\mathrm{M}(t-\mathrm{Bu}$-xantphos $)]$. . . . . 134

5.9 Calculated bond lengths and angles for $[\mathrm{M}(t$-Bu-xantphos $)(\mathrm{nb})]$. . 134

5.10 Calculated bond lengths and angles for $\mathrm{M}(t$-Bu-xantphos $\left.)\left(\eta^{2}-\mathrm{O}_{2}\right)\right] \quad 134$

5.11 Gibbs free energies calculated for the formation of [M(t-Bu-xantphos $)(n b)] \ldots \ldots \ldots$. . . . . . . . . . . . . . . . . . . . . . . . .

5.12 Gibbs free energies calculated for the formation of [M(t-Bu-xantphos $\left.)\left(\eta^{2}-\mathrm{O}_{2}\right)\right] \ldots \ldots \ldots$. . . . . . . . . . . . . . . . . . . . . . . . . .

6.1 Bond distances $(\AA)$ and angles $\left(^{\circ}\right)$ of $\left[\mathrm{Pt}(t\right.$-Bu-thixantphos $\left.) \mathrm{Cl}_{2}\right]$. . . 145

6.2 Crystallographic data and structure refinement of [ $\mathrm{Pt}(t-\mathrm{Bu}$-thixantphos $) \mathrm{Cl}_{2}$. . . . . . . . . . . . . . . . . . . . . 147

6.3 Selected NMR data for $\left[\mathrm{Pt}(\right.$ xantphos $\left.) \mathrm{Cl}_{2}\right]$ complexes . . . . . . . . . 158

6.4 Selected NMR data for $\left[\mathrm{Pt}(t-\mathrm{Bu}\right.$-xantphos $\left.) \mathrm{Cl}_{2}\right]$ and $[\mathrm{Pt}(t-\mathrm{Bu}$-xant-

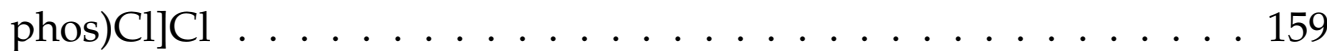

6.5 Selected NMR data for $[\mathrm{Pt}(t-\mathrm{Bu}$-xantphos $) \mathrm{Cl}] \mathrm{X}$ complexes $\ldots . . .160$

6.6 Selected $\mathrm{NMR}$ data for $\left[\mathrm{Pd}(t\right.$-Bu-xantphos $\left.) \mathrm{Cl}_{2}\right]$ complexes $\ldots . .$.

6.7 Selected NMR data for $\left[\mathrm{Pd}(t\right.$-Bu-sixantphos $\left.) \mathrm{Cl}_{2}\right] \ldots \ldots 2$

6.8 Selected $\mathrm{NMR}$ data for $\left[\mathrm{Pd}(t-\mathrm{Bu}\right.$-xantphos $) \mathrm{Cl}_{\mathrm{PF}}$ complexes . . . . 164

6.9 Selected NMR Data for $[\mathrm{Pt}(t$-Bu-xantphos)MelCl complexes. . . . . 171

6.10 Selected bond lengths and angles for $[\mathrm{Pt}(t-\mathrm{Bu} \text {-xantphos }) \mathrm{Me}]^{+} \ldots . .175$

6.11 Selected bond lengths and angles for $\left[\mathrm{Pt}\left(t-\mathrm{Bu} \text {-xantphos-k } P, \mathrm{O}_{,} \mathrm{P}^{\prime}\right) \mathrm{Me}\right]^{+} 175$

6.12 Gibbs free energies calculated for agostic and pincer $[\mathrm{Pt}(t-\mathrm{Bu}$-xantphos)Me $]^{+} \ldots \ldots \ldots \ldots \ldots \ldots$. . . . . . . . . . . . . . . . . . . . . .

6.13 Energy change for the conversion of the [ $\mathrm{Pt}(t-\mathrm{Bu}$-xantphos $) \mathrm{ClMe}]$ to the pincer complex . . . . . . . . . . . . . . . . 177

6.14 Energy change for the conversion of the [Pt(t-Bu-xantphos)ClMe] to the agostic complex . . . . . . . . . . . . 177 


\section{Acronyms}

BINAP 2,2'-bis(diphenylphosphino)-1,1'-binaphthyl

BISBI 1,1'-bis(diphenylphosphinomethyl)-2,2'-biphenyl

cod 1,5-cyclooctadiene

coe cyclooctene

CSD Cambridge Structural Database

Cyp cyclopentyl

dba dibenzylideneacetone

dbpx $\alpha, \alpha^{\prime}$-bis(di-t-butylphosphino)-o-xylene

dcype 1,2-bis(dicyclohexylphosphino)ethane

depp 1,2-bis(diethylphosphino)propane

depPE bis(2-diethylphosphinophenyl)ether

depx $\alpha, \alpha^{\prime}$-bis(diethylphosphino)-o-xylene

DFT density functional theory

diop O-isopropylidene-2,3-dihydroxy-1,4-bis(diphenylphosphino)butane

DPEphos bis(2-diphenylphosphinophenyl)ether

dppb 1,2-bis(diphenylphosphino)butane

dppe 1,2-bis(diphenylphosphino)ethane

dppf 1,1'-bis(diphenylphosphino)ferrocene

dppp 1,2-bis(diphenylphosphino)propane 
esd estimated standard deviation

HMDA hexamethylene-1,6-diamine

IR infrared

nb norbornene, bicyclo[2.2.1]hept-2-ene

NMR nuclear magnetic resonance

pta 1,3,5-triaza-7-phosphaadamantane

py 2-pyridyl

SEGphos 4,4'-Bi-1,3-benzodioxole-5,5'-diylbis(diphenylphosphane)

tht tetrahydrothiophene

TMEDA $N, N, N^{\prime}, N^{\prime}$-tetramethyl-ethane-1,2-diamine

TOF turnover frequency

TON turnover number

xantphos 4,5-bis(diphenylphosphino)-9,9-dimethylxanthene 


\section{Chapter 1}

\section{Introduction}

This thesis provides an account of research into a group of diphosphine ligands with a rigid xanthene backbone and tert-butyl substituents on the phosphorus atoms. The three ligands have different groups in the bridgehead position of the backbone $\left(\mathrm{CMe}_{2}, \mathrm{SiMe}_{2}\right.$, or S) which changes the natural bite-angle of the ligand. The coordination chemistry of these $t$-Bu-xantphos ligands with late-transition metals has been investigated with a focus on metal complexes that may form in catalytic reactions.

\subsection{Tertiary Phosphine Ligands}

Transition metal complexes have a number of different applications, from use as homogeneous catalysts, chemotherapeutics, or organic light emitting diodes. $\frac{1-3 \underline{\underline{3}}}{2}$ Tertiary phosphine ligands are some of the most ubiquitous in coordination chemistry. Phosphines coordinate to transition metals by donation of the lone pair of electrons on the phosphorus atom forming a $\sigma$-bond and $\pi$-back-donation into the P-C anti-bonding $\sigma^{*}$ orbitals which have $\pi$ symmetry. ${ }^{\underline{4}}$ This combination forms stable coordination complexes with a range of different transition metals and oxidation states. A large array of different phosphine ligands are known with a range of different steric and electronic properties. These allow the phosphines to impart different physical environments and electron densities on the metal centre, which can result in different reactivities of the complexes. Due to this, phosphine ligands are frequently used as ancillary ligands in a wide range of different 
catalytic systems including industrial-scale processes. $\cdot \underline{\underline{5}-\underline{\underline{7}}}$

\subsubsection{Electronic and Steric Properties}

As the chemical properties of the metal in a coordination complex are controlled largely by the coordinated ligands, several studies have quantified the electronic and steric properties of phosphine ligands. $\underline{\underline{8}-\underline{14}}$

Investigations of electronic properties of ligands involve the measurement of the $\mathrm{C} \equiv \mathrm{O}$ stretching frequency in a metal carbonyl complex such as $\left[\mathrm{Ni}(\mathrm{CO})_{3} \mathrm{~L}\right]$, $\left[\mathrm{Mo}(\mathrm{CO})_{5} \mathrm{~L}\right]$ or $\left[\mathrm{Rh}(\mathrm{CO}) \mathrm{ClL}_{2}\right] * \frac{* 8,9,11,14}{}$ If a metal with a spin active isotope such as rhodium or platinum is used, then the value of the one-bond metal-phosphorus coupling constant can also offer insight into the electronic properties of the phosphine ligand. $\frac{9,11,12,16}{1}$ The value of the one-bond phosphorus-selenium coupling constant in a phosphinoselenide has been used as a way to study the electronic properties of phosphines. $\frac{17,18}{12}$ The phosphinoselenides are readily synthesised, air-stable solids which avoids the need for transition metals or the use of toxic gases. A comparison of the different techniques used to measure the electronic properties has shown good correlation between the various series. $\frac{9}{}$

The steric properties of different ligands have also been examined from a quantitative perspective. The Tolman cone angle is the angle formed at the apex of a cone centred at a metal positioned $2.28 \AA$ from the phosphorus atom where the outer edges of the cone lie at the van der Waals radii of the outer most atom of the ligand..$^{14}$ The Tolman cone angle has some limitations: it was originally determined using physical molecular models with idealised bond lengths and van der Waals radii while actual molecules may vary from these values. In addition, the Tolman cone angle is a maximum cone angle which works well for approximately symmetrical spherical substituents such as $\mathrm{PMe}_{3}$ or $\mathrm{P}^{\mathrm{t}} \mathrm{Bu}_{3}$, however when planar rings, such as phenyl substituents, are present these can rotate readily, resulting in much larger changes to the cone angle than a trialkyl phosphine would exhibit. The Tolman cone angles for aryl systems tends to be larger than the crystallographically determined cone angle.

An alternative quantification of the steric properties of phosphine ligands is the

${ }^{*}$ The nomenclature used in this thesis is in accordance with the Nomenclature of Inorganic Chemistry - IUPAC Recommendations 2005. $\underline{\underline{15}}$ 
percentage buried volume which is defined as the percentage of a sphere that is occupied by a given ligand. $\underline{19}$ This sphere has a metal atom at the centre and a defined radius to allow for comparison across different ligands. The percent buried volume for a phosphine ligand is calculated using parameters obtained from the crystal structure of that ligand with hydrogen atoms omitted. The resulting values generally correlate well with the values of the Tolman cone angle $\left(\mathrm{R}^{2}=0.959\right) . \underline{\underline{20}}$ However, the values of percentage buried volume for phosphite ligands do not fit the trend, with the correlation giving calculated cone angles of 128 and $155^{\circ}$ for $\mathrm{P}(\mathrm{OMe})_{3}$ and $\mathrm{P}(\mathrm{OPh})_{3}$ respectively instead of the values from Tolman's work of 128 and $155^{\circ} \underline{\underline{14}}$

\subsubsection{Diphosphine Ligands}

Diphosphine ligands consist of two tertiary phosphines linked by a backbone. A selection of diphosphine ligands are shown in Figure 1.1. Most commonly, the backbone has a carbon skeleton such as an ethane, propane, or xylene group as found in dppe, dppp, or dbpx. Larger groups can also be used as backbones, such as the binapthyl ligand BINAP, or the BISBI system. The backbones can also include heteroatoms such as diop or DPEphos, or transition metals as in the ferrocene moiety found in dppf. The backbone can also be used to produce chiral ligands including BINAP, diop or SEGphos, which can be used for asymmetric catalysis. $\frac{5,21,22}{}$ Diphosphine ligands typically chelate to transition metals, resulting in enhanced stability through the chelate effect. The chelate effect is an entropic effect, where a complex with a bidentate ligand is observed to have increased thermodynamic stability compared to an analogous complex with similar monodentate ligands. $\frac{\underline{23}}{}$ In monophosphine complexes, the monophosphine must compete with the other components in the reaction mixture as there is no entropic driver for a second molecule of the monophosphine to coordinate over any other ligands in the system. Hence the increased thermodynamic stability of complexes with bidentate chelating ligands compared to the analogous complex with related monodentate ligands.

The electronic and steric properties of diphosphine ligands can be described using the techniques developed for monophosphines. $\underline{\underline{8}-14}$ The bite-angle, defined as the P-M-P angle in a transition-metal complex (Figure 1.2), can also be used to quantify and compare different diphosphine ligands. The natural bite-angle is 


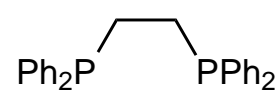

(a) dppe<smiles>c1ccc(-c2ccc3ccccc3c2-c2c(P(c3ccccc3)c3ccccc3)ccc3ccccc23)cc1</smiles>

(d) (S)-BINAP

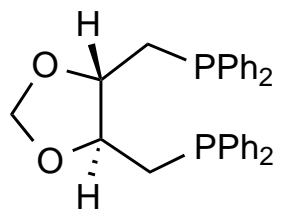

(g) $(R, R)$-diop

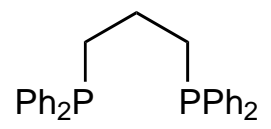

(b) dppp<smiles>c1ccc(Pc2ccccc2Cc2ccccc2-c2ccccc2)cc1</smiles>

(e) BISBI

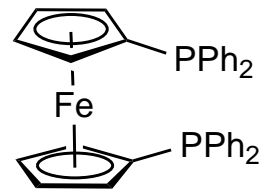

(h) dppf<smiles>CCCCCCCCCCCc1ccccc1CP</smiles>

(c) dbpx<smiles>Pc1ccccc1Oc1cccc(P)c1-c1ccccc1</smiles>

(f) DPEphos<smiles>Pc1cccc(-c2c(-c3ccccc3)ccc3c2OCO3)c1-c1ccccc1</smiles>

(i) (R)-SEGphos

Figure 1.1: Selection of diphosphine ligands. 
defined as the P-Rh-P angle calculated by molecular modelling using a rhodium atom with fixed Rh-P distances of $2.315 \AA .24$ The bite-angle for a given complex can also be measured from an X-ray crystal structure. The original development of the natural bite-angle parameter included a comparison of the natural and crystallographic bite-angles for seven different complexes, showing good correlation between the natural and crystallographic bite-angles. ${ }^{24} \mathrm{~A}$ further study in 1999 showed that, for a range of different diphosphine ligands, the crystallographic bite-angles occur within a very narrow range which correlate well with the natural bite-angles. $\underline{25}$

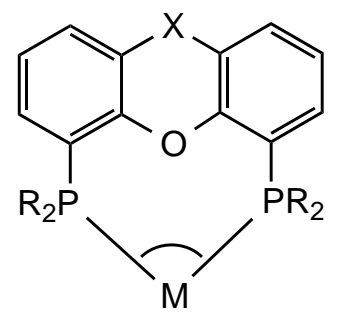

Figure 1.2: The bite angle

When used as ancillary ligands in transition metal catalysts, diphosphines can produce different product distributions compared with the analogous monophosphines. The catalytic reaction of ethene and carbon monoxide, in a methanol solution, produced short chain oligomers when $\left[\mathrm{Pd}(\mathrm{MeCN})_{4}\right]\left(\mathrm{BF}_{4}\right)_{2}$ was used as the precatalyst with an excess of a monophosphine ligand such as triphenylphosphine. ${ }^{26}$ Changing to a stoichiometric amount of a chelating diphosphine with $\left[\mathrm{Pd}(\mathrm{OAc})_{2}\right]\left(\mathrm{OAc}=\mathrm{CH}_{3} \mathrm{COO}^{-}\right)$, resulted in the co-polymerisation of carbon monoxide and ethene giving polyketone, indicating that the rate of chain termination is faster for monophosphine complexes than in the diphosphine complexes. $\frac{27}{\text { The }}$ bite-angle of the diphosphine was shown to have a significant impact on the rate of the reaction. When the $\mathrm{Ph}_{2} \mathrm{P}\left(\mathrm{CH}_{2}\right)_{\mathrm{n}} \mathrm{PPh}_{2}(\mathrm{n}=1-6)$ series of ligands was studied, the highest reaction rate was observed for 1,2-bis(diphenylphosphino)propane (dppp) ( $\mathrm{n}=3)$ with the rate decreasing as the chain length was increased or decreased. ${ }^{27}$ The steric bulk of the ligands is also important, changing the phenyl substituents on dppp for tert-butyl groups resulted in high selectivity for the mono-insertion product methyl propionate. ${ }^{28}$ Introducing further rigidity to the system by changing to the xylene backbone ligand $\alpha, \alpha^{\prime}$-bis(di- $t$-butylphosphino)$o$-xylene $(\mathrm{dbpx})$ resulted in methyl propionate exclusively. ${ }^{29}$ 


\subsubsection{Wide Bite-Angle Diphosphine Ligands}

The bite-angle of diphosphine ligands impacts the types of coordination complexes that will form upon reaction with an appropriate metal precursor. Ligands with bite-angles around $90^{\circ}$ will typically coordinate in a cis geometry in square-planar and octahedral complexes and occupy axial-equatorial positions in trigonal bipyramidal complexes. Ligands with natural bite-angles closer to $120^{\circ}$ can coordinate in a bis-equatorial arrangement in the trigonal bipyramidal complexes. $\frac{30}{\underline{3}}$ Two of the most important catalytic steps are oxidative addition and reductive elimination, which result in changes to the coordination number and

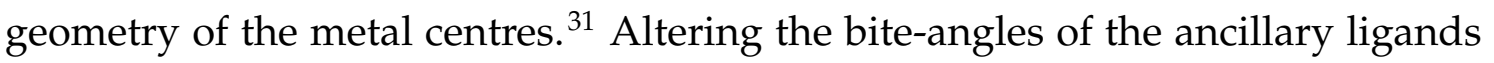
can have a significant impact on the selectivity and reactivity of these reactions, typically with larger bite-angle ligands favouring reductive elimination steps. $\underline{\underline{32}}$ The strain on ligands to coordinate with bite-angles significantly different to their natural bite-angle, can result in destabilisation of particular intermediates in catalytic cycles leading to increased selectivity. ands with large bite-angles have been reported, including dbpx, BISBI, DBFphos and Ph-xantphos (Figures, 1.1c, 1.1e, 1.3). Diphosphines with extremely large bite-angles, designed to coordinate in an exclusively trans geometry in squareplanar complexes have been reported, such as TRANSphos, SPANphos, and norphos (Figure 1.4). $\frac{25,33,34}{.3}$<smiles>CC1(C)c2cccc(-c3ccccc3)c2Oc2c(-c3ccccc3)cccc21</smiles>

(a) Ph-xantphos<smiles></smiles>

(b) DBFphos

Figure 1.3: Wide bite-angle diphosphine ligands.

\subsection{Pincer Ligands}

Diphosphine ligands such as Ph-xantphos with wide bite-angles and a heteroatom in the centre of the backbone have the potential to act as pincer ligands. Pincer ligands have attracted research attention due to the unique balance of stability and 
<smiles></smiles>

(a) TRANSphos<smiles>Cc1cc(P)c2c(c1)C(C)(C)CC1(CC(C)(C)c3cc(C)cc(-c4ccccc4)c3O1)O2</smiles>

(b) SPANphos

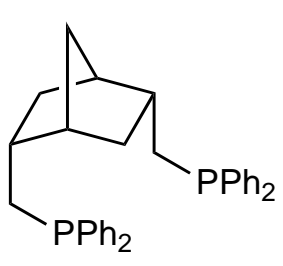

(c) norphos

Figure 1.4: Selected trans-spanning diphosphine ligands.

reactivity that they impart on transition metal complexes. $\underline{35}$ Pincer ligands are tridentate ligands that coordinate to transition metals preferentially in a meridional fashion. $\underline{\underline{36}}$ The ligands are typically named according to their donor atoms, such as PCP, POP or NCN (Figure 1.5). If the groups between the donor atoms contain heteroatoms then these may be included in the naming also, for example POCOP. The pincer ligands may be anionic (as with PCP ligands) or neutral (PNP and POP)..$\underline{37,38}$ Although phosphines are the most common donor groups, amines, $\underline{\underline{39}}$ imines, $\underline{\underline{40}}$ thioethers $\underline{41}$ and N-heterocyclic carbenes $\underline{42}$ have all been reported.

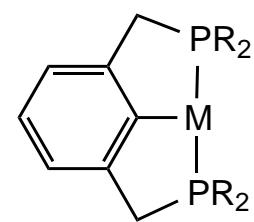

PCP

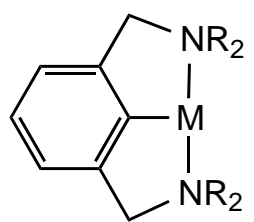

NCN<smiles>Pc1[M]ccc1</smiles>

POP<smiles>[R7]POc1cccc2c1C(P)O2</smiles>

POCOP

Figure 1.5: Naming of pincer ligands.

Pincer ligands were first reported in 1976 by Shaw $\underline{\underline{43}}$ and Alcock. $\underline{\underline{44}}$ Shaw reported a tert-butyl PCP ligand (Figure 1.6a) and introduced the naming scheme that has become commonplace for pincer ligands. When reacted with an appropriate metal precursor, complexes of the tridentate ligand formed with nickel, palladium, platinum, rhodium, and iridium with chloride, nitrile, hydride, and carbon monoxide ligands. $\underline{43}$ Alcock reported the first POP pincer ligands together with their rhodium carbonyl complexes, characterised by X-ray crystallography (Figure 1.6b). .44

The different components of pincer ligands have a significant influence on the steric and electronic properties and hence their reactivity. ${ }^{39}$ Altering the central 


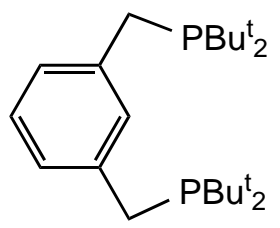

(a)

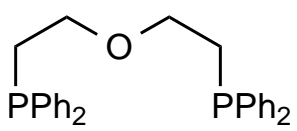

(b)

Figure 1.6: First reported pincer ligands.

donor group, X (Figure 1.7), can lead to changes in electronic effects mostly through the trans-influence. ${ }^{36}$ For example, a carbon donor ligand has a greater transinfluence than an oxygen donor. Thus ligands trans to $\mathrm{X}$ in PXP complexes will be bound more strongly when $\mathrm{X}=\mathrm{O}$ than $\mathrm{X}=\mathrm{C}$. $\stackrel{45}{ }$ The donor group $\mathrm{Y}$ controls the steric environment around the metal centre and the electron density. Changing the backbone and other remote groups gives control over the electron density on the metal and can be used to improve solubility properties. $\underline{\underline{36}}$ The tridentate coordination of pincer ligands, typically forming two five-membered metallacycles, imparts significant stability to metal complexes with pincer ligands.

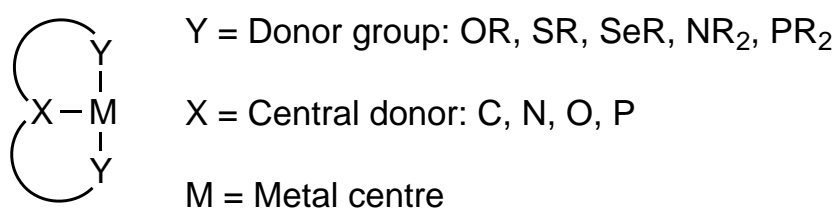

Figure 1.7: General representation of pincer complexes.

Coordination complexes of pincer ligands have a large number of applications. Platinum complexes of an NCN pincer ligand have been utilised as sensors for the detection of sulfur dioxide. $\underline{\underline{46}} \underline{\underline{48}}$ Palladium and nickel complexes of a number of pincer ligands have shown activity in cross-coupling reactions. $\underline{41,42,49}-\underline{\underline{51}}$ Theoretical studies have shown potential uses for pincer ligands in water-splitting 52 and nitrogen fixation..$\underline{53}$ However, one of the most prominent uses of pincer ligands is the activation of C-H bonds, typically as dehydrogenation catalysts. $\underline{36,48,54}$ 


\subsection{Xantphos}

First reported in 1995 by van Leeuwen et al., the xantphos $t$ class of diphosphine ligands were designed to investigate the influence of the bite-angle on catalytic reactions, in particular rhodium catalysed hydroformylation. $\underline{\underline{30}}$ The general structure and a selection of different xantphos derivatives are given in Figure 1.8. The first paper on xantphos included derivatives where the $\mathrm{CMe}_{2}$ group in the backbone of Ph-xantphos (Figure 1.8d) was replaced by a S (Ph-thixantphos, Figure 1.8f), $\mathrm{SiMe}_{2}$ (Ph-sixantphos, Figure 1.8e), a direct bond between the atoms (DBFphos, Figure 1.8c), or removed entirely (DPEphos, Figure 1.8b). Since then a vast array of derivatives have been reported. The most common position for derivatisation is the bridgehead position (occupied by a $\mathrm{CMe}_{2}$ group in $\mathrm{Ph}$-xantphos). Changes to this position can create changes in the natural bite-angles of the ligands. Another site for derivatisation is the substituents on the phosphorus atoms which have been changed for cyclic groups, chiral derivatives or alkyl chains including methyl, ethyl, isopropyl and tert-butyl groups. The phosphorus donors have also been replaced with a range of different groups including phosphonites, amines, imines, arsines, and thioethers. $\underline{55-58}$ The third site for derivatisation is the position meta to the phosphorus atoms on the backbone phenyl rings. In Phxantphos this position is occupied by hydrogen atoms, while in Ph-thixantphos methyl groups are present. Derivatives with tert-butyl or sulfate groups have also been reported. ${ }^{59-61}$ these alterations all result in changes to the bite-angle of the ligand.

The influence of the bite-angle of diphosphine ligands on the selectivity and activity of their transition metal complexes in various catalytic process has been investigated using the xantphos ligands. The hydroformylation of alkenes to give branched and linear aldehydes is an industrially important reaction with the lin-

\footnotetext{
${ }^{\dagger}$ The term xantphos is used in the literature to mean either the general class of ligands or the specific ligand 9,9-dimethyl-4,6-bis(diphenylphosphino)xanthene. For the purposes of this thesis the specific ligand will be referred to as Ph-xantphos and the term xantphos will be used to represent a generic ligand from this class.

${ }^{\ddagger}$ In the literature two different structures are commonly referred to as $t$-Bu-xantphos, one with tert-butyl substituents on the aromatic backbone and one with tert-butyl groups on the phosphorus atoms. For the purpose of this thesis the structure with the tert-butyl substituents on the phosphorus will be named $t$-Bu-xantphos and the structure with the tert-butyl groups on the aromatic backbone will be named $t$-Bu-(Ph-xantphos).
} 
<smiles>[R7]c1cc([Y20])c2c(c1)[X]c1cc([R])cc([Y20])c1O2</smiles>

(a) General structure<smiles>CC1(C)c2cccc(-c3ccccc3)c2Oc2c(-c3ccccc3)cccc21</smiles>

(d) Ph-xantphos, $111^{\circ}$<smiles>CC(C)(C)c1cc(-c2ccccc2)c2c(c1)C(C)(C)c1cc(C(C)(C)C)cc(-c3ccccc3)c1O2</smiles>

(g) $t$-Bu-(Ph-xantphos), $110^{\circ}$<smiles></smiles>

(j) Xantarsine, $113^{\circ}$<smiles>c1ccc(-c2ccccc2Oc2ccccc2-c2ccccc2)cc1</smiles>

(b) DPEphos, $102^{\circ}$<smiles>C[Si]1(C)c2cccc(-c3ccccc3)c2Oc2c(-c3ccccc3)cccc21</smiles>

(e) Ph-sixantphos, $108^{\circ}$<smiles>CCCCc1cccc2c1Oc1c(CCCC)cccc1C2(C)C</smiles>

(h) $t$-Bu-xantphos, $140^{\circ}$<smiles>CCCCOc1cccc2c1Oc1c(cccc1P(=O)(O)OC)C2(C)C</smiles><smiles></smiles>

(k) POP-xantphos, $123^{\circ}$<smiles></smiles>

(c) DBFphos, $131^{\circ}$<smiles>Cc1cc2c(c(-c3ccccc3)c1)Oc1c(cc(C)cc1-c1ccccc1)S2</smiles>

(f) Ph-thixantphos, $110^{\circ}$<smiles>C1=Cc2cccc(-c3ccccc3)c2OC(c2ccccc2)=C1c1ccccc1</smiles>

(i) Benzoxantphos, $120.6^{\circ}$

Figure 1.8: Selection of xantphos derivatives with their natural biteangles. $25,55,61,62$ 
ear aldehydes typically of higher industrial importance. ${ }^{28}$ In the hydroformylation of 1-octene using various xantphos ligands, a linear correlation was observed between the natural bite-angle of the diphosphine and the percentage of linear aldehyde product. $\underline{\underline{30}}$ From this and other kinetics studies, the bite-angle has been determined to have both a steric and electronic component. $\underline{63,64}$ In hydroformylation the hydride-migration step determines the regioselectivity of the reaction. In this case additional steric crowding of the metal centre, resulting from a larger bite-angle, can lead to a favouring of the less sterically demanding transition state - that which produces the linear aldehyde..$^{32}$ Carrying out the hydroformylation of styrene or 1-octene with a series of thixantphos complexes where the phenyl substituents on the phosphorus atoms were substituted in the para-position with various electron donating or electron withdrawing substituents, showed differences in the turnover frequency (TOF) of each reaction. $\underline{63}$ This is thought to be related to changes in the metal hybridisation, changing the reactivity of the metal towards reductive elimination. $\cdot 32,65$

The xantphos ligands were the first tertiary phosphine ligands to form active hydrocyanation catalysts on nickel. $\underline{66}$ Using styrene as a substrate, diphosphines with alkyl backbones, such as 1,2-bis(diphenylphosphino)ethane (dppe), dppp and 1,2-bis(diphenylphosphino)butane (dppb) gave poor yields $(<10 \%)$ whereas the xantphos ligands gave yields ranging from $27-95 \%$, with the highest yield for Ph-sixantphos. Ligands with bite-angles close to $105^{\circ}$ resulted in higher yields and selectivities whilst decreasing the bite-angle to $101^{\circ}$ or increasing to $110^{\circ}$ led to a much lower activity. The bite-angle can influence the selectivity of the reaction by stabilising preferred reaction intermediates and destabilising inactive species. In the hydrocyanation reaction a bite-angle close to $109^{\circ}$ destabilises square-planar $\mathrm{Ni}(\mathrm{II})$ species and stabilises the tetrahedral $\mathrm{Ni}(0)$ species, enhancing the reductive elimination step and resulting in a faster reaction. $\frac{57}{5}$ The increased reactivity of xantphos complexes compared to other diphosphines including, dppp, BINAP, dppf, and DPEphos has also been observed in a range of different cross-coupling reactions. $\frac{67}{}$

A search of the Cambridge Structural Database (CSD) indicates that of the crystallographically determined structures, most complexes with xantphos derivatives involve bidentate $\kappa P, P^{\prime}$ coordination. ${ }^{68}$ Four complexes with a 2-coordinate metal centre have been reported, all coordinated to gold. Ten 3-coordinate structures have been published. With 4-coordinate metals, tetrahedral complexes are 
the most common (31 structures), followed by pseudo-trans square-planar complexes (26), then cis square-planar geometries (20). This is particularly interesting as the natural bite-angle of Ph-xantphos ligands is closer to a $90^{\circ}$ than to $180^{\circ}$. On pentacoordinate metals 10 complexes have been reported with one square-pyramidal structure, three axial-equatorial, and six bis-equatorial trigonal bipyramidal complexes found. Six different octahedral complexes have been reported, all with the xantphos ligand displaying a cis-geometry. The xantphos ligands can also coordinate in a $\mathrm{K} P, O, P^{\prime}$ geometry in addition to the bidentate $\kappa P, P^{\prime}$ mode. This $\kappa P, O, P^{\prime}$ coordination is more common than the $\kappa P, P^{\prime}$ in octahedral complexes with 24 reported. However, it is much less common in five coordinate complexes with only four $\kappa P, O, P^{\prime}$ complexes and $10 \kappa P, O, P^{\prime}$ four-coordinate complexes. A monodentate $\kappa P$ bonding mode is also possible, though it is very rare, with only one crystal structure reported to date. $\underline{\underline{69}}$

Given the possibility of $\kappa P, O, P^{\prime}$ and $\kappa P, P^{\prime}$ coordination the xantphos ligands also have the potential for hemilability of the central donor group whereby the oxygen can bind reversibly to the metal centre in order to stabilise catalytic intermediates. This has been utilised in the hydroacylation of alkenes and alkynes using a rhodium pincer complex, where the oxygen can bind in order to stabilise important intermediates and prevent the competing decarbonylation reactions from occurring. $\underline{\underline{\underline{70}}-\underline{\underline{72}}}$ A range of rhodium complexes with Ph-xantphos or bis(2-diphenylphosphinophenyl)ether (DPEphos) as ancillary diphosphine ligands were tested for the hydroacylation reaction. $\underline{\underline{70}}$ The DPEphos complexes were more active than the dppe complex used for comparison, achieving conversions of $100 \%$ after 30 and 90 minutes respectively. However, the Ph-xantphos complex was completely inactive. The lower reactivity is thought to be a result of the increased rigidity of the Ph-xantphos backbone compared to that of DPEphos. $\frac{72}{\underline{7}}$

\subsubsection{Alkyl-Substituted Xantphos Ligands}

Despite the large number of xantphos derivatives few examples of xantphos ligands with alkyl substituents on the phosphorus atoms have been reported. The synthesis and some coordination chemistry of xantphos ligands with methyl, ethyl, isopropyl, and tert-butyl substituents has been studied. Me-xantphos was reported in 2002 and investigated for reactivity with [Pd(cod)ClMe] $(\operatorname{cod}=1,5-$ cyclooctadiene), to produce cis-[PdClMe(Me-xantphos)]. $\underline{\underline{73}}$ The cis isomer was 
also formed using Ph-sixantphos. However, using the larger bite-angle ligands $\mathrm{Ph}$-thixantphos and Ph-xantphos cis-trans isomerism was observed at room temperature. Reaction of the four chloridomethyl complexes with $\mathrm{AgSO}_{3} \mathrm{CF}_{3}$ yielded the pincer complexes $\left[\mathrm{PdMe}\left(\right.\right.$ xantphos- $\left.\left.\mathrm{K} P, \mathrm{O}, \mathrm{P}^{\prime}\right)\right]\left[\mathrm{SO}_{3} \mathrm{CF}_{3}\right]$ (Scheme 1.1).<smiles></smiles>
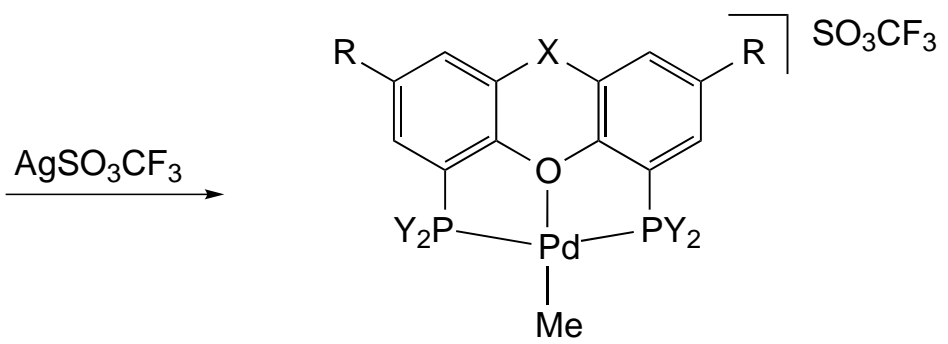

Scheme 1.1: Chloride abstraction from [PdClMe(xantphos)] xantphos $=\mathrm{Ph}$ sixantphos, Ph-thixantphos, Ph-xantphos, and Me-xantphos.

A variant of xantphos with ethyl groups on the phosphorus atoms (Et-xantphos) was reported in 2004. ${ }^{74,75}$ Palladium and platinum complexes, [M(Et-xantphos) ${ }_{2}$ ], $\left[\mathrm{M}(\text { Et-xantphos })_{2}\right]^{2+}$ and platinum complexes $\left[\mathrm{Pt}(\text { Et-xantphos })_{2} \mathrm{H}\right] \mathrm{PF}_{6}$ and $[\mathrm{Pt}($ Etxantphos $\left.)_{2}(\mathrm{H})_{2}\right]^{2+}$ have been studied for their electrochemical properties. The $\mathrm{X}-$ ray crystal structures of $\left[\mathrm{M}(\text { Et-xantphos })_{2}\right](\mathrm{M}=\mathrm{Pd}, \mathrm{Pt})$ were obtained and have a tetrahedral geometry, with average bite-angles of $108.3^{\circ}$ for both metals. The palladium(II) complex had two molecules in the asymmetric unit, one closer to a square-planar geometry (average bite-angle $=139.7^{\circ}$ ) and the other closer to a tetrahedral geometry (average bite-angle $=95.8^{\circ}$ ). The pseudo-square planar $\left[\mathrm{Pd}(\text { Et-xantphos })_{2}\right]\left(\mathrm{BF}_{4}\right)_{2}$ complex was also crystallised, and had much larger bite-angles than any of the other ethyl substituted diphosphines included in the study (1,2-bis(diethylphosphino)propane (depp), $\alpha, \alpha^{\prime}$-bis(diethylphosphino)-oxylene (depx) and bis(2-diethylphosphinophenyl)ether (depPE)) indicating the ability of the xantphos ligands to support metals in unusual geometries.

A xantphos ligand with isopropyl substituents on the phosphorus atoms, i-Prxantphos, was first reported in 2010, together with the osmium complex $\mathrm{OsCl}_{3}(i-$ Pr-xantphos- $\left.\left.\mathrm{k} P, O, P^{\prime}\right)\right]$, showing the $\kappa P, O, P^{\prime}$ coordination which is common for xantphos ligands in octahedral complexes. ${ }^{76}$ The same group, has since reported the synthesis and reactivity of $\left[\mathrm{MCl}_{2}(\mathrm{DMSO}-\kappa S)\left(i-\mathrm{Pr}\right.\right.$-xantphos- $\left.\left.\kappa P, O, P^{\prime}\right)\right](\mathrm{M}=$ $\mathrm{Os}, \mathrm{Ru}$ ) producing a number of different osmium and ruthenium complexes including various polyhydrides and bis(alkynyl)vinylidene complexes. $\underline{77,78}$ 
A study of the reactivity of $i$-Pr-xantphos towards palladium shows differences to the $\mathrm{Ph}$-xantphos ligand. $\underline{79}$ The $\left[\mathrm{Pd}\left(\mathrm{CF}_{3}\right) \mathrm{Ph}(\mathrm{Ph}\right.$-xantphos)] complex exists in a cis geometry in the solid state, and as cis and trans isomers in solution, whereas the analogous $i$-Pr-xantphos complex shows only a trans configuration. The iPr-xantphos complex was synthesised in a different manner as the $\mathrm{Ph}$-xantphos ligand was readily displaced by $\mathrm{CF}_{3}^{-}$from $\mathrm{CF}_{3} \mathrm{SiMe}_{3} / \mathrm{F}^{-}$while the $\mathrm{i}$-Pr-xantphos ligand was not, indicating the different chemistry of the two ligands. The difference in the coordination geometries also meant that the $\mathrm{PhCF}_{3}$ was more readily lost from the cis- $\left[\mathrm{Pd}\left(\mathrm{CF}_{3}\right) \mathrm{Ph}(\mathrm{Ph}\right.$-xantphos $\left.)\right]$ than the trans- $\left[\mathrm{Pd}\left(\mathrm{CF}_{3}\right) \mathrm{Ph}(i-\mathrm{Pr}\right.$-xantphos)]. A related study investigated nickel complexes of $i$-Pr-xantphos for the trifluoromethylation of aryl halides..$\underline{00}$ The $[\mathrm{NiF}(1-$ naphthyl)(i-Pr-xantphos)] complex reacted with $\mathrm{CF}_{3} \mathrm{SiMe}_{3}$ forming $\left[\mathrm{Ni}\left(\mathrm{CF}_{3}\right)\right.$ (1-naphthyl)(i-Pr-xantphos)]. Both of these complexes were found to have trans-geometries through $\mathrm{X}$-ray crystallography, and showed slow decomposition at $140{ }^{\circ} \mathrm{C}$ and $120{ }^{\circ} \mathrm{C}$ respectively, giving rise to a range of products, with no $\mathrm{C}-\mathrm{F}$ compounds or 1-trifluoromethylnaphthyl formed.

The chemistry of rhodium and iridium complexes with i-Pr-xantphos ligands has also been studied. Reaction of $i$-Pr-xantphos with $\left[\mathrm{Rh}(\mathrm{coe})_{2}\left(\eta^{2}-\mathrm{Cl}\right)\right]_{2}($ coe $=$ cyclooctene) generated the $\left[\mathrm{RhCl}\left(\mathrm{i}-\mathrm{Pr}\right.\right.$-xantphos- $\left.\left.\kappa P, O, P^{\prime}\right)\right]$ complex cleanly. ${ }^{81}$ However, reaction with the iridium analogue resulted in cyclometallation of one of

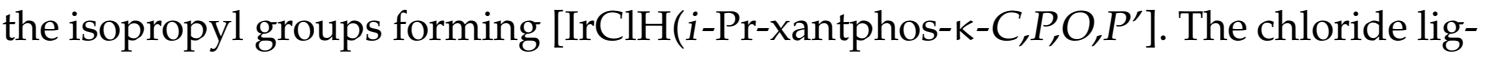
and in $\left[\mathrm{RhCl}\left(i-\operatorname{Pr}\right.\right.$-xantphos- $\left.\left.\kappa P, O, P^{\prime}\right)\right]$, was replaced with a hydride, via reaction with $\mathrm{KO}^{\mathrm{i}} \mathrm{Pr}$, producing $\mathrm{KCl}$ and acetone as by-products. The trihydride $\left[\operatorname{Ir}(\mathrm{H})_{3}(\mathrm{i}-\right.$ Pr-xantphos- $\left.\left.k P, O, P^{\prime}\right)\right]$ was produced by reaction of the metallated iridium complex with $\mathrm{KOH}$ and ${ }^{\mathrm{P}} \mathrm{PrOH}$. The rhodium chloride complex undergoes oxidative addition of $\mathrm{H}_{2}$ to form $\left[\mathrm{RhCl}(\mathrm{H})_{2}\left(i-\mathrm{Pr}\right.\right.$-xantphos- $\left.\left.\kappa P, O, P^{\prime}\right)\right]$. The analogous iridium complex was synthesised by reaction of the metallated complex with $n$ octane at $90{ }^{\circ} \mathrm{C}$. Recent research has shown that the chloride can be removed from $\left[\mathrm{RhCl}(\mathrm{H})_{2}\left(i-P r-x a n t p h o s-\kappa P, O, P^{\prime}\right)\right]$ by reaction with $\mathrm{Li}\left[\mathrm{B}\left(\mathrm{C}_{6} \mathrm{~F}_{5}\right)_{4}\right] \mathrm{OEt}_{2}$ forming $\left[\mathrm{Rh}(\mathrm{H})_{2}\left(i-\mathrm{Pr}-x a n t p h o s-\kappa P, O, P^{\prime}\right)\right]^{+} .82$ Reacting triflic acid with $[\mathrm{RhCl}(i$-Pr-xantphos- $\left.\left.\mathrm{K} P, \mathrm{O}, \mathrm{P}^{\prime}\right)\right]$ or $\left[\mathrm{IrClH}\left(\mathrm{i}-\mathrm{Pr}\right.\right.$-xantphos- $\left.\mathrm{k}-\mathrm{C}, P, \mathrm{O}, \mathrm{P}^{\prime}\right]$ results in $[\mathrm{MCl}(\mathrm{H})(\mathrm{OTf})(\mathrm{i}-\mathrm{Pr}-$ xantphos- $\left.\left.\kappa P, O, P^{\prime}\right)\right](\mathrm{M}=\mathrm{Rh}, \mathrm{Ir}) . \underline{.81}$ The reactivity of the $i$-Pr-xantphos complexes is summarised in Figure 1.2 and 1.3 for rhodium and iridium respectively.

The reactivity of the rhodium and iridium complexes towards silyl compounds has also been investigated with the results summarised in Scheme 1.4, ${ }^{83}$ Reac- 


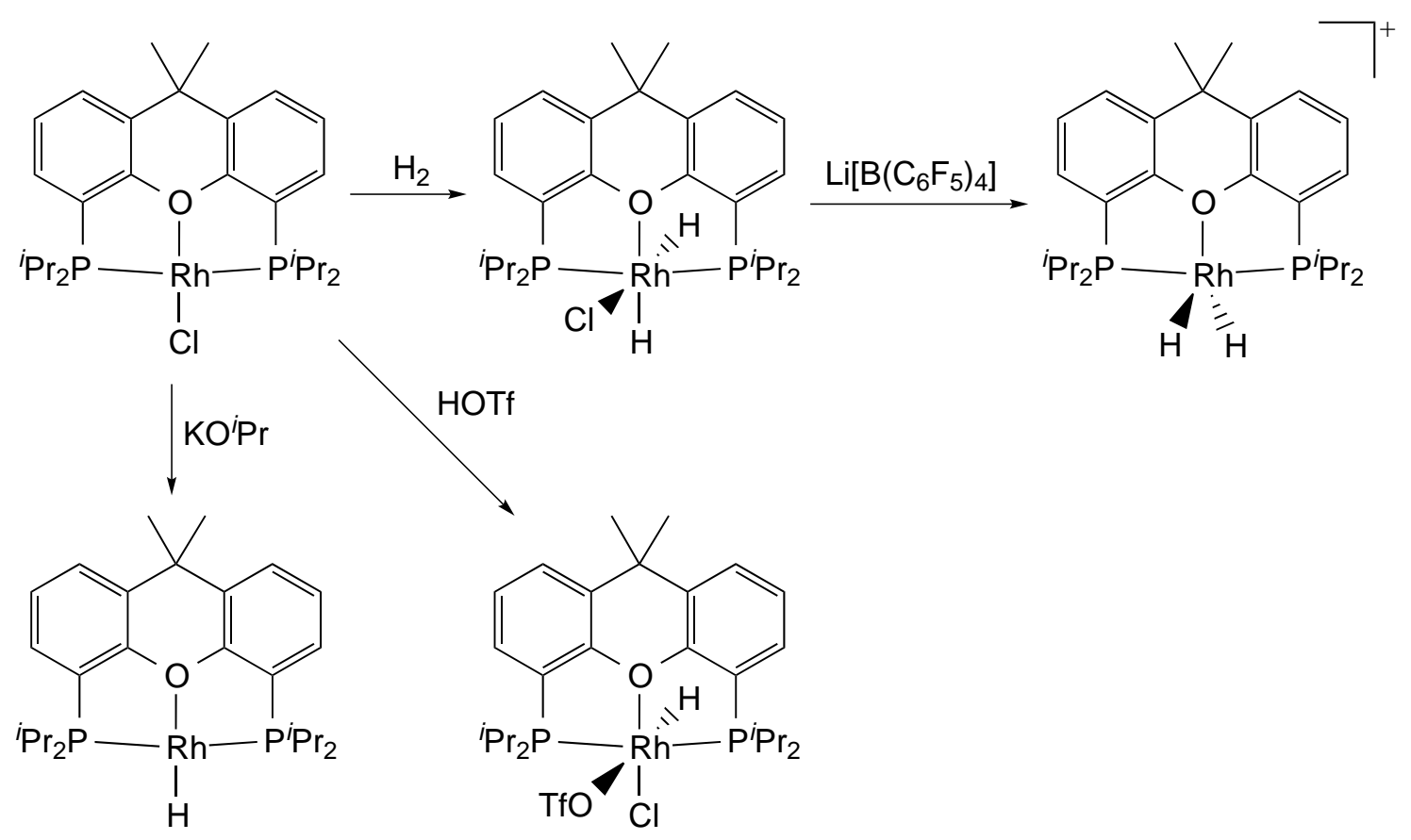

Scheme 1.2: Reactions of [Rh(i-Pr-xantphos)].
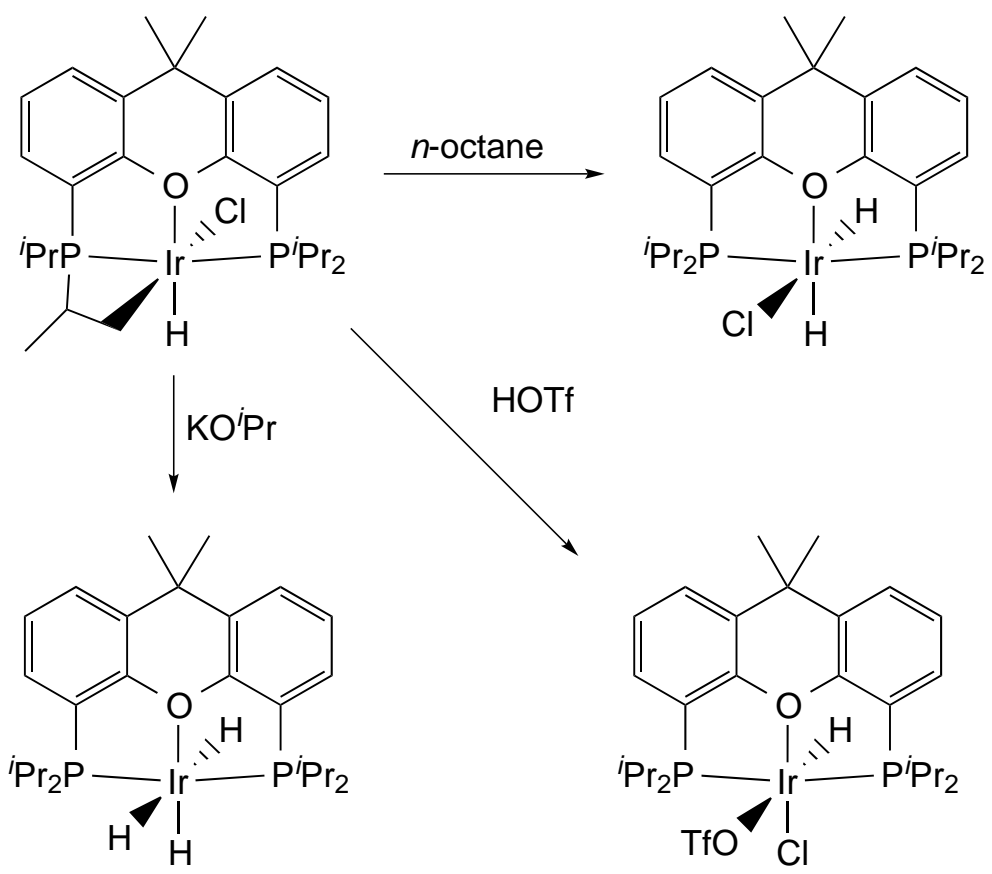

Scheme 1.3: Reactions of [Ir(i-Pr-xantphos)]. 


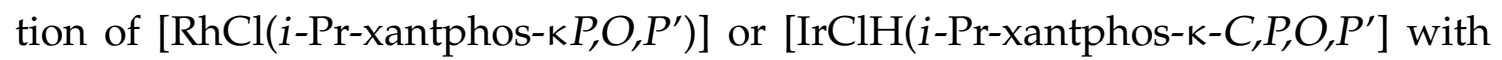
$\mathrm{SiH}_{2} \mathrm{Ph}_{2}$ or $\mathrm{SiHEt}_{3}$ resulted in $\left[\mathrm{MCl}(\mathrm{H})\left(i-\mathrm{Pr}\right.\right.$-xantphos- $\left.\left.\kappa P, O, P^{\prime}\right)\left(\mathrm{SiR}_{3}\right)\right](\mathrm{M}=\mathrm{Rh}$, Ir, $\left.\mathrm{SiR}_{3}=\mathrm{SiHPh}_{2}, \mathrm{SiEt}_{3}\right)$. The diphenylsilane complexes were unstable in solution converting to $\left[\mathrm{Rh}\left(\mathrm{SiClPh}_{2}\right)\left(i-\mathrm{Pr}\right.\right.$-xantphos- $\left.\left.\mathrm{K} P, \mathrm{O}, \mathrm{P}^{\prime}\right)\right]$ via loss of molecular hydrogen and trans-[Ir(SiClPh$\left.{ }_{2}\right)(\mathrm{H})_{2}\left(i-\operatorname{Pr}\right.$-xantphos- $\left.\left.\kappa P, O, P^{\prime}\right)\right]$. The rhodium complex $\left[\mathrm{RhH}\left(\mathrm{i}\right.\right.$-Pr-xantphos)] also underwent reaction with $\mathrm{SiHEt}_{3}$ and $\mathrm{SiHPh}_{3}$ initially forming the dihydride $\left[\mathrm{Rh}(\mathrm{H})_{2}\left(i-\mathrm{Pr}\right.\right.$-xantphos- $\left.\left.\kappa P, O, P^{\prime}\right)\left(\mathrm{SiR}_{3}\right)\right](\mathrm{R}=\mathrm{Et}, \mathrm{Ph})$ which loses molecular hydrogen to form $\left[\mathrm{Rh}\left(i-\operatorname{Pr}\right.\right.$-xantphos- $\left.\left.\kappa P, O, P^{\prime}\right)\left(\mathrm{SiR}_{3}\right)\right]$. Reaction of $\left[\mathrm{Rh}\left(\mathrm{SiClPh}_{2}\right)\left(i-\mathrm{Pr}\right.\right.$-xantphos- $\left.\left.\mathrm{K} P, \mathrm{O}, P^{\prime}\right)\right]$ with $\mathrm{Na}\left[\mathrm{BAr}_{4}^{\mathrm{F}}\right]$ in the presence of water produced $\left[\mathrm{Rh}(\mathrm{H})\left(i-\mathrm{Pr}\right.\right.$-xantphos- $\left.\left.\kappa P, O, P^{\prime}\right)\left(\mathrm{SiOHPh}_{2}\right)\right]$ which was tested as a catalyst for the alcoholysis of $\mathrm{SiH}_{2} \mathrm{Ph}_{2}$ with various alcohols in toluene at $32{ }^{\circ} \mathrm{C}$, forming $\mathrm{ROSiHPh}_{2}$ in isolated yields of $71-92 \%$ with TOFs of 4000 to $76500 \mathrm{~h}^{-1}$.

\subsection{2 $t$-Bu-Xantphos}

This thesis covers an investigation into the coordination chemistry of three different xantphos ligands with tert-butyl substituents on the phosphorus atoms. The three ligands differ in the bridgehead position: $t$-Bu-xantphos has $\mathrm{CMe}_{2}, t$-Busixantphos has $\mathrm{SiMe}_{2}$ and $t$-Bu-thixantphos has a thioether bridge. The first mention of $t$-Bu-xantphos was in 2002 with the unsuccessful attempts to synthesise $t$ Bu-xantphos from either 4,5-dilithio-9,9-(dimethyl)xanthene or 9,9-dimethyl-4,5bis(dichlorophosphino)xanthene, the researchers postulated that steric crowding was the reason for the lack of reactivity. $\underline{\underline{73}}$ A successful synthesis of $t$-Bu-xantphos from the dilithiated backbone was reported in 2005 using heptane as a solvent and heating the reaction mixture to $60^{\circ} \mathrm{C} . \underline{.84} t$-Bu-Xantphos has subsequently been studied for use as an ancillary ligand in the palladium catalysed cross-coupling of thiols and aryl bromides or triflates, the iron catalysed $\mathrm{sp}^{3}-\mathrm{sp}^{3}$ cross-coupling of alkyl halides with alkyl Grignard reagents, the platinum catalysed amination of allylic alcohols and the $\mathrm{N}$-arylation of heterocyclic diamines, all with poor yields, while higher yields were observed when Ph-xantphos was used. $\underline{84} \underline{\underline{87}}$ These studies have focussed on the addition of $t$-Bu-xantphos as a component of the catalytic system. Few complexes of $t$-Bu-xantphos have been characterised, although the difference in the catalytic results suggest differences in the coordination chemistry of Ph-xantphos and $t$-Bu-xantphos.

Prior to the start of this research, the only isolated coordination complexes of 


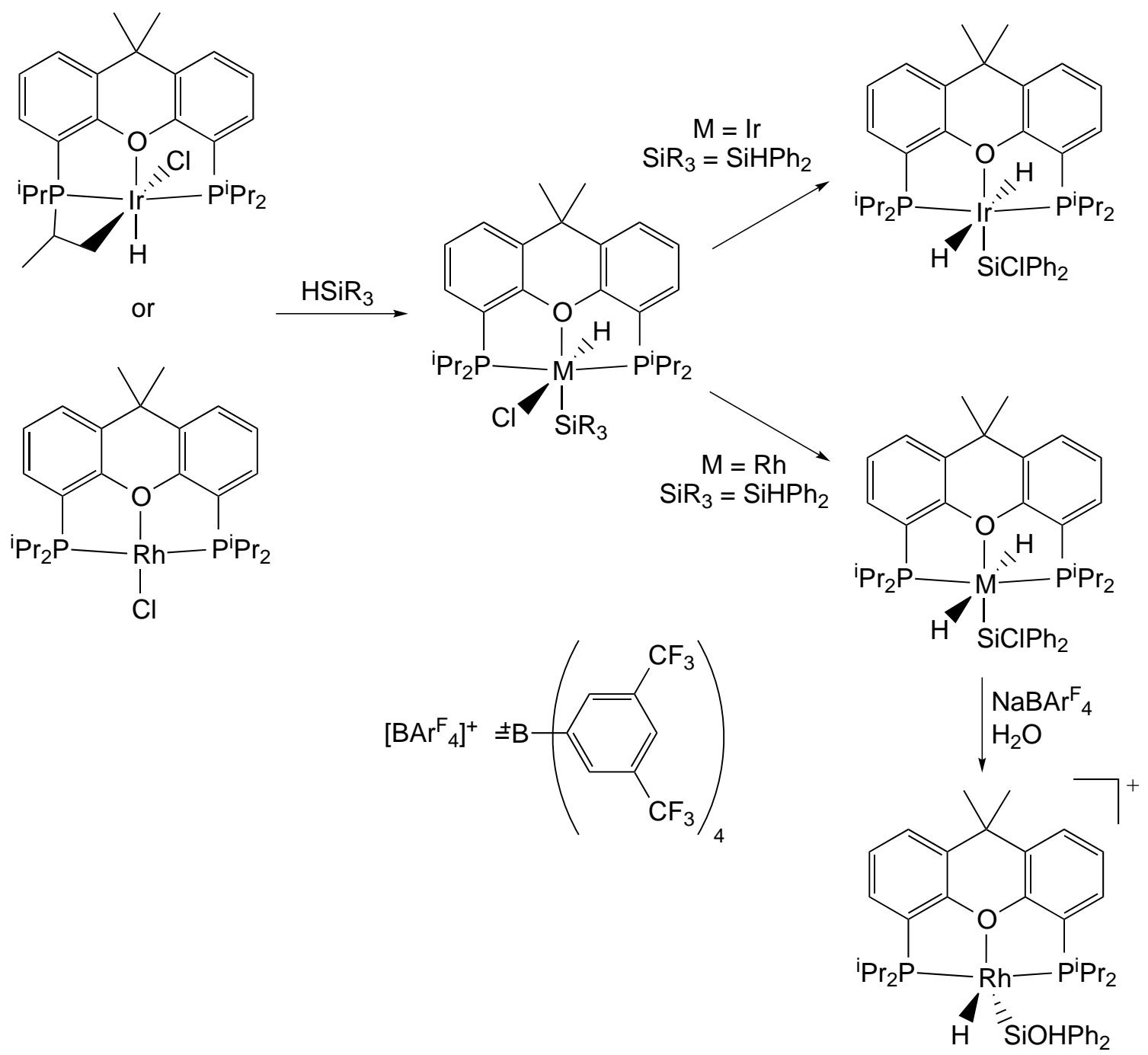

Scheme 1.4: Reactions of Rh and Ir i-Pr-xantphos complexes with silanes. $\mathrm{SiR}_{3}=$ $\mathrm{SiHPh}_{2}, \mathrm{SiEt}_{3}$. 
$t$-Bu-xantphos were $[\mathrm{Au}(t-\mathrm{Bu}$-xantphos $)]\left[\mathrm{AuX} \mathrm{X}_{2}\right](\mathrm{X}=\mathrm{Cl}, \mathrm{Br}, \mathrm{I})$, formed by reaction of $[\mathrm{AuCl}(\mathrm{tht})]$ (tht $=$ tetrahydrothiophene) with $t$-Bu-xantphos and subsequent reaction with $\mathrm{KBr}$ or $\mathrm{KI} . \underline{88}$ The analogous reaction using Ph-xantphos gave $\left[(\mathrm{AuX})_{2}(\mathrm{Ph}\right.$-xantphos)] $(\mathrm{X}=\mathrm{Cl}, \mathrm{Br}, \mathrm{I})$ with each phosphorus atom coordinated to a separate gold centre with a $\mathrm{Au}-\mathrm{Au}$ interaction. ${ }^{88,89}$ Subsequent investigations into the catalytic activity of gold xantphos complexes towards the C-F bond activation of perfluoroarenes showed turnover numbers (TONs) of 200 for [ $\mathrm{AuCl}(\mathrm{Ph}-$ xantphos)] and 1000 for [Au(t-Bu-xantphos)][AuCl$\left.{ }_{2}\right] \cdot{ }^{90}$ Mechanistic studies into the reaction showed that $\left[\mathrm{Au}(\text { xantphos) }]^{+}\right.$was an important intermediate and is in equilibrium with the inactive $\left[\mathrm{Au}(\text { xantphos })_{2}\right]$. Due to the size of $t$-Bu-xantphos the equilibrium favours the two-coordinate species while for Ph-xantphos the four-coordinate complex is preferred, resulting in lower activity. $\underline{90}$ This clearly shows the difference between the two xantphos ligands and suggests that a difference in the coordination chemistry may be the cause of the observed differences in catalytic reactivity.

Subsequent to the start of the research presented in this thesis $t$-Bu-xantphos has gained increasing attention, likely as a result of the ligand becoming commercially available. In the six years from the first successful synthesis in 2005 to the start of this research in mid-2011, only the four papers discussed above were published investigating $t$-Bu-xantphos $\$$ However, the following three years showed increasing attention with 9 papers published $\underline{82,90,93-99}$ and 10 patent applications. $\underline{100-109}$

Further investigations into the catalytic properties of $t$-Bu-xantphos have been reported, without investigation into the coordination chemistry. These include the palladium-catalysed cross-coupling of 2-(4-bromophenyl)-5-chloropyrazine with a benzimidazole boronic ester and hydroesterification of methyl oleate. $\frac{97,98}{}$ In both cases little conversion was observed when $t$-Bu-xantphos was used, though the Ph-xantphos systems showed significant reactivity. The aminocarbonylation of aryl bromides was performed with each of Ph-xantphos and $t$-Bu-xantphos in an unusual monodentate coordination mode. $\underline{\underline{93}}$ Little reactivity was observed with $t$-Bu-xantphos, while the Ph-xantphos system gave the product in $92 \%$ isolated yield. In the rhodium catalysed reductive amination of aldehydes $t$-Buxantphos showed a 59\% total conversion with only 19\% selectivity for the desired

\footnotetext{
$\S$ Two papers investigating the use of the dioxidised $t$-Bu-xantphos as a ligand on europium and samarium were also published in 2011 and 2012. ${ }^{91,92}$
} 
product, while Ph-xantphos had an $84 \%$ conversion and $93 \%$ selectivity. $\underline{\underline{96}}$

In some cases the $t$-Bu-xantphos system is more active than the Ph-xantphos system, such as the palladium catalysed N-alkylation of aniline with benzyl alcohol. ${ }^{94}$ The $t$-Bu-xantphos system gave near quantitative conversion at $100{ }^{\circ} \mathrm{C}$ while the Ph-xantphos system showed only $63 \%$ conversion at $110{ }^{\circ} \mathrm{C}$. Improved activity with $t$-Bu-xantphos compared to Ph-xantphos was also observed in the palladium catalysed methylation of alkynyl C-H bonds with dimethyl sulfonium ylides. .95 Under the same conditions a yield of $46 \%$ was obtained with $t$-Bu-xantphos while only $15 \%$ was produced using Ph-xantphos. $t$-Bu-Xantphos and Phxantphos can also have similar results, such as in the rhodium catalysed hydroamidomethylation of 1-pentene with acetamide, Ph-xantphos and $t$-Bu-xantphos showed conversions of 83 and $80 \%$ respectively with greater than $99 \%$ linearity in both cases. $\underline{99}$

Despite the increased interest in $t$-Bu-xantphos in the last four years, research has focussed on its use as an ancillary ligand for catalytic reactions, mostly with the catalyst formed in situ. A crystal structure of trans-[Pd(t-Bu-xantphos $\left.) \mathrm{Cl}_{2}\right]$, was reported via communication to the CSD in 2011 (CSD-XARXAR), no fur-

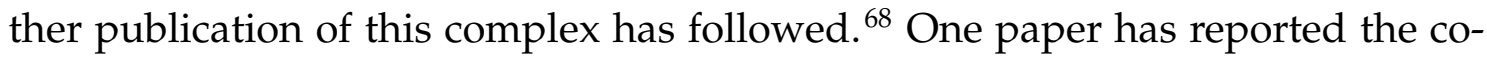
ordination behaviour of the $t$-Bu-xantphos ligand with rhodium. ${ }^{82}$ Similarly to the work with $i$-Pr-xantphos, the $t$-Bu-xantphos ligand reacts with $\left[R h(\operatorname{coe})\left(\eta^{2}-\right.\right.$ $\mathrm{Cl})]_{2}$ (coe = cyclooctene) to give $\left[\mathrm{Rh}\left(t\right.\right.$-Bu-xantphos- $\left.\left.\mathrm{K} P, \mathrm{O}, \mathrm{P}^{\prime}\right) \mathrm{Cl}\right]$. This $\mathrm{Rh}(\mathrm{I})$ chloride readily undergoes oxidative addition of hydrogen to form a [ $\mathrm{Rh}(t-\mathrm{Bu}$-xantphos- $\left.\left.\kappa P, O, P^{\prime}\right) \mathrm{Cl}(\mathrm{H})_{2}\right]$ complex. The structures of $\left[\mathrm{Rh}\left(t\right.\right.$-Bu-xantphos- $\left.\left.\kappa P, O, P^{\prime}\right) \mathrm{Cl}\right]$ and $\left[\mathrm{Rh}\left(t\right.\right.$-Bu-xantphos- $\left.\left.\mathrm{k} P, \mathrm{O}, \mathrm{P}^{\prime}\right) \mathrm{Cl}(\mathrm{H})_{2}\right]$ have been confirmed by X-ray crystallography. Chloride abstraction from $\left[\mathrm{Rh}\left(t\right.\right.$-Bu-xantphos- $\left.\left.\kappa P, O, P^{\prime}\right) \mathrm{Cl}(\mathrm{H})_{2}\right]$ using $\mathrm{AgBF}_{4}$ or $\mathrm{AgSbF}_{6}$, generates the trigonal bipyramidal complex [Rh(t-Bu-xantphos- $\left.\left.\kappa P, O, P^{\prime}\right)(\mathrm{H})_{2}\right]$, with the $t$-Bu-xantphos ligand retaining the meridional coordination typical of pincer ligands. This dihydride was found to react with ethene to give ethane and $\left[\mathrm{Rh}\left(t-\mathrm{Bu}\right.\right.$-xantphos- $\left.\left.\kappa P, O, P^{\prime}\right)\left(\mathrm{C}_{2} \mathrm{H}_{4}\right)\right]$, however no reaction with the commonly used hydrogen acceptors, $t$-butylethylene or norbornene, or with terminal alkenes was observed. [ $\mathrm{Rh}\left(t\right.$-Bu-xantphos- $\left.\left.\kappa P, O, P^{\prime}\right) \mathrm{Cl}(\mathrm{H})_{2}\right]$ reacts with $\mathrm{KO}^{t} \mathrm{Bu}$ resulting $\left[\mathrm{Rh}\left(t-\mathrm{Bu}\right.\right.$-xantphos- $\left.\left.\mathrm{K} P, \mathrm{O}, \mathrm{P}^{\prime}\right) \mathrm{H}\right]$. Addition of ethene to $[\mathrm{Rh}(t-\mathrm{Bu}-$ xantphos $) \mathrm{H}]$ resulted in the reversible formation of an ethyl complex. The $[\mathrm{Rh}(t-$ Bu-xantphos)H] complex also showed reactivity in the isomerisation of 1-hexene with a TON of 2000 after 16 hours. 
The previous work exploring $t$-Bu-xantphos and $\mathrm{Ph}$-xantphos as ancillary ligands for catalytic processes has shown significant differences in the yields of the reactions in most cases, though in one case similar activity was reported. Although a number of palladium catalysed reactions have been studied, only two palladium complexes have been reported (one being only a crystal structure). The only research into the coordination chemistry of the $t$-Bu-xantphos ligands is on gold and rhodium. $\stackrel{82.88}{ }$ The original study into the xantphos ligands focussed on changing the bridging groups of $\mathrm{Ph}$-xantphos showing the impact that subtle changes in the bite-angle can have on the catalytic activity of the transition metal complexes. $\stackrel{30}{=}$ Given the number of studies investigating the catalytic activity of $t$-Bu-xantphos without investigating the coordination chemistry, an examination of the coordination chemistry of a range of subtly different $t$-Bu-xantphos ligands with late-transition metals is of particular interest.

\subsection{Research Objectives}

The xantphos class of ligands has been the subject of a number of studies, with interesting and varied results. The initial research with $\mathrm{Ph}$-xantphos investigated the influence of changes in the bite-angle resulting from changing the group in the bridging position from the $\mathrm{CMe}_{2}$ group found in Ph-xantphos. studies have shown that small changes in the bite-angle can have a significant impact on the catalytic reactivity and selectivity of the system. Recently a large amount of research has focussed on the development of xantphos derivatives with alkyl substituents on the phosphorus atoms, particularly the i-Pr-xantphos and $t$-Bu-xantphos ligands. Prior to the start of this research only one report investigating the coordination behaviour of $t$-Bu-xantphos had been published. $\underline{88}$ The other studies all showed much lower activity in catalytic systems than with the Ph-xantphos ligand. $\underline{84}-\underline{86}$ Together this suggested that the coordination behaviour of the $t$-Bu-xantphos and $\mathrm{Ph}$-xantphos ligands is different and deserved further attention. The papers published since the inception of this research have only sought to solidify the objectives, as these have reported several examples of studies into the use of $t$-Bu-xantphos in catalytic systems with only one further study into the coordination behaviour on rhodium, despite the majority of the catalytic studies being performed on palladium. Furthermore, despite the 
much larger bite-angle and the electronic differences of $t$-Bu-xantphos compared to Ph-xantphos, and the impact small bite-angle changes have on the activity of Ph-xantphos only two xantphos ligands with tert-butyl substituents on the phosphines and $\mathrm{CMe}_{2}$ or $\mathrm{C}=\mathrm{CMe}_{2}$ in the bridgehead position have been published, and no comparative investigation has been reported.

The over-arching goal of this research is to investigate the synthesis, properties and coordination chemistry of $t$-Bu-xantphos and two derivatives in the bridgehead position ( $\mathrm{S}$ and $\mathrm{SiMe}_{2}$ ). The first stage aimed to synthesise the $t$-Bu-thixantphos and $t$-Bu-sixantphos ligands, and investigate the steric and electronic properties of all three $t$-Bu-xantphos ligands by the calculation of their natural biteangles and synthesis of non-transition metal derivatives.

Silver is a particularly interesting metal for the synthesis of coordination complexes, showing a distinct propensity to form dimers, trimers, or higher order structures, with differences dependent upon the steric bulk and flexibility of the diphosphine ligands used. $\underline{111}$ Hence, the initial coordination chemistry of the three $t$-Bu-xantphos ligands towards silver was investigated in order to gain an understanding of their coordination chemistry and gauge the impact of their large bite-angles on the coordination chemistry, and to allow comparison to the gold $t$-Bu-xantphos complexes previously published.

The remainder of the research focussed on transition metals which are commonly used in homogeneous catalysis. The xantphos class of ligands have been wellstudied for their roles as ancillary ligands in hydroformylation. aim of this research was not to investigate the catalytic properties of the three $t$-Bu-xantphos ligands, but to produce discrete transition metal complexes to gain insight into the catalytic studies that have already been performed. Hence the coordination chemistry with rhodium focussed on the synthesis of a simple rhodium(I) complex and established the reactivity towards small molecules, including hydrogen and carbon monoxide, which are the foundation for a number of different catalytic processes.

The coordination chemistry of the three $t$-Bu-xantphos ligands towards palladium and platinum in both the 0 and +2 oxidation states was also studied, in order to gain insight into the differences in the catalytic activity of systems with $t$ Bu-xantphos and Ph-xantphos. Palladium is one of the most widely used metals for homogeneous catalysis and catalytic cycles frequently involve interconver- 
sion between the two oxidation states. $\underline{31}$ Platinum complexes were synthesised prior to the palladium investigation as the presence of an NMR active isotope $\left({ }^{195} \mathrm{Pt}\right)$ coupled with the high stability of platinum complexes was beneficial to the identification and characterisation of the complexes produced. Although the coordination chemistry of Ph-xantphos with palladium is well-known, few coordination complexes of Ph-xantphos, and particularly Ph-thixantphos and Phsixantphos with platinum have been reported. This work began with a brief investigation into the coordination chemistry of $\mathrm{Ph}$-thixantphos with platinum to assist in identifying any differences in the coordination chemistry that resulted from changing the phenyl substituents to tert-butyl groups. The coordination chemistry of the three $t$-Bu-xantphos ligands with palladium and platinum in the 0 and +2 oxidation states was subsequently investigated.

Throughout this research nuclear magnetic resonance (NMR) spectroscopy was used extensively to characterise the products of the reactions and aid in the identification of any intermediates or dynamic processes that were present. X-ray crystallography was also an important tool, particularly to compare the biteangles and coordination geometries of the $t$-Bu-xantphos complexes produced with those previously reported for the Ph-xantphos. Theoretical chemistry using density functional theory (DFT) was used to gain further insight into reactions or processes when this was of benefit. 


\section{Chapter 2}

\section{Ligand Synthesis and Properties}

The $t$-Bu-xantphos ligand was first reported in 2005. $\underline{.4}$ The original paper and several since then, have tested $t$-Bu-xantphos for use as an ancillary ligand in a range of different catalytic systems with mixed results. $\underline{82,85-87,90,93-99}$ Despite the catalytic attention towards the $t$-Bu-xantphos ligand very little is known about the coordination chemistry or reasons for the very different catalytic activity compared to Ph-xantphos. Only one study comparing the coordination chemistry of the two ligands has been published. $\underline{88}$ This study found that $t$-Bu-xantphos

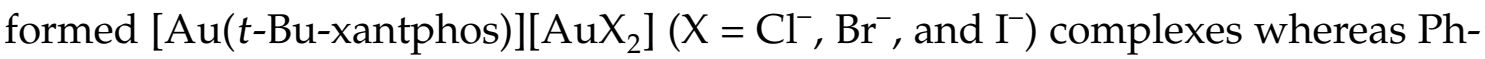
xantphos formed $\left[(\mathrm{AuX})_{2}(\mathrm{Ph}\right.$-xantphos $\left.)\right]$. A trans-[Pd(t-Bu-xantphos $\left.) \mathrm{Cl}_{2}\right]$ complex has been reported to the CSD (CSD-XARXAR), while the analogous complex with Ph-xantphos is cis, indicating a difference in the coordination chemistry of the two ligands. $\frac{68}{}$ A series of rhodium complexes with $t$-Bu-xantphos has been published, 82 though the analogous Ph-xantphos complexes have not been investigated.

The original study into the Ph-xantphos ligands investigated the effect of changing the bridging group in the backbone between $\mathrm{CMe}_{2}, \mathrm{SiMe}_{2}$, and $\mathrm{S} . \underline{\underline{30}}$ Changing this group subtly changes the natural bite-angle of the ligand and has been shown to impact the catalytic activity. $\frac{67}{}$ One derivative of $t$-Bu-xantphos in this position has been previously published with the $\mathrm{CMe}_{2}$ replaced by a $\mathrm{C}=\mathrm{CMe}_{2} \underline{117}$ Given the much larger bite-angle of the $t$-Bu-xantphos ligands the impact of small changes may be very different to those observed with the Ph-xantphos ligands. This chapter presents a study into the synthesis of $t$-Bu-sixantphos and $t$-Bu-thixantphos, and an alternative synthesis of $t$-Bu-xantphos, followed by calculation of their 
natural bite-angles and the synthesis of some non-transition metal derivatives including phosphonium salts and phosphine selenides. The goal of this chapter is to investigate the steric and electronic properties of the three $t$-Bu-xantphos ligands to form the foundation of knowledge necessary to understand their coordination chemistry, which will be presented in later chapters.

\subsection{Ligand Synthesis}

In 2002 van Leeuwen et al. reported their unsuccessful attempts to synthesise $t$-Bu-xantphos from either the dilithiated xanthene backbone or starting with 9,9-dimethyl-4,5-bis(dichlorophosphino)xanthene, suggesting that steric crowding from the presence of two tert-butyl groups on a single phosphorus atom prevented the successful coupling. $\cdot \underline{\underline{73}}$ However, in 2005 a synthesis of $t$-Bu-xant-

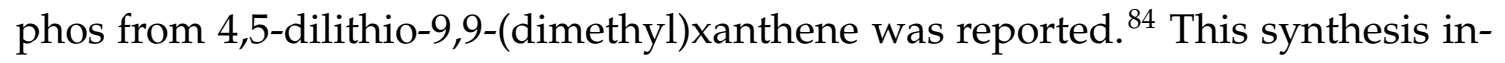
volves lithiation of the xanthene backbone using n-butyllithium and $N, N, N^{\prime}, N^{\prime}-$ tetramethyl-ethane-1,2-diamine (TMEDA) in heptane, followed by addition of $\mathrm{ClP}^{\mathrm{t}} \mathrm{Bu}_{2}$ and heating at $60{ }^{\circ} \mathrm{C}$ for 24 hours, generating the product in $38 \%$ isolated yield (Scheme 2.1).
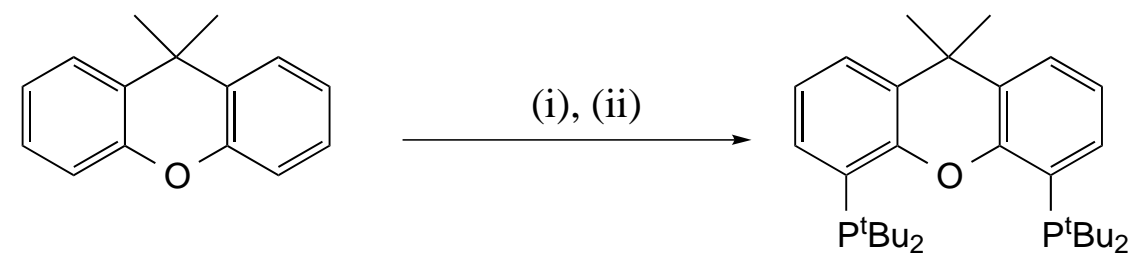

Scheme 2.1: Literature synthesis of $t$-Bu-xantphos in heptane..$^{84}$ Reagents and conditions: (i) n-BuLi, TMEDA, $15 \mathrm{~h}$, heptane. (ii): $\mathrm{ClP}^{\mathrm{t}} \mathrm{Bu}_{2}, 60^{\circ} \mathrm{C}, 24 \mathrm{~h}$.

Attempts to utilise the literature method for the synthesis of $t$-Bu-xantphos ${ }^{84}$ to synthesise $t$-Bu-thixantphos resulted in a number of unidentifiable products. An alternative method using a potassium tert-butoxide/n-butyllithium "superbase" formed a mixture of products from which separation attempts were unsuccessful. This superbase mixture forms significant amounts of organopotassium compounds, which are more active metallating agents than their organolithium counterparts. However, organopotassium compounds are also more active in cleavage 
of ethers. $\stackrel{118}{ }$ Hence the mixture of products may result from cleavage of the ether or thioether bridges resulting in undesired compounds.

The syntheses of $t$-Bu-thixantphos, $t$-Bu-sixantphos and $t$-Bu-xantphos were successfully achieved by adaptation of the reported synthesis of the Ph-xantphos

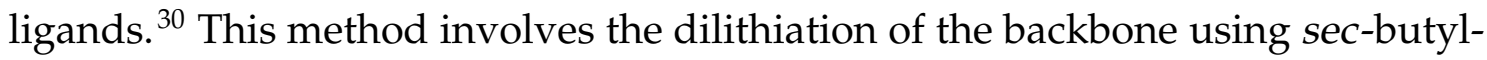
lithium and TMEDA, followed by reaction with chlorodi-tert-butyl phosphine (Scheme 2.2). Unlike the Ph-xantphos synthesis which is complete in 16 hours, the synthesis of the tert-butyl ligands required extended periods. The reactions were allowed to proceed until no further change was determined by NMR spectroscopy (typically seven days). NMR analysis of the crude reaction mixture showed the presence of both the mono and diphosphine. The diphosphine was isolated as white crystals by recrystallisation from n-propanol with yields of 14 , 85 and $37 \%$ for $t$-Bu-sixantphos, $t$-Bu-thixantphos and $t$-Bu-xantphos respectively. The remaining n-propanol solution of the monophosphine could be reduced to dryness and then reused for a second lithiation and reaction with chlorodi-tertbutyl phosphine to produce more of the desired diphosphine.

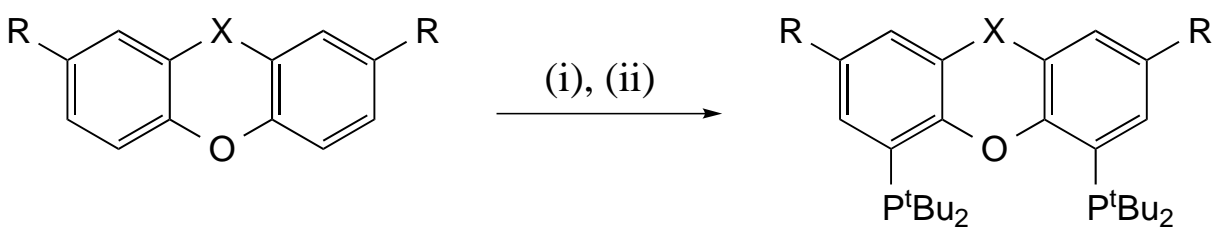

Scheme 2.2: Synthesis of $t$-Bu-xantphos $\left(X=\mathrm{CMe}_{2}, \mathrm{R}=\mathrm{H}\right), t$-Bu-thixantphos (X $=\mathrm{S}, \mathrm{R}=\mathrm{Me})$ and $t$-Bu-sixantphos $\left(\mathrm{X}=\mathrm{SiMe}_{2}, \mathrm{R}=\mathrm{H}\right)$. Reagents and conditions: (i) sec-BuLi, $\mathrm{Et}_{2} \mathrm{O}-78^{\circ} \mathrm{C} \rightarrow \mathrm{RT}, 24$ hours (ii) $\mathrm{ClP}^{\mathrm{t}} \mathrm{Bu}_{2},-78^{\circ} \mathrm{C} \rightarrow \mathrm{RT}, 7$ days.

The size of the tert-butyl groups leads to difficulties in the synthesis of the $t$-Buxantphos ligands. Reaction with one equivalent of chlorodi-tert-butyl phosphine to form the monophosphine is clean and rapid, occurring overnight. However, the steric hindrance of the monophosphine results in a much slower second addition, requiring extended reaction times to achieve significant conversion. When the reaction is allowed to proceed for longer than one week, no additional conversion is observed. This is likely due to degradation of either the lithiated monophosphine or the sec-butyllithium before the second lithiation can occur. Over extended periods organolithium reagents cleave diethyl ether resulting in 
alkanes, alkenes, and lithium ethoxide (Scheme 2.3). 118,119 sec-Butyllithium has been shown to completely react with diethyl ether in one day. ${ }^{119}$ Although the reaction between diethyl ether and phenyllithium is slower (half-time of 100 hours), the extended reaction periods required for the second phosphine addition mean that this degradation pathway may limit the yield of the diphosphine.

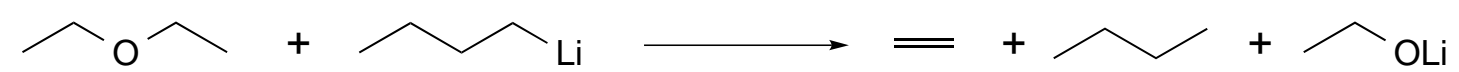

Scheme 2.3: Reaction of n-butyllithium with diethyl ether

In order to promote the dilithiation, three equivalents of a pre-formed sec-butyllithium/TMEDA complex was added to a solution of the backbone. An initial colour change to yellow ( $t$-Bu-thixantphos and $t$-Bu-sixantphos) or green $(t-B u-$ xantphos) was observed, indicative of monolithiation. After stirring overnight this changed to red or yellow respectively. This colour change gives a clear indication of successful dilithiation. In order to favour the diphosphine over the monophosphine, the lithiated backbone was then added slowly to an ethereal solution of chloro-di-t-butylphosphine. Although a mixture of the mono and diphosphine was always obtained, this order of addition was found to be more successful, resulting in increased yields.

The synthesis of these ligands utilises directed ortho metallation as first described independently by Gilman and Wittig in 1939 and 1940 respectively. 120,121 Directed ortho metallation uses a heteroatom that can coordinate to the organolithium reagent, thereby directing it to the ortho sites. In the synthesis of the $t$-Buxantphos ligands the ether linkage can act as a director for the lithiation. Once lithiated, the electron density on the oxygen stabilises the lithiated site against degradation. For $t$-Bu-xantphos and $t$-Bu-sixantphos the oxygen is the only atom present with lone pairs of electrons and thus the lithiation occurs exclusively in the desired positions ortho to the oxygen. However, the precursor to $t$-Buthixantphos, phenoxathiin, contains both an ether and a thioether group, both of which can act as ortho-directors. ${ }^{122}$ Previous research has shown that in the presence of both ether and thioether groups the lithiation will occur ortho to the oxygen. $\stackrel{123}{ }$ Once the first lithiation has taken place the oxygen is less likely to contribute significantly to the second lithiation. For $t$-Bu-xantphos and $t$-Bu-sixantphos the influence of the oxygen is still sufficient for the second lithiation to occur 
in the other ortho position. However, for phenoxathiin the thioether acts as a second directing group and the lithiation occurs ortho to the sulfur (Scheme 2.4). The addition of methyl groups in the positions meta to the thioether prevents the sulfur atom from acting as a director, resulting in the desired $t$-Bu-thixantphos ligand.

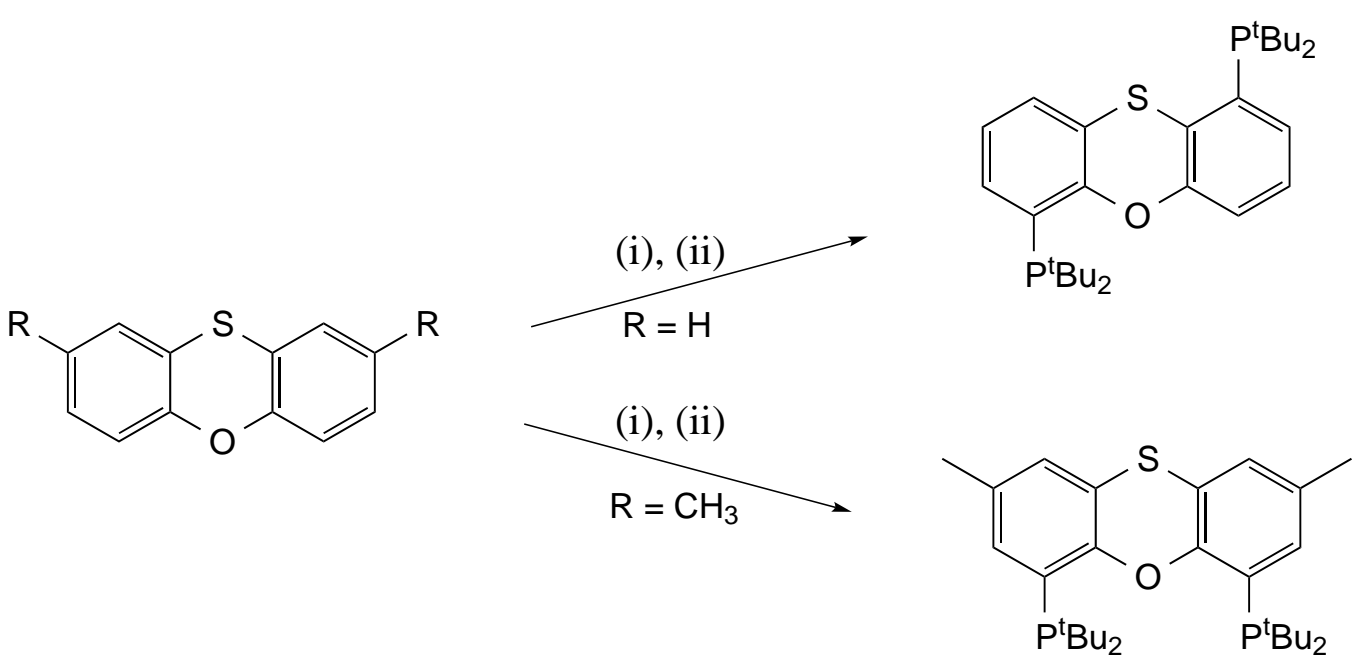

Scheme 2.4: Influence of methyl groups on the synthesis of $t$-Bu-thixantphos. Reagents and conditions: (i) sec-BuLi, $\mathrm{Et}_{2} \mathrm{O}, 24$ hours, (ii) $\mathrm{ClP}^{\mathrm{t}} \mathrm{Bu}_{2}, 24$ hours.

The $t$-Bu-xantphos ligand obtained using sec-butyllithium/TMEDA in diethyl ether had ${ }^{1} \mathrm{H}$ and ${ }^{13} \mathrm{C}$ NMR spectra consistent with the literature (Table 2.1). ${ }^{84}$ However, the ${ }^{31} \mathrm{P}$ chemical shift differed by $2.2 \mathrm{ppm}$ (10.2 ppm in this study compared to $12.4 \mathrm{ppm})$. The NMR spectra for the reported and the synthesised samples were both obtained in $\mathrm{CDCl}_{3}$ and referenced to an external $85 \% \mathrm{H}_{3} \mathrm{PO}_{4}$ standard. Hence the reason for this difference is unclear. However, as the remainder of the NMR and other characterisation data is consistent, it is likely that the two compounds are identical and a typographical error or otherwise was made in the preparation of the earlier paper.

The NMR data of the newly reported $t$-Bu-thixantphos and $t$-Bu-sixantphos ligands are consistent with expectations (Table 2.1). In both cases a plane of symmetry reduces the number of signals, showing a single peak in the ${ }^{31} \mathrm{P} N M R$. The ${ }^{31} \mathrm{P}$ NMR chemical shifts for the $t$-Bu-sixantphos and $t$-Bu-thixantphos ligands are observed as singlets at 8.4 and 9.5 ppm respectively. The ${ }^{1} \mathrm{H}$ NMR signals for the 
aromatic system are as expected, with two singlets present for $t$-Bu-thixantphos, and a doublet, a doublet of doublets and a virtual triplet observed for $t$-Bu-sixantphos and $t$-Bu-xantphos. The chemical shift of the backbone methyl substituents differs for each ligand, as expected. In $t$-Bu-sixantphos the dimethylsilyl group appears at $0.46 \mathrm{ppm}$, close to the tetramethylsilane reference. In $t$-Bu-xantphos the bridgehead methyls appear at $1.57 \mathrm{ppm}$, within the typical range for organic methyl groups. The methyl groups in $t$-Bu-thixantphos are evident at $2.25 \mathrm{ppm}$, consistent with methyl substituents attached to aryl rings.

Table 2.1: Selected NMR data for $t$-Bu-xantphos ligands in $\mathrm{CDCl}_{3}$

\begin{tabular}{|c|c|c|c|c|c|c|}
\hline \multirow{3}{*}{$\begin{array}{l}\text { Diphosphine } \\
\text {-Bu-Xantphos } \underline{84}\end{array}$} & \multirow{3}{*}{$\frac{\frac{{ }^{31} \mathbf{P}}{\delta / \mathbf{p p m}}}{12.4}$} & \multicolumn{5}{|c|}{${ }^{1} \mathbf{H}$} \\
\hline & & \multirow{2}{*}{$\frac{\delta^{\mathrm{t}} \mathbf{B u} / \mathbf{p p m}}{1.21-1.26}$} & \multirow{2}{*}{$\frac{\delta \mathbf{M e} / \mathbf{p p m}}{1.57}$} & \multicolumn{3}{|c|}{$\delta \mathbf{A r} / \mathbf{p p m}$} \\
\hline & & & & 7.02 & 7.38 & 7.60 \\
\hline$t$-Bu-Xantphos (this work) & 10.2 & $1.21-1.25$ & 1.57 & 7.03 & 7.38 & 7.60 \\
\hline$t$-Bu-Thixantphos & 9.5 & $1.22-1.24$ & 2.25 & 6.88 & 7.29 & \\
\hline t-Bu-Sixantphos & 8.4 & 1.29 & 0.46 & 7.12 & 7.53 & 7.87 \\
\hline
\end{tabular}

The ${ }^{1} \mathrm{H}$ NMR data for the $t$-Bu-xantphos ligands show a complex XAA' $X^{\prime}$ spin system for the tert-butyl protons. In a system where the two A atoms (in this case the phosphorus atoms) are strongly coupled, this leads to interaction between the three-bond and five-bond couplings (e.g. when the phosphorus atoms are in a trans configuration in a metal complex) resulting in a virtual triplet. $\underline{124}$ In the $t$-Bu-xantphos ligands there is no atom between the phosphorus atoms so we would expect a three-bond and a nine-bond coupling. A nine-bond coupling is an extremely remote coupling which we would expect to be negligible. Based on previous reports, $, 124,125$ this peak shape with two sharp outer lines and a broad inner peak occurs when the difference between the short- and long-range couplings is very small but not zero. For this to occur the phosphines must have some degree of through-space spin-spin coupling through their lone pairs of electrons (for a review of non-bonded spin-spin coupling see Hierso ${ }^{126}$ ). This is further supported by the difference in the ${ }^{1} \mathrm{H}$ NMR spectra of the three ligands; the central peak is sharpest for $t$-Bu-sixantphos followed by $t$-Bu-thixantphos. For $t$ $\mathrm{Bu}$-xantphos some further detail can be seen indicating that this has the weakest through-space coupling. This trend is consistent with the expected changes in the 
distance between the phosphines upon varying the backbone, which will impact the degree of through-space coupling that can occur, and therefore the difference in the short and long-range coupling constants.

\subsection{Bite-Angle Calculations}

The steric and electronic properties of diphosphine ligands determine their complexation behaviour with transition metals and can influence the reactivity of the complexes, particularly impacting the activity and selectivity in catalytic transformations. $\frac{32,67}{2}$ The xantphos class of ligands were initially investigated for their consistent electronic properties and steric bulk meaning that the impact of the bite-angle on rhodium catalysed hydroformylation could be studied exclusively. ${ }^{30}$ Consequently, a number of studies have investigated the bite-angle impact on a variety of catalytic conversions (for examples see $\frac{66,67,127-129}{}$ ). However, it has since been determined that the bite-angle is a result of both the steric and electronic properties of the ligand. $\frac{32}{}$

The natural bite-angle $\left(\beta_{n}\right)$ was first described by Casey and Whiteker ${ }^{24}$ as a theoretically determined parameter to indicate the preferred chelation angle of diphosphine ligands irrespective of the metal they are coordinating to. These are determined computationally by optimising the geometry of a complex with a rhodium atom at $2.315 \AA$ and measuring the resulting P-Rh-P angle. The crystallographic bite-angle for a given complex can be measured from the crystal structure. The original development of the natural bite-angle parameter included a comparison of the natural bite-angle for seven different ligands and crystallographic bite-angles for one transition metal complex of each ligand. The two biteangles were found to be correlated in accordance with the equation $\Theta_{\exp }=0.98 \beta_{n}$ $+2.13(\mathrm{R}=0.92) \cdot{ }^{24}$ Another study in 2009 showed that for a range of diphosphines the crystallographically determined bite-angles have a narrow range, which is close to the calculated natural bite-angle. $\frac{25}{5}$ This study did not however, determine a correlation between the two.

Despite the ubiquity of the natural bite-angle within diphosphine chemistry, only two studies have investigated whether the natural bite-angle has proven to be an apt predictor of the crystallographically determined bite-angle. ${ }^{24,25}$ One of these only used one complex for each ligand and the other did not calculate the math- 
ematical relationship between the two. A large number of new crystal structures have also been reported since the studies were published in the 1990 's. $\frac{68}{\text { In order }}$ to establish whether the natural bite-angle is still an apt predictor of the crystallographic bite-angle, almost 25 years after the original publication, the relationship between the two was investigated using data from the 2014 CSD. 68 The results are summarised in Table 2.2 and Figure 2.1. The median crystallographic biteangle was used to decrease the influence of outliers on the results. The natural and experimental bite-angles show a good correlation described by the equation $\Theta_{\exp }=1.10 \beta_{n}-5.70\left(R^{2}=0.92\right)$. Some of the experimental and natural bite-angles that are most relevant to this work show significant differences (for example $t$ Bu-xantphos $\beta_{n}=140^{\circ}$, experimental $=153.317^{\circ}$, and Ph-sixantphos $\beta_{n}=109^{\circ}$, experimental $=99.165^{\circ}$ ). This may be due to the low numbers of structures available for these ligands (four and five respectively) at which point the metals used will have a significant impact.

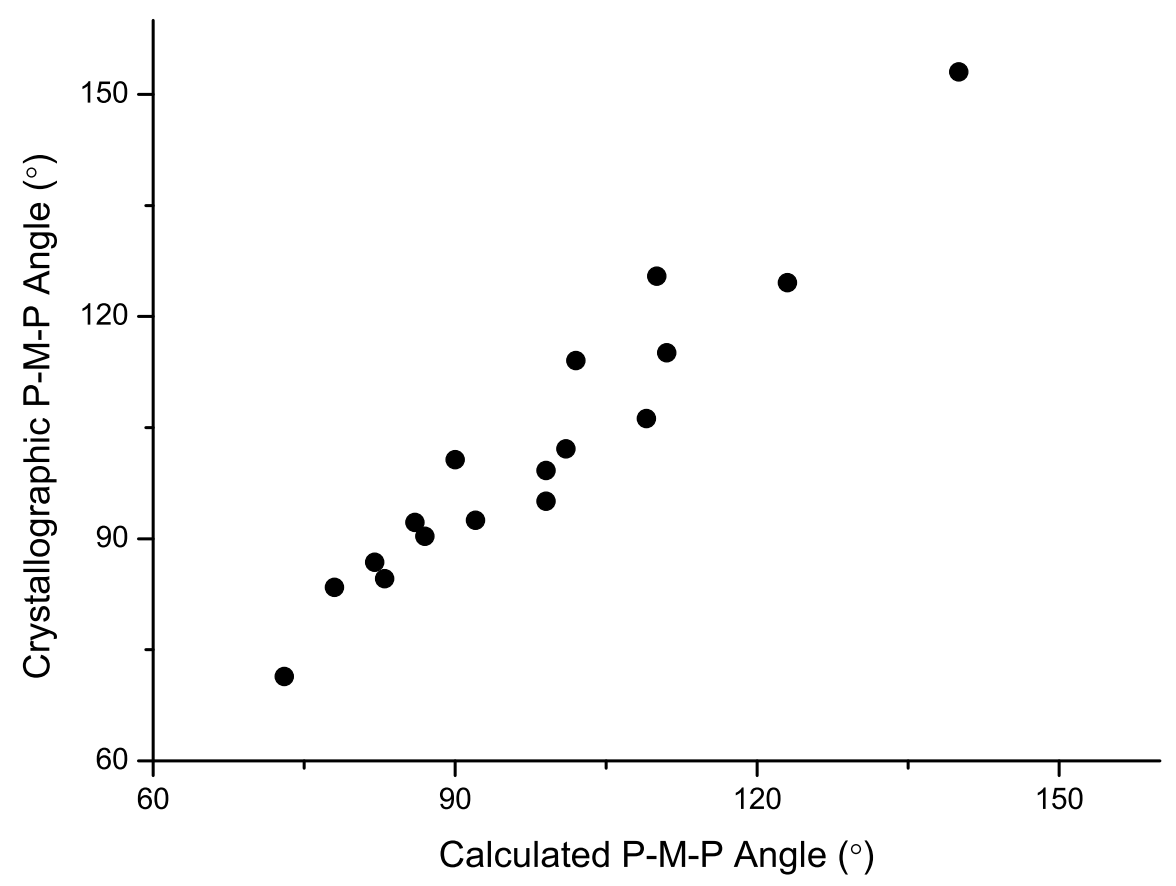

Figure 2.1: Comparison of the natural and crystallographic bite-angles. Trendline $\Theta_{\text {exp }}=1.10 \beta_{n}-5.70\left(\mathrm{R}^{2}=0.92\right)$. 
Table 2.2: Crystallographic ${ }^{68}$ and natural bite-angles ${ }^{25,64,67,130}$ for diphosphine ligands.

\begin{tabular}{cccccc}
\hline Diphosphine & Structures & $\mathbf{P}-\mathbf{P} / \AA$ & Range $^{\circ}$ & Bite-Angle $/{ }^{\circ}$ & $\beta_{\mathbf{n}} /{ }^{\circ}$ \\
\hline dppm & 384 & 2.718 & $62.757-77.706$ & 71.062 & 73 \\
dppe & 1939 & 3.095 & $71.073-92.926$ & 83.818 & 78 \\
dppp & 3237 & 3.308 & $83.333-105.420$ & 92.181 & 86 \\
BINAP & 146 & 3.296 & $85.9820-115.666$ & 91.925 & 92 \\
dppb & 127 & 3.391 & $89.447-111.491$ & 94.240 & 99 \\
DPEphos & 101 & 3.844 & $96.094-158.531$ & 112.691 & 102 \\
Ph-Xantphos & 65 & 3.908 & $98.829-153.134$ & 108.535 & 111 \\
BISBI & 4 & 4.079 & $103.533-151.951$ & 124.789 & 123 \\
Ph-Sixantphos & 5 & 3.691 & $95.250-152.149$ & 99.165 & 109 \\
Ph-Thixantphos & 3 & 4.021 & $109.528-155.050$ & 111.724 & 110 \\
$t$-Bu-xantphos & 4 & 4.527 & $152.302-153.456$ & 153.317 & 140 \\
dpp-benzene & 116 & 3.101 & $74.380-92.499$ & 84.900 & 83 \\
dppn & 25 & 3.176 & $79.867-93.270$ & 86.864 & 82 \\
dppx & 19 & 3.569 & $90.045-106.186$ & 102.636 & 90 \\
dbpe & 109 & 3.200 & $83.989-95.949$ & 90.369 & 87 \\
dbpp & 12 & 3.503 & $94.556-104.857$ & 99.175 & 99 \\
dbpx & 10 & 3.634 & $98.758-107.255$ & 101.881 & 101 \\
\hline
\end{tabular}

In order to determine the potential differences between the $t$-Bu-xantphos and $\mathrm{Ph}$-xantphos ligands, the natural bite-angles of the three $t$-Bu-xantphos ligands were calculated. Previously the $t$-Bu-xantphos bite-angle was calculated using molecular mechanics, however molecular mechanics has previously been reported as overestimating the bite-angles for diphosphines with bulky alkyl substituents, like the tert-butyl groups in the $t$-Bu-xantphos ligands. ${ }^{25}$ As the bite-angles of the $t$-Bu-xantphos ligands are an integral part of the current study, DFT was utilised to reduce the likelihood of overestimation. The angles for the Ph-xantphos ligands were also calculated to allow for a comparison between the DFT and molecular mechanics methods. The structures were optimised using the B3LYP functional, $\underline{131-134}$ with the def2-TZVP basis set. 135,136 using a Rh-P distance of $2.315 \AA$. The resulting bite-angles along with those published using molecular mechanics are given in Table 2.3, The values calculated using DFT and molecular mechanics 
are slightly larger for the Ph-xantphos ligands. The trend is consistent and molecular mechanics techniques can overestimate the impact of $\pi$-stacking in molecules with phenyl rings. The value for $t$-Bu-xantphos using DFT is much smaller than the literature value calculated with molecular mechanics. However, molecular mechanics is known to overestimate the bite-angles of bulky diphosphines which may account for this difference. $\stackrel{25}{\underline{n}}$

Table 2.3: Natural bite-angles of xantphos ligands. Molecular mechanics values taken from the literature. ${ }^{30}$

\begin{tabular}{lcc}
\hline Diphosphine & Molecular Mechanics $\left(^{\circ}\right)$ & DFT $\left(^{\circ}\right)$ \\
\hline Ph-Sixantphos & 108.7 & 111.89 \\
Ph-Thixantphos & 109.4 & 112.65 \\
Ph-Xantphos & 111.7 & 114.18 \\
$t$-Bu-Sixantphos & & 126.80 \\
$t$-Bu-Thixantphos & & 126.98 \\
$t$-Bu-Xantphos & 140 & 127.56 \\
\hline
\end{tabular}

The natural bite-angles for the $t$-Bu-xantphos series of ligands are much larger than for the Ph-xantphos ligands. Given that the remainder of the ligands are unchanged, this effect is due to the impact of the tert-butyl groups. Tert-butyl groups are more electron-donating than the phenyl groups so the phosphorus atoms will have more electron density resulting in an electrostatic repulsion of the two phosphorus atoms. The tert-butyl groups also have a larger steric impact than the planar phenyl rings. This would result in a larger bite-angle as the steric bulk of the tert-butyl substituents pushes the two phosphorus atoms further apart. The trend between the two groups is the same with the $\mathrm{CMe}_{2}$ bridged ligands having the largest bite-angles and the $\mathrm{SiMe}_{2}$ bridged having the smallest. However, the range for the $t$-Bu-xantphos ligands $\left(0.76^{\circ}\right)$ is much smaller than for the Ph-xantphos ligands $\left(2.3^{\circ}\right)$. This suggests that the tert-butyl substituents are the main contributing factor to the bite-angle and the small changes in the bridging group have a smaller effect because the sterics of the tert-butyl groups dominates.

The bite-angles of the $t$-Bu-xantphos ligands are likely to have a significant impact on their coordination chemistry. The phenyl ligands form a range of com- 
plexes favouring cis-chelation in square-planar and octahedral complexes, although trans square planar complexes have been reported. 115 With bite-angles of $126.8-127.6^{\circ}$ the $t$-Bu-xantphos ligands may prefer bis-equatorial positions in trigonal bipyramidal complexes with angles close to $120^{\circ}$. However, the biteangles are halfway between the cis- and trans-coordination angles for squareplanar complexes, which may result in mixtures of products. Mixtures of cis and trans isomers have been reported for palladium Ph-xantphos complexes, so the ratio between the two geometries may favour trans-coordination for the $t$-Buxantphos ligands. $\underline{73,115}$ Diphosphine ligands that exhibit exclusive trans-chelation have been described in a review as "elusive". 137

\subsection{Basicity}

Spectroscopic analysis of the $t$-Bu-xantphos ligands in $\mathrm{CDCl}_{3}$ showed a small amount of an impurity, characterised by a new peak in the ${ }^{31} \mathrm{P}$ NMR spectra and an unusual spin system downfield of the aromatic signals in the ${ }^{1} \mathrm{H}$ NMR spectra. The amount of the impurity increased over time, suggesting that the ligands were reacting with the solvent. Chloroform is known to undergo slow degradation forming hydrochloric acid. $\frac{138}{2}$ Tertiary phosphines can act as Brønsted bases, forming phosphonium ions that can be used as components in catalytic reactions. ${ }^{139}$ Hence, the identity of these compounds as phosphonium salts seemed likely.

The three $t$-Bu-xantphos ligands react with the strong acids $\mathrm{HCPh}\left(\mathrm{SO}_{2} \mathrm{CF}_{3}\right)_{2}\left(\mathrm{p} K_{\mathrm{a}}\right.$ $=2.0$ in DMSO) or $\mathrm{H}_{2} \mathrm{C}\left(\mathrm{SO}_{2} \mathrm{CF}_{3}\right)_{2}\left(\mathrm{pK}_{\mathrm{a}}=2.4\right.$ in DMSO$), \stackrel{140}{\underline{ }}$ resulting in immediate formation of a phosphonium salt (Scheme 2.5). These acids are useful as they are non-hygroscopic solids allowing for accurate stoichiometry. Addition of excess acid results in the same product; no evidence for an additional protonation was observed. The NMR data is consistent with the data for the impurity observed in the synthesis of the $t$-Bu-xantphos ligands, indicating that the impurity is [ $t$-Buxantphos) $\mathrm{H}]^{+}$.

Selected NMR data for the phosphonium salts [(t-Bu-xantphos) $\mathrm{H}] \mathrm{CH}\left(\mathrm{SO}_{2} \mathrm{CF}_{3}\right)_{2}$ with the three ligands is given in Table 2.4. A broad singlet is present in the ${ }^{31} \mathrm{P}$ NMR spectra for the three phosphonium ions, shifted slightly downfield from the 


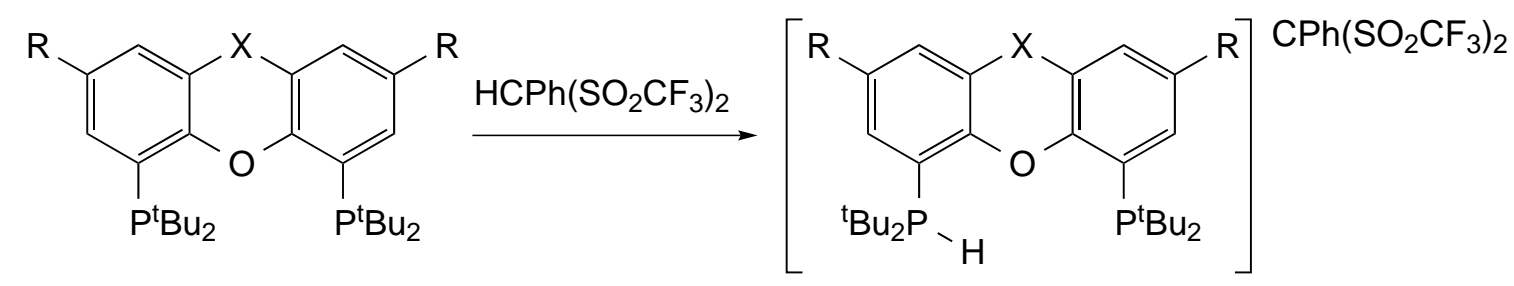

Scheme 2.5: Protonation of the ligands using a strong acid, in $\mathrm{C}_{6} \mathrm{D}_{6}$.

free ligand. The ${ }^{1} \mathrm{H}$ and ${ }^{13} \mathrm{C}$ NMR spectra show half the expected number of aromatic signals, and only one signal for the tert-butyl protons and the quaternary and terminal carbons. This indicates that although the expected complex is asymmetric the system is undergoing a dynamic process such that the two halves of the molecule are equivalent on the NMR timescale. The most likely process is the exchange of the proton between the two phosphorus atoms. The ${ }^{1} \mathrm{H}$ NMR spectra all show a complex spin system of the type $\mathrm{XAA}^{\prime} \mathrm{X}^{\prime}$ for the phosphonium proton, which is further evidence for the dynamic exchange of the proton between the two phosphorus atoms.

Table 2.4: Selected NMR data for the [(t-Bu-xantphos) $\mathrm{H}] \mathrm{CH}\left(\mathrm{SO}_{2} \mathrm{CF}_{3}\right)_{2}$ compounds in $\mathrm{CDCl}_{3}\left(\Delta \delta=\delta_{[(\text {diphosphine }) \mathrm{H}]^{+}}-\delta_{\text {diphosphine }}\right)$.

\begin{tabular}{lcccc}
\hline & \multicolumn{2}{c}{${ }^{31} \mathbf{P}$} & & ${ }^{1} \mathbf{H}$ \\
\cline { 2 - 3 } Diphosphine & $\delta / \mathbf{p p m}$ & $\Delta \delta / \mathbf{p p m}$ & & $\mathbf{H}^{+} \delta / \mathbf{p p m}$ \\
\hline t-Bu-Sixantphos & 14.3 & 5.9 & 9.57 \\
t-Bu-Thixantphos & 15.8 & 6.3 & & 8.99 \\
t-Bu-Xantphos & 17.4 & 7.2 & & 8.57 \\
\hline
\end{tabular}

The difference in the rate of exchange of the proton in the three compounds is clearly shown by their ${ }^{31} \mathrm{P}$ and ${ }^{1} \mathrm{H}$ NMR spectra. The ${ }^{31} \mathrm{P}$ NMR signal is sharpest for $t$-Bu-sixantphos followed by $t$-Bu-thixantphos while $t$-Bu-xantphos has a very broad signal. This indicates that the exchange is fastest for $t$-Bu-sixantphos and slowest for $t$-Bu-xantphos. The ${ }^{1} \mathrm{H}$ NMR spectra (Figure 2.2) show an $\mathrm{XAA}^{\prime} \mathrm{X}^{\prime}$ spin system for the phosphonium proton. The signal appears most like a virtual triplet for $t$-Bu-sixantphos, implying very little difference in the coupling constants of the proton with each phosphorus. For $t$-Bu-thixantphos the central peak 
has broadened slightly, while in $t$-Bu-xantphos the central peak is very broad, indicating that the difference in the two coupling constants is increasing. The chemical shift of the phosphonium proton is different for the three systems $(t-$ Bu-sixantphos $=9.57, t$-Bu-thixantphos $=8.99$ and $t$-Bu-xantphos $=8.57 \mathrm{ppm}$ ). This indicates that the $t$-Bu-sixantphos proton is less shielded and thus has a faster rate of exchange, while the $t$-Bu-xantphos proton is the most shielded and has the slowest rate of exchange. This trend is consistent with the bite-angles of the ligands. $t$-Bu-Sixantphos has the smallest bite-angle, so the two phosphorus atoms are closer resulting in a lower barrier to exchange, whilst $t$-Bu-xantphos has the largest bite-angle and thus the slowest exchange.

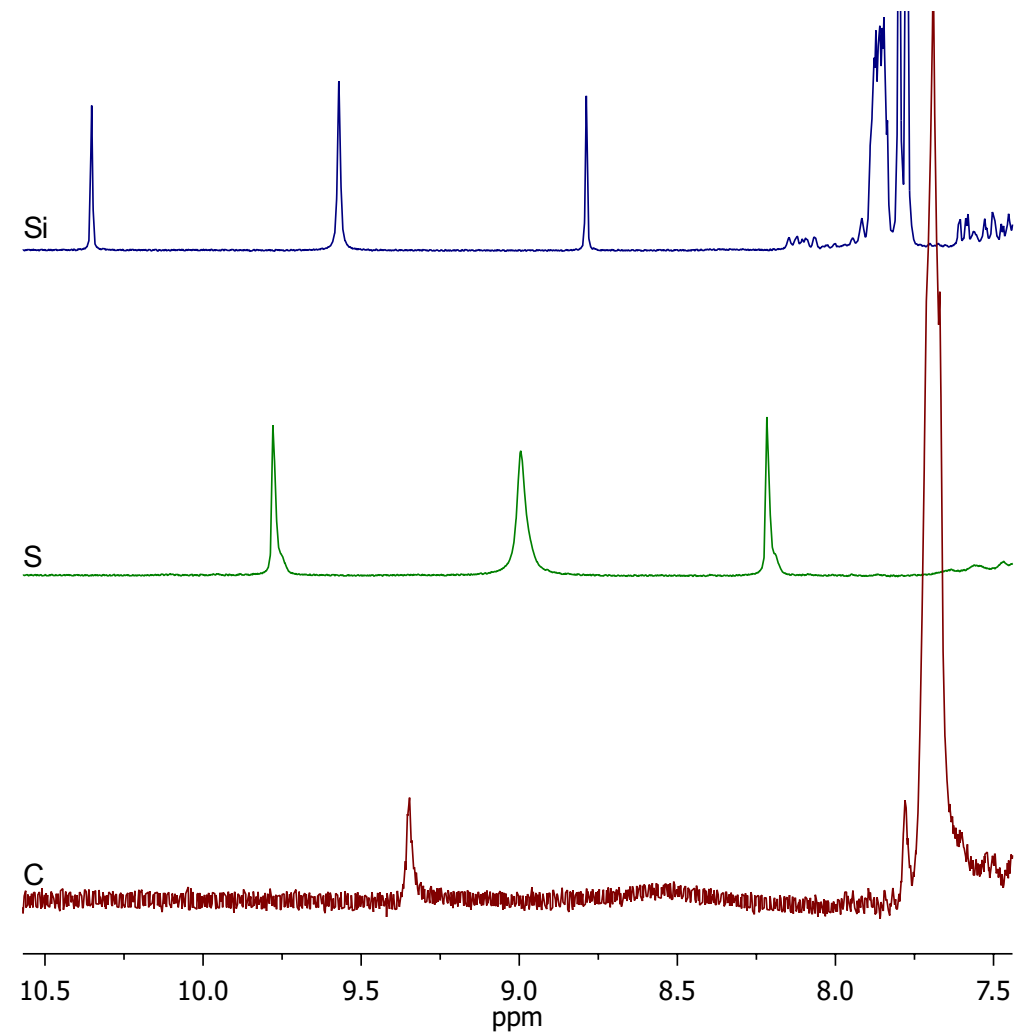

Figure 2.2: ${ }^{1} \mathrm{H}$ NMR spectra in $\mathrm{CDCl}_{3}$ at $20{ }^{\circ} \mathrm{C}$ for $[(t \text {-Bu-sixantphos }) \mathrm{H}]^{+},[(t-\mathrm{Bu}$ thixantphos $) \mathrm{H}]^{+}$, and $[(t-B u-x a n t p h o s) H]^{+}(\mathrm{Si}, \mathrm{S}$ and $\mathrm{C}$ respectively) showing the $\mathrm{P}-\mathrm{H}$ region

The dynamic behaviour of the phosphonium ions was further investigated using variable temperature ${ }^{31} \mathrm{P}$ and ${ }^{1} \mathrm{H}$ NMR experiments on $[(t$-Bu-thixantphos $) \mathrm{H}] \mathrm{CH}$ $\left(\mathrm{SO}_{2} \mathrm{CF}_{3}\right)_{2}$ (Figure 2.3). At room temperature a single peak is present in the ${ }^{31} \mathrm{P}$ 
NMR spectrum. When heated this peak shifts slightly to higher ppm and becomes sharper. This single peak indicates that the exchange of the proton between the two phosphorus atoms is occurring rapidly enough that the two phosphorus atoms appear to have the same environment on the NMR timescale. Cooling below room temperature causes the singlet to broaden significantly, with coalescence occurring around $-40^{\circ} \mathrm{C}$. At $-60^{\circ} \mathrm{C}$ two broad signals are present. One of these signals resolves into a doublet at $-80^{\circ} \mathrm{C}$, however the other peak is still very broad ranging from 11-23 ppm. Given that the protonation results in a downfield shift at room temperature, it is likely that the sharp doublet belongs to the unprotonated phosphorus and the broad peak is the protonated phosphorus.

The variable temperature ${ }^{1} \mathrm{H} \mathrm{NMR}$ data for [( $t$-Bu-thixantphos $\left.) \mathrm{H}\right] \mathrm{CH}\left(\mathrm{SO}_{2} \mathrm{CF}_{3}\right)_{2}$ (Figure 2.3) shows similar changes to the ${ }^{31} \mathrm{P}$ NMR spectra. At low temperature the proton is static on a single phosphorus atom so we observe a large doublet due to coupling with the phosphorus. As the temperature increases to $-20{ }^{\circ} \mathrm{C}$, a broad signal begins to appear in the centre of the doublet as exchange begins to occur and the signal changes from a simple doublet to a $\mathrm{XAA}^{\prime} \mathrm{X}^{\prime}$ spin system. The intensity of the central peak increases and at $20{ }^{\circ} \mathrm{C}$ all three peaks begin to broaden again with coalescence at $50{ }^{\circ} \mathrm{C}$. This broadening may be the result of the proton no longer being isolated on a single molecule but delocalised across the entire system.

Typically ${ }^{31} \mathrm{P}$ NMR spectra are proton decoupled. However, with very strongly coupled systems such as phosphonium ions the decoupler is not able to fully decouple the spin system, resulting in broadening and side bands. To further investigate the $[(t$-Bu-thixantphos $) \mathrm{H}] \mathrm{CH}\left(\mathrm{SO}_{2} \mathrm{CF}_{3}\right)_{2}$ system, proton-coupled phosphorus NMR spectra were obtained at room temperature and $-80^{\circ} \mathrm{C}$ (Figure 2.4). At room temperature a simple doublet appears, indicating rapid movement of the proton between the two phosphorus atoms thus coupling with both. When cooled to $-80^{\circ} \mathrm{C}$, the ${ }^{31} \mathrm{P}\left\{{ }^{1} \mathrm{H}\right\}$ spectrum showed a doublet and a broad singlet. In the proton-coupled phosphorus spectrum, the doublet is retained with a phosphorus-phosphorus coupling constant of $136.9 \mathrm{~Hz}$ confirming that this signal is for the non-protonated phosphorus which couples to the protonated phosphorus atom. The broad singlet resolves into a doublet of doublets with coupling constants of 481.5 and $136.9 \mathrm{~Hz}$ consistent with coupling to the proton and the other phosphorus. This further confirms that no exchange of the proton is occurring at 

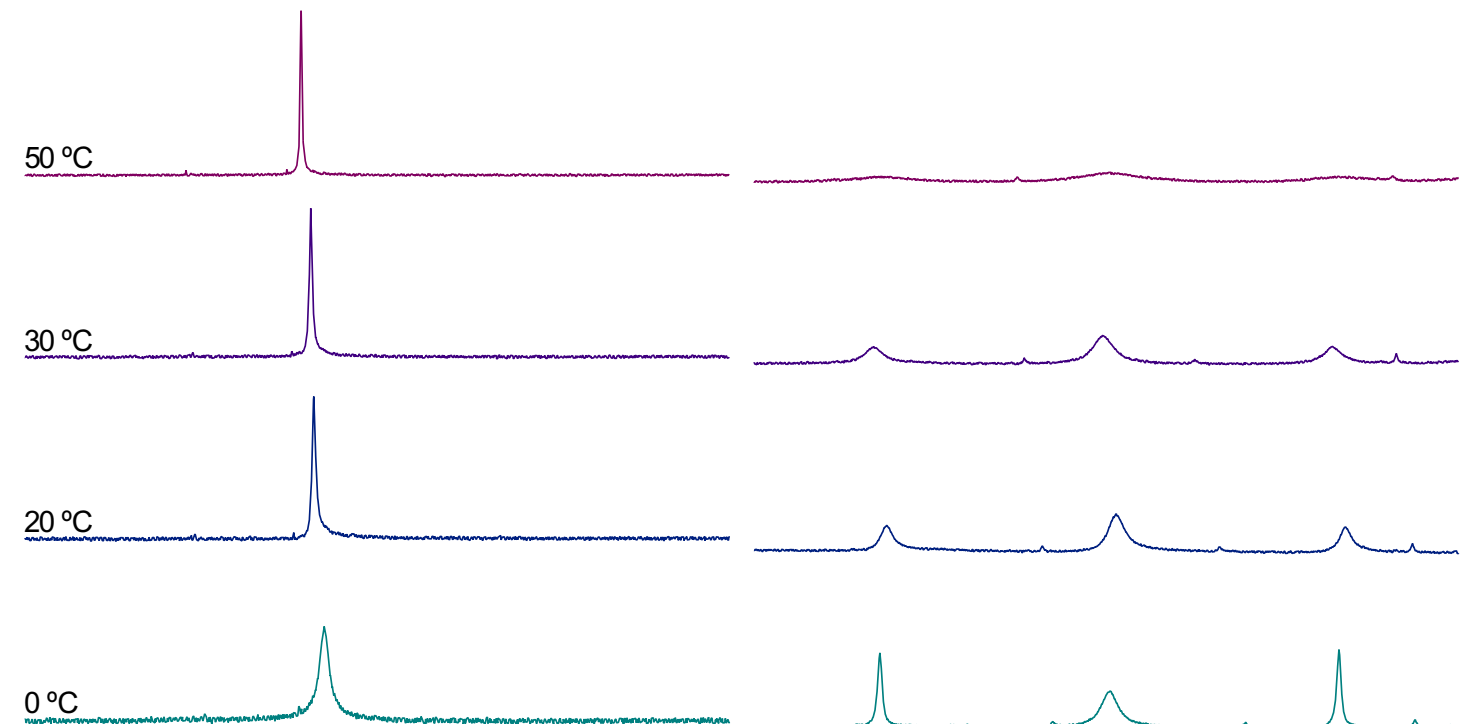

$-20^{\circ} \mathrm{C}$
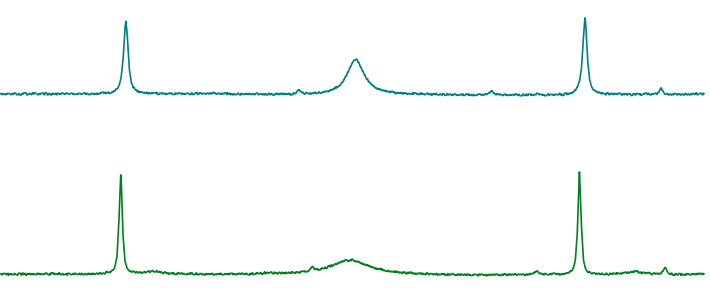

$-40^{\circ} \mathrm{C}$

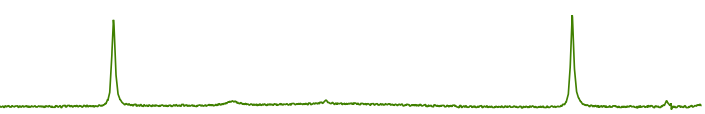

$-60^{\circ} \mathrm{C}$
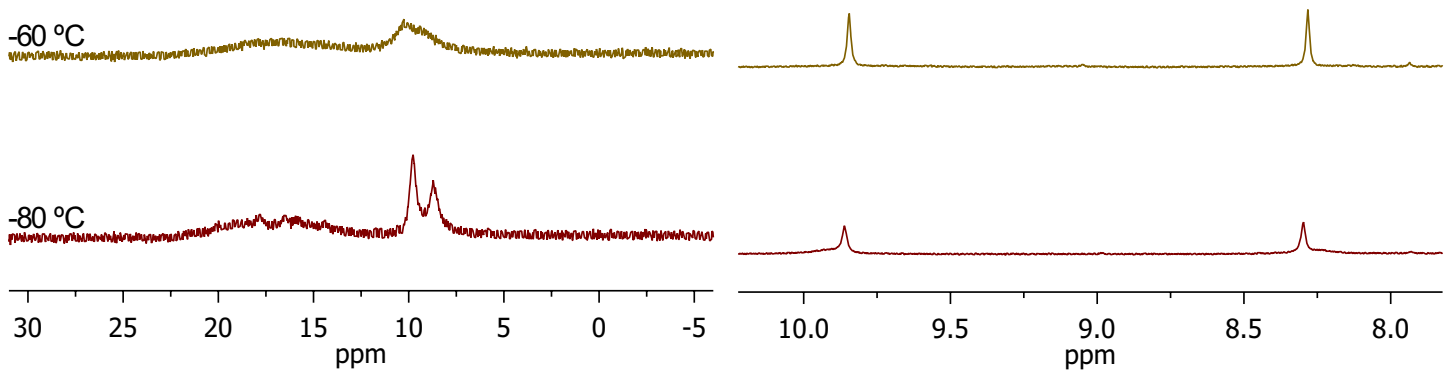

Figure 2.3: Variable temperature ${ }^{31} \mathrm{P}$ (left) and ${ }^{1} \mathrm{H}$ (right) NMR data for [( $t$-Buthixantphos) $\mathrm{H}] \mathrm{CH}\left(\mathrm{SO}_{2} \mathrm{CF}_{3}\right)_{2}$ in $\mathrm{CD}_{2} \mathrm{Cl}_{2}$.

low temperature.

Single colourless crystals of [ $t$-Bu-thixantphos $(\mathrm{H})] \mathrm{CPh}\left(\mathrm{SO}_{2} \mathrm{CF}_{3}\right)_{2}$ suitable for $\mathrm{X}$ ray diffraction were grown from the reaction mixture in benzene. The compound crystallised with a benzene solvate, as $2[t$-Bu-thixantphos $(\mathrm{H})] \mathrm{CPh}\left(\mathrm{SO}_{2} \mathrm{CF}_{3}\right)_{2} \cdot \mathrm{C}_{6} \mathrm{D}_{6}$ in the monoclinic space group $P 2_{1} / n$. Selected bond lengths and angles are summarised in Table 2.5, and crystallographic data is given in Table 2.6. Although 

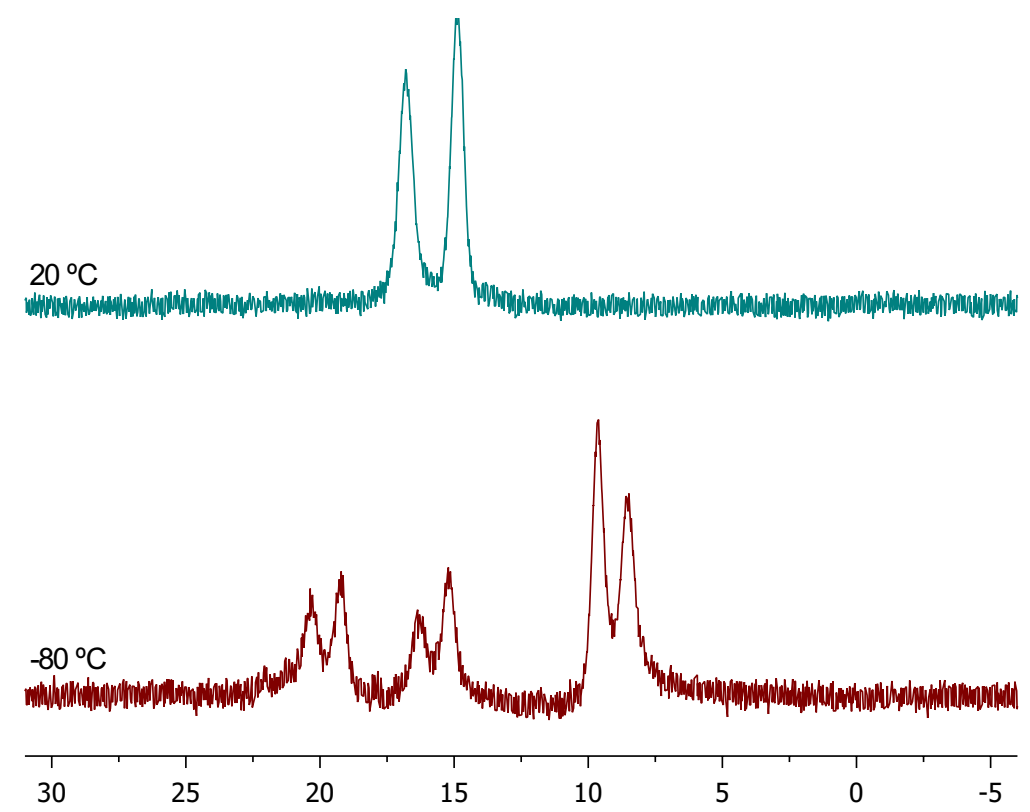

Figure 2.4: Variable-temperature proton coupled ${ }^{31} \mathrm{P}$ NMR data for [( $t$-Bu-thixantphos) $\mathrm{H}]^{+}$in $\mathrm{CD}_{2} \mathrm{Cl}_{2}$.

the cationic portion was well refined, one of the counterions is disordered with two positions for the $\mathrm{SO}_{2} \mathrm{CF}_{3}$ groups. The proton on the phosphonium ion is exclusively located on a single phosphorus atom. As a result the estimated standard deviation (esd) on the bond lengths and angles involving this proton are relatively high. The distances from the proton to the other phosphorus or to the oxygen atom are both too long to indicate any degree of interaction. The crystal structure was collected at $284.87 \mathrm{~K}$, and based on the variable temperature NMR data significant exchange of the proton is expected at this temperature. However, this is not apparent in the X-ray structure suggesting that the exchange does not occur in the solid state.

\subsection{Selenides}

The electronic properties of phosphine ligands can be investigated in a number of different ways. The most well-known quantification is the Tolman electronic parameter. ${ }^{14}$ This involves the measurement of the carbonyl stretching frequency in a $\left[\mathrm{Ni}(\mathrm{CO})_{3} \mathrm{~L}\right]$ complex. Related approaches use $\left[\mathrm{Mo}(\mathrm{CO})_{5} \mathrm{~L}\right]$ or $\left[\mathrm{Rh}(\mathrm{CO}) \mathrm{ClL}_{2}\right]$ 


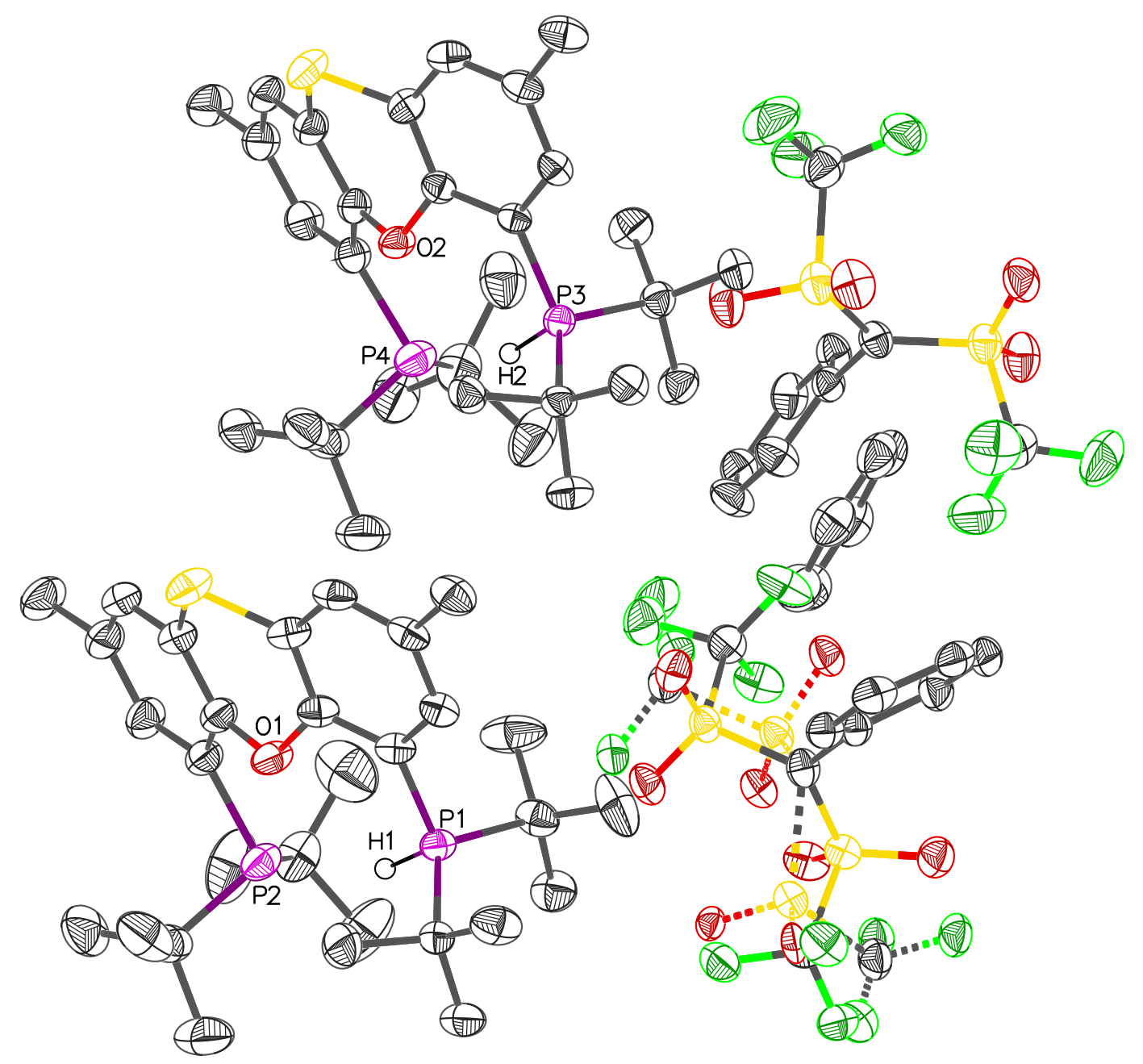

Figure 2.5: X-ray crystal structure of $2[(t$-Bu-thixantphos $) \mathrm{H}] \mathrm{CPh}\left(\mathrm{SO}_{2} \mathrm{CF}_{3}\right)_{2} \cdot \mathrm{C}_{6} \mathrm{D}_{6}$ (50\% probability thermal ellipsoids). Selected hydrogen atoms omitted for clarity. 
Table 2.5: Selected bond distances $(\AA)$ and angles $\left(^{\circ}\right)$ of 2[(t-Bu-thixantphos)$\mathrm{H}] \mathrm{CPh}\left(\mathrm{SO}_{2} \mathrm{CF}_{3}\right)_{2} \cdot \mathrm{C}_{6} \mathrm{D}_{6}$.

\begin{tabular}{llll}
\hline \multicolumn{2}{c}{ Bond distances $(\AA)$} & \multicolumn{3}{l}{ Bond angles $\left(^{\circ}\right)$} \\
\hline P1-H1 & $1.22(3)$ & P1-H1...P2 & $160(2)$ \\
P2...H1 & $2.96(3)$ & P1...O1...P2 & $89.19(6)$ \\
P1..P2 & $4.1290(11)$ & P3-H2...P4 & $154(2)$ \\
O1...H1 & $2.44(3)$ & P3...O2...P4 & $86.40(5)$ \\
P3-H2 & $1.22(3)$ & Ring 1...Ring 2 & $18.28(10)$ \\
P4...H2 & $2.91(3)$ & Ring 3...Ring 4 & $26.67(10)$ \\
P3...P4 & $4.0372(11)$ & & \\
O2...H2 & $2.43(3)$ & & \\
\hline
\end{tabular}

complexes. .11 The value of the phosphorus-metal coupling constants in platinum or rhodium complexes have also been used to quantify the electronic properties of phosphine ligands. $\frac{9,11,12,16}{}$ The values of the carbonyl stretching frequency and the phosphorus-metal coupling constants are dependent on the geometries of the metal complexes and these can be influenced significantly by steric properties, such as those imposed by large diphosphine ligands. The ${ }^{1} J_{\mathrm{PSe}}$ spin-spin coupling constants of phosphine selenides are a convenient and accurate measure of the electronic properties of tertiary phosphines. $\frac{17,141,142}{1 n}$ this case the more electron donating the phosphorus substituents are, the lower the resulting ${ }^{1} J_{\mathrm{PSe}}$.

The phosphine selenides are air-stable solids that are generally relatively straightforward to synthesise by reaction of the phosphine with either elemental selenium or potassium selenocyanide (with or without heating). $\stackrel{17,143}{=}$ The value of ${ }^{1} J_{\text {PSe, }}$ as with all coupling constants, is governed by interactions between the s-orbitals. According to Bent's rule "atomic s-character concentrates in orbitals directed towards electropositive substituents". $\underline{144}$ Thus the greater the value of ${ }^{1} J_{\mathrm{PSe}}$ the more s-character is present in the P-Se bond, indicating a greater degree of electronegative substituents, and thus the less electron donating the phosphine ligand will be.

In addition to the use of ${ }^{1} J_{\text {PSe }}$ as a measure of the electronic influence of a phosphine, it can also be used to measure the Brønsted basicity of a given phosphine. 
Table 2.6: Crystallographic data and structure refinement of 2[t-Bu-thixant$\operatorname{phos}(\mathrm{H})] \mathrm{CPh}\left(\mathrm{SO}_{2} \mathrm{CF}_{3}\right)_{2} \cdot \mathrm{C}_{6} \mathrm{D}_{6}$

\begin{tabular}{|c|c|}
\hline Empirical formula & $\mathrm{C}_{84} \mathrm{H}_{110} \mathrm{~F}_{12} \mathrm{O}_{10} \mathrm{P}_{4} \mathrm{~S}_{6}$ \\
\hline Formula weight & 1823.95 \\
\hline Temperature/K & 284.87(10) \\
\hline Crystal system & monoclinic \\
\hline Space group & $\mathrm{P} 2{ }_{1} / \mathrm{n}$ \\
\hline $\mathrm{a} / \AA$ & $14.19615(12)$ \\
\hline $\mathrm{b} / \AA$ & $39.4563(3)$ \\
\hline$c / \AA$ & $16.35832(15)$ \\
\hline$\alpha /{ }^{\circ}$ & 90 \\
\hline$\beta /{ }^{\circ}$ & $100.1751(8)$ \\
\hline$\gamma /{ }^{\circ}$ & 90 \\
\hline Volume $/ \AA^{3}$ & 9018.64(13) \\
\hline $\mathrm{Z}$ & 4 \\
\hline$\rho_{\text {calc }} \mathrm{mg} / \mathrm{mm}^{3}$ & 1.343 \\
\hline$\mu / \mathrm{mm}$ & 2.749 \\
\hline $\mathrm{F}(000)$ & 3832.0 \\
\hline Crystal size $/ \mathrm{mm}$ & $0.49 \times 0.16 \times 0.14$ \\
\hline Radiation & $\mathrm{CuK} \alpha(\lambda=1.54184)$ \\
\hline $2 \theta$ range for data collection & 5.928 to $147.832^{\circ}$ \\
\hline Index ranges & $-17 \leq \mathrm{h} \leq 17,-48 \leq \mathrm{k} \leq 48,-20 \leq 1 \leq 20$ \\
\hline Reflections collected & 69203 \\
\hline Independent reflections & $17960\left[R_{\text {int }}=0.0261, R_{\text {sigma }}=0.0219\right]$ \\
\hline Data/restraints/parameters & $17960 / 291 / 1221$ \\
\hline Goodness-of-fit on $\mathrm{F}^{2}$ & 1.068 \\
\hline Final $\mathrm{R}$ indexes $[\mathrm{I}>=2 \sigma(\mathrm{I})]$ & $\mathrm{R}_{1}=0.0549, \mathrm{w} \mathrm{R}_{2}=0.1473$ \\
\hline Final $\mathrm{R}$ indexes [all data] & $\mathrm{R}_{1}=0.0623, \mathrm{w} \mathrm{R}_{2}=0.1531$ \\
\hline Largest diff. peak/hole / e $\AA^{-3}$ & $1.11 /-0.56$ \\
\hline
\end{tabular}


A correlation between the experimentally measured $\mathrm{p} K_{\mathrm{b}}$ and the ${ }^{1} J_{\mathrm{PSe}}$ value has been reported with linear regression: ${ }^{1} J_{\mathrm{PSe}}=7.60 \times \mathrm{p} K_{\mathrm{b}}+646\left(\mathrm{R}^{2}=0.9492\right) . \underline{17}$ As such, determining the value of ${ }^{1} J_{\mathrm{PSe}}$ allows for calculation of the $\mathrm{p} K_{\mathrm{b}}$ and has shown good agreement with the experimentally determined data. One limitation of this method is the impact that sterically bulky groups can have. For $\mathrm{P}^{\mathrm{t}} \mathrm{Bu}_{3}$ the correlation suggests a $\mathrm{p} K_{\mathrm{b}}$ value of 6.0 however the experimentally determined value is 2.60 .17 This is the result of the tert-butyl groups increasing the C-P-C angles, thus decreasing the s-character of the lone pair of electrons.

The $t$-Bu-xantphos ligands showed significant resistance to selenation. Reaction directly with elemental selenium has been reported as preferable to potassium selenocyanide as the latter reacts slowly and gives lower yields. $\stackrel{17}{ }$ The previously reported $\mathrm{Ph}$-xantphos diselenide was synthesised by refluxing $\mathrm{Ph}$-xantphos and red selenium in toluene overnight resulting in diselenation. $\underline{145}$ Attempting this method with the $t$-Bu-xantphos ligands reported here showed little reaction. Attempts were also made using $\mathrm{KSeCN}$ similar to those previously reported, $\underline{143}$ however these were also unsuccessful. Successful monoselenation was obtained by refluxing the ligands with a large excess of grey selenium in toluene for three days (Scheme 2.6). Extending the reaction period did not result in the formation of any diselenide. This is likely due to the steric restraints of these ligands.<smiles></smiles>

(i)<smiles>[R]c1cc([X])c2c(c1)Oc1c(cc([R])cc1P(CCCC)C(C)CC)[X]2P=[Se]</smiles>

Scheme 2.6: Selenation of $t$-Bu-xantphos ligands, $\left(\mathrm{X}=\mathrm{CMe}_{2}, \mathrm{R}=\mathrm{H}\right), t$-Bu-thixantphos $(X=S, R=M e)$ and $t$-Bu-sixantphos $\left(X=S S_{M e}, R=H\right)$. Reagents and conditions: (i) Se, reflux, toluene, 3 days.

The cleanest reaction was observed between $t$-Bu-xantphos and selenium, with a single asymmetric product observed. For $t$-Bu-thixantphos and $t$-Bu-sixantphos a single major product was observed, also consistent with monoselenation. Selected NMR data for the selenides is given in Table 2.7. The ${ }^{31} \mathrm{P}$ NMR spectra each show two peaks in a 1:1 ratio. One peak is shifted only slightly (0.4-7.5 ppm) from the starting material while the other peak is shifted significantly (91.7- 
94.3 ppm) upfield. The asymmetry and the change in chemical shift of only one of the ${ }^{31} \mathrm{P}$ NMR signals indicates the formation of the monoselenide. The ${ }^{1} J_{\mathrm{PSe}}$ values are similar for the three ligands with a range of $12 \mathrm{~Hz}$. The monoselenated $t$-Bu-thixantphos derivative was found to have ${ }^{1} J_{\mathrm{PSe}}=698.5 \mathrm{~Hz}$, while the monoselenated $t$-Bu-xantphos has ${ }^{1} J_{\mathrm{PSe}}=696.3 \mathrm{~Hz}$. These are much lower than that reported for Ph-xantphos selenide $(749 \mathrm{~Hz}) . \stackrel{145}{ }$ This lower coupling constant is expected as tert-butyl groups are more electron donating than phenyl substituents.

Using the correlation reported by Beckmann et al., it is possible to convert the values of ${ }^{1} J_{\mathrm{PSe}}$ for the $t$-Bu-xantphos selenides and the previously reported Ph-xantphos diselenide into $\mathrm{p} K_{\mathrm{b}}$ values. ${ }^{145}$ The $\mathrm{p} K_{\mathrm{b}}$ values for the $t$-Bu-xantphos ligands range between 5.67 and 6.90 which fall between the $\mathrm{PPhMe}_{2}$ and $\mathrm{PMe}_{3}$. ${ }^{17}$ This is consistent with expectations as tert-butyl groups are more electron donating than methyl groups while the phenyl still results in a higher $\mathrm{p} K_{\mathrm{b}}$ than those for the trialkyl phosphines. The $\mathrm{p} K_{\mathrm{b}}$ value for Ph-xantphos is 13.55 , indicating that the $t$-Bu-xantphos ligands are significantly more basic. This significant difference is expected as tert-butyl groups are strongly electron donating whilst phenyl substituents are electron withdrawing. $\cdot$ This effect will dominate any other subtle effects that may arise due to bite-angle or steric considerations.

The bite-angle may play a role in the value for the ${ }^{1} J_{\mathrm{PSe}}$ coupling constant as monoselenide diphosphines have shown through space coupling to the other phosphorus. ${ }^{126}$ This would result in a lower ${ }^{1} J_{\mathrm{PSe}}$ value than otherwise expected and the effect would be more pronounced with smaller bite-angles. In this case $t$-Bu-sixantphos has the lowest $\mathrm{p} K_{\mathrm{b}}$ of the three ligands and also the smallest biteangle, so a bite-angle effect may be present. However, the differences cannot be purely the result of the bite-angle as $t$-Bu-thixantphos has the largest ${ }^{1} J_{\mathrm{PSe}}$ value but $t$-Bu-xantphos has the largest bite-angle.

In addition to the influence of the bite-angle on the ${ }^{1} J_{\mathrm{PSe}}$ coupling constant there must exist another electronic effect contributing to the difference between the ligands. Both $t$-Bu-thixantphos and $t$-Bu-xantphos groups have an ortho ether and a meta alkyl group, in addition $t$-Bu-thixantphos has the thioether bridge. Thioethers are electron donating by resonance, but being in the meta position to the phosphorus the negative charge that could be generated is unable to interact with the phosphorus atom. With sulfur being slightly more electronegative 
Table 2.7: Selected ${ }^{31} \mathrm{P}$ NMR data for $t$-Bu-xantphos selenides in 1:1 $\mathrm{CDCl}_{3:} \mathrm{CD}_{2} \mathrm{Cl}_{2}\left(\Delta \delta=\delta_{\text {phosphine selenide }}-\delta_{\text {diphosphine }}\right)$.

\begin{tabular}{lccccccc}
\hline & \multicolumn{3}{c}{$\mathbf{P}$} & & \multicolumn{4}{c}{$\mathbf{P}=\mathbf{S e}$} \\
\cline { 2 - 4 } \cline { 6 - 8 } Diphosphine & $\delta / \mathbf{p p m}$ & $\Delta \delta / \mathbf{p p m}$ & & $\delta / \mathbf{p p m}$ & $\Delta \delta / \mathbf{p p m}$ & ${ }^{1} \boldsymbol{J}_{\mathbf{P S e}} / \mathbf{H z}$ & $\mathbf{p} \boldsymbol{K}_{\mathbf{b}}$ \\
\hline t-Bu-Sixantphos & 15.9 & 7.5 & & 102.7 & 94.3 & 689.1 & 5.67 \\
t-Bu-Thixantphos & 11.7 & 2.2 & & 103.7 & 94.2 & 698.5 & 6.90 \\
$t$-Bu-Xantphos & 10.6 & 0.4 & & 101.9 & 91.7 & 697.1 & 6.72 \\
\hline
\end{tabular}


than carbon, a thioether is also slightly inductively electron withdrawing, which would result in the phosphorus being very slightly less basic in the case of $t$-Buthixantphos when compared to $t$-Bu-xantphos.

\subsection{Summary}

Two new $t$-Bu-xantphos ligands, $t$-Bu-sixantphos and $t$-Bu-thixantphos have been synthesised and an alternate synthesis of $t$-Bu-xantphos has been reported by lithiation of the appropriate backbone using sec-butyllithium with TMEDA and treatment with $\mathrm{P}^{t} \mathrm{Bu}_{2} \mathrm{Cl}$. Although this method requires long reactions times the only by-product is the mono-phosphine which can undergo the same process again to generate higher yields. NMR analysis of the three ligands showed a $\mathrm{XAA}^{\prime} \mathrm{X}^{\prime}$ spin system for the tert-butyl protons, suggestive of through-space coupling of the two phosphorus atoms.

Natural bite-angles of $126.80,126.98$, and $127.56^{\circ}$ were calculated using DFT for the $t$-Bu-sixantphos, $t$-Bu-thixantphos, and $t$-Bu-xantphos respectively. For comparative purposes the bite-angles for the Ph-xantphos ligands were also calculated: Ph-sixantphos $\left(111.89^{\circ}\right)$, Ph-thixantphos $\left(112.65^{\circ}\right)$, and Ph-xantphos $\left(114.18^{\circ}\right)$. The values for the Ph-xantphos ligands are slightly larger than those calculated using molecular mechanics, though the value for $t$-Bu-xantphos is lower than the previously reported value $\left(140^{\circ}\right)$ calculated with molecular mechanics. This is likely due to the molecular mechanics value overestimating the steric impact of the tert-butyl substituents. A comparison of the natural and median crystallographic bite-angles for a range of different diphosphine ligands showed a good correlation between the two, indicating that the natural bite-angles can be excellent predictors of coordination behaviour.

The three $t$-Bu-xantphos ligands react rapidly with one equivalent of acid to generate $[(t$-Bu-xantphos $) \mathrm{H}] \mathrm{CH}\left(\mathrm{SO}_{3} \mathrm{CF}_{3}\right)_{2}$. The proton moves between the two phosphorus atoms in room temperature solutions. This process was investigated using proton-coupled ${ }^{31} \mathrm{P}$ NMR spectroscopy and low temperature ${ }^{1} \mathrm{H}$ and ${ }^{31} \mathrm{P}$ NMR spectroscopy, revealing that the proton is isolated on a single phosphorus below $-40{ }^{\circ} \mathrm{C}$. The X-ray crystal structure of $[(t$-Bu-thixantphos $) \mathrm{H}] \mathrm{CPh}\left(\mathrm{SO}_{3} \mathrm{CF}_{3}\right)_{2}$ was obtained, and the phosphonium proton was able to be located: isolated on a single phosphorus. 
The electronic properties of the $t$-Bu-xantphos ligands were investigated by the synthesis of phosphine selenides. In all cases the monoselenide formed with no evidence for a diselenide. The values of ${ }^{1} J_{\mathrm{PSe}}$ ranged from $689.1-698.5 \mathrm{~Hz}$, which were converted into $\mathrm{p} K_{\mathrm{b}}$ values of 5.67-6.90. These fall between the values of $\mathrm{PPh}_{2} \mathrm{Me}$ and $\mathrm{PMe}_{3}$ which is consistent with the strong electron donation from the tert-butyl substituents and the electron withdrawing nature of the aryl backbone. The $\mathrm{p} K_{\mathrm{b}}$ value for $\mathrm{Ph}$-xantphos is 13.55 showing the difference in the electronic properties of the $t$-Bu-xantphos ligands.

Overall this chapter provides an interesting introduction into the chemistry of the $t$-Bu-xantphos ligands and suggests that the coordination chemistry may be different to that of the Ph-xantphos ligands as a result of the much larger natural bite-angles and more electron-rich phosphorus atoms. 


\section{Chapter 3}

\section{Coordination Complexes with Silver}

Silver is a precious metal that has been used in coins, jewellery and other ornamentation at least since 4000 B.C. Silver salts have a wide range of applications, such as photographic film, wound dressings, and water storage tanks. ${ }^{146}$ Silver coordination complexes are also biologically active: bis-diphosphine silver complexes show anti-tumour and anti-fungal activity. $\underline{147,148}$ Metallic silver is used industrially as a catalyst for the conversion of ethene to ethylene glycol, and silver salts are occasionally used as co-catalysts in cross-coupling reactions. $\underline{149}$ Complexes of silver have been used as catalysts in a number of transformations, including $\mathrm{Si}-\mathrm{H}$ activation,,$\underline{150}$ asymmetric aldol conversions $\frac{151}{}$ and allylation of benzaldehyde. $.56,152$

Silver coordination chemistry is interesting due to the wide range of different geometries available to the metal, resulting in a high degree of geometrical flexibility. The bite-angle, electronic influence of the diphosphine, and the coordination mode of any ancillary ligands can all impact the type of complex formed. Possible structures formed for an equimolar reaction of a silver salt with a diphosphine range from the straightforward monometallic complex, to multimetallic clusters with bridging diphosphines (Figure 3.1). For a detailed overview of silver coordination chemistry see Meijboom et al. $\underline{111}$

Silver complexes with xantphos ligands have been reported previously. $\cdot \frac{56,153}{}$ In all cases the complexes are monometallic, and the majority of these structures 


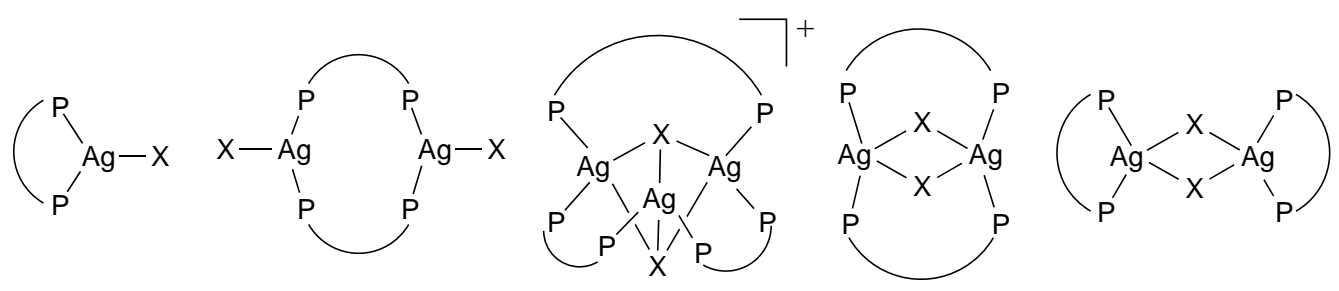

Figure 3.1: Possible structures for 1:1 silver diphosphine complexes, reproduced from Meijboom et al. $\stackrel{111}{ }$

are tetrahedral complexes of the type $\left[\mathrm{Ag}(\text { xantphos)(NN)}]^{+}\right.$where $\mathrm{NN}$ represents a bidentate nitrogen ligand such as $2,2^{\prime}$-bipyridine. These $[\operatorname{Ag}(\operatorname{xantphos})(\mathrm{NN})]^{+}$ complexes have been patented for their luminescent properties. $\underline{154} \underline{156}$ Other tetrahedral complexes with Ph-xantphos include a chelating ligand or two monodentate ligands (Figure 3.2). Only one trigonal silver Ph-xantphos complex has been reported (Figure 3.2C) $\cdot \frac{157}{}$ However, this is likely due to a lack of research rather than synthetic difficulties, as trigonal silver complexes with a chiral xantphos derivative have also been reported. $\underline{\underline{56}}$

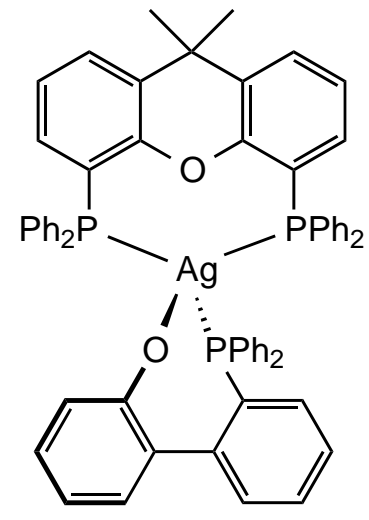

(a)<smiles></smiles>

(b)<smiles>CC1(C)c2cccc(P(Br)c3ccccc3)c2Oc2c1cccc2[PH](Br)(Br)c1ccccc1</smiles>

(c)

Figure 3.2: Tetrahedral and trigonal silver Ph-xantphos complexes.

Crystal structures of [AgBr(Ph-xantphos)] (Figure 3.3) and [AgBr(Ph-xantphos)(SpyH)] (py = 2-pyridyl, Figure 3.4) have been reported. $\frac{157}{1}$ In the trigonal structure the $\mathrm{P}-\mathrm{Ag}-\mathrm{P}$ angle is $109.38(7)^{\circ}$ which increases to $111.507(17)^{\circ}$ for $[\mathrm{AgBr}(\mathrm{Ph}-$ xantphos)(S-pyH)]. The natural bite-angle for Ph-xantphos $\left(111.7^{\circ}\right)^{67}$ is very close to the observed bite-angle. 2-Thiopyridine can act as a bidentate chelating ligand, however in this case the thione tautomer is formed and monodentate coordina- 
tion through the sulfur is observed. In order for the 2-thiopyridine to chelate, the $\mathrm{P}$-Ag-P angle would need to decrease significantly. In this instance the rigidity and steric bulk of Ph-xantphos prevents this from happening. $\frac{157}{}$

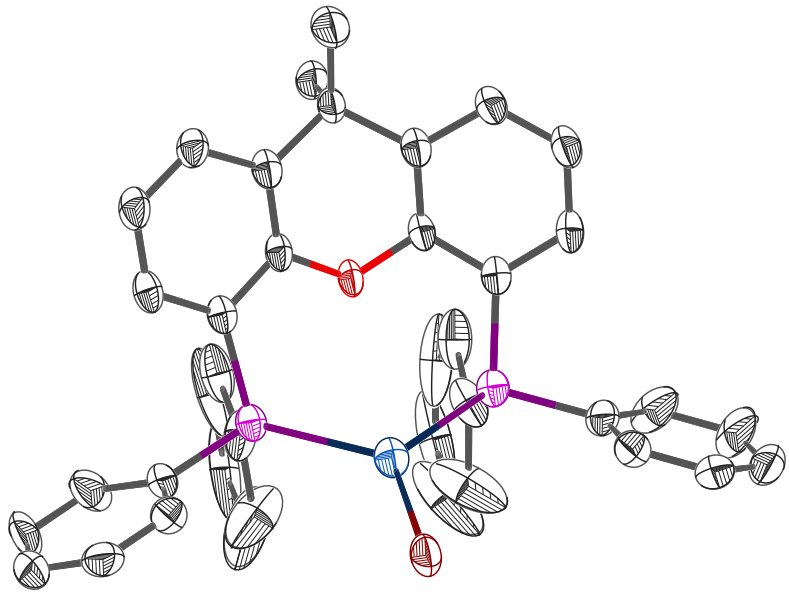

(a)

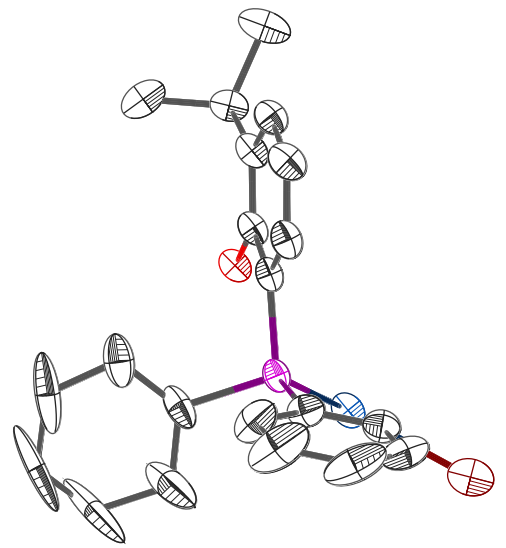

(b)

Figure 3.3: X-ray crystal structure of $[\mathrm{AgBr}(\mathrm{Ph}$-xantphos)] showing the front (a) and side (b). ${ }^{157}$ Hydrogen atoms omitted for clarity.

Silver is an excellent metal for investigating the coordination chemistry of xantphos ligands because it forms a wide range of different coordination geometries and has been shown to coordinate $\mathrm{Ph}$-xantphos close to its natural bite-angle. ${ }^{157}$ There are also a number of potential applications for silver complexes, such as catalysis, biological activity, and luminescent membranes. Silver is also especially suitable for study using ${ }^{1} \mathrm{H},{ }^{13} \mathrm{C}$, and ${ }^{31} \mathrm{P}$ NMR spectroscopy as it consists of two stable spin $1 / 2$ isotopes, ${ }^{107} \mathrm{Ag}$ and ${ }^{109} \mathrm{Ag}$, with natural abundances of 51.839 and $48.161 \%$ respectively. This allows further information to be obtained from the value of the silver-atom coupling constants. This chapter presents a study of the reactivity of the $t$-Bu-sixantphos, $t$-Bu-thixantphos, and $t$-Bu-xantphos ligands with two silver precursors: silver chloride and silver tetrafluoroborate.

\subsection{Silver Chloride Complexes}

Silver chloride complexes were synthesised by addition of each of the $t$-Bu-xantphos ligands to a suspension of silver chloride in $\mathrm{CDCl}_{3}(\mathrm{Scheme}$ 3.1), resulting in 


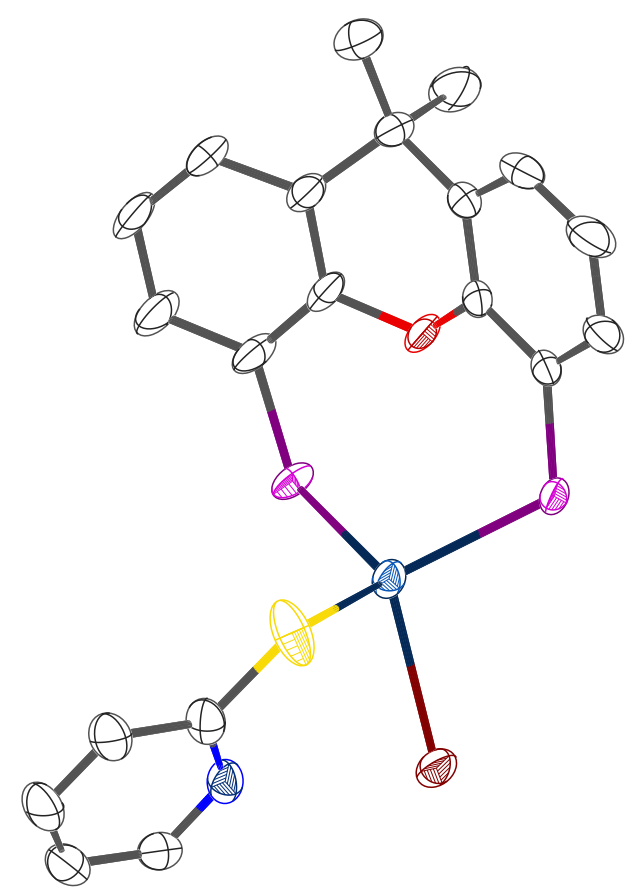

Figure 3.4: X-ray crystal structure of [Ag(Ph-xantphos)(HpyS)Br]. 157 Phenyl rings and hydrogen atom omitted for clarity.

[AgCl(diphosphine)] complexes. In all cases, electrospray ionisation - mass spectrometry showed a clear peak for $[\mathrm{M}-\mathrm{Cl}]^{+}$with no peaks observed for dimers or higher oligomers. The ${ }^{31} \mathrm{P}$ NMR spectra (see Figure 3.5 for an example) showed the expected pair of doublets in all cases, as summarised in Table 3.1. No relationship was observed between the natural bite-angle and the values of ${ }^{1} J_{\mathrm{AgP}}$. However, the change in the phosphorus chemical shift upon coordination $(\Delta \delta)$ increases with decreasing bite-angle. This indicates that the decrease in shielding is larger for smaller bite-angles.

The ${ }^{1} \mathrm{H}$ NMR spectra for the $[\mathrm{Ag}(t$-Bu-xantphos $) \mathrm{Cl}]$ complexes show half the expected number of aromatic signals, and one signal for the methyl groups and another for the tert-butyl substituents, indicating a complex with planes of symmetry in line with the backbone and perpendicular to it, running through the bridging atoms and the silver (representative spectrum for [Ag(t-Bu-sixantphos) $\mathrm{Cl}]$, Figure [3.5). The tert-butyl protons appear as a second order multiplet of the 


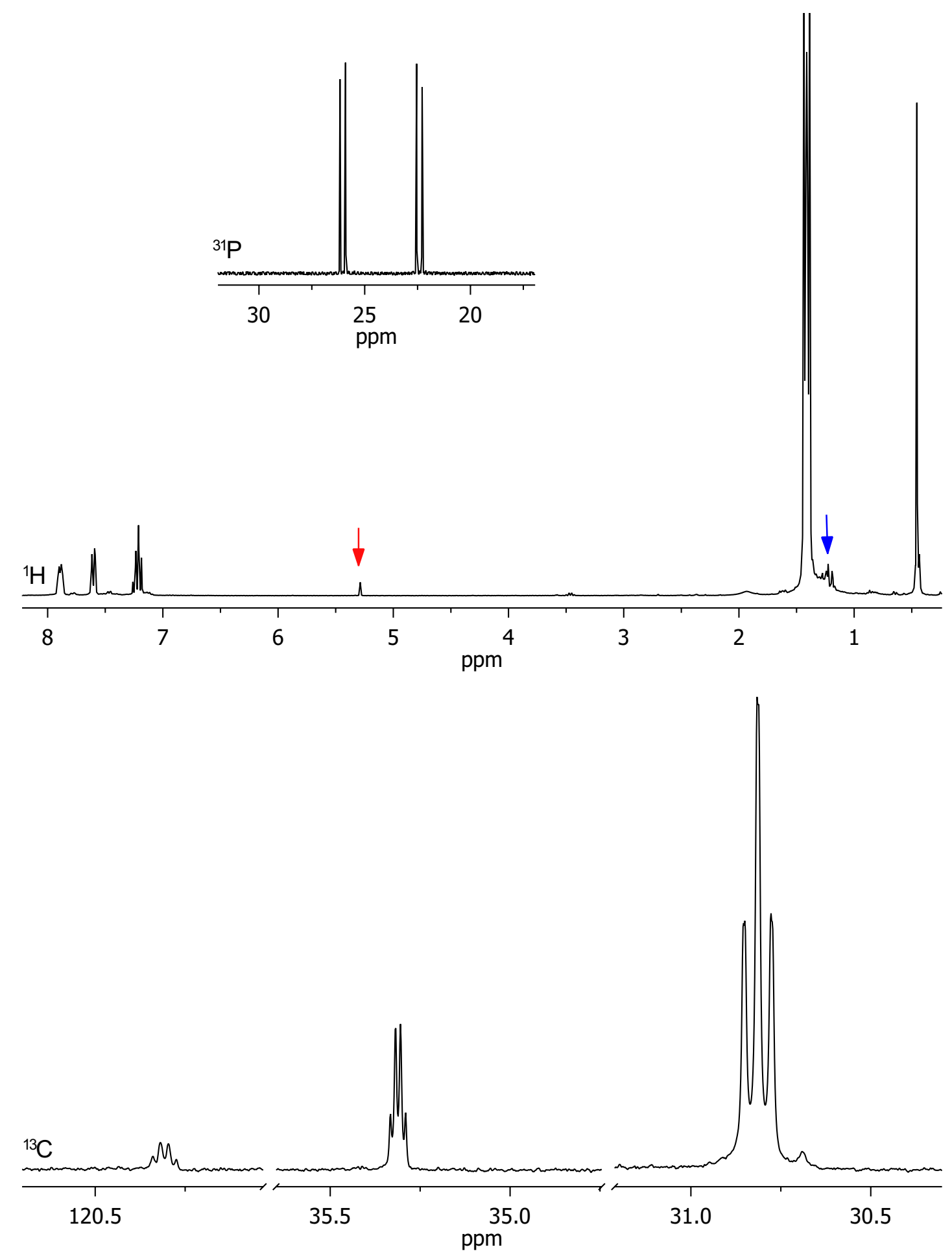

Figure 3.5: ${ }^{31} \mathrm{P},{ }^{1} \mathrm{H}$ and ${ }^{13} \mathrm{C} \mathrm{NMR}$ spectra of $[\mathrm{Ag}(t$-Bu-sixantphos $) \mathrm{Cl}]$ in $\mathrm{CDCl}_{3}$. Arrows indicate impurities, $\mathrm{CH}_{2} \mathrm{Cl}_{2}$ indicated in red. 
<smiles>[R7]c1cc([R7])c2c(c1)[X]c1cc([R7])cc(P)c1Oc1cc([R])cc([Y])c1O2</smiles>

(i)

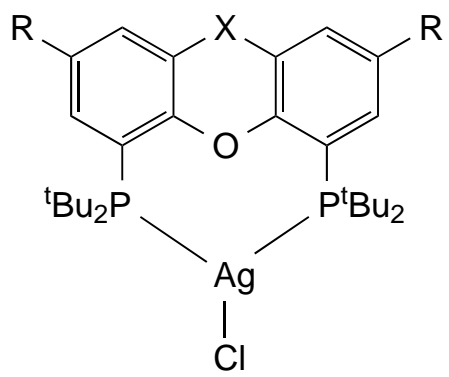

Scheme 3.1: Synthesis of [Ag(t-Bu-xantphos $) \mathrm{Cl}]$ complexes. Reagents and conditions: (i) $\mathrm{AgCl}, \mathrm{CDCl}_{3}$.

Table 3.1: Selected ${ }^{31} \mathrm{P}$ NMR data of $[\mathrm{Ag}(t$-Bu-xantphos $) \mathrm{Cl}]$ complexes in $\mathrm{CDCl}_{3}$ $\left(\Delta \delta=\delta_{\text {complex }}-\delta_{\text {free ligand }}\right)$.

\begin{tabular}{lcccc}
\hline Compound & $\delta^{31} \mathbf{P} / \mathbf{p p m}$ & $\Delta \delta / \mathbf{p p m}$ & ${ }^{1} \boldsymbol{J}_{107} \mathbf{A g P} / \mathbf{H z}$ & ${ }^{1} \boldsymbol{J}_{{ }^{009}} \mathbf{A g P} / \mathbf{H z}$ \\
\hline$t$-Bu-Sixantphos & 24.2 & 15.8 & 408.1 & 471.1 \\
$t$-Bu-Thixantphos & 21.8 & 12.3 & 406.7 & 469.6 \\
$t$-Bu-Xantphos & 20.7 & 10.5 & 409.3 & 472.2 \\
\hline
\end{tabular}

$\mathrm{X}_{18} \mathrm{AA}^{\prime} \mathrm{X}_{18}{ }^{\prime}$ type, similar to the free ligand. The ${ }^{13} \mathrm{C}$ NMR spectra of the three complexes display some very distinctive signals (representative example, $[\mathrm{Ag}(t-\mathrm{Bu}-$ sixantphos)Cl], Figure 3.5, bottom). The ipso phosphorus carbon on the aryl ring and the quaternary tert-butyl carbon appear as apparent quartets, while the signal for the terminal tert-butyl carbons is an apparent triplet of doublets. For each of these signals we would expect a doublet of virtual triplets or virtual triplet of doublets for each of the silver isotopologues. Owing to this complex spin system, values for the individual coupling constants were unable to be determined. The spectroscopic data for the three $[\mathrm{Ag}(t-\mathrm{Bu}$-xantphos $) \mathrm{Cl}]$ complexes show significant similarities, with no major discrepancies, indicating that all three complexes have the same overall geometries with only subtle differences.

Colourless crystals of [Ag(t-Bu-thixantphos $) \mathrm{Cl}]$ suitable for single-crystal X-ray diffraction were obtained by inwards diffusion of $\mathrm{Et}_{2} \mathrm{O}$ into a $\mathrm{CH}_{2} \mathrm{Cl}_{2}$ solution of the complex. The crystal structure is shown in Figure 3.6, confirming the proposed monomeric trigonal structure. Selected bond lengths and angles, and crystallographic data are given in Tables 3.2 and 3.3 respectively. The $[\mathrm{Ag}(t-$ 
Bu-thixantphos) $\mathrm{Cl}$ complex crystallised in the $\mathrm{P} 2{ }_{1}$ space group, while the similar complex $\left[\mathrm{AgBr}\left(\mathrm{Ph}\right.\right.$-xantphos)] crystallised in the higher symmetry $\mathrm{P} 2{ }_{1} / \mathrm{m}$ space group. ${ }^{157}$ The P-Ag-P angle of $130.50(7)^{\circ}$ is larger than the bite-angle for [AgBr(Ph-xantphos)] $\left(109.37(1)^{\circ}\right)$. However, both of these angles are very close to the natural bite-angle of their xantphos ligand. The natural bite-angle of $t$-Buthixantphos and Ph-xantphos were calculated to be $126.98^{\circ}$ and $114.18^{\circ}$ respectively (Section 2.2). This indicates that the natural bite-angle is a significant factor in determining the resulting P-Ag-P angle in these complexes. The silver oxygen distance of 3.007(6) A indicates there no interaction between these atoms.

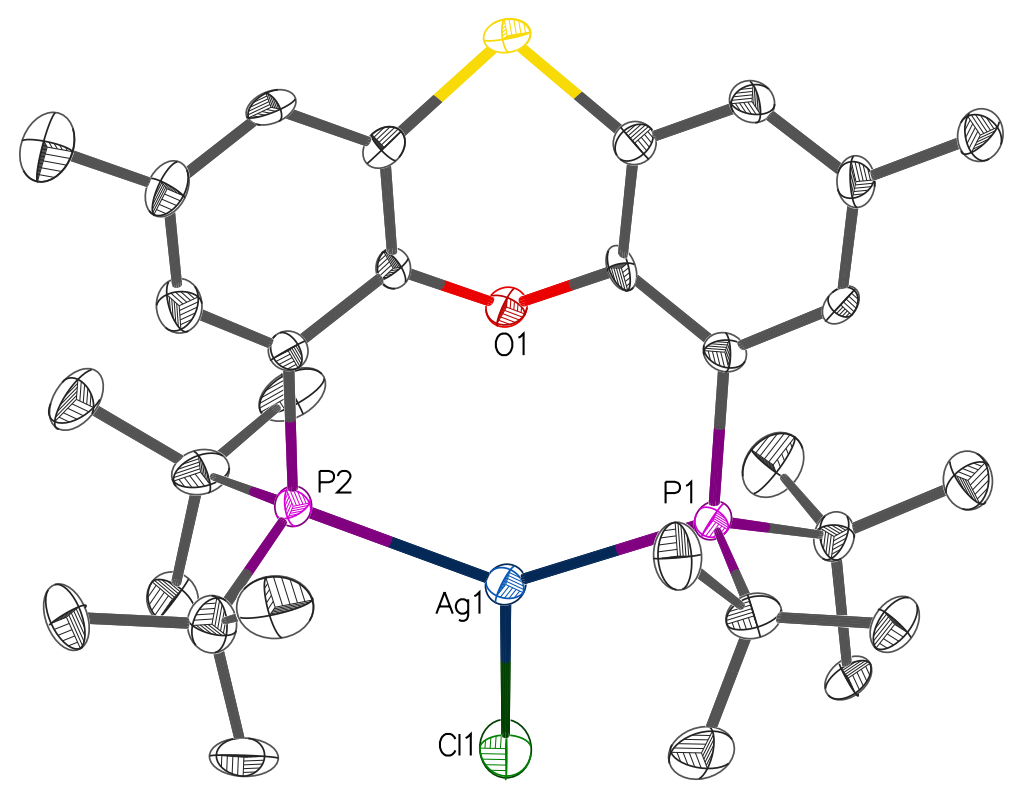

Figure 3.6: X-ray crystal structure of [Ag(t-Bu-thixantphos) $\mathrm{Cl}]$ (50\% probability thermal ellipsoids). Hydrogen atoms omitted for clarity.

A side view of $[\mathrm{Ag}(t$-Bu-thixantphos $) \mathrm{Cl}]$ given in Figure 3.7 shows the P-Ag$\mathrm{Cl}$ angle relative to the ligand backbone. The chloride is not centred, with a difference in the $\mathrm{P}-\mathrm{Ag}-\mathrm{Cl}$ angles of $3.49^{\circ}$. This difference is likely the result of the backbone twisting with the chloride occupying the least sterically hindered site. The backbone is bent, resulting in two distinct faces of the molecule. In [Ag(t-Bu-thixantphos $) \mathrm{Cl}]$ the chloride sits on the convex face of the ligand while in $[\mathrm{AgBr}(\mathrm{Ph}$-xantphos)] (Figure 3.3) the bromide ligand occupies the concave 


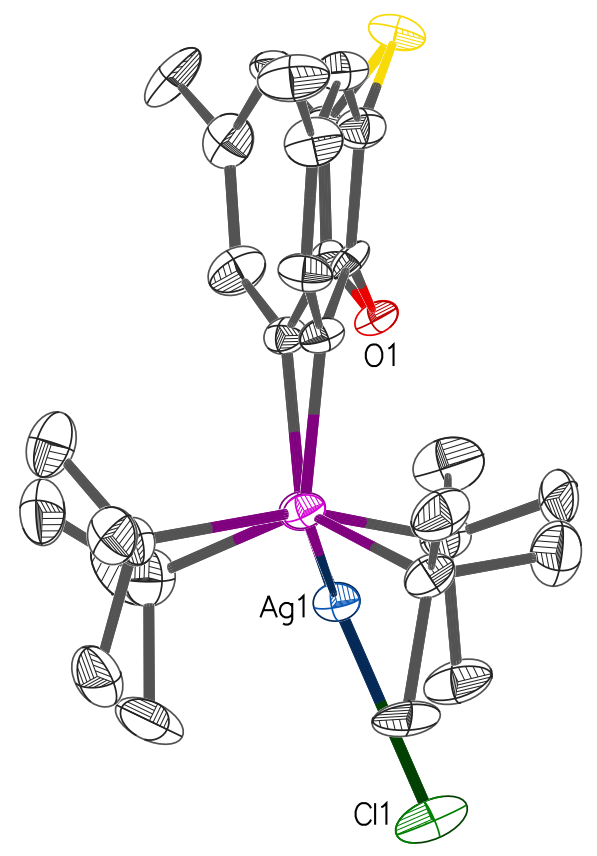

Figure 3.7: X-ray crystal structure of [Ag(t-Bu-thixantphos) $\mathrm{Cl}]$, side view $(50 \%$ probability thermal ellipsoids). Hydrogen atoms omitted for clarity.

Table 3.2: Selected bond distances $(\AA)$ and angles $\left(^{\circ}\right)$ of [Ag(t-Bu-thixantphos)Cl].

\begin{tabular}{llll}
\hline \multicolumn{2}{c}{ Bond distances $(\AA)$} & \multicolumn{2}{c}{ Bond angles $\left(^{\circ}\right)$} \\
\hline P1...P2 & $4.416(3)$ & P1-Ag1-P2 & $130.50(7)$ \\
P1-Ag & $2.430(2)$ & P1-Ag1-Cl1 & $116.46(8)$ \\
P2-Ag & $2.433(2)$ & P2-Ag1-Cl1 & $112.95(8)$ \\
Ag...O1 & $3.007(6)$ & Aryl ring 1...Aryl ring 2 & $35.7(3)$ \\
Ag-Cl1 & $2.491(2)$ & & \\
\hline
\end{tabular}


Table 3.3: Crystallographic data and structure refinement for [Ag(t-Bu-thixantphos)Cl]

\begin{tabular}{|c|c|}
\hline Empirical formula & $\mathrm{C}_{30} \mathrm{H}_{46} \mathrm{AgClOP}_{2} \mathrm{~S}$ \\
\hline Formula weight & 659.99 \\
\hline Temperature/K & 285.47(10) \\
\hline Crystal system & monoclinic \\
\hline Space group & $\mathrm{P} 2_{1}$ \\
\hline $\mathrm{a} / \AA$ & $8.6697(3)$ \\
\hline $\mathrm{b} / \AA$ & $15.4417(6))$ \\
\hline$c / \AA$ & $11.9482(4)$ \\
\hline$\alpha /^{\circ}$ & 90 \\
\hline$\beta /{ }^{\circ}$ & $99.700(3)$ \\
\hline$\gamma /{ }^{\circ}$ & 90 \\
\hline Volume $/ \AA^{3}$ & $1576.70(10)$ \\
\hline $\mathrm{Z}$ & 2 \\
\hline$\rho_{\text {calc }} \mathrm{mg} / \mathrm{mm}^{3}$ & 1.390 \\
\hline$\mu / \mathrm{mm}$ & 7.636 \\
\hline $\mathrm{F}(000)$ & 688.0 \\
\hline Crystal size $/ \mathrm{mm}$ & $0.3842 \times 0.3421 \times 0.1062$ \\
\hline Radiation & $\mathrm{CuK} \alpha(\lambda=1.54184)$ \\
\hline $2 \theta$ range for data collection & 7.506 to $147.890^{\circ}$ \\
\hline Index ranges & $-10 \leq \mathrm{h} \leq 10,-19 \leq \mathrm{k} \leq 16,-14 \leq 1 \leq 14$ \\
\hline Reflections collected & 12454 \\
\hline Independent reflections & $5818\left[R_{\text {int }}=0.0724, R_{\text {sigma }}=0.0651\right]$ \\
\hline Data/restraints/parameters & $5818 / 1 / 339$ \\
\hline Goodness-of-fit on $\mathrm{F}^{2}$ & 1.138 \\
\hline Final $\mathrm{R}$ indexes $[\mathrm{I}>=2 \sigma(\mathrm{I})]$ & $\mathrm{R}_{1}=0.0545, \mathrm{w} \mathrm{R}_{2}=0.1579$ \\
\hline Final $\mathrm{R}$ indexes [all data] & $\mathrm{R}_{1}=0.0563, \mathrm{w} \mathrm{R}_{2}=0.1618$ \\
\hline Largest diff. peak/hole / e $\AA^{-3}$ & $0.88 /-1.78$ \\
\hline Flack parameter & $-0.016(13)$ \\
\hline
\end{tabular}


face. $\frac{157}{}$ In addition, the $\mathrm{C}$ (aryl)-P-Ag-Cl dihedral angles of $159.8(3)^{\circ}$ and $147.4(3)^{\circ}$ in $[\mathrm{Ag}(t-\mathrm{Bu}$-thixantphos $) \mathrm{Cl}]$ are significantly larger then the corresponding dihedral angles in $\left[\mathrm{Ag}(\mathrm{Ph}\right.$-xantphos) $\mathrm{Br}]$ of $109.6(4)^{\circ}$. The difference in the coordination plane between the two complexes, is likely the result of the steric influence of the tert-butyl groups compared to the phenyl rings; the chloride sits below the tert-butyl groups while intercalating with the phenyl rings. The backbone bending would result in two different sets of tert-butyl groups, which would have different NMR properties. However, this is not apparent in the NMR spectra of any of the $[\mathrm{Ag}(\mathrm{t}$-Bu-xantphos) $\mathrm{Cl}]$ complexes (Figure 3.5) indicating that the backbone is likely inverting rapidly in solution (Scheme 3.2). This inversion of the xantphos backbone has recently been observed for $[\mathrm{Ru}(\mathrm{CO}) \mathrm{ClH}(\mathrm{Ph}$-xantphos $\left.)\left(\mathrm{PPh}_{3}\right)\right] \cdot \underline{158}$

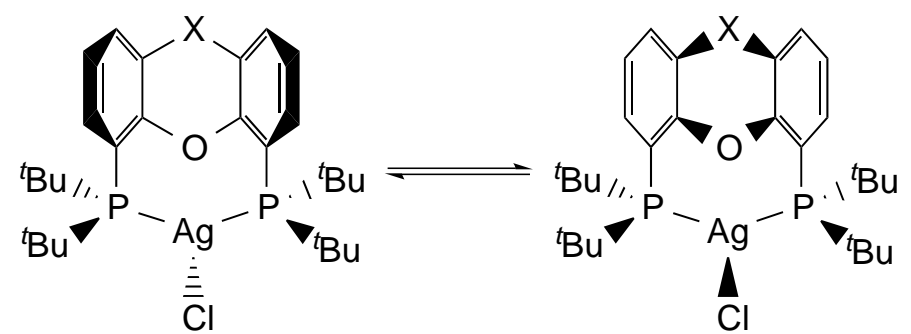

Scheme 3.2: Inversion of the xantphos backbone in [Ag(t-Bu-xantphos)Cl].

\subsection{Reactions with Silver Tetrafluoroborate}

Complexes of the type $\left[\mathrm{Ag}(t-\mathrm{Bu}\right.$-xantphos $) \mathrm{BF}_{4}$ were synthesised in order to investigate the coordination of the diphosphine ligands in the absence of other ligands. Although tetrafluoroborate can coordinate to silver, it is unusual for it to do so, and numerous examples exist of silver complexes with free coordination sites that have a non-coordinated $\mathrm{BF}_{4}{ }^{-}$counterion (Figure 3.8). $159-161$ Previous reports have shown that in the presence of an ether group and a tetrafluoroborate ion a linear diphosphine complex with mutually trans phosphorus atoms and no other ligands was synthesised. ${ }^{162}$ In addition, coordination of the tetrafluoroborate to the silver will result in a shift of peak in the ${ }^{19} \mathrm{~F}$ NMR spectrum. 


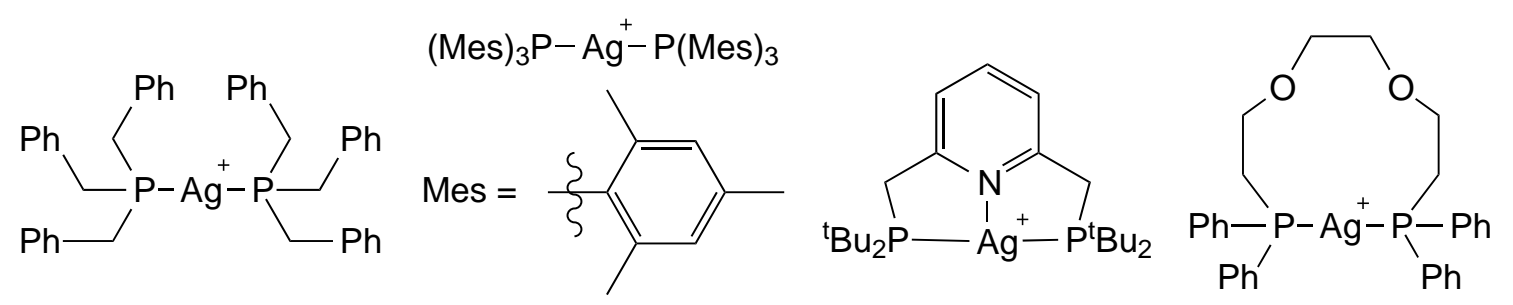

Figure 3.8: Examples of silver complexes with free coordination sites and noncoordinating $\mathrm{BF}_{4}^{-}$counterions. $\stackrel{159}{\underline{161}}$

The reaction between $\mathrm{AgBF}_{4}$ and the $t$-Bu-xantphos ligands proceeded at room temperature in $\mathrm{CH}_{2} \mathrm{Cl}_{2}$, going to completion in under one hour (Scheme 3.3). The resulting $\left[\mathrm{Ag}(t-\mathrm{Bu}\right.$-xantphos) $] \mathrm{BF}_{4}$ complexes are light-sensitive in solution and in $\mathrm{CDCl}_{3}$ solution completely degraded in 12 hours. For all three diphosphine ligands the ESI - mass spectra show a molecular ion peak corresponding to $[\mathrm{Ag} \text { (diphosphine) }]^{+}$. In all cases the ${ }^{31} \mathrm{P}$ NMR spectra (for an example see Figure 3.9) show the expected pair of doublets, indicating chelation to a single silver atom. No relationship between the bite-angle and the ${ }^{1} J_{107} \mathrm{AgP}$ or ${ }^{1} J^{109} \mathrm{AgP}$ coupling constants was observed. The ${ }^{1} J^{107} \mathrm{AgP}$ and ${ }^{1} J_{109} \mathrm{AgP}$ coupling constants are larger than those for the silver chloride complexes (Table 3.4). This is consistent with their formulation as two-coordinate silver complexes, as the values of M-P coupling constants for $\mathrm{d}^{10}$ metals generally increase with decreasing coordination number. 16

The ${ }^{19} \mathrm{~F}$ NMR spectra of the three $\left[\mathrm{Ag}(t-\mathrm{Bu}-\mathrm{xantphos}) \mathrm{BF}_{4}\right.$ complexes show a single resonances between -151.3 and -151.9 ppm, indicating non-coordinating $\mathrm{BF}_{4}^{-}$ counterions. In the ${ }^{1} \mathrm{H}$ NMR spectra the tert-butyl resonances appear as a virtual triplet. Virtual triplets are commonly observed for $X_{n} A^{\prime} A^{\prime}{ }_{n}$ when $A$ and $A^{\prime}$ are strongly coupled such that $J_{\mathrm{AA}^{\prime}}$ is very much larger than the difference between $J_{\mathrm{AX}}$ and $J_{\mathrm{AX}} \cdot \frac{124}{}$ In coordination compounds this condition is typically met if the two phosphorus atoms are in a trans configuration resulting in strong coupling of the spin systems. $\frac{16}{}$ In this case as the triplet is not a perfect 1:2:1 triplet it is likely that the phosphines are not strictly trans and the P-Ag-P angle is less than $180^{\circ}$.

The $\left[\mathrm{Ag}(t\right.$-Bu-xantphos) $) \mathrm{BF}_{4}$ complexes show distinctive peaks in their ${ }^{13} \mathrm{C} \mathrm{NMR}$ 

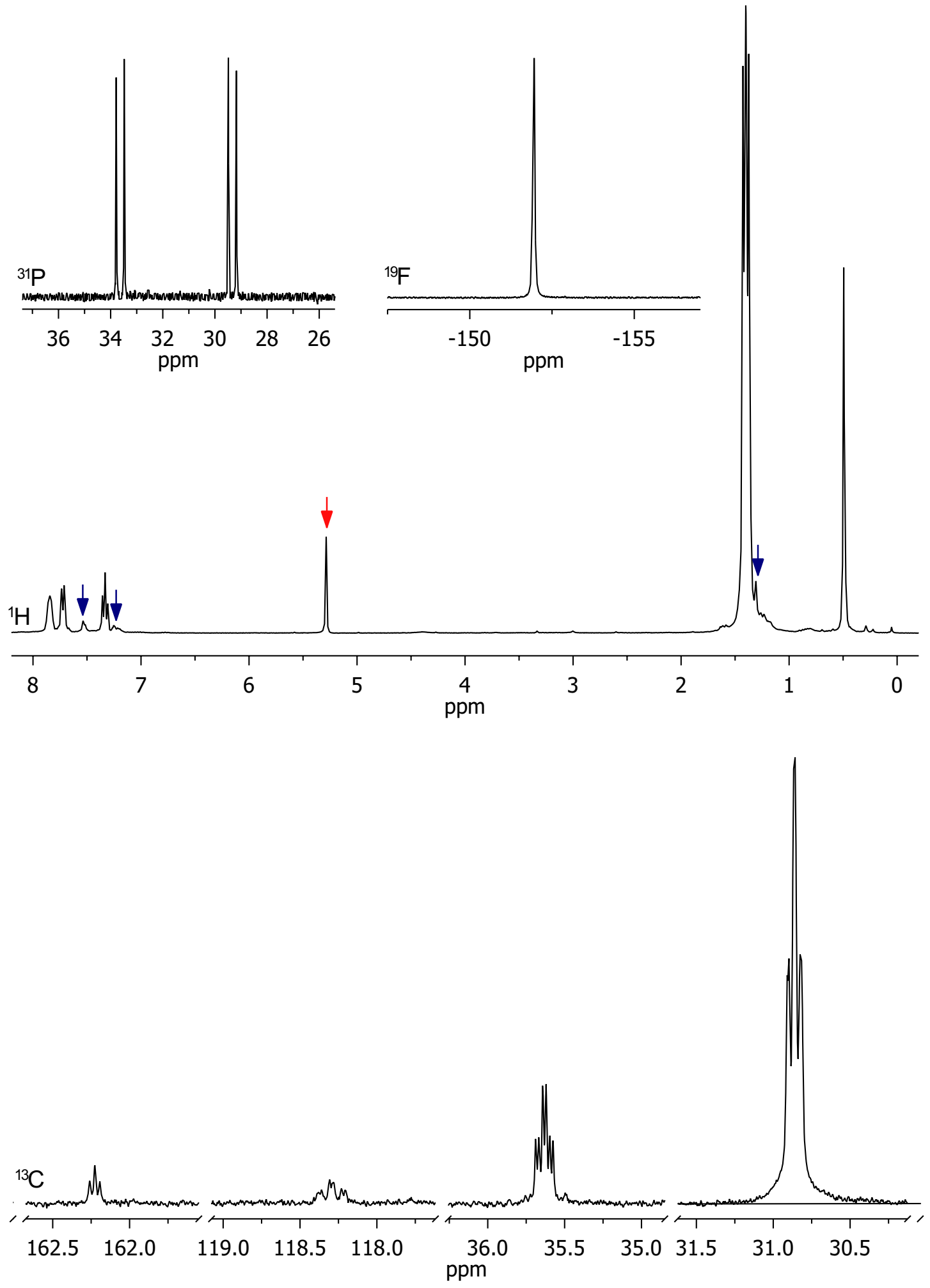

Figure 3.9: $\mathrm{NMR}$ spectra for $[\mathrm{Ag}(t-\mathrm{Bu}$-sixantphos $)] \mathrm{BF}_{4}$ in $\mathrm{CDCl}_{3}$. Arrows indicate impurities, $\mathrm{CH}_{2} \mathrm{Cl}_{2}$ indicated in red. 
R<smiles>[R]c1cc([X])c(Oc2c([Y])cc([R])cc2P(C)CC(C)(C)C)c(CCCC)c1</smiles>

(i)

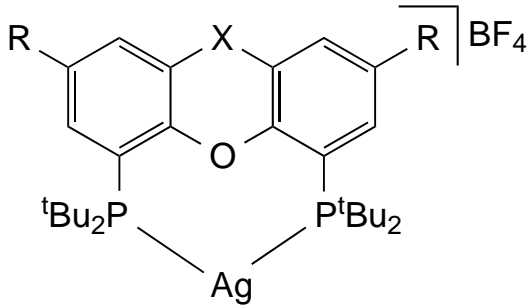

Scheme 3.3: Synthesis of $[\mathrm{Ag}(t-\mathrm{Bu}$-xantphos $)] \mathrm{BF}_{4}$ complexes. Reactions and conditions: (i) $\mathrm{AgBF}_{4}, \mathrm{CH}_{2} \mathrm{Cl}_{2}$.

Table 3.4: Selected NMR data of $[\mathrm{Ag}(t-\mathrm{Bu}$-xantphos $)] \mathrm{BF}_{4}$ complexes $\left(\Delta \delta=\delta_{\text {complex }}\right.$ - $\left.\delta_{\text {free ligand }}\right)$.

\begin{tabular}{lcccccc}
\hline & \multicolumn{4}{c}{} & & ${ }^{{ }^{31} \mathbf{P}} \mathbf{F}$ \\
\cline { 2 - 4 } Diphosphine & $\delta / \mathbf{p p m}$ & $\Delta \delta / \mathbf{p p m}$ & ${ }^{1} \boldsymbol{J}_{{ }^{107} \mathbf{A g P}} / \mathbf{H z}$ & ${ }^{1} \mathbf{J}_{109} \mathbf{A g P} / \mathbf{H z}$ & $\delta / \mathbf{p p m}$ \\
\hline t-Bu-Sixantphos & 31.5 & 23.1 & 482.9 & 557.4 & & -151.9 \\
$t$-Bu-Thixantphos & 28.4 & 18.9 & 486.7 & 562.2 & & -151.3 \\
$t$-Bu-Xantphos & 27.6 & 17.4 & 486.3 & 561.1 & & -151.9 \\
\hline
\end{tabular}

spectra (see Figure 3.9 for a representative example), similar to those observed for the $[\mathrm{Ag}(t-\mathrm{Bu}$-xantphos)Cl] complexes (Section 3.1). The most downfield signal is for the aryl carbon attached to the oxygen atom (the O-ipso carbon). This signal appears as a virtual triplet, as expected for a $\mathrm{XAA}^{\prime} \mathrm{X}^{\prime}$ system with strongly coupled phosphorus atoms. The signals for the ipso carbon attached to phosphorus, and the tert-butyl quaternary and terminal carbons, all appear as triplets of doublets due to coupling to phosphorus and both isotopes of silver. These signals would be expected to appear as a pair of virtual triplets of doublets; however, if the two leftmost peaks of one triplet of doublets overlapped with the two central peaks of the other triplet of doublets this could result in an apparent triplet of doublets (assuming that the virtual triplet is not a strict 1:2:1 triplet, which is likely applicable here as the ${ }^{1} \mathrm{H}$ NMR signal for the tert-butyl protons was not a 1:2:1 triplet).

The $[\mathrm{Ag}(t-\mathrm{Bu}$-xantphos $)] \mathrm{BF}_{4}$ complexes have two free coordination sites; however, there is no evidence for either the ligand oxygen atom or the $\mathrm{BF}_{4}{ }^{-}$acting as 
another ligand. This is likely due to the steric constraints of the larger bite-angle ligands and the bulky tert-butyl groups. Attempts were made to react the analogous $[\mathrm{Ag}(t-\mathrm{Bu}-\mathrm{xantphos})] \mathrm{PF}_{6}$ complexes with ethene, ethyne and carbon monoxide, however no reactivity was observed. $\underline{163}$

\subsubsection{Reactions with LiCCPh}

Silver acetylide complexes have gained attention recently due to their luminescent properties. $164-166$ Silver acetylides supported by phosphine ligands tend to form cluster complexes with no monomeric structures currently reported in the CSD. $\underline{68}$ Attempts to synthesise a silver acetylide complex by reaction of the $[\mathrm{Ag}(\mathrm{t}-$ Bu-xantphos) $\mathrm{BF}_{4}$ complexes with lithium phenylacetylide were marred by difficulties. The reaction was carried out in freshly distilled THF under argon, in the dark. After 12 hours the THF was removed in vacuo and the residue was extracted into a deuterated solvent and analysed by NMR spectroscopy. In $\mathrm{CDCl}_{3}$ or acetone- $\mathrm{d}_{6}$ a significant amount of DCCPh was observed in the ${ }^{13} \mathrm{C}$ NMR spectrum, indicating a reaction between the solvent and any acetylide complex that may have formed, or no reaction had occurred and the solvent was quenching the lithium acetylide. The ${ }^{31} \mathrm{P}$ NMR spectra showed a pair of doublets with different chemical shift and coupling constants to the starting material suggesting that a reaction between the $[\mathrm{Ag}(t-\mathrm{Bu}$-xantphos $)] \mathrm{BF}_{4}$ complexes and $\mathrm{LiCCPh}$ had indeed occurred.

When the reaction was repeated and extracted into $\mathrm{C}_{6} \mathrm{D}_{6}$ the resulting NMR spectra were broad, with a pair of multiplets present in the ${ }^{31} \mathrm{P}$ NMR as would be expected if a silver cluster was formed. The ${ }^{1} \mathrm{H}$ and ${ }^{13} \mathrm{C}$ NMR spectra were also broad, however no peaks for free phenyl acetylene were observed, indicating that reaction had occurred with likely coordination. Unfortunately this complex was unstable and degraded completely in 24 hours to an insoluble black material. This material may be a higher order cluster, oligomer or polymeric species which commonly form with silver acetylide complexes. Further attempts at characterisation of the intermediate complex or the resulting degradation products were unsuccessful. 


\subsection{Summary}

The coordination chemistry of $t$-Bu-sixantphos, $t$-Bu-thixantphos, and $t$-Bu-xantphos with silver(I) precursors has been investigated. [Ag(t-Bu-xantphos $) \mathrm{Cl}]$ complexes formed upon reaction of the ligands with $\mathrm{AgCl}$. The $\mathrm{X}$-ray crystal structure of $[\mathrm{Ag}(t$-Bu-thixantphos $) \mathrm{Cl}]$ showed a trigonal geometry with a P-Ag-P angle of $130.50(7)^{\circ}$, which is slightly larger than the natural bite-angle $\left(126.98^{\circ}\right)$. The biteangle in $[\mathrm{Ag}(t-\mathrm{Bu}$-thixantphos $) \mathrm{Cl}]$ is larger than the previously reported $[\mathrm{Ag}(\mathrm{Ph}$ xantphos)Br] $\left(109.37(1)^{\circ}\right)$ and chloride sits on the convex face whereas the bromide occupies the concave face. This shows the impact of the tert-butyl groups on the coordination chemistry. In solution the ligand backbones in the $[\mathrm{Ag}(t-$ Bu-xantphos)Cl] complexes are inverting rapidly, resulting in a single tert-butyl environment.

[Ag(t-Bu-xantphos) $\mathrm{BF}_{4}$ complexes were synthesised by reaction of the three $t$ $\mathrm{Bu}$-xantphos ligands with $\mathrm{AgBF}_{4}$. The NMR spectroscopy suggested that the PAg-P angle in these complexes was approaching $180^{\circ}$. Reaction of the $\mathrm{Ag}(t-\mathrm{Bu}-$ xantphos) $\mathrm{BF}_{4}$ complexes with lithium phenylacetylide in $\mathrm{CDCl}_{3}$ or acetone- $\mathrm{d}_{6}$ formed DCCPh but no change in the $[\mathrm{Ag}(t$-Bu-xantphos $)] \mathrm{BF}_{4}$ complexes. In $\mathrm{C}_{6} \mathrm{D}_{6}$ the resulting NMR spectra were broad with no peaks indicative of free phenyl acetylene. However, the product was unstable and degradation hindered characterisation. All of the characterised complexes in this chapter were monomeric species, indicating that the $t$-Bu-xantphos ligands preferentially chelate to a silver ion rather than bridging to form dimers/oligomers. The absence of any dimers and oligomers forming from the $[\mathrm{Ag}(t-\mathrm{Bu}$-xantphos $)] \mathrm{BF}_{4}$ complexes suggests that the rigid backbone and bulky tert-butyl groups are able to stabilise electron deficient metal centres. 


\section{Chapter 4}

\section{Coordination Complexes with Rhodium}

Rhodium is a very rare element forming only 0.001 ppm of the earth's crust. ${ }^{167}$ The majority of the extracted rhodium $(81 \%)$ is used in catalytic converters in cars to reduce harmful nitrogen oxides to nitrogen and oxygen. 168 Another important use of rhodium is in thermocouples where a rhodium-platinum alloyed wire is used, together with a pure platinum wire for use in high-temperature furnaces. ${ }^{167}$ Rhodium coordination complexes form active catalysts, most commonly for hydroformylation and hydrogenation. ${ }^{28,169,170}$ Wilkinson's catalyst, [ $\left.\mathrm{RhCl}\left(\mathrm{PPh}_{3}\right)_{3}\right]$, is one of the most widely known rhodium catalysts, used mainly for the hydrogenation of alkenes, but also for hydroboration of alkenes and selective hydrosilylation of $\alpha, \beta$-unsaturated carbonyl compounds. ${ }^{171}-173$

The 2001 Nobel Prize in Chemistry was awarded to Knowles, $\underline{\underline{7}}$ Noyori, $\underline{\underline{\underline{w}}}$ and Sharpless ${ }^{174}$ for their work in asymmetric catalysis, including several rhodium catalysts. A derivative of Wilkinson's catalyst, where triphenylphosphine was replaced by the chiral phosphine (-)-PMePh ${ }^{\mathrm{i}} \mathrm{Pr}$, was reported by Knowles and Sabacky in 1968. $\frac{175}{}$ This chiral version of Wilkinson's catalyst was the first asymmetric hydrogenation catalyst yielding an enantiomeric excess of $15 \%$ when hydrogenating $\alpha, \beta$-unsaturated carbonyls (Scheme 4.1). This was improved by the development of the CAMP ligand (Figure 4.1), which gives an enantiomeric excess of $80 \%$ in the hydrogenation of dehydroamino acids, a key step in the commercial production of L-DOPA, a drug used in the treatment of Parkinson's disease. $\stackrel{176}{ }$ Rhodium complexes containing chiral diphosphines also form active cat- 
alysts, for example a [Rh(cod)(S)-BINAP] (Figure 4.1) catalyst is active for the asymmetric isomerisation of allylic amines, a key step in the industrial synthesis of (-)-menthol generating over 1000 tons per year. ${ }^{-5}$<smiles>C=C(C(=O)O)c1ccccc1</smiles>

Scheme 4.1: Asymmetric catalytic hydrogenation developed by Knowles. $\underline{\underline{7}}$
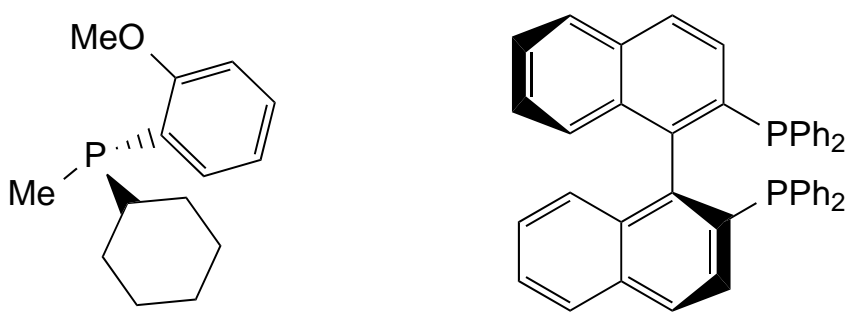

Figure 4.1: Chiral phosphine ligands CAMP (left) and (S)-BINAP (right), used in asymmetric catalysis. $\underline{\underline{5}, 176}$

The xantphos class of ligands were first studied for potential use as ancillary ligands in rhodium-catalysed hydroformylation. $\stackrel{30}{=}$ Small changes in the bite-angle can result in changes in reactivity and selectivity. For hydroformylation the larger bite-angle ligand, Ph-xantphos, showed a greater selectivity for the linear aldehyde than Ph-sixantphos or Ph-thixantphos. Xantphos ligands have the ability to coordinate to a metal centre in a number of different modes. The most common is the diphosphine $\kappa P P^{\prime}$ mode. Tridentate $\kappa P, O, P^{\prime}$ complexes of the xantphos ligands are also relatively common, especially in octahedral complexes. In this $\kappa P, O, P^{\prime}$ coordination mode, the xantphos ligand typically coordinates to the metal in a meridional manner, although facial complexes have also been reported. $\underline{177,178}$

Subsequent to the research reported in this chapter being performed, one paper has been published, reporting the coordination chemistry of the $t$-Bu-xantphos 
ligand with rhodium. $\cdot \underline{\underline{82}} t$-Bu-Xantphos was shown to react with $[\mathrm{Rh}(\mathrm{coe})(\eta-\mathrm{Cl})]_{2}$ forming a $\left[\mathrm{Rh}\left(t-\mathrm{Bu}\right.\right.$-xantphos- $\left.\left.\mathrm{\kappa} P, \mathrm{O}, \mathrm{P}^{\prime}\right) \mathrm{Cl}\right]$ complex. This complex readily splits hydrogen to form a $\left[\mathrm{Rh}\left(t-\mathrm{Bu}\right.\right.$-xantphos- $\left.\left.\mathrm{k} P, O, P^{\prime}\right) \mathrm{Cl}(\mathrm{H})_{2}\right]$ complex. Both of these complexes were also synthesised as part of the present study and comparison of the results will be discussed where relevant. Chloride abstraction from $[\mathrm{Rh}(t-\mathrm{Bu}-$ xantphos- $\left.\left.\mathrm{k} P, O, \mathrm{P}^{\prime}\right) \mathrm{Cl}(\mathrm{H})_{2}\right]$ using $\mathrm{AgBF}_{4}$ or $\mathrm{AgSbF}_{6}$ generates the trigonal bipyramidal dihydride complex $\left[\mathrm{Rh}\left(t \text {-Bu-xantphos- } \mathrm{k} P, \mathrm{O}, \mathrm{P}^{\prime}\right)(\mathrm{H})_{2}\right]^{+}$. This dihydride reacts with ethene to give ethane and $\left[\mathrm{Rh}\left(t\right.\right.$-Bu-xantphos- $\left.\left.\kappa P, O, P^{\prime}\right)\left(\mathrm{C}_{2} \mathrm{H}_{4}\right)\right]$, however no reaction with the commonly used hydrogen acceptors $t$-butylethylene or norbornene was observed, nor was any reaction with terminal alkenes. [ $\mathrm{Rh}(t-\mathrm{Bu}$ xantphos- $\left.\mathrm{K} P, O, P^{\prime}\right) \mathrm{Cl}(\mathrm{H})_{2}$ ] reacts with $\mathrm{KO}^{\mathrm{t}} \mathrm{Bu}$ resulting in a four coordinate monohydride species [Rh $\left(t\right.$-Bu-xantphos- $\left.\left.\mathrm{K} P, \mathrm{O}, P^{\prime}\right) \mathrm{H}\right]$. [Rh $\left(t-\mathrm{Bu}\right.$-xantphos- $\left.\left.\mathrm{k} P, O, P^{\prime}\right) \mathrm{H}\right]$ is an active catalyst for the isomerisation of 1-hexene, with a TON of 2000 after 16 hours. Addition of ethene to this rhodium(I) hydride resulted in the reversible formation of an ethyl complex. This paper shows some of the interesting chemistry of $t$-Bu-xantphos with rhodium. However, to date the coordination chemistry of $t$-Bu-sixantphos and $t$-Bu-thixantphos has not been reported.

This chapter presents research into the coordination chemistry of $t$-Bu-sixantphos, $t$-Bu-thixantphos, and $t$-Bu-xantphos with rhodium. The goal was the synthesis and characterisation of complexes that may form as part of catalytic reactions. As such, this work focusses on the reactivity of a simple rhodium(I) complex towards small molecules, particularly the chemistry towards hydrogen and carbon monoxide, as these are common components of catalytic systems.

\subsection{Synthesis of $\left[\mathrm{Rh}\left(\kappa P, O, P^{\prime}\right) \mathrm{Cl}\right]$ Complexes}

Chlorido-bridged rhodium alkene dimers are commonly used starting materials for the formation of rhodium phosphine complexes. These dimers can react in a number of different ways depending on the phosphine ligand used (Figure 4.2). When two equivalents of a monophosphine react with $\left[\mathrm{Rh}(\mathrm{coe})_{2} \mathrm{Cl}\right]_{2}$, (coe = cyclooctene) the phosphine displaces one coe molecule from each rhodium atom forming a symmetric dimer. $\frac{179}{1}$ These complexes are typically unstable. Further addition of phosphine displaces the remaining two coe molecules, while retaining the chlorido-bridged rhodium core. 180 Analogous complexes form when the 
reaction is carried out with diphosphines. $\frac{181}{1 n}$ some cases, bidentate ligands can cleave the dimer resulting in a $[\mathrm{Rh}(\mathrm{LL})(\mathrm{coe}) \mathrm{Cl}]$ complex. ${ }^{182}$ Tridentate ligands are also able to cleave the dimer and result in the mononuclear complexes [Rh(LLL)Cl]. 183,184 With negatively charged tridentate ligands, a trigonal bipyramidal hydride chloride complex is formed (where the hydride forms via $\mathrm{X}-\mathrm{H}$ activation of the central ligating atom). $\stackrel{185-187}{1}$ Alternatively, a hydride can be abstracted from the ligand using a strong base prior to reaction with the rhodium dimer. Silver salts can also be added to remove the bridging chloride ligands and form a monomeric complex. In this case the remaining coordination site is occupied either by the anion from the silver salt, or by a cyclooctene molecule if a non-coordinating counterion is used. 188,189

Reaction between $\left[\mathrm{Rh}(\mathrm{coe})_{2} \mathrm{Cl}_{2}\right.$ and the three $t$-Bu-xantphos ligands was carried out on an NMR scale in $\mathrm{C}_{6} \mathrm{D}_{6}$. No reaction occurred at room temperature overnight except in the case of $t$-Bu-xantphos, which displays a small amount of conversion, evident from the ${ }^{1} \mathrm{H}$ and ${ }^{31} \mathrm{P}$ NMR spectra. The lack of reactivity at room temperature may be the result of the poor solubility of $\left[\mathrm{Rh}(\mathrm{coe})_{2} \mathrm{Cl}\right]_{2}$ in $\mathrm{C}_{6} \mathrm{D}_{6}$. Upon heating to $60{ }^{\circ} \mathrm{C}$ coordination of the $t$-Bu-xantphos ligands proceeded, going to completion after 24 hours. For all three $t$-Bu-xantphos ligands the product is the expected $\left[\mathrm{Rh}\left(t-\mathrm{Bu}\right.\right.$-xantphos- $\left.\left.\kappa P, O, P^{\prime}\right) \mathrm{Cl}\right]$ mononuclear complex (Scheme 4.2). This complex is directly analogous to an i-Pr-xantphos complex reported in $2013, \underline{81}$ which was synthesised in the same manner. The $t$-Bu-xantphos ligands have a number of different possible coordination modes. In this case a meridional $\kappa P, O, P^{\prime}$ pincer coordination is observed. It is likely that the reaction proceeds by substitution of one cyclooctene ligand with one of the phosphorus atoms, followed by a second to form a chlorido-bridged dimer. However, as the $t$-Bu-xantphos ligands have such large bite-angles and the potentially coordinating ether bridge, this can readily split the dimer resulting in the desired product. None of these intermediates were observed, indicating low activation barriers once the first substitution had occurred.

The ${ }^{1} \mathrm{H},{ }^{13} \mathrm{C}$ and ${ }^{31} \mathrm{P}$ NMR spectra for the three [Rh $\left(t\right.$-Bu-xantphos- $\left.\left.\kappa P, O, P^{\prime}\right) \mathrm{Cl}\right]$ complexes are consistent with the proposed structures. Representative ${ }^{1} \mathrm{H}$ and ${ }^{31} \mathrm{P}$ NMR spectra for $\left[\mathrm{Rh}\left(t-\mathrm{Bu}\right.\right.$-xantphos- $\left.\left.\mathrm{N} P, \mathrm{O}, \mathrm{P}^{\prime}\right) \mathrm{Cl}\right]$ are shown in Figure 4.3 and selected NMR data for all three complexes is given in Table 4.1, In the ${ }^{31} \mathrm{P} N M R$ spectra the signals shift downfield by 35.8-37.5 ppm upon coordination, with the 


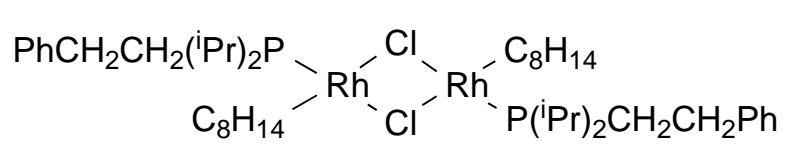

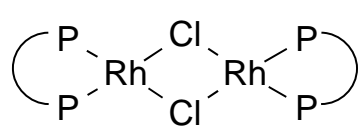

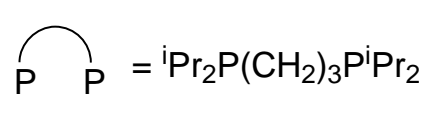<smiles>Pc1ccccc1N(c1ccccc1)[PH3+](Cl)(Cl)c1ccccc1</smiles>

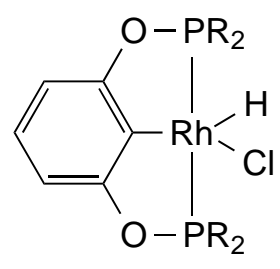

$\mathrm{R}={ }^{\mathrm{i}} \mathrm{Pr},{ }^{\mathrm{t}} \mathrm{Bu}$<smiles>CCCC[Pb]1Cc2cccc(c2[R18](Cl)(Cl)[Pb])C1</smiles>

$\mathrm{X}=\mathrm{C}, \mathrm{N}$

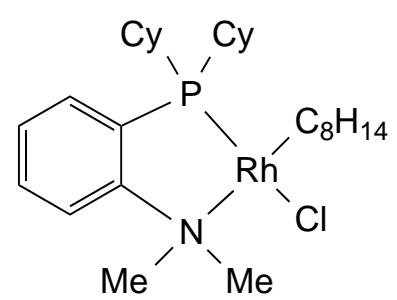

$$
\begin{aligned}
& \mathrm{R}_{3} \mathrm{P}-\mathrm{Rh}^{-} \mathrm{Cl}^{-} \mathrm{Rh}^{-} \mathrm{PR}_{3} \\
& \mathrm{R}=\mathrm{Me}, \mathrm{Et},{ }^{\mathrm{i}} \mathrm{Pr},{ }^{\mathrm{t}} \mathrm{Bu}
\end{aligned}
$$

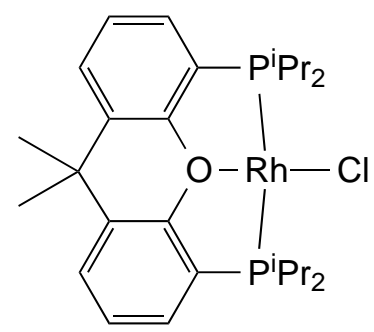

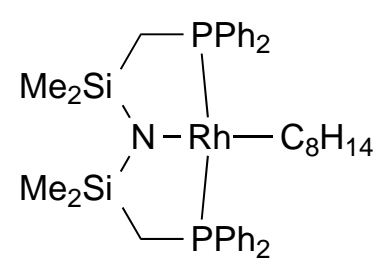

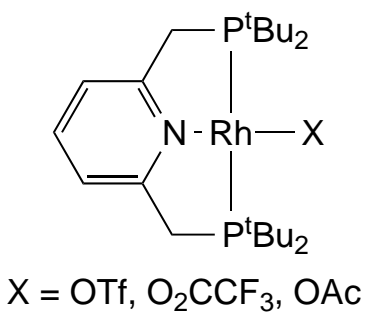

Figure 4.2: Complexes formed by reaction of $\left[\mathrm{Rh}(\mathrm{coe})_{2} \mathrm{Cl}\right]_{2}$ and phosphine ligands. First row: monophosphines in 2:1 and 4:1 ligand:[Rh(coe) $\left.{ }_{2} \mathrm{Cl}\right]_{2}$, second row: bidentate ligands, third row: tridentate ligands in the absence of other reagents, fourth row: tridentate ligands using lithiated ligand (left) or a silver salt (right).

peaks for the complexes appearing at $44.2-47.7 \mathrm{ppm}$. The signals for the complexes are doublets with rhodium coupling of $140.0-142.3 \mathrm{~Hz}$. This coupling is consistent with a rhodium(I) complex and is similar to the coupling constant for [Rh(i-Pr-xantphos- $\left.\left.\kappa P, O, P^{\prime}\right) \mathrm{Cl}\right](142.4 \mathrm{~Hz}) .{ }^{81}$ The ${ }^{1} \mathrm{H}$ and ${ }^{13} \mathrm{C}$ NMR spectra support the proposed structure, as the tert-butyl proton and carbon signals all appear as virtual triplets, indicating strongly coupled phosphorus atoms, which typically occurs in a trans coordination geometry. ${ }^{16,124}$ In the ${ }^{13} \mathrm{C}$ NMR spectrum the peak 
<smiles>[R7]c1cc(P)c2c(c1)[X]c1c([Y])cc([R])cc1O2</smiles>

(i)<smiles>[R]c1cc([X])c(OP(C)(C)(C)C)c(P(C)(C)(C)C)c1</smiles>

Scheme 4.2: Reaction of $\left[\mathrm{Rh}(\mathrm{coe})_{2} \mathrm{Cl}\right]_{2}$ and $t$-Bu-xantphos ligands. Reagents and conditions: (i) $0.5 \mathrm{eq} \cdot\left[\mathrm{Rh}(\mathrm{coe})_{2} \mathrm{Cl}_{2}, \mathrm{C}_{6} \mathrm{D}_{6}, 60^{\circ} \mathrm{C}, 24\right.$ hours.

corresponding to the $\mathrm{O}$-ipso carbon has shifted downfield relative to the free ligand, unlike the equivalent $[\mathrm{Ag}(t-\mathrm{Bu}$-xantphos $) \mathrm{Cl}]$ complexes where the oxygen is known to be non-coordinating (Table 4.2). This downfield shift is consistent with a coordinated oxygen. As the oxygen donates electron density to the metal this will inductively decrease electron density on the $O$-ipso carbon resulting in decreased shielding and thus a downfield shift of the NMR signal.
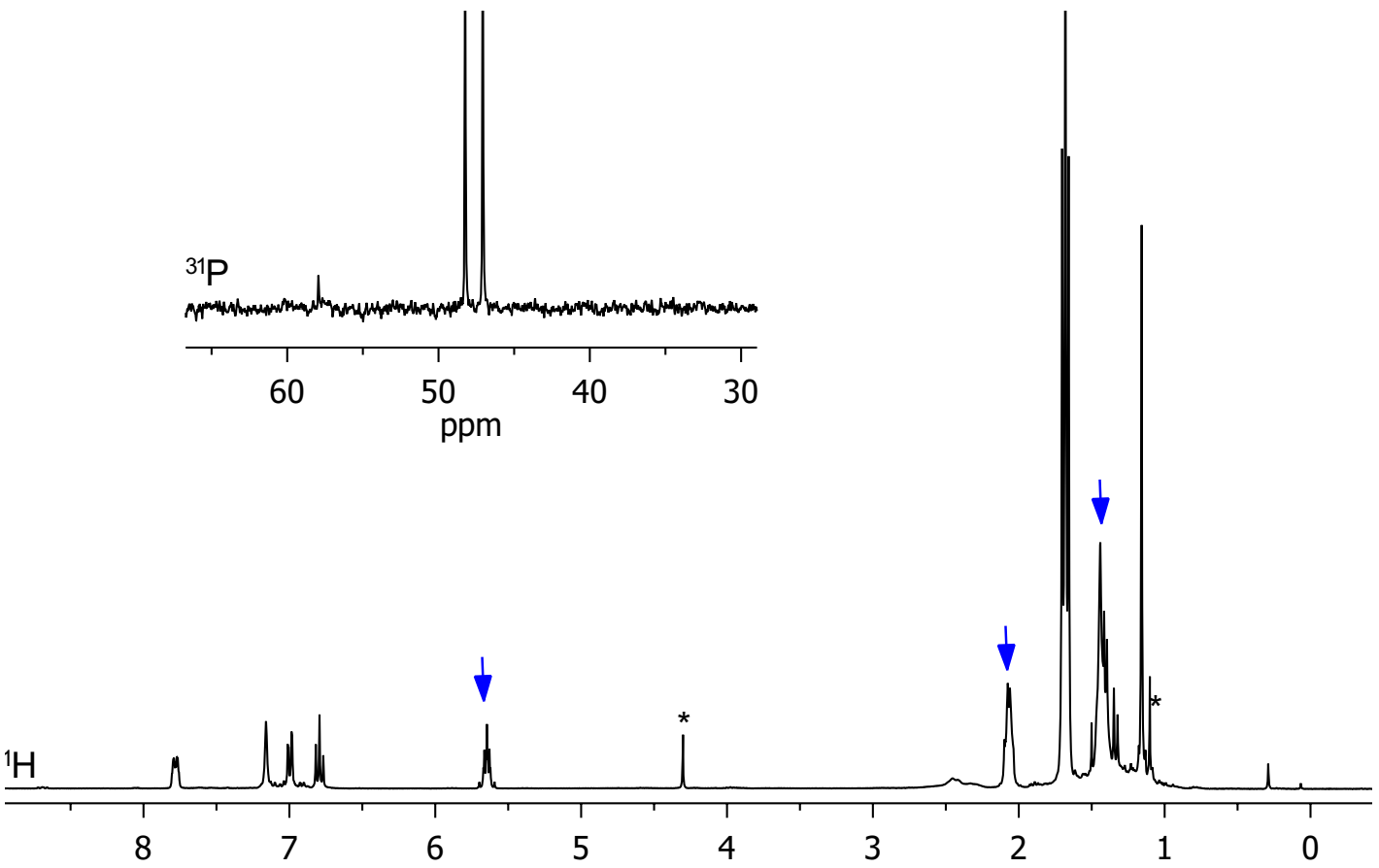

Figure 4.3: ${ }^{31} \mathrm{P}$ and ${ }^{1} \mathrm{H}$ NMR spectra for [ $\mathrm{Rh}(t$-Bu-xantphos $\left.) \mathrm{Cl}\right]$ in $\mathrm{C}_{6} \mathrm{D}_{6}$. Blue arrows indicate displaced coe, asterisks denote impurities.

Subsequent to this work the $\left[\mathrm{Rh}\left(t-\mathrm{Bu}\right.\right.$-xantphos- $\left.\left.\mathrm{k} P, \mathrm{O}, \mathrm{P}^{\prime}\right) \mathrm{Cl}\right]$ complex and $\mathrm{X}$-ray 
Table 4.1: Selected NMR data of [Rh(t-Bu-xantphos)Cl] complexes in $\mathrm{C}_{6} \mathrm{D}_{6}(\Delta \delta=$ $\left.\delta_{\text {complex }}-\delta_{\text {free ligand }}\right)$.

\begin{tabular}{ccccccc}
\hline & \multicolumn{4}{c}{${ }^{31} \mathbf{P}$} & & \multicolumn{2}{c}{${ }^{\mathbf{1}} \mathbf{H} \mathbf{t}-\mathbf{B u}$} \\
\cline { 2 - 4 } \cline { 7 - 8 } Compound & $\delta / \mathbf{p p m}$ & $\Delta \delta / \mathbf{p p m}$ & $\boldsymbol{J}_{\mathbf{R h P}} / \mathbf{H z}$ & & $\delta / \mathbf{p p m}$ & $\mathbf{J} / \mathbf{H z}$ \\
\hline t-Bu-Sixantphos & 44.2 & 35.8 & 140.0 & & 1.69 & 13.5 \\
$t$-Bu-Thixantphos & 46.5 & 37.0 & 141.5 & & 1.67 & 13.7 \\
$t$-Bu-Xantphos & 47.7 & 37.5 & 142.3 & & 1.68 & 13.4 \\
\hline
\end{tabular}

crystal structure have been reported in the literature. $\underline{82}$ The literature method also used $\left[\mathrm{Rh}(\mathrm{coe})_{2} \mathrm{Cl}\right]_{2}$ as the starting material in benzene however, their reaction was complete in 24 hours at room temperature, which suggests that the heating to 60 ${ }^{\circ} \mathrm{C}$ is unnecessary. The NMR data reported herein is consistent with the literature values. The $\mathrm{X}$-ray crystal structure of $\left[\mathrm{Rh}\left(t\right.\right.$-Bu-xantphos- $\left.\left.\kappa P, O, P^{\prime}\right) \mathrm{Cl}\right]($ Figure 4.4) shows a square-planar geometry with a $t$-Bu-xantphos- $\kappa P, O, P^{\prime}$ coordination mode.

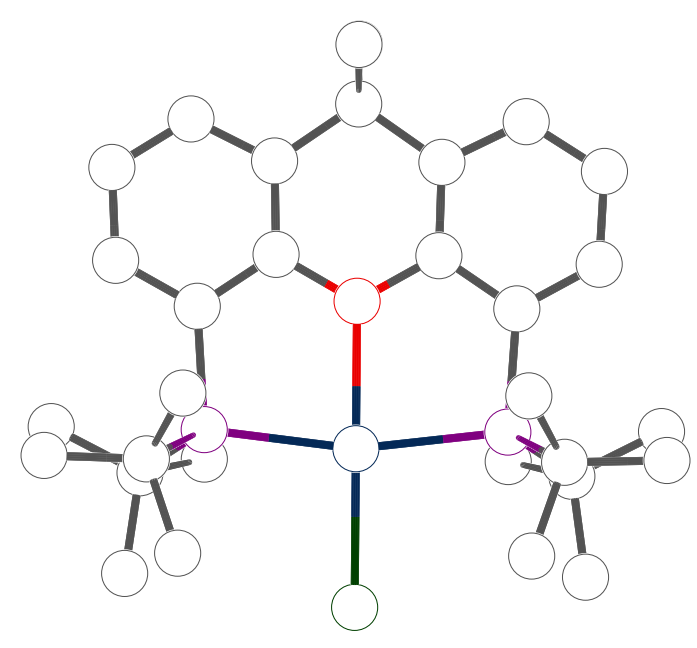

Figure 4.4: X-ray crystal structure of [Rh(t-Bu-xantphos- $\left.\left.\kappa P, O, P^{\prime}\right) \mathrm{Cl}\right]$. Hydrogen atoms omitted for clarity. .82 
Table 4.2: Chemical shift and coupling of the $O$-ipso carbon in the free ligand, $[\mathrm{Ag}(t-\mathrm{Bu}-\mathrm{xantphos}) \mathrm{Cl}]\left(\mathrm{CDCl}_{3}\right)$ and $[\mathrm{Rh}(t-$ Bu-xantphos $) \mathrm{Cl}]\left(\mathrm{C}_{6} \mathrm{D}_{6}\right)$ complexes $\left(\Delta \delta=\delta_{\text {complex }}-\delta_{\text {free ligand }}\right)$.

\begin{tabular}{|c|c|c|c|c|c|c|}
\hline \multirow[b]{2}{*}{ Ligand } & \multirow{2}{*}{$\frac{\text { Ligand }\left(\mathrm{CDCl}_{3}\right)}{\delta / \mathbf{p p m}}$} & \multirow{2}{*}{$\frac{\text { Ligand }\left(\mathbf{C}_{\mathbf{6}} \mathbf{D}_{6}\right)}{\delta / \mathbf{p p m}}$} & \multicolumn{2}{|c|}{ [Ag(t-Bu-xantphos)Cl] } & \multicolumn{2}{|c|}{$[\mathrm{Rh}(t-\mathrm{Bu}$-xantphos $) \mathrm{Cl}]$} \\
\hline & & & $\delta / \mathbf{p p m}$ & $\Delta \delta$ & $\delta / \mathbf{p p m}$ & $\Delta \delta$ \\
\hline t-Bu-Sixantphos & 164.3 & 164.5 & 163.9 & -0.4 & 169.5 & 5.0 \\
\hline$t$-Bu-Thixantphos & 155.3 & 155.9 & 155.5 & 0.2 & 157.4 & 1.5 \\
\hline$t$-Bu-Xantphos & 155.8 & 156.0 & 156.5 & 0.7 & 158.9 & 2.9 \\
\hline
\end{tabular}




\subsection{Reaction with Hydrogen}

Rhodium complexes are well-known as homogeneous hydrogenation and hydroformylation catalysts. Both of these processes involve the activation of molecular hydrogen at the rhodium centre. The catalytic cycle for hydrogenation using monophosphine ligands (Scheme 4.3) involves the addition of molecular hydrogen to $\left[\mathrm{Rh}\left(\mathrm{PR}_{3}\right)_{2} \mathrm{Cl}\right]$ generating a rhodium dihydride, which then coordinates an alkene and undergoes hydride-migration followed by reductive elimination to regenerate the starting rhodium complex and the alkane. In hydroformylation (Scheme 4.4) the alkene coordinates to an existing rhodium hydride complex. Hydride migration occurs followed by carbon monoxide coordination and migratory insertion into the rhodium alkyl bond. This is followed by the oxidative addition of dihydrogen, generating a rhodium(III) dihydride complex. The cycle concludes by reductive elimination, generating the aldehyde and the starting rhodium(I) complex. The influence of the bite-angle of Ph-xantphos complexes on the reactivity and selectivity of catalytic process was first studied for hydroformylation..$\underline{30}$ Since then hydroformylation has been studied extensively using

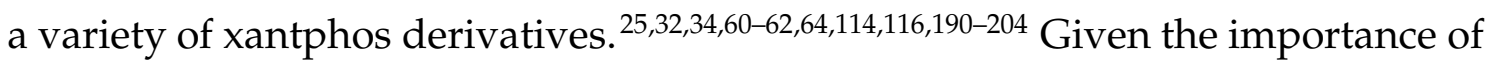
the oxidative addition of molecular hydrogen for both hydrogenation and hydroformylation, and to investigate any possible differences of the wide bite-angle and steric bulk of the $t$-Bu-xantphos ligands we investigated the reactivity of the $\left[\mathrm{Rh}\left(t-\mathrm{Bu}-x a n t p h o s-\kappa P, O, P^{\prime}\right) \mathrm{Cl}\right]$ complexes with dihydrogen.

The $\left[\mathrm{Rh}\left(t\right.\right.$-Bu-xantphos- $\left.\left.\mathrm{K} P, \mathrm{O}, \mathrm{P}^{\prime}\right) \mathrm{Cl}\right]$ complexes react readily with hydrogen, forming octahedral rhodium(III) dihydride complexes (Scheme 4.5). The dihydrogen undergoes oxidative addition forming two classical hydride ligands with a cis configuration. The $t$-Bu-xantphos ligands retain their meridional coordination, meaning that the hydride ligands are in different environments, one trans to the chloride and the other trans to the oxygen donor atom. As a result, the two faces of the $t$-Bu-xantphos ligands are now different causing two different environments for the bridgehead methyls in both $t$-Bu-sixantphos and $t$-Bu-xantphos, and the tert-butyl groups for all three $t$-Bu-xantphos ligands. However, the plane of symmetry perpendicular to the $t$-Bu-xantphos backbone is retained, such that the methyl substituents of $t$-Bu-thixantphos are in the same environment.

Two hydride resonances are evident in the ${ }^{1} \mathrm{H}$ NMR spectra of the three $[\mathrm{Rh}(t-\mathrm{Bu}$ - 


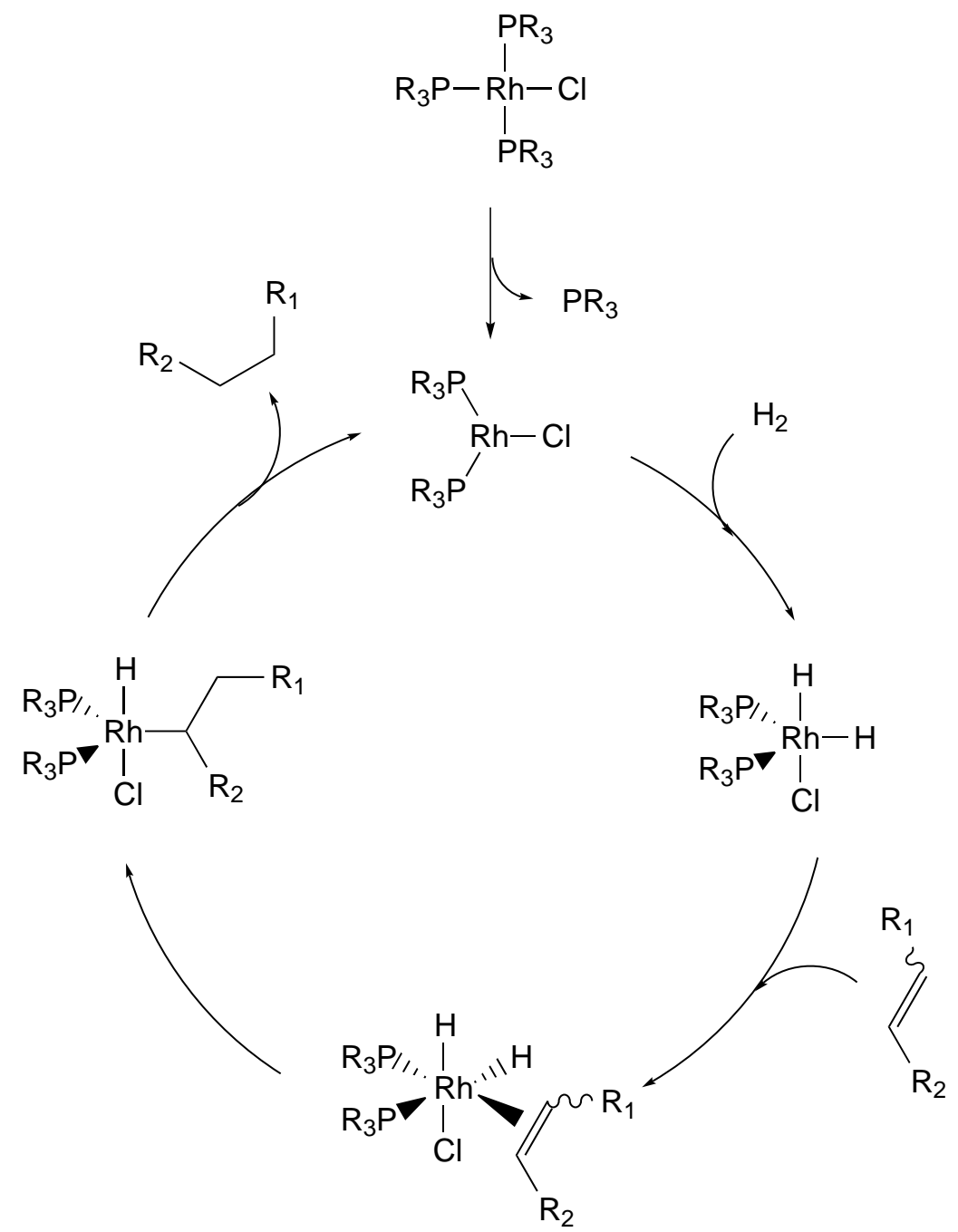

Scheme 4.3: Catalytic cycle for homogeneous hydrogenation using a rhodium chloride complex with monophosphine ligands. 


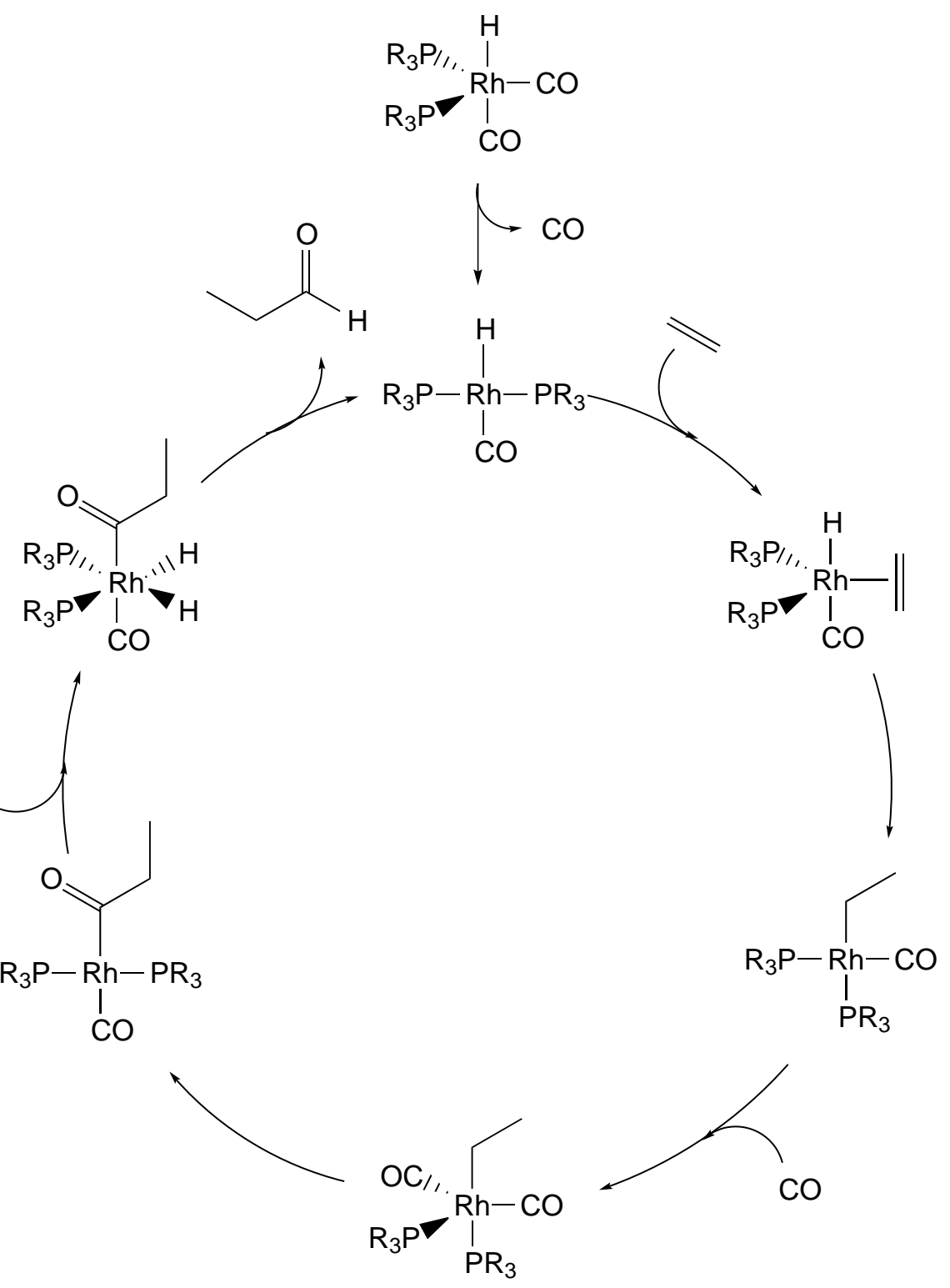

Scheme 4.4: Generic catalytic cycle for homogeneous hydroformylation. 

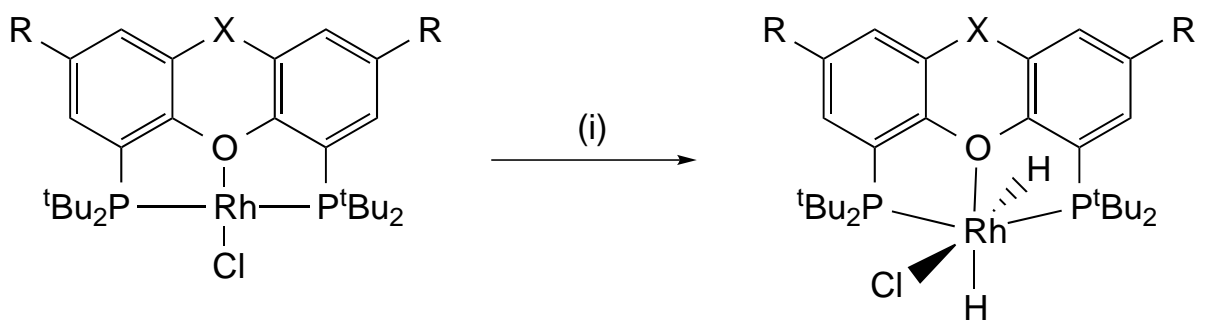

Scheme 4.5: Reaction of the $\mathrm{Rh}(t-\mathrm{Bu}$-xantphos $) \mathrm{Cl}]$ complexes with hydrogen. Reagents and conditions: (i) $\mathrm{H}_{2}, \mathrm{C}_{6} \mathrm{D}_{6}$.

xantphos- $\left.\left.\mathrm{k} P, \mathrm{O}, \mathrm{P}^{\prime}\right) \mathrm{Cl}(\mathrm{H})_{2}\right]$ complexes. The signals appear upfield as well defined doublets of triplets of doublets, coupling to the rhodium, two phosphorus atoms and each other (Figure 4.5, Table 4.3). The hydride trans to the chloride ligand (-16.92 to $-17.04 \mathrm{ppm}$ ) shows very little difference in either the chemical shift or coupling constants between the three complexes. The hydride trans to the $t$ Bu-xantphos oxygen (-20.51 to -21.12 ppm) shows more variation in the chemical shift and coupling. The value of $J_{\mathrm{RhH}}$ decreases with increasing natural bite-angle, such that the $t$-Bu-xantphos complex has the lowest value and $t$-Bu-sixantphos the largest. This suggests that the trans influence of the ether bridge is highest for $t$-Bu-xantphos and lowest for $t$-Bu-sixantphos, which may be related to the amount of strain required for the ligand to achieve tridentate coordination.

The ${ }^{31} \mathrm{P}$ NMR spectra (example in Figure 4.5 for $t$-Bu-thixantphos) for the $[\mathrm{Rh}(t-$ Bu-xantphos- $\left.\mathrm{k} P, O, P^{\prime}\right) \mathrm{Cl}(\mathrm{H})_{2}$ ] complexes each display a single resonance, downfield relative to the starting $\left[\mathrm{Rh}\left(t-\mathrm{Bu}\right.\right.$-xantphos- $\left.\left.\mathrm{K} P, \mathrm{O}, \mathrm{P}^{\prime}\right) \mathrm{Cl}\right]$ complexes, as a doublet of doublets with some further coupling evident. Although ${ }^{31} \mathrm{P} N \mathrm{NR}$ is ${ }^{1} \mathrm{H}$ decoupled, the decoupler is sometimes unable to fully decouple resonances that are so far outside the typical ${ }^{1} \mathrm{H}$ NMR range (-2 to $\left.15 \mathrm{ppm}\right)$ resulting in residual coupling. The values of $J_{\mathrm{RhP}}$ in the $\left[\mathrm{Rh}\left(t\right.\right.$-Bu-xantphos- $\left.\left.\mathrm{K} P, \mathrm{O}, \mathrm{P}^{\prime}\right) \mathrm{Cl}(\mathrm{H})_{2}\right]$ complexes are $116.1-117.8 \mathrm{~Hz}$, a decrease of $23.7-25.3 \mathrm{~Hz}$ from the starting [ $\mathrm{Rh}(t$ $\left.\left.\mathrm{Bu}-\mathrm{xantphos-} \mathrm{k} P, O, \mathrm{P}^{\prime}\right) \mathrm{Cl}\right]$ complexes. This decrease is consistent with a change in the oxidation state from rhodium(I) to rhodium(III). $\underline{16}$ Two signals are observed in the ${ }^{1} \mathrm{H}$ NMR spectra for the tert-butyl protons in all three complexes consistent with the proposed geometry. Both signals are virtual triplets, confirming the mutually trans coordination of the phosphorus atoms, consistent with the proposed meridional coordination of the ligands. Two signals were also ob- 

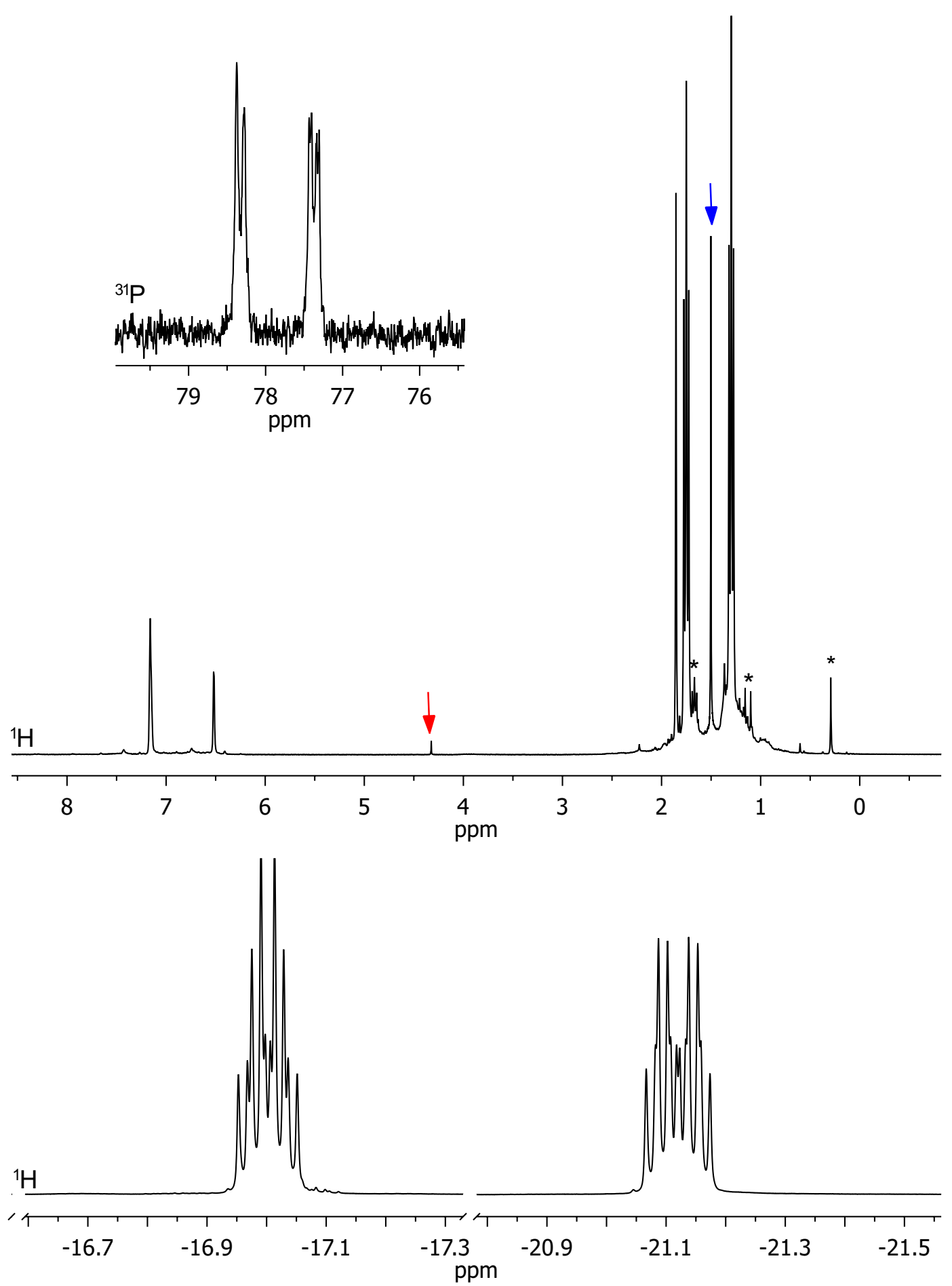

Figure 4.5: NMR spectra for $\left[\mathrm{Rh}(t\right.$-Bu-thixantphos $\left.) \mathrm{Cl}(\mathrm{H})_{2}\right]$ in $\mathrm{C}_{6} \mathrm{D}_{6}$. Impurities are indicated by asterisks, $\mathrm{CH}_{2} \mathrm{Cl}_{2}$ is indicated by a red arrow and cyclooctane by a blue arrow. 
Table 4.3: Hydride ${ }^{1} \mathrm{H}$ NMR data for $\mathrm{Rh}\left(t\right.$-Bu-xantphos- $\left.\left.\kappa P, O, P^{\prime}\right) \mathrm{Cl}(\mathrm{H})_{2}\right]$ complexes in $\mathrm{C}_{6} \mathrm{D}_{6}$.

\begin{tabular}{|c|c|c|c|c|c|c|c|c|}
\hline \multirow[b]{2}{*}{ Diphosphine } & \multicolumn{4}{|c|}{$\mathrm{H}^{-}$trans $-\mathrm{Cl}^{-}$} & \multicolumn{4}{|c|}{$\mathrm{H}^{-}$trans-O } \\
\hline & $\delta / \mathbf{p p m}$ & $J_{\mathrm{RhH}} / \mathrm{Hz}$ & $J_{\mathrm{PH}} / \mathrm{Hz}$ & $J_{\mathrm{HH}} / \mathrm{Hz}$ & $\delta / \mathbf{p p m}$ & $J_{\mathrm{RhH}} / \mathrm{Hz}$ & $J_{\mathrm{PH}} / \mathbf{H z}$ & $J_{\mathrm{HH}} / \mathrm{Hz}$ \\
\hline t-Bu-Sixantphos & -16.92 & 22.7 & 13.5 & 9.4 & -21.12 & 30.9 & 12.1 & 9.2 \\
\hline t-Bu-Thixantphos & -17.00 & 22.6 & 13.5 & 9.4 & -21.13 & 30.5 & 12.4 & 9.4 \\
\hline$t$-Bu-Xantphos & -17.04 & 22.8 & 13.4 & 9.4 & -20.51 & 28.8 & 12.2 & 9.4 \\
\hline
\end{tabular}


served in the ${ }^{1} \mathrm{H}$ NMR spectra for the bridgehead methyls in [Rh(t-Bu-xantphos$\left.\left.\kappa P, O, P^{\prime}\right) \mathrm{Cl}(\mathrm{H})_{2}\right]$ and $\left[\mathrm{Rh}\left(t-\mathrm{Bu}\right.\right.$-sixantphos- $\left.\left.\kappa P, O, P^{\prime}\right) \mathrm{Cl}(\mathrm{H})_{2}\right]$, again consistent with the proposed structure.

The $\left[\mathrm{Rh}\left(t\right.\right.$-Bu-xantphos- $\left.\left.\kappa P, O, P^{\prime}\right) \mathrm{Cl}(\mathrm{H})_{2}\right]$ complexes are similar to previously reported rhodium xantphos dihydride complexes, $\left[\mathrm{Rh}(\mathrm{H})_{2}(i\right.$-Pr-xantphos $\left.) \mathrm{X}\right](\mathrm{X}=$ $\mathrm{Cl}$, OTf $)^{81}$ and $\left[\mathrm{Rh}(\mathrm{H})_{2}(\mathrm{Ph} \text {-xantphos }) \mathrm{X}\right]^{+}\left(\mathrm{X}=\mathrm{P}(\mathrm{Cyp})_{3}\right.$ Cyp = cyclopentyl, NCMe, $\left.\mathrm{OC}\left(\mathrm{Me}_{3}\right)_{2}, \mathrm{H}_{3} \mathrm{BNMe}_{3}-\mathrm{kH}\right) \cdot \underline{72,177,205}$ In these cases the hydride trans to the oxygen atom was located in the ${ }^{1} \mathrm{H}$ NMR between -22.19 and $-19.00 \mathrm{ppm}$ with a rhodium coupling constant of 27.0-33.8 Hz. The ${ }^{1} \mathrm{H}$ NMR signals for the hydride ligands in the $\left[\mathrm{Rh}\left(t\right.\right.$-Bu-xantphos- $\left.\left.\kappa P, O, P^{\prime}\right) \mathrm{Cl}(\mathrm{H})_{2}\right]$ complexes are encompassed within these ranges (Table 4.3). For the reported $t$-Bu-xantphos complexes the ${ }^{31} \mathrm{P}$ NMR chemical shift is 77.8-79.0 ppm $\left(J_{\mathrm{RhP}}=116.1-117.8 \mathrm{~Hz}\right)$. In comparison, the literature complexes appear at 36.9-45.4 ppm $\left(J_{\mathrm{RhP}}=114-121 \mathrm{~Hz}\right)$ for Ph-xantphos and 64.8-67.2 ppm $\left(J_{\mathrm{RhP}}=113-114 \mathrm{~Hz}\right)$ for $i$-Pr-xantphos. The coupling constants are consistent for the complexes, and although the absolute chemical shift varies significantly, the change in chemical shift on reaction with hydrogen from the [ $\mathrm{Rh}(t$-Bu-xantphos)Cl] complexes is consistent (28.7-31.1 ppm for i-Pr-xantphos compared to 31.3-34.0 ppm for $t$-Bu-xantphos, [ $\mathrm{Rh}$ (Ph-xantphos) $\mathrm{Cl}$ ] has not been reported in the literature).

The $\left[\mathrm{Rh}(\mathrm{H})_{2}(\mathrm{Ph} \text {-xantphos }) \mathrm{X}\right]^{+}\left(\mathrm{X}=\mathrm{P}(\mathrm{Cyp})_{3}, \mathrm{H}_{3} \mathrm{BNMe}_{3}-\kappa H\right)$ complexes were reportedly only stable under a hydrogen atmosphere. ${ }^{177,205}$ However, the $[R h(t-$ Bu-xantphos $) \mathrm{Cl}(\mathrm{H})_{2}$ ] complexes were able to be placed under vacuum overnight with no evidence for loss of hydrogen. This difference is likely the result of the different electronics between $t$-Bu-xantphos and Ph-xantphos. The electron donating nature of the tert-butyl groups on $t$-Bu-xantphos enhances electron donation from the rhodium centre into the $\sigma^{*}$ orbital of a dihydrogen ligand, thus favouring oxidative addition and the formation of two discrete hydrides. The Ph-xantphos ligand is more electron withdrawing than $t$-Bu-xantphos, due to the phenyl substituents on the phosphorus atoms. The lower electron donation will result in less back-bonding from the metal to the dihydrogen ligand and thus the barrier towards reforming the dihydrogen will be lower.

The synthesis of the $\left[\mathrm{Rh}\left(t\right.\right.$-Bu-xantphos- $\left.\left.\mathrm{K} P, \mathrm{O}, \mathrm{P}^{\prime}\right) \mathrm{Cl}\right]$ complexes from $\left[\mathrm{Rh}(\mathrm{coe}){ }_{2} \mathrm{Cl}\right]_{2}$ generates cyclooctene as a by-product. This reaction mixture was used without further purification to determine the potential for the [Rh(t-Bu-xantphos- 
$\left.\left.\kappa P, O, P^{\prime}\right) \mathrm{Cl}\right]$ complexes to act as hydrogenation catalysts. Hydrogen gas was bubbled through a mixture of $\left[\mathrm{Rh}\left(t\right.\right.$-Bu-xantphos- $\left.\left.\mathrm{K} P, \mathrm{O}, P^{\prime}\right) \mathrm{Cl}\right]$ and cyclooctene for 10 minutes before the reaction was sealed and allowed to proceed at room temperature. No evidence for cyclooctene was observed by ${ }^{1} \mathrm{H}$ NMR spectroscopy in the reaction mixture and a peak for cyclooctane was apparent (Figure 4.5). While further work to determine the activity of these complexes needs to be performed, this result indicates the potential for the $\left[\mathrm{Rh}\left(t-\mathrm{Bu}\right.\right.$-xantphos- $\left.\left.\mathrm{k} P, \mathrm{O}, \mathrm{P}^{\prime}\right) \mathrm{Cl}\right]$ complexes to act as precatalysts for the hydrogenation of alkenes.

The $\left[\mathrm{Rh}(t\right.$-Bu-xantphos $\left.) \mathrm{Cl}(\mathrm{H})_{2}\right]$ complex and its X-ray crystal structure have subsequently been published by another research group. $\underline{\underline{82}}$ The $\mathrm{X}$-ray crystal structure confirms the $t$-Bu-xantphos ligand coordinates in a $\mathrm{k} P, O, P^{\prime}$ meridional geometry with mutually cis hydrides. The spectroscopic data contained herein is consistent with the published values.

\subsection{Rhodium Carbonyl Complexes}

Metal carbonyl complexes are some of the most well-studied transition metal complexes for many reasons. Metal carbonyls are involved in a number of different catalytic processes including hydroformylation, hydroesterification and hydrocarboxylation to introduce oxygen functionality. $28,206,207$ These reactions are of great synthetic importance and numerous industrial examples are known, such as the conversion of a benzylic alcohol to a carboxylic acid using a palladium catalyst in the synthesis of ibuprofen $\underline{208}$ and the hydroformylation of 1,3-butadiene to adipaldehyde, an intermediate for producing caprolactam and hexamethylene1,6-diamine (HMDA), key monomers for nylon-6.6 and nylon-6 manufacture. ${ }^{209}$ These catalytic processes involve the terminal coordination of the carbonyl to the metal centre. However, carbonyls can also act as bridging ligands, either solely through the carbon atom or in a side-on $\eta^{2}$ mode involving the $\pi$-system.

Transition metal carbonyl complexes have also been studied to investigate the electronic influence of other ligands. The stretching frequency of the $\mathrm{C}=\mathrm{O}$ bond is a good measure of the electronic environment as carbonyl ligands are strong $\pi$-acceptor ligands, meaning that the metal centre will donate electron density into the $\pi^{*}$-orbital. This weakens the $\mathrm{C}=\mathrm{O}$ bond and results in a shift of the infrared $\mathrm{C}=\mathrm{O}$ stretch to lower frequency. The degree to which the back-bonding 
occurs is related to the nature of the metal centre and the other ligands. As such, a number of series exist where this stretch has been used to quantify the electron donor capabilities of various ligands. These include the well-known Tolman Electronic Parameter using $\left[\mathrm{Ni}(\mathrm{CO})_{3} \mathrm{~L}\right]$ complexes, $\underline{8,14}$ and $\left[\mathrm{Mo}(\mathrm{CO})_{5} \mathrm{~L}\right]$ and $\left[\mathrm{Rh}(\mathrm{CO}) \mathrm{ClL}_{2}\right]$ series, ${ }^{\frac{9}{}}$ although other methods are utilised such as the phosphine selenide coupling constants (see Section 2.4) and the $J_{\mathrm{PtP}}$ coupling in cis- $\left[\mathrm{PtCl}_{2} \mathrm{P}_{2}\right]$ complexes. $\underline{16}$

The typical method for producing rhodium phosphine carbonyl complexes is to react the free phosphine or diphosphine ligand with either the chloro-bridged dicarbonyl dimer, $\left[\mathrm{Rh}(\mathrm{CO})_{2} \mathrm{Cl}_{2}\right.$, or by reaction with a chloro-bridged rhodium alkene dimer such as $\left[\mathrm{Rh}\left(\mathrm{C}_{2} \mathrm{H}_{4}\right)_{2} \mathrm{Cl}\right]_{2}$ or $\left[\mathrm{Rh}(\mathrm{Coe})_{2} \mathrm{Cl}_{2}\right.$ under an atmosphere of carbon monoxide. The reaction is then purified either in the air or under inert atmosphere and in the majority of cases the product is trans- $[\mathrm{Rh}(\mathrm{CO})(\mathrm{PP}) \mathrm{Cl}]$ where PP is either two monophosphine ligands or a trans-spanning diphosphine. Using smaller bite-angle diphosphine ligands results in the cis isomer.

The reactivity of the $\left[\mathrm{Rh}\left(t-\mathrm{Bu}\right.\right.$-xantphos- $\left.\left.\mathrm{k} P, \mathrm{O}, \mathrm{P}^{\prime}\right) \mathrm{Cl}\right]$ complexes towards carbon monoxide was investigated on an NMR scale by bubbling $\mathrm{CO}$ through a $\mathrm{C}_{6} \mathrm{D}_{6}$ solution of the complex for 10 minutes before sealing the NMR tube with a J. Young tap. An immediate colour change was observed, with the dark brown starting material changing to yellow ( $t$-Bu-sixantphos) or orange ( $t$-Bu-thixantphos and $t$-Bu-xantphos). NMR analysis of these samples showed the reaction was complete, forming a single product in under 15 minutes with no evidence of the starting material. All NMR spectra for these complexes were obtained under an atmosphere of $\mathrm{CO}$.

The NMR spectra for the three resulting products show some differences. The ${ }^{1} \mathrm{H}$ and ${ }^{13} \mathrm{C}$ NMR spectra for the reaction of $\left[\mathrm{Rh}\left(t\right.\right.$-Bu-thixantphos- $\left.\left.\mathrm{K} P, \mathrm{O}, \mathrm{P}^{\prime}\right) \mathrm{Cl}\right]$ with $\mathrm{CO}$ are broad, while the ${ }^{31} \mathrm{P}$ NMR spectrum shows only slight broadening. This indicates possible dynamic behaviour. However, the spectra for the reaction of $\left[\mathrm{Rh}\left(t\right.\right.$-Bu-sixantphos- $\left.\left.\kappa P, O, P^{\prime}\right) \mathrm{Cl}\right]$ and $\left[\mathrm{Rh}\left(t\right.\right.$-Bu-xantphos- $\left.\left.\kappa P, O, P^{\prime}\right) \mathrm{Cl}\right]$ with $\mathrm{CO}$ are both well resolved (Figure 4.6 shows a selection of the NMR spectra for the $t$-Buxantphos complex). In all cases the ${ }^{31} \mathrm{P}$ NMR spectrum show a single peak at 69.3-71.6 ppm, split into a doublet by rhodium coupling of 120.0-122.2 Hz (Table 4.4). These are shifted downfield relative to the $\left[\mathrm{Rh}\left(t-\mathrm{Bu}\right.\right.$-xantphos- $\left.\left.\kappa P, O, P^{\prime}\right) \mathrm{Cl}\right]$ starting material. The value of $J_{\mathrm{RhP}}$ has decreased from the starting $[\mathrm{Rh}(t-\mathrm{Bu}-$ 
xantphos-kP,O,P')Cl] complexes from $140.0-142.3 \mathrm{~Hz}$ to $120.0-122.2 \mathrm{~Hz}$.

Table 4.4: Selected NMR data of $\left[\mathrm{Rh}(t\right.$-Bu-xantphos $\left.)(\mathrm{CO})_{2} \mathrm{Cl}\right]$ complexes in $\mathrm{C}_{6} \mathrm{D}_{6}$ $\left(\Delta \delta=\delta_{\text {complex }}-\delta_{\text {free ligand }}\right)$.

\begin{tabular}{lcccccccc}
\hline & \multicolumn{4}{c}{${ }^{31} \mathbf{P}$} & & \multicolumn{3}{c}{${ }^{13} \mathbf{C} \mathbf{C O}$} \\
\cline { 2 - 4 } \cline { 7 - 9 } Ligand & $\delta / \mathbf{p p m}$ & $\Delta \delta \mathbf{p p m}$ & $\boldsymbol{J}_{\mathbf{R h P}} / \mathbf{H z}$ & & $\delta / \mathbf{p p m}$ & $\boldsymbol{J}_{\mathbf{R h C}} / \mathbf{H z}$ & $\boldsymbol{J}_{\mathbf{P C}} / \mathbf{H z}$ \\
\hline t-Bu-Sixantphos & 70.8 & 26.6 & 120.0 & & 195.5 & 84.4 & 13.0 \\
$t$-Bu-Thixantphos & 69.3 & 22.8 & 122.2 & & not observed & \\
$t$-Bu-Xantphos & 71.6 & 23.9 & 120.0 & & 194.9 & 84.4 & 12.4 \\
\hline
\end{tabular}

The NMR spectra of the products from the reaction of the $[\mathrm{Rh}(t-\mathrm{Bu}$-xantphos $) \mathrm{Cl}]$ complexes with $\mathrm{CO}$, indicate a high level of symmetry in the products. In the ${ }^{1} \mathrm{H}$ NMR spectra a single peak was observed for the tert-butyl groups and a singlet for the methyl groups for each of the three ligands. The number of aromatic signals in the ${ }^{1} \mathrm{H}$ NMR spectra indicate that two mirror planes exist, parallel to the backbone of the ligand and perpendicular to it (running through the oxygen and other bridging atom). For $t$-Bu-sixantphos and $t$-Bu-xantphos the tert-butyl groups appear as a virtual triplet indicating a trans or pseudo trans coordination geometry. For $t$-Bu-thixantphos the tert-butyl resonance displays some broadening, resulting in a singlet.

The number of signals in the ${ }^{13} \mathrm{C}$ NMR spectra is also suggestive of a highly symmetric product. Although the peaks were well resolved for the $t$-Bu-sixantphos and $t$-Bu-xantphos complexes, in the $t$-Bu-thixantphos complex all of the ${ }^{13} \mathrm{C}$ NMR peaks were broad except two, which can be attributed to the methyl groups and the aromatic carbon they are attached to, indicating that these carbons are unaffected by the dynamic process which leads to the broadening in this spectra. This process may be either exchange of the CO ligand with the uncoordinated $\mathrm{CO}$, or a change in geometry such as a trigonal bipyramidal to square-planar equilibrium.

The reaction of the $[\mathrm{Rh}(t$-Bu-xantphos $) \mathrm{Cl}]$ complexes with carbon monoxide was carried out with natural abundance $\mathrm{CO}$. Despite this, a peak was observed for the coordinated carbonyl in the ${ }^{13} \mathrm{C}$ NMR spectra for the $t$-Bu-sixantphos and $t$-Bu- 

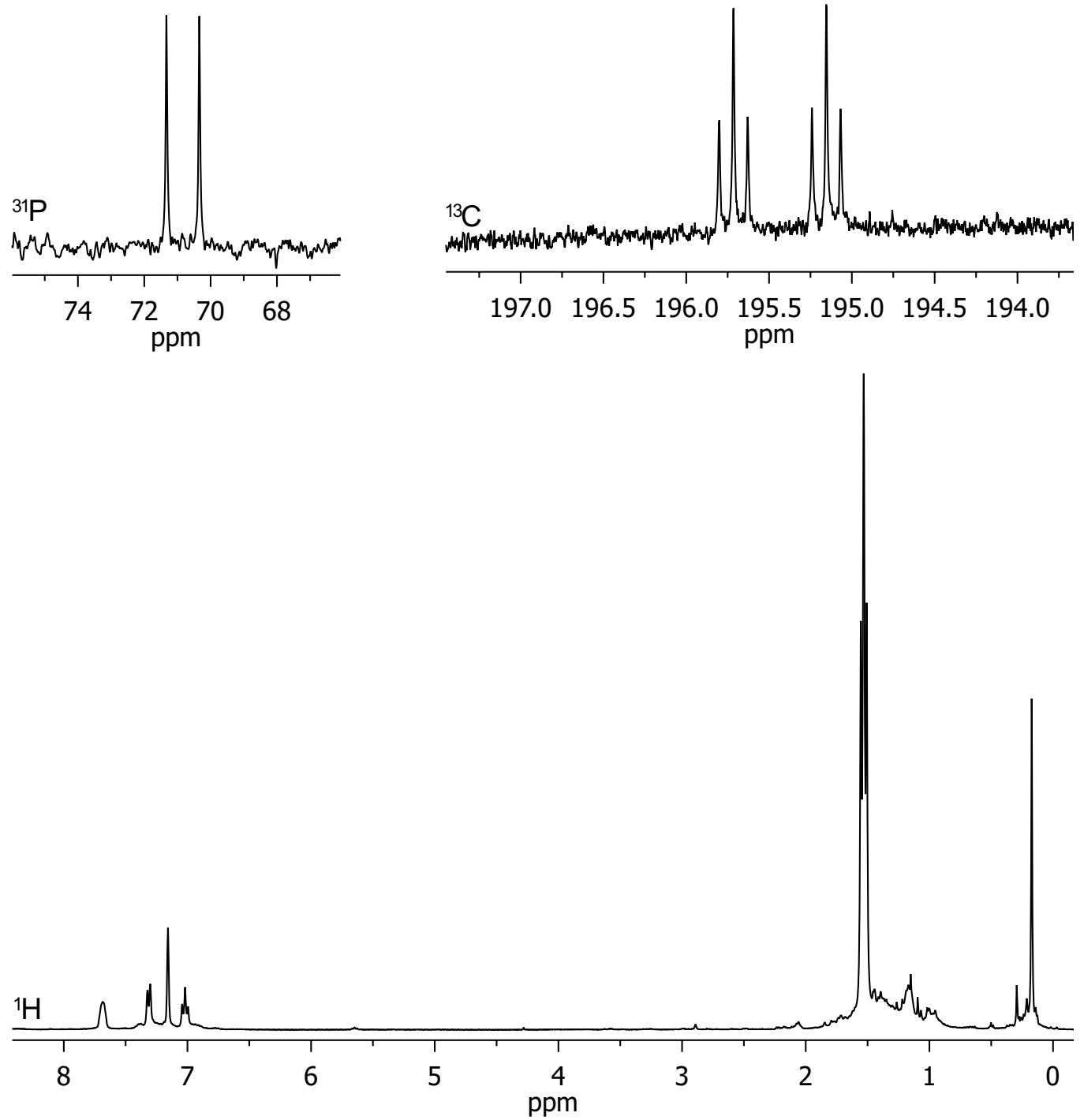

Figure 4.6: ${ }^{31} \mathrm{P},{ }^{13} \mathrm{C}$ and ${ }^{1} \mathrm{H}$ NMR spectra of the reaction of [Rh $(t$-Bu-sixantphos)Cl] with $\mathrm{CO}$ in $\mathrm{C}_{6} \mathrm{D}_{6}$. 
xantphos products (Table 4.4 and Figure 4.6). This peak occurs at $195.5 \mathrm{ppm}$ for $t$-Bu-sixantphos and 194.9 ppm for $t$-Bu-xantphos as a well resolved doublet of triplets indicating coupling to rhodium $\left(J_{\mathrm{RhC}}=84.4 \mathrm{~Hz}\right.$ for both) and two equivalent phosphorus atoms $\left(J_{\mathrm{PC}}=13.0\right.$ and $12.4 \mathrm{~Hz}$ for $t$-Bu-sixantphos and $t$-Buxantphos respectively). These values are within the expected ranges for rhodium carbonyl complexes.

There are a number of different possible products for the reaction of the $[\mathrm{Rh}(t-$ Bu-xantphos- $\left.\left.\kappa P, O, P^{\prime}\right) C l\right]$ complexes with carbon monoxide, due to the ability of rhodium(I) to form both square planar and trigonal bipyramidal structures, combined with the potential hemilability of the $t$-Bu-xantphos oxygen resulting in a $\kappa P, P^{\prime}$ bonding geometry. In addition, it is possible for either one or two carbonyl ligands to coordinate, displacing either the oxygen or the chloride ligand. The number of possibilities is decreased by the inability for the $t$-Bu-xantphos ligands to coordinate in a cis geometry on square planar centres (See Chapter 6 for further discussion) thus also eliminating trigonal bipyramidal products with the $t$-Bu-xantphos complex coordinated in a $\kappa P, P^{\prime}$ axial-equatorial position. Possible products from the reaction are outlined in Figure 4.7 .

The majority of the possible structures outlined in Figure 4.7 can be discounted as they do no meet the symmetry requirements determined from the NMR spectra. The complexes consistent with the NMR spectra are the square planar complexes, $\left[\mathrm{Rh}\left(t\right.\right.$-Bu-xantphos- $\left.\left.\kappa P, O, P^{\prime}\right) \mathrm{CO}\right] \mathrm{Cl}(\mathrm{B})$ and $\left[\mathrm{Rh}\left(t\right.\right.$-Bu-xantphos- $\left.\left.\kappa P, P^{\prime}\right)(\mathrm{CO})_{2}\right] \mathrm{Cl}(\mathrm{C})$; and the trigonal bipyramidal complexes, $\left[\mathrm{Rh}\left(t\right.\right.$-Bu-xantphos- $\left.\left.\kappa P, O, P^{\prime}\right)(\mathrm{CO})_{2}\right] \mathrm{Cl}(\mathrm{D})$ and trans-[Rh $\left(t-\mathrm{Bu}\right.$-xantphos- $\left.\left.\kappa P, P^{\prime}\right)(\mathrm{CO})_{2} \mathrm{Cl}\right](\mathrm{J})$. The mass spectra of the products from the reaction of the $[\mathrm{Rh}(t-\mathrm{Bu}$-xantphos $) \mathrm{Cl}]$ complexes with $\mathrm{CO}$ show a single major ion cluster. This cluster only differs for the three ligands due to the differences in the ligand structure. The cluster is consistent with a [Rh(t-Bu-xantphos $)(\mathrm{CO})]^{+}$ion in both mass/charge ratio and isotopic distribution. While this supports the formulation of the product as a monocarbonyl entity, this ion cluster could result from any of the possible structures given in Figure 4.7 as mass spectrometry is performed under high vacuum, which may result in loss of CO from the dicarbonyl structures, and ionisation by loss of chloride ligands is very common for coordination complexes. $\stackrel{210}{ }$

As previously discussed (Section 4.1) the position of the $O$-ipso carbon peak in the ${ }^{13} \mathrm{C}$ NMR spectra for $t$-Bu-xantphos complexes can be used as a guide for the 


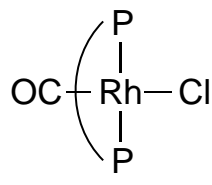

A<smiles>CC[In]1P2COCP1P2C=O</smiles>

B<smiles>CC[I+]I1CPC2(CO2)PC1=O</smiles>

C

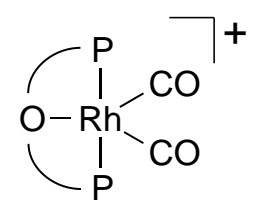

D<smiles>O=C[Pb]1(Cl)PCOCP1</smiles>

E<smiles></smiles>

$\mathrm{F}$<smiles></smiles>

G

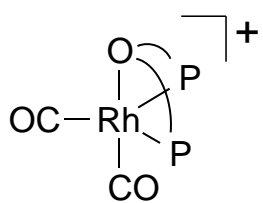

$\mathrm{H}$<smiles>CP1([O-])(Cl)CCPC1=O</smiles><smiles>O=C[Pb](Cl)(Cl)P1CCPC1</smiles>

I

J

Figure 4.7: Possible products from reaction of [Rh( $t$-Bu-xantphos- $\left.\left.\kappa P, O, P^{\prime}\right) \mathrm{Cl}\right]$ complexes with $\mathrm{CO}$. First row: square planar complexes, second row: trigonal bipyramidal complexes with $\kappa P, O, P^{\prime} t$-Bu-xantphos, and third row: trigonal bipyramidal complexes with $\kappa P, P^{\prime} t$-Bu-xantphos ligands.

bonding mode of the ligand. A peak for the $O$-ipso carbon that has not shifted significantly from the $O$-ipso carbon peak for the free ligand is indicative of a $\kappa P, P^{\prime}$ coordination, while a peak shifted downfield by more than $2.0 \mathrm{ppm}$ is indicative of a $\kappa P, O, P^{\prime}$ coordination. The products from this reaction show very little change in the position of the $O$-ipso peak compared to the free ligand (Table 4.5). Thus indicating that the coordination mode of the three $t$-Bu-xantphos ligands in these carbonyl complexes is most likely to be a bidentate $\kappa P, P^{\prime}$ mode.

The two possible square planar complexes [ $\mathrm{Rh}\left(t\right.$-Bu-xantphos- $\left.\left.\kappa P, O, P^{\prime}\right) \mathrm{CO}\right] \mathrm{Cl}(\mathrm{B})$ and $\left[\mathrm{Rh}\left(t\right.\right.$-Bu-xantphos- $\left.\left.\kappa P, P^{\prime}\right)(\mathrm{CO})_{2}\right] \mathrm{Cl}(\mathrm{C})$ and one of the trigonal bipyramidal structures $\left[\mathrm{Rh}\left(t-\mathrm{Bu}\right.\right.$-xantphos- $\left.\left.\kappa P, O, P^{\prime}\right)(\mathrm{CO})_{2}\right] \mathrm{Cl}(\mathrm{D})$ are positively charged. As such, poor solubility in $\mathrm{C}_{6} \mathrm{D}_{6}$ would be expected. However, the product of this reaction remains in $\mathrm{C}_{6} \mathrm{D}_{6}$ solution with no signs of precipitation over a period of several weeks. This supports the formulation of the product as the trigonal bipyramidal trans-[ $\mathrm{Rh}(t$-Bu-xantphos $\left.)(\mathrm{CO})_{2} \mathrm{Cl}\right](\mathrm{J})$. Rhodium dicarbonyl com- 
Table 4.5: ${ }^{13} \mathrm{C}$ Chemical shift and coupling for the $O$-ipso carbon in $t$-Bu-xantphos, $\left[\mathrm{Rh}\left(t\right.\right.$-Bu-xantphos- $\left.\left.\mathrm{k} P, O, P^{\prime}\right) \mathrm{Cl}\right]$ and carbonyl complexes $\left(\Delta \delta=\delta_{\text {complex }}-\delta_{\text {free ligand }}\right)$.

\begin{tabular}{|c|c|c|c|c|c|c|c|c|}
\hline \multirow[b]{2}{*}{ Ligand } & \multicolumn{2}{|c|}{ Free ligand } & \multicolumn{3}{|c|}{ [Rh(t-Bu-xantphos- $\left.\left.\mathrm{K} P, O, P^{\prime}\right) \mathrm{Cl}\right]$} & \multicolumn{3}{|c|}{$\left[\mathrm{Rh}(t-\mathrm{Bu}\right.$-xantphos $\left.)(\mathrm{CO})_{2} \mathrm{Cl}\right]$} \\
\hline & $\delta^{13} \mathrm{C} / \mathrm{ppm}$ & $J / H z$ & $\delta^{13} \mathrm{C} / \mathrm{ppm}$ & $\Delta \delta$ & $J / \mathbf{H z}$ & $\delta^{13} \mathrm{C} / \mathrm{ppm}$ & $\Delta \delta$ & $J / \mathbf{H z}$ \\
\hline$t$-Bu-Sixantphos & 164.3 & 11.3 & 169.5 & 5.2 & 14.4 & 164.3 & 0.0 & 8.1 \\
\hline$t$-Bu-Thixantphos & 155.3 & 13.0 & 157.4 & 2.1 & 16.8 & 154.9 & -0.4 & n.o. \\
\hline$t$-Bu-Xantphos & 155.8 & 12.0 & 158.9 & 3.1 & 16.3 & 155.6 & -0.2 & 10.4 \\
\hline
\end{tabular}


plexes are typically only stable below room temperature and under an atmosphere of carbon monoxide, readily losing CO under vacuum. 211,212 No change was observed in the ${ }^{31} \mathrm{P}$ or ${ }^{1} \mathrm{H}$ NMR spectra for the complexes in a $\mathrm{C}_{6} \mathrm{D}_{6}$ solution under an argon atmosphere after being under vacuum overnight. Likewise no change was observed for these samples after several weeks in a $\mathrm{C}_{6} \mathrm{D}_{6}$ solution under argon.

The infrared (IR) spectra of the $\left[\mathrm{Rh}(t\right.$-Bu-xantphos $\left.)(\mathrm{CO})_{2} \mathrm{Cl}\right]$ complexes were obtained to determine any differences in the electronic properties of the three ligands. The $\mathrm{C}=\mathrm{O}$ stretching frequency is sensitive to changes in the electron density on the metal centre. More electron rich metal centres will enhance the backdonation from the metal into the $\pi^{*}$-orbital on the carbonyl. As this is an antibonding orbital, increased electron density will result in decreased bond order observed as a lower $\mathrm{C}=\mathrm{O}$ stretching frequency in the IR spectra. The IR spectra for the three $\left[\mathrm{Rh}(t\right.$-Bu-xantphos $\left.)(\mathrm{CO})_{2} \mathrm{Cl}\right]$ complexes showed multiple stretches in the region where $\mathrm{C}=\mathrm{O}$ stretches are observed. Indicative of multiple carbonyl ligands. The major $\mathrm{C}=\mathrm{O}$ stretch occurs at $1979 \mathrm{~cm}^{-1}$ for all of the complexes irrespective of the $t$-Bu-xantphos ligand present. This indicates that the ligands are comparable in their electron donation capabilities in the $\kappa P, P^{\prime}$ coordination mode.

Previous reports described the formation of trans-[ $\mathrm{Rh}(\mathrm{CO}) \mathrm{Cl}($ diphosphine)] complexes upon reaction of $\mathrm{Ph}$-xantphos and DPEphos with $\left[\mathrm{Rh}(\mathrm{CO})_{2} \mathrm{Cl}\right]_{2} \cdot \underline{213}$ These complexes appeared in the ${ }^{31} \mathrm{P}$ NMR spectra at 27.3 and $22.6 \mathrm{ppm}$ with $J_{\mathrm{RhP}}=119.2$ and $123.5 \mathrm{~Hz}$ for Ph-xantphos and DPEphos respectively. In the ${ }^{13} \mathrm{C}$ NMR spectra singlets were observed for the carbonyl ligands at 180.9 and $183.3 \mathrm{ppm}$ for Phxantphos and DPEphos respectively. The position of the carbonyl carbon in the $t$ Bu-xantphos complexes is at 195.5 or 194.9 ppm, indicating less shielding, which may be a result of the more electron rich rhodium centre in a five-coordinate complex. The rhodium chemistry of a more sterically demanding version of $\mathrm{Ph}$ xantphos where the phenyl rings are replaced with o-tolyl groups has also been reported. ${ }^{214}$ Again, this complex forms a trans-[ $\mathrm{Rh}(\mathrm{CO}) \mathrm{Cl}($ diphosphine)] complex. However, the analogous complexes with an iodide replacing the chloride ligand forms a square pyramidal structure with the oxygen coordinated to the rhodium. The $\mathrm{C}=\mathrm{O}$ stretching frequencies for the $t$-Bu-xantphos carbonyl complexes $\left(1979 \mathrm{~cm}^{-1}\right)$ occur between those for the trans-[Rh(CO)Cl(Ph-xantphos)] $\left(1974 \mathrm{~cm}^{-1}\right)$ and trans-[Rh(CO)Cl(DPEphos) $]\left(1985 \mathrm{~cm}^{-1}\right)$. The $\mathrm{C}=\mathrm{O}$ stretch is 
similar to $\left[\mathrm{Rh}(\mathrm{CO}) \mathrm{Cl}\left(\mathrm{PR}_{3}\right)_{2}\right]$ with $\mathrm{PR}_{3}=\mathrm{PPh}_{3}\left(1979 \mathrm{~cm}^{-1}\right)$ however, the strongly electron donating tert-butyl substituents on the $t$-Bu-xantphos ligands should result in a lower stretching frequency such as that where $\mathrm{PR}_{3}=\mathrm{PPhMe}_{2}(1968$ $\left.\mathrm{cm}^{-1}\right) . \underline{9}$ This is further evidence that the $t$-Bu-xantphos complexes are not trans[Rh(t-Bu-xantphos)(CO)Cl].

Based on this evidence we propose that the reaction of the [ $\mathrm{Rh}(t-\mathrm{Bu}$-xantphos $) \mathrm{Cl}]$ complexes results in a trigonal bipyramidal structure with the $t$-Bu-xantphos ligand occupying two of the equatorial sites in a $\kappa P, P^{\prime}$ coordination mode. The remaining equatorial site is occupied by a chloride ligand and carbonyl ligands take up the two axial positions (Scheme 4.6). Trigonal bipyramidal structures (particularly those with carbonyl ligands) are known to undergo facile rearrangement to square pyramids. $\stackrel{212}{2}$ This may be the cause of the broadening of the spectra for the $t$-Bu-thixantphos system, and the multiple peaks observed in the IR spectra. Ph-xantphos and DPEphos both form trans-[Rh(CO)Cl(diphosphine)], which would have a larger P-Rh-P angle than the $\left[\mathrm{Rh}(t-\mathrm{Bu}\right.$-xantphos $\left.)(\mathrm{CO})_{2} \mathrm{Cl}\right]$ complexes. This is counterintuitive as the natural bite-angle is larger for the $t$-Bu-xantphos ligands than for Ph-xantphos. However, it is possible that the trans-[ $\mathrm{Rh}(\mathrm{CO}) \mathrm{Cl}($ diphosphine)] are actually square pyramidal complexes with a weak interaction of the ether bridge in the diphosphine ligands, thus relieving the strain of such a wide bite-angle.

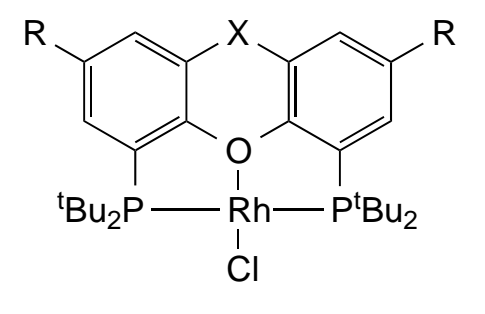
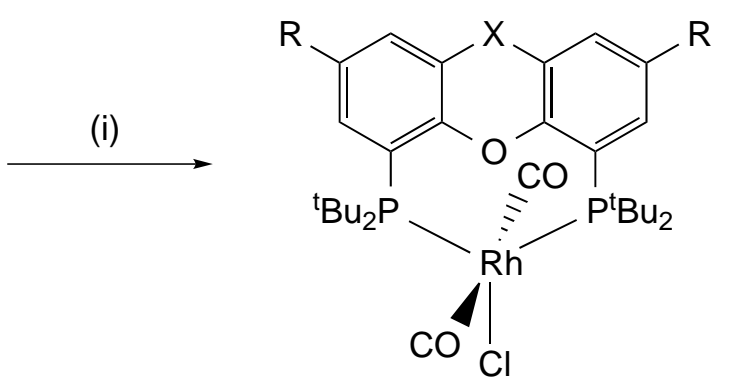

Scheme 4.6: Reaction of [ $\mathrm{Rh}(t-\mathrm{Bu}$-xantphos $) \mathrm{Cl}]$ with carbon monoxide. Reagents and conditions: (i) 10 mins $\mathrm{CO}, \mathrm{C}_{6} \mathrm{D}_{6}, 3$ days.

Dicarbonyl complexes have been reported for Ph-xantphos ligands, for example, Ph-sixantphos, Ph-thixantphos and Ph-xantphos form [ $\mathrm{Rh}(\mathrm{CO})_{2} \mathrm{H}(\mathrm{Ph}$-xantphos)] complexes. ${ }^{30}$ The diphosphine ligands coordinate in a bis-equatorial- $\kappa P, P^{\prime}$ mode, while the hydride occupies an axial site and the two carbonyls are in one equa- 
torial and one axial site. Little difference is observed between the three ligands with the ${ }^{31} \mathrm{P}$ NMR peaks between 21.1 and $23.4 \mathrm{ppm}\left(J_{\mathrm{RhP}}=123.9-127.9 \mathrm{~Hz}\right)$. Interestingly, although the two carbonyl ligands are inequivalent, only one carbonyl peak was reported, occurring as a doublet of triplets at $201.1 \mathrm{ppm}$ with $J_{\mathrm{RhC}}=65.7$ $\mathrm{Hz}$, and $J_{\mathrm{PC}}=10.6 \mathrm{~Hz}$ for the Ph-xantphos complex. No explanation was given for this but it may be due to the rapid exchange of one of the carbonyl ligands with free $\mathrm{CO}$ leading to broadening of the second ${ }^{13} \mathrm{C}$ peak.

\subsection{Dioxygen and Oxo Complexes}

The reaction of rhodium(I) complexes with oxygen is well established with numerous examples published. $\stackrel{215,216}{ }$ Many aspects of the oxidation of Wilkinson's complex, $\left[\mathrm{RhCl}\left(\mathrm{PPh}_{3}\right)_{3}\right]$, to form a distorted octahedral complex with a side-on $\eta^{2}$ $\mathrm{O}_{2}$ ligand have been reported, including the synthesis, $, 217,218$ degradation ${ }^{219}$ and use as an oxidation catalyst. $\stackrel{220,221}{ }$ Numerous other rhodium dioxygen complexes have been reported with monophosphine ${ }^{222-231}$ and diphosphine ${ }^{232-239}$ ligands. Reports of rhodium dioxygen complexes with heterobidentate $\underline{240-243}$ and tridentate $244-250$ ligands are also numerous. However, despite the number of rhodium complexes of $\mathrm{Ph}$-xantphos and other xantphos derivatives, no rhodium dioxygen complexes of these ligands have been reported. To address this, the reactivity of the $[\mathrm{Rh}(t-\mathrm{Bu}$-xantphos $) \mathrm{Cl}]$ and $\left[\mathrm{Rh}(t\right.$-Bu-xantphos $\left.) \mathrm{Cl}(\mathrm{H})_{2}\right]$ complexes with all three ligands towards oxygen was investigated.

Bubbling air through $\mathrm{C}_{6} \mathrm{D}_{6}$ solution of each of the $\left[\mathrm{Rh}\left(t\right.\right.$-Bu-xantphos- $\left.\left.\mathrm{k} P, \mathrm{O}, \mathrm{P}^{\prime}\right) \mathrm{Cl}\right]$ complexes resulted in the rapid formation of a new complex as expected (Scheme 4.7). The dihydride complexes, $\left[\mathrm{Rh}\left(t\right.\right.$-Bu-xantphos- $\left.\left.\kappa P, O, P^{\prime}\right) \mathrm{Cl}(\mathrm{H})_{2}\right]$, also reacted rapidly with air to form the same complexes. The ${ }^{31} \mathrm{P}$ NMR spectra of the resulting $\left[\mathrm{Rh}(t\right.$-Bu-xantphos $\left.) \mathrm{Cl}\left(\eta^{2}-\mathrm{O}_{2}\right)\right]$ complexes showed a single doublet (39.440.5) shifted upfield from the [ $\mathrm{Rh}(t$-Bu-xantphos $) \mathrm{Cl}]$ complex by between 4.8 and 7.5 ppm (Table 4.6). The value of $J_{\mathrm{RhP}}$ decreased by $37.8-41.6 \mathrm{~Hz}$, to $100.7-102.2$ $\mathrm{Hz}$. This is consistent with a change in the oxidation state from rhodium(I) to rhodium(III) and the corresponding decrease in the s-character of the metal hybrid orbital, resulting from the change in coordination geometry from square planar to pseudo-octahedral. 
Table 4.6: Selected NMR data for $\left[\mathrm{Rh}\left(t\right.\right.$-Bu-xantphos- $\left.\left.\kappa P, O, P^{\prime}\right) \mathrm{Cl}\left(\eta^{2}-\mathrm{O}_{2}\right)\right]$ in $\mathrm{C}_{6} \mathrm{D}_{6}\left(\Delta \delta=\delta_{\text {complex }}-\delta_{\text {free ligand }}\right)$.

\begin{tabular}{|c|c|c|c|c|c|c|c|}
\hline \multirow[b]{2}{*}{ Diphosphine } & \multicolumn{4}{|c|}{${ }^{31} \mathbf{P}$} & \multicolumn{3}{|c|}{${ }^{13} \mathrm{C} O$-ipso } \\
\hline & $\delta / \mathbf{p p m}$ & $\Delta \delta / \mathbf{p p m}$ & $J_{\mathrm{RhP}} / \mathbf{H z}$ & $\Delta J_{\mathrm{RhP}} / \mathrm{Hz}$ & $\delta / \mathbf{p p m}$ & $\Delta \delta / \mathbf{p p m}$ & $J / H z$ \\
\hline t-Bu-Sixantphos & 39.4 & 4.8 & 102.2 & 37.8 & 166.7 & 2.4 & 9.8 \\
\hline$t$-Bu-Thixantphos & 39.0 & 7.5 & 101.5 & 40.0 & 155.9 & 0.6 & 12.5 \\
\hline t-Bu-Xantphos & 40.5 & 7.2 & 100.7 & 41.6 & 157.3 & 1.5 & 11.5 \\
\hline
\end{tabular}



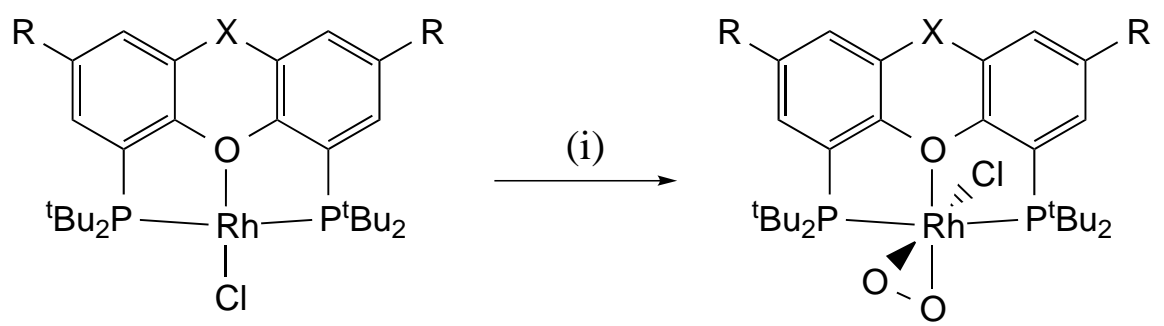

Scheme 4.7: Reaction of [Rh(t-Bu-xantphos $) \mathrm{Cl}]$ complexes with oxygen. Reagents and conditions: (i) 10 mins Air, $\mathrm{C}_{6} \mathrm{D}_{6}, 24$ hours.

The ${ }^{1} \mathrm{H}$ and ${ }^{13} \mathrm{C}$ NMR spectra for the three $\left[\mathrm{Rh}(t\right.$-Bu-xantphos $\left.) \mathrm{Cl}\left(\eta^{2}-\mathrm{O}_{2}\right)\right]$ complexes showed a loss of symmetry compared to the starting [ $\mathrm{Rh}(t$-Bu-xantphos $) \mathrm{Cl}]$ complexes. A single peak was observed for the methyl substituents on the aromatic rings of the $t$-Bu-thixantphos. However, two peaks were present for the methyl substituents on the carbon or silicon bridging atoms in both the $t$-Buxantphos and $t$-Bu-sixantphos complexes. In all three complexes, two different sets of peaks for the tert-butyl groups were observed. Together, this data indicates the presence of a plane of symmetry perpendicular to the backbone through the bridging atoms, and the absence of a plane of symmetry parallel to the backbone. Interestingly, the two methyl groups in the $t$-Bu-sixantphos and $t$-Bu-xantphos complexes occur in very different positions; one of the methyl groups is shifted upfield and the other downfield in both the ${ }^{1} \mathrm{H}$ and ${ }^{13} \mathrm{C}$ NMR spectra, compared to the $\left[\mathrm{Rh}\left(t-\mathrm{Bu}\right.\right.$-xantphos- $\left.\left.\mathrm{K} P, \mathrm{O}, \mathrm{P}^{\prime}\right) \mathrm{Cl}\right]$ starting material and $[\mathrm{Rh}(t-\mathrm{Bu}$ xantphos- $\left.\left.\kappa P, O, P^{\prime}\right) \mathrm{Cl}(\mathrm{H})_{2}\right]$. The effect is more pronounced for $t$-Bu-xantphos than $t$-Bu-sixantphos. In addition, one of the tert-butyl groups in each of the three $t$-Bu-xantphos ligands is well resolved as a virtual triplet for all the ${ }^{1} \mathrm{H}$ and ${ }^{13} \mathrm{C}$ NMR environments, while the other tert-butyl group has a well resolved virtual triplet for the quaternary carbon while the terminal carbon and protons are broad.

In the ${ }^{13} \mathrm{C}$ NMR spectra for the $\left[\mathrm{Rh}(t\right.$-Bu-xantphos $\left.) \mathrm{Cl}\left(\eta^{2}-\mathrm{O}_{2}\right)\right]$ complexes, the shift in the position of the $\mathrm{O}$-ipso carbon peak from the free ligand was lower than for the $[\mathrm{Rh}(t$-Bu-xantphos $) \mathrm{Cl}]$ complexes (Table 4.6). In particular, the $t$-Bu-thixantphos dioxygen complex exhibited a shift in position of only $0.6 \mathrm{ppm}$. However, the effect is typically reduced for the $t$-Bu-thixantphos ligand system, in fact for $\left[\mathrm{Rh}(t\right.$-Bu-thixantphos $\left.) \mathrm{Cl}(\mathrm{H})_{2}\right]$ a shift of only $0.1 \mathrm{ppm}$ was observed despite a 
$\kappa P, O, P^{\prime}$ coordination geometry. This difference may be due to the electron donation from the methyl groups on the aromatic system of $t$-Bu-thixantphos, which may mitigate the loss of electron density in the O-ipso carbon upon oxygen coordination. The shifts for $t$-Bu-sixantphos and $t$-Bu-xantphos are both consistent with $\mathrm{k} P, \mathrm{O}, \mathrm{P}^{\prime}$ coordination in the $\left[\mathrm{Rh}(t\right.$-Bu-xantphos $\left.) \mathrm{Cl}\left(\eta^{2}-\mathrm{O}_{2}\right)\right]$ complexes.

Slow evaporation of a $\mathrm{C}_{6} \mathrm{D}_{6}$ solution of $\left[\mathrm{Rh}\left(t-\mathrm{Bu}\right.\right.$-xantphos- $\left.\left.\mathrm{k} P, \mathrm{O}, \mathrm{P}^{\prime}\right) \mathrm{Cl}\left(\eta^{2}-\mathrm{O}_{2}\right)\right]$ produced dark red crystals suitable for X-ray diffraction. The crystal structure (Figure 4.8) displayed some substitutional disorder with the dioxygen ligand replaced in approximately $15 \%$ of the sites by an oxo ligand (Figure 4.9). The complexes cocrystallised in the orthorhombic space group Pbca. Crystallographic data is given in Table 4.7 and selected bond lengths and angles are given in Table 4.8 .

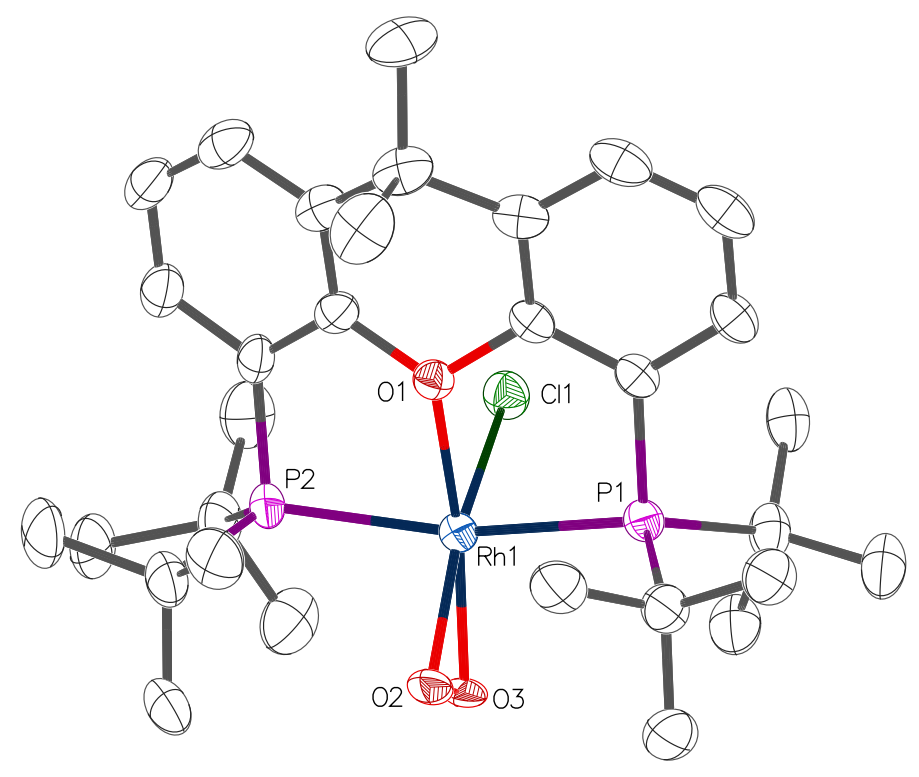

Figure 4.8: X-ray crystal structure of $\left[\mathrm{Rh}(t\right.$-Bu-xantphos $\left.) \mathrm{Cl}\left(\eta^{2}-\mathrm{O}_{2}\right)\right](50 \%$ probability thermal ellipsoids). Hydrogen atoms omitted for clarity.

The O-O bond lengthens upon coordination due to back-bonding from the metal into the anti-bonding $\pi^{*}$-orbital of the ligand. In the crystal structure of [Rh(t-Buxantphos- $\left.\left.\mathrm{P}, \mathrm{O}, \mathrm{P}^{\prime}\right) \mathrm{Cl}\left(\eta^{2}-\mathrm{O}_{2}\right)\right]$, the O2-O3 bond length is 1.424(5) $\AA$, lengthened by $0.21 \AA$ from the bond length in molecular oxygen of $1.21 \AA .251$ This length is typical for rhodium dioxygen complexes with the average $\mathrm{O}-\mathrm{O}$ bond length being $1.423 \AA .68$ The Rh-O bond lengths for the dioxygen ligand are different, with the oxygen trans to chloride having a longer bond by $0.054 \AA$. The oxygen trans to 
Table 4.7: Crystallographic Data and Structure Refinement of [Rh(t-Bu-xantphos $\left.) \mathrm{Cl}\left(\eta^{2}-\mathrm{O}_{2}\right)\right]$.

\begin{tabular}{|c|c|}
\hline Empirical formula & $\mathrm{C}_{31} \mathrm{H}_{48} \mathrm{ClO}_{2.85} \mathrm{P}_{2} \mathrm{Rh}$ \\
\hline Formula weight & 666.53 \\
\hline Temperature/K & $120.01(10)$ \\
\hline Crystal system & orthorhombic \\
\hline Space group & Pbca \\
\hline $\mathrm{a} / \AA$ & $11.84223(17)$ \\
\hline $\mathrm{b} / \AA$ & $20.2961(3)$ \\
\hline$c / \AA$ & $26.8069(4)$ \\
\hline$\alpha /{ }^{\circ}$ & 90 \\
\hline$\beta /{ }^{\circ}$ & 90 \\
\hline$\gamma /{ }^{\circ}$ & 90 \\
\hline Volume $/ \AA^{3}$ & 6443.06(17) \\
\hline $\mathrm{Z}$ & 8 \\
\hline$\rho_{\text {calc }} \mathrm{mg} / \mathrm{mm}^{3}$ & 1.374 \\
\hline$\mu / \mathrm{mm}$ & 6.206 \\
\hline $\mathrm{F}(000)$ & 2790.0 \\
\hline Crystal size $/ \mathrm{mm}$ & $0.1092 \times 0.0738 \times 0.0159$ \\
\hline Radiation & $\mathrm{CuK} \alpha(\lambda=1.54184)$ \\
\hline $2 \theta$ range for data collection & 6.594 to $147.8^{\circ}$ \\
\hline Index ranges & $-14 \leq \mathrm{h} \leq 13,-25 \leq \mathrm{k} \leq 21,-30 \leq 1 \leq 33$ \\
\hline Reflections collected & 45616 \\
\hline Independent reflections & $6481\left[R_{\text {int }}=0.0631, R_{\text {sigma }}=0.0332\right]$ \\
\hline Data/restraints/parameters & $6481 / 399 / 367$ \\
\hline Goodness-of-fit on $\mathrm{F}^{2}$ & 1.035 \\
\hline Final $\mathrm{R}$ indexes $[\mathrm{I}>=2 \sigma(\mathrm{I})]$ & $\mathrm{R}_{1}=0.0439, \mathrm{w} \mathrm{R}_{2}=0.1145$ \\
\hline Final $\mathrm{R}$ indexes [all data] & $\mathrm{R}_{1}=0.0550, \mathrm{w} \mathrm{R}_{2}=0.1226$ \\
\hline Largest diff. peak/hole / e $\AA^{-3}$ & $1.40 /-0.78$ \\
\hline
\end{tabular}




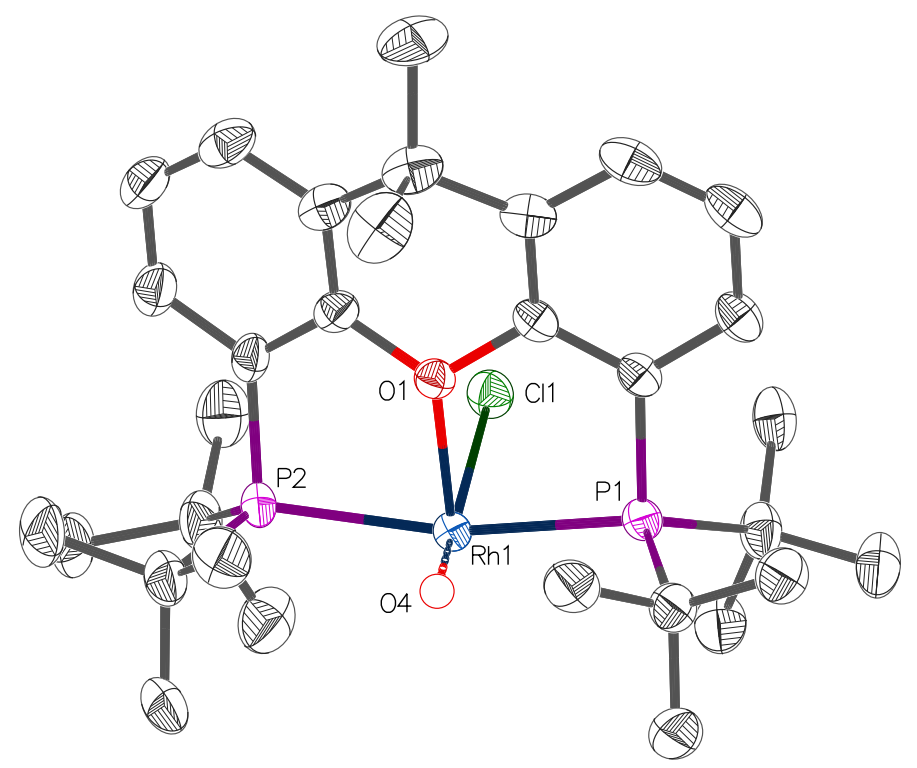

Figure 4.9: X-ray crystal structure of $\left[\mathrm{Rh}\left(t-\mathrm{Bu}-x a n t p h o s-\kappa P, O, P^{\prime}\right) \mathrm{Cl}(\mathrm{O})\right](50 \%$ probability thermal ellipsoids). Hydrogen atoms omitted for clarity.

Table 4.8: Selected bond distances $(\AA)$ and angles $\left(^{\circ}\right)$ of $\left[\mathrm{Rh}(t-\mathrm{Bu}\right.$-xantphos $) \mathrm{Cl}\left(\eta^{2}-\right.$ $\left.\mathrm{O}_{2}\right)$.

\begin{tabular}{llll}
\hline \multicolumn{2}{c}{ Bond distances $(\AA)$} & \multicolumn{2}{c}{ Bond angles $\left(^{\circ}\right)$} \\
\hline P1-Rh & $2.3391(8)$ & P1-Rh-P2 & $166.01(3)$ \\
P2-Rh & $2.3237(9)$ & Cl1-Rh-O2 & $158.18(10)$ \\
O1-Rh & $2.193(2)$ & Cl1-Rh-O4 & $161.2(3)$ \\
O2-Rh & $2.016(3)$ & O1-Rh-O3 & $161.33(12)$ \\
O3-Rh & $1.962(3)$ & Ring1-Ring2 & $26.65(12)$ \\
Cl1-Rh & $2.3871(9)$ & O1-C(bridge)-CH & $165.9(3)$ \\
O2-O3 & $1.424(5)$ & O1-C(bridge)-CH $\mathrm{CH}_{3}$ & 94.73 \\
O4-Rh & $1.669(10)$ & & \\
\hline
\end{tabular}


chloride has a typical Rh-O bond length for rhodium dioxygen complexes (average $=2.017 \AA$ ), while the oxygen trans to the ether bridge is shorter than average with a length of 1.962(3) $\AA$. This shorter bond results from the low trans-influence of the ether oxygen. $\frac{252,253}{}$ Only three complexes with shorter Rh-O bonds in a dioxygen complex have been reported (Figure 4.10). ${ }^{254-256}$ These complexes have the dioxygen atoms trans to nitrogen or oxygen donor atoms, which are known to have low trans-influences. ${ }^{252,253}$

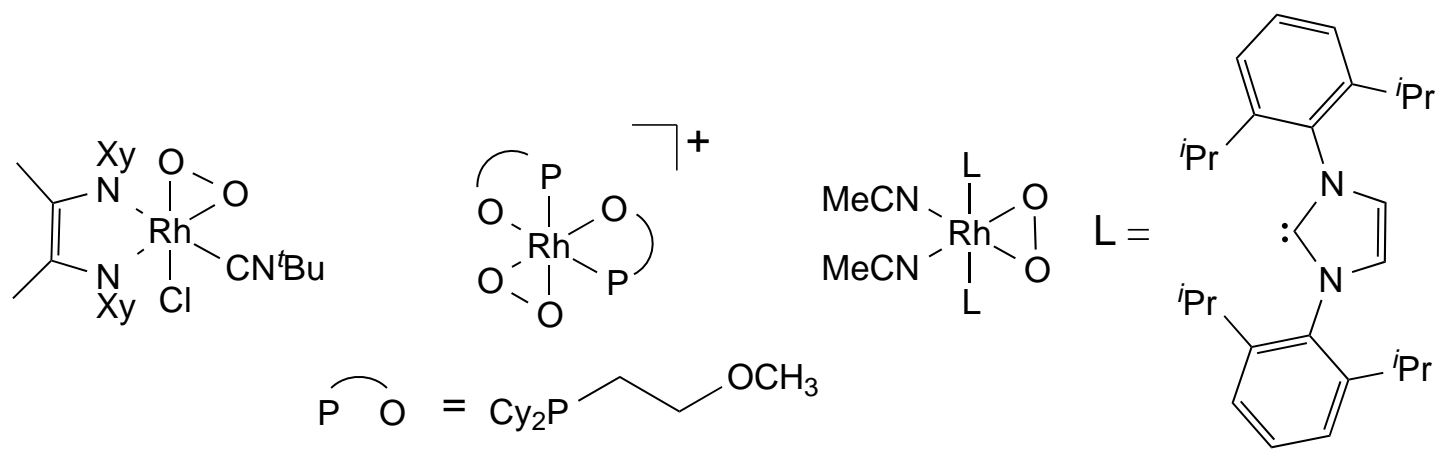

Figure 4.10: Rhodium dioxygen complexes with shorter $\mathrm{Rh}-\mathrm{O}$ bonds than $[\mathrm{Rh}(t-$ Bu-xantphos-k $\left.\left.P, O, P^{\prime}\right) \mathrm{Cl}\left(\eta^{2}-\mathrm{O}_{2}\right)\right]$.

The $\left[\mathrm{Rh}\left(t\right.\right.$-Bu-xantphos- $\left.\left.\mathrm{K} P, \mathrm{O}, \mathrm{P}^{\prime}\right) \mathrm{Cl}\left(\eta^{2}-\mathrm{O}_{2}\right)\right]$ complexes, with $t$-Bu-sixantphos or $t$ Bu-xantphos, show two distinct methyl environments in the ${ }^{1} \mathrm{H}$ and ${ }^{13} \mathrm{C}$ NMR spectra for the bridgehead methyls. This indicates a loss of symmetry, giving two distinct faces of the $t$-Bu-xantphos ligands. From the crystal structure (Figure 4.8) the chloride ligand sits on the same side as the concave face of the ligand while the dioxygen ligand occupies the position pseudo-trans to the oxygen bridge of the ligand. This geometry leaves more space trans to the chloride, resulting in the curvature of the ligand to occupy this free space and the tipping of the methyl groups away from the chloride. The angle $\mathrm{O} 1-\mathrm{C}($ bridge $)-\mathrm{CH}_{3}$ is very different for the two methyl groups; 165.9(3) and $94.7(3)^{\circ}$ for the concave and convex methyls, respectively and dihedral angles to the first $\mathrm{C}-\mathrm{H}$ in the aromatic ring of 24.8 and $42.8^{\circ}$. This positioning results in quite different chemical environments for the two methyl groups, resulting in the different chemical shifts in the NMR spectra.

Although rhodium xantphos complexes are well studied due to their high catalytic activity and selectivity for hydroformylation, to the best of our knowledge, 
no rhodium dioxygen complexes have been reported with any xantphos ligands. Only one transition metal complex with a xantphos related ligand and an $\eta^{2}-\mathrm{O}_{2}$ ligand has been reported; $\left[\mathrm{Ru}(\mathrm{Ph}-\mathrm{xantphos})\left(\mathrm{PPh}_{3}\right)\left(\eta^{2}-\mathrm{O}_{2}\right) \mathrm{H}\right]\left[\mathrm{BAr}_{4}^{\mathrm{F}}\right] .{ }^{257}$ This complex formed by reaction of $\left[\mathrm{Ru}(\mathrm{Ph}\right.$-xantphos $\left.)\left(\mathrm{PPh}_{3}\right) \mathrm{HCl}\right]$ with $\mathrm{Na}\left[\mathrm{BAr}_{4}^{\mathrm{F}}\right]$ followed by filtration then stirring in the air for 10 minutes. Similarly to the rhodium $t$ $\mathrm{Bu}$-xantphos complexes, the mer- $\kappa P, O, P^{\prime}$ coordination is retained upon reaction with oxygen. The dioxygen ligand occupies the site trans to the hydride, where the chloride had previously been located.

The X-ray crystal structure of $\left[\mathrm{Rh}(t\right.$-Bu-xantphos $\left.) \mathrm{Cl}\left(\eta^{2}-\mathrm{O}_{2}\right)\right]$ contains substitutional disorder with approximately $15 \%$ of the dioxygen molecules replaced by an oxo ligand (Figure 4.9). The oxo complex shows a slightly distorted squarebased pyramid structure with the ether bridge of the $t$-Bu-xantphos ligand occupying the apex, and trans coordination of the phosphorus atoms. The rhodium oxo bond (1.669(10) $\AA$ ) is much shorter than the rhodium oxygen bond lengths in $\left[\mathrm{Rh}(t\right.$-Bu-xantphos $\left.) \mathrm{Cl}\left(\eta^{2}-\mathrm{O}_{2}\right)\right]$, consistent with a higher bond order. In addition, the rhodium oxo bond is the shortest crystallographically determined Rh-O bond.

To the best of our knowledge, this is the first example of crystallographic evidence for a rhodium(III) oxo complex. Only one previous crystal structure has been reported for a rhodium oxo complex (Figure 4.11). ${ }^{258}$ The literature structure contains a rhodium(V) dimer with each rhodium atom coordinated to two oxo ligands, a DMSO ligand and an oxygen-nitrogen-sulfur heterotridentate ligand. The oxo trans to the DMSO ligand has a bond length of 1.701(5) $\AA$, while the oxo trans to the nitrogen donor is slightly longer at 1.712(5) $\AA$. Both of these are slightly longer than the Rh-O bond length in the [ $\mathrm{Rh}(t-\mathrm{Bu}$-xantphos $) \mathrm{Cl}(\mathrm{O})]$ structure $(1.669(10) \AA)$. The average bond length for a transition metal oxo is 1.689 $\AA$ with a lower quartile at $1.671 \AA .68$ This indicates that the bond length for the rhodium(III) oxo is in the lowest quarter of crystallographically determined bond lengths for transition metal oxo complexes; however, the bond is well within the range (1.106-2.956 ̊).

Transition metal oxo complexes have long been studied as they have been impli-

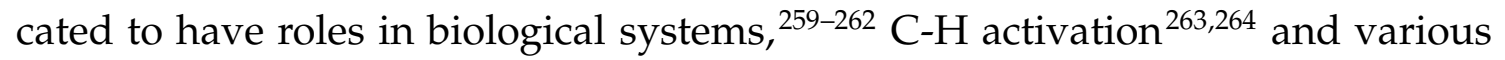
oxidation reactions. $\frac{219,265,266}{A}$ terminal oxo ligand is a very strong $\pi$-donor ligand, thus the strongest coordination occurs with high oxidation state, early tran- 


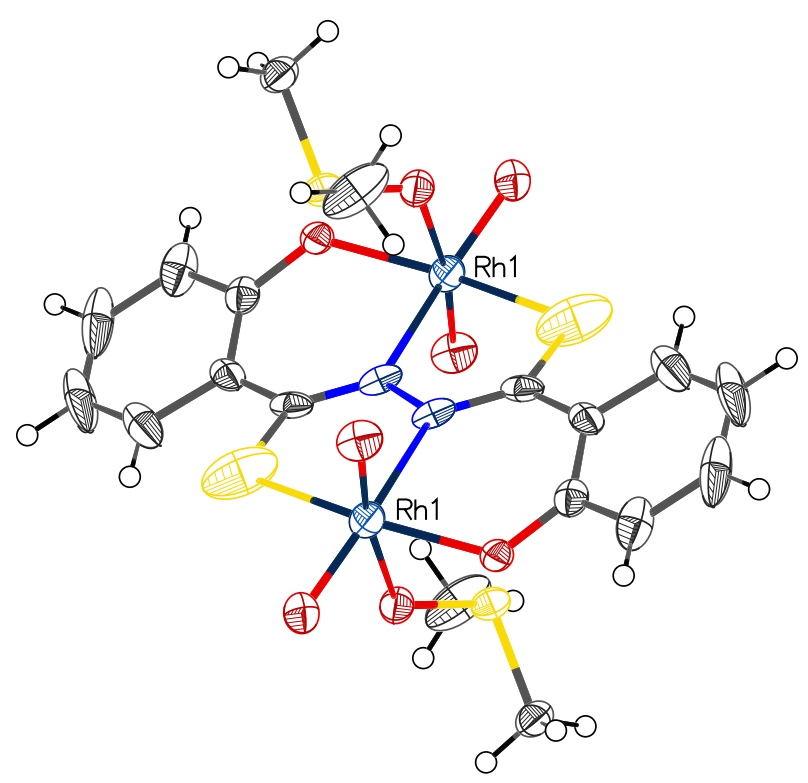

Figure 4.11: An X-ray crystal structure for the rhodium(V) oxo complex $\left[\left(\mathrm{RhO}_{2}\right)_{2}\left(\mathrm{C}_{6} \mathrm{H}_{4}(\mathrm{O})-\mathrm{C}(=\mathrm{S})-\mathrm{N}=\mathrm{N}-\mathrm{C}(=\mathrm{S})(\mathrm{O}) \mathrm{C}_{6} \mathrm{H}_{4}\right)(\mathrm{DMSO})_{2}\right] .258$

sition metals. $\frac{267}{}$ A review of transition metal oxo complexes published in 1987 stated that " $\mathrm{M}=\mathrm{O}$ groups are stabilized at metal centres with an oxidation state of no less than +4 and no more than four $d$ electrons". 265 This has led to the proposition of an "oxo-wall," a barrier between groups 8 and 9 of the periodic table, explaining the lack of tetragonal oxo complexes for group 9-11 transition metals. ${ }^{268}$ This effect arises as the orbital splitting in an octahedral oxo complex lowers the symmetry of the $t_{2 g}$ orbitals to $b_{2}\left(d_{x y}\right)$ and $e\left(d_{x z}, d_{y z}\right)$ and the metal-ligand antibonding $e_{g}$ orbitals to $b_{1}\left(d_{x^{2}-y^{2}}\right)$ and $a_{1}\left(d_{z^{2}}\right)$ with the $e\left(d_{x x}, d_{y z}\right)$ and $d_{z^{2}}$, orbitals destabilised. ${ }^{269}$ The more d-electrons that are present, the lower the bond order to the oxo, resulting in decreased stability. The $\left[\mathrm{Rh}\left(t\right.\right.$-Bu-xantphos- $\left.\left.\mathrm{K} P, \mathrm{O}, \mathrm{P}^{\prime}\right) \mathrm{Cl}(\mathrm{O})\right]$ complex is of square pyramidal geometry so does not violate the oxo-wall proposition. The poor electron donation from the central ether bridge may lead to increased stability compared to other square pyramidal structures.

Despite the instability of terminal oxo complexes of the late transition metals, some examples do exist (Figure 4.12). A platinum(II) complex with a PCN pincer ligand was reacted with a freshly prepared acetone solution of dioxirane, resulting in formation of a platinum(IV) oxo complex. ${ }^{266}$ This complex degrades over a period of 7-10 hours via an intramolecular oxygen transfer reaction from the 
platinum to the phosphorus atom. The oxo also readily reacts with water (forming a bishydroxy, aquo complex), hydrogen (liberating water), carbon monoxide (forming $\mathrm{CO}_{2}$ and a carbonyl complex), potassium hydride (to form a hydroxy complex) and oxidises triphenyl phosphine. This demonstrates a range of different potential uses for late transition metal oxo complexes. An iridium(V) oxo complex has also been reported, in this case the oxo complex was synthesised by reaction of $\left[\operatorname{Ir}(\mathrm{mes})_{3}\right]$ (mes = mesityl) with trimethylamine oxide (Figure 4.12). ${ }^{270}$ A rhodium oxo complex was recently reported by Caulton et al. from reaction of a rhodium(III) hydride complex containing a metalled PNP ligand reacting with trimethylamine oxide, pyridine $N$-oxide or $\mathrm{N}_{2} \mathrm{O} . \stackrel{249,271}{ }$ This complex undergoes oxo transfer reactions with trimethylphosphine and carbon monoxide.
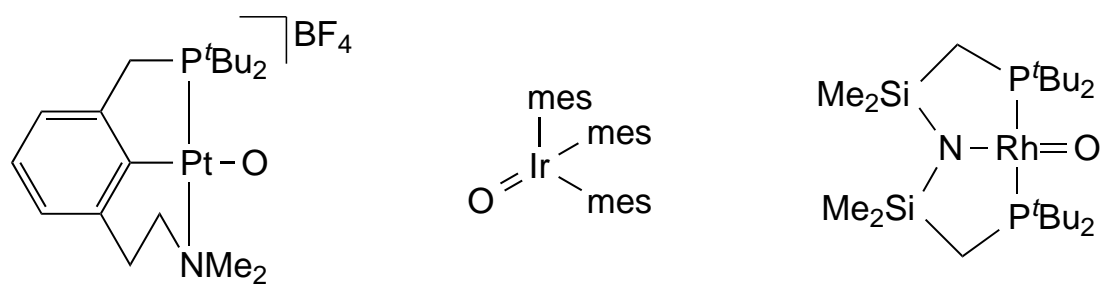

Figure 4.12: Previously reported late transition metal oxo complexes, mes $=2,4,6-$ trimethylphenyl. 249,266,271

Given the novelty of the proposed rhodium(III) oxo complex, the synthesis of $\left[\mathrm{Rh}\left(t\right.\right.$-Bu-xantphos- $\left.\left.\mathrm{B} P, \mathrm{O}, \mathrm{P}^{\prime}\right) \mathrm{Cl}(\mathrm{O})\right]$ complexes with all three $t$-Bu-xantphos ligands was attempted using trimethylamine oxide, as this has been used previously in the synthesis of late transition metal oxo complexes, $\stackrel{270,271}{=}$ and the by-product (trimethylamine) should be a poor ligand and result in little reactivity. Reaction between $\left[\mathrm{Rh}\left(t\right.\right.$-Bu-sixantphos- $\left.\left.\mathrm{K} P, \mathrm{O}, \mathrm{P}^{\prime}\right) \mathrm{Cl}\right]$ and trimethylamine oxide resulted in the immediate formation of the $\left[\mathrm{Rh}\left(t\right.\right.$-Bu-sixantphos- $\left.\left.\kappa P, O, P^{\prime}\right) \mathrm{Cl}\left(\eta^{2}-\mathrm{O}_{2}\right)\right]$ complex, together with a small amount of the ligand oxide and uncoordinated $t$-Bu-sixantphos, over time peaks due to the $\left[\mathrm{Rh}\left(t\right.\right.$-Bu-sixantphos- $\left.\left.\kappa P, O, P^{\prime}\right) \mathrm{Cl}\right]$ complex began to reappear. This result may be due to the incomplete dissolution of $[\mathrm{Rh}(t-\mathrm{Bu}-$ sixantphos- $\left.\left.\kappa P, O, P^{\prime}\right) C l\right]$ in the $\mathrm{CD}_{2} \mathrm{Cl}_{2}$ reaction solvent, meaning that the amount in solution was over-oxidised before the remainder dissolved. Unfortunately, as $\left[\mathrm{Rh}\left(t\right.\right.$-Bu-sixantphos- $\left.\left.\mathrm{k} P, O, P^{\prime}\right) \mathrm{Cl}\right]$ is a dark brown colour it is challenging to gauge full dissolution. After four days at room temperature a small amount of product was evident in the ${ }^{31} \mathrm{P}$ NMR spectrum at $70.5 \mathrm{ppm}\left(J_{\mathrm{RhP}}=114.1 \mathrm{~Hz}\right)$. 
Reacting $\left[\mathrm{Rh}\left(t\right.\right.$-Bu-thixantphos- $\left.\left.\kappa P, O, P^{\prime}\right) \mathrm{Cl}\right]$ and trimethylamine oxide resulted in a mixture of compounds. Analysis by ${ }^{31} \mathrm{P}$ NMR showed $22.9 \%$ free $t$-Bu-thixantphos ligand indicating possible degradation of the rhodium complexes formed,

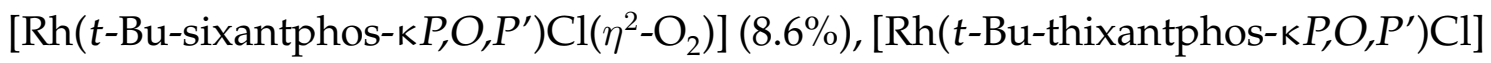
starting material $(64.4 \%)$, and a new complex at $40.9 \mathrm{ppm}\left(J_{\mathrm{RhP}}=90.4 \mathrm{~Hz}\right)(4.1 \%)$. After 10 days no [Rh( $t$-Bu-thixantphos- $\left.\left.\kappa P, O, P^{\prime}\right) \mathrm{Cl}\right]$ or unidentified complex remained and the reaction mixture was primarily the dioxygen complex and a further new complex at $74.1 \mathrm{ppm}\left(J_{\mathrm{RhP}}=116.3 \mathrm{~Hz}\right)$.

The reaction of $\left[\mathrm{Rh}\left(t-\mathrm{Bu}\right.\right.$-xantphos- $\left.\left.\mathrm{k} P, \mathrm{O}, \mathrm{P}^{\prime}\right) \mathrm{Cl}\right]$ with trimethylamine oxide was initially carried out at $-80{ }^{\circ} \mathrm{C}$ in order to prevent over-oxidation. However, initially the slow generation of the dioxygen species was observed with no evidence for any intermediate or otherwise. The reaction was allowed to warm to room temperature and after 24 hours a small signal was observed at $42.6 \mathrm{ppm}\left(J_{\mathrm{RhP}}\right.$ $=90.4 \mathrm{~Hz}$ ) comprising $4.2 \%$ of the reaction mixture. However, around $70.5 \%$ of the reaction mixture was the dioxygen complex and the remainder was the $\mathrm{Rh}(\mathrm{I})$ starting material. Similarly to the $t$-Bu-thixantphos reaction, this reaction slowly converted into a species at $75.1 \mathrm{ppm}\left(J_{\mathrm{RhP}}=116.3 \mathrm{~Hz}\right)$.

The initial unknown complexes formed by the oxidation of [Rh $(t-B u$-xantphos$\left.\left.\kappa P, O, P^{\prime}\right) \mathrm{Cl}\right]$ and $\left[\mathrm{Rh}\left(t\right.\right.$-Bu-thixantphos- $\left.\left.\kappa P, O, P^{\prime}\right) \mathrm{Cl}\right]$ had similar NMR spectra. In the ${ }^{31} \mathrm{P}$ NMR spectra a doublet was observed at 40.9 or $42.6 \mathrm{ppm}$, slightly downfield of the dioxygen complex. The values of $J_{\mathrm{RhP}}$ were identical with either ligand $(90.4 \mathrm{~Hz})$. The decrease from the values in the rhodium(I) starting materials (142.3 and $140.0 \mathrm{~Hz}$ for $t$-Bu-xantphos and $t$-Bu-thixantphos respectively) clearly indicates oxidation to a rhodium(III) species. ESI - Mass spectrometry was performed on the reaction mixtures for all three $t$-Bu-xantphos ligands. In all cases a molecular ion peak was observed consistent in both mass/charge ratio and isotopic pattern, indicative of an oxo complex ionising via loss of a chloride ligand.

Late transition metal oxo complexes are generally considered unstable and likely to undergo further reaction. The reactions of $\left[\mathrm{Rh}\left(t-\mathrm{Bu}\right.\right.$-xantphos- $\left.\left.\kappa P, O, P^{\prime}\right) \mathrm{Cl}\right]$ with trimethylamine oxide were performed in $\mathrm{CD}_{2} \mathrm{Cl}_{2}$. As previously discussed (see Section 2.3) halocarbon molecules are not stable for extended periods of time in the light, undergoing a photo-catalysed degradation forming small amounts of hydrochloric acid (deuterium chloride in the case of NMR solvents). $\frac{138}{}$ Hereby we propose that the oxo complex likely reacted with the small amounts of deu- 


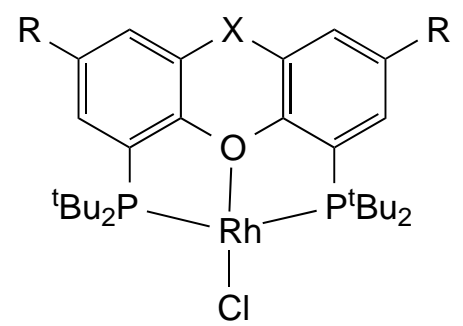

\section{(i)}

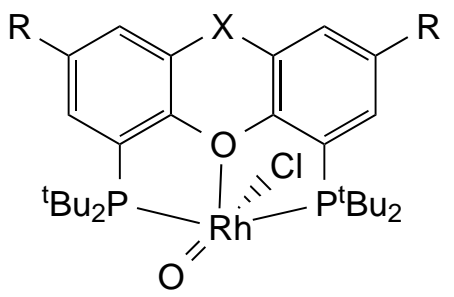

Scheme 4.8: Reaction of [ $\mathrm{Rh}(t$-Bu-xantphos $) \mathrm{Cl}]$ with trimethylamine oxide. Reagents and conditions: 1eq. $\mathrm{ONMe}_{3}, \mathrm{CD}_{2} \mathrm{Cl}_{2}$.

terium chloride that formed over time, thus producing a hydroxy ligand (Scheme 4.9). The remaining coordination site on the metal would then likely be occupied by the chloride ion. The platinum oxo complex reported by Milstein et al. underwent reaction with water to form a bishydroxy complex while the rhodium oxo reported by Caulton et al. underwent metallation of one of the tert-butyl substituents on the phosphorus donor also forming a hydroxy ligand. ${ }^{249}$<smiles></smiles>

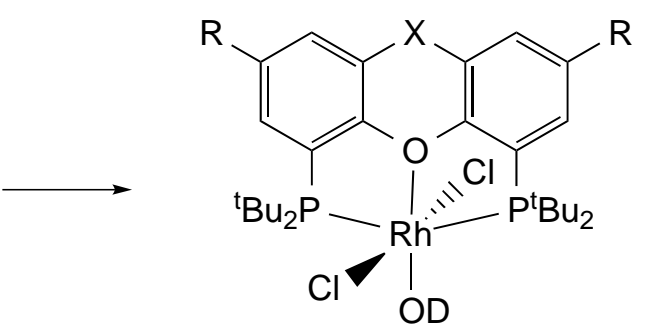

Scheme 4.9: Proposed reaction of $[\mathrm{Rh}(t-\mathrm{Bu}-x a n t p h o s) \mathrm{Cl}(\mathrm{O})]$ with $\mathrm{DCl}$.

Unfortunately, due to time constraints, the formation of the rhodium oxo complexes was not studied in more depth.

\subsection{Summary}

The coordination chemistry of $t$-Bu-sixantphos, $t$-Bu-thixantphos, and $t$-Bu-xantphos with rhodium in both the +1 and +3 oxidation states was explored. The three ligands reacted readily with $\left[\mathrm{Rh}(\mathrm{coe}){ }_{2} \mathrm{Cl}\right]_{2}$ forming $\left[\mathrm{Rh}\left(t\right.\right.$-Bu-xantphos- $\left.\left.\kappa P, O, P^{\prime}\right) \mathrm{Cl}\right]$ 
complexes. These complexes readily split hydrogen to form [Rh(t-Bu-xantphos$\left.\left.\mathrm{k} P, \mathrm{O}, \mathrm{P}^{\prime}\right) \mathrm{Cl}(\mathrm{H})_{2}\right]$ complexes with a meridional $t$-Bu-xantphos ligand and cis hydrides. Little difference in the position and coupling constants of the hydride trans to the chloride was observed in the ${ }^{1} \mathrm{H}$ NMR spectra of the three complexes. The values of $J_{\mathrm{RhH}}$ for the hydride trans to the oxygen donor decreased with increasing bite-angle. Suggesting a trans influence series of $t$-Bu-xantphos $>t$-Buthixantphos $>t$-Bu-sixantphos. This difference may be related to the ease with which the ligands form wide bite-angle complexes.

The three $\left[\mathrm{Rh}\left(t\right.\right.$-Bu-xantphos- $\left.\left.\kappa P, O, P^{\prime}\right) \mathrm{Cl}\right]$ complexes were reactive towards carbon monoxide, forming [ $\mathrm{Rh}(t-\mathrm{Bu}$-xantphosk-P,P' $\left.)(\mathrm{CO})_{2} \mathrm{Cl}\right]$. In contrast, Ph-xantphos and DPEphos formed trans-[Rh(CO)Cl(diphosphine)] complexes. The $t$-Buxantphos ligands were coordinated in a $k-P, P^{\prime}$ bisequatorial mode with an equatorial chloride and two axial carbonyl ligands. This shows a clear difference in the reactivity of the $t$-Bu-xantphos ligands when compared with Ph-xantphos.

The rhodium dioxygen complexes, $\left[\mathrm{Rh}(t\right.$-Bu-xantphos $\left.) \mathrm{Cl}\left(\eta^{2}-\mathrm{O}_{2}\right)\right]$ with the $t$-Buxantphos ligands were synthesised by reaction of [ $\mathrm{Rh}\left(t\right.$-Bu-xantphos-k $\left.\left.P, O, P^{\prime}\right) \mathrm{Cl}\right]$ with the air. These are the first rhodium dioxygen complexes with xantphos ligands and only the second with any transition-metals. The X-ray crystal structure of $\left[\mathrm{Rh}(t-\mathrm{Bu}\right.$-xantphos $\left.) \mathrm{Cl}\left(\eta^{2}-\mathrm{O}_{2}\right)\right]$ was reported showing a distorted octahedral configuration with a meridional coordination of the $t$-Bu-xantphos ligand. The O-O bond length was typical for rhodium dioxygen complexes. The Rh-O bond length for the oxygen trans to the oxygen donor of the $t$-Bu-xantphos ligand was the fourth shortest Rh-O length for a dioxygen complex that have been reported, indicating the low trans-influence of the $t$-Bu-xantphos oxygen.

The X-ray crystal structure of $\left[\mathrm{Rh}(t\right.$-Bu-xantphos $\left.) \mathrm{Cl}\left(\eta^{2}-\mathrm{O}_{2}\right)\right]$ was disordered with the dioxygen ligand replaced in around $15 \%$ of sites by an oxo ligand. To the best of our knowledge this is the first crystallographic evidence of a rhodium(III) oxo complex. The Rh-O bond length was 1.669(10) $\AA$ which is the shortest reported rhodium oxo bond length. The complex is a distorted square pyramid, so does not violate the "oxo-wall" theory. Attempts to synthesise [Rh(t-Bu-xantphos- $\left.\left.\kappa P, O, P^{\prime}\right) \mathrm{Cl}(\mathrm{O})\right]$ by reaction of $\left[\mathrm{Rh}\left(t\right.\right.$-Bu-xantphos- $\left.\left.\kappa P, O, P^{\prime}\right) \mathrm{Cl}\right]$ with trimethylamine oxide were promising for $t$-Bu-thixantphos and $t$-Bu-xantphos, showing a new peak in the ${ }^{31} \mathrm{P}$ NMR spectra, which slowly converted into a different species, and mass spectra consistent with $\left[\mathrm{Rh}\left(t-\mathrm{Bu} \text {-xantphos- } \mathrm{K} P, \mathrm{O}, \mathrm{P}^{\prime}\right)(\mathrm{O})\right]^{+}$. 


\section{Chapter 5}

\section{Coordination Complexes with Platinum(0) and Palladium(0)}

Coordination complexes of palladium and platinum have a range of uses. Perhaps the most well-known platinum complex is $\left[\mathrm{PtCl}_{2}\left(\mathrm{NH}_{3}\right)_{2}\right]$ (cisplatin). Cisplatin, distributed under the trade name Platinol, is a widely used chemotherapeutic which is used in conjunction with other therapies for the treatment of various cancers, most commonly tumours including sarcomas, carcinomas and lymphomas. $\frac{2,3}{3}$ A further two platinum complexes (carboplatin and oxaliplatin) have been approved for use in chemotherapy worldwide, with other complexes approved in select countries.

Palladium complexes are some of the most common coordination complexes used for homogeneous catalysis, with applications in a wide-range of reactions including cross-coupling, allylic alkylation and various carbonylation reactions among many others. $\underline{6,32,149,273}-276$ These transformations are highly important for industrial processes and laboratory scale total syntheses. $.277-279$ The high reactivity of these species can make palladium complexes and their catalytic processes difficult to study. Platinum complexes are typically more inert than their corresponding palladium analogues to processes such as ligand exchange and redox changes. $\frac{280}{}$ The spin active isotope platinum-195 (34\% abundant) can result in platinum coupling in the ${ }^{1} \mathrm{H},{ }^{13} \mathrm{C}$, and ${ }^{31} \mathrm{P}$ NMR spectra, the coupling constants of which, can give additional information regarding the geometry and bonding in the complex. Platinum and palladium are otherwise very similar metals, which makes the additional insight gained from platinum models valuable when exam- 
ining similar chemistry with palladium.

Prior to the start of this work four studies had been published investigating the catalytic activity of $t$-Bu-xantphos. $\stackrel{84-87}{ }$ Two of these studies investigated the catalytic activity of $t$-Bu-xantphos palladium systems and one investigated platinum systems. In all cases little to no reactivity was observed with $t$-Bu-xantphos systems, whereas the corresponding Ph-xantphos reactions gave $40-99 \%$ conversions. This suggests differences in the coordination chemistry of the two ligands towards palladium and platinum. However, no studies have investigated the coordination chemistry of $t$-Bu-xantphos with either palladium or platinum, in order to explain the different activities. A crystal structure of $[\mathrm{Pd}(t-\mathrm{Bu}-x a n t-$ phos) $\mathrm{Cl}_{2}$ ] was reported to the CSD in 2011 (CSD-XARXAR). $\underline{68}$ The crystal structure has three molecules in the asymmetric unit with bite-angles of 160.59(4), 161.97(3) and $161.31(4)^{\circ}$, compared to $100.61(5)$ and $100.92(7)^{\circ}$ for $[\mathrm{Pd}(\mathrm{Ph}-x a n t-$ phos) $\mathrm{Cl}_{2}$ ] with different solvates. $.68,145,281$ The difference in the bite-angles and geometries of the complexes that form may explain the difference in the catalytic reactivity of the complexes.

Since the start of this work, five further papers have been published investigating the catalytic activity of palladium complexes with $t$-Bu-xantphos. $\underline{93-95,97,98}$ In the palladium-catalysed Suzuki cross-coupling and the hydroesterification of methyl oleate little activity was observed with $t$-Bu-xantphos, whereas the Ph-xantphos complexes gave high yields. ${ }^{97,98}$ However, in the $N$-alkylation of amines with primary and secondary alcohols, the $t$-Bu-xantphos system was more active than the $\mathrm{Ph}$-xantphos system, with $t$-Bu-xantphos showing near quantitative conversion at $100{ }^{\circ} \mathrm{C}$, while the $\mathrm{Ph}$-xantphos system gave only $63 \%$ conversion at $110{ }^{\circ} \mathrm{C}$. Similarly, the methylation of alkynyl C-H bonds with dimethyl sulfonium ylides using a palladium complex with $t$-Bu-xantphos went to $46 \%$ conversion, while Phxantphos gave only $15 \% .95$ A palladium complex with a monodentate Ph-xantphos or $t$-Bu-xantphos ligand was tested in the aminocarbonylation of heteroaryl bromides, and showed no reactivity with $t$-Bu-xantphos while $\mathrm{Ph}$-xantphos gave a $92 \%$ isolated yield. ${ }^{93}$ Together these studies support the premise that the coordination chemistry of $t$-Bu-xantphos and $\mathrm{Ph}$-xantphos are distinct, likely as a result of the differences in the bite-angles and steric bulk between the ligands. However, no research into this area has yet been reported.

The coordination chemistry of Ph-xantphos with palladium has been the subject 
of a number of different studies, $\underline{\underline{73}-75,79,115,282,283}$ though no research into the coordination chemistry of the $t$-Bu-xantphos ligands with platinum has been reported. The main focus of this chapter is the investigation into the coordination chemistry of the three $t$-Bu-xantphos ligands with a range of platinum( 0$)$ and palladium( $(0)$ precursors. Some reactivity of the resulting complexes towards small molecules is also presented. A brief investigation into the coordination chemistry of Phthixantphos with platinum(0) will also be presented for comparative purposes. The subsequent chapter will address the coordination chemistry of the $t$-Bu-xantphos ligands with platinum(II) and palladium(II) starting materials.

\subsection{Reactions of Ph-thixantphos with Platinum(0) Precursors}

The 1:1 reaction between $\left[\mathrm{Pt}(\mathrm{nb})_{3}\right]$ ( $\mathrm{nb}=$ norbornene, bicyclo[2.2.1]hept-2-ene) and $\mathrm{Ph}$-thixantphos in $\mathrm{C}_{6} \mathrm{D}_{6}$ rapidly formed two products but did not progress further. The ${ }^{31} \mathrm{P}$ NMR spectrum shows one sharp peak at $20.0 \mathrm{ppm}\left(J_{\mathrm{PtP}}=3470\right.$ $\mathrm{Hz}, 39.6 \%)$ and two overlapping broad singlets ranging from 0 to $-10 \mathrm{ppm}(60.4 \%)$. The platinum-phosphorus coupling constant on the sharp signal is consistent with a mono-alkene complex such as, [Pt(nb)(Ph-thixantphos)]. ${ }^{284}$ The ${ }^{1} \mathrm{H}$ NMR spectrum showed a number of aromatic signals together with peaks that can be ascribed to $\left[\mathrm{Pt}(\mathrm{nb})_{3}\right]$ and uncoordinated norbornene, as well as new alkyl and alkene proton signals. This suggests that the complex appearing at $20.0 \mathrm{ppm}$ in the ${ }^{31} \mathrm{P}$ NMR spectrum is [ $\mathrm{Pt}(\mathrm{nb})(\mathrm{Ph}$-thixantphos)], while the other complex has more than one molecule of Ph-thixantphos per platinum atom. Attempts to synthesise $[\mathrm{Pt}(\mathrm{nb})(\mathrm{Ph}$-thixantphos)] exclusively were unsuccessful, as were attempts to isolate the complex from the product mixture. As such, this species was not characterised fully.

The reaction between $\mathrm{Ph}$-thixantphos and $\left[\mathrm{Pt}(\mathrm{nb})_{3}\right]$ was repeated using two equivalents of Ph-thixantphos per platinum. In this case only the broad species between 0 and -10 ppm was observed by ${ }^{31} \mathrm{P}$ NMR spectroscopy. Upon removal of the displaced norbornene from the system, the ${ }^{1} \mathrm{H}$ NMR spectrum displayed only aromatic signals. This indicates that the complex formed under these conditions does not contain an alkene ligand, nor is there any evidence for uncoordinated 
Ph-thixantphos ligand. This is consistent with the formation of [ $\mathrm{Pt}(\mathrm{Ph}$-thixantphos) $)_{2}$ ] (Scheme 5.1).
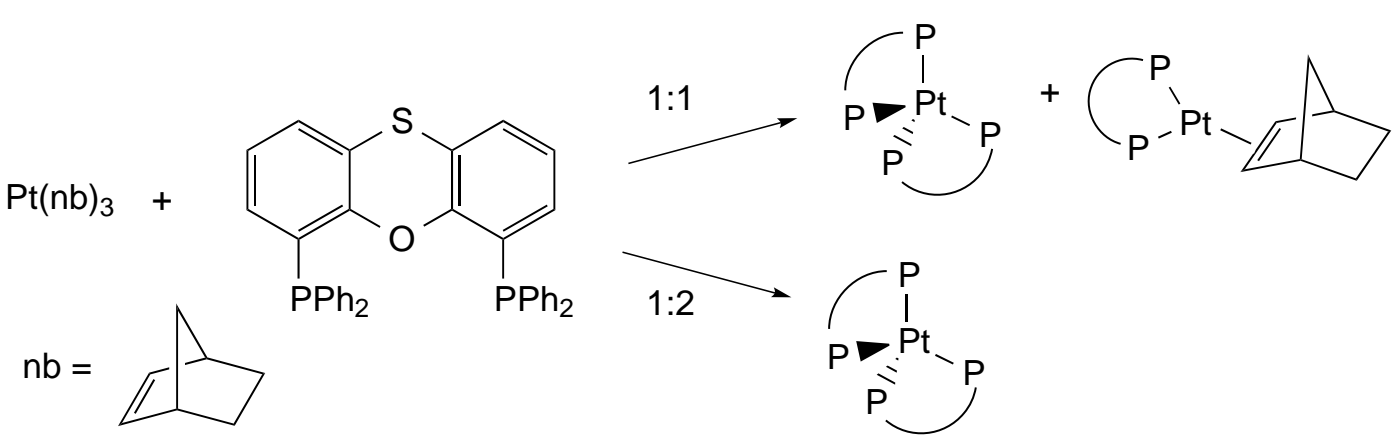

Scheme 5.1: Reaction of Ph-thixantphos with tris(norbornene)platinum.

Yellow crystals of $\left[\mathrm{Pt}(\mathrm{Ph} \text {-thixantphos) })_{2}\right] \cdot 2.5 \mathrm{CH}_{2} \mathrm{Cl}_{2}$ were grown by inwards diffusion of diethyl ether into a dichloromethane solution of the complex. The complex crystallised in the monoclinic space group $C 2 / c$ with a well-defined main structure, though some disorder was present in the dichloromethane solvate. The crystal structure is shown in Figure 5.1, with Figure 5.2 showing the same structure without the phenyl substituents on the phosphorus atoms. Selected bond lengths and angles are given in Table 5.1, and crystallographic information is given in Table 5.2 .

The X-ray crystal structure of $\left[\mathrm{Pt}(\mathrm{Ph} \text {-thixantphos })_{2}\right]$ shows a distorted tetrahedral configuration with angles ranging from $103.741(18)$ to $119.313(17)^{\circ}$. This is likely due to the restrictions caused by having two rigid $\mathrm{Ph}$-xantphos ligands around a single metal centre. There are two different phosphorus environments present in the crystal structure, such that each ligand has each of the two environments. One phosphine on each ligand is nearer to the adjacent ligand backbone whilst the other phosphine is nearer to the adjacent phenyl substituents. The backbone is bent away from planarity to allow the ligand to coordinate with bite angles of $106.377(16)$ and $108.059(17)^{\circ}$. These angles are close to the natural bite-angle calculated for this ligand of $109.4^{\circ}$ from molecular mechanics and are slightly smaller than the DFT calculated value of $112.65^{\circ} .67$

Tetrahedral complexes of platinum with four phosphorus donors are well-known with numerous crystallographically determined structures. $\frac{68}{}$ One such structure with a xantphos ligand derivatised with ethyl groups on the phosphorus atoms was reported in 2004 (Figure [5.3a).$\underline{74}$ The [Pt(Et-xantphos) ${ }_{2}$ ] complex shows a 


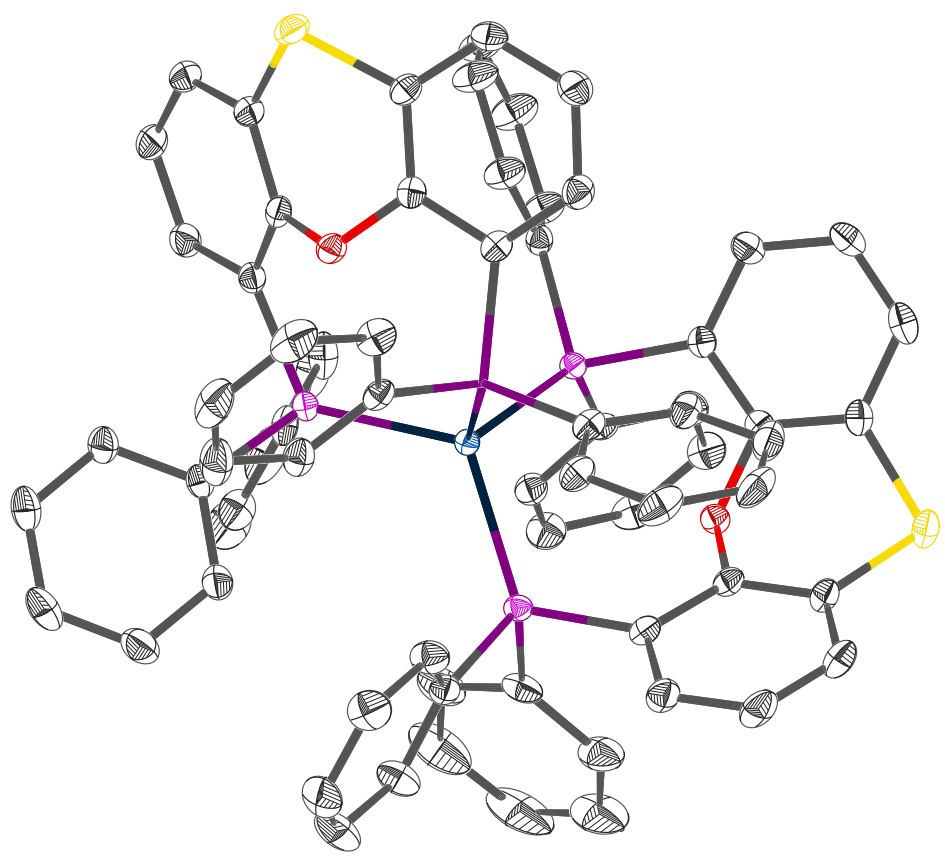

Figure 5.1: X-ray crystal structure of $\left[\mathrm{Pt}(\mathrm{Ph} \text {-thixantphos })_{2}\right]$ (50\% probability thermal ellipsoids). Hydrogen atoms and dichloromethane solvate omitted for clarity.

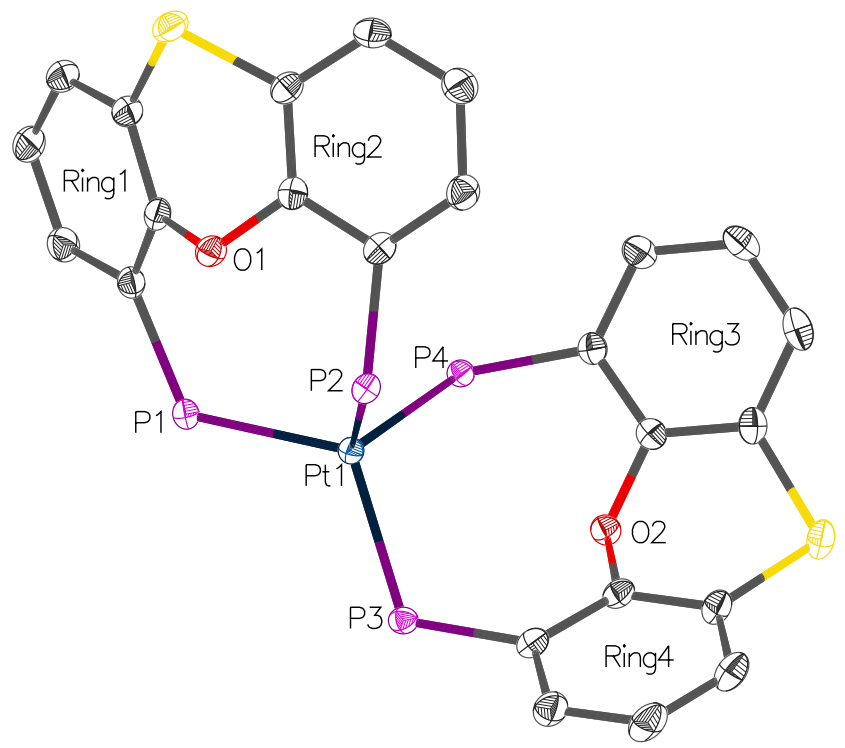

Figure 5.2: X-ray crystal structure of $\left[\mathrm{Pt}(\mathrm{Ph} \text {-thixantphos })_{2}\right]$ (50\% probability thermal ellipsoids). Hydrogen atoms, dichloromethane solvate and phenyl rings omitted for clarity. 
Table 5.1: Selected bond distances $(\AA)$ and angles $\left(^{\circ}\right)$ of $\left[\mathrm{Pt}(\mathrm{Ph} \text {-thixantphos })_{2}\right]$.

\begin{tabular}{llll}
\hline \multicolumn{2}{c}{ Bond distances $(\AA)$} & \multicolumn{2}{c}{ Bond angles $\left({ }^{\circ}\right)$} \\
\hline P1-Pt1 & $2.3325(6)$ & P1-Pt1-P2 & $108.059(17)$ \\
P2-Pt1 & $2.3209(5)$ & P1-Pt1-P3 & $105.501(17)$ \\
P3-Pt1 & $2.3242(4)$ & P1-Pt1-P4 & $119.313(17)$ \\
P4-Pt1 & $2.3249(5)$ & P2-Pt1-P3 & $114.224(19)$ \\
P1..P2 & $3.7661(7)$ & P2-Pt1-P4 & $103.741(18)$ \\
P1..P3 & $3.7068(7)$ & P3-Pt1-P4 & $106.377(16)$ \\
P1..P4 & $4.0194(7)$ & Ring 1-Ring 2 & $35.57(8)$ \\
P2..P3 & $3.9007(7)$ & Ring 3-Ring 4 & $45.39(8)$ \\
P2...P4 & $3.6544(7)$ & & \\
P3..P4 & $3.7221(6)$ & & \\
Pt1...O1 & $3.4977(14)$ & & \\
Pt1...O2 & $3.5635(14)$ & & \\
\hline
\end{tabular}

similar structure to $\left[\mathrm{Pt}(\mathrm{Ph} \text {-thixantphos })_{2}\right]$. The bite-angles for the ethyl complex are $108.07(7)$ and $108.44(7)^{\circ}$, which are close to the Ph-thixantphos complex of 108.059(17) and $106.377(16)^{\circ}$. The P-Pt bond distances are slightly shorter for $\left[\mathrm{Pt}(\text { Et-xantphos })_{2}\right](2.312(2), 2.3119(16), 2.2916(17)$ and $2.289(2) \AA)$ than for $[\mathrm{Pt}(\mathrm{Ph}-$ thixantphos) ${ }_{2}$ ] (2.3325(6), 2.3209(5), 2.3242(4) and 2.3249(5) $\AA$ ) this is likely the result of the higher electron donation of ethyl substituents compared to phenyl rings. The bite-angles are also very similar to a $\left[\mathrm{Pd}\left(\mathrm{Ph}\right.\right.$-xantphos) $\left.{ }_{2}\right]$ complex (Figure 5.3b), which has P-Pd-P angles of $106.34(2)^{\circ} .285$ Given these similarities it is likely that the geometry of the complexes and the bite-angles of the ligands in these complexes are controlled primarily by valence angles.

The X-ray crystal structure of $\left[\mathrm{Pt}(\mathrm{Ph} \text {-thixantphos })_{2}\right]$ shows two different phosphorus environments for each Ph-thixantphos ligand (Figure 5.2). The backbone of the Ph-thixantphos ligand is bent resulting in one of the phosphorus atoms on one of the ligands sitting within the concave face of the other ligand's backbone while the other phosphorus atom sits away from the ligand backbone and has closer contacts with the phenyl substituents than the backbone. This indicates that while the two ligands are related overall, the two halves of a single ligand are in quite different spatial environments. 
Table 5.2: Crystallographic data and structure refinement of $[\mathrm{Pt}(\mathrm{Ph}-$ thixantphos) ${ }_{2}$ ].

\begin{tabular}{|c|c|}
\hline Empirical formula & $\mathrm{C}_{149} \mathrm{H}_{114} \mathrm{Cl}_{10} \mathrm{O}_{4} \mathrm{P}_{8} \mathrm{Pt}_{2} \mathrm{~S}_{4}$ \\
\hline Formula weight & 3089.07 \\
\hline Temperature/K & 296.0 \\
\hline Crystal system & monoclinic \\
\hline Space group & $\mathrm{C} 2 / \mathrm{c}$ \\
\hline $\mathrm{a} / \AA$ & $46.8743(16)$ \\
\hline $\mathrm{b} / \AA$ & $13.1000(4)$ \\
\hline$c / \AA$ & $21.7852(7)$ \\
\hline$\alpha /^{\circ}$ & 90 \\
\hline$\beta /{ }^{\circ}$ & $105.208(2)$ \\
\hline$\gamma /{ }^{\circ}$ & 90 \\
\hline Volume $/ \AA^{3}$ & $12908.8(7)$ \\
\hline Z & 4 \\
\hline$\rho_{\text {calc }} \mathrm{mg} / \mathrm{mm}^{3}$ & 1.589 \\
\hline$\mu / \mathrm{mm}$ & 2.594 \\
\hline $\mathrm{F}(000)$ & 6200.0 \\
\hline Crystal size $/ \mathrm{mm}$ & $0.61 \times 0.18 \times 0.15$ \\
\hline Radiation & $\operatorname{MoK} \alpha(\lambda=0.71073)$ \\
\hline $2 \theta$ range for data collection & 3.602 to $60.916^{\circ}$ \\
\hline Index ranges & $-66 \leq \mathrm{h} \leq 66,-18 \leq \mathrm{k} \leq 17,-30 \leq 1 \leq 30$ \\
\hline Reflections collected & 172570 \\
\hline Independent reflections & $19386\left[R_{\text {int }}=0.0370, R_{\text {sigma }}=0.0207\right]$ \\
\hline Data/restraints/parameters & $19386 / 0 / 808$ \\
\hline Goodness-of-fit on $\mathrm{F}^{2}$ & 1.232 \\
\hline Final $\mathrm{R}$ indexes $[\mathrm{I}>=2 \sigma(\mathrm{I})]$ & $\mathrm{R}_{1}=0.0219, \mathrm{w} \mathrm{R}_{2}=0.0565$ \\
\hline Final $\mathrm{R}$ indexes [all data] & $\mathrm{R}_{1}=0.0338, \mathrm{w} \mathrm{R}_{2}=0.0716$ \\
\hline Largest diff. peak/hole / e $\AA^{-3}$ & $1.08 /-1.07$ \\
\hline
\end{tabular}




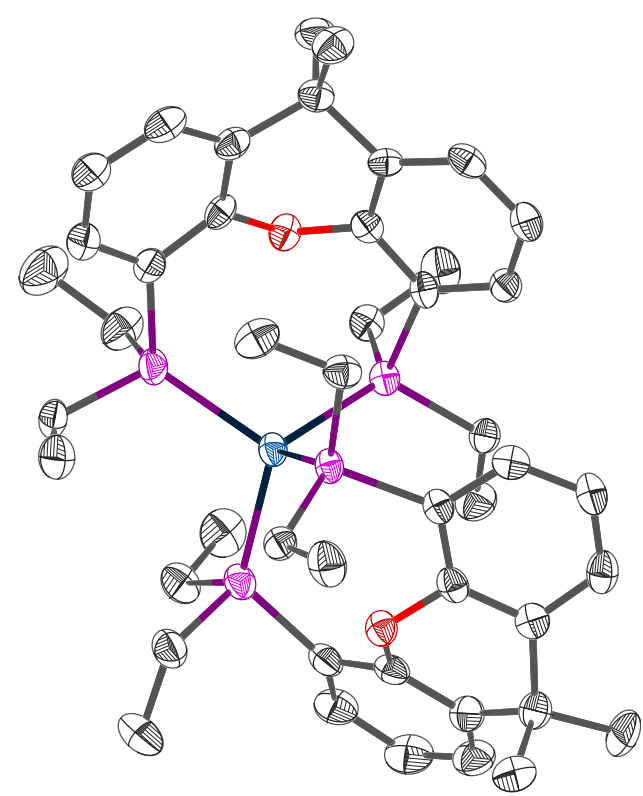

(a)

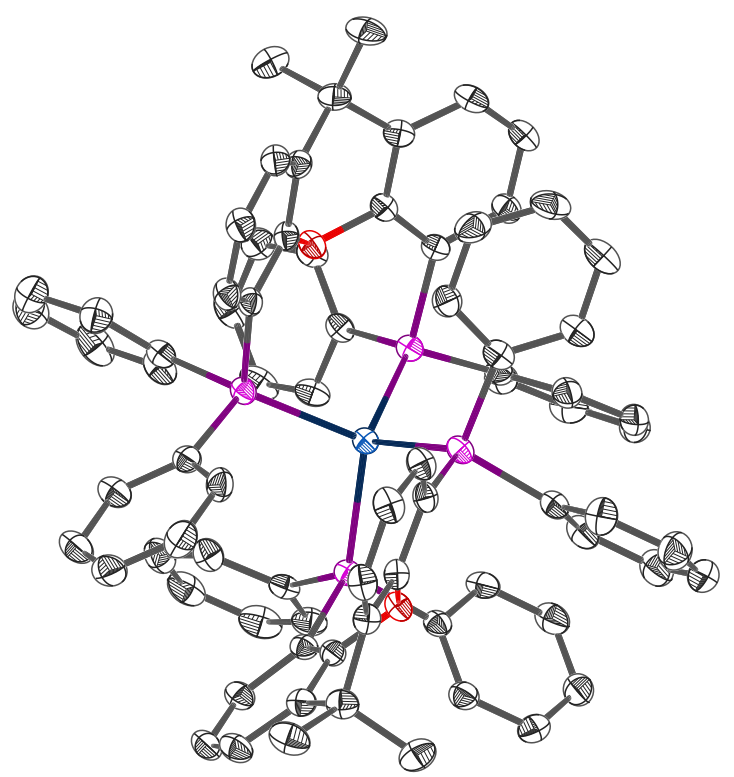

(b)

Figure 5.3: X-ray crystal structures of $\left[\mathrm{Pt}(\text { Et-xantphos })_{2}\right]^{285}$ (a) and $[\mathrm{Pd}(\mathrm{Ph}-\mathrm{xant}-$ phos $\left.)_{2}\right]^{74}(b)$.

From the X-ray crystal structure of $\left[\mathrm{Pt}(\mathrm{Ph} \text {-thixantphos })_{2}\right]$, we would expect the ${ }^{31} \mathrm{P}$ NMR spectrum to show two different phosphorus environments. At room temperature both the ${ }^{1} \mathrm{H}$ and ${ }^{31} \mathrm{P}$ NMR spectra are very broad. While two environments are observed in the ${ }^{31} \mathrm{P}$ NMR spectrum, no coupling can be readily discerned, suggesting that a dynamic process is occurring. This behaviour was investigated using variable temperature ${ }^{1} \mathrm{H}$ and ${ }^{31} \mathrm{P} \mathrm{NMR}$ analysis in $\mathrm{CD}_{2} \mathrm{Cl}_{2}$ (Figures 5.4 and 5.5). Lowering the temperature to $0{ }^{\circ} \mathrm{C}$ causes the ${ }^{31} \mathrm{P} \mathrm{NMR}$ spectrum to sharpen with phosphorus coupling and platinum satellites resolved. The ${ }^{31} \mathrm{P}$ NMR spectrum continues to sharpen down to around $-40{ }^{\circ} \mathrm{C}$, below which no further changes are observed. The low temperature ${ }^{1} \mathrm{H}$ NMR spectra show a similar pattern: sharpening upon cooling to $0{ }^{\circ} \mathrm{C}$, which continues down to $-40{ }^{\circ} \mathrm{C}$. However, unlike the ${ }^{31} \mathrm{P}$ NMR spectra, the ${ }^{1} \mathrm{H}$ NMR spectra at -60 and $-80{ }^{\circ} \mathrm{C}$ begin to broaden once more.

The xantphos backbones frequently bend in order to achieve the coordination angles desired by the metal. However, while this is seen in the crystal structures of the complexes, the effects are not observed in the NMR spectra due to rapid 
5.1. Reactions of Ph-thixantphos with Platinum(0)

Precursors

$20^{\circ} \mathrm{C}$

H.t.m.

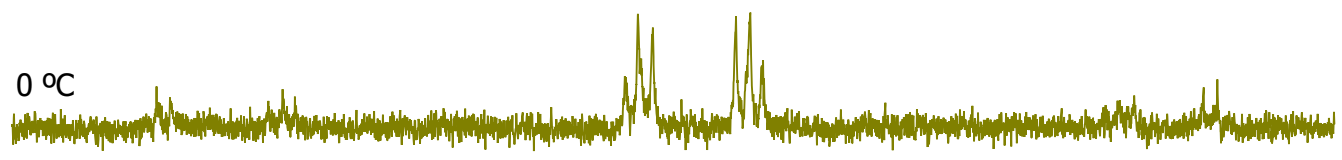

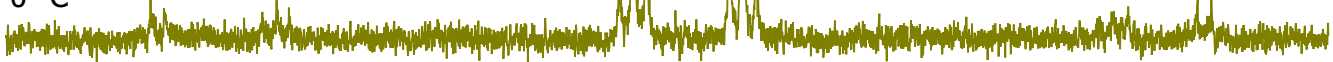
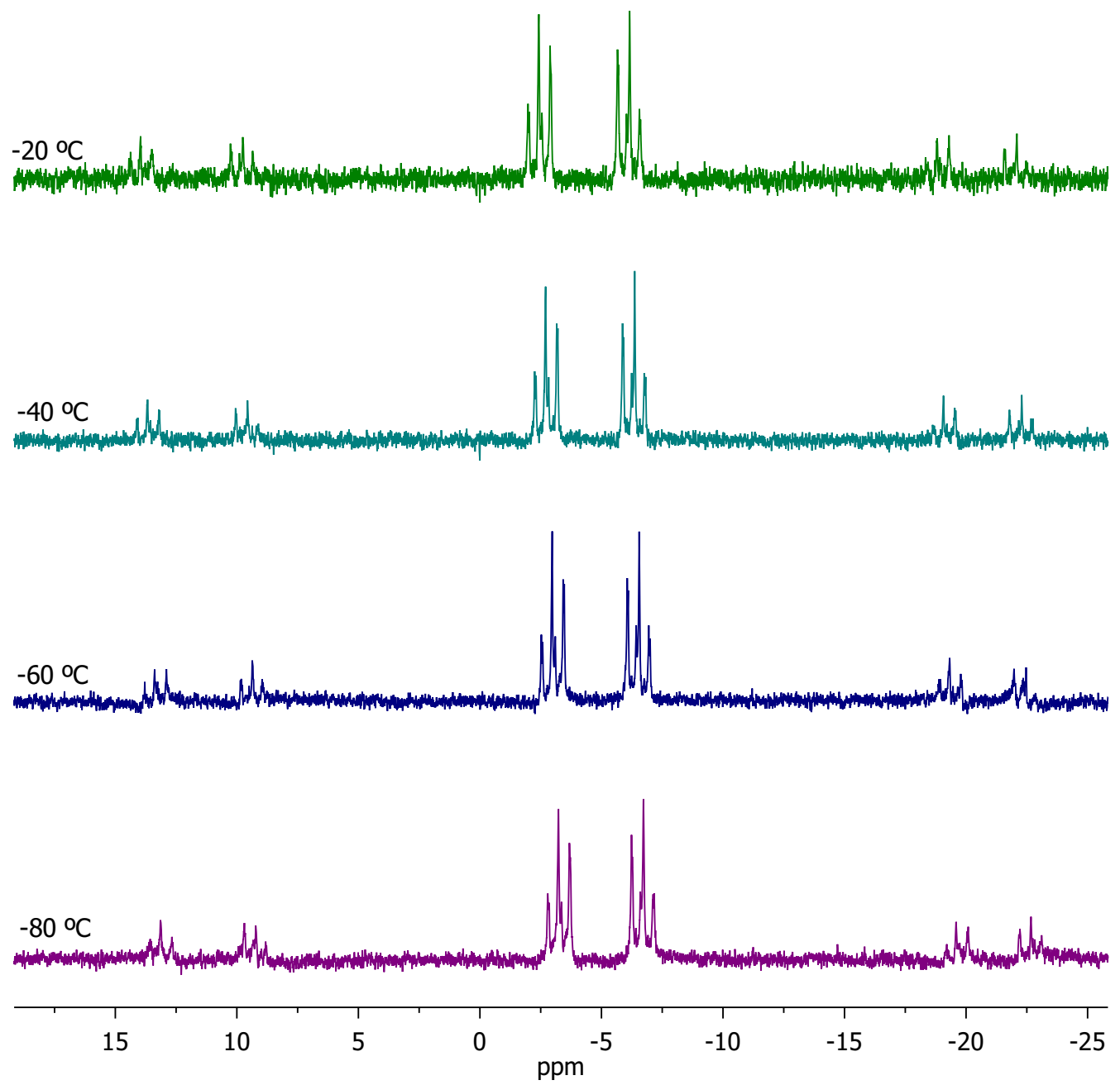

Figure 5.4: Low temperature ${ }^{31} \mathrm{P}$ NMR spectra of $\left[\mathrm{Pt}(\mathrm{Ph} \text {-thixantphos })_{2}\right]$ in $\mathrm{CD}_{2} \mathrm{Cl}_{2}$. 

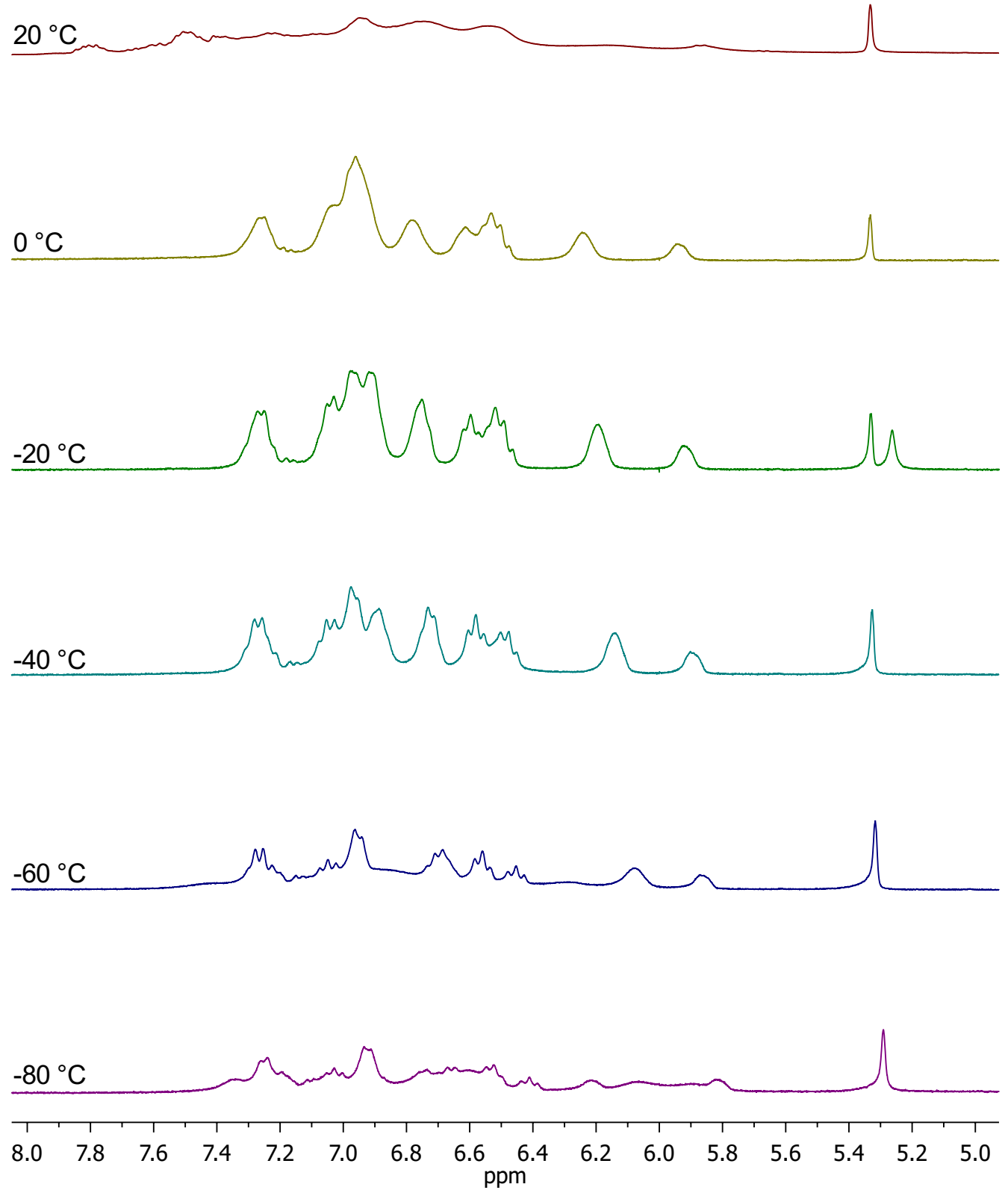

Figure 5.5: Low temperature ${ }^{1} \mathrm{H}$ NMR spectra of $\left[\mathrm{Pt}(\mathrm{Ph} \text {-thixantphos })_{2}\right]$ in $\mathrm{CD}_{2} \mathrm{Cl}_{2}$, showing the aromatic region. 


\subsection{Reactions of Ph-thixantphos with Platinum(0)}

Precursors

inversion of the ligand such that both faces of the backbone are averaged on the NMR timescale. In the case of $\left[\mathrm{Pt}(\mathrm{Ph} \text {-thixantphos })_{2}\right]$, the X-ray crystal structure shows two different spatial phosphorus environments, which should result in different chemical shifts in the ${ }^{31} \mathrm{P}$ NMR spectrum. This system is sufficiently crowded around the metal centre to reduce the ability of the xantphos system to invert. As such, cooling to $0{ }^{\circ} \mathrm{C}$ is sufficient to lock the backbones of $[\mathrm{Pt}(\mathrm{Ph}$ thixantphos $)_{2}$ ] in a single configuration, thus allowing the two different ${ }^{31} \mathrm{P}$ environments to become visible. Even at room temperature two different signals are apparent, indicating that although the inversion is occurring, the rate is slow enough compared to the NMR timescale to allow some distinction between the two phosphorus atoms. The inversion of the xantphos backbones stops, on the NMR timescale, upon cooling to around $-20{ }^{\circ} \mathrm{C}$. However, while the ${ }^{31} \mathrm{P}$ NMR spectrum remains sharp below this temperature, the ${ }^{1} \mathrm{H}$ NMR spectrum begins to broaden again. This may be the result of restricted rotation of some or all of the phenyl rings, which may occur due to the steric demands of the system.

The ${ }^{31} \mathrm{P}$ NMR spectrum of $\left[\mathrm{Pt}(\mathrm{Ph} \text {-thixantphos })_{2}\right]$ at $-80{ }^{\circ} \mathrm{C}$ shows some interesting features. Two peaks are observed as triplets at -2.43 and -6.16 ppm with phosphorus-phosphorus coupling of $55.0 \mathrm{~Hz}$ and platinum-phosphorus coupling of 3976 and $3864 \mathrm{~Hz}$ respectively. The value for the phosphorus-phosphorus coupling was slightly larger than that reported for the analogous [Pt(Et-xantphos $\left.)_{2}\right]$ complex, which showed two triplets at -6.82 and -19.65 ppm with two-bond phosphorus-phosphorus coupling of $48 \mathrm{~Hz} \cdot \underline{\underline{74}}$ The phosphorus-platinum coupling constants were also similar between the two complexes. The Ph-thixantphos complex had coupling constants of 3976 and $3864 \mathrm{~Hz}$, whereas the Et-xantphos complex $J_{\mathrm{PtP}}=4086$ and $3602 \mathrm{~Hz}$. However, the ${ }^{31} \mathrm{P}$ NMR spectrum of [Pt(Etxantphos) $)_{2}$ ] was sharp at room temperature in toluene- $d_{8}$, while the spectrum for $\left[\mathrm{Pt}(\mathrm{Ph} \text {-thixantphos })_{2}\right]$ did not fully resolve until $-40{ }^{\circ} \mathrm{C}$, indicating that the propensity for the ligands in these complexes to undergo inversion differs. $\underline{\underline{74}}$

A useful platinum $(0)$ starting material is $\left[\mathrm{Pt}\left(\mathrm{C}_{2} \mathrm{H}_{4}\right)_{3}\right]$, as the ethene ligands are readily displaced by other ligands, although $\left[\mathrm{Pt}\left(\mathrm{C}_{2} \mathrm{H}_{4}\right)_{3}\right]$ is only stable under an ethene atmosphere. ${ }^{286,287}$ Transition metal ethene complexes are important from an industrial perspective as ethene is a common feedstock used in industrialscale syntheses. ${ }^{288,289}$ The 1:1 reaction between $\left[\mathrm{Pt}\left(\mathrm{C}_{2} \mathrm{H}_{4}\right)_{3}\right]$ and Ph-thixantphos was carried out on an NMR scale to allow the identification of any intermediates that may form. The reaction was rapid and the solution turned orange after 
only 10 minutes at room temperature. The ${ }^{31} \mathrm{P}$ NMR spectrum showed $[\mathrm{Pt}(\mathrm{Ph}-$ thixantphos $)_{2}$ ] was the major product accounting for $93 \%$ of the phosphorus containing species, while the remaining $7 \%$ was $\left[\mathrm{Pt}\left(\mathrm{C}_{2} \mathrm{H}_{4}\right)(\mathrm{Ph}\right.$-thixantphos)], analogous to the $\left[\mathrm{Pt}(\mathrm{nb})\left(\mathrm{Ph}\right.\right.$-thixantphos)] formed in reaction with $\left[\mathrm{Pt}(\mathrm{nb})_{3}\right]$. Interestingly, the ratio of the alkene to bis complex formed appears to be dependent on the alkene used. The small amount of $\left[\mathrm{Pt}\left(\mathrm{C}_{2} \mathrm{H}_{4}\right)(\mathrm{Ph}\right.$-thixantphos)] formed suggests that the free ligand prefers to react with the alkene complex to form $[\mathrm{Pt}(\mathrm{Ph}-$ thixantphos $\left.)_{2}\right]$ rather than react with $\left[\mathrm{Pt}\left(\mathrm{C}_{2} \mathrm{H}_{4}\right)_{3}\right]$. This indicates that the initial coordination of the diphosphine to form $\left[\mathrm{Pt}\left(\mathrm{C}_{2} \mathrm{H}_{4}\right)(\mathrm{Ph}\right.$-thixantphos)] activates the system to react further with free Ph-thixantphos. However, in the norbornene case we observe $60.4 \%$ [Pt(Ph-thixantphos $\left.)_{2}\right]$, indicating that while the uncoordinated diphosphine does prefer to react with $[\mathrm{Pt}(\mathrm{nb})(\mathrm{Ph}$-thixantphos)] over the $\left[\mathrm{Pt}(\mathrm{nb})_{3}\right]$, the effect is not as significant as for the ethene system.

\subsection{Reaction of $t$-Bu-xantphos Ligands with $\left[\mathrm{Pt}(\mathrm{nb})_{3}\right]$}

The equimolar reaction of $\left[\mathrm{Pt}(\mathrm{nb})_{3}\right]$ with Ph-thixantphos instantaneously formed $\left[\mathrm{Pt}(\mathrm{Ph} \text {-thixantphos })_{2}\right]$ and $[\mathrm{Pt}(\mathrm{nb})(\mathrm{Ph}$-thixantphos) $]$ as the major and minor products respectively. The same reaction with $t$-Bu-thixantphos in $\mathrm{C}_{6} \mathrm{D}_{6}$ was slower, requiring heating at $60^{\circ} \mathrm{C}$ for four days in order to go to completion. In a similar manner to the reaction with Ph-thixantphos, the reaction of $t$-Bu-thixantphos with $\left[\mathrm{Pt}(\mathrm{nb})_{3}\right]$ also produces two products. One of these products is the same in both cases, $[\mathrm{Pt}(\mathrm{nb})$ (xantphos)] (xantphos $=$ Ph-thixantphos, $t$-Bu-thixantphos). However, rather than a bis(diphosphine) complex forming, as observed with $\mathrm{Ph}$-thixantphos, using $t$-Bu-thixantphos generated a reactive, 14-electron $[\mathrm{Pt}(t-$ Bu-thixantphos)] complex (60.8\%, Scheme 5.2). Although 14-electron complexes are electron-deficient, several examples have been reported, with $\left[\mathrm{Pt}\left(\mathrm{PPh}_{3}\right)_{2}\right]$ first reported in $1966^{290}$ followed by $\left[\mathrm{Pt}\left(\mathrm{PCy}_{3}\right)_{2}\right], \stackrel{291}{=}\left[\mathrm{Pt}\left(\mathrm{P}^{\mathrm{t}} \mathrm{Bu}_{3}\right)_{2}\right],\left[\mathrm{Pt}\left(\mathrm{P}^{\mathrm{t}} \mathrm{Bu}{ }_{2} \mathrm{Ph}\right)_{2}\right]$, and $\left[\mathrm{Pt}\left(\mathrm{P}^{\mathrm{i}} \mathrm{Pr}_{3}\right)_{2}\right] .292$

The $[\mathrm{Pt}(t-\mathrm{Bu}$-thixantphos)(nb)] complex exhibits a broad resonance at $55.6 \mathrm{ppm}$ with platinum satellites in the ${ }^{31} \mathrm{P}$ NMR spectrum. The value of $J_{\mathrm{PtP}}(3612 \mathrm{~Hz})$ is typical for trigonal platinum alkene complexes. $\stackrel{284}{2}$ The alkene protons appear at $2.37 \mathrm{ppm}$ in the ${ }^{1} \mathrm{H}$ NMR spectrum as a broad singlet with no discernable phosphorus coupling but platinum coupling of $67.8 \mathrm{~Hz}$. A similarly broad singlet in 


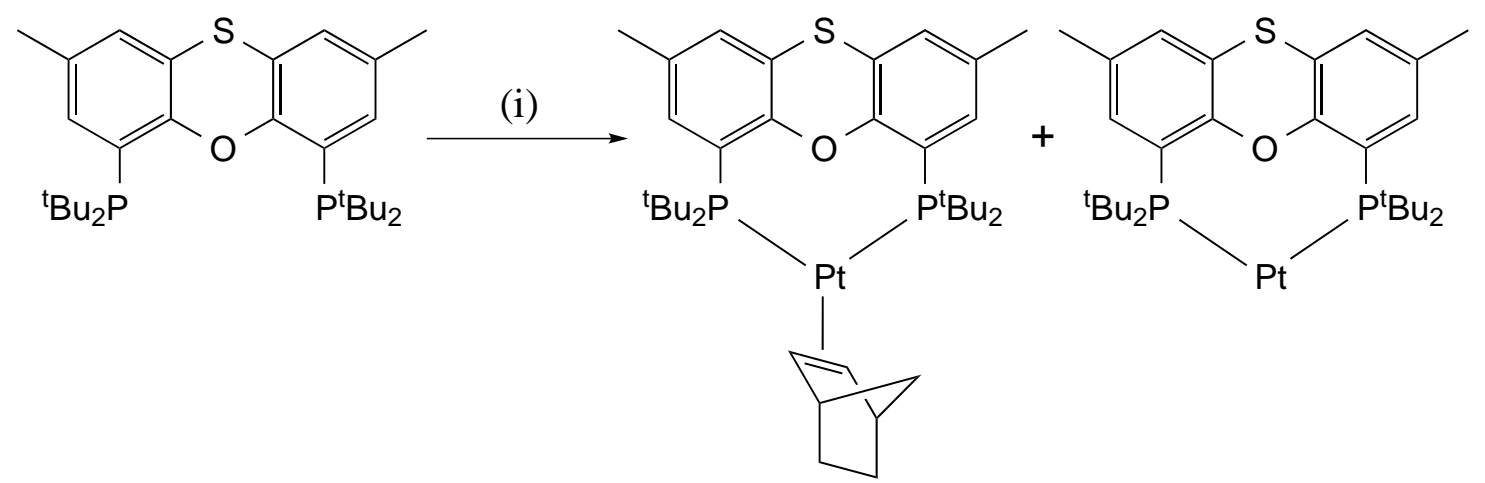

Scheme 5.2: Reaction between $\mathrm{tBu}$-thixantphos and $\left[\mathrm{Pt}(\mathrm{nb})_{3}\right]$. Reagents and conditions: (i) $\left[\mathrm{Pt}(\mathrm{nb})_{3}\right], \mathrm{C}_{6} \mathrm{D}_{6}, 60^{\circ} \mathrm{C}$.

the ${ }^{13} \mathrm{C}$ NMR spectrum at $51.9 \mathrm{ppm}$ with $J_{\mathrm{PtC}}=343.9 \mathrm{~Hz}$ was observed for the alkene carbons. Half of the expected number of norbornene and $t$-Bu-thixantphos NMR signals are observed indicating a symmetrical complex.

The reaction between $t$-Bu-sixantphos and $\left[\mathrm{Pt}(\mathrm{nb})_{3}\right]$ under the same conditions formed an analogous 14-electron [ $\mathrm{Pt}(t-\mathrm{Bu}$-sixantphos)] complex as the major product after 24 hours at $60{ }^{\circ} \mathrm{C}$, appearing in the ${ }^{31} \mathrm{P}$ NMR spectrum at $79.5 \mathrm{ppm}\left(J_{\mathrm{PtP}}\right.$ $=4827 \mathrm{~Hz})$. Two other products are also observed, appearing at $59.3 \mathrm{ppm}\left(J_{\mathrm{PtP}}=\right.$ $3572 \mathrm{~Hz})$ and $34.7 \mathrm{ppm}\left(J_{\mathrm{PtP}}=2677 \mathrm{~Hz}\right)$. As the complex at $59.3 \mathrm{ppm}$ is similar in both chemical shift and coupling constant to [ $\mathrm{Pt}(t-\mathrm{Bu}$-thixantphos)(nb)], it is proposed to be $[\mathrm{Pt}(t-\mathrm{Bu}$-sixantphos)(nb)]. The features of the complex at $34.7 \mathrm{ppm}$ are surprising. The value of $J_{\mathrm{PtP}}$ is similar to a number of $\mathrm{Pt}(\mathrm{II})$ complexes with trans phosphorus atoms (for examples see Chapter 6). Unfortunately the complexes could not be separated to gain further insight into their structures.

The major product in the reaction between $t$-Bu-xantphos and $\left[\mathrm{Pt}(\mathrm{nb})_{3}\right]$ was different to the other xantphos ligands. A small amount of a complex with a signal at $78.9 \mathrm{ppm}$ in the ${ }^{31} \mathrm{P}$ NMR spectrum was formed, consistent with a $[\mathrm{Pt}(t-\mathrm{Bu}-$ xantphos)] complex (the signal was too weak to observe any platinum coupling). However, the major product, and the only species present after 24 hours at $60^{\circ} \mathrm{C}$ was a complex in the ${ }^{31} \mathrm{P}$ NMR spectrum at $46.7 \mathrm{ppm}\left(J_{\mathrm{PtP}}=3246 \mathrm{~Hz}\right)$. The ${ }^{1} \mathrm{H}$ NMR spectrum of this complex showed a triplet at $-18.49 \mathrm{ppm}$, with $J_{\mathrm{PH}}$ of 13.1 $\mathrm{Hz}$ and $J_{\mathrm{PtH}}$ of $1107 \mathrm{~Hz}$. The chemical shift of this signal and the value of $J_{\mathrm{PtH}}$ are consistent with a hydride positioned trans to a ligand with a low trans-influence. 
The value of $J_{\mathrm{PH}}$ is consistent with a cis arrangement of the hydride with the two phosphorus atoms. The ${ }^{1} \mathrm{H}$ and ${ }^{13} \mathrm{C}$ NMR signals for the tert-butyl substituents are broad which may suggest a dynamic process is occurring. The O-ipso carbon shows a downfield shift of $2.8 \mathrm{ppm}$ which is consistent with coordination of the oxygen atom. This suggests a platinum(II) complex of the type $[\mathrm{Pt}(t-\mathrm{Bu}-$ xantphos- $\left.\left.\kappa P, O, P^{\prime}\right) \mathrm{H}\right] \mathrm{X}$ (Scheme [5.3). However, complexes of this type typically have platinum-phosphorus coupling constants of between 2300 and $2800 \mathrm{~Hz}$ (see Chapter (6), while the value of $J_{\mathrm{PtP}}$ for this complex is higher at $3246 \mathrm{~Hz}$. The nature of the counterion and the hydride source were not determined.
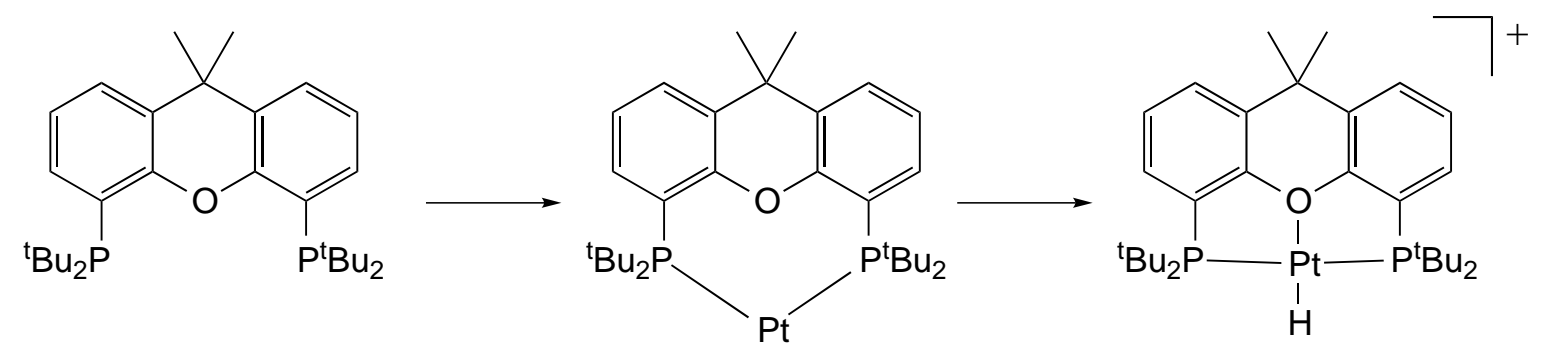

Scheme 5.3: Reaction between $t$-Bu-xantphos and $\left[\mathrm{Pt}(\mathrm{nb})_{3}\right] \cdot$ Reagents and conditions: $\left[\mathrm{Pt}(\mathrm{nb})_{3}\right], \mathrm{C}_{6} \mathrm{D}_{6}, 60^{\circ} \mathrm{C}, 24$ hours.

The formation of the proposed $\left[\mathrm{Pt}\left(t-\mathrm{Bu}\right.\right.$-xantphos- $\left.\left.\kappa P, O, P^{\prime}\right) \mathrm{H}\right] \mathrm{X}$ complex likely results from the $[\mathrm{Pt}(t-\mathrm{Bu}-\mathrm{xantphos})]$ complex undergoing reaction with another molecule in the system. Despite the number of $\left.[\mathrm{Pt} \text { (monophosphine })_{2}\right]$ complexes, isolable [Pt(diphosphine)] complexes are relatively rare as diphosphine ligands typically provide less protection of the metal centre due to the restrictions of their bite-angle. Although [Pt(diphosphine)] complexes are frequently proposed as intermediates, few have been isolated. ${ }^{293}-295[\mathrm{Pt}($ diphosphine) $]$ complexes will typically coordinate to any possible ligands that are present. The proposed 14electron complex [Pt(dcype)] (dcype = 1,2-bis(dicyclohexylphosphino)ethane) reacts with alkenes and alkynes to form [Pt(alkene)(dcype)] or [Pt(alkyne) (dcype)], and reacts with alkanes to form [Pt(alkyl)(dcype)H] complexes. ${ }^{294}$ [Pt(dcype)] also reacts with benzene to form $\left[\mathrm{Pt}\left(\mathrm{C}_{6} \mathrm{H}_{5}\right)(\mathrm{dcype}) \mathrm{H}\right]$. Although in the $[\mathrm{Pt}(t-\mathrm{Bu}-$ xantphos- $\left.\left.\kappa P, O, P^{\prime}\right) \mathrm{H}\right] \mathrm{X}$ system no evidence of a coordinated alkyl, phenyl or other fragment was observed, this may explain the formation of the hydride species, which then loses the other ligand to form a counterion. The hydride is unlikely to result from water as all solvents that were used were dried over molecular 
sieves prior to use and the water content of solvents dried in this manner is too small to account for the complete transformation to a hydride complex. ${ }^{296}$

The $[\mathrm{Pt}(t-\mathrm{Bu}$-thixantphos $)]$ complex can be isolated by removal of the norbornene from the sample, under reduced pressure, while the analogous $t$-Bu-sixantphos complex only formed as a mixture and the $t$-Bu-xantphos complex went on to form $\left[\mathrm{Pt}\left(t-\mathrm{Bu}\right.\right.$-xantphos- $\left.\left.\mathrm{k} P, O, P^{\prime}\right) \mathrm{H}\right]$ with full conversion after 24 hours preventing their isolation and full characterisation. The ${ }^{31} \mathrm{P}$ NMR data for the complexes is given in Table 5.3. The complexes all exhibit a single peak in the ${ }^{31} \mathrm{P}$ NMR spectra shifted downfield from the free ligand by 68-71 ppm $(\Delta \delta)$. A study of the ${ }^{31} \mathrm{P}$ NMR properties of $\left[\mathrm{Pt}\left(\mathrm{PR}_{3}\right)_{2}\right]$ complexes showed that all of the 14-electron complexes of this type that were studied display a downfield shift of the phosphorus signal by between 48 and 58 ppm upon coordination, with platinum-phosphorus

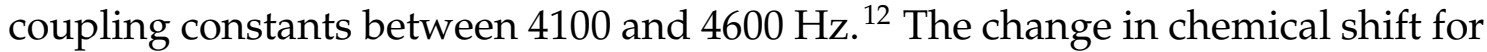
the $t$-Bu-xantphos complexes is larger than that reported for monophosphines. This may be the result of strain placed on the system to achieve such a large biteangle, or the result of a slightly bent complex which may form to allow bidentate coordination. The platinum-phosphorus coupling is also larger than those previously reported, by over $200 \mathrm{~Hz}$. The largest previously reported Pt-P coupling for these complexes is $4592 \mathrm{~Hz}$ for $\left[\mathrm{Pt}\left(\mathrm{P}^{t} \mathrm{Bu}_{2} \mathrm{Ph}\right)_{2}\right]$, and coupling constants of between 4370 and $4424 \mathrm{~Hz}$ have been reported by our group for substituted $\left[\mathrm{Pt}\left(\mathrm{P}^{\mathrm{t}} \mathrm{Bu}_{2} \mathrm{Bn}\right)_{2}\right]$ complexes. ${ }^{297,298}$ The larger coupling constants for the $t$-Bu-xantphos species likely indicates a distortion of the system from the linearity observed with monophosphine systems.

Table 5.3: ${ }^{31} \mathrm{P}$ NMR data for [Pt( $t$-Bu-xantphos $\left.)\right]$ complexes. $J_{\mathrm{PtP}}$ was not observed for $t$-Bu-xantphos $\left(\Delta \delta=\delta_{\text {complex }}-\delta_{\text {free ligand }}\right)$.

\begin{tabular}{lccc}
\hline & \multicolumn{3}{c}{${ }^{31} \mathbf{P}$} \\
\cline { 2 - 4 } Diphosphine & $\delta / \mathbf{p p m}$ & $\Delta \delta / \mathbf{p p m}$ & $\boldsymbol{J}_{\mathbf{P t P}} / \mathbf{H z}$ \\
\hline t-Bu-Sixantphos & 79.5 & 71.1 & 4827 \\
t-Bu-Thixantphos & 78.6 & 69.1 & 4810 \\
t-Bu-Xantphos & 78.9 & 68.7 & n.o. \\
\hline
\end{tabular}




\subsection{Reaction of $t$-Bu-thixantphos with $\left[\operatorname{Pt}(\operatorname{cod})_{2}\right]$}

$\left[\mathrm{Pt}(\operatorname{cod})_{2}\right](\operatorname{cod}=1,5$-cyclooctadiene $)$ is a commonly used starting material for the formation of platinum(0) phosphine complexes as the cod ligands can be readily displaced by phosphines. ${ }^{291,299}$ The reaction between $t$-Bu-thixantphos and $\left[\mathrm{Pt}(\mathrm{cod})_{2}\right]$ was carried out on an NMR scale in $\mathrm{C}_{6} \mathrm{D}_{6}$. The only phosphorus containing product from the reaction was [ $\mathrm{Pt}(t-\mathrm{Bu}$-thixantphos)]. However, conversion of only $28 \%$ was achieved. After 48 hours at room temperature the reaction had become black and despite heating no further reaction was observed. Together with the absence of unreacted $\left[\mathrm{Pt}(\operatorname{cod})_{2}\right]$ in the ${ }^{1} \mathrm{H}$ NMR spectrum this suggests that the $\left[\mathrm{Pt}(\operatorname{cod})_{2}\right]$ was unstable under the reaction conditions. Analysis of the reaction mixture by ${ }^{1} \mathrm{H}$ and ${ }^{13} \mathrm{C}$ NMR spectroscopy showed the presence of 1,3and 1,4-cyclooctadiene in addition to 1,5-cyclooctadiene. A weak signal in the ${ }^{1} \mathrm{H}$ NMR spectrum was observed at $-18.2 \mathrm{ppm}$ as a triplet $\left(J_{\mathrm{PH}}=13.7 \mathrm{~Hz}\right)$ with platinum satellites $\left(J_{\mathrm{PtH}}=1100 \mathrm{~Hz}\right)$. This signal is in a similar position and has similar coupling constants to the peak in the ${ }^{1} \mathrm{H}$ NMR of $[\mathrm{Pt}(t-\mathrm{Bu}$-xantphos) $\mathrm{H}] \mathrm{X}$. This may indicate that the 14-electron $[\mathrm{Pt}(t$-Bu-thixantphos)] complex is able to catalyse the isomerisation of 1,5-cyclooctadiene. This process may involve the oxidative addition of an allylic $\mathrm{C}-\mathrm{H}$ of the cod ligand to the platinum forming an $\eta^{3}$-allyl and a hydride ligand, which could then reductively eliminate to form 1,3-cyclooctadiene. Given the poor reactivity of $\left[\mathrm{Pt}(\mathrm{cod})_{2}\right]$ with $t$-Bu-thixantphos the analogous reactions with $t$-Bu-sixantphos and $t$-Bu-xantphos were not attempted.

\subsection{Reaction of $t$-Bu-xantphos Ligands and $\left[\mathrm{Pt}\left(\mathrm{C}_{2} \mathrm{H}_{4}\right)_{3}\right]$}

Both ethene and norbornene are monodentate alkene ligands whilst cod is a bidentate ligand which typically chelates to metal centres. Hence, the reactivity of the $t$-Bu-xantphos ligands with $\left[\mathrm{Pt}\left(\mathrm{C}_{2} \mathrm{H}_{4}\right)_{3}\right]$ is expect to be more similar to that observed with $\left[\mathrm{Pt}(\mathrm{nb})_{3}\right]$ than that with $\left[\mathrm{Pt}(\mathrm{cod})_{2}\right]$. Furthermore ethene is more readily displaced than norbornene as the coordination of norbornene relieves the strain associated with the bicyclic system. However, ethene is a smaller ligand than norbornene. Hence investigating the reactivity of the $t$-Bu-xantphos ligands with $\left[\mathrm{Pt}\left(\mathrm{C}_{2} \mathrm{H}_{4}\right)_{3}\right]$ gives an opportunity to probe the relative importance of elec- 
tronic and steric effects in controlling the coordination of alkene ligands to $[\mathrm{Pt}(t-$ Bu-xantphos)] complexes. The three $t$-Bu-xantphos ligands were reacted on an NMR scale in $\mathrm{C}_{6} \mathrm{D}_{6}$ with freshly prepared $\left[\mathrm{Pt}\left(\mathrm{C}_{2} \mathrm{H}_{4}\right)_{3}\right]$, made from $\left[\mathrm{Pt}(\operatorname{cod})_{2}\right]$ under an ethene atmosphere. All three ligands initially formed a complex of the type [Pt(t-Bu-xantphos) $\left.\left(\mathrm{C}_{2} \mathrm{H}_{4}\right)\right]$ (Scheme 5.4). However, while the ethene complex was the only product for the reaction with $t$-Bu-thixantphos, the reaction with $t$-Bu-xantphos progressed to form $[\mathrm{Pt}(t-\mathrm{Bu}$-xantphos $) \mathrm{H}] \mathrm{X}$. Again the nature of the counterion in this species was unable to be determined. The reaction between $t$-Bu-sixantphos and $\left[\mathrm{Pt}\left(\mathrm{C}_{2} \mathrm{H}_{4}\right)_{3}\right]$ showed $11.1 \%$ of $\left[\mathrm{Pt}(t\right.$-Bu-sixantphos $\left.)\left(\mathrm{C}_{2} \mathrm{H}_{4}\right)\right]$ after four hours. However, the reaction did not progress further.

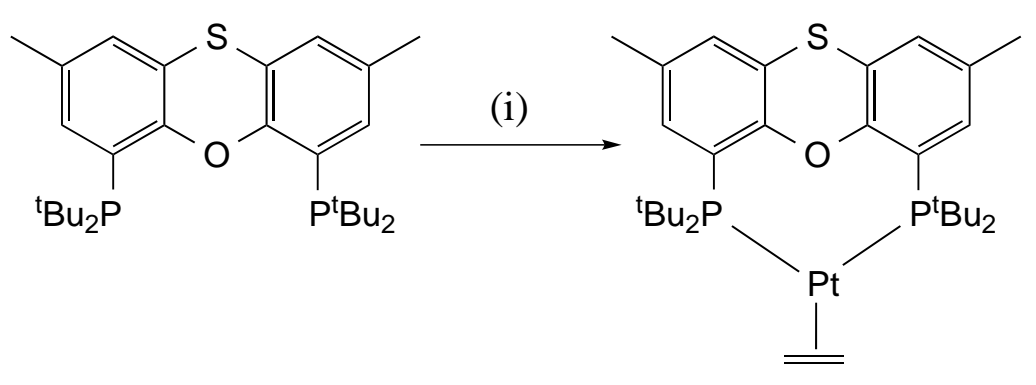

Scheme 5.4: Reaction between $t$-Bu-thixantphos and $\left[\mathrm{Pt}\left(\mathrm{C}_{2} \mathrm{H}_{4}\right)_{3}\right]$. Reagents and conditions: (i) $\left[\mathrm{Pt}\left(\mathrm{C}_{2} \mathrm{H}_{4}\right)_{3}\right], \mathrm{C}_{6} \mathrm{D}_{6}, 48$ hours, under $\mathrm{C}_{2} \mathrm{H}_{4}$.

Selected NMR data for the $\left[\mathrm{Pt}(t\right.$-Bu-xantphos $\left.)\left(\mathrm{C}_{2} \mathrm{H}_{4}\right)\right]$ complexes is given in Table 5.4. In all cases the ${ }^{31} \mathrm{P}$ NMR spectrum showed a singlet between 53.4 and 55.7 ppm, shifted downfield by between 43.2 and 46.2 ppm from the corresponding free ligand. All of the $J_{\mathrm{PtP}}$ values are within the expected range for complexes of this type. $\stackrel{16}{=}\left[\mathrm{Pt}(t\right.$-Bu-sixantphos $\left.)\left(\mathrm{C}_{2} \mathrm{H}_{4}\right)\right]$ has a $J_{\mathrm{PtP}}$ value almost $400 \mathrm{~Hz}$ lower than that observed for the corresponding $t$-Bu-thixantphos and $t$-Bu-xantphos complexes. The lower coupling constant may indicate that the ethene ligand is more strongly coordinated to the platinum centre in $\left[\mathrm{Pt}(t-\mathrm{Bu}\right.$-sixantphos $\left.)\left(\mathrm{C}_{2} \mathrm{H}_{4}\right)\right]$. This may be the result of the smaller bite-angle of the $t$-Bu-sixantphos ligand allowing more room for the ethene ligand to interact with the platinum. The ${ }^{1} \mathrm{H}$ NMR spectra showed peaks at 2.50 and $2.52 \mathrm{ppm}$ corresponding to the ethene protons for $\left[\mathrm{Pt}(t\right.$-Bu-thixantphos $\left.)\left(\mathrm{C}_{2} \mathrm{H}_{4}\right)\right]$ and $\left[\mathrm{Pt}(t\right.$-Bu-xantphos $\left.)\left(\mathrm{C}_{2} \mathrm{H}_{4}\right)\right]$ respectively. The peak corresponding to coordinated ethene was obscured in the ${ }^{1} \mathrm{H}$ NMR spectrum for the $t$-Bu-sixantphos complex. The dramatic upfield shift of 
the ${ }^{1} \mathrm{H}$ signal for the ethene ligand is consistent with coordination to a metal centre such at platinum (uncoordinated ethene appears at $5.25 \mathrm{ppm}^{300}$ ). As both the $t$-Bu-sixantphos and $t$-Bu-xantphos ethene complexes reacted further to produce mixtures, the ${ }^{13} \mathrm{C}$ NMR peak for the ethene ligand was only observed in $[\mathrm{Pt}(t-\mathrm{Bu}$ thixantphos $\left.)\left(\mathrm{C}_{2} \mathrm{H}_{4}\right)\right]$. The ethene carbon signal appeared at $34.2 \mathrm{ppm}$ as a broad singlet with a $J_{\mathrm{PtC}}$ value of $223.2 \mathrm{~Hz}$.

Table 5.4: ${ }^{31} \mathrm{P}$ NMR data for $\left[\mathrm{Pt}(t\right.$-Bu-xantphos $\left.)\left(\mathrm{C}_{2} \mathrm{H}_{4}\right)\right]$ complexes in $\mathrm{C}_{6} \mathrm{D}_{6}(\Delta \delta=$ $\left.\delta_{\text {complex }}-\delta_{\text {free ligand }}\right)$.

\begin{tabular}{lcccccc}
\hline & \multicolumn{4}{c}{${ }^{31} \mathbf{P}$} & & \multicolumn{2}{c}{${ }^{\mathbf{1}} \mathbf{H}$} \\
\cline { 2 - 4 } \cline { 7 - 8 } Diphosphine & $\delta / \mathbf{p p m}$ & $\Delta \delta / \mathbf{p p m}$ & $\boldsymbol{J}_{\mathbf{P t P}} / \mathbf{H z}$ & & $\delta / \mathbf{p p m}$ & $\boldsymbol{J}_{\mathbf{P t H}} / \mathbf{H z}$ \\
\hline t-Bu-Sixantphos & 53.7 & 45.3 & 3499 & & \\
t-Bu-Thixantphos & 55.7 & 46.2 & 3899 & & 2.50 & 59.5 \\
t-Bu-Xantphos & 53.4 & 43.2 & 3878 & & 2.52 & 58.0 \\
\hline
\end{tabular}

The reaction of $t$-Bu-thixantphos with the three different platinum alkene complexes showed markedly different properties: $\left[\mathrm{Pt}(\mathrm{nb})_{3}\right]$ forms an equilibrium between $[\mathrm{Pt}(t-\mathrm{Bu}$-thixantphos $)(\mathrm{nb})]$ and $[\mathrm{Pt}(t-\mathrm{Bu}$-thixantphos $)] ;\left[\mathrm{Pt}(\mathrm{cod})_{2}\right]$ forms $[\mathrm{Pt}$ (t-Bu-thixantphos)]; and $\left[\mathrm{Pt}\left(\mathrm{C}_{2} \mathrm{H}_{4}\right)_{3}\right]$ forms [Pt(t-Bu-thixantphos $\left.)\left(\mathrm{C}_{2} \mathrm{H}_{4}\right)\right]$. [Pt $(t-$ Bu-thixantphos)(nb)] was converted entirely to the [Pt(t-Bu-thixantphos)] complex by placing a sample under vacuum for one hour, while the $[\mathrm{Pt}(t-\mathrm{Bu}$-thixantphos $)\left(\mathrm{C}_{2} \mathrm{H}_{4}\right)$ ] complex showed no change under the same conditions. Bubbling argon through a solution of $\left[\mathrm{Pt}(t\right.$-Bu-thixantphos $\left.)\left(\mathrm{C}_{2} \mathrm{H}_{4}\right)\right]$ for 10 mins, resulted in the formation of only small amounts of [ $\mathrm{Pt}(t$-Bu-thixantphos)]. This indicates that ethene is more strongly coordinated to the platinum centre than norbornene and is less prone to dissociation. In general dialkyl substituted alkenes coordinate more weakly than ethene, as shown in the straightforward synthesis of $\left[\mathrm{Pt}\left(\mathrm{C}_{2} \mathrm{H}_{4}\right)_{3}\right]$ from $\left[\mathrm{Pt}(\operatorname{cod})_{2}\right] \cdot{ }^{291,301}$ However, coordination of norbornene relieves some of the ring-strain which means it coordinates more strongly than cod. $\left[\mathrm{Pt}(\mathrm{nb})_{3}\right]$ is an air-stable solid, while $\left[\mathrm{Pt}\left(\mathrm{C}_{2} \mathrm{H}_{4}\right)_{3}\right]$ degrades if not stored under ethene, indicating that norbornene coordinates more strongly than ethene. 


\subsection{Formation of Platinum Dioxygen Complexes}

$\left[\mathrm{Pt}\left(\mathrm{P}_{2}\right)\right]$ complexes $\left(\mathrm{P}=\right.$ monophosphine, $\mathrm{P}_{2}=$ diphosphine $)$ are reactive towards small molecules, particularly those with diphosphine ligands as was observed in the formation of $[\mathrm{Pt}(t-\mathrm{Bu}$-xantphos $) \mathrm{H}] X .^{294}$ The reactivity of $[\mathrm{Pt}(t-\mathrm{Bu}$-thixantphos)] is lower than the reactivity of the corresponding $t$-Bu-sixantphos and $t$ $\mathrm{Bu}$-xantphos complexes. Both the $t$-Bu-sixantphos and $t$-Bu-xantphos complexes were unstable in solution, while the $t$-Bu-thixantphos complex was stable in $\mathrm{C}_{6} \mathrm{D}_{6}$, under argon, at room temperature for at least several days. However, $[\mathrm{Pt}(t-\mathrm{Bu}-$ thixantphos)] reacts rapidly with oxygen forming $\left[\mathrm{Pt}(t-\mathrm{Bu}\right.$-thixantphos $\left.)\left(\eta^{2}-\mathrm{O}_{2}\right)\right]$ upon exposure to air (Scheme 5.5). Bubbling air through a sample containing $[\mathrm{Pt}(t-\mathrm{Bu}$-thixantphos $)]$ and $[\mathrm{Pt}(t$-Bu-thixantphos $)(\mathrm{nb})]$ or a sample of $[\mathrm{Pt}(t-\mathrm{Bu}$ thixantphos) $\left.\left(\mathrm{C}_{2} \mathrm{H}_{4}\right)\right]$ resulted in complete conversion (by ${ }^{31} \mathrm{P}$ NMR spectroscopy) to $\left[\mathrm{Pt}(t-\mathrm{Bu}\right.$-thixantphos $\left.)\left(\eta^{2}-\mathrm{O}_{2}\right)\right]$. This reaction is quite common for complexes of the type $\left[\mathrm{Pt}\left(\mathrm{PR}_{3}\right)_{2}\right] \cdot \underline{302,303}$<smiles>CCCCPc1cc(C)cc2c1Oc1c(PCCCC)cc(C)cc1S2</smiles>

or

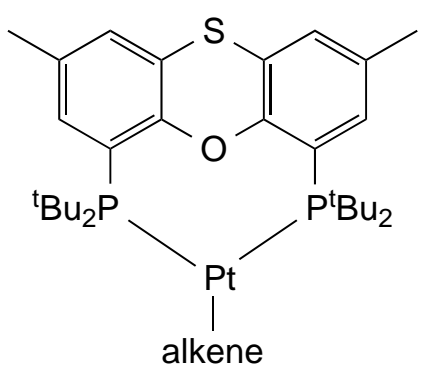

(i)

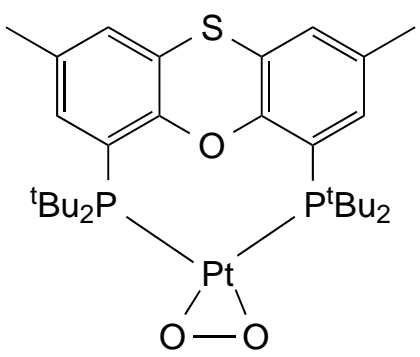

Scheme 5.5: Reaction of [Pt(alkene)(t-Bu-thixantphos)] and [Pt( $t$-Bu-thixantphos)] with air. Alkene $=\mathrm{C}_{2} \mathrm{H}_{4}$ or norbornene. Reagents and conditions: (i) Air, 10 mins, $\mathrm{C}_{6} \mathrm{D}_{6}$.

Upon reaction of $[\mathrm{Pt}(t-\mathrm{Bu}$-thixantphos $)]$ with oxygen, the ${ }^{31} \mathrm{P} \mathrm{NMR}$ signal shifts 
upfield from 78.6 to $38.4 \mathrm{ppm}$ with an associated reduction in the magnitude of the phosphorus-platinum coupling from 4810 to $4488 \mathrm{~Hz}$. The reduction in coupling constant is due to the decrease in the s-character of the platinum bond orbitals upon changing the geometry of the complex. ${ }^{16}$ The value of the $J_{\mathrm{PtP}}$ coupling constant is larger than those previously reported $(3930-4112 \mathrm{~Hz}) .{ }^{297,302} \mathrm{Sim}-$ ilarly to the NMR data obtained for [ $\mathrm{Pt}(t$-Bu-thixantphos)], these values may be different from the literature data due to the constraints of the diphosphine ligand over the monophosphines reported in the literature. The ${ }^{1} \mathrm{H}$ and ${ }^{13} \mathrm{C} \mathrm{NMR}$ spectra of $\left[\mathrm{Pt}(t\right.$-Bu-thixantphos $\left.)\left(\eta^{2}-\mathrm{O}_{2}\right)\right]$ show doublets for the tert-butyl proton and carbon environments, rather than the virtual triplets observed for $[\mathrm{Pt}(t-\mathrm{Bu}-$ thixantphos)]. This is consistent with the change in geometry as virtual triplets indicate strongly coupled phosphorus atoms which generally occurs with trans coordination. $\underline{124}$

Colourless single crystals of $\left[\mathrm{Pt}(t\right.$-Bu-thixantphos $\left.)\left(\eta^{2}-\mathrm{O}_{2}\right)\right]$ were obtained by allowing oxygen to diffuse slowly into a $\mathrm{C}_{6} \mathrm{D}_{6}$ solution containing a mixture of [Pt(t-Bu-thixantphos)(nb)] and [Pt(t-Bu-thixantphos)]. The complex crystallised in the Pbca space group with two molecules of $\mathrm{C}_{6} \mathrm{D}_{6}$ as solvate. The crystal structure is shown in Figure 5.6 with a side view in Figure 5.7. Selected bond lengths and angles for the complex are summarised in Table 5.5 and crystallographic data is given in Table 5.6 .

Table 5.5: Selected bond distances $(\AA)$ and angles $\left(^{\circ}\right)$ of $\left[\mathrm{Pt}(t\right.$-Bu-thixantphos $)\left(\eta^{2}-\right.$ $\left.\left.\mathrm{O}_{2}\right)\right] \cdot 2 \mathrm{C}_{6} \mathrm{D}_{6}$.

\begin{tabular}{llll}
\hline \multicolumn{2}{c}{ Bond distances $(\AA)$} & \multicolumn{2}{c}{ Bond angles $\left({ }^{\circ}\right)$} \\
\hline P1-Pt & $2.3159(13)$ & P1-Pt-P2 & $117.26(5)$ \\
P2-Pt & $2.3056(13)$ & P1-Pt-O2 & $101.26(14)$ \\
O1-Pt & $3.383(3)$ & P2-Pt-O3 & $100.11(13)$ \\
O2-Pt & $2.024(4)$ & O2-Pt-O3 & $41.38(18)$ \\
O3-Pt & $2.022(4)$ & Ring 1 - Ring 2 & $133.90(18)$ \\
O1-O2 & $1.429(6)$ & & \\
\hline
\end{tabular}

The crystal structure of $\left[\mathrm{Pt}(t-\mathrm{Bu}\right.$-thixantphos $\left.)\left(\eta^{2}-\mathrm{O}_{2}\right)\right]$ shows a planar geometry around the platinum with the sum of the angles $=360.01^{\circ}$. The backbone of 
Table 5.6: Crystallographic data and structure refinement of $[\mathrm{Pt}(t-\mathrm{Bu}$-thixantphos $\left.)\left(\eta^{2}-\mathrm{O}_{2}\right)\right] \cdot 2 \mathrm{C}_{6} \mathrm{D}_{6}$.

\begin{tabular}{|c|c|}
\hline Empirical formula & $\mathrm{C}_{42} \mathrm{H}_{46} \mathrm{D}_{12} \mathrm{O}_{3} \mathrm{P}_{2} \mathrm{PtS}$ \\
\hline Formula weight & 912.04 \\
\hline Temperature/K & $120.01(10)$ \\
\hline Crystal system & orthorhombic \\
\hline Space group & $\mathrm{Pbca}$ \\
\hline $\mathrm{a} / \AA$ & 17.7892(3) \\
\hline $\mathrm{b} / \AA$ & $15.8129(3)$ \\
\hline$c / \AA$ & $28.4025(5)$ \\
\hline$\alpha /^{\circ}$ & 90 \\
\hline$\beta /{ }^{\circ}$ & 90 \\
\hline$\gamma /{ }^{\circ}$ & 90 \\
\hline Volume $/ \AA^{3}$ & $7989.6(2)$ \\
\hline Z & 8 \\
\hline$\rho_{\text {calc }} \mathrm{mg} / \mathrm{mm}^{3}$ & 1.516 \\
\hline$\mu / \mathrm{mm}$ & 3.682 \\
\hline $\mathrm{F}(000)$ & 3664.0 \\
\hline Crystal size $/ \mathrm{mm}$ & $0.21 \times 0.16 \times 0.15$ \\
\hline Radiation & $\operatorname{MoK} \alpha(\lambda=0.71073)$ \\
\hline $2 \theta$ range for data collection & 5.348 to $65.882^{\circ}$ \\
\hline Index ranges & $-27 \leq \mathrm{h} \leq 26,-22 \leq \mathrm{k} \leq 24,-43 \leq 1 \leq 40$ \\
\hline Reflections collected & 118275 \\
\hline Independent reflections & $14395\left[R_{\text {int }}=0.0668, R_{\text {sigma }}=0.0383\right]$ \\
\hline Data/restraints/parameters & $14395 / 0 / 456$ \\
\hline Goodness-of-fit on $\mathrm{F}^{2}$ & 1.101 \\
\hline Final $\mathrm{R}$ indexes $[\mathrm{I}>=2 \sigma(\mathrm{I})]$ & $\mathrm{R}_{1}=0.0638, \mathrm{w} \mathrm{R}_{2}=0.1563$ \\
\hline Final $\mathrm{R}$ indexes [all data] & $\mathrm{R}_{1}=0.0824, \mathrm{wR}_{2}=0.1718$ \\
\hline Largest diff. peak/hole / e $\AA^{-3}$ & $7.62 /-3.06$ \\
\hline
\end{tabular}




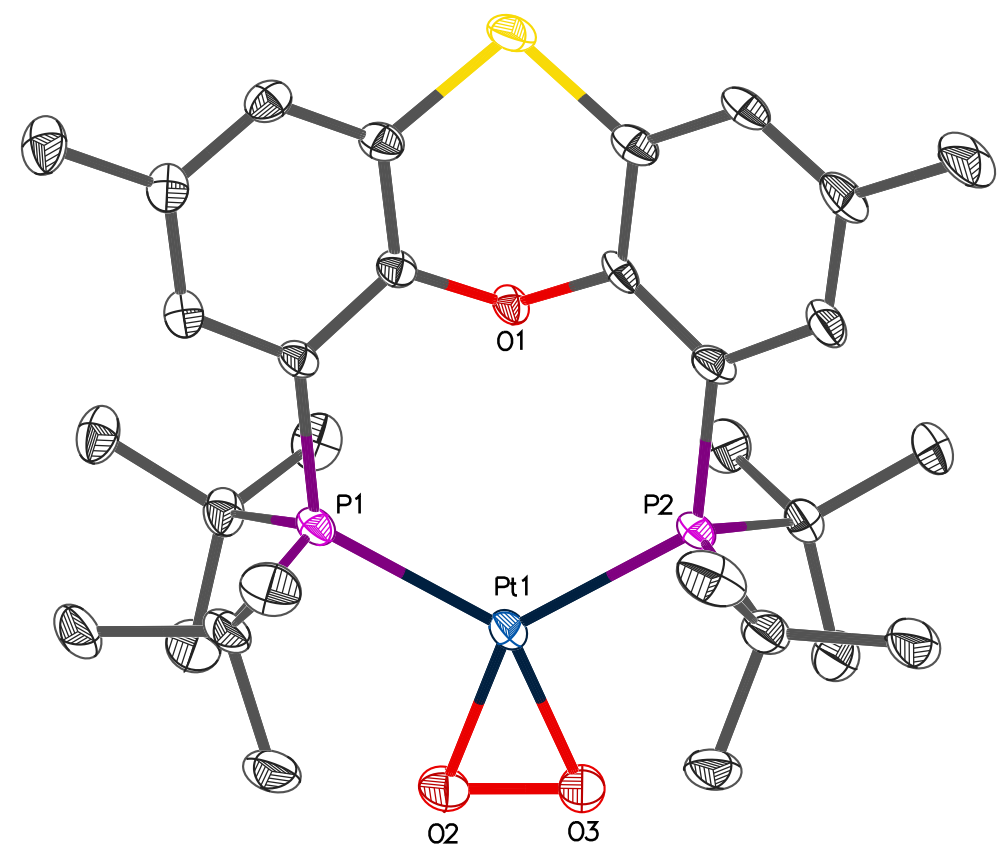

Figure 5.6: $\left[\mathrm{Pt}(t-\mathrm{Bu}\right.$-thixantphos $\left.)\left(\eta^{2}-\mathrm{O}_{2}\right)\right] \cdot 2 \mathrm{C}_{6} \mathrm{D}_{6}$ (50\% probability thermal ellipsoids). Hydrogen atoms and solvent molecules omitted for clarity.

the ligand is bent to achieve a bite-angle of $117.26(5)^{\circ}$, smaller than the calculated bite-angle of $126.98^{\circ}$ (Section 2.2). The bite-angle of the $t$-Bu-thixantphos ligand in $\left[\mathrm{Pt}(t\right.$-Bu-thixantphos $\left.)\left(\eta^{2}-\mathrm{O}_{2}\right)\right]$ is similar to the bite-angle in $[\mathrm{Ag}(t-\mathrm{Bu}$ thixantphos)Cl] $\left(116.46(8)^{\circ}\right.$, Table 3.2). No interaction between the ether-bridge oxygen and the platinum is observed, with a distance of 3.383(3) A between the two atoms. All of the bond lengths and angles have typical values. Surprisingly only four crystal structures of platinum dioxygen complexes have been reported in the CSD, three of which are for $\left[\mathrm{Pt}\left(\eta^{2}-\mathrm{O}_{2}\right)\left(\mathrm{PPh}_{3}\right)_{2}\right]$ with different solvates, $\left(\mathrm{C}_{6} \mathrm{H}_{6}, \mathrm{CHCl}_{3}\right.$ and $\left.\mathrm{C}_{7} \mathrm{H}_{8}\right) \cdot 304-306$ However, the data quality for the structure with a toluene solvate was poor. $\frac{306}{2}$ The other reported crystal structure is for $\left[\mathrm{Pt}\left(\eta^{2}-\mathrm{O}_{2}\right)\left(\mathrm{P}^{\mathrm{t}} \mathrm{Bu}_{2} \mathrm{Ph}\right)_{2}\right] \cdot \underline{307}$ The $\mathrm{P}^{\mathrm{t}} \mathrm{Bu}_{2} \mathrm{Ph}$ ligand has similar steric and electronic properties to the $t$-Bu-xantphos ligands, though the $t$-Bu-xantphos ligands are restricted by the backbone. The P-Pt-P angle in $\left[\mathrm{Pt}\left(\eta^{2}-\mathrm{O}_{2}\right)\left(\mathrm{P}^{\mathrm{t}} \mathrm{Bu}_{2} \mathrm{Ph}\right)_{2}\right]\left(113.1(2)^{\circ}\right)$ is smaller than that observed for $\left[\mathrm{Pt}(t\right.$-Bu-thixantphos $\left.)\left(\eta^{2}-\mathrm{O}_{2}\right)\right]$, indicating the role of the rigid backbone in determining the bite-angle of transition metal complexes. The Pt-O, and O-O bond lengths, and the O-Pt-O angle show little difference 


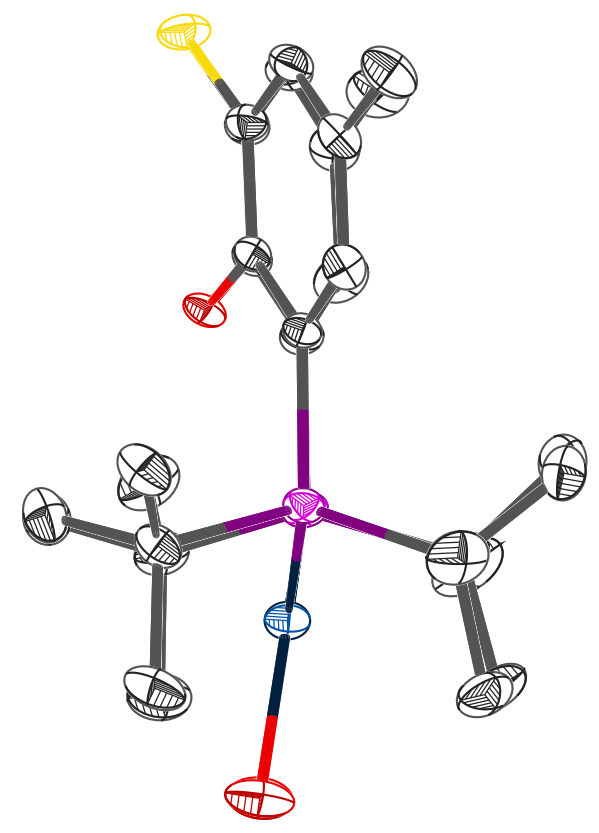

Figure 5.7: $\left[\mathrm{Pt}(t-\mathrm{Bu}\right.$-thixantphos $\left.)\left(\eta^{2}-\mathrm{O}_{2}\right)\right] \cdot 2 \mathrm{C}_{6} \mathrm{D}_{6}$, side view (50\% probability thermal ellipsoids). Hydrogen atoms and solvent molecules omitted for clarity.

between the three reported structures and $\left[\mathrm{Pt}(t-\mathrm{Bu}\right.$-thixantphos $\left.)\left(\eta^{2}-\mathrm{O}_{2}\right)\right]$ (Table 5.7).

The O-O bond length $(1.429(6) \AA)$ in $\left[\mathrm{Pt}(t\right.$-Bu-thixantphos $\left.)\left(\eta^{2}-\mathrm{O}_{2}\right)\right]$ is longer than the bond length of molecular oxygen $(1.21 \AA)$ indicating a reduction in the bond order upon coordination to the metal centre. Transition metal complexes with $\mathrm{O}$ O bond lengths of 1.4-1.5 $\AA$ are regarded formally as peroxide complexes with an $\mathrm{O}_{2}{ }^{2-}$ ligand and a platinum(II) centre. $\stackrel{308}{3}$ The $\mathrm{O}-\mathrm{O}$ bond length is slightly shorter in $\left[\mathrm{Pt}(t-\mathrm{Bu}\right.$-thixantphos $\left.)\left(\eta^{2}-\mathrm{O}_{2}\right)\right]$ than the analogous $\mathrm{PPh}_{3}$ or $\mathrm{P}^{\mathrm{t}} \mathrm{Bu}_{2} \mathrm{Ph}$ complexes (although within error for the $\mathrm{P}^{\mathrm{t}} \mathrm{Bu}_{2} \mathrm{Ph}$ value). $\stackrel{304-307}{=} t$-Bu-Thixantphos would be expected to be more electron-donating than $\mathrm{PPh}_{3}$, and to have similar electronic properties to $\mathrm{P}^{t} \mathrm{Bu}_{2} \mathrm{Ph}$. A longer $\mathrm{O}-\mathrm{O}$ bond length is expected if more electrondonating ligands are used as this would add to the $\mathrm{O}_{2}$ anti-bonding $\pi^{*}$-orbital through back-donation, which should reduce the bond order and thus increase the $\mathrm{O}-\mathrm{O}$ bond length. However, the P-Pt-P angle is larger for $t$-Bu-thixantphos than the other ligands. This may indicate that the $\mathrm{O}-\mathrm{O}$ bond length is restrained by the sterics of the $t$-Bu-thixantphos ligand. Hence the $\mathrm{O}-\mathrm{O}$ bond length in these 
Table 5.7: Selected bond distances $(\AA)$ and angles $\left(^{\circ}\right)$ of $\left[\mathrm{Pt}(\mathrm{PP})\left(\eta^{2}-\mathrm{O}_{2}\right)\right]$.

\begin{tabular}{lcccccc}
\hline & \multicolumn{3}{c}{ Bond distances $(\AA)$} & & \multicolumn{2}{c}{ Bond angles ( $\left.{ }^{\circ}\right)$} \\
\cline { 2 - 3 } Compound & O-O & Pt-O & Pt-O' & & O-Pt-O' & P-Pt-P \\
\hline$\left.\left[\mathrm{Pt}\left(\eta^{2}-\mathrm{O}_{2}\right)\left(\mathrm{P}^{\mathrm{t}} \mathrm{Bu}_{2} \mathrm{Ph}\right)_{2}\right)\right]$ & $1.42(2)$ & $2.02(1)$ & $2.02(1)$ & & $41.5(5)$ & $113.1(2)$ \\
{$\left[\mathrm{Pt}\left(\eta^{2}-\mathrm{O}_{2}\right)\left(\mathrm{PPh}_{3}\right)_{2}\right] \cdot \mathrm{C}_{6} \mathrm{H}_{6}$} & $1.45(4)$ & $2.01(3)$ & $2.01(2)$ & & $43(1)$ & $101.2(4)$ \\
{$\left[\mathrm{Pt}\left(\eta^{2}-\mathrm{O}_{2}\right)\left(\mathrm{PPh}_{3}\right)_{2}\right] \cdot \mathrm{CHCl}_{3}$} & $1.505(16)$ & $2.006(7)$ & $2.006(7)$ & & $44.06(40)$ & $101.23(12)$ \\
{$\left[\mathrm{Pt}(t-\mathrm{Bu}\right.$-thixantphos $\left.)\left(\eta^{2}-\mathrm{O}_{2}\right)\right] \cdot 2 \mathrm{C}_{6} \mathrm{D}_{6}$} & $1.429(6)$ & $2.024(4)$ & $2.022(4)$ & & $41.38(18)$ & $117.26(5)$ \\
\hline
\end{tabular}


complexes is likely the result of a combination of both steric and electronic effects.

\subsection{Reactions of $\left[\mathrm{Pt}(t-B u-t h i x a n t p h o s)\left(\eta^{2}-\mathrm{O}_{2}\right)\right]$}

The bond length of the dioxygen molecule increases upon coordination, indicating a decrease in the bond order and strength. As such, the oxygen molecule should be activated, and more likely to undergo reactions with small molecules, with several examples reported in the literature. 215,216,231,237,302,309-312 Some examples of reversible coordination of dioxygen to a palladium centre have also been reported, with removal of the dioxygen ligand either by vacuum, substitution or reaction with other ligands. $\frac{215,307,313}{2}$ As such the reversibility of the formation of $\left[\mathrm{Pt}(t\right.$-Bu-thixantphos $\left.)\left(\eta^{2}-\mathrm{O}_{2}\right)\right]$ was investigated by vacuum and by reaction with $\mathrm{C}_{2} \mathrm{H}_{4}$, and the activation of the oxygen ligand was explored by reaction with various small molecules; $\mathrm{C}_{2} \mathrm{H}_{2}, \mathrm{H}_{2}, \mathrm{CO}, \mathrm{CO}_{2}, \mathrm{NH}_{4} \mathrm{PF}_{6}$ and 1,3,5-triaza-7phosphaadamantane (pta).

The $\left[\mathrm{Pd}\left(\eta^{2}-\mathrm{O}_{2}\right)\left(\mathrm{P}^{\mathrm{t}} \mathrm{Bu}_{2} \mathrm{Ph}\right)_{2}\right]$ complex readily loses dioxygen under vacuum, although the dioxygen ligand in $\left[\mathrm{Pt}\left(\eta^{2}-\mathrm{O}_{2}\right)\left(\mathrm{P}^{\mathrm{t}} \mathrm{Bu}_{2} \mathrm{Ph}\right)_{2}\right]$ does not dissociate under the same conditions. ${ }^{307}$ Recent work in our group showed that $\left[\mathrm{Pt}\left(\eta^{2}-\mathrm{O}_{2}\right)\left(\mathrm{P}^{\mathrm{t}} \mathrm{Bu}_{2} \mathrm{Bn}\right)\right]$ can also lose oxygen under vacuum. ${ }^{297,298}$ The dioxygen ligand in the $[\mathrm{Pt}(t-\mathrm{Bu}-$ thixantphos $\left.)\left(\eta^{2}-\mathrm{O}_{2}\right)\right]$ complex was not observed to dissociate despite heating the reaction under vacuum. This result is not unexpected as the $t$-Bu-thixantphos system is more sterically and electronically similar to $\mathrm{P}^{\mathrm{t}} \mathrm{Bu}_{2} \mathrm{Ph}$ than $\mathrm{P}^{\mathrm{t}} \mathrm{Bu} \mathrm{u}_{2} \mathrm{Bn}$. However, the larger P-Pt-P angle in the $t$-Bu-thixantphos complex $\left(117.26(5)^{\circ}\right)$ compared to the $\mathrm{P}^{\mathrm{t}} \mathrm{Bu}_{2} \mathrm{Ph}$ complex $\left(113.1(2)^{\circ}\right)$ may been expected to promote the loss of the dioxygen ligand. $\underline{307}$

[Pt $(t$-Bu-thixantphos $\left.)\left(\eta^{2}-\mathrm{O}_{2}\right)\right]$ can also be formed through reaction of $[\mathrm{Pt}(t-\mathrm{Bu}-$ thixantphos $\left.\left(\mathrm{C}_{2} \mathrm{H}_{4}\right)\right]$ with air. However, bubbling ethene through an NMR sample of $\left[\mathrm{Pt}(t\right.$-Bu-thixantphos $\left.)\left(\eta^{2}-\mathrm{O}_{2}\right)\right]$ in $\mathrm{C}_{6} \mathrm{D}_{6}$ for 10 minutes showed no conversion to the ethene complex. Leaving the sample under an atmosphere of ethene for a further 48 hours showed no evidence by NMR spectroscopy for conversion to $\left[\mathrm{Pt}(t-\mathrm{Bu}\right.$-thixantphos $\left.)\left(\mathrm{C}_{2} \mathrm{H}_{4}\right)\right]$, indicating that the reaction is not readily reversible. 
Complexes $\left[\mathrm{Pd}\left(\eta^{2}-\mathrm{O}_{2}\right)\left(\mathrm{P}^{\mathrm{t}} \mathrm{Bu}_{2}{ }^{\mathrm{n}} \mathrm{Bu}\right)_{2}\right]$ and $\left[\mathrm{Pd}\left(\eta^{2}-\mathrm{O}_{2}\right)\left(\mathrm{PAd}_{2}{ }^{\mathrm{n}} \mathrm{Bu}\right)_{2}\right](\mathrm{Ad}=1$-adamantyl $)$ have been used as stable precatalysts for palladium catalysed formylations and alkoxycarbonylations due to their reduction to the 14-electron palladium complexes by reaction with hydrogen $(5 \mathrm{bar})$ at $40^{\circ} \mathrm{C}$ for 16 hours. $\frac{313}{\text { Reaction of }}$ $\left[\mathrm{Pt}(t\right.$-Bu-thixantphos $\left.)\left(\eta^{2}-\mathrm{O}_{2}\right)\right]$ with hydrogen gas was attempted by bubbling the gas through the reaction mixture then sealing under an atmosphere of hydrogen. No reaction was observed after three days at room temperature, indicating the stability of the dioxygen complex. However, this cannot be taken as a direct comparison of stability between $\left[\mathrm{Pt}(t\right.$-Bu-thixantphos $\left.)\left(\eta^{2}-\mathrm{O}_{2}\right)\right]$ and the $\left[\mathrm{Pd}\left(\eta^{2}-\right.\right.$ $\left.\left.\mathrm{O}_{2}\right)\left(\mathrm{P}^{\mathrm{t}} \mathrm{Bu}_{2}{ }^{\mathrm{n}} \mathrm{Bu}\right)_{2}\right]$ and $\left[\mathrm{Pd}\left(\eta^{2}-\mathrm{O}_{2}\right)\left(\mathrm{PAd}_{2}{ }^{\mathrm{n}} \mathrm{Bu}\right)_{2}\right](\mathrm{Ad}=1$-adamantyl $)$ complexes, as the reaction was not attempted at the higher temperatures and pressures used in the literature method. ${ }^{313}$

Having established that the reaction of $[\mathrm{Pt}(t-\mathrm{Bu}$-thixantphos $)]$ with dioxygen is not readily reversible, the reactivity of the coordinated dioxygen ligand was investigated. The platinum dioxygen complexes $\left[\mathrm{Pt}\left(\eta^{2}-\mathrm{O}_{2}\right)\left(\mathrm{PR}_{3}\right)_{2}\right]\left(\mathrm{PR}_{3}=\mathrm{PCy}_{3}\right.$, $\mathrm{P}^{\mathrm{i}} \mathrm{Pr}_{3}, \mathrm{P}^{\mathrm{t}} \mathrm{Bu}_{2}{ }^{\mathrm{n}} \mathrm{Bu}, \mathrm{P}^{\mathrm{t}} \mathrm{Bu}_{2} \mathrm{Me}, \mathrm{PPh}_{3}$ ) readily react with alkynes showing 1,2-addition of the dioxygen across the triple bond forming metallacyclic compounds. ${ }^{309}$ Reaction of $\left[\mathrm{Pt}(t-\mathrm{Bu}\right.$-thixantphos $\left.)\left(\eta^{2}-\mathrm{O}_{2}\right)\right]$ with ethyne showed no reaction after two days at room temperature. The absence of any reactivity may be due to the inactivity of ethyne towards dioxygen complexes (the literature examples used electron poor alkynes for this reaction), rather than any inherent stability of the dioxygen complex.

Platinum dioxygen complexes can react with carbon monoxide to form carbonate complexes and with carbon dioxide to form peroxycarbonates. ${ }^{302}$ No reaction was observed between $\left[\mathrm{Pt}(t-\mathrm{Bu}\right.$-thixantphos $\left.)\left(\eta^{2}-\mathrm{O}_{2}\right)\right]$ and carbon dioxide, possibly due to the steric demands of the $t$-Bu-thixantphos ligand. However, the reaction with carbon monoxide proved to be more complex than those previously reported, proceeding through several intermediates before forming a single stable species after seven days at room temperature. In order to assist in identification of these intermediates, the reaction was repeated using ${ }^{13} \mathrm{C}$-enriched $\mathrm{CO}$ and the reaction was followed by ${ }^{1} \mathrm{H},{ }^{13} \mathrm{C}$ and ${ }^{31} \mathrm{P}$ NMR spectroscopy. However, due to the complexity of the system, little information could be gained from the ${ }^{1} \mathrm{H}$ NMR spectra.

The first step in the reaction of $\left[\mathrm{Pt}(t-\mathrm{Bu}\right.$-thixantphos $\left.)\left(\eta^{2}-\mathrm{O}_{2}\right)\right]$ with carbon monox- 
ide results in the expected product, with the carbon monoxide inserting into the $\mathrm{O}-\mathrm{O}$ bond to form a $\mathrm{K}^{2} \mathrm{O}^{1}, \mathrm{O}^{2}$-carbonate ligand. The ${ }^{13} \mathrm{C}$ NMR spectrum contains a triplet peak at $167.9 \mathrm{ppm}\left(J_{\mathrm{PC}}=3.9 \mathrm{~Hz}\right)$ with platinum satellites $\left(J_{\mathrm{PtC}}=64.4 \mathrm{~Hz}\right)$. The ${ }^{31} \mathrm{P}$ NMR spectrum shows an associated peak at $15.6 \mathrm{ppm}$ with $J_{\mathrm{PtP}}$ of 4055 $\mathrm{Hz}$. Previously, this reaction has been observed with $\left[\mathrm{Pt}\left(\eta^{2}-\mathrm{O}_{2}\right)\left(\mathrm{P}^{\mathrm{t}} \mathrm{BuPh}_{2}\right)_{2}\right]$ and $\left[\mathrm{Pt}\left(\eta^{2}-\mathrm{O}_{2}\right)\left(\mathrm{P}^{\mathrm{t}} \mathrm{Bu}_{2}{ }^{\mathrm{n}} \mathrm{Bu}\right)_{2}\right]$ which experienced upfield shifts of 9.7 and $16.3 \mathrm{ppm}$, and a decrease in the value of $J_{\mathrm{PtP}}$ by 364 and $330 \mathrm{~Hz}$ respectively upon reaction with $\mathrm{CO} . \underline{302}$ In the $t$-Bu-thixantphos reaction an upfield shift of $22.8 \mathrm{ppm}$ and a decrease in the value of $J_{\mathrm{PtP}}$ of $433 \mathrm{~Hz}$ was observed. While the shift is greater than expected for the $t$-Bu-thixantphos complex, the chemical shift of $\left[\mathrm{Pt}\left(\eta^{2}-\mathrm{CO}_{3}\right)(\mathrm{PP})\right]$ complexes with mono, or diphosphines varies substantially (-12.0-58.7 ppm), and although reported coupling constants $(3377-3697 \mathrm{~Hz})^{16,302}$ are smaller than for $\left[\mathrm{Pt}(t\right.$-Bu-thixantphos $\left.)\left(\mathrm{CO}_{3}-\mathrm{K}^{2} \mathrm{O}^{1}, \mathrm{O}^{2}\right)\right](4055 \mathrm{~Hz})$ it has been shown throughout this chapter that the $t$-Bu-xantphos platinum complexes typically have larger $J_{\mathrm{PtP}}$ values than other complexes of the same type.

The $\left[\mathrm{Pt}(t\right.$-Bu-thixantphos $\left.)\left(\mathrm{CO}_{3}-\mathrm{K}^{2} \mathrm{O}^{1}, \mathrm{O}^{2}\right)\right]$ complex converts over the course of several days into another intermediate. This intermediate has two different signals in the ${ }^{31} \mathrm{P}$ NMR spectrum in a 1:1 ratio at 50.5 and $-38.7 \mathrm{ppm}$ with platinum satellites of 1893 and $2854 \mathrm{~Hz}$ respectively. This shows a loss of the symmetry of the $t$-Bu-thixantphos ligand with the two phosphorus atoms now appearing in different environments. The large upfield shift $(48.2 \mathrm{ppm}$ from the free ligand) is typical for formation of a four-membered ring including the phosphorus atom. ${ }^{314}$ The phosphorus atom at 50.5 ppm has a coupling constant indicative of a trans ligand with a high trans-influence such as an alkyl group. From this a cis arrangement of the phosphorus atoms with metallation of one of the tert-butyl substituents creating an alkyl group trans to the non-metallated phosphorus can be proposed. If metallation had occurred via a C-H activation an upfield signal in the ${ }^{1} \mathrm{H}$ NMR spectrum would be expected indicative of a platinum hydride. No such peak was observed, instead a strongly downfield ${ }^{1} \mathrm{H}$ signal was observed at $17.6 \mathrm{ppm}$ indicating the presence of an acidic proton. From this information the conversion of the $\mathrm{k}^{2} \mathrm{O}^{1}, \mathrm{O}^{2}$-carbonate ligand into a $\mathrm{k}^{2} \mathrm{O}^{1}, \mathrm{O}^{2}$-bicarbonate incorporating the hydrogen from the metallated tert-butyl group is proposed. Unfortunately this complex is too short-lived to investigate by ${ }^{13} \mathrm{C}$ NMR spectroscopy

The final product in the reaction of $\left[\mathrm{Pt}(t-\mathrm{Bu}\right.$-thixantphos $\left.)\left(\eta^{2}-\mathrm{O}_{2}\right)\right]$ with carbon 
monoxide forms by loss of carbon dioxide gas (observed in the ${ }^{13} \mathrm{C} \mathrm{NMR} \mathrm{spec-}$ trum) from the $\left[\mathrm{Pt}\left(t-\mathrm{Bu}\right.\right.$-thixantphos- $\left.\left.\mathrm{H}-\kappa P, P^{\prime}, C\right)\left(\mathrm{O}_{2} \mathrm{COH}-\kappa^{2} \mathrm{O}^{1}, \mathrm{O}^{2}\right)\right]$ complex. The final product shows two peaks in the ${ }^{31} \mathrm{P} N M R$ spectrum: one at $-49.2 \mathrm{ppm}\left(J_{\mathrm{PtP}}=\right.$ $3943 \mathrm{~Hz})$ and the other at $38.2 \mathrm{ppm}\left(J_{\mathrm{PtP}}=1794 \mathrm{~Hz}\right)$. This indicates the retention of the $t$-Bu-thixantphos ligand with one metallated tert-butyl group from the bicarbonate complex. The platinum-phosphorus coupling constants show that the non-metallated phosphorus is trans to the metallated tert-butyl group, while the metallated phosphorus is trans to a ligand with a weak trans influence. This ligand is proposed to be a hydroxyl, as this would result from loss of $\mathrm{CO}_{2}$ from a bicarbonate, and hydroxyl ligands have a low trans influence. The final product shows a signal in the ${ }^{13} \mathrm{C}$ NMR spectrum for the metallated carbon at $15.7 \mathrm{ppm}$ as a doublet of doublets with coupling of $81.6 \mathrm{~Hz}$ to the trans phosphorus and $35.6 \mathrm{~Hz}$ to the cis phosphorus, though no platinum satellites could be observed. The position of this peak is consistent with other platinum alkyls that occur trans to phosphorus. ${ }^{315}-\underline{317}$ No signal for the hydroxyl proton was observed in the ${ }^{1} \mathrm{H}$ NMR spectrum, which may indicate exchange with a deuterium from the solvent $\left(\mathrm{CD}_{2} \mathrm{Cl}_{2}\right)$. The mass spectrum was also unable to confirm the presence of a hydroxyl ligand as a peak was observed for $[\mathrm{M}-\mathrm{OH}]^{+}$, as transition metal complexes frequently ionise by loss of weakly coordinated ligands. 1210 The overall reaction showing the intermediates is given in Scheme 5.6.

The final two structures in the reaction of $\left[\mathrm{Pt}(t-\mathrm{Bu}\right.$-thixantphos $\left.)\left(\eta^{2}-\mathrm{O}_{2}\right)\right]$ with carbon monoxide (Scheme 5.6) are particularly noteworthy due to the cis coordination of the phosphines. Metallation of i-Pr-xantphos has been reported on iridium $\underline{\underline{ }}$ with $i$-Pr-xantphos coordinating in a $\kappa P, P^{\prime}, O, C$ mode. However, the present example of $\left[\mathrm{Pt}\left(t-\mathrm{Bu}\right.\right.$-thixantphos- $\left.\mathrm{H}-\kappa\left(P, P^{\prime}, C\right) \mathrm{OH}\right]$ is the only example of $\kappa P, P^{\prime}, C$ coordination of a xantphos ligand. Sterically bulky ligands show a distinct preference for trans coordination (discussed further in Chapter 6). The metallacyclobutane must coordinate in two cis sites. However, the $t$-Bu-xantphos could still maintain a trans coordination. It is plausible that metallation of the tert-butyl group relieves the steric constraints of the system allowing for cis chelation of the phosphorus atoms. The P-Pt-C angle in the metallacyclobutane is also likely to be less than $90^{\circ}$ resulting in additional room around the platinum centre.

Despite such unexpected chemistry with $\mathrm{CO},\left[\mathrm{Pt}(t\right.$-Bu-thixantphos $\left.)\left(\eta^{2}-\mathrm{O}_{2}\right)\right]$ shows 


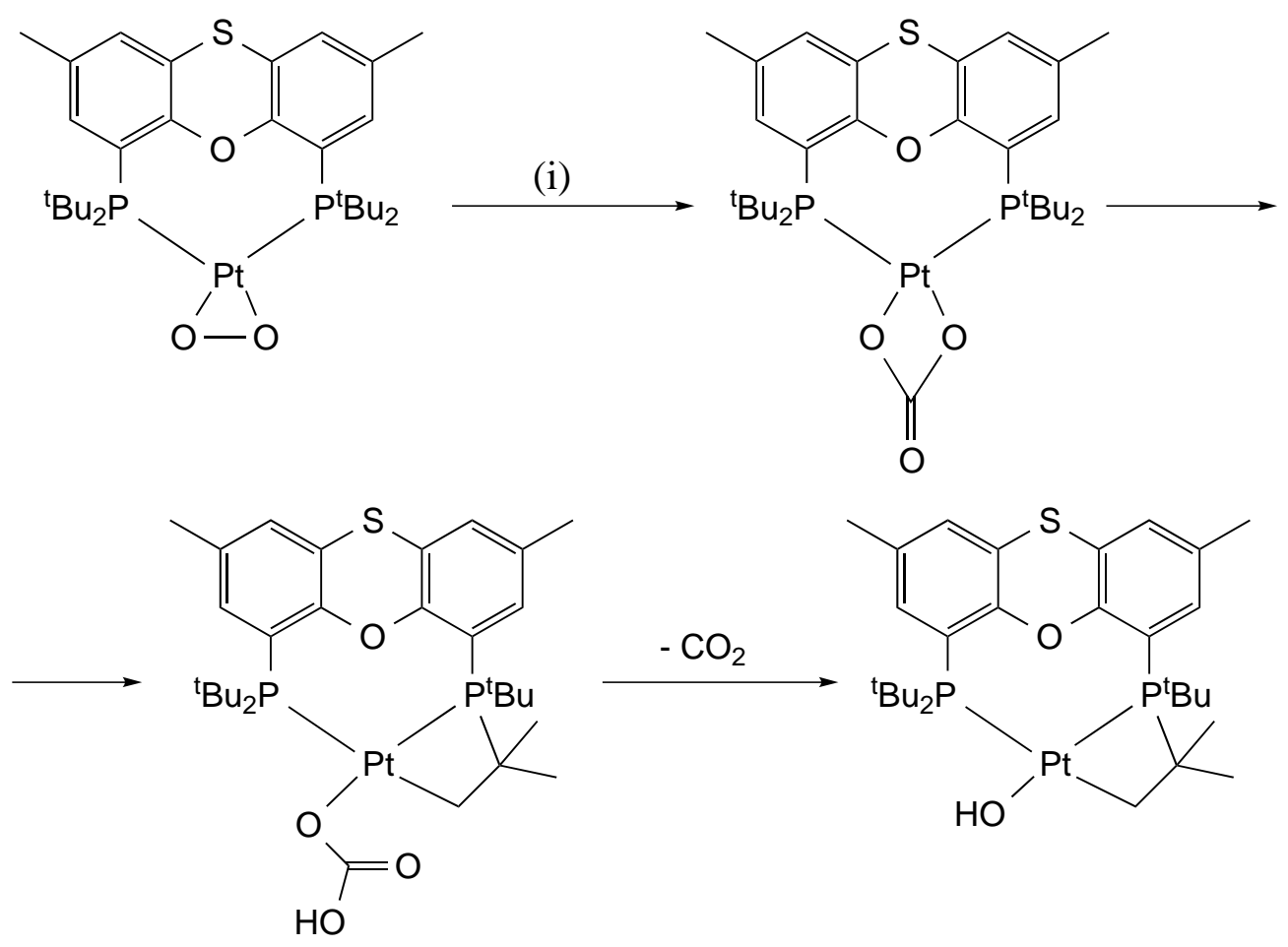

Scheme 5.6: Reaction between $\left[\mathrm{Pt}(t\right.$-Bu-thixantphos $\left.)\left(\eta^{2}-\mathrm{O}_{2}\right)\right]$ and CO.

a distinct stability towards other small molecules. No reaction with ammonium hexafluorophosphate or with methane was observed. However, a straightforward reaction does occur with pta. Pta is a phosphine ligand with a very small cone angle making it an ideal choice to react with the sterically encumbered system. ${ }^{318}$ Addition of pta to a solution of $\left[\mathrm{Pt}(t-\mathrm{Bu}\right.$-thixantphos $\left.)\left(\eta^{2}-\mathrm{O}_{2}\right)\right]$ showed immediate changes in the ${ }^{31} \mathrm{P}$ NMR spectrum, with new peaks appearing at 9.5 and $-74.1 \mathrm{ppm}\left(J_{\mathrm{PtP}}=3562 \mathrm{~Hz}\right)$. The peak at $9.5 \mathrm{ppm}$ did not show any coupling to platinum and is consistent with an uncoordinated $t$-Bu-thixantphos ligand. The peak at $-74.1 \mathrm{ppm}$ is consistent with the literature reported $\left[\mathrm{Pt}(\mathrm{pta})_{4}\right]$ complex. ${ }^{319}$ The ${ }^{1} \mathrm{H}$ and ${ }^{13} \mathrm{C}$ NMR data confirm these assignments. No intermediates were observed due to the speed of the reaction. This suggests that pta is able to displace both the dioxygen and the $t$-Bu-thixantphos ligand from the platinum, and thus is too reactive to gain any insight into the activation of the dioxygen moiety. 


\subsection{Reactions with Palladium(0) Precursors}

Platinum complexes are frequently synthesised as models for the analogous palladium species as they are frequently more stable, and the presence of ${ }^{195} \mathrm{Pt}$ allows for additional information in the NMR spectra, which can be useful in characterisation. However, palladium complexes are of particular interest due to their use in a wide range of catalytic processes. These systems often involve both palladium(0) and palladium(II) complexes. A number of papers regarding the catalytic activity of palladium $t$-Bu-xantphos systems have also been reported though no investigation into the coordination chemistry has been published. As such, the reactivity of the $t$-Bu-xantphos ligands towards palladium(0) starting materials was investigated. All reactions discussed in this section were carried out on an NMR scale.

Initial investigations focussed on the reactivity of $t$-Bu-thixantphos towards palladium(0) precursors commonly used in catalysis. Allyl-(cyclopentadienyl)palladium(II) $\left[\mathrm{Pd}\left(\mathrm{C}_{3} \mathrm{H}_{5}\right) \mathrm{Cp}\right]$ can react with phosphine ligands to give palladium(0) complexes. The reaction between $t$-Bu-thixantphos and $\left[\mathrm{Pd}\left(\mathrm{C}_{3} \mathrm{H}_{5}\right) \mathrm{Cp}\right]$ in $\mathrm{C}_{6} \mathrm{D}_{6}$ was slow. After 24 hours at room temperature no changes in the NMR spectra were observed. After 48 hours at $50{ }^{\circ} \mathrm{C}$ no unreacted $t$-Bu-thixantphos was observed in the ${ }^{31} \mathrm{P}$ NMR spectrum. However, the ${ }^{31} \mathrm{P}$ and ${ }^{1} \mathrm{H}$ NMR spectra showed a number of different products, which were unable to be separated or analysed further.

$\left[\mathrm{Pd}_{2}(\mathrm{dba})_{3}\right](\mathrm{dba}=$ dibenzylideneacetone $)$ is another common palladium $(0)$ complex used as a precatalyst. The reaction between $t$-Bu-thixantphos and $\left[\operatorname{Pd}_{2}(\mathrm{dba})_{3}\right]$ generated a mixture of products. However, a single major product was observed at 40.0 ppm in the ${ }^{31} \mathrm{P}$ NMR spectrum $\left(\mathrm{C}_{6} \mathrm{D}_{6}\right)$, after heating at $40{ }^{\circ} \mathrm{C}$ for four days. The major product integrated for $67 \%$ of the ${ }^{31} \mathrm{P}$ containing species while uncoordinated $t$-Bu-thixantphos accounted for $17 \%$ of the reaction mixture. The species was not $[\mathrm{Pd}(t-\mathrm{Bu}$-thixantphos)] (this will be discussed shortly). However, the product was unable to be isolated from the reaction mixture and the presence of large amounts of uncoordinated dba hampered the further characterisation of this species. It is possible that the species was $[\mathrm{Pd}(t$-Bu-thixantphos $)(\mathrm{dba})]$ although little evidence to support this could be obtained.

Platinum alkene complexes, such as the air-stable solids $\left[\mathrm{Pt}(\operatorname{cod})_{2}\right]$ and $\left[\mathrm{Pt}(\mathrm{nb})_{3}\right]$, 
make excellent starting materials for the formation of platinum(0) complexes. ${ }^{291,299}$ Although $\left[\mathrm{Pt}\left(\mathrm{C}_{2} \mathrm{H}_{4}\right)_{3}\right]$ is only stable under an ethene atmosphere it is readily synthesised in situ, via reaction of ethene with $\left[\mathrm{Pt}(\mathrm{cod})_{2}\right] \cdot \frac{320}{}$ Unfortunately the corresponding palladium complexes are typically unstable at room temperature in solution and are not practical to be used as starting materials. ${ }^{321}$ However, given the slow reactivity of $t$-Bu-thixantphos with $\left[\mathrm{Pd}\left(\mathrm{C}_{3} \mathrm{H}_{5}\right) \mathrm{Cp}\right]$ and $\left[\mathrm{Pd}_{2}(\mathrm{dba})_{3}\right]$, coupled with the desire to investigate the analogues of the platinum alkene complexes discussed earlier, the reactivity of $t$-Bu-thixantphos towards $\left[\mathrm{Pd}(\mathrm{nb})_{3}\right]$ was studied. $\left[\mathrm{Pd}(\mathrm{nb})_{3}\right]$, like other palladium alkene complexes, is unstable in solution, with significant degradation observed at room temperature. ${ }^{321}$ However, the amount of degradation can be reduced by addition of excess norbornene to the reactions.

Reaction of $t$-Bu-thixantphos with $\left[\mathrm{Pd}(\mathrm{nb})_{3}\right]$ was rapid compared to the other palladium(0) starting materials, going to completion in 72 hours at room temperature. The reaction formed a mixture of $[\mathrm{Pd}(t-\mathrm{Bu}$-thixantphos)(nb)] (38.0\%) and $[\mathrm{Pd}(t$-Bu-thixantphos)] (62.0\%). As such, the reaction was also carried out with $t$ Bu-sixantphos and $t$-Bu-xantphos. The reaction of $t$-Bu-xantphos with $\left[\mathrm{Pd}(\mathrm{nb})_{3}\right]$ was complete in under one hour forming a mixture including, $[\mathrm{Pd}(t-\mathrm{Bu}$-xantphos)(nb)] (12.6\%) and [Pd(t-Bu-xantphos)] (73.1\%). Using $t$-Bu-sixantphos resulted in only $43.4 \%$ product in the ${ }^{31} \mathrm{P}$ NMR spectrum after 24 hours (the remainder being free ligand). No further progress in the reaction between $t$-Bu-sixantphos and $\left[\mathrm{Pd}(\mathrm{nb})_{3}\right]$ was observed by NMR spectroscopy after 48 hours. However, after five months at room temperature the reaction had progressed to $68 \%$ complex indicating that the lack of reactivity was not necessarily due to degradation of the $\left[\mathrm{Pd}(\mathrm{nb})_{3}\right]$.

The major products from the reaction of the $t$-Bu-xantphos ligands with $\left[\mathrm{Pd}(\mathrm{nb})_{3}\right]$ appear at 41.9-42.9 ppm in the ${ }^{31} \mathrm{P}$ NMR spectra. Given the similarity in the ${ }^{31} \mathrm{P}$ chemical shift and the inability to isolate the $t$-Bu-sixantphos and $t$-Bu-xantphos complexes, the $t$-Bu-thixantphos complex was the only product explored in depth. Once the product was formed, the norbornene was removed under vacuum with no degradation of the resulting complex. The complex displays no resonances in the ${ }^{1} \mathrm{H}$ and ${ }^{13} \mathrm{C}$ NMR spectra apart from those corresponding to a coordinated $t$-Bu-thixantphos ligand. The quarternary and terminal tert-butyl carbon and proton signals appear as virtual triplets, consistent with a trans coordination of phosphorus atoms. Hence, the complexes formed in these three 
reactions are $[\mathrm{Pd}(t-\mathrm{Bu}-\mathrm{xantphos})]$ species (Scheme 5.7).<smiles>[R6]c1cc([R6])c2c(c1)[X]c1cc([R])cc(P)c1O2</smiles>

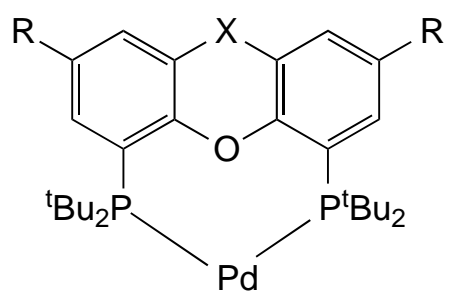

Scheme 5.7: Formation of the major product in the reaction between the $t$-Buxantphos ligands and $\left[\mathrm{Pd}(\mathrm{nb})_{3}\right]$. Reagents and conditions: (i) $\left[\mathrm{Pd}(\mathrm{nb})_{3}\right], \mathrm{C}_{6} \mathrm{D}_{6}$.

\subsubsection{Reaction of [Pd(t-Bu-thixantphos)] with Oxygen}

The $[\mathrm{Pt}(t-\mathrm{Bu}$-thixantphos $)]$ complex reacts rapidly and irreversibly with dioxygen to form [Pt $\left(t-\mathrm{Bu}\right.$-thixantphos) $\left.\left(\eta^{2}-\mathrm{O}_{2}\right)\right]$ (Section [5.5). Previous examples have indicated that the coordination of dioxygen to palladium is frequently reversible even if the platinum analogue is not. $\frac{307}{} \operatorname{Reaction}$ of $[\mathrm{Pd}(t-\mathrm{Bu}$-thixantphos)] with oxygen occurs rapidly forming a green $\left[\mathrm{Pd}(t-\mathrm{Bu}\right.$-thixantphos $\left.)\left(\eta^{2}-\mathrm{O}_{2}\right)\right]$ complex. The ${ }^{31} \mathrm{P}$ NMR spectrum shows a smaller change in chemical shift than is observed with the platinum analogue (9.1 compared to $40.2 \mathrm{ppm}$ ) and the shift is downfield rather than upfield as in the platinum case. This is likely due to marked differences in the 14-electron complexes rather than the dioxygen complexes as the ${ }^{31} \mathrm{P}$ NMR signal for [ $\mathrm{Pt}(t-\mathrm{Bu}$-thixantphos $)]$ appears at $78.6 \mathrm{ppm}$, whereas the signal for $[\mathrm{Pd}(t$-Bu-thixantphos $)]$ is at $42.9 \mathrm{ppm}$. The tert-butyl signals in the ${ }^{1} \mathrm{H}$ and ${ }^{13} \mathrm{C}$ NMR spectra of $\left[\mathrm{Pd}(t-\mathrm{Bu}\right.$-thixantphos $\left.)\left(\eta^{2}-\mathrm{O}_{2}\right)\right]$ appear as doublets indicating the loss of the trans arrangement of the phosphorus atoms.

The reaction of $[\mathrm{Pd}(t-\mathrm{Bu}$-thixantphos $)]$ with dioxygen was observed to be readily reversible. Removal of the solvent from the NMR sample under vacuum for a few minutes was sufficient for the dioxygen to dissociate and undergo complete reversion to $[\mathrm{Pd}(t-\mathrm{Bu}$-thixantphos $)]$. This is consistent with the literature, which indicates that coordination of dioxygen to palladium complexes is typically reversible. $\frac{307}{\sqrt{3}}$ This may be due to the reduced back-bonding of palladium compared to platinum, which results in less activation of the O-O bond upon coordination, such that on palladium the dioxygen is more like a neutral $\mathrm{O}_{2}$ ligand, 
whereas on platinum the dioxygen becomes more like a peroxy $\mathrm{O}_{2}{ }^{2-}$.

\subsection{Computational Results}

It has been observed throughout this chapter that the differences in the coordination chemistry of the three $t$-Bu-xantphos ligands with platinum and palladium is subtle, often forming mixtures of products and some readily reversible systems. However, a number of the complexes were unable to be isolated and fully characterised. As such, we have investigated the conversion of the [M( $t$-Bu-xantphos $)]$ complexes to $[\mathrm{M}(t$-Bu-xantphos $)(\mathrm{nb})]$ or $\left[\mathrm{M}(t\right.$-Bu-xantphos $\left.)\left(\eta^{2}-\mathrm{O}_{2}\right)\right](\mathrm{M}=\mathrm{Pd}, \mathrm{Pt})$ using DFT. Structural models of the complexes were optimised and their vibrational frequencies calculated using a B3LYP functional,, $131-134$ with the def2-TZVP basis set. 135,136

Selected bond lengths and angles are given in Tables 5.8, 5.9 and 5.10] for [M(t-Buxantphos $)],[\mathrm{M}(t$-Bu-xantphos $)(\mathrm{nb})]$ and $\left[\mathrm{M}(t-\mathrm{Bu}\right.$-xantphos $\left.)\left(\eta^{2}-\mathrm{O}_{2}\right)\right]$ respectively. The P-M-P angles are larger in the $[\mathrm{M}(t-\mathrm{Bu}-\mathrm{xantphos})]$ complexes than in the $[\mathrm{M}(t$-Bu-xantphos $)(\mathrm{nb})]$ and $\left[\mathrm{M}(t\right.$-Bu-xantphos $\left.)\left(\eta^{2}-\mathrm{O}_{2}\right)\right]$ as expected. However, the P-M-P angles in the $[\mathrm{M}(t-\mathrm{Bu}$-xantphos $)]$ complexes are smaller than the idealised $180^{\circ}$ expected for a two-coordination platinum( 0 ) complex. The rigid backbone of the $t$-Bu-xantphos ligands likely prevents larger bite-angles from forming without introducing coordination to the oxygen bridge in the backbone. In this case the $\mathrm{M}-\mathrm{O}$ distances are larger than expected for $\mathrm{Pt}-\mathrm{O}$ or $\mathrm{Pd}-\mathrm{O}$ bonds, indicating that no bonding interaction is present. The $\mathrm{C}=\mathrm{C}$ and $\mathrm{O}-\mathrm{O}$ distances for the norbornene in $[\mathrm{M}(t-\mathrm{Bu}$-xantphos $)(\mathrm{nb})]$ and the dioxygen in $[\mathrm{M}(t-\mathrm{Bu}$-xantphos $)\left(\eta^{2}-\mathrm{O}_{2}\right)$ ] are longer on platinum than palladium, consistent with the higher $\pi$-aciditiy of platinum compared to palladium. ${ }^{280}$

The Gibbs free energies of the complexes were calculated and the values of $\Delta G$ for the reaction of the $[\mathrm{M}(t-\mathrm{Bu}$-xantphos $)](\mathrm{M}=\mathrm{Pd}, \mathrm{Pt})$ complexes with norbornene were calculated (Table 5.11). The value of $\Delta G$ for the reaction between the $[\mathrm{M}(t-\mathrm{Bu}-\mathrm{xantphos})]$ complexes and norbornene is positive, indicating that the reaction would not occur spontaneously and the 14-electron complex is the lower energy system. This is consistent with the experimental results for the palladium complexes. However, in the platinum system mixtures of the norbornene and the 14-electron complexes were observed for both $t$-Bu-sixantphos and $t$ - 
Table 5.8: Calculated bond lengths and angles for [M(t-Bu-xantphos)].

\begin{tabular}{lcccc}
\hline Ligand & $\mathbf{P - P t - P} /{ }^{\circ}$ & $\mathbf{P - P d - P} /{ }^{\circ}$ & $\mathbf{P t}-\mathbf{O} / \AA$ & $\mathbf{P d - O} / \AA$ \\
\hline$t$-Bu-Sixantphos & 152.5 & 150.2 & 2.7982 & 2.7954 \\
$t$-Bu-Thixantphos & 151.8 & 149.4 & 2.8290 & 2.8283 \\
$t$-Bu-Xantphos & 151.5 & 149.3 & 2.7791 & 2.7723 \\
\hline
\end{tabular}

Table 5.9: Calculated bond lengths and angles for [M(t-Bu-xantphos)(nb)].

\begin{tabular}{lcccc}
\hline Ligand & $\mathbf{P - P t - P} /{ }^{\circ}$ & $\mathbf{P - P d - P} /{ }^{\circ}$ & $\mathbf{C}=\mathbf{C}(\mathbf{P t}) / \AA$ & $\mathbf{C}=\mathbf{C}(\mathbf{P d}) / \AA$ \\
\hline$t$-Bu-Sixantphos & 114.8 & 115.4 & 1.4359 & 1.4055 \\
$t$-Bu-Thixantphos & 115.6 & 116.2 & 1.4353 & 1.4046 \\
$t$-Bu-Xantphos & 116.0 & 116.6 & 1.4355 & 1.4055 \\
\hline
\end{tabular}

Table 5.10: Calculated bond lengths and angles for [M(t-Bu-xantphos $\left.)\left(\eta^{2}-\mathrm{O}_{2}\right)\right]$.

\begin{tabular}{lcccc}
\hline Ligand & $\mathbf{P}-\mathbf{P t}-\mathbf{P} /{ }^{\circ}$ & $\mathbf{P - P d}-\mathbf{P} /{ }^{\circ}$ & $\mathbf{O}-\mathbf{O}(\mathbf{P t}) / \AA$ & $\mathbf{O - O}(\mathbf{P d}) / \AA$ \\
\hline$t$-Bu-Sixantphos & 115.3 & 118.7 & 1.4083 & 1.3714 \\
$t$-Bu-Thixantphos & 116.8 & 118.6 & 1.4078 & 1.3710 \\
$t$-Bu-Xantphos & 117.2 & 119.6 & 1.4070 & 1.3705 \\
\hline
\end{tabular}


Bu-thixantphos. In the case of $t$-Bu-xantphos, the [Pt(t-Bu-xantphos)] complex was formed initially but this converted to $\left[\mathrm{Pt}\left(t-\mathrm{Bu} \text {-xantphos- } k P, O, P^{\prime}\right) \mathrm{H}\right]^{+}$. This hydride complex is likely the result of the 14-electron complex undergoing reaction with another component of the system. Although the [Pt( $t$-Bu-xantphos)] complexes are formed as the major products experimentally, an equilibrium with the norbornene complex is likely present. These calculations do not take into account the effect of having a large excess of norbornene present in solution, which may result in an equilibrium between the $[\mathrm{Pt}(t-\mathrm{Bu}$-xantphos $)]$ and $[\mathrm{Pt}(t-\mathrm{Bu}$-xantphos)(nb)] complexes.

The values for the Gibbs free energies of the $\left[\mathrm{M}(t-\mathrm{Bu}\right.$-xantphos $\left.)\left(\eta^{2}-\mathrm{O}_{2}\right)\right](\mathrm{M}=\mathrm{Pd}$, $\mathrm{Pt})$ complexes were also calculated together with the $\Delta G$ values for their formation from the $[\mathrm{M}(t$-Bu-xantphos)] complexes (Table 5.12). All of the $\Delta G$ values are negative, indicating that the formation of the dioxygen complex is spontaneous. The $\Delta G$ values for the palladium complexes are more negative than the platinum ones. This suggests that the palladium complexes should be more stable than the platinum system to reversibility. However, this was not observed experimentally for the $t$-Bu-thixantphos system where the platinum dioxygen complex was very stable and the palladium dioxygen complex readily converted to the $[\mathrm{Pd}(t$-Bu-thixantphos)] species. From Table 5.10 we can see that the P-M-P angle is consistently larger in the palladium complexes than the platinum, while the $\mathrm{O}-\mathrm{O}$ bond length is smaller for the palladium complexes. This suggests that there is less back-donation from the metal centre into the oxygen $\pi^{*}$-orbital. Hence, the dioxygen ligand on palladium is less strongly coordinated than on platinum and may have a lower activation barrier towards dissociation.

\subsection{Summary}

Ph-Thixantphos was shown to react with $\left[\mathrm{Pt}(\mathrm{nb})_{3}\right]$ and $\left[\mathrm{Pt}\left(\mathrm{C}_{2} \mathrm{H}_{4}\right)_{3}\right]$ forming $[\mathrm{Pt}(\mathrm{Ph}$ thixantphos $)_{2}$ ] as the major product in both 1:1 and 2:1 reactions. [ $\mathrm{Pt}(\mathrm{Ph}$-thixantphos)(alkene)] (alkene $=\mathrm{nb}, \mathrm{C}_{2} \mathrm{H}_{4}$ ) were present as minor components in the 1:1 reactions. [Pt(Ph-thixantphos)] was characterised by $\mathrm{X}$-ray crystallography showing a tetrahedral environment around the platinum. The ${ }^{1} \mathrm{H}$ and ${ }^{31} \mathrm{P}$ NMR spectra of $[\mathrm{Pt}(\mathrm{Ph}$-thixantphos $)]$ at room temperature are broad, possibly due to inversion of the backbone. However, cooling to $-40^{\circ} \mathrm{C}$ resolved a pair of triplets 
Table 5.11: Gibbs free energies calculated for reaction of $[\mathrm{M}(t-\mathrm{Bu}-\mathrm{xantphos})]$ with norbornene $(\mathrm{M}=\mathrm{Pd}, \mathrm{Pt})$. All values are in $\mathrm{kJ} \mathrm{mol}^{-1}$, Norbornene $G=-715998.2 \mathrm{~kJ} \mathrm{~mol}^{-1}$.

\begin{tabular}{lcccccc}
\hline $\mathbf{P P}$ & {$[\mathbf{P d}(\mathbf{P P})]$} & {$[\mathbf{P d}(\mathbf{P P})(\mathbf{n b})]$} & $\mathbf{P d} \Delta \boldsymbol{G}$ & {$[\mathbf{P t}(\mathbf{P P})]$} & {$[\mathbf{P t}(\mathbf{P P})(\mathbf{n b})]$} & $\mathbf{P t} \Delta \boldsymbol{G}$ \\
\hline$t$-Bu-Sixantphos & -6163451.1 & -6879350.9 & 98.4 & -6140995.8 & -6856897.4 & 96.6 \\
$t$-Bu-Thixantphos & -6445645.2 & -7161551.4 & 92.1 & -6423187.2 & -7139098.3 & 87.1 \\
$t$-Bu-Xantphos & -5503258.5 & -6219169.0 & 87.8 & -5480799.2 & -6196709.7 & 87.7 \\
\hline
\end{tabular}


Table 5.12: Gibbs free energies calculated for reaction of $[\mathrm{M}(t-\mathrm{Bu}$-xantphos $)]$ with oxygen $(\mathrm{M}=\mathrm{Pd}, \mathrm{Pt})$. All values are in kJ mol ${ }^{-1}$ oxygen $G=-394857.8 \mathrm{~kJ} \mathrm{~mol}^{-1}$.

\begin{tabular}{lcccccc}
\hline $\mathbf{P P}$ & {$[\mathbf{P d}(\mathbf{P P})]$} & {$\left[\mathbf{P d}\left(\eta-\mathbf{O}_{2}\right)(\mathbf{P P})\right]$} & $\mathbf{P d} \Delta \mathbf{G}$ & {$[\mathbf{P t}(\mathbf{P P})]$} & {$\left[\mathbf{P t}\left(\eta-\mathbf{O}_{2}\right)(t-B u-x a n t p h o s)\right]$} & $\mathbf{P t} \Delta \boldsymbol{G}$ \\
\hline$t$-Bu-Sixantphos & -6163451.1 & -6558323.8 & -14.8 & -6140995.8 & -6535862.4 & -8.8 \\
$t$-Bu-Thixantphos & -6445645.2 & -6840520.9 & -17.8 & -6423187.2 & -6818062.4 & -17.4 \\
$t$-Bu-Xantphos & -5503258.5 & -5898134.9 & -18.6 & -5480799.2 & -5875674.2 & -17.2 \\
\hline
\end{tabular}


with platinum satellites.

No evidence for a bis(diphosphine) complex was observed in the reactions of the $t$-Bu-xantphos ligands with $\left[\mathrm{Pt}(\mathrm{nb})_{3}\right],\left[\mathrm{Pt}(\mathrm{cod})_{2}\right],\left[\mathrm{Pt}\left(\mathrm{C}_{2} \mathrm{H}_{4}\right)_{3}\right]$, and $\left[\mathrm{Pd}(\mathrm{nb})_{3}\right]$ starting materials. Reaction with $\left[\mathrm{Pd}(\mathrm{nb})_{3}\right]$ formed mixtures with $[\mathrm{Pd}(t-\mathrm{Bu}$-xantphos)] as the major product. Similarly, reaction of $t$-Bu-thixantphos with $\left[\mathrm{Pt}(\mathrm{nb})_{3}\right]$ formed a mixture of [Pt( $t$-Bu-thixantphos)] and $[\mathrm{Pt}(t-\mathrm{Bu}$-thixantphos $)(\mathrm{nb})]$. Analogues of these two complexes were also observed in the analogous reaction with $t$-Bu-sixantphos, in addition to an unknown complex with a coupling constant indicative of platinum(II). The reaction between $\left[\mathrm{Pt}(\mathrm{nb})_{3}\right]$ and $t$-Bu-xantphos formed $[\mathrm{Pt}(t-\mathrm{Bu}-\mathrm{xantphos})]$ as a minor component which converted into [Pt(t-Bu-xantphos)H]X.

The reactions of the $t$-Bu-xantphos ligands with $\left[\mathrm{Pt}\left(\mathrm{C}_{2} \mathrm{H}_{4}\right)_{3}\right]$ resulted in $[\mathrm{Pt}(t$ Bu-xantphos $)\left(\mathrm{C}_{2} \mathrm{H}_{4}\right)$ ] complexes. [ $\mathrm{Pt}(t$-Bu-xantphos $)\left(\mathrm{C}_{2} \mathrm{H}_{4}\right)$ ] went on to produce $\left[\mathrm{Pt}\left(t\right.\right.$-Bu-xantphos)H]X. Reacting $t$-Bu-thixantphos with $\left[\mathrm{Pt}(\operatorname{cod})_{2}\right]$ formed only [Pt(t-Bu-thixantphos)], though the yield was low due to degradation.

[Pd(t-Bu-thixantphos)], [Pt(t-Bu-thixantphos)], and [Pt(t-Bu-thixantphos $\left.)\left(\mathrm{C}_{2} \mathrm{H}_{4}\right)\right]$ all react rapidly with air forming $\left[\mathrm{M}(t\right.$-Bu-thixantphos $\left.)\left(\eta^{2}-\mathrm{O}_{2}\right)\right](\mathrm{M}=\mathrm{Pd}, \mathrm{Pt})$. With palladium this reaction was readily reversibly in a few minutes under vacuum. On platinum the coordination of oxygen was not reversible either by vacuum, or reaction with $\mathrm{C}_{2} \mathrm{H}_{4}$ or $\mathrm{H}_{2}$. [ $\mathrm{Pt}(t$-Bu-thixantphos $)\left(\eta^{2}-\mathrm{O}_{2}\right)$ ] was characterised by Xray crystallography, displaying a planar molecule with a bent $t$-Bu-thixantphos backbone. The bite-angle was 117.26(5), smaller than the natural bite-angle of $\left(126.98^{\circ}\right)$, but the largest P-Pt-P angle of the five $\left[\mathrm{Pt}\left(\eta^{2}-\mathrm{O}_{2}\right)\left(\mathrm{P}_{2}\right)\right]$ complexes that have been characterised crystallographically.

[Pt $(t$-Bu-thixantphos $\left.)\left(\eta^{2}-\mathrm{O}_{2}\right)\right]$ was tested for reactivity towards a range of different small molecules. No reaction was observed with ethyne, ammonium hexafluorophosphate or carbon dioxide. Reaction with the small phosphine pta produce uncoordinated $t$-Bu-thixantphos and $\left[\mathrm{Pt}(\mathrm{pta})_{4}\right]$, going to completion when four equivalents of pta were used. Reaction of $\left[\mathrm{Pt}(t\right.$-Bu-thixantphos $\left.)\left(\eta^{2}-\mathrm{O}_{2}\right)\right]$ with carbon monoxide initially formed the expected carbonate complex. However, this continued to react, undergoing metallation of a tert-butyl methyl to form a bicarbonate, which loses carbon dioxide leaving a hydroxyl ligand coordinated to the platinum centre. 
The $[\mathrm{M}(t$-Bu-xantphos $)],[\mathrm{M}(t$-Bu-xantphos $)(\mathrm{nb})]$, and [M(t-Bu-xantphos $\left.)\left(\eta^{2}-\mathrm{O}_{2}\right)\right]$ $(\mathrm{M}=\mathrm{Pd}, \mathrm{Pt})$ complexes were explored computationally. The $\mathrm{O}-\mathrm{O}$ bond were longer in the platinum complexes than in the palladium, which may explain the ready dissociation of the dioxygen from the palladium complex while the platinum complex was not reversible. The $\Delta G$ values for the formation of the norbornene and the dioxygen complexes from the 14-electron complexes were also calculated. The results indicated that the formation of the dioxygen complex is spontaneous, although the formation of the norbornene complex from the $[\mathrm{Pt}(t-$ Bu-xantphos)] complexes is not. While this does not take into account the effect of having excess norbornene in solution which may effect the position of the equilibrium.

Overall this chapter provides an account into the coordination chemistry of $t$ $\mathrm{Bu}$-sixantphos, $t$-Bu-thixantphos, and $t$-Bu-xantphos with platinum( $(0)$ and palla$\operatorname{dium}(0)$ and the reactivity of $\mathrm{Ph}$-thixantphos towards $\left[\mathrm{Pt}(\mathrm{nb})_{3}\right]$ and $\left[\mathrm{Pt}\left(\mathrm{C}_{2} \mathrm{H}_{4}\right)_{3}\right]$. Several new complexes have been synthesised including two characterised by $\mathrm{X}$ ray crystallography. In a number of cases the reactivity of the three $t$-Bu-xantphos ligands was different, indicating the role of the bite-angle on the coordination chemistry of these compounds. 


\section{Chapter 6}

\section{Coordination Complexes with Platinum(II) and Palladium(II)}

Coordination complexes of palladium are used as catalysts for a wide range of reactions including cross-coupling, allylic alkylation and various carbonylation reactions, which are important on both laboratory and industrial scales. $., 32,149,273-279$ The high reactivity of palladium complexes can render them difficult to characterise and study. Platinum and palladium have similar overall reactivity patterns. However, platinum complexes are typically more inert than their corresponding palladium analogues to processes such as ligand exchange and redox changes. ${ }^{280}$ The presence of a spin active isotope ${ }^{195} \mathrm{Pt}$ (34\% abundant) can result in coupling in the NMR spectra, which can give additional information about the nature of the complexes. This makes platinum a useful model for the chemistry of palladium.

Seven reports have been published investigating the catalytic activity of palladium complexes using $t$-Bu-xantphos. These include the $\mathrm{N}$-arylation and alkylation of amines, cross-coupling of thiols and aryl bromides or triflates, Suzuki cross-coupling, hydroesterificaton of methyl oleate, aminocarbonylation of hetero(aryl) bromides, and the methylation of alkynyl C-H bonds with dimethyl sulfonium ylides. ${ }^{84,87,93-95,97,98}$ In the $\mathrm{N}$-arylation of heterocyclic diamines the $t$ Bu-xantphos system showed little activity $(<1 \%)$, whereas the Ph-xantphos system was more successful (40\%). $\frac{87}{=}$ In the $\mathrm{N}$-alkylation of aniline with benzyl alcohol the $t$-Bu-xantphos system gave $100 \%$ conversion at $100{ }^{\circ} \mathrm{C}$ while the Ph-xantphos reaction at $110{ }^{\circ} \mathrm{C}$ only gave $63 \% .94$ The cross-coupling of butane thiol with 
phenyl triflates in xylene at $140{ }^{\circ} \mathrm{C}$ produced only trace amounts of the product using $t$-Bu-xantphos while an $80 \%$ yield was obtained with Ph-xantphos. ${ }^{84}$ The $\mathrm{Ph}$-xantphos complex was also more reactive in the Suzuki cross-coupling of 2(4-bromophenyl)-5-chloropyrazine with a benzimidazole boronic ester giving a $83 \%$ yield compared to only $5 \%$ with $t$-Bu-xantphos. ${ }^{97}$ The same lack of activity was observed for the hydroesterification of methyl oleate, with no conversion obtained for $t$-Bu-xantphos compared to $70 \%$ for Ph-xantphos. In the methylation of alkynyl C-H bonds with dimethyl sulfonium ylides, the $t$-Bu-xantphos ligand produced a greater yield than the Ph-xantphos ligand (46\% compared to $15 \%$ ). ${ }^{95}$ Together, these studies suggest differences in the coordination chemistry and reactivity of the $t$-Bu-xantphos and $\mathrm{Ph}$-xantphos ligands. However, little research into the coordination chemistry of $t$-Bu-xantphos has been performed.

Only two palladium complexes of $t$-Bu-xantphos have been reported. The Xray crystal structure of $\left[\mathrm{Pd}(t-\mathrm{Bu}\right.$-xantphos $\left.) \mathrm{Cl}_{2}\right]$ was reported to the CSD in 2011 (CSD-XARXAR), $\underline{68}$ however a research paper including synthetic details has not been published. The crystal structure (Figure 6.1) has three molecules in the asymmetric unit each showing a distorted trans geometry with P-Pd-P angles of 160.59(4), 161.97(3) and 161.31(4) ${ }^{\circ}$. These are larger than the 100.61(5) and $100.92(7)^{\circ}$ for $\left[\mathrm{Pd}(\mathrm{Ph}\right.$-xantphos $\left.) \mathrm{Cl}_{2}\right]$ with different solvates. $.68,145,281$ The difference in the bite-angles and geometries of the complexes that form may explain the difference in the catalytic reactivity of the complexes. The other complex reported for $t$-Bu-xantphos showed an unusual monodentate coordination mode (Figure 6.2). .93 This is not a common coordination mode for the xantphos ligands and the same complex was also formed with Ph-xantphos. In an aminocarbonylation reaction the Ph-xantphos complex gave a $92 \%$ isolated yield, while no product was observed using the $t$-Bu-xantphos system. The reason for this difference was not addressed in the publication.

Metal halide complexes are ubiquitous in coordination chemistry. They form a number of complexes that are active catalysts, and are widely used as starting points for the synthesis of more complex systems. Platinum halide complexes are also of interest due to their potential as chemotherapeutics. Complexes such as, cis-platin $\left[\mathrm{PtCl}_{2}\left(\mathrm{NH}_{3}\right)_{2}\right]$ and its derivatives are now an important part of several treatment protocols and have improved the prognosis for many cancer patients, for example, the cure rate of testicular cancer has increased from less than $10 \%$ to over $90 \%$. 

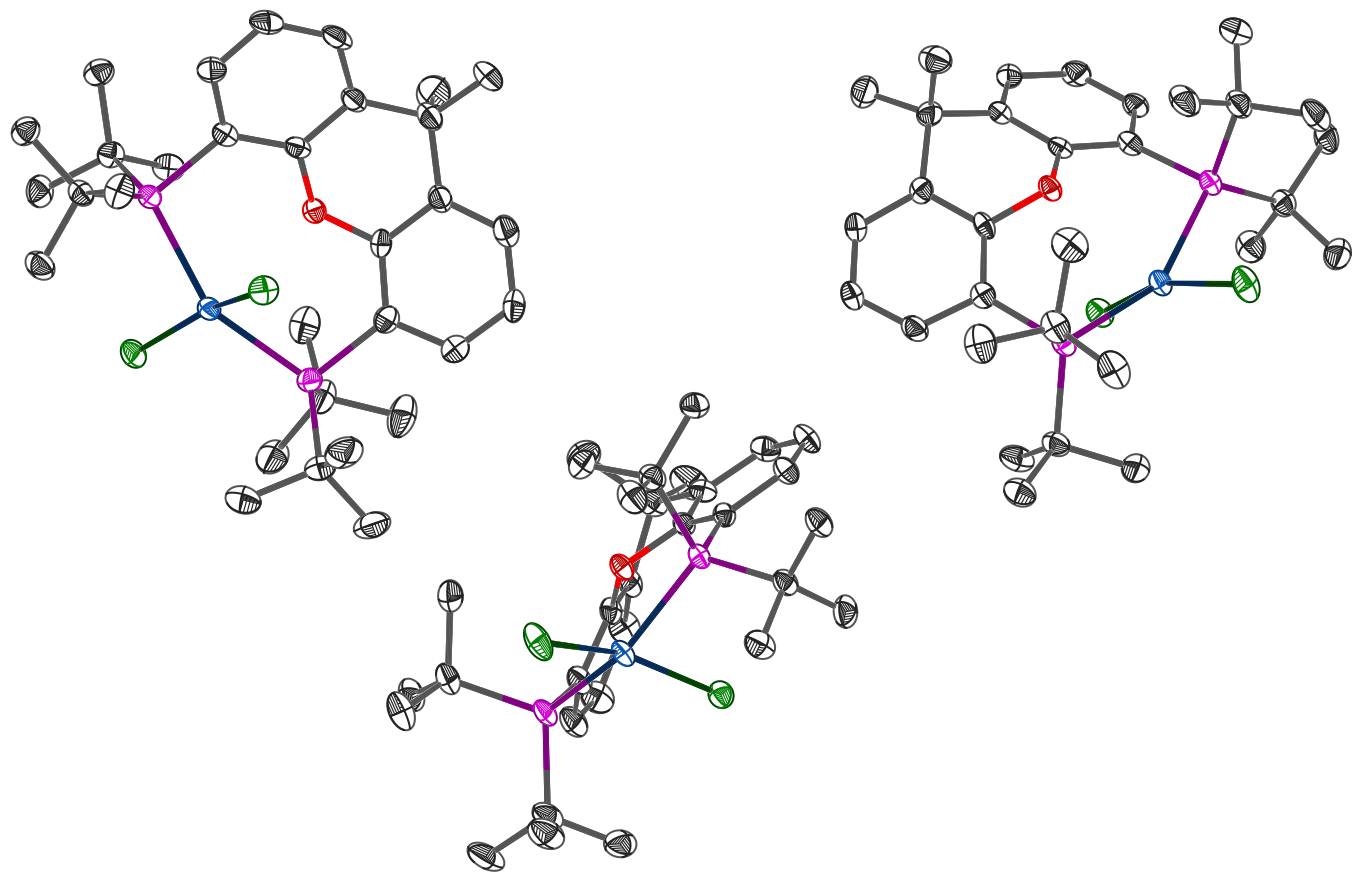

Figure 6.1: X-ray crystal structure of $\left[\mathrm{Pd}(t-B u-x a n t p h o s) \mathrm{Cl}_{2}\right]$ (CSD-XARXAR). Hydrogen atoms and solvent of crystallisation omitted for clarity.

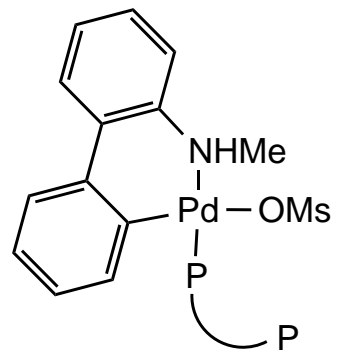

Figure 6.2: Previously reported palladium complex with $\mathrm{PP}=t$-Bu-xantphos or Ph-xantphos. $\underline{\underline{93}}$ 
The coordination chemistry of Ph-xantphos on palladium has been the subject of a number of studies, $, \underline{73}-75,79,115,282,283$ though few have investigated the coordination chemistry with platinum. Catalytic systems frequently involve palladium in both the 0 and +2 oxidation states, hence it is important that both are studied. $\underline{\underline{3}}$ Investigation into the coordination chemistry of the $t$-Bu-xantphos ligands with platinum and palladium(0) was reported in Chapter 5. This chapter will present work on the coordination chemistry of the three $t$-Bu-xantphos ligands with platinum and palladium(II) in order to investigate the differences and similarities in the chemistry of the $t$-Bu-xantphos ligands with the $\mathrm{Ph}$-xantphos ligands.

\subsection{Reactions with Platinum Dichloride Starting Materials}

The reactivity of the $t$-Bu-xantphos ligands towards platinum dichloride starting materials was initially investigated by studying the reaction of $t$-Bu-thixantphos with a range of different starting materials in both cis and trans geometries: $\left[\mathrm{PtCl}_{2}(\right.$ hex $\left.)\right]$, (hex = hexa-1,5-diene) cis- $\left[\mathrm{PtCl}_{2}\left(\mathrm{SEt}_{2}\right)_{2}\right]$, cis- $\left[\mathrm{PtCl}_{2}\left(\mathrm{MeCN}_{2}\right]\right.$, trans- $\left[\mathrm{PtCl}_{2}(\mathrm{MeCN})\right]$, and trans $-\left[\mathrm{PtCl}_{2}\left({ }^{\mathrm{t}} \mathrm{BuCN}\right)_{2}\right]$. In a number of cases, the reaction was very slow and protonation of the $t$-Bu-thixantphos ligand occurred faster than the formation of a platinum complex. If a platinum complex was produced, it was the same species irrespective of the starting material or conditions of the reaction. The most successful result went to completion after 72 hours in toluene at $50{ }^{\circ} \mathrm{C}$ using $\left[\mathrm{PtCl}_{2}(\right.$ hex $\left.)\right]$, which contains a readily displaced diene ligand. In $\mathrm{C}_{6} \mathrm{D}_{6}$ the product appears in the ${ }^{31} \mathrm{P} \mathrm{NMR}$ spectrum as a singlet at $32.9 \mathrm{ppm}$ with a $J_{\mathrm{PtP}}$ value of $2700 \mathrm{~Hz}$. The value of the one-bond phosphorusplatinum coupling constant can give important information regarding the geometry of the complex. In particular, the value of $J_{\mathrm{PtP}}$ is highly dependent on the trans-influence of the ligand trans to the phosphorus atom. ${ }^{16}$ A value of $2700 \mathrm{~Hz}$ for $J_{\mathrm{PtP}}$ is characteristic of mutually trans phosphorus atoms, rather than phosphorus trans to a chloride $\left(J_{\mathrm{PtP}}=3200-3800 \mathrm{~Hz}\right) \cdot{ }^{252,253,322}$ Hence, the product of the reaction between $t$-Bu-thixantphos and $\left[\mathrm{PtCl}_{2}(\right.$ hex $\left.)\right]$ is trans-[Pt $(t-B u$-thixantphos) $\mathrm{Cl}_{2}$ ] (Scheme 6.1).

[Pt $(t$-Bu-thixantphos $\left.) \mathrm{Cl}_{2}\right]$ is dark red in both $\mathrm{C}_{6} \mathrm{D}_{6}$ solution and the solid state. 
<smiles>CCCCc1cc(C)cc2c1Oc1c(P)cc(C)cc1S2</smiles><smiles>ClP1(Cl)=C2CCC=C1CC2</smiles>

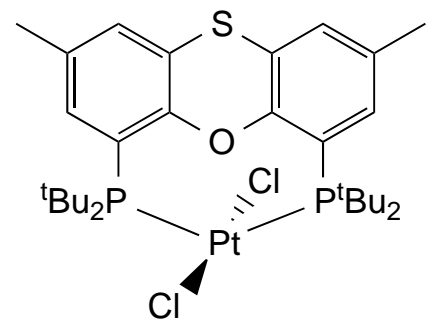

Scheme 6.1: Synthesis of [Pt(t-Bu-thixantphos $\left.) \mathrm{Cl}_{2}\right]$. Reagents and conditions: (i) toluene, $50^{\circ} \mathrm{C}, 3$ days.

This is unusual as platinum dichloride complexes are typically pale, or yellow in colour. Indeed the previously reported $\left[\mathrm{Pt}(\mathrm{Ph}\right.$-xantphos $\left.) \mathrm{Cl}_{2}\right]$ complex is a white solid. $\underline{.6}$ This suggests that $\left[\mathrm{Pt}(t\right.$-Bu-thixantphos $\left.) \mathrm{Cl}_{2}\right]$ is unusual from an electronic perspective, which may be the result of a strained coordination geometry.

Dark red crystals of $\left[\mathrm{Pt}(t-\mathrm{Bu}\right.$-thixantphos $\left.) \mathrm{Cl}_{2}\right]$ suitable for $\mathrm{X}$-ray diffraction were grown by inwards diffusion of diethyl ether into a dichloromethane solution of the complex. The structure is shown in Figure 6.3, with a side-on view in Figure 6.4. The complex crystallises in the monoclinic crystal system in the space group $\mathrm{P} 2{ }_{1} / \mathrm{c}$. Selected bond lengths and angles are given in Table 6.1, and the crystallographic data is given in Table 6.2 .

Table 6.1: Selected bond distances $(\AA)$ and angles $\left(^{\circ}\right)$ of $\left[\mathrm{Pt}(t-\mathrm{Bu}\right.$-thixantphos $\left.) \mathrm{Cl}_{2}\right]$.

\begin{tabular}{llll}
\hline \multicolumn{2}{c}{ Bond distances $(\AA)$} & \multicolumn{2}{c}{ Bond angles $\left(^{\circ}\right)$} \\
\hline P1..P2 & $4.5037(6)$ & P1-Pt-P2 & $151.722(15)$ \\
P1-Pt & $2.3205(4)$ & Cl1-Pt-Cl2 & $164.235(15)$ \\
P2-Pt & $2.3239(4)$ & P1-Pt-Cl1 & $87.11(15)$ \\
Pt-Cl1 & $2.3209(4)$ & P1-Pt-Cl2 & $95.939(16)$ \\
Pt-Cl2 & $2.3098(4)$ & P2-Pt-Cl1 & $86.699(14)$ \\
Pt...O & $2.8016(11)$ & P2-Pt-Cl2 & $96.917(15)$ \\
& & Ring 1-Ring 2 & $139.11(6)$ \\
\hline
\end{tabular}

The crystal structure of $\left[\mathrm{Pt}\left(t-\mathrm{Bu}\right.\right.$-thixantphos) $\left.\mathrm{Cl}_{2}\right]$ (Figure 6.3) is distorted from the square-planar geometry typically observed for $\mathrm{Pt}(\mathrm{II})$ complexes. The $\tau_{5}$ pa- 


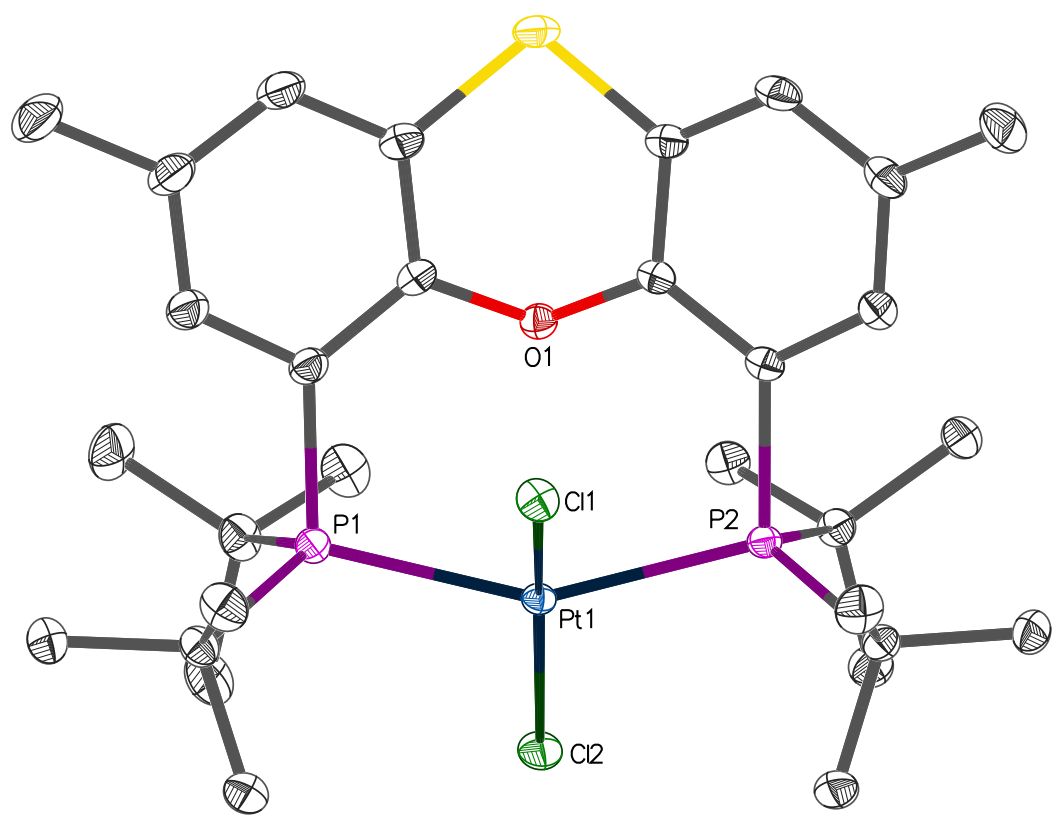

Figure 6.3: X-ray crystal structure of $\left[\mathrm{Pt}(t\right.$-Bu-thixantphos $\left.) \mathrm{Cl}_{2}\right](50 \%$ probability thermal ellipsoids). Hydrogen atoms omitted for clarity.

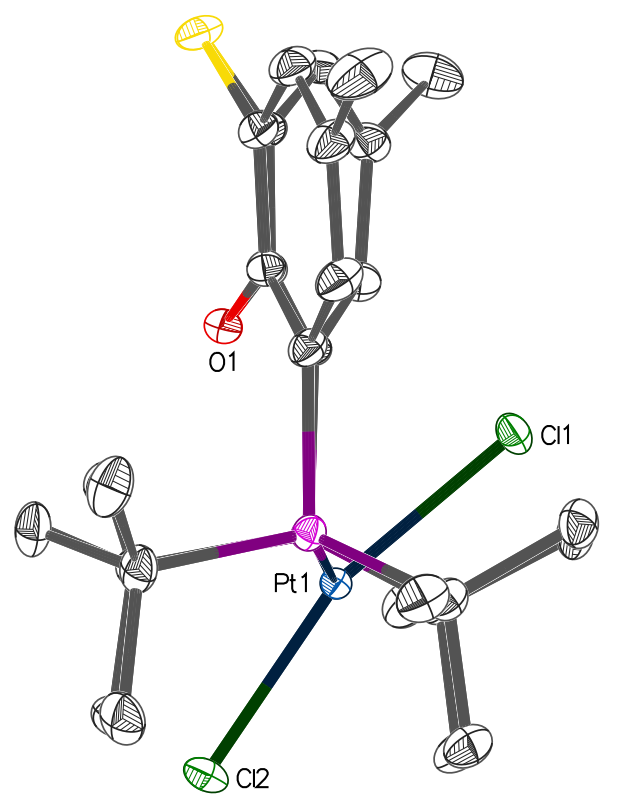

Figure 6.4: X-ray crystal structure of $\left[\mathrm{Pt}(t-\mathrm{Bu}\right.$-thixantphos $\left.) \mathrm{Cl}_{2}\right]$, side view $(50 \%$ probability thermal ellipsoids). Hydrogen atoms omitted for clarity. 
Table 6.2: Crystallographic data and structure refinement of $[\mathrm{Pt}(t-\mathrm{Bu}$-thixantphos) $\left.\mathrm{Cl}_{2}\right]$.

\begin{tabular}{|c|c|}
\hline Empirical formula & $\mathrm{C}_{30} \mathrm{H}_{46} \mathrm{Cl}_{2} \mathrm{OP}_{2} \mathrm{PtS}$ \\
\hline Formula weight & 782.66 \\
\hline Temperature/K & $284.87(10)$ \\
\hline Crystal system & monoclinic \\
\hline Space group & $\mathrm{P} 2_{1} / \mathrm{c}$ \\
\hline $\mathrm{a} / \AA$ & $15.26769(8)$ \\
\hline $\mathrm{b} / \AA$ & $10.98650(7)$ \\
\hline$c / \AA$ & $19.05134(13)$ \\
\hline$\alpha /{ }^{\circ}$ & 90 \\
\hline$\beta /{ }^{\circ}$ & $95.2318(5)$ \\
\hline$\gamma /{ }^{\circ}$ & 90 \\
\hline Volume $/ \AA^{3}$ & $3182.33(3)$ \\
\hline $\mathrm{Z}$ & 4 \\
\hline$\rho_{\text {calc }} \mathrm{mg} / \mathrm{mm}^{3}$ & 1.634 \\
\hline$\mu / \mathrm{mm}$ & 4.766 \\
\hline $\mathrm{F}(000)$ & 1568.0 \\
\hline Crystal size $/ \mathrm{mm}$ & $0.27 \times 0.14 \times 0.08$ \\
\hline Radiation & $\operatorname{MoK} \alpha(\lambda=0.71073)$ \\
\hline $2 \theta$ range for data collection & 5.264 to $75.522^{\circ}$ \\
\hline Index ranges & $-25 \leq \mathrm{h} \leq 25,-15 \leq \mathrm{k} \leq 18,-32 \leq 1 \leq 31$ \\
\hline Reflections collected & 49341 \\
\hline Independent reflections & $16242\left[R_{\text {int }}=0.0277, R_{\text {sigma }}=0.0333\right]$ \\
\hline Data/restraints/parameters & $16242 / 0 / 348$ \\
\hline Goodness-of-fit on $\mathrm{F}^{2}$ & 1.076 \\
\hline Final $\mathrm{R}$ indexes $[\mathrm{I}>=2 \sigma(\mathrm{I})]$ & $\mathrm{R}_{1}=0.0240, \mathrm{wR}_{2}=0.0445$ \\
\hline Final $\mathrm{R}$ indexes [all data] & $\mathrm{R}_{1}=0.0351, \mathrm{w} \mathrm{R}_{2}=0.0486$ \\
\hline Largest diff. peak/hole / e $\AA^{-3}$ & $1.46 /-1.27$ \\
\hline
\end{tabular}


rameter has been used for many years to describe the shapes of five-coordinate complexes. $\stackrel{323}{=}$ In 2007 a related $\tau_{4}$ parameter was introduced to describe the distortion of four-coordinate copper complexes between the ideal tetrahedral and square planar geometries. $\stackrel{324}{2}$ The parameter is defined in Equation 6.1 where $\alpha$ and $\beta$ are the two largest L-M-L angles in the four-coordinate complex. The value of $\tau_{4}$ vary between 0 for an ideal square-planar complex to 1 for a tetrahedral complex. Using the two largest angles for $\left[\mathrm{Pt}(t\right.$-Bu-thixantphos $\left.) \mathrm{Cl}_{2}\right](\mathrm{P} 1-\mathrm{Pt}-\mathrm{P} 2=$ 151.722 and $\mathrm{Cl} 1-\mathrm{Pt}-\mathrm{Cl} 2=164.235)$ gives a value for $\tau_{4}$ of 0.31 . This indicates severe distortion from the expected square-planar geometry with the structure closest to a seesaw geometry.

$$
\tau_{4}=\frac{360-(\alpha+\beta)}{141}
$$

The bite-angle of the $t$-Bu-thixantphos ligand is $151.722(15)^{\circ}$ in $[\mathrm{Pt}(t$-Bu-thixantphos) $\mathrm{Cl}_{2}$ ], which is significantly larger than the natural bite-angle determined by DFT (126.98 ${ }^{\circ}$, Section 2.2). This deviation is likely due to the influence of the platinum centre, which prefers a coordination geometry close to square-planar. The bite-angle is unusual for complexes of the type $\left[\mathrm{Pt}(\mathrm{PP}) \mathrm{Cl}_{2}\right]$, where $\mathrm{PP}$ is either two monophosphine ligands or one diphosphine ligand. A search of the CSD showed that the P-Pt-P angles of these complexes are very bi-modal, showing clear preference for the cis $90^{\circ}$ or the trans $180^{\circ}$ (Figure 6.5). thixantphos angle $\left(151.722(15)^{\circ}\right)$ is situated between these two regions, with the closest bis-monophosphine complex at 167.261, and the closest chelating diphosphine at $171.9(4)^{\circ}$. Seven other trans-dichloridoplatinum complexes which have chelating diphosphines have been reported (Figure 6.6) $33,325-330$ All of these have larger P-Pt-P angles than the $t$-Bu-thixantphos complex $\left(171.9(4)-180.00(0)^{\circ}\right)$. The chelate ring size for these molecules (Figure 6.6) ranges from 10-14 atoms, with the cyclodextrin-derived system having a ring size of 21 atoms (Figure 6.6g). In contrast, the $t$-Bu-thixantphos chelate ring contains only 8 atoms. This relatively small chelation ring size, combined with the rigidity of the backbone is likely the reason for the distortion around the platinum centre as the trans chelation puts strain on the $t$-Bu-thixantphos ligand.

The P-Pt-P angle of $\left[\mathrm{Pt}(t\right.$-Bu-thixantphos $\left.) \mathrm{Cl}_{2}\right]\left(151.722(15)^{\circ}\right)$ is much larger than in the closest cis- $\left[\mathrm{PtCl}_{2}\left(\mathrm{P}_{2}\right)\right]\left(\mathrm{P}_{2}=\right.$ chelating diphosphine $)$ complexes $(113.39(3))^{\circ}$ 


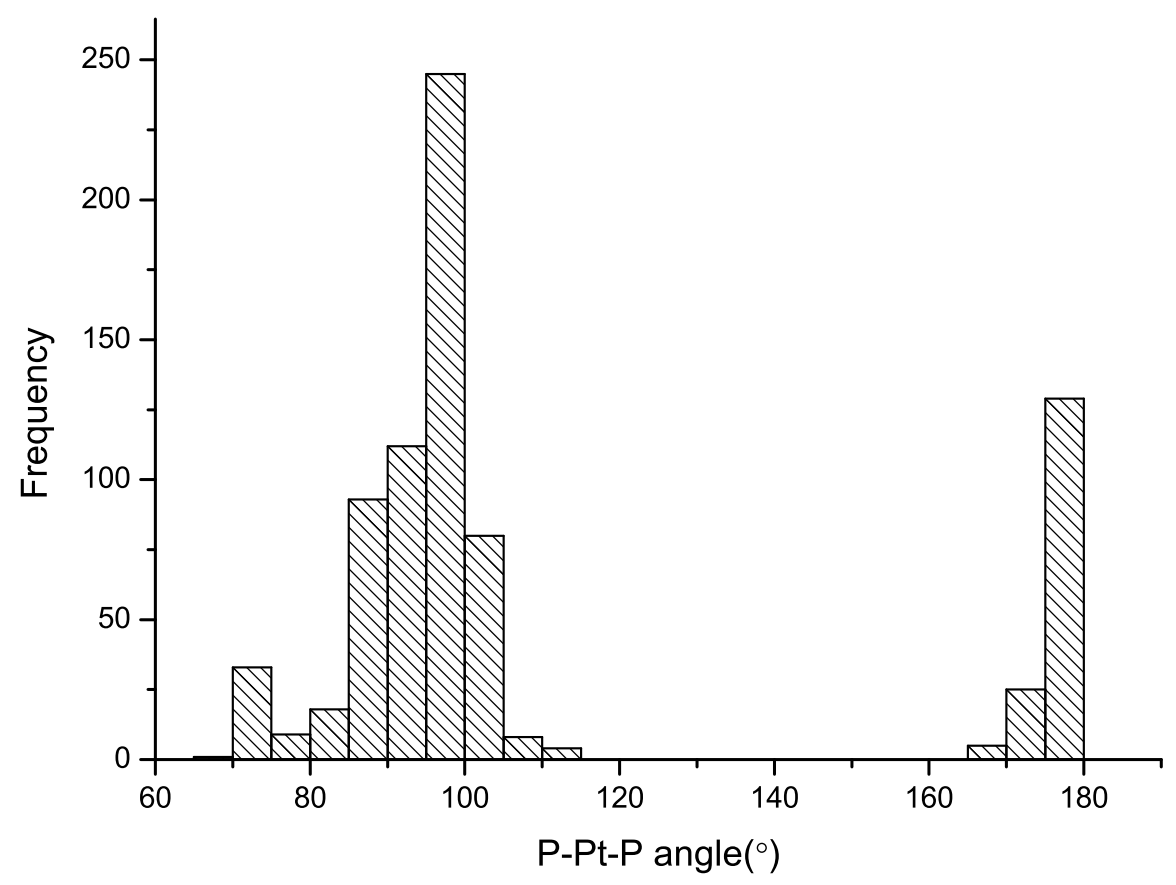

Figure 6.5: Distribution of the P-Pt-P angles in crystal structures of $\left[\mathrm{PtCl}_{2}\left(\mathrm{P}_{2}\right)\right]$ where $\mathrm{P}=$ tertiary phosphine ligand, $\mathrm{P}_{2}=$ chelating diphosphine ligand.

and $\left.113.43(6)^{\circ}\right)$. Both of these complexes contain xantphos ligands, indicating that the rigid xantphos backbone plays a role in forming species with larger bite-angles. ${ }^{331,332}$ In addition to the two already mentioned, three other crystal structures for $\left[\mathrm{PtCl}_{2}\right.$ (xantphos)] complexes have been reported (Figure 6.7). These complexes have crystallographically determined bite-angles of 99.17(3), 99.64(4) and $100.87(8)^{\circ}$. Although these are all cis dichloride complexes it can be seen that the xantphos ligands have access to larger bite-angles than the ideal $90^{\circ}$ of the square-planar platinum(II) complexes. In [ $\mathrm{Pt}(t$-Bu-thixantphos $\left.) \mathrm{Cl}_{2}\right]$ it is likely that the trans coordination of the chloride ligands is the result of the increased steric demand of the tert-butyl substituents, which results in the larger observed bite-angle.

The angle of the coordination plane relative to the backbone of the $\left[\mathrm{PtCl}_{2}\right.$ (xantphos)] complexes appears to be related to the bite-angle. The three xantphos complexes with bite-angles of $99-100^{\circ}$ (Figure 6.8) have a coordination plane 


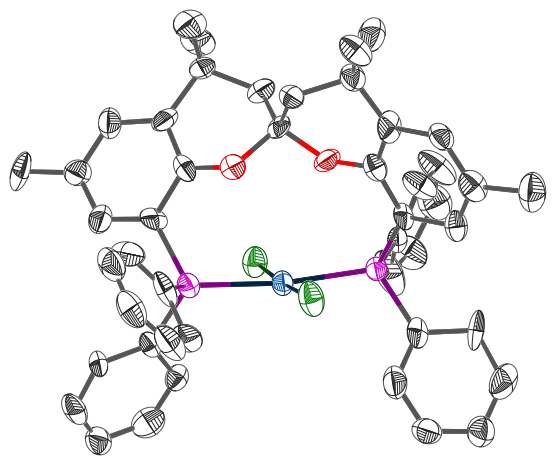

(a) 10 atoms, $171.9(4)^{\circ} \underline{33}$

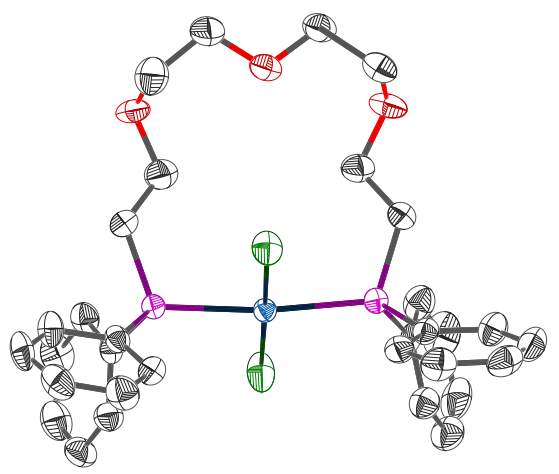

(c) 14 atoms, $173.01(6)^{\circ} \stackrel{328}{ }$

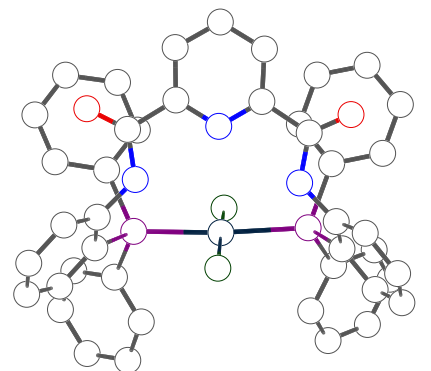

(b) 14 atoms, $172.25(4)^{\circ} \stackrel{329}{ }$

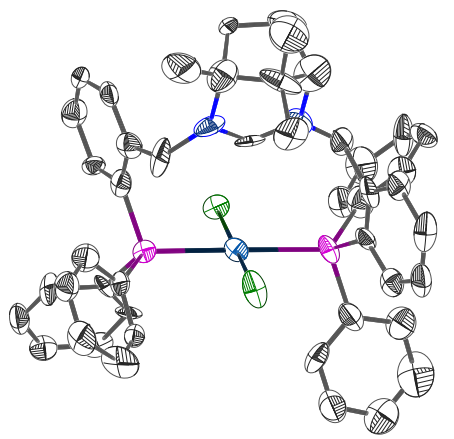

(d) 12 atoms, $174.9(3)^{\circ} \stackrel{327}{ }$

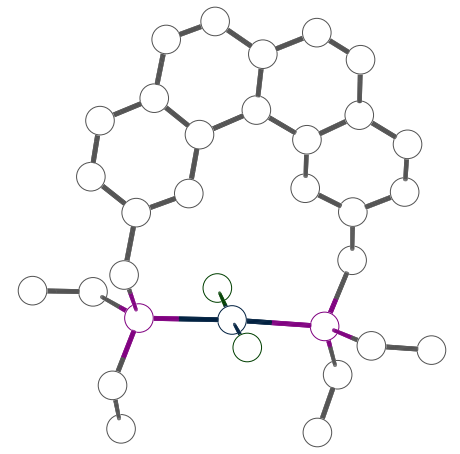

(e) 12 atoms, $177.1(3)^{\circ} \underline{\underline{225}}$

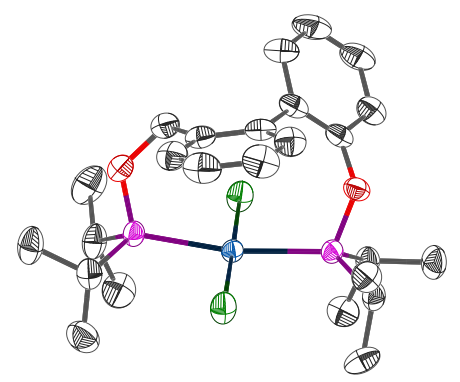

(f) 10 atoms, $177.77(6)^{\circ} \underline{\underline{330}}$

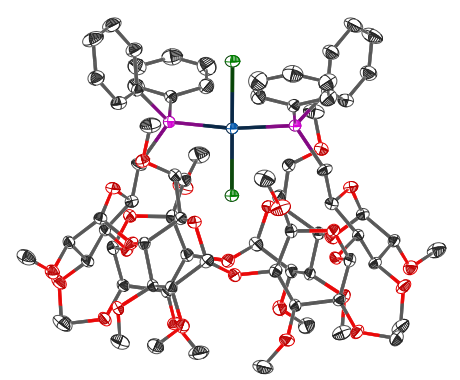

(g) 21 atoms, $180.00(0)^{\circ} \stackrel{326}{ }$

Figure 6.6: X-ray crystal structures of dichloridoplatinum complexes with transspanning diphosphine ligands. The values given are the P-Pt-P angles. 


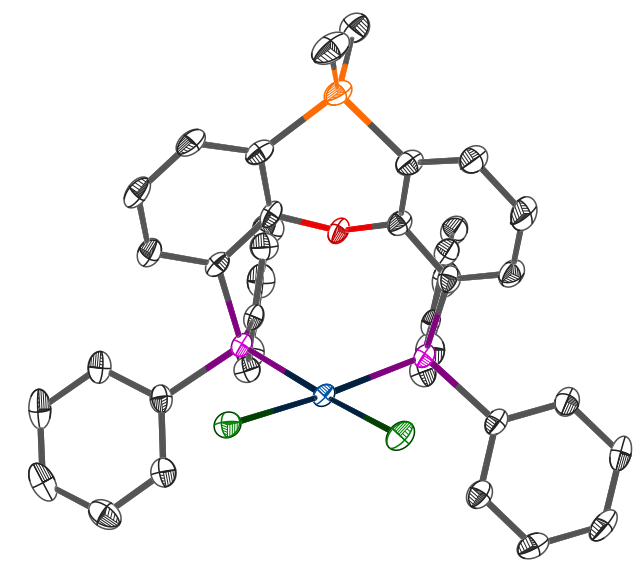

(a) $99.17(3)^{\circ} \underline{34}$

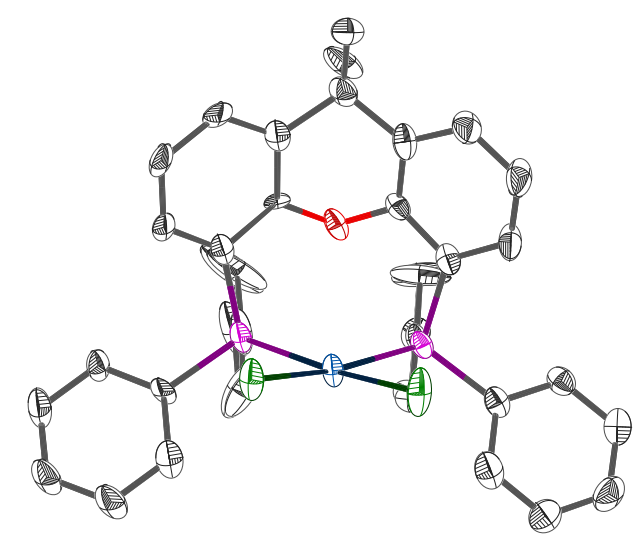

(c) $100.87(8)^{\circ} \underline{335}$

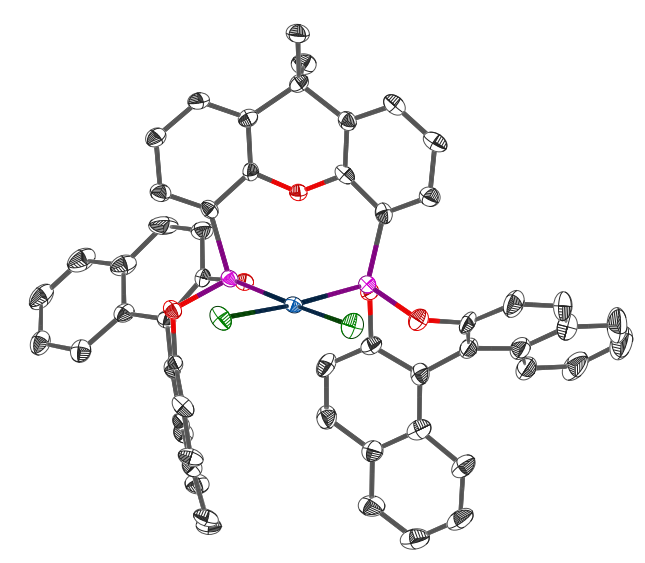

(b) $99.64(4)^{\circ} \underline{333}$

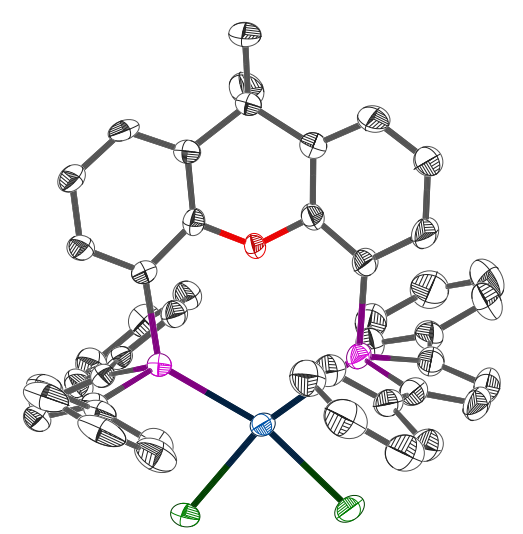

(d) $113.39(3)^{\circ} \underline{332}$

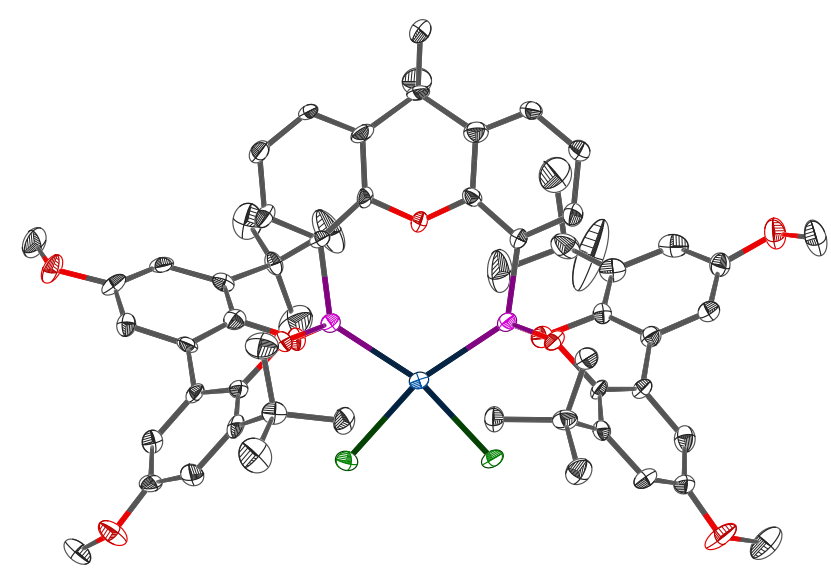

(e) $113.43(6)^{\circ} \underline{331}$

Figure 6.7: X-ray crystal structures of complexes of the type [ $\mathrm{PtCl}_{2}$ (xantphos)]. The P-Pt-P angle is indicated. 
that is almost perpendicular to the plane of the xantphos backbone $\left(105.90(12)^{\circ}\right.$, $74.42(13)^{\circ}$ and $\left.77.0(4)^{\circ}\right)$. In contrast, the two xantphos complexes with bite-angles of $113.39(3)$ and $113.43(6)^{\circ}$ show coordination planes that are in-line with the xantphos backbone $\left(176.9(2)^{\circ}\right.$ and $\left.178.63(10)^{\circ}\right)$. This is likely the result of the cyclic bulky substituents on the phosphorus atoms of these complexes. In contrast, the $\left[\mathrm{Pt}(t-\mathrm{Bu}\right.$-thixantphos $\left.) \mathrm{Cl}_{2}\right]$ complex, with a much larger bite-angle of $151.722(15)^{\circ}$, shows an angle of $140.48(5)^{\circ}$ between the P-Ar bonds and the Pt coordination plane. This shows the marked difference between the $[\mathrm{Pt}(t-\mathrm{Bu}$-thixantphos) $\left.\mathrm{Cl}_{2}\right]$ complex and the other $\left[\mathrm{PtCl}_{2}\right.$ (xantphos)] complexes. The change in the position of the coordination plane relative to the backbone is due to the bulky tert-butyl-substituents causing a distorted trans coordination geometry. In all of the xantphos crystal structures the ligand backbone is bent, which is clear in the side-on view (Figures 6.4 and 6.8). This bending results in a concave face that is less sterically hindered than the convex face. Hence the coordination plane tilts such that the chloride on the concave face is closer to the backbone than the chloride on the convex face.

While the geometry of $\left[\mathrm{Pt}(t\right.$-Bu-thixantphos $\left.) \mathrm{Cl}_{2}\right]$ is unusual when compared to other platinum complexes, there are 805 bis-phosphine dichloridopalladium complexes in the CSD, with 18 of these having P-Pd-P angles between 150 and $167^{\circ}$ (no platinum complexes of this type with a bite-angle in this region have been reported). $\underline{\underline{68}}$ All of these complexes include diphosphine ligands, indicating the importance of the ligand in controlling the coordination geometry of the complex. Of particular interest is the structure of $\left[\mathrm{Pd}(t-\mathrm{Bu}\right.$-xantphos $\left.\left.) \mathrm{Cl}_{2}\right]\right]^{68}$ This structure has three distinct molecules of $\left[\mathrm{Pd}(t-\mathrm{Bu}\right.$-xantphos $\left.) \mathrm{Cl}_{2}\right]$ within the unit cell, with bite-angles of 152.30(4), 152.91(4) and 152.83(4) ${ }^{\circ}$. These are all slightly larger than the P-Pt-P angle in $\left[\mathrm{Pt}(t-\mathrm{Bu}\right.$-thixantphos $\left.) \mathrm{Cl}_{2}\right]$, which is expected due to the larger natural bite-angle of $t$-Bu-xantphos. In other respects the crystal structures are very similar.

The bond lengths for the coordinating atoms in $\left[\mathrm{Pt}(t-\mathrm{Bu}\right.$-thixantphos $\left.) \mathrm{Cl}_{2}\right]$ are all close to the average values determined from the Cambridge Crystallographic Data Centre (Table 6.1). The Pt-O distance is 2.8016(11) $\AA$, significantly longer than the average reported Pt-O bond length (2.053 $\AA$ ) indicating that there is no interaction between the two atoms. However, this is shorter than the five other complexes with xantphos derived backbones (3.174-3.466 $\AA$ ) indicating that there is a relationship between the bite-angle of the diphosphine and the proximity of 


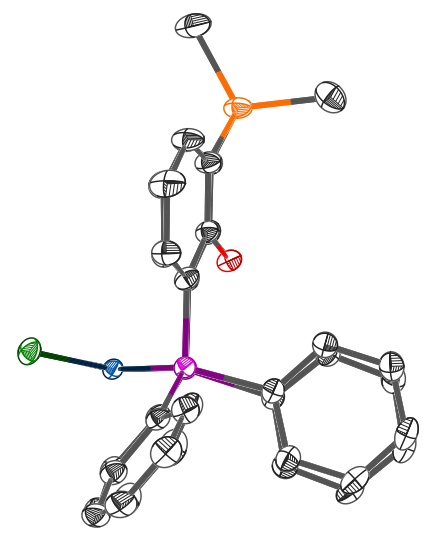

(a) $105.90(12)^{\circ} 334$

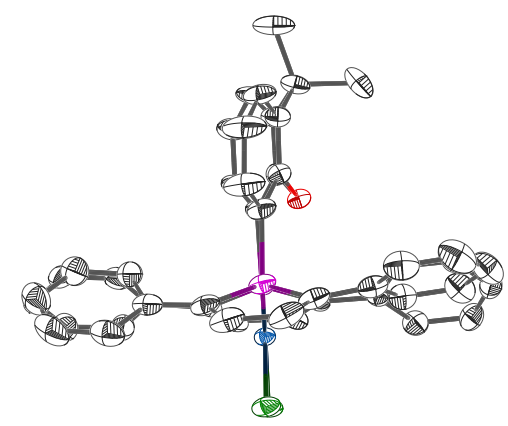

(d) $178.63(10)^{\circ} \underline{32}$

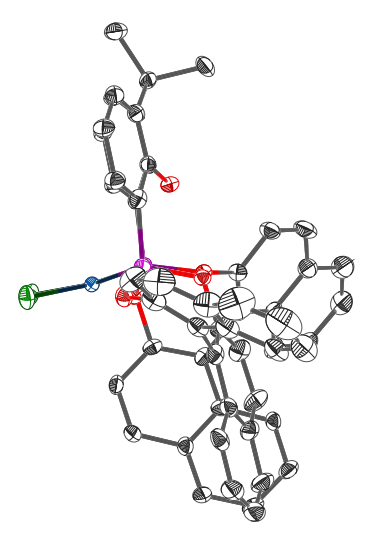

(b) $74.42(13)^{\circ} \underline{333}$

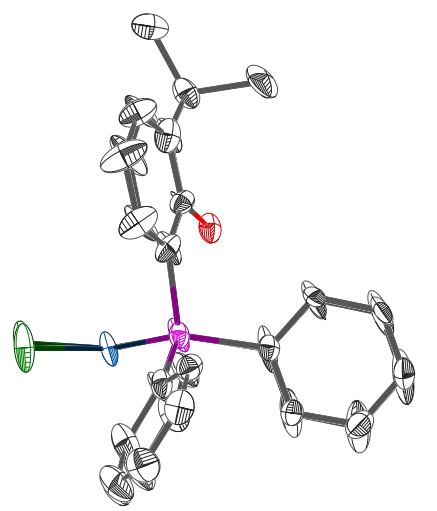

(c) $77.0(4)^{\circ} \underline{335}$

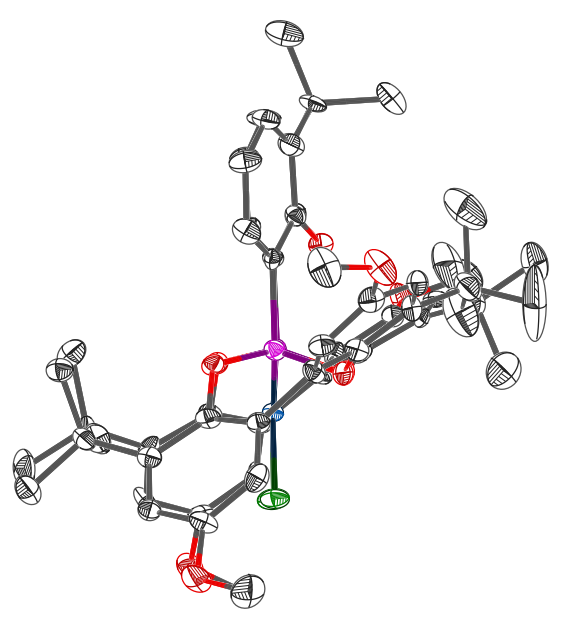

(e) $176.9(2)^{\circ} \underline{331}$

Figure 6.8: Side-on view of X-ray crystal structures of complexes of the type $\left[\mathrm{PtCl}_{2}\right.$ (xantphos)] showing the coordination plane relative to the xantphos backbone. The value of the angle between the mean coordination plane and the Pbackbone bond is given beneath each structure. 
the oxygen atom.

The ${ }^{1} \mathrm{H}$ and ${ }^{13} \mathrm{C}$ NMR spectra of trans-[Pt(t-Bu-thixantphos $\left.) \mathrm{Cl}_{2}\right]$ in $\mathrm{C}_{6} \mathrm{D}_{6}$ show two different sets of tert-butyl carbon and proton resonances. One of the sets appears as clearly resolved virtual triplets for both carbon environments and the proton environment. The other set has a well resolved virtual triplet for the quaternary carbon, whereas the other carbon and the proton signal are broad singlets. The reason for the appearance of two different tert-butyl environments is apparent from the solid-state structure (Figures 6.3 and 6.4). The backbone of the $t$-Bu-thixantphos ligand is bent, resulting in a concave and a convex side. If the ligand is not inverting in solution then this will result in two different tert-butyl environments: the two on the concave side, and the two on the convex side. The broadness that is observed for the methyl groups in one of the sets may be due to the steric constraints of this structure.

The trans-[Pt(t-Bu-thixantphos $\left.) \mathrm{Cl}_{2}\right]$ complex showed a solvent dependent NMR spectrum. In $\mathrm{C}_{6} \mathrm{D}_{6}$ a single sharp peak was observed at $32.9 \mathrm{ppm}\left(J_{\mathrm{PtP}}=2700 \mathrm{~Hz}\right)$ in the ${ }^{31} \mathrm{P}$ NMR spectrum. However, in acetone- $\mathrm{d}_{6}$ this peak became very broad, at $35 \mathrm{ppm}$, while in $\mathrm{CD}_{2} \mathrm{Cl}_{2}$ or $\mathrm{CDCl}_{3}$ the peak shifted to $46.4 \mathrm{ppm}$. The colour of the complex changes dramatically depending on the solvent. In the solid state the crystals have been determined to be trans-[Pt( $t$-Bu-thixantphos $\left.) \mathrm{Cl}_{2}\right]$ without any solvent of crystallisation and are deep red in colour. Benzene and toluene solutions retain this colour. However, acetone solutions are orange, while in chloroform or dichloromethane the solution is yellow. Although colour changes for systems with solvent interactions or strong intermolecular interactions are well-known this is unlikely to be the case here. Instead it is likely that solventdependent coordination is occurring. In non-polar solvents such as benzene and toluene $\left[\mathrm{Pt}(t-\mathrm{Bu}\right.$-thixantphos $\left.) \mathrm{Cl}_{2}\right]$ is present. However, polar solvents like acetone or chloroform are more likely than benzene or toluene to stabilise charged species. Hence, in polar solvents it is proposed that one of the chloride ligands dissociates and the ether oxygen associates to form $[\mathrm{Pt}(t-\mathrm{Bu}$-thixantphos$\left.\left.\kappa P, O, P^{\prime}\right) \mathrm{Cl}\right] \mathrm{Cl}$ (Scheme 6.2). In acetone the broadness of the spectrum combined with the intermediate colour likely indicates the rapid interconversion of the complexes.

To test the chloride dissociation theory, the $\left[\mathrm{Pt}(t\right.$-Bu-thixantphos $\left.) \mathrm{Cl}_{2}\right]$ complex was dissolved in acetone and reacted with ammonium hexafluorophosphate. The 

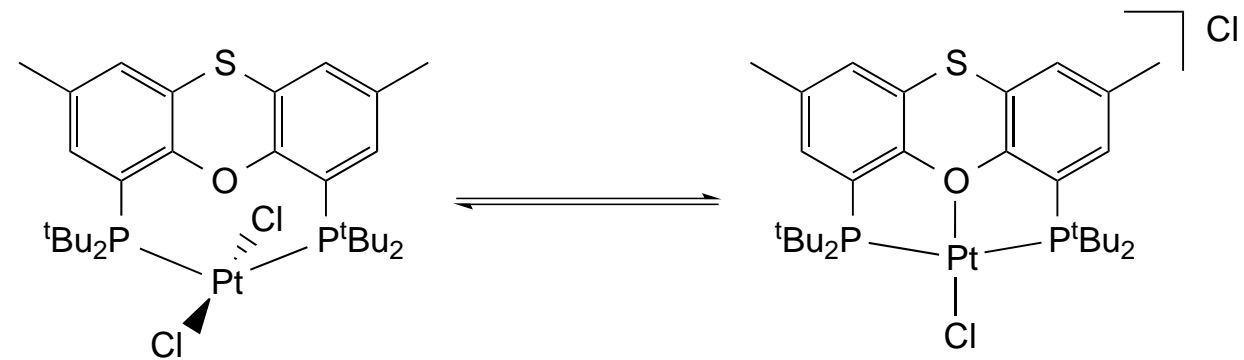

Scheme 6.2: Equilibrium between $\left[\mathrm{Pt}(t-\mathrm{Bu}\right.$-thixantphos $\left.) \mathrm{Cl}_{2}\right]$ and $\mathrm{Pt}(t-\mathrm{Bu}$-thixantphos)Cl]Cl.

solution changed colour over 30 minutes from deep orange to yellow, and a precipitate of ammonium chloride formed. The product appeared at $46.4 \mathrm{ppm}\left(J_{\mathrm{PtP}}=\right.$ $2347 \mathrm{~Hz})$ in the ${ }^{31} \mathrm{P} N M R$ spectrum with an associated septet at $-144.5\left(J_{\mathrm{PF}}=710.6\right)$ ppm indicative of $\mathrm{PF}_{6}$. The value of $J_{\mathrm{PtP}}$ had decreased from the dichloride complex by $353 \mathrm{~Hz}$, which may be the result of changes in the coordination geometry of the platinum centre. A shift of the signal in the ${ }^{13} \mathrm{C}$ NMR spectrum for the O-ipso carbon occurred from $155.8 \mathrm{ppm}$ to $157.4 \mathrm{ppm}$, indicative of the oxygen coordinating to the metal centre.

\subsubsection{Reactions of $\left[\mathrm{PtCl}_{2}(\right.$ hex) $]$ with $t$-Bu-sixantphos and $t$-Bu- xantphos}

Several starting materials were investigated for the synthesis of trans-[Pt $(t-\mathrm{Bu}-$ thixantphos) $\left.\mathrm{Cl}_{2}\right]$. The best results occurred using $\left[\mathrm{PtCl}_{2}(\right.$ hex $\left.)\right]$, which proceeded in $\mathrm{C}_{6} \mathrm{D}_{6}$ at $40{ }^{\circ} \mathrm{C}$ showing $75 \%$ conversion after 72 hours, or in toluene at $50{ }^{\circ} \mathrm{C}$ with $100 \%$ conversion in the same time. Due to the success of these conditions over other choices of starting material, temperature, and solvent, the synthesis of analogous trans-[ $\left[\mathrm{PtCl}_{2} \mathrm{P}_{2}\right]$ complexes with $t$-Bu-sixantphos and $t$-Bu-xantphos was attempted using $\left[\mathrm{PtCl}_{2}(\right.$ hex $\left.)\right]$, in toluene at $50{ }^{\circ} \mathrm{C}$.

The reaction between $t$ - $\mathrm{Bu}$-xantphos and $\left[\mathrm{PtCl}_{2}(\mathrm{hex})\right]$ proceeded as expected. After 72 hours at $50^{\circ} \mathrm{C}$ in toluene an orange solid was isolated which was further purified by recrystallisation, via inwards diffusion of diethyl ether into a dichloromethane solution of the product. The ${ }^{31} \mathrm{P}$ NMR spectrum showed one major product at $32.4 \mathrm{ppm}\left(\mathrm{JPtP}_{\mathrm{P}}=2721 \mathrm{~Hz}, \mathrm{C}_{6} \mathrm{D}_{6}\right)$. The downfield shift of the peak 
in the ${ }^{31} \mathrm{P}$ NMR spectrum is indicative of complexation. The value of $2721 \mathrm{~Hz}$ is consistent with a phosphorus trans to another phosphorus donor, rather than trans to a chloride ligand. $\frac{252,253,322}{2}$ The position of the peak and the value of the one-bond platinum-phosphorus coupling constant are very similar to those for trans-[Pt $(t-B u$-thixantphos $\left.) \mathrm{Cl}_{2}\right]\left(32.9 \mathrm{ppm}, J_{\mathrm{PtP}}=2700 \mathrm{~Hz}\right)$, suggesting the formation of an analogous product, trans-[Pt(t-Bu-xantphos $\left.) \mathrm{Cl}_{2}\right]$.

The reaction between $t$-Bu-sixantphos and $\left[\mathrm{PtCl}_{2}(\mathrm{hex})\right]$ was not straightforward. After 72 hours at $50{ }^{\circ} \mathrm{C}$ the reaction contained unreacted $t$-Bu-sixantphos $(53.4 \%)$, $[(t \text {-Bu-sixantphos }) \mathrm{H}]^{+}(22.8 \%)$, and a platinum complex. Although the protonation of $t$-Bu-thixantphos did occur in a number of the reactions with various different $\left[\mathrm{PtCl}_{2} \mathrm{~L}_{2}\right]$ starting materials, this side-reaction was only observed when chlorinated solvents were used. Hence the formation of $[(t-B u \text {-thixantphos }) \mathrm{H}]^{+}$ can be attributed to the formation of small amounts of hydrochloric acid due to the UV-catalysed degradation of chlorinated solvents. However, the formation of $[(t-B u-s i x a n t p h o s) H]^{+}$also occurred in toluene and $\mathrm{C}_{6} \mathrm{D}_{6}$. There are two possible proton sources, either small amounts of water which may have entered the reaction, or reaction with the surface of the glassware. The solvents were all degassed and dried over molecular sieves in accordance with Armarego and Chai. $\stackrel{336}{ }$ This should result in a water concentration of 0.9 ppm for toluene, which is insufficient to account for the conversion. ${ }^{296}$ Furthermore, the $t$-Bu-sixantphos ligand synthesis involves a dichloromethane/water liquid/liquid separation and no protonation was evident in the ${ }^{1} \mathrm{H}$ or ${ }^{31} \mathrm{P} \mathrm{NMR}$ spectra of the $t$-Bu-sixantphos ligand after this step. This makes it unlikely that water is the proton source in this reaction.

Analysis of the $t$-Bu-xantphos selenides (Section 2.4) showed that $t$-Bu-sixantphos is the most basic of all of the ligands with a $\mathrm{p} K_{\mathrm{b}}$ of 5.67 compared to 6.90 and 6.72 for $t$-Bu-thixantphos and $t$-Bu-xantphos respectively. Hence the $t$-Busixantphos ligand is significantly more susceptible to protonation, leading to the observed difficulties. The $t$-Bu-sixantphos ligand has the smallest calculated biteangle of the three ligands (Section 2.2) which would result in additional strain when forming the trans- $\left[\mathrm{Pt}(t-\mathrm{Bu}\right.$-sixantphos $\left.) \mathrm{Cl}_{2}\right]$ complex compared to the other two ligands. The reaction with $t$-Bu-thixantphos requires 72 hours at $50{ }^{\circ} \mathrm{C}$ to reach completion. It is likely that the reaction with $t$-Bu-sixantphos would require additional time or harsher conditions to go to completion. Hence the formation of trans-[Pt $(t-B u$-sixantphos $\left.) \mathrm{Cl}_{2}\right]$ has a larger activation barrier compared with the 
$t$-Bu-thixantphos or $t$-Bu-xantphos ligands, making the alternative protonation reaction more likely to occur.

Despite the significant amounts of $[(t-B u \text {-sixantphos }) \mathrm{H}]^{+}$that formed, one species that displays platinum coupling in the ${ }^{31} \mathrm{P}$ NMR spectrum is formed. This species was not isolated and due to the complexity of the ${ }^{1} \mathrm{H}$ and ${ }^{13} \mathrm{C}$ NMR spectra for the reaction mixture these were unable to be fully assigned. The peak for the platinum containing species appears in the ${ }^{31} \mathrm{P}$ NMR spectrum at $34.7 \mathrm{ppm}\left(J_{\mathrm{PtP}}\right.$ $\left.=2686 \mathrm{~Hz}, \mathrm{C}_{6} \mathrm{D}_{6}\right)$. The value of $J_{\mathrm{PtP}}$ is indicative of a trans arrangement of phosphorus atoms. ${ }^{252,253,322}$ The position of the ${ }^{31} \mathrm{P}$ NMR signal and the value of $J_{\mathrm{PtP}}$ are similar to the trans-[ $\mathrm{Pt}(t$-Bu-thixantphos $\left.) \mathrm{Cl}_{2}\right]$ complex which appears at 32.9 $\operatorname{ppm}\left(J_{\mathrm{PtP}}=2700 \mathrm{~Hz}, \mathrm{C}_{6} \mathrm{D}_{6}\right)$. Based on this evidence the product of the reaction between $t$-Bu-sixantphos and $\left[\mathrm{PtCl}_{2}(\right.$ hex $\left.)\right]$ is trans-[Pt(t-Bu-sixantphos $\left.) \mathrm{Cl}_{2}\right]$. The mass spectrum shows a peak with a consistent mass and isotopic distribution for $\left[\mathrm{C}_{30} \mathrm{H}_{48} \mathrm{ClOP}_{2} \mathrm{PtSi}\right]^{+}$, which could result from loss of a chloride from trans-[Pt $(t-$ Bu-sixantphos) $\left.\mathrm{Cl}_{2}\right] \cdot \frac{210}{}$

[PtCl ${ }_{2}$ (xantphos)] complexes have been reported for Ph-sixantphos, Ph-thixantphos and Ph-xantphos. ${ }^{337}$ Unlike the trans-[Pt(t-Bu-xantphos $\left.) \mathrm{Cl}_{2}\right]$ complexes the $\mathrm{Ph}$-xantphos complexes form cis-[Pt(Ph-xantphos) $\left.\mathrm{Cl}_{2}\right]$ with $J_{\mathrm{PtP}}$ values over 3600 $\mathrm{Hz}$ (Table 6.3), indicative of phosphorus trans to a chloride ligand. The $J_{\mathrm{PtP}}$ values for the trans-[Pt $(t-B u$-xantphos $\left.) \mathrm{Cl}_{2}\right]$ complexes are almost $1000 \mathrm{~Hz}$ lower, indicative of trans coordination of the phosphorus atoms. The cis geometries of the Ph-xantphos complexes have been confirmed by X-ray crystallography of cis$\left[\mathrm{Pt}(\mathrm{Ph} \text {-sixantphos }) \mathrm{Cl}_{2}\right]^{334}$ and cis-[Pt(Ph-xantphos $\left.) \mathrm{Cl}_{2}\right] \cdot \frac{335}{3}$ The pseudo-trans geometry of trans-[Pt(t-Bu-thixantphos $\left.) \mathrm{Cl}_{2}\right]$ has been determined crystallographically (Figure 6.3).

The ${ }^{1} \mathrm{H}$ and ${ }^{13} \mathrm{C}$ NMR spectra for all three trans-[Pt(t-Bu-xantphos $\left.) \mathrm{Cl}_{2}\right]$ complexes display broad signals for the tert-butyl proton and carbon environments. The NMR spectra of trans-[Pt( $t$-Bu-thixantphos $\left.) \mathrm{Cl}_{2}\right]$ showed two different sets of tertbutyl peaks. Based on the crystal structure these were determined to be on the concave or convex side of the bent backbone. For the $t$-Bu-xantphos complex the backbone may be inverting on a similar timescale to the NMR spectroscopy. This results in a single signal for the tert-butyl groups but broadened due to the similar timescale to the NMR analysis. The ${ }^{13} \mathrm{C}$ NMR signals for the $O$-ipso carbon shifted very slightly upon coordination (less than $1.1 \mathrm{ppm}$ ), which indicates that 
the oxygen atom is not coordinated to the metal centre, consistent with the crystal structure of trans-[ $\mathrm{Pt}(t-\mathrm{Bu}-$ thixantphos $\left.) \mathrm{Cl}_{2}\right]$.

Table 6.3: Selected NMR data for $\left[\mathrm{Pt}(\right.$ xantphos $\left.) \mathrm{Cl}_{2}\right]$ complexes. Values for $\mathrm{Ph}-$ xantphos complexes are in $\mathrm{CDCl}_{3} \frac{110}{1}$ and values for $t$-Bu-xantphos complexes are in $\mathrm{C}_{6} \mathrm{D}_{6} \cdot{ }^{13} \mathrm{C}$ data for the Ph-xantphos complexes was not reported $\left(\Delta \delta=\delta_{\text {complex }}\right.$ - $\left.\delta_{\text {free ligand }}\right)$.

\begin{tabular}{lcccccc}
\hline & \multicolumn{3}{c}{${ }^{31} \mathbf{P}$} & & \multicolumn{2}{c}{${ }^{13} \mathbf{C}$ O-ipso } \\
\cline { 2 - 4 } Diphosphine & $\delta / \mathbf{p p m}$ & $\Delta \delta / \mathbf{p p m}$ & $\boldsymbol{J}_{\mathbf{P t P}}$ & & $\delta / \mathbf{p p m}$ & $\Delta \delta / \mathbf{p p m}$ \\
\hline Ph-Sixantphos & 7.6 & 25.2 & 3663 & & \\
Ph-Thixantphos & 8.4 & 25.7 & 3641 & & \\
Ph-Xantphos & 6.6 & 24.1 & 3695 & & \\
$t$-Bu-Sixantphos & 34.7 & 26.3 & 2686 & & \\
$t$-Bu-Thixantphos & 32.9 & 23.4 & 2700 & & 155.8 & -0.1 \\
$t$-Bu-Xantphos & 32.4 & 22.2 & 2721 & 157.1 & 1.1 \\
\hline
\end{tabular}

The solvent-dependent coordination that was observed in the synthesis of $[\mathrm{Pt}(t-$ Bu-thixantphos $\left.) \mathrm{Cl}_{2}\right]$ was also observed for $\left[\mathrm{Pt}(t-\mathrm{Bu}\right.$-xantphos $\left.) \mathrm{Cl}_{2}\right]$, and was studied in more depth for this complex. Selected NMR data for [ $\mathrm{Pt}(t-\mathrm{Bu}$-xantphos $\left.) \mathrm{Cl}_{2}\right]$ dissolved in $\mathrm{CDCl}_{3}, \mathrm{CD}_{2} \mathrm{Cl}_{2}$ and $\mathrm{C}_{6} \mathrm{D}_{6}$ are given in Table 6.4. The complex was also analysed in acetone- $\mathrm{d}_{6}$ however the ${ }^{1} \mathrm{H},{ }^{13} \mathrm{C}$ and ${ }^{31} \mathrm{P}$ NMR spectra were very broad likely indicating the rapid interconversion between $\left[\mathrm{Pt}(t\right.$-Bu-xantphos $\left.) \mathrm{Cl}_{2}\right]$ and $\left[\mathrm{Pt}\left(t-\mathrm{Bu}\right.\right.$-xantphos- $\left.\left.\mathrm{k} P, \mathrm{O}, \mathrm{P}^{\prime}\right) \mathrm{Cl}\right] \mathrm{Cl}$. The peak in the ${ }^{31} \mathrm{P}$ NMR spectrum shifts by around $15 \mathrm{ppm}$ upon changing the solvent from $\mathrm{C}_{6} \mathrm{D}_{6}$ to $\mathrm{CDCl}_{3}$ or $\mathrm{CD}_{2} \mathrm{Cl}_{2}$. The value of $J_{\mathrm{PtP}}$ is lower by more than $370 \mathrm{~Hz}$, consistent with coordination of the oxygen to the metal centre. The pseudo-trans geometry of the trans- $[\operatorname{Pt}(t-$ Bu-xantphos) $\mathrm{Cl}_{2}$ ] complexes results in significant strain in the $t$-Bu-xantphos ligands. Coordination of the oxygen to the metal centre can relieve the strain and allow much larger P-M-P angles to form. With a larger P-Pt-P angle the phosphorus atoms are closer to a mutually trans configuration. The closer to trans the atoms become, the greater the influence of the other phosphorus atom and thus the value of $J_{\text {PtP }}$.

The ${ }^{1} \mathrm{H}$ and ${ }^{13} \mathrm{C}$ NMR data for $\left[\mathrm{Pt}(t-\mathrm{Bu}\right.$-xantphos $\left.) \mathrm{Cl}_{2}\right]$ and $[\mathrm{Pt}(t$-Bu-xantphos $) \mathrm{Cl}] \mathrm{Cl}$ all show virtual triplet peaks for the tert-butyl protons and carbons. This indi- 
Table 6.4: Selected NMR data for $\left[\mathrm{Pt}(t-\mathrm{Bu}\right.$-xantphos $\left.) \mathrm{Cl}_{2}\right]$ and $[\mathrm{Pt}(t-\mathrm{Bu}-\mathrm{xant}$ phos $) \mathrm{Cl}] \mathrm{Cl}\left(\Delta \delta=\delta_{\text {complex }}-\delta_{\text {free ligand }}\right)$.

\begin{tabular}{|c|c|c|c|c|c|c|}
\hline \multirow[b]{2}{*}{ Solvent } & \multirow[b]{2}{*}{ Complex } & \multicolumn{3}{|c|}{${ }^{31} \mathbf{P}$} & \multicolumn{2}{|c|}{${ }^{13} \mathrm{C} O$-ipso } \\
\hline & & $\delta / \mathbf{p p m}$ & $\Delta \delta / \mathbf{p p m}$ & $J_{\mathrm{PtP}}$ & $\delta / \mathbf{p p m}$ & $\Delta \delta / \mathbf{p p m}$ \\
\hline $\mathrm{C}_{6} \mathrm{D}_{6}$ & {$\left[\mathrm{Pt}(t-\mathrm{Bu}\right.$-xantphos $\left.) \mathrm{Cl}_{2}\right]$} & 32.4 & 22.2 & 2721.0 & 157.1 & 1.1 \\
\hline $\mathrm{CDCl}_{3}$ & {$[\mathrm{Pt}(t-\mathrm{Bu}$-xantphos $) \mathrm{Cl}] \mathrm{Cl}$} & 47.7 & 37.5 & 2349.6 & 158.5 & 2.7 \\
\hline $\mathrm{CD}_{2} \mathrm{Cl}_{2}$ & {$[\mathrm{Pt}(t-\mathrm{Bu}$-xantphos $) \mathrm{Cl}] \mathrm{Cl}$} & 47.8 & 37.6 & 2345.3 & 158.8 & 3.0 \\
\hline
\end{tabular}

cates strongly coupled phosphorus atoms, which typically occurs with mutually trans phosphorus atoms. However, the ${ }^{13} \mathrm{C}$ peak for the $\mathrm{O}$-ipso carbon in the $t$-Bu-xantphos ligand shows a shift of only $1.1 \mathrm{ppm}$ (from the free ligand) when in $\mathrm{C}_{6} \mathrm{D}_{6}$, but a shift $(\Delta \delta)$ of 2.7 or $3.0 \mathrm{ppm}$ in $\mathrm{CDCl}_{3}$ and $\mathrm{CD}_{2} \mathrm{Cl}_{2}$ respectively. This shift has been observed for a number of rhodium complexes with the $t$-Bu-xantphos ligands coordinated as tridentate $\kappa P, O, P^{\prime}$ pincer ligands (Chapter 4). The downfield shift upon coordination of the oxygen is due to the oxygen donating electron density to the metal, which inductively decreases the electron density on the adjacent carbon atoms, resulting in decreased shielding.

The solvent dependent formation of [ $\mathrm{Pt}(t-\mathrm{Bu}-x a n t p h o s) \mathrm{Cl}] \mathrm{Cl}$ complexes was confirmed by reaction of $[\mathrm{Pt}(t-\mathrm{Bu}$-xantphos $) \mathrm{Cl}] \mathrm{Cl}$ and $[\mathrm{Pt}(t-\mathrm{Bu}$-thixantphos $) \mathrm{Cl}] \mathrm{Cl}$ with $\mathrm{NH}_{4} \mathrm{PF}_{6}$. [Pt $(t-\mathrm{Bu}$-sixantphos $\left.) \mathrm{Cl}_{2}\right]$ could not be isolated and therefore was not investigated for this reaction. The two complexes react visibly with $\mathrm{NH}_{4} \mathrm{PF}_{6}$, changing a dichloromethane solution from orange/red to yellow over a one hour period. The excess $\mathrm{NH}_{4} \mathrm{PF}_{6}$ and $\mathrm{NH}_{4} \mathrm{Cl}$ by-product are readily removed by concentration of the solution and filteration through a plug of alumina. Selected NMR data is given in Table 6.5. The ${ }^{31} \mathrm{P}$ NMR spectra clearly show the presence of the $\mathrm{PF}_{6}$ counterion appearing as a septet at $-144.5 \mathrm{ppm}\left(J_{\mathrm{PF}}=710.5 \mathrm{~Hz}\right)$ for both $t$-Buthixantphos and $t$-Bu-xantphos. The ${ }^{19} \mathrm{~F}$ NMR spectra further confirms this with a clear doublet at $-73.4 \mathrm{ppm}\left(J_{\mathrm{PF}}=710.6 \mathrm{~Hz}\right)$.

The ${ }^{1} \mathrm{H}$ and ${ }^{13} \mathrm{C}$ NMR spectra for the $\left[\mathrm{Pt}\left(t-\mathrm{Bu}\right.\right.$-xantphos- $\left.\left.\kappa P, O, P^{\prime}\right) \mathrm{Cl}\right] \mathrm{PF}_{6}$ complexes show virtual triplet peaks for all of the tert-butyl environments, indicative of trans coordination of the phosphorus atoms. The position of the $\mathrm{O}$-ipso carbon has also shifted relative to both the free ligand and the $\left[\mathrm{Pt}(t-\mathrm{Bu}\right.$-xantphos $\left.) \mathrm{Cl}_{2}\right]$ 
Table 6.5: Selected NMR data for $[\mathrm{Pt}(t$-Bu-xantphos $) \mathrm{Cl}] \mathrm{X}$ complexes $\left(\mathrm{X}=\mathrm{Cl}, \mathrm{PF}_{6}\right)$ in $\mathrm{CD}_{2} \mathrm{Cl}_{2}\left(\Delta \delta=\delta_{\text {complex }}-\delta_{\text {free ligand }}\right)$.

\begin{tabular}{|c|c|c|c|c|c|c|c|}
\hline \multirow[b]{2}{*}{ Complex } & \multicolumn{5}{|c|}{${ }^{31} \mathbf{P}$} & \multicolumn{2}{|c|}{${ }^{13} \mathrm{C} O$-ipso } \\
\hline & $\delta / \mathbf{p p m}$ & $\Delta \delta / \mathbf{p p m}$ & $J_{\mathrm{PtP}} / \mathrm{Hz}$ & $\delta / \mathbf{p p m}$ & $J_{\mathrm{PF}} / \mathrm{Hz}$ & $\delta / \mathbf{p p m}$ & $\Delta \delta / \mathbf{p p m}$ \\
\hline$[\mathrm{Pt}(t-\mathrm{Bu}$-thixantphos $) \mathrm{Cl}] \mathrm{PF}_{6}$ & 46.4 & 36.9 & 2347 & -144.5 & 710.5 & 157.4 & 2.1 \\
\hline$[\mathrm{Pt}(t-\mathrm{Bu}-\mathrm{xantphos}) \mathrm{Cl}] \mathrm{PF}_{6}$ & 47.8 & 37.6 & 2350 & -144.5 & 710.4 & 158.8 & 3.0 \\
\hline$[\mathrm{Pt}(t-\mathrm{Bu}-\mathrm{xantphos}) \mathrm{Cl}] \mathrm{Cl}$ & 47.8 & 37.6 & 2345 & $\mathrm{~N} / \mathrm{A}$ & $\mathrm{N} / \mathrm{A}$ & 158.8 & 3.0 \\
\hline
\end{tabular}


complexes (Table 6.5). The shift from the free ligand is in excess of $2.0 \mathrm{ppm}$, indicative of the decreased shielding of the $\mathrm{O}$-ipso carbon that occurs upon coordination of the oxygen to a transition metal.

\subsection{Reactions with $\left[\mathrm{Pd}(\operatorname{cod}) \mathrm{Cl}_{2}\right]$}

Palladium dichloride complexes are ubiquitous in coordination chemistry, the CSD contains over 3000 X-ray crystal structures of palladium dichlorides. $\underline{68}$ The interest in these complexes is due to their relative ease of synthesis and high reactivity, which enables their wide use as pre-catalysts in a range of different catalytic processes such as the many palladium catalysed cross-coupling reactions. $\underline{\underline{31}}$

The reactivity of the $t$-Bu-xantphos ligands with palladium(II) was explored with a common palladium(II) starting material, $\left[\mathrm{Pd}(\operatorname{cod}) \mathrm{Cl}_{2}\right]$ ( $\operatorname{cod}=1,5$-cyclooctadiene). This complex is very similar to the $\left[\mathrm{PtCl}_{2}(\mathrm{hex})\right]$ that was used to explore the analogous chemistry with platinum; hexa-1,5-diene and cod are both readily displaced by phosphine ligands. The reactions between the $t$-Bu-xantphos ligands and $\left[\mathrm{Pd}(\mathrm{cod}) \mathrm{Cl}_{2}\right]$ were straightforward, producing only one product in all cases, determined to be the trans-[Pd(t-Bu-xantphos) $\left.\mathrm{Cl}_{2}\right]$ complex (Figure 6.3).<smiles>[R7]c1cc(F)c2c(c1)[X]c1cc(F)cc(CCCC)c1Oc1cc([R])cc([X])c1O2</smiles>

(i)

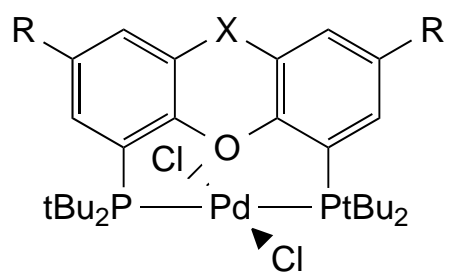

Scheme 6.3: Synthesis of $\left[\mathrm{Pd}(t-\mathrm{Bu}\right.$-xantphos $\left.) \mathrm{Cl}_{2}\right]$ complexes. Reagents and conditions: (i) $\left[\mathrm{Pd}(\operatorname{cod}) \mathrm{Cl}_{2}\right]$, toluene, $40^{\circ} \mathrm{C}, 3$ days.

Selected NMR data for the trans-[Pd(t-Bu-xantphos $\left.) \mathrm{Cl}_{2}\right]$ complexes is given in Table 6.6. The ${ }^{31} \mathrm{P}$ NMR spectra each show one singlet, while the ${ }^{1} \mathrm{H}$ and ${ }^{13} \mathrm{C}$ NMR spectra display half the total expected number of peaks indicating the presence of a plane of symmetry. The chemical shift of the ${ }^{31} \mathrm{P}$ signal varies between 30.5 and 43.9 ppm for the three complexes. A downfield shift of this magnitude is consistent with coordination to a transition metal. $\underline{16} \operatorname{In}\left[\mathrm{Pd}(t-\mathrm{Bu}\right.$-xantphos $\left.) \mathrm{Cl}_{2}\right]$ both 
of the tert-butyl carbon signals and the proton signal appear as virtual triplets, consistent with a trans coordination. However, the complexes with $t$-Bu-sixantphos and $t$-Bu-thixantphos both have resolved virtual triplets for the tert-butyl protons and quaternary carbon environments, while the terminal carbon signals display some broadening. The position of the $O$-ipso carbon can be indicative of tridentate coordination of the xantphos ligands. In this case the shift upon coordination ranges from -0.2 to $1.1 \mathrm{ppm}$. These are all smaller than would be expected if a chloride had dissociated and a pincer complex had formed.

Table 6.6: Selected NMR data for $\left[\mathrm{Pd}(t-\mathrm{Bu}\right.$-xantphos $\left.) \mathrm{Cl}_{2}\right]$ complexes in $\mathrm{CD}_{2} \mathrm{Cl}_{2}$ $\left(\Delta \delta=\delta_{\text {complex }}-\delta_{\text {free ligand }}\right)$.

\begin{tabular}{lcccccc}
\hline & \multicolumn{2}{c}{${ }^{31} \mathbf{P}$} & & \multicolumn{2}{c}{${ }^{13} \mathbf{C}$ O-ipso } \\
\cline { 2 - 3 } \cline { 6 - 7 } Diphosphine & $\delta / \mathbf{p p m}$ & $\Delta \delta / \mathbf{p p m}$ & & $\delta / \mathbf{p p m}$ & $\Delta \delta / \mathbf{p p m}$ \\
\hline t-Bu-Sixantphos & 46.7 & 38.3 & & 165.4 & 1.1 \\
t-Bu-Thixantphos & 40.0 & 30.5 & & 155.1 & -0.2 \\
$t$-Bu-Xantphos & 54.1 & 43.9 & & 156.6 & 0.8 \\
\hline
\end{tabular}

Unlike the $\left[\mathrm{Pt}(t-\mathrm{Bu}\right.$-xantphos $\left.) \mathrm{Cl}_{2}\right]$ complexes, the $\left[\mathrm{Pd}(t-\mathrm{Bu}\right.$-xantphos $\left.) \mathrm{Cl}_{2}\right]$ complexes do not display solvent dependent coordination. NMR spectroscopy of the complexes in $\mathrm{CDCl}_{3}, \mathrm{CD}_{2} \mathrm{Cl}_{2}$, and $\mathrm{C}_{6} \mathrm{D}_{6}$ showed no substantial changes in either the chemical shift of the ${ }^{31} \mathrm{P}$ NMR signal or the $O$-ipso carbon. Selected NMR data for $t$-Bu-sixantphos in different solvents is given in Table6.7.

Table 6.7: Selected NMR data for $\left[\mathrm{Pt}(t-\mathrm{Bu}\right.$-sixantphos $\left.) \mathrm{Cl}_{2}\right]$ in different solvents $\left(\Delta \delta=\delta_{\text {complex }}-\delta_{\text {free ligand }}\right)$.

\begin{tabular}{lccccc}
\hline & \multicolumn{2}{c}{${ }^{31} \mathbf{P}$} & & \multicolumn{2}{c}{${ }^{13} \mathbf{C}$ O-ipso } \\
\cline { 2 - 3 } \cline { 5 - 6 } Solvent & $\delta / \mathbf{p p m}$ & $\Delta \delta / \mathbf{p p m}$ & & $\delta / \mathbf{p p m}$ & $\Delta \delta / \mathbf{p p m}$ \\
\hline $\mathrm{C}_{6} \mathrm{D}_{6}$ & 42.0 & 33.6 & & 164.9 & 0.4 \\
$\mathrm{CDCl}_{3}$ & 45.7 & 37.3 & & 165.1 & 0.8 \\
$\mathrm{CD}_{2} \mathrm{Cl}_{2}$ & 46.7 & 38.3 & & 165.4 & 1.1 \\
\hline
\end{tabular}




\subsubsection{Formation of $\left[\mathrm{Pd}\left(t-\mathrm{Bu}-\mathrm{xantphos}-\kappa P, O, P^{\prime}\right) \mathrm{Cl}\right] \mathrm{PF}_{6}$ Complexes}

The $\left[\mathrm{Pt}(t-\mathrm{Bu}\right.$-xantphos $\left.) \mathrm{Cl}_{2}\right]$ complexes displayed solvent dependent dissociation of one of the chloride ligands. The pincer complex $\left[\mathrm{Pt}\left(t-\mathrm{Bu} \text {-xantphos- } \mathrm{k} P, \mathrm{O}, \mathrm{P}^{\prime}\right) \mathrm{Cl}\right]^{+}$ was trapped by counterion exchange with $\mathrm{NH}_{4} \mathrm{PF}_{6}$. Although the $[\mathrm{Pd}(t-\mathrm{Bu}$-xantphos) $\mathrm{Cl}_{2}$ ] complexes do not display the same solvent dependent coordination, reaction with $\mathrm{NH}_{4} \mathrm{PF}_{6}$ resulted in the analogous $[\mathrm{Pd}(t-\mathrm{Bu}$-xantphos $) \mathrm{Cl}] \mathrm{PF}_{6}$ complexes (Scheme 6.4). An associated colour change from the deep red dichloride complexes to bright yellow pincer species was observed for all three of the ligands.

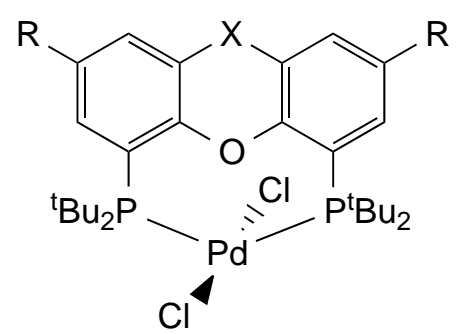

(i)

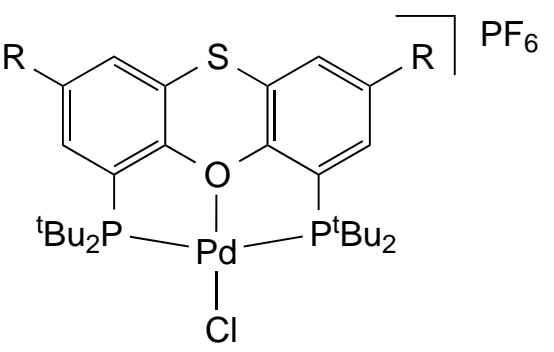

Scheme 6.4: Synthesis of $\left[\mathrm{Pd}\left(t-\mathrm{Bu}\right.\right.$-xantphos- $\left.\left.\mathrm{\kappa} P, \mathrm{O}, \mathrm{P}^{\prime}\right) \mathrm{Cl}\right] \mathrm{PF}_{6}$. Reagents and conditions: (i) $\mathrm{NH}_{4} \mathrm{PF}_{6}, \mathrm{CH}_{2} \mathrm{Cl}_{2}, 24$ hours.

Selected NMR data of the $\left[\mathrm{Pd}\left(t-\mathrm{Bu}\right.\right.$-xantphos- $\left.\left.\mathrm{N} P, \mathrm{O}, \mathrm{P}^{\prime}\right) \mathrm{Cl}\right] \mathrm{PF}_{6}$ complexes is given in Table 6.8. The $\mathrm{PF}_{6}{ }^{-}$counterion is clearly apparent as a septet in the ${ }^{31} \mathrm{P} \mathrm{NMR}$ spectrum at $-144.5 \mathrm{ppm}$ and a doublet in the ${ }^{19} \mathrm{~F}$ NMR spectrum at $-73.4 \mathrm{ppm}$, indicating a non-coordinating counterion. The ${ }^{31} \mathrm{P}$ NMR signals for the $t$-Bu-xantphos ligands have all shifted downfield. The tert-butyl ${ }^{1} \mathrm{H}$ and ${ }^{13} \mathrm{C}$ environments for all three complexes now appear as well-resolved virtual triplets indicating trans chelation of the phosphorus atoms. A reaction has occurred in all cases indicated by a shift in the ${ }^{31} \mathrm{P}$ NMR spectrum and the changes of other peaks in the ${ }^{1} \mathrm{H}$ and ${ }^{13} \mathrm{C}$ NMR spectra. Although the change in the chemical shift of the $\mathrm{O}$ ipso carbon of $t$-Bu-sixantphos from the uncoordinated ligand $(\Delta \delta)$ is indicative of oxygen coordination, the values for $t$-Bu-thixantphos and $t$-Bu-xantphos are not. This may mean that the oxygen is only very weakly coordinating to the palladium centre and in solution a T-shaped palladium complex is present, or that the complex may be stabilised by other interactions such as weak agostic interactions with the tert-butyl methyl groups or between the palladium atom 
and the $\mathrm{PF}_{6}$ counterion.

Table 6.8: Selected NMR data for $[\mathrm{Pd}(t-B u-x a n t p h o s) C l] \mathrm{PF}_{6}$ in $\mathrm{CD}_{2} \mathrm{Cl}_{2}(\Delta \delta=$ $\left.\delta_{\text {complex }}-\delta_{\text {free ligand }}\right)$.

\begin{tabular}{lccccccc}
\hline & \multicolumn{5}{c}{} & & \multicolumn{2}{c}{${ }^{13} \mathbf{C}$} \\
\cline { 2 - 4 } \cline { 8 - 9 } Ligand & $\delta / \mathbf{p p m}$ & $\Delta \delta / \mathbf{p p m}$ & $\delta / \mathbf{p p m}$ & $\mathbf{J}_{\mathbf{P F}} / \mathbf{H z}$ & & $\delta / \mathbf{p p m}$ & $\Delta \delta / \mathbf{p p m}$ \\
\hline t-Bu-Sixantphos & 52.4 & 5.7 & -144.5 & 710.3 & & 168.4 & 4.1 \\
t-Bu-Thixantphos & 56.4 & 16.4 & -144.5 & 710.5 & & 154.9 & -0.4 \\
$t$-Bu-Xantphos & 58.3 & 4.2 & -144.5 & 710.4 & & 156.7 & 0.9 \\
\hline
\end{tabular}

\subsection{Computational Results}

The $\left[\mathrm{Pt}(t\right.$-Bu-xantphos $\left.) \mathrm{Cl}_{2}\right]$ complexes displayed solvent dependent coordination forming $\left[\mathrm{Pt}\left(t-\mathrm{Bu}\right.\right.$-xantphos- $\left.\left.\mathrm{k} P, O, P^{\prime}\right) \mathrm{Cl}\right] \mathrm{Cl}$. This pincer complex was trapped by the exchange of the counterion using ammonium hexafluorophosphate. The palladium analogue did not display the same solvent-induced dissociation, although the $\left[\mathrm{Pd}\left(t-\mathrm{Bu}\right.\right.$-xantphos- $\left.\left.\mathrm{k} P, \mathrm{O}, \mathrm{P}^{\prime}\right) \mathrm{Cl}\right] \mathrm{Cl}$ complexes could be readily synthesised by reaction with ammonium hexafluorophosphate. This reactivity, particularly the solvent dependent exchange was investigated further using density functional theory. The geometries of all structures were optimised and frequencies were calculated using a B3LYP functional, 131-134 with the def2-TZVP basis set. 135,136 The energies of the systems were then calculated with incorporation of a solvent model (SMD).

Structures were optimised for the complexes $\left[\mathrm{M}(t-\mathrm{Bu}\right.$-xantphos $\left.) \mathrm{Cl}_{2}\right]$ and $[\mathrm{M}(t$ Bu-xantphos- $\left.\left.k P, O, P^{\prime}\right) \mathrm{Cl}\right]^{+}(\mathrm{M}=\mathrm{Pd}, \mathrm{Pt})$ with the three different $t$-Bu-xantphos ligands. The change in energy for the dissociation of a chloride ligand was calculated and the results for each of these reactions are summarised in Figure 6.9. Typical DFT calculations are performed for molecules in the gas phase. However, this is not always an appropriate description of the system, particularly when conversions between uncharged and charged species are involved as solvent stabilisation can become important. This is apparent for the dichloride to pincer conversion. In the gas phase, the systems show that the pincer complexes are 
around $400 \mathrm{~kJ} \mathrm{~mol}^{-1}$ higher in energy than the corresponding dichloride complex. This indicates that the reaction would be highly endothermic, although it may still be favoured entropically.

Figure 6.9: Energy change on conversion from the $\left[\mathrm{M}(t-\mathrm{Bu}-\mathrm{xantphos}) \mathrm{Cl}_{2}\right]$ to $[\mathrm{M}(t-$ Bu-xantphos)Cl]Cl.

\begin{tabular}{|c|c|c|c|c|c|}
\hline \multirow[b]{2}{*}{ Ligand } & \multicolumn{5}{|c|}{$\Delta \boldsymbol{E}, \mathrm{kJ} \mathrm{mol}^{-1}$} \\
\hline & Solvent Free & $\left(\mathrm{CH}_{3}\right)_{2} \mathrm{CO}$ & $\mathrm{C}_{6} \mathrm{H}_{6}$ & $\mathrm{CHCl}_{3}$ & $\mathrm{CH}_{2} \mathrm{Cl}_{2}$ \\
\hline$t$-Bu-sixantphos & 393.5 & -2.2 & 150.7 & 59.0 & 20.4 \\
\hline t-Bu-thixantphos & 400.2 & 4.3 & 157.9 & 66.0 & 27.3 \\
\hline$t$-Bu-xantphos & 384.5 & -14.6 & 140.1 & 47.7 & 8.6 \\
\hline \multicolumn{6}{|c|}{$\left[\mathrm{Pd}(\right.$ diphosphine $\left.) \mathrm{Cl}_{2}\right] \rightarrow[\mathrm{Pd}(\mathrm{POP}) \mathrm{Cl}]+\mathrm{Cl}^{-}$} \\
\hline & \multicolumn{5}{|c|}{$\Delta \boldsymbol{E}, \mathrm{kJ} \mathrm{mol}^{-1}$} \\
\hline Ligand & Solvent Free & $\left(\mathrm{CH}_{3}\right)_{2} \mathrm{CO}$ & $\mathrm{C}_{6} \mathrm{H}_{6}$ & $\mathrm{CHCl}_{3}$ & $\mathrm{CH}_{2} \mathrm{Cl}_{2}$ \\
\hline$t$-Bu-sixantphos & 397.4 & 0.8 & 155.0 & 62.7 & 23.7 \\
\hline t-Bu-thixantphos & 405.3 & 7.9 & 163.1 & 70.3 & 31.4 \\
\hline t-Bu-xantphos & 391.7 & -7.9 & 83.2 & 55.0 & 15.6 \\
\hline
\end{tabular}

Single point energy calculations for the $\left[\mathrm{M}(t-\mathrm{Bu}\right.$-xantphos $\left.) \mathrm{Cl}_{2}\right]$ and $[\mathrm{M}(t-\mathrm{Bu}$-xantphos- $\left.\left.\mathrm{k} P, \mathrm{O}, \mathrm{P}^{\prime}\right) \mathrm{Cl}\right]^{+}$in a range of solvents were performed on the structures that had been optimised in the gas phase (Figure 6.9). The results show that the introduction of any solvent reduces the energy required by more than half. For both the platinum and palladium complexes, the reaction in benzene requires the most energy of any of the solvents studied. This is consistent with the experimental results, as the platinum complexes are present as $\left[\mathrm{Pt}(t-\mathrm{Bu}-x a n t p h o s) \mathrm{Cl}_{2}\right]$ in benzene. For the platinum complexes conversion to [ $\mathrm{Pt}\left(t-\mathrm{Bu}\right.$-xantphos- $\left.\left.k P, O, P^{\prime}\right) \mathrm{Cl}\right] \mathrm{Cl}$ was observed experimentally in chloroform and dichloromethane and the spectrum in acetone was broad, likely indicating some conversion. Interestingly, the computational results indicate that the lowest energy change occurs for the reaction in acetone, as these values are either close to zero or even negative, while the dichloromethane values are $8.6-27.3 \mathrm{~kJ} \mathrm{~mol}^{-1}$ and the chloroform values are 
higher still 47.7-66.0 $\mathrm{kJ} \mathrm{mol}^{-1}$. These values do not include entropic effects which are likely to be significant as the disorder is increased. Although the calculations suggest that the chloride dissociation is an endothermic process, the reactions may be spontaneous due to entropy.

The results for the palladium complexes show similar overall trends to the platinum complexes (Figure 6.9). However, most of the palladium reactions require slightly more energy than the platinum complexes. This is consistent with the observation that the reaction is spontaneous for platinum (under certain conditions) while it is not for palladium. The energy for the palladium complexes in dichloromethane are lower than the energy for the platinum reaction in chloroform, which was observed to be spontaneous experimentally, again indicating that entropy is likely playing a significant role for this reaction.

\subsection{Reactions with Platinum Dimethyl Starting Materials}

Transition metal alkyl complexes are among the most important organometallic complexes as they can be intermediates in a wide range of catalytic transformations, including polymerisation and carbonylation reactions. $\underline{113,338}$ The synthesis of isolatable transition metal alkyl complexes is important, as studying their chemistry can give insight into the reactions occurring in catalytic systems. ${ }^{339}$ Methyl complexes have gained attention in recent years, as protonation of a rhodium methyl complex led to a $\sigma$-methane complex which was characterised by NMR spectroscopy. ${ }^{340}$ Platinum methyl complexes were investigated as part of this study as the stronger $\sigma$-donor character of the methyl ligand may lead to different reactivity than that of the platinum dichloride complexes. ${ }^{252}$

The reactivity of the $t$-Bu-xantphos ligands with $\left[\mathrm{Pt}(\right.$ hex $\left.\left.) \mathrm{Me}_{2}\right)\right]$ was first studied using the $t$-Bu-thixantphos ligand. $t$-Bu-Thixantphos was combined with [Pt(hex) $\left.\mathrm{Me}_{2}\right]$ in an NMR tube in $\mathrm{C}_{6} \mathrm{D}_{6}$ under argon to enable the progress to be followed by NMR spectroscopy. After 24 hours at room temperature no reaction was observed, so the sample was heated to $60{ }^{\circ} \mathrm{C}$. The progress of the sample was monitored at regular intervals. However, after 28 days at $60{ }^{\circ} \mathrm{C}$ no changes were observed. The ${ }^{31} \mathrm{P}$ NMR spectrum showed a single peak indicative 
of uncoordinated $t$-Bu-thixantphos, and the ${ }^{1} \mathrm{H}$ NMR spectrum showed a mixture with $\left[\mathrm{Pt}(\mathrm{hex}) \mathrm{Me}_{2}\right]$. This indicates that the lack of reactivity was not the result of degradation of either the platinum starting material or the $t$-Bu-thixantphos ligand.

One equivalent of the strong acid $\mathrm{CHPh}\left(\mathrm{SO}_{2} \mathrm{CF}_{3}\right)_{2}$ was added to the mixture of $t$-Bu-thixantphos and $\left[\mathrm{Pt}(\mathrm{hex}) \mathrm{Me}_{2}\right]$ in an attempt to protonate the platinum complex and remove one of the methyl ligands as methane, thus forming a free coordination site to promote reaction with the $t$-Bu-thixantphos. As discussed in Section 2.3, the $t$-Bu-xantphos ligands can act as Brønsted bases and have $\mathrm{p} K_{\mathrm{b}}$ values of 5.67-6.90. As such, the $t$-Bu-thixantphos ligand reacted with the acid quickly and completely forming [(t-Bu-thixantphos) $\mathrm{H}] \mathrm{CPh}\left(\mathrm{SO}_{2} \mathrm{CF}_{3}\right)_{2}$ (Scheme 6.5). Although the mixture was subsequently followed for several days by NMR spectroscopy, no reaction was observed between the protonated $t$-Bu-thixantphos ligand and the $\left[\mathrm{Pt}(\mathrm{hex}) \mathrm{Me}_{2}\right]$ complex.<smiles>CCCCCc1cc(C)cc2c1Oc1c(CCCC)cc(C)cc1Cc1cc(C)cc(c1OCC(C)C)S2</smiles>

(ii)

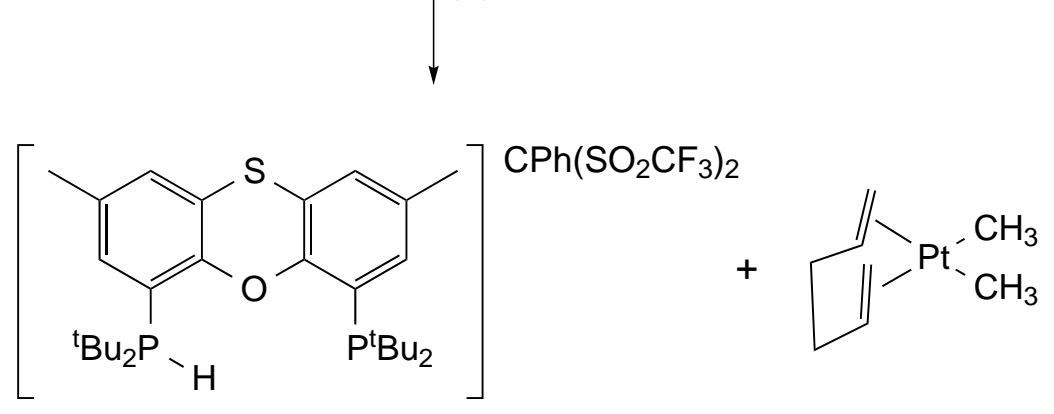

Scheme 6.5: Attempted reaction of $\left[\mathrm{Pt}(\mathrm{hex}) \mathrm{Me}_{2}\right]$ and $t$-Bu-thixantphos. Reagents and conditions: (i) $\mathrm{C}_{6} \mathrm{D}_{6}, 60^{\circ} \mathrm{C}$, (ii) $\mathrm{HCPh}\left(\mathrm{SO}_{2} \mathrm{CF}_{3}\right)_{2}$.

When the $t$-Bu-xantphos ligands were reacted with $\left[\mathrm{PtCl}_{2} \mathrm{~L}_{2}\right]$ complexes $(\mathrm{L}=$ $\mathrm{SEt}_{2}, \mathrm{MeCN}, t$-BuCN, $\mathrm{L}_{2}=1,5$-hexadiene) no cis products were observed regardless of the starting material, and the reaction was fastest when the cis complex $\left[\mathrm{PtCl}_{2}(\right.$ hex $\left.)\right]$ was used due to the lability of the diene. Due to the steric bulk of the 
tert-butyl substituents on the phosphorus atoms and the rigidity of the $t$-Bu-xantphos backbone these ligands would be expected to act as trans-spanning diphosphines or tridentate $\kappa P, O, P^{\prime}$ pincer ligands rather than forming cis chelates. Thus, in order for a reaction to occur between $t$-Bu-xantphos and $\left[\mathrm{Pt}(\mathrm{hex}) \mathrm{Me}_{2}\right]$ the system must undergo a cis-trans isomerism process. For the dichlorides this is easily facilitated by loss of one of the chloride ligands followed by re-coordination in a trans configuration. However, methyl ligands are strong $\sigma$-donors and coordinate very strongly to $\mathrm{Pt}(\mathrm{II}) .{ }^{252}$ The first step of the reaction likely proceeds with one of the phosphorus donors displacing one half of the diene (Scheme 6.6). From this position the other phosphorus needs to displace the alkene forming a cis-tBu-xantphos complex. This could then undergo cis-trans isomerism; or the complex could isomerise first and then the phosphine could displace the alkene. In the first case, the cis-t-Bu-xantphos complex would be highly disfavoured due to the very large natural bite-angle of these ligands. In either case, for the cis-trans isomerism to occur, the complex must lose a methyl ligand and these are too strongly coordinated for this to occur, especially combined with the low transinfluence of the hexa-1,5-diene ligand and the moderate trans-influence of the phosphorus donors. $\stackrel{252,253,322}{2}$

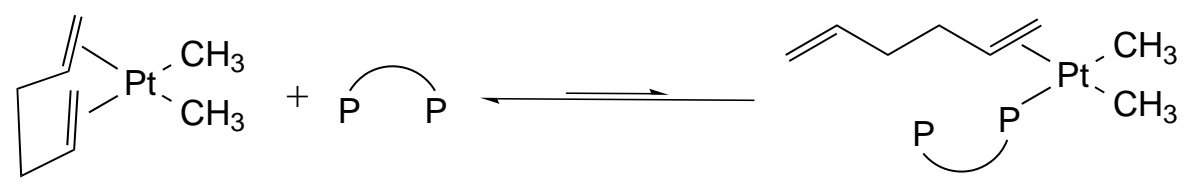

Scheme 6.6: Proposed reaction between $\left[\mathrm{Pt}(\mathrm{hex}) \mathrm{Me}_{2}\right]$ and a $t$-Bu-xantphos diphosphine ligand (PP).

\subsection{Reactions with $[\mathrm{PtCl}(\mathrm{hex}) \mathrm{Me}]$}

Chloridomethylplatinum complexes are interesting due to the different properties of the chloride and methyl ligands. The methyl ligand is a much stronger $\sigma$ donor than a chloride ligand, meaning that the methyl imparts a much stronger trans-influence. $252,253,322$ The difference in the donor properties of the chloride and methyl ligands can result in asymmetric reactivity as the chloride will undergo substitution more readily than the methyl ligand. If a cis-[PtClMe(PP)] 
complex was formed, the two phosphorus atoms would be in different environments, which could lead to asymmetric reactions of the diphosphine. In the more likely scenario for the $t$-Bu-xantphos ligand system, that trans-[Pt $(t-B u-$ xantphos)ClMe] complex forms, then the strong trans-influence of the methyl group may promote the dissociation of the chloride ligand

$[\mathrm{PtCl}($ hex)Me] was chosen as the starting material due to the success of the reactions of the $t$-Bu-xantphos ligands with $\left[\mathrm{PtCl}_{2}(\mathrm{hex})\right]$. Reaction between the $t$-Buxantphos ligands and $\left[\mathrm{PtCl}\left(\right.\right.$ hex)Me] was performed on an NMR-scale in $\mathrm{C}_{6} \mathrm{D}_{6}$ to allow the reactions to be studied as they progressed and identify any intermediates that may form. As the reaction progressed the product precipitated out as an off-white solid. This solid was insoluble in most common solvents except acetone- $\mathrm{d}_{6}$. The $t$-Bu-xantphos ligands and $[\mathrm{PtCl}($ hex)Me] are insoluble in acetone, meaning that this was not suitable as the reaction solvent. In all cases, the solution was decanted from the precipitate and the solid was dissolved in acetone- $\mathrm{d}_{6}$, where only one species, $([\mathrm{Pt}(t-\mathrm{Bu}$-xantphos $) \mathrm{Me}] \mathrm{Cl})$ was observed, indicating complete reaction (Scheme 6.7).

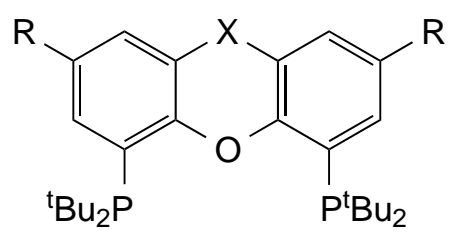

(i)

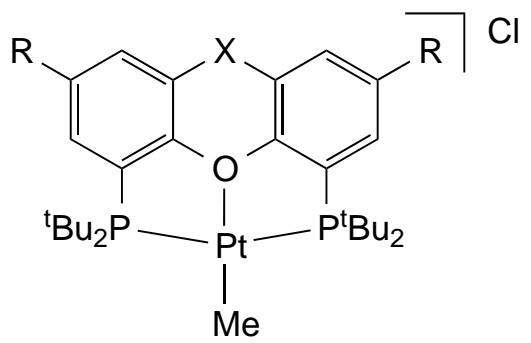

Scheme 6.7: Reaction between [ $\mathrm{PtCl}(\mathrm{hex}) \mathrm{Me}]$ and $t$-Bu-xantphos ligands. Reagents and conditions: (i) [ $\mathrm{PtCl}\left(\right.$ hex)Me], 24 hours, room temperature $\left(50{ }^{\circ} \mathrm{C}\right.$ t-Bu-sixantphos).

The speed of the reaction varied between the three ligands. With $t$-Bu-xantphos the reaction was fastest, reaching completion in 48 hours at room temperature. $t$ Bu-Thixantphos reacted slightly more slowly, with completion observed after 72 hours. $t$-Bu-Sixantphos was the slowest of the three systems; little reaction was observed after 72 hours at room temperature, however after $24 \mathrm{hrs}$ of heating at $50{ }^{\circ} \mathrm{C}$ the reaction was complete by NMR spectroscopy. This difference in reactivity is likely the result of the bite-angle of the ligand and the amount of energy 
required to achieve a trans-geometry. With the larger calculated bite-angle of the $t$-Bu-xantphos ligand the amount of energy required to achieve a pincer geometry would be lower than that for the other ligands. Similarly $t$-Bu-sixantphos, with the smallest of the three natural bite-angles, would require more energy in order to achieve the coordination geometry of the product.

Selected NMR data for the three $[\mathrm{Pt}(t-\mathrm{Bu}$-xantphos) $\mathrm{Me}] \mathrm{Cl}$ complexes is summarised in Table 6.9. In the ${ }^{31} \mathrm{P}$ NMR spectra only one signal is observed for each of the complexes, indicating identical environments for both phosphorus atoms which implies symmetrical complexes. Given that $[\mathrm{PtCl}($ hex $) \mathrm{Me}]$ is asymmetrical and a symmetrical complex is formed, this is evidence for trans-coordination of the $t$-Bu-xantphos ligands. The ${ }^{31} \mathrm{P}$ NMR signal has shifted downfield by around 40 ppm to $48.7,50.5$, or 51.0 ppm for $t$-Bu-sixantphos, $t$-Bu-thixantphos and $t$-Buxantphos, respectively. The peak for each complex is a singlet with $J_{\mathrm{PtP}}$ coupling ranging from 2763 to $2793 \mathrm{~Hz}$. If the phosphorus atom was trans to a chloride we would typically expect coupling constants larger than $3000 \mathrm{~Hz}$, while if it was trans to a methyl ligand we would expect values below $2000 \mathrm{~Hz}$. A coupling constant of around $2800 \mathrm{~Hz}$ is generally indicative of phosphorus trans to an atom with a similar trans-influence, in this case another phosphorus. Furthermore this coupling is of similar magnitude to the coupling constant observed for the $\left[\mathrm{Pt}(t\right.$-Bu-xantphos $\left.) \mathrm{Cl}_{2}\right]$ complexes $(2686-2721 \mathrm{~Hz})$. Both of these points strongly indicate a trans-coordination geometry for the complex.

The position of the $\mathrm{O}$-ipso carbon in the ${ }^{13} \mathrm{C}$ NMR spectrum can be indicative of coordination of the oxygen to a metal centre. In this particular case the peak for $t$-Bu-sixantphos moves 3.0 ppm downfield from the uncoordinated ligand, while the peak for $t$-Bu-xantphos shifts by only $0.3 \mathrm{ppm}$ downfield and the peak for $t$-Bu-thixantphos shifts 1.5 ppm upfield comparing the free ligand with the $[\mathrm{Pt}(t-\mathrm{Bu}-x a n t p h o s) \mathrm{Me}] \mathrm{Cl}$ complexes. This is no clear indication of whether the oxygen is coordinated to the metal centre or not. One of the reasons for this inconsistency is the insolubility of the free ligand in acetone, meaning that we are comparing the data for the free ligand in $\mathrm{CDCl}_{3}$ with the complex in acetone- $\mathrm{d}_{6}$. Peaks frequently shift depending on the solvent, meaning that no conclusions can be drawn from the position of the O-ipso ${ }^{13} \mathrm{C}$ NMR signal in this case. The solubility of the complex is indicative of the chloride dissociating from the metal centre. The $[\mathrm{Pt}(t-\mathrm{Bu}$-xantphos) $\mathrm{Me}] \mathrm{Cl}$ complexes are insoluble in the non-polar $\mathrm{C}_{6} \mathrm{D}_{6}$, whereas they are very soluble in polar acetone- $\mathrm{d}_{6}$. This is consistent with a 


\begin{tabular}{|c|c|c|c|c|c|}
\hline \multirow[b]{2}{*}{ Diphosphine } & \multicolumn{2}{|c|}{${ }^{31} \mathbf{P}$} & \multirow{2}{*}{$\frac{{ }^{1} \mathbf{H}}{\mathbf{P t}-\mathrm{CH}_{3} \delta / \mathbf{p p m}}$} & \multicolumn{2}{|c|}{${ }^{13} \mathrm{C}$} \\
\hline & $\delta / \mathbf{p p m}$ & $\Delta \delta / \mathbf{p p m}$ & & $\mathbf{P t}-\mathrm{CH}_{3} \delta / \mathbf{p p m}$ & $\Delta \delta \mathbf{C - O} / \mathbf{p p m}$ \\
\hline t-Bu-Sixantphos & $48.7(2763)$ & 40.3 & $2.01(98.6)$ & $-22.7(780.5)$ & 3.0 \\
\hline t-Bu-Thixantphos & $50.5(2793)$ & 41.0 & $1.94(97.4)$ & $-23.8(777.2)$ & -1.5 \\
\hline$t$-Bu-Xantphos & $51.0(2788)$ & 40.8 & $1.92(97.4)$ & $-23.9(774.6)$ & 0.3 \\
\hline
\end{tabular}


charged species forming in solution which could result from the loss of the chloride ligand. Furthermore, in the $\left[\mathrm{Pt}(t-\mathrm{Bu}\right.$-xantphos $\left.) \mathrm{Cl}_{2}\right]$ complexes the chloride was observed to dissociate readily. In this case the high trans-influence character of the methyl ligand would promote the loss of the chloride ligand. In addition, the strong $\sigma$-donor ability of the methyl ligand would mean that the oxygen atom would be coordinated in an extremely weak fashion and would donate less electron density when in $[\mathrm{Pt}(t-\mathrm{Bu}$-xantphos $) \mathrm{Me}] \mathrm{Cl}$ compared with $[\mathrm{Pt}(t-\mathrm{Bu}$-xantphos)Cl]Cl.

The peaks for the methyl ligand in the ${ }^{1} \mathrm{H}$ and the ${ }^{13} \mathrm{C}$ NMR spectra can give additional information about the nature of the complex. In the ${ }^{1} \mathrm{H}$ NMR spectrum the peak appears between 1.92 and 2.01 ppm as a triplet with platinum satellites. Coupling to two phosphorus atoms in the same environment generates the triplet with a three-bond $J_{\mathrm{PH}}$ coupling constant of $5.4-5.5 \mathrm{~Hz}$, indicative of a cis relationship between the phosphorus atoms and the methyl ligand, consistent with a trans-spanning diphosphine ligand. The value of the two-bond platinum-proton coupling constant on the methyl ligand ranges from $97.4 \mathrm{~Hz}$ for both $t$-Bu-xantphos and $t$-Bu-thixantphos, to $98.6 \mathrm{~Hz}$ for $t$-Bu-sixantphos. This is a very large coupling constant for a platinum methyl ligand and is indicative of a ligand trans to the methyl which has a very low trans-influence. This is also reflected in the ${ }^{13} \mathrm{C}$ NMR spectrum where the methyl ligands appear at between -22.7 and $-23.9 \mathrm{ppm}$ with coupling constants of 774.6-780.5 Hz. The large upfield shift of the methyl ligand from the typical position of an organic methyl substituent (5-30 ppm ${ }^{341}$ ) arises from the additional electron density as a result of coordination. The chemical shift of the methyl carbon, together with the value of $J_{\mathrm{PC}}$ is largely the result of the ligand in the trans position. Previous work both in our group ${ }^{315,316}$ and by others ${ }^{342}$ has shown that methyl ligands trans to a moderate $\sigma$ donor such as a phosphorus appear at $5-15 \mathrm{ppm}$ in the ${ }^{13} \mathrm{C}$ NMR spectrum, with a platinumcarbon coupling constant of around $700 \mathrm{~Hz}$. In contrast, when the methyl is trans to a weak $\sigma$-donor ligand such as a nitrogen donor the methyl shifts upfield to around -10- -25 ppm with larger coupling constants of around $800 \mathrm{~Hz}$. In this case values of -22.7 to -23.9 for the ${ }^{13} \mathrm{C}$ chemical shift and values of $J_{\mathrm{PtC}}$ between 774.6-780.5 Hz clearly indicate that the methyl ligand is trans to a weak $\sigma$-donor such as a chloride or an oxygen. The value of $J_{\mathrm{PtC}}$ is lowest for $t$-Bu-xantphos and highest for $t$-Bu-sixantphos suggesting a series of O-trans influence for the three ligands of $t$-Bu-sixantphos $<t$-Bu-thixantphos $<t$-Bu-xantphos. 
Based on the relative reactivity of the $t$ - $\mathrm{Bu}$-xantphos ligands with $[\mathrm{PtCl}(\mathrm{hex}) \mathrm{Me}]$ and the NMR data, $[\mathrm{Pt}(t-\mathrm{Bu}$-xantphos) $\mathrm{Me}] \mathrm{Cl}$ is proposed as the product. However, the coordination of the oxygen has not been established conclusively. Two related complexes have been reported: $\left[\mathrm{PdMe}\left(\mathrm{P}^{\mathrm{t}} \mathrm{Bu}_{3}\right)_{2}\right]^{+}$and $\left[\mathrm{PtMe}\left(\mathrm{P}^{\mathrm{i}} \mathrm{Pr}_{3}\right)_{2}\right]^{+}$, the solid state structures of which have been determined by X-ray crystallography (Figure 6.10). 343,344 Both complexes are stabilised in the solid-state by an agostic interaction from one of the isopropyl or tert-butyl methyl substituents, although they were synthesised in the presence of coordinating solvents. The NMR spectra of each showed symmetrical molecules with no evidence for an agostic interaction, even down to $-80^{\circ} \mathrm{C}$, indicating rapid exchange of the agostic interaction between the numerous $\mathrm{C}-\mathrm{H}$ bonds available. Low temperature ${ }^{1} \mathrm{H}$ and ${ }^{31} \mathrm{P}$ $\mathrm{NMR}$ analysis of $\left[\mathrm{Pt}\left(t-\mathrm{Bu}\right.\right.$-sixantphos)Me] was carried out from 20 to $-80^{\circ} \mathrm{C}$. No changes in the spectra were observed, except for a slight broadening of the signals due to precipitation. This indicates that if an agostic-bond is present, the presence of 12 different tert-butyl $\mathrm{CH}_{3}$ groups (i.e. 36 different agostic possibilities) means there is ample opportunity for exchange, resulting in the apparent symmetry at room temperature.

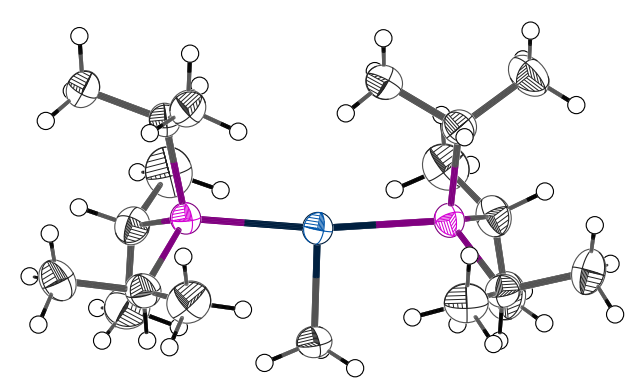

(a) $\left[\mathrm{PtMe}\left(\mathrm{P}^{\mathrm{i}} \mathrm{Pr}_{3}\right)_{2}\right]^{+}$

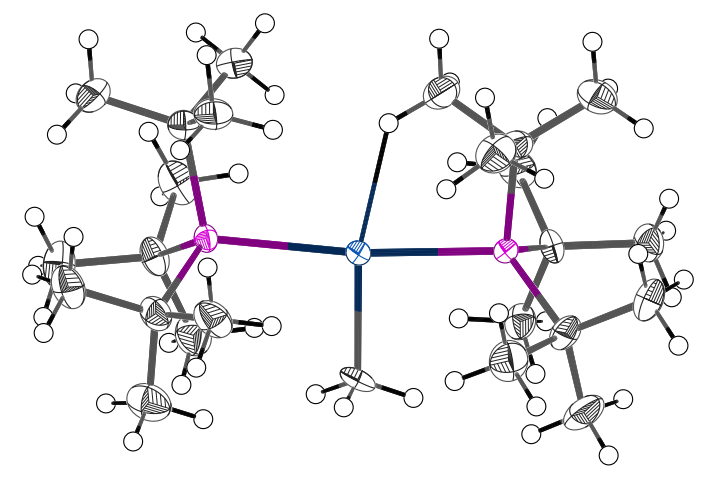

(b) $\left[\mathrm{PdMe}\left(\mathrm{P}^{\mathrm{t}} \mathrm{Bu}_{3}\right)_{2}\right]^{+}$

Figure 6.10: X-ray crystal structures of $\left[\mathrm{PtMe}\left(\mathrm{P}^{\mathrm{i}} \mathrm{Pr}_{3}\right)_{2}\right]^{+\underline{343}}$ and $\left[\mathrm{PdMe}\left(\mathrm{P}^{\mathrm{t}} \mathrm{Bu}_{3}\right)_{2}\right]^{+} . \underline{344}$ Counterions omitted for clarity.

On the basis of the spectroscopic analysis and the structures of similar compounds there are three plausible structures for the product from reaction between $[\mathrm{PtCl}($ hex) $\mathrm{Me}]$ and the $t$-Bu-xantphos ligands; all contain the $t$-Bu-xantphos ligand in a trans-configuration and a methyl group. The fourth coordination site on the platinum could either be occupied by a chloride ligand, an agostic interaction from one of the many tert-butyl C-H bonds, or by a very weak interaction 
with the oxygen in the backbone of the $t$-Bu-xantphos ligands. The absence of the chloride ligand was confirmed by reaction between $[\mathrm{Pt}(t-\mathrm{Bu}$-xantphos)Me]Cl and $\mathrm{NH}_{4} \mathrm{PF}_{6}$ on an NMR-scale. Over the course of several days no change was observed in the ${ }^{1} \mathrm{H},{ }^{13} \mathrm{C}$ or ${ }^{31} \mathrm{P}$ NMR spectra except for the appearance of a septet in the ${ }^{31} \mathrm{P}$ NMR spectrum and a doublet in the ${ }^{19} \mathrm{~F}$ NMR spectrum indicative of a non-coordinating $\mathrm{PF}_{6}{ }^{-}$counterion $\left(\mathrm{NH}_{4} \mathrm{PF}_{6}\right.$ is insoluble in acetone- $\left.\mathrm{d}_{6}\right)$. As no change was observed in the NMR spectra upon cooling to $-80^{\circ} \mathrm{C}$, the fourth coordination site could be occupied either a rapidly exchanging agostic interaction or the oxygen in the backbone (Scheme 6.8).
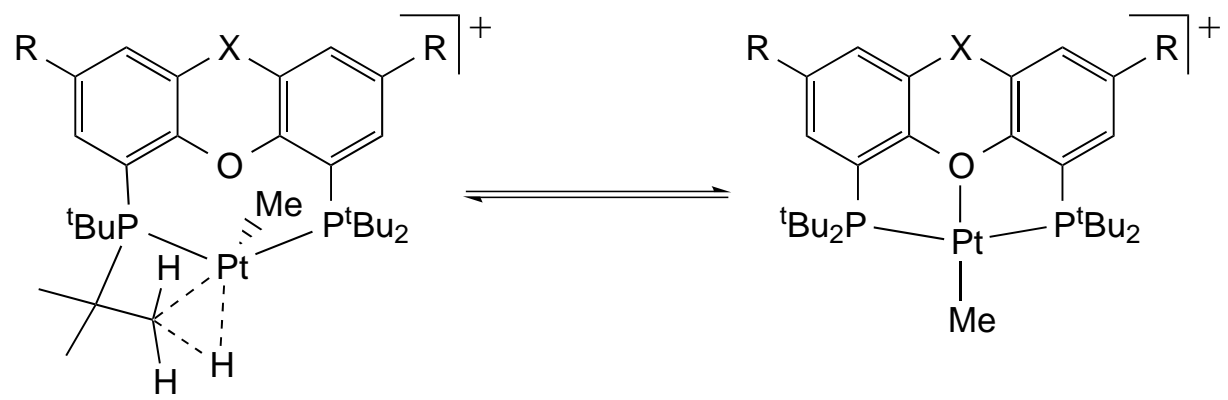

Scheme 6.8: Equilibrium between $[\mathrm{Pt}(t-\mathrm{Bu}-x a n t p h o s) \mathrm{Me}]$ and $[\mathrm{Pt}(t-\mathrm{Bu}-\mathrm{xantphos}-$ $\left.\left.\kappa P, O, P^{\prime}\right) \mathrm{Me}\right]$.

\subsubsection{Computational Results}

Computational modelling was used to determine whether the three $[\mathrm{Pt}(t-\mathrm{Bu}$ xantphos)Me]X complexes take the form of a pincer complex [Pt $(t-B u-x a n t p h o s-$ $\left.\kappa P, O, P^{\prime}\right) \mathrm{Me}^{+}$, or whether an agostic interaction from the tert-butyl groups is formed instead. Structural models of both of these isomers were optimised and their vibrational frequencies were calculated using density functional theory using a B3LYP functional $\stackrel{131-134}{ }$ with the def2-TZVP basis set. 135,136 Selected bond lengths and angles are given in Table 6.10 and 6.11 for the agostic and pincer complexes respectively.

Little variation is present in the bond lengths and bite-angles for the three ligands in either the pincer or the agostic complex. The platinum-methyl bond length varies by only $0.001 \AA$ between the three agostic and the three pincer complexes, 
Table 6.10: Selected bond lengths and angles calculated for the agostic complexes $[\mathrm{Pt}(t-\mathrm{Bu}-x a n t p h o s) \mathrm{Me}]^{+}$.

\begin{tabular}{lccc}
\hline Ligand & Pt-Me $/ \AA$ & Pt-H $/ \AA$ & P-Pt-P / \\
\hline$t$-Bu-Sixantphos & 2.051 & 2.763 & 153.9 \\
$t$-Bu-Thixantphos & 2.052 & 2.828 & 153.7 \\
$t$-Bu-Xantphos & 2.051 & 2.868 & 153.2 \\
\hline
\end{tabular}

Table 6.11: Selected bond lengths and angles calculated for the pincer complexes $\left[\mathrm{Pt}\left(t-\mathrm{Bu}-\text { xantphos-k } P, \mathrm{O}, \mathrm{P}^{\prime}\right) \mathrm{Me}\right]^{+}$.

\begin{tabular}{lccc}
\hline Ligand & Pt-Me $/ \AA$ & Pt-O $/ \AA$ & P-Pt-P $/{ }^{\circ}$ \\
\hline$t$-Bu-Sixantphos & 2.052 & 2.323 & 165.7 \\
$t$-Bu-Thixantphos & 2.053 & 2.277 & 164.4 \\
$t$-Bu-Xantphos & 2.053 & 2.256 & 164.4 \\
\hline
\end{tabular}


and by only $0.002 \AA$ across all six of the structures. Similarly, the bite-angles show little difference among the complexes despite the difference in the natural bite-angles for the ligands. The platinum-oxygen bond length for the pincer complex shows a decrease with the increasing natural bite-angle of the ligand such that the $t$-Bu-sixantphos complex has the longest $\mathrm{Pt}-\mathrm{O}$ bond. A similar trend is present for the $\mathrm{Pt}-\mathrm{H}$ bond length in the agostic complexes, where the longest $\mathrm{Pt}$ $\mathrm{H}$ distance is for the $t$-Bu-sixantphos system and the shortest $\mathrm{Pt}-\mathrm{H}$ distance is for the $t$-Bu-xantphos complex. These are consistent with the observed changes in the ${ }^{13} \mathrm{C}$ NMR spectrum for the methyl ligand, whereby the $t$-Bu-sixantphos complex has the largest value of $J_{\mathrm{PtC}}$ and $t$-Bu-xantphos has the smallest. Decreasing the $\mathrm{Pt}-\mathrm{O}$ or $\mathrm{Pt}-\mathrm{H}$ bond lengths will increase the trans influence of the ligand as a result of increased $\sigma$ donation from the ligand to the platinum, leading to a lower platinum-carbon coupling constant.

The Gibbs free energy for the each of the complexes was also calculated (Table 6.12). The agostic complexes were consistently higher in energy than the pincer complexes, indicating that conversion from the agostic to the pincer is an exothermic process generating 106, 111, or $122 \mathrm{~kJ} \mathrm{~mol}^{-1}$ of energy. This suggests that the pincer complex is likely the thermodynamically favoured product in the reaction between $[\mathrm{PtCl}($ hex)Me] and the three $t$-Bu-xantphos ligands (Scheme 6.8). The value for $\Delta G$ appears to be correlated with the natural bite-angle of the ligands. This indicates that although the three diphosphine ligands produce complexes with similar bite-angles, the natural bite-angle of the ligand can impact the stability of the starting and final complexes.

Table 6.12: Gibbs free energies calculated for the agostic and pincer complexes $\left[\mathrm{Pt}\left(t-\mathrm{Bu}-\mathrm{xantphos}-\kappa P, O, P^{\prime}\right) \mathrm{Me}\right]^{+}$. All values are in $\mathrm{kJ} \mathrm{mol}^{-1}$

\begin{tabular}{lccc}
\hline Ligand & Agostic $\boldsymbol{G}$ & Pincer $\boldsymbol{G}$ & $\Delta \boldsymbol{G}$ \\
\hline$t$-Bu-Sixantphos & -6245006 & -6245113 & -106 \\
$t$-Bu-Thixantphos & -6527190 & -6527301 & -111 \\
$t$-Bu-Xantphos & -5584828 & -5584950 & -122 \\
\hline
\end{tabular}

While the relative thermodynamic preference between the pincer and the agostic has been established, the reaction occurring is actually dissociation of a chlo- 
ride from the $[\mathrm{Pt}(t-\mathrm{Bu}-x a n t p h o s) C l M e]$ complexes forming either the pincer or the agostic complex. The energy changes for these reactions are given in Tables 6.13 and 6.14. In this case we see that under standard gas-phase DFT conditions, neither the pincer nor the agostic complex is lower in energy than the $[\mathrm{Pt}(t-\mathrm{Bu}-$ xantphos)ClMe] starting material. However, significantly less energy is required for the conversion to the pincer than to the agostic.

Table 6.13: Energy change for the conversion of the $[\mathrm{Pt}(t-\mathrm{Bu}-\mathrm{xantphos}) \mathrm{ClMe}$ to the pincer complex.

\begin{tabular}{|c|c|c|c|c|c|}
\hline \multirow[b]{2}{*}{ Ligand } & \multicolumn{5}{|c|}{$\Delta \mathrm{E}, \mathrm{kJ} \mathrm{mol}^{-1}$} \\
\hline & Solvent Free & $\left(\mathrm{CH}_{3}\right)_{2} \mathrm{CO}$ & $\mathrm{C}_{6} \mathrm{H}_{6}$ & $\mathrm{CHCl}_{3}$ & $\mathrm{CH}_{2} \mathrm{Cl}_{2}$ \\
\hline t-Bu-sixantphos & 341.2 & -45.6 & 100.4 & 11.7 & -24.7 \\
\hline t-Bu-thixantphos & 349.7 & -38.3 & 109.2 & 20.0 & -17.0 \\
\hline t-Bu-xantphos & 337.5 & -52.7 & 97.2 & 7.4 & -30.4 \\
\hline
\end{tabular}

Table 6.14: Energy change for the conversion of the [Pt(t-Bu-xantphos)ClMe] to the agostic complex $[\mathrm{Pt}(t-\mathrm{Bu}-x a n t p h o s) \mathrm{Me}]^{+}$.

\begin{tabular}{lccccc}
\multicolumn{5}{c}{$[\mathrm{Pt}(t$-Bu-xantphos $) \mathrm{ClMe}] \rightarrow[\mathrm{Pt}(t \text {-Bu-xantphos }) \mathrm{Me}]^{+}+\mathrm{Cl}^{-}$} \\
\hline \multirow{2}{*}{ Ligand } & Solvent Free & $\left(\mathbf{C H}_{3}\right)_{\mathbf{2}} \mathbf{C O}$ & $\mathbf{C}_{6} \mathbf{H}_{\mathbf{6}}$ & $\mathbf{C H C l}_{3}$ & $\mathbf{C H}_{\mathbf{2}} \mathbf{C l}_{\mathbf{2}}$ \\
\cline { 2 - 6 }$t$-Bu-sixantphos & 454.2 & 54.9 & 210.0 & 117.0 & 78.2 \\
$t$-Bu-thixantphos & 462.4 & 59.8 & 217.6 & 123.8 & 84.1 \\
$t$-Bu-xantphos & 462.7 & 60.4 & 218.9 & 124.9 & 84.8 \\
\hline
\end{tabular}

Typical DFT computations are carried out for single molecules in the gas phase. While this is applicable to a range of applications, when dealing with conversions between uncharged and charged molecules the solvent can have a significant impact on the thermodynamics of the reaction. It has been observed experimentally with the platinum and palladium dichloride complexes that the solvent can also play a role in the formation of the pincer complexes. As such, single-point energy calculations with a solvent correction were performed for the conversion of $[\mathrm{Pt}(t-$ $\mathrm{Bu}$-xantphos)ClMe] into the pincer or the agostic complexes. The energy changes 
are summarised in Tables 6.13 and 6.14 for the pincer and the agostic respectively. The inclusion of any of the solvents drastically decreases the amount of energy required to form the product, thus indicating that the solvent is important for these reactions. Under experimental conditions the reaction is performed in benzene from which the product precipitates as a white solid that is only soluble in acetone (of the solvents examined).

The energy change for conversion of the [ $\mathrm{Pt}(t-\mathrm{Bu}$-xantphos)ClMe] complexes into the pincer is much lower than that required to form the agostic complex, regardless of solvent. This indicates that the pincer is thermodynamically preferred over the agostic complex. Interestingly, for the pincer complex the reaction in benzene requires the most energy, whereas the reaction in chloroform, although still an overall positive $\Delta \mathrm{E}$ value, only requires at most $20.0 \mathrm{~kJ} \mathrm{~mol}^{-1}$. Based on the computational results the proposed product in the reaction between the $t$-Bu-xantphos ligands and $[\mathrm{PtCl}(\mathrm{hex}) \mathrm{Me}]$ is the pincer $\left[\mathrm{Pt}\left(t-\mathrm{Bu}\right.\right.$-xantphos- $\left.\left.\kappa P, O, P^{\prime}\right) \mathrm{Me}\right] \mathrm{Cl}$ complex.

The reaction of a number of xantphos ligands with $[\mathrm{Pd}(\mathrm{cod}) \mathrm{ClMe}$ has previously been studied experimentally. $\underline{\underline{73}}$ Me-xantphos is a xantphos derivative with methyl substituents on the phosphorus atoms. Reaction of $[\mathrm{Pd}(\mathrm{cod}) \mathrm{ClMe}]$ with Me-xantphos produces exclusively cis-[PdClMe(Me-xantphos)]. All of the Phxantphos ligands formed [PdClMe(Ph-xantphos)] complexes that displayed cistrans-isomerism at room temperature. At low temperature both the cis and trans isomers were observed for Ph-thixantphos and Ph-xantphos, while only the cis complex was observed for Ph-xantphos. Reaction of the [PdClMe(xantphos)] complexes with $\mathrm{AgSO}_{3} \mathrm{CF}_{3}$ produces the pincer complexes [PdClMe(xantphos$\left.\left.\kappa P, O, P^{\prime}\right)\right]\left[\mathrm{SO}_{3} \mathrm{CF}_{3}\right]$ with all four ligands that were studied. The difference in the coordination behaviour of these ligands and the $t$-Bu-xantphos complexes indicates that the direct formation of the $[\mathrm{Pt}(t-\mathrm{Bu}$-xantphos) $\mathrm{Me}] \mathrm{Cl}$ complexes is most likely the result of the higher steric demands of the $t$-Bu-xantphos ligands compared to the Ph-xantphos or Me-xantphos ligands.

\subsection{Summary}

$t$-Bu-Xantphos and $t$-Bu-thixantphos were shown to react with $\left[\mathrm{PtCl}_{2}(\right.$ hex $\left.)\right]$ forming exclusively trans-[Pt(t-Bu-xantphos) $\left.\mathrm{Cl}_{2}\right]$ complexes, unlike the $\mathrm{Ph}$-xantphos 
ligands which form exclusively cis dichlorides. The same reaction with $t$-Busixantphos produced the trans-[Pt(t-Bu-sixantphos $\left.) \mathrm{Cl}_{2}\right]$ complex together with $t$-Bu-sixantphos $\mathrm{H}^{+}$, even in toluene and benzene, possibly due to reaction with the glass. All of the dichloride complexes were dark red in colour, although most platinum dichloride complexes are pale or yellow. This colour is possibly due to the unusual coordination geometry of the platinum, shown by X-ray crystallography of $\left[\mathrm{Pt}(t-\mathrm{Bu}\right.$-thixantphos $\left.) \mathrm{Cl}_{2}\right]$. The complex has a bite-angle of $151.722(15)^{\circ}$ and the sum of the angles around the platinum is $499.006^{\circ}$ showing significant distortion from the ideal square-planar geometry typically observed for platinum(II). Analogous $\left[\mathrm{Pd}(t-\mathrm{Bu}\right.$-xantphos $\left.) \mathrm{Cl}_{2}\right]$ complexes were synthesised for $t$-Bu-sixantphos, $t$-Bu-thixantphos, and $t$-Bu-xantphos by reaction with $\left[\mathrm{Pd}(\operatorname{cod}) \mathrm{Cl}_{2}\right]$.

The $\left[\mathrm{Pt}(t\right.$-Bu-xantphos $\left.) \mathrm{Cl}_{2}\right]$ and $\left[\mathrm{Pt}(t\right.$-Bu-thixantphos $\left.) \mathrm{Cl}_{2}\right]$ complexes showed dissociation of a chloride ligand, forming $\left[\mathrm{Pt}\left(t-\mathrm{Bu}\right.\right.$-xantphos- $\left.\left.\mathrm{K}, \mathrm{O}, \mathrm{P}^{\prime}\right) \mathrm{Cl}\right] \mathrm{Cl}$ in $\mathrm{CDCl}_{3}$ and $\mathrm{CD}_{2} \mathrm{Cl}_{2}$. The same behaviour was not observed for the palladium analogues. The pincer complexes $\left[\mathrm{M}\left(t \text {-Bu-xantphos- } \kappa P, O, P^{\prime}\right) \mathrm{Cl}\right]^{+}$could be formed for both platinum and palladium by counterion exchange with $\mathrm{NH}_{4} \mathrm{PF}_{6}$. Computational investigation into the chloride dissociation showed that although the formation of the pincer complexes is endothermic in almost all cases, the presence of any solvent more than halved the required energy and the reactions in chloroform and dichloromethane were less endothermic than in benzene. The reactions in acetone were close to neutral energy requirements with some exothermic results. The palladium reactions required slightly more energy in all cases, consistent with the experimental results. The calculations do not include entropy which is likely to be significantly in favour of the pincer complexes.

The pincer complexes $\left[\mathrm{Pt}\left(t-\mathrm{Bu}\right.\right.$-xantphos- $\left.\left.\kappa P, O, P^{\prime}\right) \mathrm{Me}\right] \mathrm{Cl}$ were observed to form as the sole product from reaction of the $t$-Bu-xantphos ligands with [ $\mathrm{PtCl}(\mathrm{hex}) \mathrm{Me}]$. The NMR data was unclear regarding the coordination of the oxygen atom to the platinum and previous literature suggested that the platinum centre may be stabilised by an agostic interaction with one of the tert-butyl C-H bonds. Computational investigation showed that for all three $t$-Bu-xantphos ligands the pincer complex is lower in energy suggesting that this the fourth coordination site on the platinum is occupied by the backbone oxygen. From the values of $J_{\mathrm{PC}}$ for the methyl ligand the $t$-Bu-sixantphos ligand has the weakest trans influence and the $t$-Bu-xantphos ligand has the strongest. This suggests that although compu- 
tationally the bite-angles of the three ligands are the same, the natural bite-angle can predict the donor properties of the backbone oxygen.

This chapter has presented a number of new platinum and palladium complexes with the $t$-Bu-sixantphos, $t$-Bu-thixantphos, and $t$-Bu-xantphos. No cis complexes were observed at any point, indicating the influence of tert-butyl groups on the coordination modes of the ligands. The trans complexes have the smallest observed bite-angles for square-planar complexes with trans-spanning diphosphine ligands. A number of $t$-Bu-xantphos pincer complexes were also reported, with $[\mathrm{Pt}(t-\mathrm{Bu} \text {-xantphos }) \mathrm{Cl}]^{+}$and $[\mathrm{Pt}(t-\mathrm{Bu}$-xantphos) $\mathrm{Me}] \mathrm{Cl}$ forming spontaneously with appropriate solvent choice. 


\section{Chapter 7}

\section{Conclusion}

The $t$-Bu-xantphos ligand has been tested in a number of catalytic reactions with varying results. $\underline{\underline{84}-87,90,93,96-98}$ The conversions are typically very different to those obtained with $\mathrm{Ph}$-xantphos suggesting differences in their coordination behaviour. However, few studies had investigated the coordination chemistry of the $t$-Buxantphos ligand. This thesis aimed to address that deficit by synthesising two new $t$-Bu-xantphos ligands, and investigating the properties and coordination chemistry of the three ligands, with a particular focus on transition metals used in catalysis.

Two new $t$-Bu-xantphos ligands with a $\mathrm{SiMe}_{2}(t$-Bu-sixantphos) or a sulfur atom ( $t$-Bu-thixantphos) in place of the $\mathrm{CMe}_{2}$ bridging group in $t$-Bu-xantphos were synthesised. The synthetic method for $t$-Bu-sixantphos and $t$-Bu-thixantphos was also used successfully to synthesise $t$-Bu-xantphos. This synthesis is advantageous as the only by-products are the monophosphines, which can be reused in a further cycle to produce additional diphosphine. The bite-angles of the $t$-Bu-xantphos and Ph-xantphos were calculated using DFT methods with the $t$-Bu-xantphos ligands found to have larger bite-angles $\left(126.80-127.56^{\circ}\right)$ than the Ph-xantphos ligands (111.89-114.18 $)$. The values calculated for the Ph-xantphos ligands using DFT are consistent with the literature values calculated using molecular mechanics.

The $t$-Bu-xantphos ligands are both Brønsted and Lewis bases. The three ligands reacted rapidly with strong acids forming ( $t$-Bu-xantphos) $\mathrm{H}^{+}$with a single proton that exchanged rapidly between the two phosphorus atoms at room temper- 
ature. This process has a coalescense temperature of around $-40{ }^{\circ} \mathrm{C}$ in solution. Synthesis of the $t$-Bu-xantphos selenides and analysis of their ${ }^{1} J_{\mathrm{PSe}}$ values gave $\mathrm{p} K_{\mathrm{b}}$ values of 5.67 for $t$-Bu-sixantphos, 6.90 for $t$-Bu-thixantphos, and 6.72 for $t$-Bu-xantphos. These are much lower than the value for Ph-xantphos of 13.55, as expected due to greater electron density on the phosphorus atoms in $t$-Bu-xantphos resulting from the tert-butyl substituents.

The coordination chemistry of the $t$-Bu-xantphos ligands was investigated with silver, rhodium, platinum, and palladium. Two types of silver complexes were reported, $[\mathrm{Ag}(t-\mathrm{Bu}$-xantphos $) \mathrm{Cl}]$ and $[\mathrm{Ag}(t-\mathrm{Bu}$-xantphos $)] \mathrm{BF}_{4}$. Both are monomeric despite the free-coordination site in $[\mathrm{Ag}(t-\mathrm{Bu}$-xantphos $)] \mathrm{BF}_{4}$. The $t$-Bu-thixantphos ligand in the $\mathrm{x}$-ray crystal structure of [Ag(t-Bu-thixantphos)Cl] had a biteangle of $130.50(7)^{\circ}$, which is larger than the bite-angle in the previously reported [Ag(Ph-xantphos) $\mathrm{Br}]$ complex $\left(109.37(1)^{\circ}\right)$. Both of these are close to the natural bite-angle of the xantphos ligand, indicating that the coordination geometry around the silver is controlled by the diphosphine ligand. Although the crystal structure of $[\mathrm{Ag}(t-\mathrm{Bu}$-thixantphos $) \mathrm{Cl}]$ suggested that the tert-butyl substituents should have different NMR signals, this was not observed spectroscopically, likely due to the rapid inversion of the xantphos backbone.

Rhodium complexes are used as catalysts for hydrogenation and hydroformylation which are performed under hydrogen or a mixed hydrogen/carbon monoxide atmosphere. [ $\mathrm{Rh}\left(t-\mathrm{Bu}\right.$-xantphos- $\left.\left.\mathrm{k} P, O, P^{\prime}\right) \mathrm{Cl}\right]$ complexes were formed for all three $t$-Bu-xantphos ligands. The reactivity of these complexes towards $\mathrm{H}_{2}, \mathrm{CO}$, and $\mathrm{O}_{2}$ was investigated, forming $\left[\mathrm{Rh}\left(t-\mathrm{Bu}\right.\right.$-xantphos- $\left.\left.\mathrm{k} P, \mathrm{O}, \mathrm{P}^{\prime}\right) \mathrm{Cl}(\mathrm{H})_{2}\right],[\mathrm{Rh}(t-\mathrm{Bu}-$ xantphos $\left.)(\mathrm{CO})_{2} \mathrm{Cl}\right]$, and $\left[\mathrm{Rh}\left(t-\mathrm{Bu}\right.\right.$-xantphos- $\left.\left.\mathrm{k} P, \mathrm{O}, \mathrm{P}^{\prime}\right) \mathrm{Cl}\left(\eta^{2}-\mathrm{O}_{2}\right)\right]$ complexes. The value of the $J_{\mathrm{RhH}}$ coupling constant for the hydride trans to the oxygen atom in the $\left[\mathrm{Rh}\left(t\right.\right.$-Bu-xantphos- $\left.\left.\mathrm{K} P, \mathrm{O}, P^{\prime}\right) \mathrm{Cl}(\mathrm{H})_{2}\right]$ complexes was largest for the $t$-Bu-sixantphos complex, followed by $t$-Bu-thixantphos then $t$-Bu-xantphos. This shows that the ligands have differing trans influences; $t$-Bu-sixantphos $<t$-Bu-thixantphos $<t$-Bu-xantphos. The X-ray crystal structure of $\left[\mathrm{Rh}\left(t-\mathrm{Bu}\right.\right.$-xantphos- $\left.\mathrm{k} P, \mathrm{O}, \mathrm{P}^{\prime}\right)$ $\left.\mathrm{Cl}\left(\eta^{2}-\mathrm{O}_{2}\right)\right]$ was disordered with the dioxygen ligand replaced in $15 \%$ of the sites by an oxo. This is the first crystallographic evidence for a rhodium(III) oxo complex, and only the third rhodium oxo complex that has been reported. Attempts to synthesis the oxo complex directly were promising, with a new peak present in the ${ }^{31} \mathrm{P}$ NMR spectrum for a short-lived species. However, further research and characterisation is necessary. 
Upon reaction of Ph-thixantphos with $\left[\mathrm{Pt}(\mathrm{nb})_{3}\right]$ or $\left[\mathrm{Pt}\left(\mathrm{C}_{2} \mathrm{H}_{4}\right)_{3}\right]$ the major product was [Pt(Ph-thixantphos) $)_{2}$; this was the only product observed in a 2:1 reaction, and was characterised crystallographically. The coordination chemistry with platinum( $(0)$ and palladium( 0 ) showed some differences between the three $t$-Bu-xantphos ligands. $t$-Bu-Thixantphos reacts with $\left[\mathrm{Pt}(\mathrm{nb})_{3}\right]$ to form a mixture of $[\mathrm{Pt}(t-\mathrm{Bu}$-thixantphos $)]$ and $[\mathrm{Pt}(t-\mathrm{Bu}$-thixantphos $)(\mathrm{nb})]$. Analogues of these complexes were formed in the reaction between $\left[\mathrm{Pt}(\mathrm{nb})_{3}\right]$ and $t$-Bu-sixantphos. However, over time a complex with NMR data consistent with platinum(II) is observed. Using $t$-Bu-xantphos small amounts of [ $\mathrm{Pt}(t-\mathrm{Bu}$-xantphos $)]$ forms, but the final product is $[\mathrm{Pt}(t-\mathrm{Bu}$-xantphos $) \mathrm{H}] \mathrm{X}$, possibly through reaction of the 14electron complex with a component of the reaction mixture. The ability to isolate $[\mathrm{Pt}(t-\mathrm{Bu}$-thixantphos $)]$, indicates the protection that the wide bite-angle and size of the tert-butyl substituents can impart to a metal centre. Isolable complexes of the type [Pt(diphosphine)] are rare, although examples with monophosphines are known. Reaction of the $t$-Bu-xantphos ligands with $\left[\mathrm{Pd}(\mathrm{nb})_{3}\right]$ formed $[\mathrm{Pd}(t-$ Bu-xantphos)] and $[\mathrm{Pd}(t-\mathrm{Bu}$-xantphos $)(\mathrm{nb})]$ complexes for all three ligands. $[\mathrm{Pt}(t-$ Bu-xantphos $\left.)\left(\mathrm{C}_{2} \mathrm{H}_{4}\right)\right]$ complexes via reaction with $\left[\mathrm{Pt}\left(\mathrm{C}_{2} \mathrm{H}_{4}\right)_{3}\right]$. [ $\mathrm{Pd}(t$-Bu-thixantphos $)]$, [ $\mathrm{Pt}(t$-Bu-thixantphos $)]$, and $\left[\mathrm{Pt}(t\right.$-Bu-thixantphos $\left.)\left(\mathrm{C}_{2} \mathrm{H}_{4}\right)\right]$ all react with oxygen. On palladium the oxygen is readily removed in vacuo, whereas the platinum complex does not lose oxygen under vacuum. [ $\mathrm{Pt}(t-\mathrm{Bu}$-thixantphos $)\left(\eta^{2}\right.$ $\left.\mathrm{O}_{2}\right)$ ] is unreactive towards $\mathrm{C}_{2} \mathrm{H}_{2}, \mathrm{H}_{2}, \mathrm{CO}_{2}, \mathrm{NH}_{4} \mathrm{PF}_{6}$ and pta. However, $\mathrm{CO}$ can insert into the $\mathrm{O}-\mathrm{O}$ bond forming a carbonate, complex which progresses through two intermediates resulting in $\left[\mathrm{Pt}\left(t-\mathrm{Bu}\right.\right.$-thixantphos- $\left.\left.\mathrm{H}-\mathrm{k}-C, P, P^{\prime}\right) \mathrm{OH}\right]$. This reactivity is unprecedented in the literature.

The coordination chemistry of the $t$-Bu-xantphos ligands with platinum(II) and palladium(II) starting materials was also explored. Regardless of the geometry of the starting material exclusively trans-[M(t-Bu-xantphos $\left.) \mathrm{Cl}_{2}\right](\mathrm{M}=\mathrm{Pd}, \mathrm{Pt})$ complexes were formed. The reaction of $t$-Bu-sixantphos with $\left[\mathrm{PtCl}_{2}(\mathrm{hex})\right]$ was hindered due to the competing protonation of the ligand due to the higher basicity of $t$-Bu-sixantphos compared to $t$-Bu-thixantphos and $t$-Bu-xantphos. The $\mathrm{X}$ ray crystal structure of $\left[\mathrm{Pt}(t-\mathrm{Bu}\right.$-xantphos $\left.) \mathrm{Cl}_{2}\right]$ is unique among $\left[\mathrm{PtCl}_{2}(\mathrm{PP})\right]$ complexes, with a P-Pt-P angle of $151.722(15)^{\circ}$, with no similar angles reported in the CSD. The platinum dichloride complexes underwent solvent-dependent dissociation of a chloride ligand, forming $\left[\mathrm{Pt}\left(t-\mathrm{Bu} \text {-xantphos- } \mathrm{k} P, \mathrm{O}, \mathrm{P}^{\prime}\right) \mathrm{Cl}\right]^{+}$in $\mathrm{CDCl}_{3}$ and $\mathrm{CD}_{2} \mathrm{Cl}_{2}$. The solvent-dependent behaviour was not observed for the palladium 
complexes. Computational investigations showed that, although the pincer complexes are consistently higher in energy than the dichloride complexes, the energy difference is much lower in polar solvents. Entropy would also promote the dissociation of the chloride ligand leading to the experimentally observed spontaneity. No reaction occurred between $t$-Bu-thixantphos and $\left[\mathrm{Pt}(\mathrm{hex}) \mathrm{Me}_{2}\right]$, likely due to the stronger coordination of the methyl ligands. The reactions of the $t$-Buxantphos ligands with $[\mathrm{PtCl}($ hex $) \mathrm{Me}]$ formed only $\left[\mathrm{Pt}(t-\mathrm{Bu} \text {-xantphos)Me }]^{+}\right.$pincer complexes with no chlorido-methyl species observed. This is likely the result of the high trans-influence of the methyl ligand promoting loss of the chloride. Computational investigation showed that the fourth coordination site is occupied by the oxygen in the backbone of the $t$-Bu-xantphos ligands. The value of the $J_{\mathrm{PtC}}$ coupling constant is largest for $t$-Bu-sixantphos, then $t$-Bu-thixantphos, then $t$-Bu-xantphos consistent with the O-trans influence series observed in the rhodium complexes; $t$-Bu-sixantphos $<t$-Bu-thixantphos $<t$-Bu-xantphos.

Overall this thesis provides an account into the synthesis, properties and coordination chemistry of $t$-Bu-sixantphos, $t$-Bu-thixantphos, and $t$-Bu-xantphos with four late-transition metals; silver, rhodium, platinum, and palladium. The ligands showed: formation of monomeric silver complexes with $k-P, P^{\prime}$ coordination; rhodium complexes with meridional $\kappa P, O, P^{\prime}$ coordination in square-planar, octahedral and trigonal bipyramidal complexes, and $k-P, P^{\prime}$ coordination in trigonal bipyramidal species; platinum(0) and palladium(0) complexes with $\mathrm{k}-P, P^{\prime}$ coordination in the presence and absence of other ligands and; exclusive trans- $\mathrm{K}-$ $P, P^{\prime}$ coordination to platinum(II) and palladium(II) and $\kappa P, O, P^{\prime}$ bonding modes. The final mode observed was the metallation of the tert-butyl group on platinum to form a $\mathrm{k}-C, P, P^{\prime}$ ligand. X-ray crystallography showed that the $t$-Bu-thixantphos ligand can achieve $\kappa-P, P^{\prime}$ coordination with bite-angles ranging from 117$151^{\circ}$. 


\section{Chapter 8}

\section{Experimental}

\subsection{General Procedures}

All reactions and manipulation of products and reagents were carried out under an inert nitrogen or argon atmosphere using standard Schlenk line techniques unless stated otherwise. All reactions using silver compounds were performed in the dark. Unless otherwise stated the starting materials used in this work were obtained from Sigma-Aldrich, Merck Chemical Companies, Thermo Fisher Scientific, and BOC Industrial Gases, and used without further purification. Analytical grade reagents and high purity solvents were degassed and purged with argon before use, and dried over molecular sieves. Diethyl ether and tetrahydrofuran were distilled under an argon atmosphere from sodium benzophenone ketyl immediately prior to use. 1,3,5-triaza-7-phosphaadamantane,

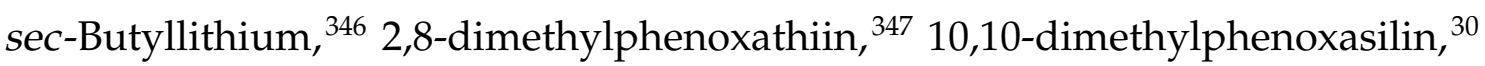

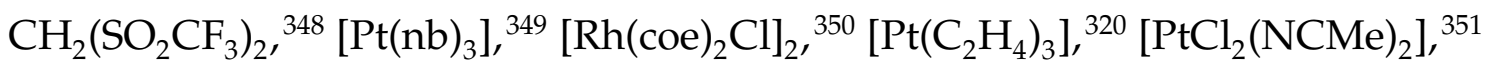

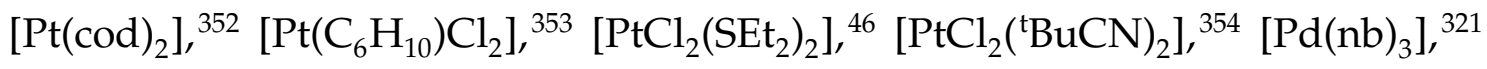
$\left[\mathrm{Pt}\left(\mathrm{C}_{6} \mathrm{H}_{10}\right) \mathrm{ClMe}\right], \underline{355}\left[\mathrm{Pd}(\mathrm{cod}) \mathrm{Cl}_{2}\right]$, and $\left[\mathrm{Pt}\left(\mathrm{C}_{6} \mathrm{H}_{10}\right) \mathrm{Me}_{2}\right]$, , 349 were prepared according to literature procedures.

NMR spectra were recorded using a Varian Unity Inova $300\left(300 \mathrm{MHz}\right.$ for ${ }^{1} \mathrm{H}$, $75 \mathrm{MHz}$ for ${ }^{13} \mathrm{C}, 121 \mathrm{MHz}$ for ${ }^{31} \mathrm{P}$ and $282 \mathrm{MHz}$ for ${ }^{19} \mathrm{~F}$ ), a Varian Unity Inova 500 (500 MHz for ${ }^{1} \mathrm{H}$ and $125 \mathrm{MHz}$ for ${ }^{13} \mathrm{C}$ ), or a Varian DirectDrive $600(600 \mathrm{MHz}$ for ${ }^{1} \mathrm{H}$ and $150 \mathrm{MHz}$ for ${ }^{13} \mathrm{C}$ ) spectrometer. The $600 \mathrm{MHz}$ instrument was equipped with a Varian inverse-detected triple-resonance $\mathrm{HCN}$ cold probe operating at 
$25 \mathrm{~K}$. All direct-detected ${ }^{1} \mathrm{H}$ and ${ }^{13} \mathrm{C}$ chemical shifts were referenced to the residual solvent peak. .300 NMR samples were prepared under an inert nitrogen or argon atmosphere unless otherwise stated, using $\mathrm{C}_{6} \mathrm{D}_{6}, \mathrm{CDCl}_{3}, \mathrm{CD}_{2} \mathrm{Cl}_{2}$, acetone- $\mathrm{d}_{6}$ and toluene- $\mathrm{d}_{8}$. Variable temperature NMR spectroscopy was carried out using a Varian Unity Inova $300 \mathrm{MHz}$ NMR spectrometer. Infrared spectra were recorded with a PerkinElmer Spectrum One FT-IR spectrophotometer in absorbance mode. Electrospray ionisation mass spectrometry was recorded using an Agilent 6530 QTOF mass spectrometer, or performed by the Carbohydrate Chemistry Group at Industrial Research Limited, Lower Hutt, using a Waters Q-TOF Premier Tandem mass spectrometer.

Single crystal X-ray diffraction data was solved using the Superflip program $\frac{356}{36}$ (except for [Ag( $t$-Bu-thixantphos)Cl] which was solved using the SHELX program) and refined using the SHELX program ${ }^{357}$ with OLEX2 (version 1.2.5) $\frac{358}{5}$ as a front-end. Unless stated otherwise the hydrogen atoms were placed in calculated positions. Crystallographic data was collected by the X-ray Crystallography Laboratory at the University of Canterbury, using either a Bruker SMART APEX-II CCD diffractometer or an Agilent SuperNova (Dual Source) CCD diffractometer. Data were reduced using Bruker SAINT or Agilent CrysAlisPro software. Absorption correction was performed using SADABS or SCALE3 ABSPACK. Theoretical calculations were performed using the Gaussian 09 Revision B.01 program $\underline{359,360}$ running on the Victoria University of Wellington School of Chemical and Physical Sciences Heisenberg cluster, consisting of sixteen processing nodes with two $2.53 \mathrm{GHz}$ Xeon X3440 quad-core processors per node and $1 \mathrm{~GB}$ of memory per core. All searches of the Cambridge Structural Databank used version 5.35 (November 2013). $\underline{\underline{68}}$

\subsection{Ligands and Non-Transition Metal Derivatives}

\section{2,8-Dimethyl-4,6-bis(di-tert-butylphosphino)phenoxathiin ( $t$-Bu-thixantphos)}

sec-Butyllithium (5.32 mL, 1.2 M in cyclohexane) was added dropwise to a stirred solution of 2,8-dimethylphenoxathiin $(0.71 \mathrm{~g}, 3.1 \mathrm{mmol})$ and TMEDA $(0.92 \mathrm{~mL}$, $6.2 \mathrm{mmol})$ in diethyl ether $(30 \mathrm{~mL})$ at $-78^{\circ} \mathrm{C}$. The resulting yellow solution was al- 
lowed to warm to room temperature and stirred for a further 16 hours over which time a dark red colour developed. The reaction was cooled to $-78^{\circ} \mathrm{C}$ and chlorodi$t$-butylphosphine $(1.18 \mathrm{~mL}, 6.2 \mathrm{mmol})$ was added dropwise. The reaction mixture was stirred for a further seven days resulting in a yellow solution with a white precipitate of lithium chloride. The solvent was removed in vacuo giving an orange oil. This oil was dissolved in dichloromethane $(25 \mathrm{~mL})$ and washed with water $(3 \times 15 \mathrm{~mL})$. The organic layer was passed through a column of magnesium sulfate and solvent was removed in vacuo. The product was recrystallised from n-propanol giving small white crystals (1.36 g, 85\%). This compound can be handled in the air for short periods however, should be stored under an inert atmosphere. ${ }^{31} \mathrm{P} \mathrm{NMR}\left(121 \mathrm{MHz}, \mathrm{CDCl}_{3}\right): \delta 9.5$ (s). ${ }^{1} \mathrm{H} \mathrm{NMR}(500 \mathrm{MHz}$, $\mathrm{CDCl}_{3}$ ): $\delta 7.29$ (s, PC(Ar)CH), 6.88 (s, SCCH), 2.25 (s, $\left.\mathrm{C}(\mathrm{Ar}) \mathrm{CH}_{3}\right), 1.22-1.24(\mathrm{~m}$, $\left.\mathrm{PC}\left(\mathrm{CH}_{3}\right)_{3}\right) .{ }^{13} \mathrm{C} \mathrm{NMR}\left(125 \mathrm{MHz}, \mathrm{CDCl}_{3}\right): \delta 155.3$ (vt, $\left.J_{\mathrm{PC}}=13.0 \mathrm{~Hz}, \mathrm{PCCO}\right), 134.6$ (s, PC(Ar)CH), 131.9 (s, C(Ar) $\mathrm{CH}_{3}$ ), 128.0 (dd, $J=22.5,17.7 \mathrm{~Hz}, \mathrm{PC}(\mathrm{Ar})$ ), 127.4 (s, $\mathrm{SCCH}), 120.1\left(\mathrm{vt}, J_{\mathrm{PC}}=2.4 \mathrm{~Hz}, \mathrm{SCCH}\right), 32.9\left(\mathrm{dd}, J=15.8,13.4 \mathrm{~Hz}, \mathrm{PC}\left(\mathrm{CH}_{3}\right)_{3}\right), 30.8$ (vt, $\left.J_{\mathrm{PC}}=18.2 \mathrm{~Hz}, \mathrm{PC}\left(\mathrm{CH}_{3}\right)_{3}\right), 20.9\left(\mathrm{~s}, \mathrm{C}(\mathrm{Ar}) \mathrm{CH}_{3}\right)$. HRMS calcd for $\mathrm{C}_{30} \mathrm{H}_{47} \mathrm{OP}_{2} \mathrm{~S}$ $[\mathrm{M}+\mathrm{H}]^{+} \mathrm{m} / \mathrm{z}=517.2817$; found $=517.2819$.

\section{4,6-Bis(di-tert-butylphosphino)-10,10-dimethylphenoxasilin (t-Bu-sixantphos)}

This compound was prepared similarly to $t$-Bu-thixantphos using 10,10-dimethylphenoxasilin $(0.40 \mathrm{~g}, 1.8 \mathrm{mmol})$ giving the title compound as white crystals $(0.127$ g, 14\%).

${ }^{31} \mathrm{P} \mathrm{NMR}\left(121 \mathrm{MHz}, \mathrm{CD}_{2} \mathrm{Cl}_{2}\right): \delta 8.4\left(\mathrm{~s},{ }^{4} J_{\mathrm{PSi}}=4.8 \mathrm{~Hz}\right) .{ }^{1} \mathrm{H} \mathrm{NMR}\left(500 \mathrm{MHz}, \mathrm{CD}_{2} \mathrm{Cl}_{2}\right)$ : $\delta 7.87(\mathrm{~d}, J=7.4 \mathrm{~Hz}, \mathrm{PC}(\mathrm{Ar}) \mathrm{CH}), 7.53(\mathrm{~d}, J=7.1 \mathrm{~Hz}, \operatorname{SiCCH}), 7.12(\mathrm{t}, J=7.5 \mathrm{~Hz}$, $\mathrm{PCCCH}), 1.29\left(\mathrm{vt}, J_{\mathrm{PH}}=5.6 \mathrm{~Hz}, \mathrm{PC}\left(\mathrm{CH}_{3}\right)_{3}\right), 0.46\left(\mathrm{~s}, \mathrm{Si}\left(\mathrm{CH}_{3}\right)_{2}\right),{ }^{13} \mathrm{C} \mathrm{NMR}(125 \mathrm{MHz}$, $\left.\mathrm{CD}_{2} \mathrm{Cl}_{2}\right): \delta 164.3\left(\mathrm{vt}, J_{\mathrm{PC}}=11.3 \mathrm{~Hz}, \mathrm{PCCO}\right), 138.5$ (s, PC(Ar)CH), $134.8(\mathrm{~s}, \mathrm{SiCCH})$, 128.0 (s, PC(Ar)), 121.4 (s, PCCCH), 119.4 (s, SiCCH), 33.2 (dd, $J_{\mathrm{PC}}=16.3,13.9 \mathrm{~Hz}$, $\left.\mathrm{PC}\left(\mathrm{CH}_{3}\right)_{3}\right), 31.0\left(\mathrm{vt}, J_{\mathrm{PC}}=9.6 \mathrm{~Hz}, \mathrm{PC}\left(\mathrm{CH}_{3}\right)_{3}\right),-0.09\left(\mathrm{~s}, \mathrm{Si}\left(\mathrm{CH}_{3}\right)_{2}\right)$. HRMS calcd for $\mathrm{C}_{30} \mathrm{H}_{49} \mathrm{OP}_{2} \mathrm{Si}[\mathrm{M}+\mathrm{H}]^{+} \mathrm{m} / \mathrm{z}=515.3022$; found $=515.3021$. 


\section{9,9-Dimethyl-4,6-bis(diphenylphosphino)xanthene}

\section{( $t$-Bu-xantphos)}

9,9-dimethylxanthene (0.50 g, $2.38 \mathrm{mmol})$ and TMEDA (1.07 $\mathrm{mL}, 7.13 \mathrm{mmol})$ were dissolved in diethyl ether $(20 \mathrm{~mL})$. Added s-BuLi dropwise causing the reaction to change to yellow then a deep red. After stirring for 24 hours chlorodi(tertbutyl)phosphine $(1.36 \mathrm{~mL}, 7.13 \mathrm{mmol}$ ) was added dropwise. After six days of stirring a white precipitate had formed and a pale yellow solution remained. The solvent was removed in vacuo and the resulting yellow oil was taken up in dichloromethane $(20 \mathrm{~mL})$ and washed with degassed water $(10 \mathrm{~mL})$. The aqueous layer was further extracted with dichloromethane $(20 \mathrm{~mL})$ and the combined organic layers were washed with water $(3 \times 10 \mathrm{~mL})$. The organic layers were dried over magnesium sulfate and the solvent was removed in vacuo. The resulting pale yellow solid was recrystallised from n-propanol yielding the title compound as fine white needles $(0.44 \mathrm{~g}, 37 \%)$.

The ${ }^{1} \mathrm{H}$ and ${ }^{13} \mathrm{C}$ NMR data are consistent with the literature values. ${ }^{84}$ However, the literature reported ${ }^{31} \mathrm{P}$ chemical shift is $12.4 \mathrm{ppm}$. Due to this discrepancy full characterisation data for this compound is given below.

${ }^{31} \mathrm{P}$ NMR (121 MHz, $\left.\mathrm{CDCl}_{3}\right): \delta 10.2$ (s), ${ }^{1} \mathrm{H}$ NMR (500 MHz, $\left.\mathrm{CDCl}_{3}\right): \delta 7.60$ (d, $J=7.6 \mathrm{~Hz}, \mathrm{PC}(\mathrm{Ar}) \mathrm{CH}), 7.38(\mathrm{dd}, 7.8,1.5 \mathrm{~Hz}, \mathrm{C}$ (bridge)CCH), $7.03(\mathrm{t}, J=7.6 \mathrm{~Hz}$, PCCCH $), 1.57$ (s, C(bridge) $\left.\left(\mathrm{CH}_{3}\right)_{2}\right), 1.21-1.25\left(\mathrm{~m}, \mathrm{PC}\left(\mathrm{CH}_{3}\right)_{3}\right) .{ }^{13} \mathrm{C} \mathrm{NMR}(125 \mathrm{MHz}$, $\left.\mathrm{CDCl}_{3}\right): \delta 155.8\left(\mathrm{vt}, J_{\mathrm{PC}}=12.0 \mathrm{~Hz}, \mathrm{PCCO}\right), 133.7$ (bs, PC(Ar)CH), 130.7 (vt, $J_{\mathrm{PC}}=2.0 \mathrm{~Hz}, \mathrm{C}($ bridge $\left.) C \mathrm{CH}\right), 126.6\left(\mathrm{dd}, J_{\mathrm{PC}}=21.6,15.4 \mathrm{~Hz}, \mathrm{PC}(\mathrm{Ar})\right), 125.5(\mathrm{~s}$, $\mathrm{C}$ (bridge)CCH), 121.5 (s, PCCCH), 35.0 (bs, $C$ (bridge)), 32.7 (dd, $J_{\mathrm{PC}}=16.1$, $\left.12.7 \mathrm{~Hz}, \mathrm{PC}\left(\mathrm{CH}_{3}\right)_{3}\right), 31.1$ (s, C(bridge) $\left.\left(\mathrm{CH}_{3}\right)_{2}\right), 30.8\left(\mathrm{vt}, J_{\mathrm{PC}}=18.8 \mathrm{~Hz}, \mathrm{PC}\left(\mathrm{CH}_{3}\right)_{3}\right)$. HRMS calcd for $\mathrm{C}_{31} \mathrm{H}_{49} \mathrm{OP}_{2}[\mathrm{M}+\mathrm{H}]^{+} \mathrm{m} / \mathrm{z}=499.3253$; found $=499.3241$.

\section{2,6-bis(di-tert-butylphosphino)phenoxathiin}<smiles>CCCCc1cccc2c1Sc1cccc(PCCC)c1O2</smiles> 
Phenoxathiin $(0.20 \mathrm{~g}, 1.00 \mathrm{mmol})$ and TMEDA $(0.43 \mathrm{~mL}, 2.27 \mathrm{mmol})$ were dissolved in heptane $(6 \mathrm{~mL})$. A solution of sec-butyllithium $(1.0 \mathrm{M}$ in cyclohexane, $2.27 \mathrm{~mL}$ ) was added dropwise. The mixture was stirred for 24 hours resulting in a yellow solution with a white precipitate. $\mathrm{P}^{\mathrm{t}} \mathrm{Bu}_{2} \mathrm{Cl}(0.55 \mathrm{~mL}, 2.90 \mathrm{mmol})$ was added dropwise to the reaction and the resulting mixture was heated at $60{ }^{\circ} \mathrm{C}$ for 24 hours. The solvent was removed under reduced pressure and the resulting yellow oil was taken up in dichloromethane $(10 \mathrm{~mL})$ and washed with degassed water $(2 \times 10 \mathrm{~mL})$. The organic layer was dried by passing through a plug of $\mathrm{MgSO}_{4}$ before removed the solvent in vacuo. The resulting oil was recrystallised from hot n-propanol yielding the title compound as a yellow microcrystalline solid $(0.316 \mathrm{~g}, 65 \%)$.

${ }^{31} \mathrm{P}$ NMR (121 MHz, $\left.\mathrm{CDCl}_{3}\right): \delta 17.7(\mathrm{~d}, J=2.2 \mathrm{~Hz}, \mathrm{PCCO}) 11.7(\mathrm{~d}, J=2.3 \mathrm{~Hz}$, PCCS) ${ }^{1} \mathrm{H}$ NMR (500 MHz, $\left.\mathrm{CDCl}_{3}\right): \delta 7.49(\mathrm{~d}, J=7.6 \mathrm{~Hz}, 1 \mathrm{H}, \mathrm{Ar}), 7.40(\mathrm{~d}, J=7.3$ $\mathrm{Hz}, 1 \mathrm{H}, \mathrm{Ar}), 7.17$ (dd, J=7.7 Hz, 1.3 Hz, 1H, Ar), 7.13 (d, J= 7.6 Hz, 1H, Ar), 7.07 (t, $J=7.7 \mathrm{~Hz}, 1 \mathrm{H}, \mathrm{Ar}), 6.94(\mathrm{t}, J=7.6 \mathrm{~Hz}, 1 \mathrm{H}, \mathrm{Ar}), 1.21\left(\mathrm{~d}, J=12.3 \mathrm{~Hz}, 18 \mathrm{H}, \mathrm{PC}\left(\mathrm{CH}_{3}\right)_{3}\right)$, $1.20\left(\mathrm{~d}, J=12.0 \mathrm{~Hz}, 18 \mathrm{H}, \mathrm{PC}\left(\mathrm{CH}_{3}\right)_{3}\right) .{ }^{13} \mathrm{C} \mathrm{NMR}\left(150 \mathrm{MHz}, \mathrm{CDCl}_{3}\right): \delta 156.4\left(\mathrm{~d}, J_{\mathrm{PC}}=\right.$ $17.9 \mathrm{~Hz}, 1 \mathrm{C}, \mathrm{Ar}), 151.6\left(\mathrm{~d}, J_{\mathrm{PC}}=10.4 \mathrm{~Hz}, 1 \mathrm{C}, \mathrm{Ar}\right), 134.1$ (s, 1C, Ar), 134.0 (s, 1C, Ar), 133.8 (s, 1C, Ar), 130.5 (d, JPC $=3.4 \mathrm{~Hz}, 1 \mathrm{C}, \mathrm{Ar}), 127.9$ (s, 1C, Ar), 126.2 (d, $\left.J_{\mathrm{PC}}=31.8 \mathrm{~Hz}, 1 \mathrm{C}, \mathrm{Ar}\right), 125.6\left(\mathrm{~d}, J_{\mathrm{PC}}=7.0 \mathrm{~Hz}, 1 \mathrm{C}, \mathrm{Ar}\right), 122.8$ (s, 1C, Ar), 122.3 (d, $\left.J_{\mathrm{PC}}=17.9 \mathrm{~Hz}, 1 \mathrm{C}, \mathrm{Ar}\right), 119.4(\mathrm{~s}, 1 \mathrm{C}, \mathrm{Ar}) 33.1\left(\mathrm{~d}, J_{\mathrm{PC}}=21.4 \mathrm{~Hz}, \mathrm{PC}\left(\mathrm{CH}_{3}\right)_{3}\right), 32.4(\mathrm{~d}$, $\left.J_{\mathrm{PC}}=23.7 \mathrm{~Hz}, \mathrm{PC}\left(\mathrm{CH}_{3}\right)_{3}\right), 30.7\left(\mathrm{~d}, J_{\mathrm{PC}}=15.1 \mathrm{~Hz}, \mathrm{PC}\left(\mathrm{CH}_{3}\right)_{3}\right), 30.4\left(\mathrm{~d}, J_{\mathrm{PC}}=14.4 \mathrm{~Hz}\right.$, $\left.\mathrm{PC}\left(\mathrm{CH}_{3}\right)_{3}\right)$.

\section{Synthesis of $[(t-B u-x a n t p h o s) \mathrm{H}] \mathrm{CH}\left(\mathrm{SO}_{2} \mathrm{CF}_{3}\right)$}

A solution of $\mathrm{CH}_{2}\left(\mathrm{SO}_{2} \mathrm{CF}_{3}\right)_{2}(0.009 \mathrm{~g}, 0.032 \mathrm{mmol})$ in $\mathrm{CDCl}_{3}(0.5 \mathrm{~mL})$ was added to $t$-Bu-xantphos $(0.016 \mathrm{~g}, 0.032 \mathrm{mmol})$ in an NMR tube. The reaction was instantaneous and quantitative conversion was observed by NMR spectroscopy. Removal of the solvent in vacuo gave the product as a white solid in quantitative yield.

${ }^{31} \mathrm{P} \mathrm{NMR}\left(121 \mathrm{MHz}, \mathrm{CDCl}_{3}\right): \delta 17.4$ (bs). ${ }^{1} \mathrm{H} \mathrm{NMR}\left(600 \mathrm{MHz}, \mathrm{CDCl}_{3}\right): \delta 9.05-$ 8.09 (m, PH), 7.67-7.74 (m, 4H, Ar), 7.39 (bs, 2H, Ar), 4.06 (bs, $\left.\mathrm{CH}\left(\mathrm{SO}_{2} \mathrm{CF}_{3}\right)_{2}\right)$, 1.65 (s, C(bridge) $\left.\left(\mathrm{CH}_{3}\right)_{2}\right), 1.37-1.43\left(\mathrm{~m}, \mathrm{PC}\left(\mathrm{CH}_{3}\right)_{3}\right) .{ }^{19} \mathrm{~F} \mathrm{NMR}\left(282 \mathrm{MHz}, \mathrm{CDCl}_{3}\right)$ : $\delta$-80.9 (s, $\left.\mathrm{CF}_{3}\right){ }^{13} \mathrm{C} \mathrm{NMR}\left(150 \mathrm{MHz}, \mathrm{CDCl}_{3}\right): \delta$ 153.6-153.8 (m, PCCO), 132.5 
(bs), 130.3 (bs), 125.0 (bs), 121.0 (quartet, $\left.J_{\mathrm{CF}}=317.9 \mathrm{~Hz}, \mathrm{CH}\left(\mathrm{SO}_{2} \mathrm{CF}_{3}\right)_{2}\right), 54.1$ (s, $\left.\mathrm{CH}\left(\mathrm{SO}_{2} \mathrm{CF}_{3}\right)_{2}\right), 35.5$ (s, $\mathrm{C}$ (bridge) ), 34.0 (bs, $\left.\mathrm{PC}\left(\mathrm{CH}_{3}\right)_{3}\right), 30.8$ (s, C(bridge $\left.\left(\mathrm{CH}_{3}\right)_{2}\right)$, 29.4 (bs, $\left.\mathrm{PC}\left(\mathrm{CH}_{3}\right)_{3}\right)$. HRMS calcd for $\mathrm{C}_{31} \mathrm{H}_{49} \mathrm{OP}_{2}[\mathrm{M}]^{+} \mathrm{m} / \mathrm{z}=499.3253$; found = 499.3254.

\section{Synthesis of $[(t-B u-t h i x a n t p h o s) \mathrm{H}] \mathrm{CH}\left(\mathrm{SO}_{2} \mathrm{CF}_{3}\right)$}

This product was synthesised similarly to $[t-\mathrm{Bu}$-xantphos $(\mathrm{H})] \mathrm{CH}\left(\mathrm{SO}_{2} \mathrm{CF}_{3}\right)_{2}$ using $t$-Bu-thixantphos $(0.041 \mathrm{~g}, 0.079 \mathrm{mmol})$ giving the title compound in quantitative yield as a pale yield solid. X-ray quality crystals were grown by performing the reaction in $\mathrm{C}_{6} \mathrm{D}_{6}$ and allowing the solvent to slowly evaporate.

${ }^{31} \mathrm{P}$ NMR (121 MHz, $\left.\mathrm{CD}_{2} \mathrm{Cl}_{2}\right): \delta 15.8$ (s) ${ }^{1} \mathrm{H} \mathrm{NMR}\left(500 \mathrm{MHz}, \mathrm{CD}_{2} \mathrm{Cl}_{2}\right): \delta 8.99(\mathrm{~m}$, $\mathrm{PH}), 7.29$ (s, SCCH), 7.22 (s, PC(Ar)CH), 3.83 (s, $\left.\mathrm{CH}\left(\mathrm{SO}_{2} \mathrm{CF}_{3}\right)\right), 2.36$ (s, $\left.\mathrm{C}(\mathrm{Ar}) \mathrm{CH}_{3}\right)$, 1.41-1.44 (m, PC $\left.\left(\mathrm{CH}_{3}\right)_{3}\right) .{ }^{13} \mathrm{C} \mathrm{NMR}\left(125 \mathrm{MHz}, \mathrm{CD}_{2} \mathrm{Cl}_{2}\right): \delta 152.9$ (s, PCCO), 136.2 (s, $\left.\mathrm{C}(\mathrm{Ar}) \mathrm{CH}_{3}\right), 132.5$ (s, $\left.\mathrm{SCCH}\right), 131.8$ (s, PC(Ar)CH), 122.2 (s, SCCH), 121.5 (quartet, $\left.J_{\mathrm{CF}}=325.6 \mathrm{~Hz}, \mathrm{CF}_{3}\right), 115.3(\mathrm{~s}, \mathrm{PC}(\mathrm{Ar})), 34.4\left(\mathrm{~s}, \mathrm{PC}\left(\mathrm{CH}_{3}\right)_{3}\right), 29.5\left(\mathrm{~s}, \mathrm{PC}\left(\mathrm{CH}_{3}\right)_{3}\right)$. One peak $\left(\mathrm{CH}\left(\mathrm{SO}_{2} \mathrm{CF}_{3}\right)\right)$ obscured by solvent. HRMS calcd for $\mathrm{C}_{30} \mathrm{H}_{47} \mathrm{OP}_{2} \mathrm{~S}[\mathrm{M}]^{+} \mathrm{m} / z$ $=517.2817$; found $=517.2817$.

\section{Synthesis of $[(t-B u$-sixantphos $) \mathrm{H}] \mathrm{CH}\left(\mathrm{SO}_{2} \mathrm{CF}_{3}\right)$}

[t-Bu-Sixantphos $(\mathrm{H})] \mathrm{CH}\left(\mathrm{SO}_{2} \mathrm{CF}_{3}\right)_{2}$ was synthesised in a similar manner to [ $t$ Bu-xantphos $(\mathrm{H})] \mathrm{CH}\left(\mathrm{SO}_{2} \mathrm{CF}_{3}\right)$ using $t$-Bu-sixantphos $(0.020 \mathrm{~g}, 0.039 \mathrm{mmol})$. The product was produced as a white solid in quantitative yield.

${ }^{31} \mathrm{P}$ NMR (121 MHz, $\left.\mathrm{CDCl}_{3}\right): \delta 14.3$ (s) ${ }^{1} \mathrm{H}$ NMR (500 MHz, $\left.\mathrm{CDCl}_{3}\right): \delta 9.57(\mathrm{~m}$, $\mathrm{PH})$, 7.85-7.87 (m, PC(Ar)CH), $7.79(\mathrm{~d}, 6.6 \mathrm{~Hz}, \mathrm{SiCCH}), 7.41(\mathrm{t}, 7.5 \mathrm{~Hz}, \mathrm{PCCCH})$, 4.06 (bs, $\left.\mathrm{CH}\left(\mathrm{SO}_{2} \mathrm{CF}_{3}\right)_{2}\right), 1.43\left(\mathrm{vt}, J_{\mathrm{PH}}=7.5 \mathrm{~Hz}, \mathrm{PC}\left(\mathrm{CH}_{3}\right)_{3}\right), 0.53\left(\mathrm{~s}, \mathrm{Si}\left(\mathrm{CH}_{3}\right)_{2}\right)$. ${ }^{19} \mathrm{~F} \mathrm{NMR}\left(282 \mathrm{MHz}, \mathrm{CDCl}_{3}\right): \delta-80.8\left(\mathrm{~s}, \mathrm{CF}_{3}\right){ }^{13} \mathrm{C} \mathrm{NMR}\left(125 \mathrm{MHz}, \mathrm{CDCl}_{3}\right): \delta$ $161.5\left(\mathrm{vt}, J_{\mathrm{PC}}=5.3 \mathrm{~Hz}, \mathrm{PCCO}\right), 139.1(\mathrm{~s}, \mathrm{SiCCH}), 136.9$ (s, PC(Ar)CH), 124.0 $\left(\mathrm{vt}, J_{\mathrm{PC}}=2.4 \mathrm{~Hz}, \mathrm{PCCCH}\right), 121.1(\mathrm{~s}, \mathrm{SiCCH}), 121.1$ (quartet, $J_{\mathrm{CF}}=327.2 \mathrm{~Hz}$, $\left.\mathrm{CH}\left(\mathrm{SO}_{2} \mathrm{CF}_{3}\right)_{2}\right), 115.0$ (bs, $\left.\mathrm{PC}(\mathrm{Ar})\right), 53.6\left(\mathrm{~s}, \mathrm{CH}\left(\mathrm{SO}_{2} \mathrm{CF}_{3}\right)_{2}\right), 34.2\left(\mathrm{vt}, J_{\mathrm{PC}}=3.9 \mathrm{~Hz}\right.$, $\left.\mathrm{PC}\left(\mathrm{CH}_{3}\right)_{3}\right), 29.5\left(\mathrm{vt}, J_{\mathrm{PC}}=4.3 \mathrm{~Hz}, \mathrm{PC}\left(\mathrm{CH}_{3}\right)_{3}\right),-0.4\left(\mathrm{~s}, \mathrm{Si}\left(\mathrm{CH}_{3}\right)_{2}\right)$. HRMS calcd for $\mathrm{C}_{30} \mathrm{H}_{49} \mathrm{OP}_{2} \mathrm{Si}[\mathrm{M}]^{+} \mathrm{m} / \mathrm{z}=515.3022$; found $=515.3023$. 


\section{t-Bu-SixantphosSe}

A solution of $t$-Bu-xantphos $(0.031 \mathrm{~g}, 0.060 \mathrm{mmol})$ in toluene $(5 \mathrm{~mL})$ was added to grey selenium $(0.095 \mathrm{~g}, 1.20 \mathrm{mmol})$ in toluene $(5 \mathrm{~mL})$. The reaction was heated to reflux with stirring for 3 days. The resulting yellow solution was allowed to cool, filtered and reduced in vacuo to give a pale yellow solid $(0.036 \mathrm{~g}, 100 \%)$.

${ }^{31} \mathrm{P}$ NMR (121 MHz, 1:1, $\left.\mathrm{CDCl}_{3}: \mathrm{CD}_{2} \mathrm{Cl}_{2}\right): \delta 15.9(\mathrm{~s}, \mathrm{P}), 102.7\left(\mathrm{~s},{ }^{1} J_{\mathrm{PSe}}=689.1 \mathrm{~Hz}\right.$, $\mathrm{P}=\mathrm{Se}) .{ }^{1} \mathrm{H} \mathrm{NMR}\left(300 \mathrm{MHz}, 1: 1, \mathrm{CDCl}_{3}: \mathrm{CD}_{2} \mathrm{Cl}_{2}\right): \delta 9.13$ (bs, $\left.1 \mathrm{H}, \mathrm{Ar}\right), 7.81$ (d, $J=$ $7.5 \mathrm{~Hz}, 1 \mathrm{H}, \mathrm{Ar}), 7.66-7.16$ (m, 4H, Ar), 1.61 (d, $\left.J=22.5 \mathrm{~Hz}, \mathrm{P}(=\mathrm{Se}) \mathrm{C}(\mathrm{CH} 3)_{3}\right), 1.10$ $\left(\mathrm{d}, J=11.5 \mathrm{~Hz}, \mathrm{PC}\left(\mathrm{CH}_{3}\right)_{3}\right), 0.36\left(\mathrm{~s}, \mathrm{Si}\left(\mathrm{CH}_{3}\right)_{2}\right), 0.07\left(\mathrm{~s}, \mathrm{Si}\left(\mathrm{CH}_{3}\right)_{2}\right)$. HRMS calcd for $\mathrm{C}_{30} \mathrm{H}_{49} \mathrm{OP}_{2} \mathrm{SeSi}[\mathrm{M}+\mathrm{H}]^{+} \mathrm{m} / \mathrm{z}=595.2190$; found $=595.2172$.

\section{t-Bu-ThixantphosSe}

A solution of $t$-Bu-thixantphos $(0.042 \mathrm{~g}, 0.081 \mathrm{mmol})$ in toluene $(5 \mathrm{~mL})$ was added to grey selenium $(0.128 \mathrm{~g}, 1.62 \mathrm{mmol})$ in toluene $(5 \mathrm{~mL})$. The reaction was heated to reflux with stirring for 3 days. The resulting yellow solution was allowed to cool, filtered and reduced in vacuo to give the title compound as a yellow solid $(0.047 \mathrm{~g}, 98 \%)$.

${ }^{31} \mathrm{P}$ NMR (121 MHz, $\left.\mathrm{CDCl}_{3}\right): \delta 11.7(\mathrm{~s}, \mathrm{P}), 101.9$ (s, $\left.{ }^{1} J_{\mathrm{PSe}}=698.5 \mathrm{~Hz}, \mathrm{P}=\mathrm{Se}\right) .{ }^{1} \mathrm{H}$ NMR (300 MHz, CDCl $): \delta 8.85(\mathrm{~d}, J=16.2 \mathrm{~Hz}, \mathrm{P}(=\mathrm{Se}) \mathrm{CCH}), 7.26(\mathrm{~s}, 1 \mathrm{H}, \mathrm{Ar})$, 6.96 (s, 1H, Ar), 6.89 (s, 1H, Ar), 2.27 (s, 3H, C(Ar) $\left.\mathrm{CH}_{3}\right), 2.25$ (s, 3H, C(Ar)CH $\mathrm{CH}_{3}$, $1.62\left(\mathrm{~d}, J=16.6 \mathrm{~Hz}, 18 \mathrm{H}, \mathrm{P}(=\mathrm{Se}) \mathrm{C}(\mathrm{CH} 3)_{3}\right), 1.15\left(\mathrm{~d}, J=11.9 \mathrm{~Hz}, 18 \mathrm{H}, \mathrm{PC}\left(\mathrm{CH}_{3}\right)_{3}\right)$. HRMS calcd for $\mathrm{C}_{31} \mathrm{H}_{49} \mathrm{OP}_{2} \mathrm{SSe}[\mathrm{M}+\mathrm{H}]^{+} \mathrm{m} / z=597.1984$; found = 597.1919.

\section{t-Bu-XantphosSe}

A solution of $t$-Bu-xantphos $(0.041 \mathrm{~g}, 0.082 \mathrm{mmol})$ in toluene $(5 \mathrm{~mL})$ was added to grey selenium $(0.130 \mathrm{~g}, 1.65 \mathrm{mmol})$ in toluene $(5 \mathrm{~mL})$. The reaction was heated to reflux with stirring for 3 days. The resulting yellow solution was allowed to cool, filtered and reduced in vacuo to give a pale yellow solid $(0.038 \mathrm{~g}, 80 \%)$.

${ }^{31} \mathrm{P}$ NMR (121 MHz, 1:1, $\left.\mathrm{CDCl}_{3}: \mathrm{CD}_{2} \mathrm{Cl}_{2}\right): \delta 10.6$ (s, P), 101.9 (s, ${ }^{1} J_{\mathrm{PSe}}=$ $697.1 \mathrm{~Hz}, \mathrm{P}=\mathrm{Se}) .{ }^{1} \mathrm{H}$ NMR $\left(500 \mathrm{MHz}, 1: 1, \mathrm{CDCl}_{3}: \mathrm{CD}_{2} \mathrm{Cl}_{2}\right): \delta 9.26(\mathrm{dd}, 17.4$, 
$7.8 \mathrm{~Hz}, \mathrm{P}(=\mathrm{Se}) \mathrm{CCH}), 7.67$ (d, $J=7.6 \mathrm{~Hz}, \mathrm{PC}(\mathrm{Ar}) \mathrm{CH}), 7.60(\mathrm{~d}, J=7.5 \mathrm{~Hz}$, $\mathrm{P}(=\mathrm{Se}) \mathrm{CCCCH}), 7.50(\mathrm{~d}, J=7.5 \mathrm{~Hz}, \mathrm{PCCCCH}), 7.24(\mathrm{t}, J=7.8 \mathrm{~Hz}, \mathrm{P}(=\mathrm{Se}) \mathrm{CCCH})$, $7.19(\mathrm{t}, J=7.6 \mathrm{~Hz}, \mathrm{PCCCH}), 1.66\left(\mathrm{~d}, J=16.6 \mathrm{~Hz}, \mathrm{P}(=\mathrm{Se}) \mathrm{C}(\mathrm{CH} 3)_{3}\right), 1.57$ (s, C(bridge) $\left.\left(\mathrm{CH}_{3}\right)_{2}\right), 1.19\left(\mathrm{~d}, J=11.8 \mathrm{~Hz}, \mathrm{PC}\left(\mathrm{CH}_{3}\right)_{3}\right) .{ }^{13} \mathrm{C}$ NMR (125 MHz, 1:1 $\left.\mathrm{CDCl}_{3}: \mathrm{CD}_{2} \mathrm{Cl}_{2}\right): \delta 157.0\left(\mathrm{~d}, J_{\mathrm{PC}}=21.1 \mathrm{~Hz}, \mathrm{PCCO}\right), 154.2(\mathrm{~s}, \mathrm{P}(=\mathrm{Se}) \mathrm{CCO}), 143.5$ (d, $\left.J_{\mathrm{PC}}=11.6 \mathrm{~Hz}, \mathrm{P}(=\mathrm{Se}) \mathrm{CCH}\right), 136.0$ (s, PC(Ar)CH), 132.8 (s, PCCCC(bridge)), 132.0 $\left(\mathrm{d}, J_{\mathrm{PC}}=4.8 \mathrm{~Hz}, \mathrm{P}(=\mathrm{Se}) \mathrm{CCCC}(\right.$ bridge $\left.)\right), 129.2\left(\mathrm{~d}, J_{\mathrm{PC}}=2.4 \mathrm{~Hz}, \mathrm{P}(=\mathrm{Se}) \mathrm{CCCCH}\right)$, 126.3 (s, PCCCCH), 125.3 (d, $J_{\mathrm{PC}}=35 \mathrm{~Hz}, \mathrm{PC}(\mathrm{Ar})$ ), 123.2 (s, 2C, P(=Se)CCCH, $\mathrm{PCCCH}), 115.4\left(\mathrm{~d}, J_{\mathrm{PC}}=39.8 \mathrm{~Hz}, \mathrm{P}(=\mathrm{Se}) C(\mathrm{Ar})\right), 39.2\left(\mathrm{~d}, J_{\mathrm{PC}}=34.6 \mathrm{~Hz}\right.$, $\mathrm{P}(=\mathrm{Se}) C(\mathrm{CH} 3) 3), 35.3$ (s, $C$ (bridge) ), $33.5\left(\mathrm{~d}, J_{\mathrm{PC}}=26.9 \mathrm{~Hz}, \mathrm{PC}\left(\mathrm{CH}_{3}\right)_{3}\right), 31.2$ $\left(\mathrm{d}, J_{\mathrm{PC}}=15.4 \mathrm{~Hz}, \mathrm{PC}\left(\mathrm{CH}_{3}\right)_{3}\right), 30.9\left(\mathrm{dd}, 7.7,2.0 \mathrm{~Hz}, \mathrm{P}(=\mathrm{Se}) \mathrm{C}\left(\mathrm{CH}_{3}\right)_{3}\right) .30 .8$ (s, $\mathrm{C}$ (bridge) $\mathrm{CH}_{3}$ ). HRMS calcd for $\mathrm{C}_{31} \mathrm{H}_{49} \mathrm{OP}_{2} \mathrm{Se}[\mathrm{M}+\mathrm{H}]^{+} \mathrm{m} / z=579.2421$; found $=579.2381$.

\subsection{Silver Complexes}

\section{[Ag(t-Bu-thixantphos)Cl]}

This reaction was carried out in the dark. $t$-Bu-Thixantphos $(88 \mathrm{mg}, 0.17 \mathrm{mmol})$ and silver chloride $(24 \mathrm{mg}, 0.17 \mathrm{mmol})$ were combined $\mathrm{CH}_{2} \mathrm{Cl}_{2}(4 \mathrm{~mL})$ in a Schlenk tube. After 5 days stirring the solution was passed through a plug of alumina, washing with dichloromethane $(4 \times 1 \mathrm{~mL})$. The solvent was removed in vacuo giving a cloudy oil. The oil was triturated with hexane $(2 \mathrm{~mL})$ yielding the title compound as a white powder $(94 \mathrm{mg}, 84 \%)$. The resulting silver complex is light sensitive and care should be taken to exclude light.

${ }^{31} \mathrm{P}$ NMR $\left(121 \mathrm{MHz}, \mathrm{CDCl}_{3}\right): \delta 21.81\left(\mathrm{~d},{ }^{1} J^{107} \mathrm{AgP}=406.7 \mathrm{~Hz}, \mathrm{~d},{ }^{1} J_{109} \mathrm{AgP}=469.6 \mathrm{~Hz}\right)$. ${ }^{1} \mathrm{H}$ NMR $\left(600 \mathrm{MHz}, \mathrm{CDCl}_{3}\right): \delta 7.39\left(\mathrm{~d}, J_{\mathrm{PH}}=1.0 \mathrm{~Hz}, \mathrm{PC}(\mathrm{Ar}) \mathrm{CH}\right), 7.11(\mathrm{~d}$, $\left.J_{\mathrm{PH}}=1.6 \mathrm{~Hz}, \mathrm{SCCH}\right), 2.31\left(\mathrm{~s}, \mathrm{C}(\mathrm{Ar}) \mathrm{CH}_{3}\right) 1.41\left(\mathrm{~m}, \mathrm{PC}\left(\mathrm{CH}_{3}\right)_{3}\right) .{ }^{13} \mathrm{C} \mathrm{NMR}(150 \mathrm{MHz}$, $\left.\mathrm{CDCl}_{3}\right): \delta 155.5\left(\mathrm{vt}, J_{\mathrm{PC}}=6.6 \mathrm{~Hz}, \mathrm{PCCO}\right), 134.8\left(\mathrm{~d}, J_{\mathrm{PC}}=4.9 \mathrm{~Hz}, \mathrm{PC}(\mathrm{Ar}) C \mathrm{H}\right), 133.1$ $\left(\mathrm{d}, J_{\mathrm{PC}}=1.5 \mathrm{~Hz}, C(\mathrm{Ar}) \mathrm{CH}_{3}\right), 130.3(\mathrm{~s}, \mathrm{SCCH}), 122.8\left(\mathrm{vt}, J_{\mathrm{PC}}=3.0 \mathrm{~Hz}, \mathrm{SCCH}\right)$, $120.9(\mathrm{~m}, \mathrm{PC}(\mathrm{Ar})) 35.3\left(\mathrm{~m}, \mathrm{PC}\left(\mathrm{CH}_{3}\right)_{3}\right) 30.9\left(\mathrm{vt}, J_{\mathrm{PC}}=5.6 \mathrm{~Hz}, \mathrm{PC}\left(\mathrm{CH}_{3}\right)_{3}\right), 20.8$ (s, $\left.\mathrm{C}(\mathrm{Ar}) \mathrm{CH}_{3}\right)$. HRMS calcd for $\mathrm{C}_{30} \mathrm{H}_{46} \mathrm{OP}_{2} \mathrm{SAg}[\mathrm{M}-\mathrm{Cl}]^{+} \mathrm{m} / z=623.1796$; found = 623.1805. 


\section{[Ag(t-Bu-sixantphos)Cl]}

This reaction was carried out in the dark. $t$-Bu-Sixantphos and silver chloride were combined in an NMR tube, dissolved in $\mathrm{CDCl}_{3}$, and sonicated for 5 mins. After four days the reaction was sonicated for $6 \times 5$ mins. The solution was decanted and the resulting solid was washed with dichloromethane $(3 \times 1 \mathrm{~mL})$. The solvent was removed in vacuo yielding the title compound as a white solid (31 $\mathrm{mg}, 97 \%$ ). The resulting silver complex is light sensitive and care should be taken to exclude light.

${ }^{31} \mathrm{P}$ NMR $\left(121 \mathrm{MHz}, \mathrm{CDCl}_{3}\right): \delta 24.2\left(\mathrm{~d},{ }^{1} J^{107} \mathrm{AgP}=408.1 \mathrm{~Hz}, \mathrm{~d},{ }^{1} J^{109} \mathrm{AgP}=471.1 \mathrm{~Hz}\right)$ ${ }^{1} \mathrm{H}$ NMR (600 MHz, $\left.\mathrm{CDCl}_{3}\right): \delta 7.88$ (m, PC(Ar)CH), $7.61(\mathrm{dd}, 7.0,1.8 \mathrm{~Hz}, \mathrm{SiCCH})$, $7.21(\mathrm{t}, 7.3 \mathrm{~Hz}, \mathrm{PCCCH}), 1.42\left(\mathrm{~m}, \mathrm{PC}\left(\mathrm{CH}_{3}\right)_{3}\right), 0.46\left(\mathrm{~s}, \mathrm{Si}\left(\mathrm{CH}_{3}\right)_{2}\right) .{ }^{13} \mathrm{C}$ NMR $(150$ $\left.\mathrm{MHz} \mathrm{CDCl}_{3}\right): \delta 163.9\left(\mathrm{vt}, J_{\mathrm{PC}}=5.2 \mathrm{~Hz}, \mathrm{PCCO}\right), 138.2\left(\mathrm{~d}, J_{\mathrm{PC}}=4.4 \mathrm{~Hz}, \mathrm{PC}(\mathrm{Ar}) C \mathrm{H}\right)$, 136.3 (s, SiCCH), 122.2 (s, SiCCH), 122.1 (s, PCCCH), 120.5 (m, PC(Ar)) 35.5 (m, $\left.\mathrm{PC}\left(\mathrm{CH}_{3}\right)_{3}\right) 31.0\left(\mathrm{vt}, J_{\mathrm{PC}}=5.6 \mathrm{~Hz}, \mathrm{PC}\left(\mathrm{CH}_{3}\right)_{3}\right),-1.3\left(\mathrm{~s}, \mathrm{C}(\mathrm{Ar}) \mathrm{CH}_{3}\right)$. HRMS calcd for $\mathrm{C}_{30} \mathrm{H}_{48} \mathrm{OP}_{2} \mathrm{Ag}[\mathrm{M}-\mathrm{Cl}]^{+} \mathrm{m} / \mathrm{z}=621.2001$; found $=621.2021$.

\section{[Ag(t-Bu-xantphos)Cl]}

This reaction was carried out in the dark. $t$-Bu-Xantphos $(0.017 \mathrm{~g}, 0.034 \mathrm{mmol})$ and silver chloride $(0.005 \mathrm{~g}, 0.035 \mathrm{mmol})$ were combined in an NMR tube and dissolved in $\mathrm{CDCl}_{3}$. After 48 hours the reaction mixture was sonicated $10 \times 5$ mins. The solution was decanted and the resulting solid was dried under reduced pressure leaving the title compound as a white powder $(0.017 \mathrm{~g}, 78 \%)$. The resulting silver complex is light sensitive and care should be taken to exclude light.

${ }^{31} \mathrm{P}$ NMR $\left(121 \mathrm{MHz}, \mathrm{CDCl}_{3}\right): \delta 20.7\left(\mathrm{~d},{ }^{1} J^{107} \mathrm{AgP}=409.3 \mathrm{~Hz}, \mathrm{~d},{ }^{1} J^{109} \mathrm{AgP}=472.2 \mathrm{~Hz}\right)$ ${ }^{1} \mathrm{H}$ NMR (500 MHz, $\left.\mathrm{CDCl}_{3}\right): \delta 7.68\left(\mathrm{~d}, J_{\mathrm{PH}}=6.9 \mathrm{~Hz}, \mathrm{PC}(\mathrm{Ar}) \mathrm{CH}\right), 7.53(\mathrm{dd}, 7.6$, $1.2 \mathrm{~Hz}, \mathrm{C}($ bridge $) \mathrm{CCH}), 7.19(\mathrm{t}, 7.7 \mathrm{~Hz}, \mathrm{PCCCH}), 1.56$ (s, C(bridge) $\left.\left(\mathrm{CH}_{3}\right)_{2}\right), 1.40$ $1.43\left(\mathrm{~m}, \mathrm{PC}\left(\mathrm{CH}_{3}\right)_{3}\right) .{ }^{13} \mathrm{C} \mathrm{NMR}\left(125 \mathrm{MHz}, \mathrm{CDCl}_{3}\right): \delta 156.5\left(\mathrm{vt}, J_{\mathrm{PC}}=6.5 \mathrm{~Hz}, \mathrm{PCCO}\right)$, 133.7 (m, PC(Ar)CH), 130.7 (s, C(bridge)CCH), 126.8 (s, C(bridge)CCH), 122.7 (s, PCCCH), 119.3 (m, PC(Ar)), 35.6 (m, C(bridge)), 35.1 (m, PC $\left.\left(\mathrm{CH}_{3}\right)_{3}\right), 30.8$ (vt, $\left.J_{\mathrm{PC}}=5.6 \mathrm{~Hz}, \mathrm{PC}\left(\mathrm{CH}_{3}\right)_{3}\right), 28.5\left(\mathrm{~s}, \mathrm{C}(\right.$ bridge $\left.)\left(\mathrm{CH}_{3}\right)_{2}\right)$. HRMS calcd for $\mathrm{C}_{31} \mathrm{H}_{48} \mathrm{OP}_{2} \mathrm{Ag}$ $[\mathrm{M}-\mathrm{Cl}]^{+} \mathrm{m} / \mathrm{z}=605.2226$; found $=605.2163$. 


\section{$\left[\mathrm{Ag}(t-\mathrm{Bu}-\right.$ sixantphos $) \mathrm{BF}_{4}$}

This reaction was carried out in the dark. $t$-Bu-Sixantphos $(0.027 \mathrm{~g}, 0.052 \mathrm{mmol})$ and silver tetrafluoroborate $(0.010,0.052 \mathrm{mmol})$ were combined in an NMR tube and dissolved in $\mathrm{CDCl}_{3}$. After 3 days the reaction was complete by NMR spectroscopy. The solution was removed under reduced pressure yielding the title compound as a white solid (0.024 g, $0.034 \mathrm{mmol}, 64 \%)$.

${ }^{31} \mathrm{P}$ NMR $\left(121 \mathrm{MHz}, \mathrm{CDCl}_{3}\right): \delta 31.5\left(\mathrm{~d},{ }^{1} J^{107} \mathrm{AgP}=482.9 \mathrm{~Hz}, \mathrm{~d},{ }^{1} J^{109} \mathrm{AgP}=557.4 \mathrm{~Hz}\right)$. ${ }^{1} \mathrm{H}$ NMR (500 MHz, $\mathrm{CDCl}_{3}$ ): $\delta 7.84$ (bs, PC(Ar)CH), 7.73 (d, $\left.6.8 \mathrm{~Hz}, \mathrm{SiCCH}\right), 7.34$ $(\mathrm{t}, 7.3 \mathrm{~Hz}, \mathrm{PCCCH}), 1.40\left(\mathrm{vt}, J_{\mathrm{PH}}=15.9 \mathrm{~Hz}, \mathrm{PC}\left(\mathrm{CH}_{3}\right)_{3}\right), 0.49\left(\mathrm{~s}, \mathrm{Si}\left(\mathrm{CH}_{3}\right)_{2}\right) .{ }^{13} \mathrm{C}$ $\operatorname{NMR}\left(125 \mathrm{MHz}, \mathrm{CDCl}_{3}\right): \delta 162.3\left(\mathrm{vt}, J_{\mathrm{PC}}=8.7 \mathrm{~Hz}, \mathrm{PCCO}\right), 138.2(\mathrm{~s}, \mathrm{SiCCH}), 138.1$ $\left(\mathrm{d}, J_{\mathrm{PC}}=6.7 \mathrm{~Hz}, \mathrm{PC}(\mathrm{Ar}) C \mathrm{H}\right), 123.1\left(\mathrm{~d}, J_{\mathrm{PC}}=1.9 \mathrm{~Hz}, \mathrm{PCCCH}\right), 122.1(\mathrm{~s}, \mathrm{SiCCH})$, $118.4(\mathrm{~m}, \mathrm{PC}(\mathrm{Ar})), 35.7\left(\mathrm{~m}, \mathrm{PC}\left(\mathrm{CH}_{3}\right)_{3}\right), 30.9\left(\mathrm{~m}, \mathrm{PC}\left(\mathrm{CH}_{3}\right)_{3}\right),-0.6\left(\mathrm{~s}, \mathrm{Si}\left(\mathrm{CH}_{3}\right)_{2}\right) .{ }^{19} \mathrm{~F}$ $\operatorname{NMR}\left(282 \mathrm{MHz}, \mathrm{CDCl}_{3}\right): \delta-151.9\left(\mathrm{~s}, \mathrm{BF}_{4}^{-}\right)$. HRMS calcd for $\mathrm{C}_{30} \mathrm{H}_{48} \mathrm{OP}_{2} \mathrm{AgSi}[\mathrm{M}-$ $\left.\mathrm{BF}_{4}^{-}\right]^{+} \mathrm{m} / \mathrm{z}=621.2001$; found $=621.2032$.

\section{$[\mathrm{Ag}(t-\mathrm{Bu}-$ thixantphos $)] \mathrm{BF}_{4}$}

This reaction was carried out in the dark. A solution of $t$-Bu-thixantphos $(0.026$ $\mathrm{g}, 0.050 \mathrm{mmol})$ in $0.5 \mathrm{~mL}$ of $\mathrm{CDCl}_{3}$ was added to silver tetrafluoroborate $(0.010$ $\mathrm{g}, 0.051 \mathrm{mmol}$ ) in an NMR tube. After 3 days the reaction NMR showed complete conversion into the title complex. The solvent was removed under reduced pressure yielding the product as a white solid ( $0.030 \mathrm{~g}, 0.042 \mathrm{mmol}, 84 \%)$.

${ }^{31} \mathrm{P}$ NMR $\left(121 \mathrm{MHz}, \mathrm{CDCl}_{3}\right): \delta 28.4\left(\mathrm{~d},{ }^{1} J^{107} \mathrm{AgP}=486.7 \mathrm{~Hz}, \mathrm{~d},{ }^{1} J^{109} \mathrm{AgP}=562.2 \mathrm{~Hz}\right)$. ${ }^{1} \mathrm{H}$ NMR (500 MHz, $\mathrm{CDCl}_{3}$ ): $\delta 7.32$ (s, PC(Ar)CH), 7.16 (s, SCCH), 2.33 (s, $\left.\mathrm{C}(\mathrm{Ar}) \mathrm{CH}_{3}\right), 1.40\left(\mathrm{vt}, J_{\mathrm{PH}}=15.8 \mathrm{~Hz}, \mathrm{PC}\left(\mathrm{CH}_{3}\right)_{3}\right) .{ }^{13} \mathrm{C} \mathrm{NMR}\left(125 \mathrm{MHz}, \mathrm{CDCl}_{3}\right): \delta$ $153.9\left(\mathrm{vt}, J_{\mathrm{PC}}=11.0 \mathrm{~Hz}, \mathrm{PCCO}\right), 134.4\left(\mathrm{~d}, J_{\mathrm{PC}}=1.7 \mathrm{~Hz}, C(\mathrm{Ar}) \mathrm{CH}_{3}\right), 134.2(\mathrm{~d}$, $\left.J_{\mathrm{PC}}=6.30 \mathrm{~Hz}, \mathrm{PC}(\mathrm{Ar}) C \mathrm{H}\right), 131.1(\mathrm{~s}, \mathrm{SCCH}), 122.3(\mathrm{~s}, \mathrm{SCCH}), 119.4(\mathrm{~m}, \mathrm{PC}(\mathrm{Ar}))$, $35.5\left(\mathrm{~m}, \mathrm{PC}\left(\mathrm{CH}_{3}\right)_{3}\right), 30.8\left(\mathrm{~m}, \mathrm{PC}\left(\mathrm{CH}_{3}\right)_{3}\right), 20.7\left(\mathrm{~s}, \mathrm{C}(\mathrm{Ar}) \mathrm{CH}_{3}\right) .{ }^{19} \mathrm{~F} \mathrm{NMR}(282 \mathrm{MHz}$,

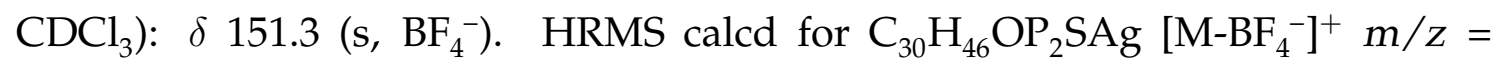
623.1796 ; found $=623.1826$. 


\section{$\left[\mathrm{Ag}\left(t-\mathrm{Bu}-x_{\text {-xantphos })}\right] \mathrm{BF}_{4}\right.$}

This reaction was carried out in the dark as silver compounds are typically light sensitive. $t$-Bu-Xantphos $(0.017 \mathrm{~g}, 0.034 \mathrm{mmol})$ and silver tetrafluoroborate $(0.008$ $\mathrm{g}$, $0.041 \mathrm{mmol}$ ) were combined in an NMR tube and dissolved in $\mathrm{CDCl}_{3}$. After 48 hours the reaction was complete by NMR spectroscopy. The solvent was removed under reduced pressed yielding a white solid in quantitative yield.

${ }^{31} \mathrm{P}$ NMR $\left(121 \mathrm{MHz}, \mathrm{CDCl}_{3}\right): \delta 27.6\left(\mathrm{~d},{ }^{1} J^{107} \mathrm{AgP}=486.3 \mathrm{~Hz}, \mathrm{~d},{ }^{1} J^{109} \mathrm{AgP}=561.1 \mathrm{~Hz}\right)$ ${ }^{1} \mathrm{H}$ NMR (500 MHz, $\left.\mathrm{CDCl}_{3}\right): \delta$ 7.63-7.67 (m, C(bridge)CCH, PC(Ar)CH), $7.29(\mathrm{t}$, $7.7 \mathrm{~Hz}, \mathrm{PCCCH}), 1.59$ (s, C(bridge) $\left.\left(\mathrm{CH}_{3}\right)_{2}\right), 1.39-1.42\left(\mathrm{~m}, \mathrm{PC}\left(\mathrm{CH}_{3}\right)_{3}\right) .{ }^{13} \mathrm{C} \mathrm{NMR}$ $\left(125 \mathrm{MHz}, \mathrm{CDCl}_{3}\right): \delta 154.9\left(\mathrm{vt}, J_{\mathrm{PC}}=5.6 \mathrm{~Hz}, \mathrm{PCCO}\right), 133.5\left(\mathrm{vt}, J_{\mathrm{PC}}=1.9 \mathrm{~Hz}\right.$, $\mathrm{PC}(\mathrm{Ar}) \mathrm{CH}), 133.5\left(\mathrm{~d}, J_{\mathrm{PC}}=5.8 \mathrm{~Hz}, \mathrm{C}(\right.$ bridge $\left.) C \mathrm{CH}\right), 128.4$ (s, C(bridge)CCH), 123.8 (s, PCCCH), 117.8 (s, PC(Ar)), 35.6 (vtd, $J_{\mathrm{PC}}=5.3,2.4 \mathrm{~Hz}, C$ (bridge)), $35.3\left(\mathrm{~m}, \mathrm{PC}\left(\mathrm{CH}_{3}\right)_{3}\right), 30.6\left(\mathrm{~m}, \mathrm{PC}\left(\mathrm{CH}_{3}\right)_{3}\right), 29.4$ (s, $\mathrm{C}$ (bridge) $\left.\left(\mathrm{CH}_{3}\right)_{2}\right) .{ }^{19} \mathrm{~F} \mathrm{NMR}(282$ $\left.\mathrm{MHz}, \mathrm{CDCl}_{3}\right): \delta-151.9\left(\mathrm{~s}, \mathrm{BF}_{4}^{-}\right) \mathrm{HRMS}$ calcd for $\mathrm{C}_{31} \mathrm{H}_{48} \mathrm{OP}_{2} \mathrm{Ag}\left[\mathrm{M}-\mathrm{BF}_{4}^{-}\right]^{+} \mathrm{m} / \mathrm{z}=$ 605.2226; found $=605.2217$.

\section{Reaction of $[\mathrm{Ag}(t-\mathrm{Bu}$-sixantphos $)] \mathrm{BF}_{4}$ with $\mathrm{LiCCPh}$}

A solution of $n$-butyllithium in cyclohexanes (1.6 M $0.12 \mathrm{~mL})$ was added to a solution of phenylacetylene $(0.021 \mathrm{~mL})$ in THF $(40 \mathrm{~mL})$. The mixture was stirred for 10 minutes, then a solution of $\left[\mathrm{Ag}(t-\mathrm{Bu}\right.$-sixantphos $) \mathrm{BF}_{4}(0.34 \mathrm{~g})$ in THF $(2.0$ $\mathrm{mL}$ ) was added. The reaction mixture was stirred in the dark for 2 hours. The solvent was removed in vacuo and the residue taken up in acetone- $\mathrm{d}_{6}$ for NMR analysis.

${ }^{31} \mathrm{P}$ NMR (121 MHz, acetone $\left.-\mathrm{d}_{6}\right): \delta 26.4\left(\mathrm{~d},{ }^{1} \mathrm{~J}_{107} \mathrm{AgP}=418.5 \mathrm{~Hz}, \mathrm{~d}\right.$, $\left.{ }^{1} J_{109} \mathrm{AgP}=482.9 \mathrm{~Hz}\right){ }^{1} \mathrm{H}$ NMR $\left(500 \mathrm{MHz}\right.$, acetone $\left.-\mathrm{d}_{6}\right): \delta 8.06(\mathrm{~m}, 2 \mathrm{H}, \mathrm{Ar}), 7.86$ $(\mathrm{dd}, J=7.1,1.5 \mathrm{~Hz}, 2 \mathrm{H}, \mathrm{Ar}), 7.41(\mathrm{t}, J=7.4 \mathrm{~Hz}, \mathrm{PCCCH}), 1.47-1.45\left(\mathrm{~m}, \mathrm{PC}\left(\mathrm{CH}_{3}\right)_{3}\right)$, $0.51\left(\mathrm{~s}, \mathrm{Si}\left(\mathrm{CH}_{3}\right)_{2}\right) .{ }^{19} \mathrm{~F} \mathrm{NMR}\left(282 \mathrm{MHz}\right.$, acetone $\left.-\mathrm{d}_{6}\right): \delta-152.3\left(\mathrm{~s}, \mathrm{BF}_{4}^{-}\right)$.

\section{Reaction of $[\mathrm{Ag}(t-\mathrm{Bu}$-thixantphos $)] \mathrm{BF}_{4}$ with $\mathrm{LiCCPh}$}

A solution of n-butyllithium in cyclohexanes $(1.6 \mathrm{M} 0.11 \mathrm{~mL})$ was added to a solution of phenylacetylene $(0.020 \mathrm{~mL})$ in THF $(40 \mathrm{~mL})$. The mixture was stirred 
for 10 minutes, then $17 \mathrm{~mL}$ of the solution was added to a solution of [Ag(t-Buthixantphos) $\mathrm{BF}_{4}$ in $(0.055 \mathrm{~g})$ in THF $(3.0 \mathrm{~mL})$. The reaction mixture was stirred in the dark for 12 hours. The solvent was removed in vacuo and the residue taken up in acetone- $\mathrm{d}_{6}$ or $\mathrm{C}_{6} \mathrm{D}_{6}$ for NMR analysis.

${ }^{31} \mathrm{P}$ NMR (121 MHz, $\left.\mathrm{C}_{6} \mathrm{D}_{6}\right): \delta 23.4$, (bs) 19.9, (bs) ${ }^{1} \mathrm{H}$ NMR (300 MHz, $\left.\mathrm{C}_{6} \mathrm{D}_{6}\right): \delta$ 7.7-6.8 (m, 6H, Ar), 1.96 (bs, C (Ar) $\left.\mathrm{CH}_{3}\right), 1.42$ (bs, PC $\left.\left(\mathrm{CH}_{3}\right)_{3}\right) .{ }^{19} \mathrm{~F}$ NMR $(282 \mathrm{MHz}$, $\left.\mathrm{C}_{6} \mathrm{D}_{6}\right): \delta-156.9\left(\mathrm{~s}, \mathrm{BF}_{4}^{-}\right)$

${ }^{31} \mathrm{P}$ NMR (121 MHz, acetone- $\left.\mathrm{d}_{6}\right): \delta 23.1\left(\mathrm{~d},{ }^{1} J_{107} \mathrm{AgP}=410.3 \mathrm{~Hz}, \mathrm{~d}\right.$, $\left.{ }^{1} J_{109} \mathrm{AgP}=474.1 \mathrm{~Hz}\right) .{ }^{1} \mathrm{H} \mathrm{NMR}\left(300 \mathrm{MHz}\right.$, acetone $\left.-\mathrm{d}_{6}\right): \delta 7.61(\mathrm{~s}, 2 \mathrm{H}, \mathrm{Ar}) 7.36(\mathrm{~s}, 2 \mathrm{H}$, Ar) $2.39\left(\mathrm{~s}, \mathrm{C}(\mathrm{Ar}) \mathrm{CH}_{3}\right), 1.51-1.46\left(\mathrm{~m}, \mathrm{PC}\left(\mathrm{CH}_{3}\right)_{3}\right) .{ }^{19} \mathrm{~F} \mathrm{NMR}\left(282 \mathrm{MHz}\right.$, acetone $\left.-\mathrm{d}_{6}\right)$ : $\delta-152.1\left(\mathrm{~s}, \mathrm{BF}_{4}^{-}\right)$.

\section{Reaction of [Ag(t-Bu-xantphos) $] \mathrm{BF}_{4}$ with $\mathrm{LiCCPh}$}

A solution of $\mathrm{n}$-butyllithium in cyclohexanes $(1.6 \mathrm{M} 0.11 \mathrm{~mL})$ was added to a solution of phenylacetylene $(0.020 \mathrm{~mL})$ in THF $(40 \mathrm{~mL})$. The mixture was stirred for 10 minutes, then $16.2 \mathrm{~mL}$ of the solution was added to a solution of [Ag(t-Buxantphos) $\mathrm{BF}_{4}$ in $(0.051 \mathrm{~g})$ in THF $(3.0 \mathrm{~mL})$. The reaction mixture was stirred in the dark for 12 hours. The solvent was removed in vacuo and the residue taken up in acetone- $\mathrm{d}_{6}$ for NMR analysis.

${ }^{31} \mathrm{P}$ NMR (121 MHz, acetone- $\left.\mathrm{d}_{6}\right): \delta 20.7\left(\mathrm{~d},{ }^{1} J_{107} \mathrm{AgP}=407.4 \mathrm{~Hz}, \mathrm{~d}\right.$, $\left.{ }^{1} J_{109} \mathrm{AgP}=471.1 \mathrm{~Hz}\right) .{ }^{1} \mathrm{H} \mathrm{NMR}\left(500 \mathrm{MHz}\right.$, acetone $\left.-\mathrm{d}_{6}\right): \delta 7.86(\mathrm{~d}, J=7.5 \mathrm{~Hz}$, 2H, Ar), $7.76(\mathrm{dd}, J=7.6,0.9 \mathrm{~Hz}, 2 \mathrm{H}, \mathrm{Ar}), 7.37$ (t, $J=7.6 \mathrm{~Hz}, \mathrm{PCCCH}), 1.61$ (s, $\mathrm{C}($ bridge $\left.)\left(\mathrm{CH}_{3}\right)_{2}\right), 1.46-1.44\left(\mathrm{~m}, \mathrm{PC}\left(\mathrm{CH}_{3}\right)_{3}\right) .{ }^{19} \mathrm{~F} \mathrm{NMR}\left(282 \mathrm{MHz}\right.$, acetone $\left.-\mathrm{d}_{6}\right): \delta$ $-152.6\left(\mathrm{~s}, \mathrm{BF}_{4}^{-}\right)$.

\subsection{Rhodium Complexes}

\section{[Rh(t-Bu-sixantphos- $\left.\left.\mathrm{R}, O, P^{\prime}\right) \mathrm{Cl}\right]$}

A solution of $t$-Bu-sixantphos $(0.032 \mathrm{~g}, 0.062 \mathrm{mmol})$ in $\mathrm{C}_{6} \mathrm{D}_{6}$ was added to solid $\left[\mathrm{Rh}(\mathrm{coe})_{2} \mathrm{Cl}\right]_{\mathrm{n}}(0.027 \mathrm{~g}, 0.075 \mathrm{mmol}, 1.2 \mathrm{eq}$. of $\mathrm{Rh})$ in a Young's tap NMR tube. The tube was sealed under argon and heated to $60{ }^{\circ} \mathrm{C}$ for 24 hours. The solution was 
decanted and the solvent removed in vacuo to yield the title compound as a dark red solid in quantitative yield $(0.041 \mathrm{~g}, 0.062 \mathrm{mmol}, 100 \%)$.

${ }^{31} \mathrm{P}$ NMR $\left(121 \mathrm{MHz}, \mathrm{C}_{6} \mathrm{D}_{6}\right): \delta 44.2\left(\mathrm{~d}, J_{\mathrm{RhP}}=140.0 \mathrm{~Hz}\right) .{ }^{1} \mathrm{H} \mathrm{NMR}\left(600 \mathrm{MHz}, \mathrm{C}_{6} \mathrm{D}_{6}\right)$ : $\delta 8.05(\mathrm{~m}, \mathrm{PC}(\mathrm{Ar}) \mathrm{CH}), 7.13(\mathrm{dd}, J=6.8,1.8 \mathrm{~Hz}, \mathrm{SiCCH}), 6.84(\mathrm{t}, J=7.2 \mathrm{~Hz}$, $\mathrm{PCCCH}), 1.69\left(\mathrm{vt}, J_{\mathrm{PH}}=13.5 \mathrm{~Hz}, \mathrm{PC}\left(\mathrm{CH}_{3}\right)_{3}\right), 0.07\left(\mathrm{~s}, \mathrm{Si}\left(\mathrm{CH}_{3}\right)_{2}\right) .{ }^{13} \mathrm{C} \mathrm{NMR}(125$ $\left.\mathrm{MHz}, \mathrm{C}_{6} \mathrm{D}_{6}\right): \delta 169.5\left(\mathrm{PCCO}, J_{\mathrm{PC}}=, \mathrm{Hz}\right.$, ) (s, 138.4)PC(Ar)CH, 136.0 (s, SiCCH), 127.0 (m, PC(Ar)), 122.8 (s, PCCCH), $121.5\left(\mathrm{SiCCH}, J_{\mathrm{PC}}=, \mathrm{Hz},\right)\left(37.7, J_{\mathrm{PC}}=\mathrm{vt} \mathrm{Hz}\right.$, 9.9) PC $\left(\mathrm{CH}_{3}\right)_{3}, 31.0\left(\mathrm{vt}, J_{\mathrm{PC}}=8.1 \mathrm{~Hz}, \mathrm{PC}\left(\mathrm{CH}_{3}\right)_{3}\right),-0.7\left(\mathrm{~s}, \mathrm{Si}\left(\mathrm{CH}_{3}\right)_{2}\right)$. HRMS calcd for $\mathrm{C}_{30} \mathrm{H}_{48} \mathrm{OP}_{2} \mathrm{RhSi}[\mathrm{M}-\mathrm{Cl}]^{+} \mathrm{m} / z=617.1999$; found $=617.1998$.

\section{[Rh(t-Bu-thixantphos-к $\left.\left.P, O, P^{\prime}\right) \mathrm{Cl}\right]$}

This compound was synthesised similarly to [ $\mathrm{Rh}(t$-Bu-sixantphos $) \mathrm{Cl}] \mathrm{using}, t$ Bu-thixantphos $(0.025 \mathrm{~g}, 0.048 \mathrm{mmol})$ and $\left[\mathrm{Rh}(\mathrm{coe})_{2} \mathrm{Cl}\right]_{\mathrm{n}}(0.021 \mathrm{~g}, 0.059 \mathrm{mmol})$. The title compound was obtained as an air-sensitive brown solid in quantitative yield (0.032 g, $0.048 \mathrm{mmol}, 100 \%)$.

${ }^{31} \mathrm{P}$ NMR $\left(121 \mathrm{MHz}, \mathrm{C}_{6} \mathrm{D}_{6}\right): \delta 46.5\left(\mathrm{~d}, J_{\mathrm{RhP}}=141.5 \mathrm{~Hz}\right){ }^{1} \mathrm{H} \mathrm{NMR}\left(600 \mathrm{MHz}, \mathrm{C}_{6} \mathrm{D}_{6}\right)$ : $\delta 7.43(\mathrm{~d}, J=1.5 \mathrm{~Hz}, \mathrm{PC}(\mathrm{Ar}) \mathrm{CH}), 6.39$ (s, SCCH), 1.81 (s, C(Ar)CH $\left.\mathrm{CH}_{3}\right), 1.67$ (vt, $\left.J=13.6 \mathrm{~Hz}, \mathrm{PC}\left(\mathrm{CH}_{3}\right)_{3}\right) .{ }^{13} \mathrm{C} \mathrm{NMR}\left(150 \mathrm{MHz}, \mathrm{C}_{6} \mathrm{D}_{6}\right): \delta 157.4\left(\mathrm{vt}, J_{\mathrm{PC}}=16.8 \mathrm{~Hz}\right.$, PCCO), 134.0 (vt, $\left.J_{\mathrm{PC}}=3.6 \mathrm{~Hz}, C(\mathrm{Ar}) \mathrm{CH}_{3}\right), 133.7$ (s, PC(Ar)CH), $128.5(\mathrm{~s}, \mathrm{SCCH})$, $127.4\left(\mathrm{vt}, J_{\mathrm{PC}}=8.1 \mathrm{~Hz}, \mathrm{SCCH}\right), 117.9\left(\mathrm{vt}, J_{\mathrm{PC}}=7.6 \mathrm{~Hz}, \mathrm{PC}(\mathrm{Ar})\right), 37.7\left(\mathrm{vt}, J_{\mathrm{PC}}=9.2 \mathrm{~Hz}\right.$, $\left.\mathrm{PC}\left(\mathrm{CH}_{3}\right)_{3}\right), 30.9\left(\mathrm{vt}, J_{\mathrm{PC}}=7.5 \mathrm{~Hz}, \mathrm{PC}\left(\mathrm{CH}_{3}\right)_{3}\right), 19.8\left(\mathrm{~s}, \mathrm{C}(\mathrm{Ar}) \mathrm{CH}_{3}\right)$. HRMS calcd for $\mathrm{C}_{30} \mathrm{H}_{46} \mathrm{OP}_{2} \mathrm{RhS}[\mathrm{M}-\mathrm{Cl}]^{+} \mathrm{m} / \mathrm{z}=619.1794$; found $=619.1795$.

\section{$\left[\mathrm{Rh}\left(t-\mathrm{Bu}-x a n t p h o s-\kappa P, O, P^{\prime}\right) \mathrm{Cl}\right]$}

The compound was synthesised similarly to [ $\mathrm{Rh}(t$-Bu-sixantphos $) \mathrm{Cl}]$ using $t$-Buxantphos $(0.019 \mathrm{~g}, 0.038 \mathrm{mmol})$ and $\left[\mathrm{Rh}(\mathrm{coe}){ }_{2} \mathrm{Cl}\right]_{\mathrm{n}}(0.016 \mathrm{~g}, 0.038 \mathrm{mmol})$. A dark red solid was obtained in quantitative yield $(0.024 \mathrm{~g}, 0.038 \mathrm{mmol}, 98.9 \%)$.

${ }^{31} \mathrm{P}$ NMR $\left(121 \mathrm{MHz}, \mathrm{C}_{6} \mathrm{D}_{6}\right): \delta 47.7\left(\mathrm{~d}, J_{\mathrm{RhP}}=142.3 \mathrm{~Hz}\right){ }^{1} \mathrm{H} \mathrm{NMR}\left(500 \mathrm{MHz}, \mathrm{C}_{6} \mathrm{D}_{6}\right)$ : $\delta 7.78(\mathrm{~d}, J=7.3 \mathrm{~Hz}, \mathrm{PC}(\mathrm{Ar}) \mathrm{CH}), 7.01(\mathrm{~d}, J=7.6 \mathrm{~Hz}, \mathrm{C}$ (bridge)CCH), $6.80(\mathrm{t}$, $J=7.7 \mathrm{~Hz}, \mathrm{PCCCH}), 1.68\left(\mathrm{vt}, J_{\mathrm{PH}}=13.4 \mathrm{~Hz}, \mathrm{PC}\left(\mathrm{CH}_{3}\right)_{3}\right), 1.16\left(\mathrm{~s}, \mathrm{C}(\right.$ bridge $\left.)\left(\mathrm{CH}_{3}\right)_{2}\right)$. ${ }^{13} \mathrm{C} \mathrm{NMR}\left(125 \mathrm{MHz}, \mathrm{C}_{6} \mathrm{D}_{6}\right): \delta 158.9\left(\mathrm{vt}, J_{\mathrm{PC}}=16.3 \mathrm{~Hz}, \mathrm{PCCO}\right), 133.6(\mathrm{~s}, \mathrm{PC}(\mathrm{Ar}) C \mathrm{H})$, 
$131.3\left(\mathrm{vt}, J_{\mathrm{PC}}=6.23 \mathrm{~Hz}, \mathrm{C}(\right.$ bridge $\left.) C \mathrm{CH}\right), 127.7$ (s, C(bridge) $\left.\mathrm{CCH}\right), 125.7$ (vt, $\left.J_{\mathrm{PC}}=12.0 \mathrm{~Hz}, \mathrm{PC}(\mathrm{Ar})\right), 123.8(\mathrm{~s}, \mathrm{PCCCH}), 37.6\left(\mathrm{vt}, J_{\mathrm{PC}}=10.1 \mathrm{~Hz}, \mathrm{PC}\left(\mathrm{CH}_{3}\right)_{3}\right)$, 32.5 (s, C(bridge)), 33.8 (s, C(bridge) $\left.\left(\mathrm{CH}_{3}\right)_{2}\right), 30.8$ (vt, $\left.J_{\mathrm{PC}}=7.7 \mathrm{~Hz}, \mathrm{PC}\left(\mathrm{CH}_{3}\right)_{3}\right)$. HRMS calcd for $\mathrm{C}_{31} \mathrm{H}_{48} \mathrm{OP}{ }_{2} \mathrm{Rh}[\mathrm{M}-\mathrm{Cl}]^{+} \mathrm{m} / \mathrm{z}=601.2230$; found = 601.2222.

\section{$(O C-6-43)-\left[\mathrm{Rh}\left(t-B u-s i x a n t p h o s-\kappa P, O, P^{\prime}\right) \mathrm{Cl}(\mathrm{H})_{2}\right]$}

$\mathrm{C}_{6} \mathrm{D}_{6}(0.5 \mathrm{~mL})$ was stirred vigorously under hydrogen for $10 \mathrm{mins}$, before using to dissolve [Rh( $t$-Bu-sixantphos $) \mathrm{Cl}](0.037 \mathrm{~g}, 0.057 \mathrm{mmol})$ in a Young's tap NMR tube. Hydrogen was bubbled through the solution for $10 \mathrm{mins}$ before sealing the tube under hydrogen. After 48 hours removal of the solvent in vacuo yielded the title compound as a brown solid in quantitative yield $(0.037 \mathrm{~g}, 0.056 \mathrm{mmol})$.

${ }^{31} \mathrm{P}$ NMR $\left(121 \mathrm{MHz}, \mathrm{C}_{6} \mathrm{D}_{6}\right): \delta 78.2\left(\mathrm{ddd}, J_{\mathrm{RhP}}=116.1, J_{\mathrm{PH}}=12.1,2.7 \mathrm{~Hz}\right) .{ }^{1} \mathrm{H}$ $\operatorname{NMR}\left(600 \mathrm{MHz}, \mathrm{C}_{6} \mathrm{D}_{6}\right): \delta 7.71(\mathrm{~m}, \mathrm{PC}(\mathrm{Ar}) \mathrm{CH}), 7.23(\mathrm{dd}, J=7.1,1.7 \mathrm{~Hz}, \mathrm{SiCCH})$, $6.93(\mathrm{t}, J=7.4 \mathrm{~Hz}, \mathrm{PCCCH}), 1.75\left(\mathrm{vt}, J=14.7 \mathrm{~Hz}, \mathrm{PC}\left(\mathrm{CH}_{3}\right)_{3}\right), 1.29(\mathrm{vt}, J=13.7 \mathrm{~Hz}$, $\left.\mathrm{PC}\left(\mathrm{CH}_{3}\right)_{3}\right), 0.17\left(\mathrm{~s}, \mathrm{Si}\left(\mathrm{CH}_{3}\right)_{2}\right), 0.10\left(\mathrm{~s}, \mathrm{Si}\left(\mathrm{CH}_{3}\right)_{2}\right),-16.92\left(\mathrm{dtd}, J_{\mathrm{RhH}}=22.7, J_{\mathrm{PH}}=13.5\right.$, $J_{\mathrm{HH}} 9.4, \mathrm{H}^{-}$trans $\left.\mathrm{Cl}^{-}\right),-21.12\left(\mathrm{dtd}, J_{\mathrm{RhH}}=30.9, J_{\mathrm{PH}}=12.1, J_{\mathrm{HH}} 9.2, \mathrm{H}^{-}\right.$trans $\left.\mathrm{O}\right) .{ }^{13} \mathrm{C}$ $\operatorname{NMR}\left(150 \mathrm{MHz}, \mathrm{C}_{6} \mathrm{D}_{6}\right): \delta 165.6(\mathrm{vt}, J=11.0 \mathrm{~Hz}, \mathrm{PCCO}), 137.5$ (s, PC(Ar)CH), 136.4 (s, SiCCH), 126.5 (vt, $J=15.0 \mathrm{~Hz}, \mathrm{PC}(\mathrm{Ar})), 122.6$ (vt, $J=4.6 \mathrm{~Hz}, \mathrm{PCCCH}$ ), 122.0 (s, SiCCH), 38.4 (vt, $\left.J=10.9 \mathrm{~Hz}, \mathrm{PC}\left(\mathrm{CH}_{3}\right)_{3}\right), 36.7(\mathrm{vtt}, J=20.8,2.8 \mathrm{~Hz}$, $\left.\mathrm{PC}\left(\mathrm{CH}_{3}\right)_{3}\right), 33.5\left(\mathrm{vt}, J=8.5 \mathrm{~Hz}, \mathrm{PC}\left(\mathrm{CH}_{3}\right)_{3}\right), 30.0\left(\mathrm{bs}, \mathrm{PC}\left(\mathrm{CH}_{3}\right)_{3}\right),-0.7\left(\mathrm{~s}, \mathrm{Si}\left(\mathrm{CH}_{3}\right)_{2}\right)$, $-0.9\left(\mathrm{~s}, \mathrm{Si}\left(\mathrm{CH}_{3}\right)_{2}\right)$. HRMS calcd for $\mathrm{C}_{30} \mathrm{H}_{50} \mathrm{OP}_{2} \mathrm{RhSi}[\mathrm{M}-\mathrm{Cl}]^{+} \mathrm{m} / z=619.2156$; found $=619.2137$.

\section{$(O C-6-43)-\left[\mathrm{Rh}\left(t-\mathrm{Bu}-\right.\right.$ thixantphos- $\left.\left.\kappa P, O, P^{\prime}\right) \mathrm{Cl}(\mathrm{H})_{2}\right]$}

The reaction was performed using the same method as the synthesis of $[\mathrm{Rh}(t-\mathrm{Bu}$ sixantphos $\left.) \mathrm{Cl}(\mathrm{H})_{2}\right]$, using $t$-Bu-thixantphos $(0.034 \mathrm{~g}$, $0.052 \mathrm{mmol})$. NMR analysis after 48 hours showed the title compound as the only product. The solvent was removed under reduced pressure, yielding the title compound as a brown solid in quantitative yield $(0.034 \mathrm{~g})$.

${ }^{31} \mathrm{P}$ NMR $\left(121 \mathrm{MHz}, \mathrm{C}_{6} \mathrm{D}_{6}\right): \delta 77.8\left(\mathrm{ddd}, J_{\mathrm{RhP}}=117.8, J_{\mathrm{PH}}=11.9,3.7\right) .{ }^{1} \mathrm{H} \mathrm{NMR}(600$ $\left.\mathrm{MHz}, \mathrm{C}_{6} \mathrm{D}_{6}\right): \delta 7.15(\mathrm{~d}, J=2.1 \mathrm{~Hz}, \mathrm{PC}(\mathrm{Ar}) \mathrm{CH}), 6.51$ (s, SCCH), 1.85 (s, C (Ar)CH $\mathrm{CH}_{3}$, $1.75\left(\mathrm{vt}, J=14.6 \mathrm{~Hz}, \mathrm{PC}\left(\mathrm{CH}_{3}\right)_{3}\right), 1.29\left(\mathrm{vt}, J=13.9 \mathrm{~Hz}, \mathrm{PC}\left(\mathrm{CH}_{3}\right)_{3}\right),-17.00\left(\mathrm{dtd}, J_{\mathrm{RhH}}\right.$ 
$=22.6, J_{\mathrm{PH}}=13.5, J_{\mathrm{HH}} 9.4, \mathrm{H}^{-}$trans $\left.\mathrm{Cl}^{-}\right),-21.13\left(\mathrm{dtd}, J_{\mathrm{RhH}}=30.5, J_{\mathrm{PH}}=12.4, J_{\mathrm{HH}}\right.$ 9.4, $\mathrm{H}^{-}$trans $\left.\mathrm{O}\right) .{ }^{13} \mathrm{C} \mathrm{NMR}\left(150 \mathrm{MHz}, \mathrm{C}_{6} \mathrm{D}_{6}\right): \delta 155.4(\mathrm{vt}, J=13.8 \mathrm{~Hz}, \mathrm{PCCO})$, 133.8 (vt, $\left.J=4.6 \mathrm{~Hz}, C(\mathrm{Ar}) \mathrm{CH}_{3}\right), 133.1$ (s, PC(Ar)CH), 129.1 (s, SCCH), 126.7 (vt, $J=16.2 \mathrm{~Hz}, \mathrm{SCCH}), 120.2$ (vt, $J=6.9 \mathrm{~Hz}, \mathrm{PC}(\mathrm{Ar})), 38.3$ (vt, $J=10.9 \mathrm{~Hz}$, $\left.\mathrm{PC}\left(\mathrm{CH}_{3}\right)_{3}\right), 36.5\left(\mathrm{vtt}, J=19.1,2.5 \mathrm{~Hz}, \mathrm{PC}\left(\mathrm{CH}_{3}\right)_{3}\right), 33.2\left(\mathrm{vt}, J=8.1 \mathrm{~Hz}, \mathrm{PC}\left(\mathrm{CH}_{3}\right)_{3}\right)$, 30.1 (bs, $\left.\mathrm{PC}\left(\mathrm{CH}_{3}\right)_{3}\right), 20.1$ (s, $\left.\mathrm{C}(\mathrm{Ar}) \mathrm{CH}_{3}\right)$.

\section{$(O C-6-43)-\left[\mathrm{Rh}\left(t-\mathrm{Bu}-x a n t p h o s-\kappa P, O, P^{\prime}\right) \mathrm{Cl}(\mathrm{H})_{2}\right]$}

This reaction was performed using the same method as the synthesis of $[\mathrm{Rh}(t-\mathrm{Bu}-$ sixantphos $\left.) \mathrm{Cl}(\mathrm{H})_{2}\right]$, using [ $\mathrm{Rh}(t$-Bu-xantphos $\left.) \mathrm{Cl}\right](0.023 \mathrm{~g}, 0.036 \mathrm{mmol})$. After 48 hours the solvent was removed in vacuo yielding the title compound as a brown solid in quantitative yield $(0.023 \mathrm{~g})$.

${ }^{31} \mathrm{P}$ NMR $\left(121 \mathrm{MHz}, \mathrm{C}_{6} \mathrm{D}_{6}\right): \delta 79.0\left(\mathrm{ddd}, J_{\mathrm{RhP}}=117.0, J_{\mathrm{PH}}=11.9,5.2\right) .{ }^{1} \mathrm{H}$ NMR $\left(600 \mathrm{MHz}, \mathrm{C}_{6} \mathrm{D}_{6}\right): \delta 7.48(\mathrm{~m}, \mathrm{PC}(\mathrm{Ar}) \mathrm{CH}), 7.08(\mathrm{~d}, J=7.5 \mathrm{~Hz}, \mathrm{C}($ bridge $) \mathrm{CCH}), 6.88$ $(\mathrm{t}, J=7.6 \mathrm{~Hz}, \mathrm{PCCCH}), 1.77\left(\mathrm{vt}, J=14.8 \mathrm{~Hz}, \mathrm{PC}\left(\mathrm{CH}_{3}\right)_{3}\right), 1.30(\mathrm{vt}, J=13.7 \mathrm{~Hz}$, $\left.\mathrm{PC}\left(\mathrm{CH}_{3}\right)_{3}\right), 1.25\left(\mathrm{~s}, \mathrm{C}(\right.$ bridge $\left.)\left(\mathrm{CH}_{3}\right)_{2}\right), 1.22\left(\mathrm{~s}, \mathrm{C}(\right.$ bridge $\left.)\left(\mathrm{CH}_{3}\right)_{2}\right),-17.04\left(\mathrm{dtd}, J_{\mathrm{RhH}}=\right.$ $22.8, J_{\mathrm{PH}}=13.4, J_{\mathrm{HH}} 9.4, \mathrm{H}^{-}$trans $\left.\mathrm{Cl}^{-}\right),-20.51\left(\mathrm{dtd}, J_{\mathrm{RhH}}=28.8, J_{\mathrm{PH}}=12.2, J_{\mathrm{HH}} 9.4\right.$, $\mathrm{H}^{-}$trans $\left.\mathrm{O}\right) .{ }^{13} \mathrm{C} \mathrm{NMR}\left(150 \mathrm{MHz}, \mathrm{C}_{6} \mathrm{D}_{6}\right): \delta 156.2(\mathrm{vt}, J=13.3 \mathrm{~Hz}, \mathrm{PCCO}), 132.9$ (s, C(bridge)CCH), 132.4 (vt, $J=5.2 \mathrm{~Hz}, \mathrm{C}($ bridge $) C \mathrm{CH}) 127.8(\mathrm{vt}, J=8.1 \mathrm{~Hz}$, PC(Ar)CH), 125.1 (vt, J = 17.9 Hz, PC(Ar)), 123.5 (vt, $J=4.6 \mathrm{~Hz}, \mathrm{PCCCH}), 38.1$ (vt, $\left.J=11.6 \mathrm{~Hz}, \mathrm{PC}\left(\mathrm{CH}_{3}\right)_{3}\right), 36.5\left(\mathrm{vtt}, J=19.1 \mathrm{~Hz}, \mathrm{PC}\left(\mathrm{CH}_{3}\right)_{3}\right), 34.8$ (s, C(bridge)), 33.5 (vt, $\left.J=7.5 \mathrm{~Hz}, \mathrm{PC}\left(\mathrm{CH}_{3}\right)_{3}\right), 32.4$ (s, C(bridge) $\left.\left(\mathrm{CH}_{3}\right)_{2}\right), 30.4$ (s, C(bridge) $\left.\left(\mathrm{CH}_{3}\right)_{2}\right)$, 30.0 (bs, $\left.\mathrm{PC}\left(\mathrm{CH}_{3}\right)_{3}\right)$.

\section{$(T B P Y-5-33)-\left[R h\left(t-B u-s i x a n t p h o s-\kappa^{2}-P, P^{\prime}\right)(C O)_{2} \mathrm{Cl}\right]$}

[Rh(t-Bu-sixantphos)Cl] $(0.041 \mathrm{~g}, 0.063 \mathrm{mmol})$ was dissolved in $\mathrm{C}_{6} \mathrm{D}_{6}(0.5 \mathrm{~mL})$ in a Young's tap NMR tube, under an argon atmosphere. Carbon monoxide was bubble through the solution for 10 mins, before the tube was sealed under a carbon monoxide atmosphere. After three days the reaction was complete by ${ }^{31} \mathrm{P}$ NMR spectroscopy. The orange solution was decanted and the solvent was removed under reduced pressure, yielding the title complex as an orange solid (0.027 $\mathrm{g}$, $0.038 \mathrm{mmol}, 60 \%$ ). 
${ }^{31} \mathrm{P}$ NMR $\left(121 \mathrm{MHz}, \mathrm{C}_{6} \mathrm{D}_{6}\right): \delta 70.8\left(\mathrm{~d}, J_{\mathrm{RhP}}=120.0 \mathrm{~Hz}\right) .{ }^{1} \mathrm{H}$ NMR $(600 \mathrm{MHz}$, $\left.\mathrm{C}_{6} \mathrm{D}_{6}\right): \delta 7.69(\mathrm{~m}, \mathrm{PC}(\mathrm{Ar}) \mathrm{CH}), 7.32(\mathrm{~d}, J=6.8 \mathrm{~Hz}, \operatorname{SiCCH}), 7.02(\mathrm{t}, J=7.2 \mathrm{~Hz}$, $\mathrm{PCCCH}), 1.53\left(\mathrm{vt}, J=14.0 \mathrm{~Hz}, \mathrm{PC}\left(\mathrm{CH}_{3}\right)_{3}\right), 0.18\left(\mathrm{~s}, \mathrm{Si}\left(\mathrm{CH}_{3}\right)_{2}\right) .{ }^{13} \mathrm{C} \mathrm{NMR}(150 \mathrm{MHz}$, $\left.\mathrm{C}_{6} \mathrm{D}_{6}\right): \delta 195.5(\mathrm{dt}, J=84.4,13.0 \mathrm{~Hz}, \mathrm{RhCO}) 164.3(\mathrm{vt}, J=8.1 \mathrm{~Hz}, \mathrm{PCCO}), 138.1$ (s, PC(Ar)CH), 136.5 (s, SiCCH), 124.9 (vt, J = 24.2 Hz, PC(Ar)), 123.2 (s, SiCCH), 122.4 (s, PCCCH), 38.4 (vt, $\left.J=13.9 \mathrm{~Hz}, \mathrm{PC}\left(\mathrm{CH}_{3}\right)_{3}\right), 31.5$ (bs, $\left.\mathrm{PC}\left(\mathrm{CH}_{3}\right)_{3}\right),-1.5$ (bs, $\left.\mathrm{Si}\left(\mathrm{CH}_{3}\right)_{2}\right)$. HRMS calcd for $\mathrm{C}_{31} \mathrm{H}_{48} \mathrm{O}_{2} \mathrm{P}_{2} \mathrm{RhSi}[\mathrm{M}-\mathrm{COCl}]^{+} \mathrm{m} / \mathrm{z}=645.1948$; found $=$ 645.1959.

\section{(TBPY-5-33)-[Rh(t-Bu-thixantphos- $\left.\left.\kappa^{2}-P, P^{\prime}\right)(C O)_{2} \mathrm{Cl}\right]$}

This compound was synthesised similarly to $\left[\mathrm{Rh}(t\right.$-Bu-sixantphos $\left.)(\mathrm{CO})_{2} \mathrm{Cl}\right]$, using [ $\mathrm{Rh}(t$-Bu-thixantphos $) \mathrm{Cl}](0.032 \mathrm{~g}, 0.049 \mathrm{mmol})$ generating [Rh(t-Bu-thixantphos $)(\mathrm{CO})_{2} \mathrm{Cl}$ ] as a yellow solid, $(0.025 \mathrm{~g}, 0.035 \mathrm{mmol}, 72 \%)$.

${ }^{31} \mathrm{P}$ NMR $\left(121 \mathrm{MHz}, \mathrm{C}_{6} \mathrm{D}_{6}\right): \delta 69.3\left(\mathrm{~d}, J_{\mathrm{RhP}}=122.2 \mathrm{~Hz}\right){ }^{1} \mathrm{H} \mathrm{NMR}\left(600 \mathrm{MHz}, \mathrm{C}_{6} \mathrm{D}_{6}\right)$ : $\delta 7.15$ (s, Ar), 6.88 (bs, Ar), 1.88 (s, C(Ar)CH $\left.\mathrm{CH}_{3}\right), 1.52$ (bs, PC $\left.\left(\mathrm{CH}_{3}\right)_{3}\right) .{ }^{13} \mathrm{C} \mathrm{NMR} \mathrm{(150}$ $\mathrm{MHz}, \mathrm{C}_{6} \mathrm{D}_{6}$ ): $\delta 154.9$ (bs, PCCO), 134.1 (s, Ar), 132.5 (bs, Ar), 129.5 (bs, Ar), 125.6 (bs, Ar), 121.7 (bs, Ar), 38.2 (bs, C(bridge) $\left.\left(\mathrm{CH}_{3}\right)_{2}\right), 31.5$ (bs, PC $\left.\left(\mathrm{CH}_{3}\right)_{3}\right), 20.3$ (s, $\mathrm{C}$ (bridge)). HRMS calcd for $\mathrm{C}_{31} \mathrm{H}_{46} \mathrm{O}_{2} \mathrm{P}_{2} \mathrm{RhS}$ [M-COCl] ${ }^{+} \mathrm{m} / \mathrm{z}=647.1743$; found $=647.1754$.

\section{(TBPY-5-33)-[Rh(t-Bu-thixantphos- $\left.\left.\kappa^{2}-P, P^{\prime}\right)(\mathrm{CO})_{2} \mathrm{Cl}\right]$}

This compound was synthesised similarly to [ $\mathrm{Rh}(t$-Bu-sixantphos $\left.)(\mathrm{CO})_{2} \mathrm{Cl}\right]$, using [Rh(t-Bu-xantphos $) \mathrm{Cl}](0.024 \mathrm{~g}, 0.038 \mathrm{mmol})$ generating the title compound as a yellow solid, $(0.018 \mathrm{~g}, 0.026 \mathrm{mmol}, 67 \%)$.

${ }^{31} \mathrm{P}$ NMR (121 MHz, $\left.\mathrm{C}_{6} \mathrm{D}_{6}\right): \delta 71.6\left(\mathrm{~d}, J_{\mathrm{RhP}}=120.0 \mathrm{~Hz}\right) .{ }^{1} \mathrm{H}$ NMR $(600 \mathrm{MHz}$, $\left.\mathrm{C}_{6} \mathrm{D}_{6}\right): \delta 7.46(\mathrm{~m}, \mathrm{PC}(\mathrm{Ar}) \mathrm{CH}), 7.15$ (partially obscured by residual solvent peak, $\mathrm{C}$ (bridge)CCH), $6.94(\mathrm{t}, J=7.6 \mathrm{~Hz}, \mathrm{PCCCH}), 1.54\left(\mathrm{vt}, J=13.8 \mathrm{~Hz}, \mathrm{PC}\left(\mathrm{CH}_{3}\right)_{3}\right), 1.24$ (s, C(bridge) $\left.\left(\mathrm{CH}_{3}\right)_{2}\right) .{ }^{13} \mathrm{C} \mathrm{NMR}\left(150 \mathrm{MHz}, \mathrm{C}_{6} \mathrm{D}_{6}\right): \delta 194.9(\mathrm{dt}, J=84.4,12.4 \mathrm{~Hz}$, RhCO), 155.6 (vt, $J=10.4 \mathrm{~Hz}, \mathrm{PCCO}), 133.4$ (vt, $J=4.6 \mathrm{~Hz}, \mathrm{C}$ (bridge)CCH), 133.3 (s, PC(Ar)CH), 127.0 (s, C(bridge)CCH), 123.9 (vt, J = 25.4 Hz, PC(Ar)), 122.7 (vt, $J=5.3 \mathrm{~Hz}, \mathrm{PCCCH}), 38.1\left(\mathrm{vt}, J=13.9 \mathrm{~Hz}, \mathrm{PC}\left(\mathrm{CH}_{3}\right)_{3}\right), 35.3$ (s, C(bridge)), 
31.4 (bs, $\left.\mathrm{PC}\left(\mathrm{CH}_{3}\right)_{3}\right), 29.5$ (bs, $\mathrm{C}($ bridge $\left.)\left(\mathrm{CH}_{3}\right)_{2}\right)$. HRMS calcd for $\mathrm{C}_{32} \mathrm{H}_{48} \mathrm{O}_{2} \mathrm{P}_{2} \mathrm{Rh}$ $[\mathrm{M}-\mathrm{COCl}]^{+} \mathrm{m} / \mathrm{z}=629.2179$; found $=629.2186$.

\section{$\left[\mathbf{R h}(\boldsymbol{t}\right.$-Bu-sixantphos $\left.) \mathrm{Cl}\left(\eta^{2}-\mathrm{O}_{2}\right)\right]$}

Air was bubbled through an NMR sample of [Rh(t-Bu-sixantphos)Cl] (0.050 g) in $\mathrm{C}_{6} \mathrm{D}_{6}$ for 10 mins. After 24 hours at room temperature, NMR spectroscopy showed quantitative conversion to $\left[\mathrm{Rh}(t-\mathrm{Bu}\right.$-sixantphos $\left.) \mathrm{Cl}\left(\eta^{2}-\mathrm{O}_{2}\right)\right]$.

${ }^{31} \mathrm{P}$ NMR $\left(121 \mathrm{MHz}, \mathrm{C}_{6} \mathrm{D}_{6}\right): \delta 39.4\left(\mathrm{~d}, J_{\mathrm{RhP}}=102.2 \mathrm{~Hz}\right) .{ }^{1} \mathrm{H}$ NMR $(600 \mathrm{MHz}$, $\left.\mathrm{C}_{6} \mathrm{D}_{6}\right): \delta 7.62(\mathrm{~m}, \mathrm{PC}(\mathrm{Ar}) \mathrm{CH}), 7.18(\mathrm{dd}, J=7.0,1.6 \mathrm{~Hz}, \mathrm{SiCCH}), 6.89(\mathrm{t}, J=7.2 \mathrm{~Hz}$, $\mathrm{PCCCH}), 1.87\left(\mathrm{vt}, J=14.4 \mathrm{~Hz}, \mathrm{PC}\left(\mathrm{CH}_{3}\right)_{3}\right), 1.40\left(\mathrm{bs}, \mathrm{PC}\left(\mathrm{CH}_{3}\right)_{3}\right), 0.19\left(\mathrm{~s}, \mathrm{Si}\left(\mathrm{CH}_{3}\right)_{2}\right)$, $0.01\left(\mathrm{~s}, \mathrm{Si}\left(\mathrm{CH}_{3}\right)_{2}\right) .{ }^{13} \mathrm{C} \mathrm{NMR}\left(150 \mathrm{MHz}, \mathrm{C}_{6} \mathrm{D}_{6}\right): \delta 166.7(\mathrm{vt}, J=9.8 \mathrm{~Hz}, \mathrm{PCCO})$, 138.7 (s, PC(Ar)CH), 136.5 (s, SiCCH), 124.6 (vt, J = 20.8 Hz, PC(Ar)), 123.2 (s, $\mathrm{SiCCH}), 122.9$ (vt, $J=4.7 \mathrm{~Hz}, \mathrm{PCCCH}), 39.1\left(\mathrm{vt}, J=10.4 \mathrm{~Hz}, \mathrm{PC}\left(\mathrm{CH}_{3}\right)_{3}\right), 38.9$ (vt, $\left.J=13.8 \mathrm{~Hz}, \mathrm{PC}\left(\mathrm{CH}_{3}\right)_{3}\right), 33.4\left(\mathrm{vt}, J=5.8 \mathrm{~Hz}, \mathrm{PC}\left(\mathrm{CH}_{3}\right)_{3}\right), 29.5$ (bs, $\left.\mathrm{PC}\left(\mathrm{CH}_{3}\right)_{3}\right), 0.8$ $\left(\mathrm{s}, \mathrm{Si}\left(\mathrm{CH}_{3}\right)_{2}\right),-4.2\left(\mathrm{~s}, \mathrm{Si}\left(\mathrm{CH}_{3}\right)_{2}\right)$, HRMS calcd for $\mathrm{C}_{30} \mathrm{H}_{48} \mathrm{O}_{3} \mathrm{P}_{2} \mathrm{RhSi}[\mathrm{M}-\mathrm{Cl}]^{+} \mathrm{m} / z=$ 649.1898; found $=649.1917$.

\section{[Rh(t-Bu-thixantphos) $\left.\mathrm{Cl}\left(\eta^{2}-\mathrm{O}_{2}\right)\right]$}

This compound was synthesised as for $\left[\mathrm{Rh}(t\right.$-Bu-sixantphos $\left.) \mathrm{Cl}\left(\eta^{2}-\mathrm{O}_{2}\right)\right]$ using $[\mathrm{Rh}(t-$ Bu-thixantphos)Cl] (0.050 g).

${ }^{31} \mathrm{P}$ NMR $\left(121 \mathrm{MHz}, \mathrm{C}_{6} \mathrm{D}_{6}\right): \delta 39.0\left(\mathrm{~d}, J_{\mathrm{RhP}}=101.5 \mathrm{~Hz}\right) .{ }^{1} \mathrm{H}$ NMR $(500 \mathrm{MHz}$, $\left.\mathrm{C}_{6} \mathrm{D}_{6}\right): \delta 7.07\left(\mathrm{~d}, J_{\mathrm{PH}}=2.2 \mathrm{~Hz}, \mathrm{PC}(\mathrm{Ar}) \mathrm{CH}\right), 6.50(\mathrm{~s}, \mathrm{SCCH}), 1.83(\mathrm{vt}, J=14.5 \mathrm{~Hz}$, $\left.\mathrm{PC}\left(\mathrm{CH}_{3}\right)_{3}\right), 1.79$ (s, $\left.\mathrm{C}(\mathrm{Ar}) \mathrm{CH}_{3}\right), 1.44$ (bs, PC $\left.\left(\mathrm{CH}_{3}\right)_{3}\right) .{ }^{13} \mathrm{C} \mathrm{NMR}\left(125 \mathrm{MHz}, \mathrm{C}_{6} \mathrm{D}_{6}\right)$ : $\delta 155.9$ (vt, $J=12.5 \mathrm{~Hz}, \mathrm{PCCO}$ ), 134.6 (s, PC(Ar)CH), 134.0 (vt, $J=5.3 \mathrm{~Hz}$, $\left.\mathrm{C}(\mathrm{Ar}) \mathrm{CH}_{3}\right), 129.8$ (s, SCCH), 125.2 (vt, $\left.J=19.7 \mathrm{~Hz}, \mathrm{PC}(\mathrm{Ar})\right), 119.9$ (vt, $J=7.2 \mathrm{~Hz}$, $\mathrm{SCCH}), 39.1\left(\mathrm{vt}, J=10.6 \mathrm{~Hz}, \mathrm{PC}\left(\mathrm{CH}_{3}\right)_{3}\right), 38.9\left(\mathrm{vt}, J=13.5 \mathrm{~Hz}, \mathrm{PC}\left(\mathrm{CH}_{3}\right)_{3}\right), 33.2$ (vt, $\left.J=5.5 \mathrm{~Hz}, \mathrm{PC}\left(\mathrm{CH}_{3}\right)_{3}\right), 28.9$ (bs, $\left.\mathrm{PC}\left(\mathrm{CH}_{3}\right)_{3}\right), 19.9$ (s, $\left.\mathrm{C}(\mathrm{Ar}) \mathrm{CH}_{3}\right)$. HRMS calcd for $\mathrm{C}_{30} \mathrm{H}_{46} \mathrm{O}_{3} \mathrm{P}_{2} \mathrm{Rh}[\mathrm{M}-\mathrm{Cl}]^{+} \mathrm{m} / \mathrm{z}=651.1692$; found $=651.1695$. 


\section{$\left[\mathbf{R h}(t-B u-x a n t p h o s) \mathrm{Cl}\left(\eta^{2}-\mathrm{O}_{2}\right)\right]$}

This compound was synthesised as for $\left[\mathrm{Rh}(t\right.$-Bu-sixantphos $\left.) \mathrm{Cl}\left(\eta^{2}-\mathrm{O}_{2}\right)\right]$ using $[\mathrm{Rh}(t-$ Bu-xantphos)Cl] (0.050 g).

${ }^{31} \mathrm{P}$ NMR $\left(121 \mathrm{MHz}, \mathrm{C}_{6} \mathrm{D}_{6}\right): \delta 40.5\left(\mathrm{~d}, J_{\mathrm{RhP}}=100.7 \mathrm{~Hz}\right){ }^{1} \mathrm{H}$ NMR $(600 \mathrm{MHz}$, $\left.\mathrm{C}_{6} \mathrm{D}_{6}\right): \delta 7.40(\mathrm{~m}, \mathrm{PC}(\mathrm{Ar}) \mathrm{CH}), 7.08(\mathrm{~d}, J=7.5 \mathrm{~Hz}, \mathrm{C}($ bridge $) \mathrm{CCH}), 6.87(\mathrm{t}$, $J=7.7 \mathrm{~Hz}, \mathrm{PCCCH}), 1.83\left(\mathrm{vt}, J=14.4 \mathrm{~Hz}, \mathrm{PC}\left(\mathrm{CH}_{3}\right)_{3}\right), 1.43\left(\mathrm{bs}, \mathrm{PC}\left(\mathrm{CH}_{3}\right)_{3}\right)$, 1.32 (s, C(bridge) $\left.\left(\mathrm{CH}_{3}\right)_{2}\right), 1.00\left(\mathrm{~s}, \mathrm{C}(\right.$ bridge $\left.)\left(\mathrm{CH}_{3}\right)_{2}\right) .{ }^{13} \mathrm{C} \mathrm{NMR}\left(150 \mathrm{MHz}, \mathrm{C}_{6} \mathrm{D}_{6}\right)$ : $\delta 157.3$ (vt, $J=11.5 \mathrm{~Hz}, \mathrm{PCCO}$ ), 133.9 (s, PC(Ar)CH), 133.1 (vt, $J=5.8 \mathrm{~Hz}$, $\mathrm{C}$ (bridge)CCH), 127.4 (s, C(bridge)CCH), 123.9 (vt, J = 4.7 Hz, PCCCH), 123.3 (vt, $J=21.6 \mathrm{~Hz}, \mathrm{PC}(\mathrm{Ar})), 38.9\left(\mathrm{vt}, J=11.0 \mathrm{~Hz}, \mathrm{PC}\left(\mathrm{CH}_{3}\right)_{3}\right), 38.7(\mathrm{vt}, J=14.0 \mathrm{~Hz}$, $\left.\mathrm{PC}\left(\mathrm{CH}_{3}\right)_{3}\right), 35.5$ (s, $\mathrm{C}($ bridge $\left.)\left(\mathrm{CH}_{3}\right)_{2}\right), 34.7$ (s, $\mathrm{C}$ (bridge)), 33.3 (vt, $J=5.8 \mathrm{~Hz}$, PC $\left.\left(\mathrm{CH}_{3}\right)_{3}\right), 29.0$ (bs, PC $\left.\left(\mathrm{CH}_{3}\right)_{3}\right), 24.2$ (s, C(bridge) $\left.\left(\mathrm{CH}_{3}\right)_{2}\right)$. HRMS calcd for $\mathrm{C}_{31} \mathrm{H}_{48} \mathrm{O}_{3} \mathrm{P}_{2} \mathrm{Rh}[\mathrm{M}-\mathrm{Cl}]^{+} \mathrm{m} / \mathrm{z}=633.2128$; found $=633.2140$.

\section{Reaction of [Rh(t-Bu-sixantphos)Cl] with $\mathrm{ONMe}_{3}$}

In a glovebox, solid $\mathrm{ONMe}_{3}(0.004 \mathrm{~g}, 0.05 \mathrm{mmol})$ was added to an NMR tube containing a $\mathrm{CD}_{2} \mathrm{Cl}_{2}$ solution of [ $\mathrm{Rh}(t$-Bu-sixantphos $\left.) \mathrm{Cl}\right](0.035 \mathrm{~g}, 0.05 \mathrm{mmol})$. The NMR tube was sealed with a J. Young tap. By ${ }^{31} \mathrm{P}$ NMR spectroscopy the mixture contained uncoordinated $t$-Bu-sixantphos $(18.6 \%), t$-Bu-sixantphos oxide $(12.5 \%)$ and $\left[\mathrm{Rh}(t-\mathrm{Bu}\right.$-sixantphos $\left.) \mathrm{Cl}\left(\eta^{2}-\mathrm{O}_{2}\right)\right](68.9 \%)$. After four days the reaction contained uncoordinated $t$-Bu-sixantphos $(6.1 \%), t$-Bu-sixantphos oxide (5.3\%), [Rh(t-Bu-sixantphos) $\left.\mathrm{Cl}\left(\eta^{2}-\mathrm{O}_{2}\right)\right]$ (36.7\%), [Rh(t-Bu-sixantphos) $\left.\mathrm{Cl}\right](46.2 \%)$ and an unidentified species possibly $\left[\mathrm{Rh}(t\right.$-Bu-sixantphos $\left.) \mathrm{Cl}_{2}(\mathrm{OH})\right](5.8 \%)$.

\section{[Rh(t-Bu-sixantphos) $\left.\mathrm{Cl}_{2}(\mathrm{OH})\right]$}

${ }^{31} \mathrm{P}$ NMR $\left(121 \mathrm{MHz}, \mathrm{CD}_{2} \mathrm{Cl}_{2}\right): \delta 70.5\left(\mathrm{~d}, J_{\mathrm{RhP}}=114.1 \mathrm{~Hz}\right)$

\section{Reaction of [Rh(t-Bu-thixantphos)Cl] with $\mathrm{ONMe}_{3}$}

In a glovebox, solid $\mathrm{ONMe}_{3}(0.008 \mathrm{~g}, 0.11 \mathrm{mmol})$ was added to an NMR tube containing a $\mathrm{C}_{6} \mathrm{D}_{6}$ solution of $[\mathrm{Rh}(t$-Bu-thixantphos $) \mathrm{Cl}](0.066 \mathrm{~g}, 0.10 \mathrm{mmol})$. 
The NMR tube was sealed with a J. Young tap. Solid was evident in the NMR tube so the solvent was removed in vacuo and replaced with $\mathrm{CD}_{2} \mathrm{Cl}_{2}$. By ${ }^{31} \mathrm{P}$ NMR the mixture contained uncoordinated $t$-Bu-thixantphos $(22.9 \%),[R h(t-B u-$ thixantphos- $\left.\left.\mathrm{k} P, \mathrm{O}, \mathrm{P}^{\prime}\right) \mathrm{Cl}\left(\eta^{2}-\mathrm{O}_{2}\right)\right](8.6 \%),\left[\mathrm{Rh}\left(t\right.\right.$-Bu-thixantphos- $\left.\left.\mathrm{K} P, \mathrm{O}, \mathrm{P}^{\prime}\right) \mathrm{Cl}\right](64.4 \%)$ and a new compound proposed as $\left[\mathrm{Rh}\left(t\right.\right.$-Bu-thixantphos- $\left.\left.\mathrm{P}, \mathrm{O}, \mathrm{P}^{\prime}\right) \mathrm{Cl}(\mathrm{O})\right](7.1 \%)$.

\section{[Rh(t-Bu-thixantphos- $\left.\left.\kappa P, O, P^{\prime}\right) \mathrm{Cl}(\mathrm{O})\right]$}

${ }^{31} \mathrm{P}$ NMR $\left(121 \mathrm{MHz}, \mathrm{CD}_{2} \mathrm{Cl}_{2}\right): \delta 40.9\left(\mathrm{~d}, J_{\mathrm{RhP}}=90.4 \mathrm{~Hz}\right)$.

After 10 days no starting material remained and the reaction mixture contained $\left[\mathrm{Rh}\left(t\right.\right.$-Bu-thixantphos- $\left.\left.\mathrm{P}, \mathrm{O}, \mathrm{P}^{\prime}\right) \mathrm{Cl}\left(\eta^{2}-\mathrm{O}_{2}\right)\right](66.7 \%),[(t \text {-Bu-thixantphos }) \mathrm{H}]^{+}(14.2 \%)$, $t$-Bu-thixantphos (5.3\%) and a new complex proposed as [Rh( $t$-Bu-thixantphos)$\left.\mathrm{Cl}_{2}(\mathrm{OH})\right]$.

\section{$\left[\mathrm{Rh}(t-\mathrm{Bu}\right.$-thixantphos $\left.) \mathrm{Cl}_{2}(\mathrm{OH})\right]$}

${ }^{31} \mathrm{P}$ NMR $\left(121 \mathrm{MHz}, \mathrm{CD}_{2} \mathrm{Cl}_{2}\right): \delta 74.1\left(\mathrm{~d}, J_{\mathrm{RhP}}=116.3 \mathrm{~Hz}\right)$.

\section{Reaction of $\left[\mathrm{Rh}\left(t-\mathrm{Bu}\right.\right.$-xantphos)Cl] with $\mathrm{ONMe}_{3}$}

Solid $\mathrm{ONMe}_{3}(0.007 \mathrm{~g}, 0.088 \mathrm{mmol})$ was added to a solution of [Rh(t-Bu-xantphos)Cl] $(0.056 \mathrm{~g}, 0.088 \mathrm{mmol})$ in $\mathrm{CD}_{2} \mathrm{Cl}_{2}$ at $-78{ }^{\circ} \mathrm{C}$. The NMR tube was sealed with a J. Young tap, and immediately transferred to an NMR spectrometer at $80^{\circ} \mathrm{C}$. The reaction contained unreacted [ $\mathrm{Rh}(t$-Bu-xantphos $\left.) \mathrm{Cl}\right](85.7 \%)$ and $[\mathrm{Rh}(t-$ Bu-xantphos $\left.) \mathrm{Cl}\left(\eta^{2}-\mathrm{O}_{2}\right)\right](14.3 \%)$. Upon warming to room temperature over 24 hours the reaction mixture contained [ $\mathrm{Rh}(t$-Bu-xantphos $) \mathrm{Cl}](25.4 \%),[\mathrm{Rh}(t-\mathrm{Bu}$ xantphos $\left.) \mathrm{Cl}\left(\eta^{2}-\mathrm{O}_{2}\right)\right](70.5 \%)$, and a new set of peaks proposed as [ $\mathrm{Rh}(t$-Bu-xantphos $) \mathrm{Cl}(\mathrm{O})](4.2 \%)$.

\section{[Rh(t-Bu-xantphos) $\mathrm{Cl}(\mathrm{O})]$}

${ }^{31} \mathrm{P} \mathrm{NMR}\left(121 \mathrm{MHz}, \mathrm{CD}_{2} \mathrm{Cl}_{2}\right): \delta 42.6\left(\mathrm{~d}, J_{\mathrm{RhP}}=90.4 \mathrm{~Hz}\right)$. 
After 14 days at room temperature the reaction mixture contained [ $\mathrm{Rh}(t$-Bu-xantphos) $\mathrm{Cl}(\mathrm{O})](42.8 \%),\left[\mathrm{Rh}(t-\mathrm{Bu}-\mathrm{xantphos}) \mathrm{Cl}\left(\eta^{2}-\mathrm{O}_{2}\right)\right](49.9 \%)$ and a new complex proposed as $\left[\mathrm{Rh}(t-\mathrm{Bu}-\mathrm{xantphos}) \mathrm{Cl}_{2}(\mathrm{OH})\right](7.3 \%)$.

[Rh(t-Bu-xantphos $\left.) \mathrm{Cl}_{2}(\mathrm{OH})\right]$

${ }^{31} \mathrm{P}$ NMR $\left(121 \mathrm{MHz}, \mathrm{CD}_{2} \mathrm{Cl}_{2}\right): \delta 75.1\left(\mathrm{~d}, J_{\mathrm{RhP}}=116.3 \mathrm{~Hz}\right)$

\subsection{Platinum Complexes}

\section{1:1 Reaction of Ph-thixantphos with $\left[\mathrm{Pt}(\mathrm{nb})_{3}\right]$}

A solution of Ph-thixantphos $(0.020 \mathrm{~g}, 0.035 \mathrm{mmol})$ in $\mathrm{C}_{6} \mathrm{D}_{6}$ was added to an NMR tube containing tris(norbornene)platinum $(0.017 \mathrm{~g}, 0.035 \mathrm{mmol})$. Immediate ${ }^{1} \mathrm{H}$ and ${ }^{31} \mathrm{P}$ NMR spectroscopy showed [Pt(nb)(Ph-thixantphos)] (39.6\%) and [Pt(Phthixantphos $\left.)_{2}\right](60.4 \%)$. No change was observed in the ratio over 7 days.

\section{[Pt(Ph-thixantphos)(nb)]}

${ }^{31} \mathrm{P}$ NMR $\left(121 \mathrm{MHz}, \mathrm{C}_{6} \mathrm{D}_{6}\right): \delta 20.0\left(\mathrm{~s}, J_{\mathrm{PtP}}=3470 \mathrm{~Hz}\right)$.

\section{$\left[\mathrm{Pt}(\mathrm{Ph}-\text { thixantphos })_{2}\right]$}

A solution of Ph-thixantphos $(0.200 \mathrm{~g}, 0.35 \mathrm{mmol})$ in toluene $(5 \mathrm{~mL})$ was added to tris(norbornene)platinum $(0.084 \mathrm{~g}, 0.18 \mathrm{mmol})$ in toluene $(5 \mathrm{~mL})$. The reaction was stirred for one hour before removing the solvent under reduced pressure. The yellow powder was purified by recrystallisation from a mixture of toluene and diethyl ether yielding the title compound as a yellow microcrystalline solid ( 0.066 $\mathrm{g}, 14 \%$ ). Single X-ray quality crystals were grown by inwards diffusion of diethyl ether into a dichloromethane solution of the complex over three days.

Data at $-80{ }^{\circ} \mathrm{C}:{ }^{31} \mathrm{P}$ NMR $\left(121 \mathrm{MHz}, \mathrm{CD}_{2} \mathrm{Cl}_{2}\right): \delta-2.4\left(\mathrm{t}, J=55.0, J_{\mathrm{PtP}}=3976 \mathrm{~Hz},\right)$, $-6.2\left(\mathrm{t}, J=55.0, J_{\mathrm{PtP}}=3864 \mathrm{~Hz},\right) .{ }^{1} \mathrm{H}$ NMR $\left(300 \mathrm{MHz}, \mathrm{CD}_{2} \mathrm{Cl}_{2}\right): \delta 5.7-7.4(\mathrm{~m}, \mathrm{Ar})$. HRMS calcd for $\mathrm{C}_{72} \mathrm{H}_{52} \mathrm{O}_{2} \mathrm{P}_{4} \mathrm{~S}_{2} \mathrm{Pt}[\mathrm{M}]^{+} \mathrm{m} / \mathrm{z}=1330.2002$; found $=1330.1986$. 


\section{Reaction of Ph-thixantphos with $\left[\mathrm{Pt}\left(\mathrm{C}_{2} \mathrm{H}_{4}\right)_{3}\right]$}

A solution of Ph-thixantphos $(0.017 \mathrm{~g}, 0.029 \mathrm{mmol})$ in $\mathrm{C}_{6} \mathrm{D}_{6}$ was added to an NMR tube containing tris(ethene)platinum $(0.008 \mathrm{~g}, 0.029 \mathrm{mmol})$. The reaction became orange after 10 minutes. By ${ }^{31} \mathrm{P} \mathrm{NMR}$ analysis $\left[\mathrm{Pt}(\mathrm{Ph} \text {-thixantphos })_{2}\right.$ ] was the major product $(93 \%)$ with a small amount of $\left[\mathrm{Pt}\left(\mathrm{C}_{2} \mathrm{H}_{4}\right)(\mathrm{Ph}\right.$-thixantphos) $](7 \%)$ as a minor component.

$\left[\mathrm{Pt}\left(\mathrm{C}_{2} \mathrm{H}_{4}\right)(\mathrm{Ph}\right.$-thixantphos)]

${ }^{31} \mathrm{P}$ NMR (121 MHz, $\left.\mathrm{C}_{6} \mathrm{D}_{6}\right): \delta 20.9\left(\mathrm{~s}, J_{\mathrm{PtP}}=3659 \mathrm{~Hz}\right) .{ }^{1} \mathrm{H} \mathrm{NMR}\left(300 \mathrm{MHz}, \mathrm{C}_{6} \mathrm{D}_{6}\right)$ : $\delta 2.29\left(\mathrm{~s}, J_{\mathrm{PtH}}=61.0 \mathrm{~Hz}, \mathrm{C}=\mathrm{C}-\mathrm{H}\right)$.

\section{Reaction of $t$-Bu-thixantphos with $\left[\operatorname{Pt}(\mathrm{nb})_{3}\right]$}

In a glovebox, $t$-Bu-thixantphos $(0.057 \mathrm{~g}, 0.110 \mathrm{mmol})$ in $\mathrm{C}_{6} \mathrm{D}_{6}(0.5 \mathrm{~mL})$ was added to a J. Young's tap NMR tube containing $\left[\mathrm{Pt}(\mathrm{nb})_{3}\right](0.053 \mathrm{~g}, 0.110 \mathrm{mmol})$. The NMR tube was closed under a nitrogen atmosphere and the reaction was heated to $60{ }^{\circ} \mathrm{C}$ until the reaction was deemed complete by the absence of $t$-Bu-thixantphos from the ${ }^{31} \mathrm{P}$ and ${ }^{1} \mathrm{H} \mathrm{NMR}$ spectra (4-6 days). At this stage, ${ }^{1} \mathrm{H},{ }^{13} \mathrm{C}$ and ${ }^{31} \mathrm{P}$ NMR analysis was carried out to characterise [ $\mathrm{Pt}(t$-Bu-thixantphos)(nb)] complex $(39.2 \%)$. The solution was filtered through a plug of diatomaceous earth washing through with toluene $(2 \times 1 \mathrm{~mL})$. The solvent was removed under reduced pressure for one hour leaving [ $\mathrm{Pt}(t-\mathrm{Bu}$-thixantphos)] as a brown expanded oil (0.073 g, 93\%).

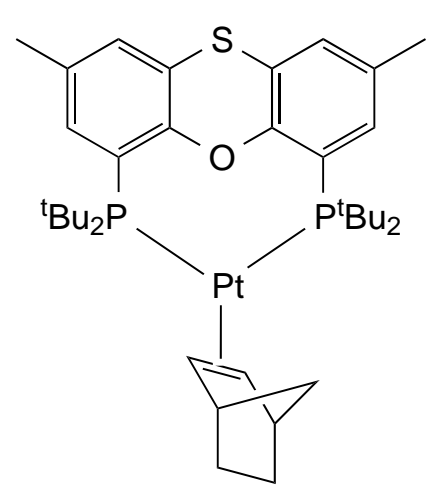


${ }^{31} \mathrm{P}$ NMR $\left(121 \mathrm{MHz}, \mathrm{C}_{6} \mathrm{D}_{6}\right): \delta 55.6\left(\mathrm{~s}, J_{\mathrm{PtP}}=3612 \mathrm{~Hz}\right){ }^{1} \mathrm{H} \mathrm{NMR}\left(600 \mathrm{MHz}, \mathrm{C}_{6} \mathrm{D}_{6}\right): \delta$ 7.61 (s, PC(Ar)CH), 6.95 (s, SCCH), 3.12 (s, nb CH), 2.37 (bs, ${ }^{1} J_{\mathrm{PtH}}=67.8 \mathrm{~Hz}, \mathrm{nb}$ $=\mathrm{CH}), 1.96\left(\mathrm{~s}, \mathrm{C}(\mathrm{Ar}) \mathrm{CH}_{3}\right), 1.93$ (obscured, assigned by COSY correlation, $2 \mathrm{H}, \mathrm{nb}$ $\left.\mathrm{CH}_{2}\right), 1.42-1.56\left(\mathrm{~m}, \mathrm{PC}\left(\mathrm{CH}_{3}\right)_{3}\right), 1.32$ (obscured, assigned by COSY correlation, $3 \mathrm{H}$, nb $\mathrm{CH}_{2}$, bridge $\left.\mathrm{CH}_{2}\right), 0.62\left(\mathrm{~d}, 15.3 \mathrm{~Hz}, 1 \mathrm{H}\right.$, nb bridge $\left.\mathrm{CH}_{2}\right) .{ }^{13} \mathrm{C} \mathrm{NMR}(150 \mathrm{MHz}$, $\left.\mathrm{C}_{6} \mathrm{D}_{6}\right): \delta 158.9\left(\mathrm{vt}, J_{\mathrm{PC}}=9.8 \mathrm{~Hz}, \mathrm{PCCO}\right), 135.4\left(\mathrm{~s},{ }^{2} J_{\mathrm{PtC}}=26.6 \mathrm{~Hz}, \mathrm{PC}(\mathrm{Ar}) C \mathrm{H}\right)$, 130.9 (bs, $\left.C(\mathrm{Ar}) \mathrm{CH}_{3}\right), 130.7$ (bs, SCCH), 127.6 (vt, $\left.J_{\mathrm{PC}}=5.8 \mathrm{~Hz}, \mathrm{SCCH}\right), 125.9$ (bs, $\mathrm{PC}(\mathrm{Ar})), 51.9\left(\mathrm{bs},{ }^{1} \mathrm{JtC}_{\mathrm{PtC}}=343.9 \mathrm{~Hz}, \mathrm{nb}=\mathrm{CH}\right), 44.9(\mathrm{~s}, \mathrm{nb} \mathrm{CH}), 38.5\left(\mathrm{~m}, \mathrm{PC}\left(\mathrm{CH}_{3}\right)_{3}\right)$, 38.0-38.5 (obscured, assigned by HSQC correlation, nb bridge $\mathrm{CH}_{2}$ ), 31.7 (bs, $\left.\mathrm{PC}\left(\mathrm{CH}_{3}\right)_{3}\right), 31.4\left(\mathrm{bs},{ }^{3} J_{\mathrm{PtC}}=61.4 \mathrm{~Hz}, \mathrm{nb} \mathrm{CH}_{2}\right), 20.7\left(\mathrm{~s}, \mathrm{C}(\mathrm{Ar}) \mathrm{CH}_{3}\right)$.

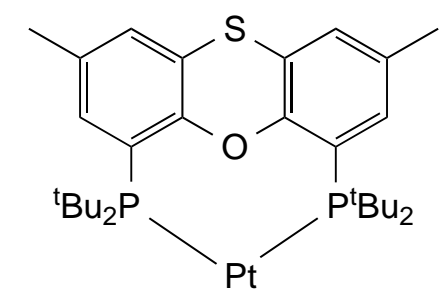

${ }^{31} \mathrm{P}$ NMR $\left(121 \mathrm{MHz}, \mathrm{C}_{6} \mathrm{D}_{6}\right): \delta 78.6\left(\mathrm{~s}, J_{\mathrm{PtP}}=4809.5 \mathrm{~Hz}\right){ }^{1} \mathrm{H} \mathrm{NMR}(600 \mathrm{MHz}$, $\left.\mathrm{C}_{6} \mathrm{D}_{6}\right): \delta 7.32\left(\mathrm{~d}, J_{\mathrm{PH}}=1.8 \mathrm{~Hz}, \mathrm{PC}(\mathrm{Ar}) \mathrm{CH}\right), 6.87(\mathrm{~s}, \mathrm{SCCH}), 1.95\left(\mathrm{~s}, \mathrm{C}(\mathrm{Ar}) \mathrm{CH}_{3}\right)$, $1.52\left(\mathrm{vt}, J_{\mathrm{PH}}=13.8 \mathrm{~Hz}, \mathrm{PC}\left(\mathrm{CH}_{3}\right)_{3}\right) .{ }^{13} \mathrm{C} \mathrm{NMR}\left(150 \mathrm{MHz}, \mathrm{C}_{6} \mathrm{D}_{6}\right): \delta 155.9(\mathrm{vt}$, $\left.J_{\mathrm{PC}}=10.4 \mathrm{~Hz}, \mathrm{PCCO}\right), 133.3(\mathrm{~s}, \mathrm{PC}(\mathrm{Ar}) \mathrm{CH}), 132.1\left(\mathrm{vt}, J_{\mathrm{PC}}=5.2 \mathrm{~Hz}, C(\mathrm{Ar}) \mathrm{CH}_{3}\right)$, $128.8(\mathrm{~s}, \mathrm{SCCH}), 126.6\left(\mathrm{vt}, J_{\mathrm{PC}}=28.9 \mathrm{~Hz}, \mathrm{PC}(\mathrm{Ar})\right), 126.1\left(\mathrm{vt}, J_{\mathrm{PC}}=5.8 \mathrm{~Hz}, \mathrm{SCCH}\right)$, $37.8\left(\mathrm{vt}, J_{\mathrm{PC}}=15 \mathrm{~Hz}, \mathrm{PC}\left(\mathrm{CH}_{3}\right)_{3}\right), 31.7\left(\mathrm{vt}, J_{\mathrm{PC}}=10.4 \mathrm{~Hz}, \mathrm{PC}\left(\mathrm{CH}_{3}\right)_{3}\right), 20.5(\mathrm{~s}$, $\left.\mathrm{C}(\mathrm{Ar}) \mathrm{CH}_{3}\right)$. HRMS calcd for $\mathrm{C}_{30} \mathrm{H}_{47} \mathrm{OP}_{2} \mathrm{PtS}\left[\mathrm{M}^{+} \mathrm{H}\right]^{+} \mathrm{m} / \mathrm{z}=711.2438$; found = 711.2450 .

\section{Reaction of $t$-Bu-sixantphos with $\left[\mathrm{Pt}(\mathrm{nb})_{3}\right]$}

A solution of $t$-Bu-sixantphos $(0.19 \mathrm{~g}, 0.37 \mathrm{mmol})$ in $\mathrm{C}_{6} \mathrm{D}_{6}(0.5 \mathrm{~mL})$ was added to $\left[\mathrm{Pt}(\mathrm{nb})_{3}\right](0.018 \mathrm{~g}, 0.37 \mathrm{mmol})$ in a J. Young's tap NMR tube. After 24 hours at $60{ }^{\circ} \mathrm{C}$ the ${ }^{31} \mathrm{P}$ NMR spectrum showed a mixture of unreacted $t$-Bu-sixantphos (75.0\%), [Pt(t-Bu-sixantphos)] (15.8\%), and [Pt(t-Bu-sixantphos)(nb)] (9.2\%). After five days at $60{ }^{\circ} \mathrm{C}$ the reaction mixture contained unreacted $t$-Bu-sixantphos (11.1\%), [Pt(t-Bu-sixantphos)] (32.5\%), [Pt(t-Bu-sixantphos)(nb)] (24.1\%) and an unidentified $\mathrm{Pt}(\mathrm{II})$ species $(18.2 \%)$. 
[Pt(t-Bu-sixantphos)]

${ }^{31} \mathrm{P}$ NMR $\left(121 \mathrm{MHz}, \mathrm{C}_{6} \mathrm{D}_{6}\right): \delta 79.5\left(\mathrm{~s}, J_{\mathrm{PtP}}=4826.6 \mathrm{~Hz}\right)$

[Pt(t-Bu-sixantphos)(nb)]

${ }^{31} \mathrm{P}$ NMR $\left(121 \mathrm{MHz}, \mathrm{C}_{6} \mathrm{D}_{6}\right): \delta 59.3\left(\mathrm{~s}, J_{\mathrm{PtP}}=3571.8 \mathrm{~Hz}\right)$

\section{$\operatorname{Pt}(\mathrm{II}) \mathrm{t}$-Bu-sixantphos species}

${ }^{31} \mathrm{P}$ NMR $\left(121 \mathrm{MHz}, \mathrm{C}_{6} \mathrm{D}_{6}\right): \delta 34.7\left(\mathrm{~s}, J_{\mathrm{PtP}}=2676.9 \mathrm{~Hz}\right)$

\section{$[\operatorname{Pt}(t-B u-x a n t p h o s) H] X$}

A solution of $t$-Bu-xantphos $(0.016 \mathrm{~g}, 0.032 \mathrm{mmol})$ in $\mathrm{C}_{6} \mathrm{D}_{6}(0.5 \mathrm{~mL})$ was added to tris-(norbornene)-platinum $(0.015 \mathrm{~g}, 0.032 \mathrm{mmol})$ in an Young's tap NMR tube. The tube was closed under argon and heated to $60{ }^{\circ} \mathrm{C}$ overnight. After 24 hours no free ligand remained and the major product is proposed as $[\mathrm{Pt}(t-\mathrm{Bu}$-xantphos$\left.\left.\kappa P, O, P^{\prime}\right) \mathrm{H}\right] X$. After 7 days at room temperature small yellow crystals had formed. The solution was decanted and the product was isolated $(0.020 \mathrm{~g})$.

${ }^{31} \mathrm{P}$ NMR (121 MHz, $\left.\mathrm{C}_{6} \mathrm{D}_{6}\right): \delta 46.7\left(\mathrm{~s}, J_{\mathrm{PtP}}=3246 \mathrm{~Hz}\right){ }^{1} \mathrm{H}$ NMR $(600 \mathrm{MHz}$, $\left.\mathrm{C}_{6} \mathrm{D}_{6}\right): \delta 7.43(\mathrm{~m}, \mathrm{PC}(\mathrm{Ar}) \mathrm{CH}), 7.11(\mathrm{~d}, J=7.5 \mathrm{~Hz}, \mathrm{C}($ bridge $) \mathrm{CCH}), 6.84(\mathrm{t}$, $J=7.6 \mathrm{~Hz}, \mathrm{PCCCH}), 1.7$ (bs, C(bridge) $\left.\left(\mathrm{CH}_{3}\right)_{2}\right), 1.5$ (bs, $\left.\mathrm{PC}\left(\mathrm{CH}_{3}\right)_{3}\right),-18.49(\mathrm{t}, 13.1$ $\left.\mathrm{Hz},{ }^{1} J_{\mathrm{PtH}}=1107 \mathrm{~Hz}, \mathrm{Pt}-\mathrm{H}\right) .{ }^{13} \mathrm{C} \mathrm{NMR}\left(150 \mathrm{MHz}, \mathrm{C}_{6} \mathrm{D}_{6}\right): \delta 158.6(\mathrm{vt}, J=8.0 \mathrm{~Hz}$, PCCO), 138.4 (vt, $J=4.1 \mathrm{~Hz}, \mathrm{C}($ bridge)CCH), 132.1 (s, PC(Ar)CH), 124.8 (vtd, $J=39.3,2.9 \mathrm{~Hz}, \mathrm{PC}(\mathrm{Ar})), 125.8$ (s, C(bridge)CCH), 121.8 (vt, $J=5.7 \mathrm{~Hz}, \mathrm{PCCCH})$, 38.0 (vt, $\left.J=17.5 \mathrm{~Hz}, \mathrm{PC}\left(\mathrm{CH}_{3}\right)_{3}\right), 36.7$ (s, $\mathrm{C}$ (bridge)), 30.7 (bs, PC $\left.\left(\mathrm{CH}_{3}\right)_{3}\right)$. HRMS calcd for $\mathrm{C}_{31} \mathrm{H}_{49} \mathrm{OP}_{2} \mathrm{Pt}[\mathrm{M}-\mathrm{X}]^{+} \mathrm{m} / \mathrm{z}=689.2858$; found $=689.2864$.

\section{Reaction of $t$-Bu-thixantphos with $\left[\mathrm{Pt}(\operatorname{cod})_{2}\right]$}

A solution of $t$-Bu-thixantphos $(0.026 \mathrm{~g}, 0.050 \mathrm{mmol})$ in $\mathrm{C}_{6} \mathrm{D}_{6}(0.3 \mathrm{~mL})$ was added to a solution of bis-(1,5-cyclooctadiene)platinum $(0.020 \mathrm{~g}, 0.050 \mathrm{mmol})$ in $\mathrm{C}_{6} \mathrm{D}_{6}$ $(0.3 \mathrm{~mL})$. The NMR tube was closed with a septum and the reaction was followed 
by ${ }^{1} \mathrm{H}$ and ${ }^{31} \mathrm{P}$ NMR spectroscopy. After $48 \mathrm{hrs}$ at room temperature the reaction had become black and contained $72 \%$ unreacted $t$-Bu-thixantphos with the remainder being [ $\mathrm{Pt}\left(t-\mathrm{Bu}\right.$-thixantphos)]. The reaction was heated to $40{ }^{\circ} \mathrm{C}$ for 24 hours, then $60{ }^{\circ} \mathrm{C}$ for 24 hours, but no further reaction was observed.

\section{Reaction of $t$-Bu-sixantphos with $\left[\mathrm{Pt}\left(\mathrm{C}_{2} \mathrm{H}_{4}\right)_{3}\right]$}

$\mathrm{C}_{6} \mathrm{D}_{6}(0.5 \mathrm{~mL})$ which had been previously dried and degassed then stored under $\mathrm{Ar}$, was sparged by bubbling ethene through the liquid for $10 \mathrm{mins}$, then stirring vigorously under an ethene atmosphere for a further $10 \mathrm{mins}$. $\left[\mathrm{Pt}(\operatorname{cod})_{2}\right](0.026$ $\mathrm{g}$, $0.063 \mathrm{mmol}$ ) was placed under ethene and $0.03 \mathrm{~mL}$ of the $\mathrm{C}_{6} \mathrm{D}_{6}$ was added. The solution was stirred vigorously for $30 \mathrm{mins}$ to ensure complete conversion to $\left[\mathrm{Pt}\left(\mathrm{C}_{2} \mathrm{H}_{4}\right)_{3}\right]$. t-Bu-Sixantphos $(0.032 \mathrm{~g}, 0.063 \mathrm{mmol})$ was placed in a Young's tap NMR tube under an ethene atmosphere and dissolved in the remaining $\mathrm{C}_{6} \mathrm{D}_{6}$. The $\left[\operatorname{Pt}\left(\mathrm{C}_{2} \mathrm{H}_{4}\right)_{3}\right]$ solution was added to the NMR tube, which was closed under ethene and shaken to ensure mixing of the solutions. After four hours at room temperature $\left[\mathrm{Pt}(t-\mathrm{Bu}\right.$-sixantphos $\left.)\left(\mathrm{C}_{2} \mathrm{H}_{4}\right)\right](11.1 \%)$ had been produced. The reaction did not proceed further.

${ }^{31} \mathrm{P}$ NMR $\left(121 \mathrm{MHz}, \mathrm{C}_{6} \mathrm{D}_{6}\right): \delta 53.7\left(\mathrm{~s}, J_{\mathrm{PtP}}=3499 \mathrm{~Hz}\right)$.

\section{[Pt $(t$-Bu-thixantphos $\left.)\left(\mathrm{C}_{2} \mathrm{H}_{4}\right)\right]$}

$\mathrm{C}_{6} \mathrm{D}_{6}(0.5 \mathrm{~mL})$ which had been previously dried and degassed then stored under Ar, was sparged by bubbling ethene through the liquid for 10 mins, then stirring vigorously under an ethene atmosphere for a further 10 mins. Bis-(1,5cyclooctadiene)platinum $(0.032 \mathrm{~g}, 0.078 \mathrm{mmol})$ was placed under ethene and 0.03 $\mathrm{mL}$ of the $\mathrm{C}_{6} \mathrm{D}_{6}$ was added. The solution was stirred vigorously for 30 mins to ensure complete conversion to $\left[\mathrm{Pt}\left(\mathrm{C}_{2} \mathrm{H}_{4}\right)_{3}\right]$. $t$-Bu-Thixantphos $(0.040 \mathrm{~g}, 0.077 \mathrm{mmol})$ was placed in a Young's tap NMR tube under an ethene atmosphere and dissolved in the remaining $\mathrm{C}_{6} \mathrm{D}_{6}$. The $\left[\mathrm{Pt}\left(\mathrm{C}_{2} \mathrm{H}_{4}\right)_{3}\right]$ solution was added to the NMR tube, which was closed under ethene and shaken to ensure mixing of the solutions. After 48 hours at room temperature the only product was [ $\mathrm{Pt}(t-\mathrm{Bu}$-thixantphos) $\left.\left(\mathrm{C}_{2} \mathrm{H}_{4}\right)\right]$ (100 \% by NMR). 
${ }^{31} \mathrm{P}$ NMR (121 MHz, $\left.\mathrm{C}_{6} \mathrm{D}_{6}\right): \delta 55.7\left(\mathrm{~s}, J_{\mathrm{PtP}}=3899 \mathrm{~Hz}\right){ }^{1} \mathrm{H} \mathrm{NMR}\left(600 \mathrm{MHz}, \mathrm{C}_{6} \mathrm{D}_{6}\right)$ : $\delta 7.63$ (s, PC(Ar)CH), $6.96(\mathrm{~s}, \mathrm{SCCH}), 2.50\left(\mathrm{bs},{ }^{2} J_{\mathrm{PtH}}=59.5 \mathrm{~Hz}, \mathrm{C}=\mathrm{CH}_{2}\right), 1.97$ (s, $\left.\mathrm{C}(\mathrm{Ar}) \mathrm{CH}_{3}\right), 1.38-1.40\left(\mathrm{~m}, \mathrm{PC}\left(\mathrm{CH}_{3}\right)_{3}\right) .{ }^{13} \mathrm{C} \mathrm{NMR}\left(150 \mathrm{MHz}, \mathrm{C}_{6} \mathrm{D}_{6}\right): \delta 158.7$ (bs, PCCO), 135.3 (s, ${ }^{4} J_{\mathrm{PtC}}=29.5 \mathrm{~Hz}, \mathrm{PC}(\mathrm{Ar}) \mathrm{CH}$ ), 131.0 (bs, $\left.\mathrm{C}(\mathrm{Ar}) \mathrm{CH}_{3}\right), 130.5$ (bs, $\mathrm{SCCH}), 128.5\left(\mathrm{vt}, J_{\mathrm{PC}}=5.8 \mathrm{~Hz}, \mathrm{SCCH}\right), 125.7$ (bs, PC(Ar)), $38.7\left(\mathrm{~m}, \mathrm{PC}\left(\mathrm{CH}_{3}\right)_{3}\right)$, $34.2\left(\mathrm{bs},{ }^{1} J_{\mathrm{PtC}}=223.2 \mathrm{~Hz}, \mathrm{C}=\mathrm{C}\right) 31.6\left(\mathrm{bs}, \mathrm{PC}\left(\mathrm{CH}_{3}\right)_{3}\right), 20.7\left(\mathrm{~s}, \mathrm{C}(\mathrm{Ar}) \mathrm{CH}_{3}\right)$.

\section{Reaction of $t$-Bu-xantphos with $\left[\mathrm{Pt}\left(\mathrm{C}_{2} \mathrm{H}_{4}\right)_{3}\right]$}

$\mathrm{C}_{6} \mathrm{D}_{6}(0.5 \mathrm{~mL})$ which had been previously dried and degassed then stored under Ar, was sparged by bubbling ethene through the liquid for $10 \mathrm{mins}$, then stirring vigorously under an ethene atmosphere for a further $10 \mathrm{mins}$. [ $\left.\mathrm{Pt}(\mathrm{cod})_{2}\right](0.015$ $\mathrm{g}, 0.036 \mathrm{mmol}$ ) was placed under ethene and $0.03 \mathrm{~mL}$ of the $\mathrm{C}_{6} \mathrm{D}_{6}$ was added. The solution was stirred vigorously for 30 mins to ensure complete conversion to $\left[\mathrm{Pt}\left(\mathrm{C}_{2} \mathrm{H}_{4}\right)_{3}\right]$. $t$-Bu-Xantphos $(0.018 \mathrm{~g}, 0.036 \mathrm{mmol})$ was placed in a Young's tap NMR tube under an ethene atmosphere and dissolved in the remaining $\mathrm{C}_{6} \mathrm{D}_{6}$. The $\left[\mathrm{Pt}\left(\mathrm{C}_{2} \mathrm{H}_{4}\right)_{3}\right]$ solution was added to the NMR tube, which was closed under ethene and shaken to ensure mixing of the solutions. Both $\left[\mathrm{Pt}(t\right.$-Bu-xantphos $\left.)\left(\mathrm{C}_{2} \mathrm{H}_{4}\right)\right]$ $(41.7 \%)$ and $[\mathrm{Pt}(t-\mathrm{Bu}-\mathrm{xantphos}) \mathrm{H}] \mathrm{X}(8.6 \%))$ were evident after four hours at room temperature. After five days $[\mathrm{Pt}(t-\mathrm{Bu}$-xantphos $) \mathrm{H}] \mathrm{X}$ was the only phosphorus containing compound in solution.

\section{$\left[\operatorname{Pt}(t-B u-x a n t p h o s)\left(\mathrm{C}_{2} \mathrm{H}_{4}\right)\right]$}

${ }^{31} \mathrm{P}$ NMR (121 MHz, $\left.\mathrm{C}_{6} \mathrm{D}_{6}\right): \delta 53.4\left(\mathrm{~s}, J_{\mathrm{PtP}}=3878 \mathrm{~Hz}\right){ }^{1} \mathrm{H}$ NMR $\left(600 \mathrm{MHz}, \mathrm{C}_{6} \mathrm{D}_{6}\right): \delta$ $7.79(\mathrm{~d}, J=6.8 \mathrm{~Hz}, \mathrm{PC}(\mathrm{Ar}) \mathrm{CH}), 7.09(\mathrm{dd}, J=7.6,1.1 \mathrm{~Hz}, \mathrm{C}($ bridge $) C C H), 6.87$ (t, $J=7.7 \mathrm{~Hz}, \mathrm{PCCCH}), 2.52\left(\mathrm{bs}^{2} \mathrm{~J}_{\mathrm{PtH}}=58.0 \mathrm{~Hz}, \mathrm{C}=\mathrm{CH}_{2}\right), 1.43\left(\mathrm{~s}, \mathrm{C}(\right.$ bridge $\left.)\left(\mathrm{CH}_{3}\right)_{2}\right)$, $1.42\left(\mathrm{~d}, J=13.0 \mathrm{~Hz}, \mathrm{PC}\left(\mathrm{CH}_{3}\right)_{3}\right) .{ }^{13} \mathrm{C} \mathrm{NMR}\left(150 \mathrm{MHz}, \mathrm{C}_{6} \mathrm{D}_{6}\right): \delta 161.0(\mathrm{~m}$, PCCO), 138.4 (s, C(bridge)CCH), $133.6\left(\mathrm{~s},{ }^{4} J_{\mathrm{PtC}}=28.1 \mathrm{~Hz}, \mathrm{PC}(\mathrm{Ar}) \mathrm{CH}\right), 124.6$ (s, $\mathrm{C}($ bridge $) \mathrm{CCH}), 124.1$ (m, PC(Ar)), $121.2(\mathrm{~s}, \mathrm{PCCCH}), 38.4\left(\mathrm{vt}, J=18.5,{ }^{2} J_{\mathrm{PtC}}=\right.$ $\left.34.1 \mathrm{~Hz}, \mathrm{PC}\left(\mathrm{CH}_{3}\right)_{3}\right), 37.4$ (s, $\mathrm{C}$ (bridge)), $31.6\left(\mathrm{vt}, J=8.1,{ }^{3} J_{\mathrm{PtC}}=17.3 \mathrm{~Hz}, \mathrm{PC}\left(\mathrm{CH}_{3}\right)_{3}\right)$ 25.6 (s, $\mathrm{C}($ bridge $\left.)\left(\mathrm{CH}_{3}\right)_{2}\right)$. 


\section{$\left[\mathrm{Pt}(\boldsymbol{t}\right.$-Bu-thixantphos $\left.)\left(\eta^{2}-\mathrm{O}_{2}\right)\right]$}

A solution of $t$-Bu-thixantphos $(0.117 \mathrm{~g}, 0.23 \mathrm{mmol})$ in toluene $(1 \mathrm{~mL})$ was added to a Schlenk tube containing tris-(norbornene)platinum $(0.108 \mathrm{~g}, 0.023 \mathrm{mmol})$ in toluene $(3 \mathrm{~mL})$. The reaction was stirred at $40{ }^{\circ} \mathrm{C}$ for 3 days. The solvent was reduced in vacuo and the reaction mixture was placed under air. The title compound was isolated by cooling the reaction mixture for two weeks at $-20{ }^{\circ} \mathrm{C}$ forming $\left[\mathrm{Pt}(t\right.$-Bu-thixantphos $\left.)\left(\eta^{2}-\mathrm{O}_{2}\right)\right]$ as a pale peach solid $(0.109 \mathrm{~g}, 65 \%)$. Xray quality crystals were grown by slow diffusion of air into a $\mathrm{C}_{6} \mathrm{D}_{6}$ solution of [Pt(t-Bu-thixantphos)].

${ }^{31} \mathrm{P}$ NMR $\left(121 \mathrm{MHz}, \mathrm{CD}_{2} \mathrm{Cl}_{2}\right): \delta 38.4\left(\mathrm{~s}, J_{\mathrm{PtP}}=4488 \mathrm{~Hz}\right){ }^{1} \mathrm{H}$ NMR $(600 \mathrm{MHz}$, $\left.\mathrm{CD}_{2} \mathrm{Cl}_{2}\right): \delta 7.59(\mathrm{dd}, J=5.9,1.0 \mathrm{~Hz}, \mathrm{a}), 7.32(\mathrm{~s}, \mathrm{c}), 2.34(\mathrm{~s}, \mathrm{~g}), 1.43\left(\mathrm{~d}, J_{\mathrm{PH}}=14.4 \mathrm{~Hz}\right.$, $\left.\mathrm{C}(\mathrm{Ar}) \mathrm{CH}_{3}\right) .{ }^{13} \mathrm{C} \mathrm{NMR}\left(125 \mathrm{MHz}, \mathrm{CD}_{2} \mathrm{Cl}_{2}\right): \delta 156.6$ (bs, $\left.\mathrm{CO}\right), 133.8\left(\mathrm{~s},{ }^{2} \mathrm{~J}_{\mathrm{PtC}}=37.9 \mathrm{~Hz}\right.$, $\mathrm{PC}(\mathrm{Ar}) \mathrm{CH}), 133.1\left(\mathrm{~d}, J_{\mathrm{PC}}=5.3 \mathrm{~Hz}, \mathrm{C}(\mathrm{Ar}) \mathrm{CH}_{3}\right), 131.7$ (bs, $\left.\operatorname{SCCC}\left(\mathrm{CH}_{3}\right)\right), 128.5$ (s, CS), $119.3\left(\mathrm{~d}, J_{\mathrm{PC}}=27.8 \mathrm{~Hz}, \mathrm{PC}(\mathrm{Ar})\right), 39.3\left(\mathrm{~d}, J_{\mathrm{PC}}=23.5 \mathrm{~Hz}, \mathrm{PCCH}_{3}\right), 31.2$ $\left(\mathrm{d}, J_{\mathrm{PC}}=5.3 \mathrm{~Hz}, \mathrm{PCCH}_{3}\right), 21.2\left(\mathrm{~s}, \mathrm{C}(\mathrm{Ar}) \mathrm{CH}_{3}\right)$. HRMS calcd for $\mathrm{C}_{30} \mathrm{H}_{47} \mathrm{O}_{3} \mathrm{P}_{2} \mathrm{PtS}$ $[\mathrm{M}+\mathrm{H}]^{+} \mathrm{m} / \mathrm{z}=743.2326$; found $=743.2291$.

\section{Reaction of $\left[\mathrm{Pt}(t-\mathrm{Bu}\right.$-thixantphos $\left.)\left(\mathrm{C}_{2} \mathrm{H}_{4}\right)\right]$ with Air}

Air was bubbled through a solution of $\left[\mathrm{Pt}(t\right.$-Bu-thixantphos $\left.)\left(\mathrm{C}_{2} \mathrm{H}_{4}\right)\right](0.020 \mathrm{~g}$, $0.027 \mathrm{mmol})$ in $\mathrm{C}_{6} \mathrm{D}_{6}(0.4 \mathrm{~mL})$ for a total of $10 \mathrm{mins}$. The sample was sealed under air with a septum and analysed by NMR spectroscopy immediately. [ $\mathrm{Pt}(t-\mathrm{Bu}-$ thixantphos $\left.)\left(\eta^{2}-\mathrm{O}_{2}\right)\right]$ was produced in quantitative yield by NMR spectroscopy.

\section{Reaction of $\left[\mathrm{Pt}(t-\mathrm{Bu}\right.$-thixantphos $\left.)\left(\mathrm{C}_{2} \mathrm{H}_{4}\right)\right]$ with argon}

Argon was bubbled through a solution of $\left[\mathrm{Pt}(t\right.$-Bu-thixantphos $\left.)\left(\mathrm{C}_{2} \mathrm{H}_{4}\right)\right](0.020 \mathrm{~g}$, $0.027 \mathrm{mmol})$ in $\mathrm{C}_{6} \mathrm{D}_{6}(0.4 \mathrm{~mL})$ for 10 mins. The sample was sealed under argon and NMR analysis showed slight conversion to [Pt(t-Bu-thixantphos)]. 


\section{Reaction of $\left[\mathrm{Pt}(t-\mathrm{Bu}\right.$-thixantphos $\left.)\left(\eta^{2}-\mathrm{O}_{2}\right)\right]$ with $\mathrm{CO}$}

Carbon monoxide was bubbled through a solution of [Pt $(t$-Bu-thixantphos $)\left(\eta^{2}-\right.$ $\left.\mathrm{O}_{2}\right)$ ] $(0.032 \mathrm{~g}, 0.043 \mathrm{mmol})$ in $\mathrm{CD}_{2} \mathrm{Cl}_{2}(0.4 \mathrm{~mL})$ for $10 \mathrm{mins}$. The reaction was followed by NMR spectroscopy and no further changes were observed after 7 days. The solution was passed through an alumina plug (washing with $3 \times 1 \mathrm{~mL}$ $\mathrm{CH}_{2} \mathrm{Cl}_{2}$ ) and the solvent removed in vacuo to give the metallated complex shown below as a brown solid $(0.010 \mathrm{~g}, 32 \%)$.

Reaction with ${ }^{13} \mathrm{CO}$ : $\left[\mathrm{Pt}(t\right.$-Bu-thixantphos $\left.)\left(\eta^{2}-\mathrm{O}_{2}\right)\right](0.030 \mathrm{~g}, 0.040 \mathrm{mmol})$ was dissolved in $\mathrm{CD}_{2} \mathrm{Cl}_{2}$ and transferred to an NMR tube and sealed with a septum. The sample was frozen in liquid nitrogen and attached by cannula to a solid potassium hydroxide trap, which in turn was attached to a sample vial containing ${ }^{13} \mathrm{C}$ sodium formate. The system was placed under vacuum, using syringes attached to both the NMR tube and the sample vial. The system was closed under vacuum and the NMR tube was transferred to a dry ice/acetone bath and allowed to melt. The vacuum syringe on the NMR tube was replaced with a gas-tight syringe to collect any excess carbon monoxide that may be produced. Conc. sulfuric acid $(1 \mathrm{~mL})$ was added dropwise into the sample vial containing sodium formate producing rapid bubbling. The set-up was left until the bubbling had mostly abated (approx. 10 mins). All syringes and the cannula were removed from the sample vial and the septum was secured with Parafilm. No unexpected differences were observed in the NMR spectra.

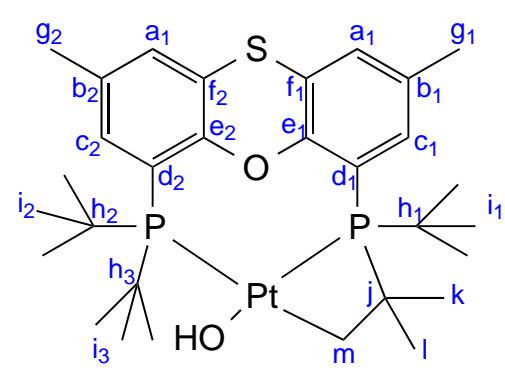

${ }^{31} \mathrm{P}$ NMR $\left(121 \mathrm{MHz}, \mathrm{CD}_{2} \mathrm{Cl}_{2}\right): \delta 38.2\left(\mathrm{~s}, J_{\mathrm{PtP}}=1794 \mathrm{~Hz}, \mathrm{P}_{-}{ }^{\mathrm{t}} \mathrm{Bu}_{2}\right),-49.6\left(\mathrm{~s}, J_{\mathrm{PtP}}=3943 \mathrm{~Hz}\right.$, PCCPt). ${ }^{1} \mathrm{H}$ NMR (600 MHz, $\left.\mathrm{CD}_{2} \mathrm{Cl}_{2}\right): \delta 7.60\left(\mathrm{~d}, J_{\mathrm{PH}}=3.8 \mathrm{~Hz}, \mathrm{c}_{2}\right), 7.25\left(\mathrm{~s}, \mathrm{a}_{1}\right), 7.16$ $\left(\mathrm{s}, \mathrm{a}_{2}\right), 6.95\left(\mathrm{~d}, J_{\mathrm{PH}}=6.5 \mathrm{~Hz}, \mathrm{c}_{1}\right), 2.34\left(\mathrm{~s}, \mathrm{~g}_{1}\right), 2.32\left(\mathrm{~s}, \mathrm{~g}_{2}\right), 1.71\left(\mathrm{~d}, J_{\mathrm{PH}}=12.9 \mathrm{~Hz}, \mathrm{i}_{3}\right)$, $1.41(\mathrm{~d}, J=15.9 \mathrm{~Hz}, \mathrm{k}), 1.36(\mathrm{~d}, J=14.9 \mathrm{~Hz}, \mathrm{l}), 1.34\left(\mathrm{~d}, J_{\mathrm{PH}}=13.2 \mathrm{~Hz}, \mathrm{i}_{2}\right), 1.2-1.5$, obscured $(\mathrm{m}, \mathrm{m}), 1.03\left(\mathrm{~d}, J_{\mathrm{PH}}=15.0 \mathrm{~Hz}, \mathrm{i}_{1}\right) \cdot{ }^{13} \mathrm{C} \mathrm{NMR}\left(150 \mathrm{MHz}, \mathrm{CD}_{2} \mathrm{Cl}_{2}\right): \delta, 157.1$ $\left(\mathrm{d}, J_{\mathrm{PC}}=9.0 \mathrm{~Hz}, \mathrm{e}_{2}\right), 153.9\left(\mathrm{~d}, J_{\mathrm{PC}}=4.3 \mathrm{~Hz}, \mathrm{e}_{1}\right), 134.2\left(\mathrm{~d}, J_{\mathrm{PC}}=3.2 \mathrm{~Hz}, \mathrm{c}_{2}\right), 133.8(\mathrm{~d}$, 
$\left.J_{\mathrm{PC}}=6.3 \mathrm{~Hz}, \mathrm{~b}_{1}\right), 133.4\left(\mathrm{~d}, J_{\mathrm{PC}}=3.2 \mathrm{~Hz}, \mathrm{~b}_{2}\right), 132.8\left(\mathrm{~s}, \mathrm{c}_{1}\right), 130.5\left(\mathrm{~d}, J_{\mathrm{PC}}=1.6 \mathrm{~Hz}, \mathrm{a}_{1}\right)$, $129.6\left(\mathrm{~d}, J_{\mathrm{PC}}=1.6 \mathrm{~Hz}, \mathrm{a}_{2}\right), 128.5\left(\mathrm{dd}, J_{\mathrm{PC}}=4.2,1.6 \mathrm{~Hz}, \mathrm{f}_{1}\right), 124.0\left(\mathrm{~d}, J_{\mathrm{PC}}=4.3 \mathrm{~Hz}, \mathrm{f}_{2}\right)$, $121.3\left(\mathrm{~d}, J_{\mathrm{PC}}=13.8 \mathrm{~Hz}, \mathrm{~d}_{2}\right), 116.6\left(\mathrm{dd}, J_{\mathrm{PC}}=30.7,1.6 \mathrm{~Hz}, \mathrm{~d}_{1}\right), 46.7\left(\mathrm{~d}, J_{\mathrm{PC}}=37.6 \mathrm{~Hz}\right.$, j) $40.8\left(\mathrm{~d}, J_{\mathrm{PC}}=10.1 \mathrm{~Hz}, \mathrm{~h}_{2}\right), 36.8\left(\mathrm{~d}, J_{\mathrm{PC}}=8.0 \mathrm{~Hz}, \mathrm{~h}_{3}\right), 36.0\left(\mathrm{~d}, J_{\mathrm{PC}}=22.3 \mathrm{~Hz}\right.$, $\left.\mathrm{h}_{1}\right), 33.2\left(\mathrm{~d}, J_{\mathrm{PC}}=5.8 \mathrm{~Hz}, \mathrm{i}_{3}\right), 32.6(\mathrm{~s}, \mathrm{l}), 32.1\left(\mathrm{dd}, J_{\mathrm{PC}}=10.1,3.7 \mathrm{~Hz}, \mathrm{k}\right), 31.3(\mathrm{~d}$, $\left.J_{\mathrm{PC}}=5.3 \mathrm{~Hz}, \mathrm{i}_{2}\right), 28.8\left(\mathrm{bs}, \mathrm{i}_{1}\right), 21.3\left(\mathrm{~s}, \mathrm{~g}_{2}\right), 21.0\left(\mathrm{~s}, \mathrm{~g}_{1}\right), 15.7(\mathrm{dd}, J=81.6,35.5 \mathrm{~Hz}$, $\mathrm{m})$.

\section{Attempted reaction of $\left[\mathrm{Pt}(t-\mathrm{Bu}\right.$-thixantphos $\left.)\left(\eta^{2}-\mathrm{O}_{2}\right)\right]$ with $\mathrm{CO}_{2}$}

Carbon dioxide was bubbled through a solution of $\left[\mathrm{Pt}(t\right.$-Bu-thixantphos $\left.)\left(\eta^{2}-\mathrm{O}_{2}\right)\right]$ $(0.030 \mathrm{~g}, 0.040 \mathrm{mmol})$ in $\mathrm{CD}_{2} \mathrm{Cl}_{2}(0.4 \mathrm{~mL})$ for $10 \mathrm{mins}$. The NMR tube was sealed under carbon dioxide with a septum and the reaction was followed by ${ }^{1} \mathrm{H},{ }^{13} \mathrm{C}$ and ${ }^{31} \mathrm{P}$ NMR. No reaction was observed after four days at room temperature.

\section{Attempted reaction of $\left[\mathrm{Pt}(t-\mathrm{Bu}\right.$-thixantphos $\left.)\left(\eta^{2}-\mathrm{O}_{2}\right)\right]$ with $\mathrm{CH}_{4}$}

Methane was bubbled through a solution of $\left[\mathrm{Pt}(t\right.$-Bu-thixantphos $\left.)\left(\eta^{2}-\mathrm{O}_{2}\right)\right](0.015$ $\mathrm{g}, 0.020 \mathrm{mmol})$ in $\mathrm{C}_{6} \mathrm{D}_{6}(0.5 \mathrm{~mL})$ for 10 mins. The NMR tube was sealed under methane with a septum and the reaction was followed by ${ }^{1} \mathrm{H},{ }^{13} \mathrm{C}$ and ${ }^{31} \mathrm{P} \mathrm{NMR}$. No reaction was observed after 14 days at room temperature.

\section{Attempted reaction of $\left[\mathrm{Pt}(t-\mathrm{Bu}\right.$-thixantphos $\left.)\left(\eta^{2}-\mathrm{O}_{2}\right)\right]$ with $\mathrm{C}_{2} \mathrm{H}_{2}$}

Ethyne was bubbled through a solution of $\left[\mathrm{Pt}(t\right.$-Bu-thixantphos $\left.)\left(\eta^{2}-\mathrm{O}_{2}\right)\right](0.037$ $\mathrm{g}, 0.050 \mathrm{mmol})$ in $\mathrm{C}_{6} \mathrm{D}_{6}(0.5 \mathrm{~mL})$ for 10 mins. The NMR tube was sealed under ethyne with a septum and the reaction was followed by ${ }^{1} \mathrm{H}$ and ${ }^{31} \mathrm{P}$ NMR spectroscopy. No reaction was observed after 48 hours.

\section{Attempted reaction of $\left[\mathrm{Pt}(t-\mathrm{Bu}\right.$-thixantphos $\left.)\left(\eta^{2}-\mathrm{O}_{2}\right)\right]$ with $\mathrm{C}_{2} \mathrm{H}_{4}$}

Ethene was bubbled through a solution of $\left[\mathrm{Pt}(t\right.$-Bu-thixantphos $\left.)\left(\eta^{2}-\mathrm{O}_{2}\right)\right](0.037$ $\mathrm{g}, 0.050 \mathrm{mmol})$ in $\mathrm{C}_{6} \mathrm{D}_{6}(0.5 \mathrm{~mL})$ for $10 \mathrm{mins}$. The NMR tube was sealed under 
ethene with a septum and the reaction was followed by ${ }^{1} \mathrm{H}$ and ${ }^{31} \mathrm{P}$ NMR spectroscopy. No reaction was observed after 48 hours.

\section{Attempted reaction of $\left[\mathrm{Pt}(t-\mathrm{Bu}\right.$-thixantphos $\left.)\left(\eta^{2}-\mathrm{O}_{2}\right)\right]$ with $\mathrm{NH}_{4} \mathrm{PF}_{6}$}

A solution of $\mathrm{NH}_{4} \mathrm{PF}_{6}(0.007 \mathrm{~g}, 0.043 \mathrm{mmol})$ in THF $(2 \mathrm{~mL})$ was added to a suspension of $\left[\mathrm{Pt}(t\right.$-Bu-thixantphos $\left.)\left(\eta^{2}-\mathrm{O}_{2}\right)\right](0.029 \mathrm{~g}, 0.039 \mathrm{mmol})$ in THF $(3 \mathrm{~mL})$. The reaction was stirred at room temperature for 24 hours. The solvent was removed under reduced pressure and the brown solid was taken up in $\mathrm{d}_{6}$-acetone for NMR. No reaction was observed so the sample was heated to $40{ }^{\circ} \mathrm{C}$ for four days. No evidence of reaction was present.

\section{Attempted reaction of $\left[\mathrm{Pt}(t-\mathrm{Bu}\right.$-thixantphos $\left.)\left(\eta^{2}-\mathrm{O}_{2}\right)\right]$ with $\mathrm{H}_{2}$}

Hydrogen gas was bubbled through a solution of $\left[\mathrm{Pt}(t-\mathrm{Bu}\right.$-thixantphos $\left.)\left(\eta^{2}-\mathrm{O}_{2}\right)\right]$ $(0.032 \mathrm{~g}, 0.043 \mathrm{mmol})$ in $\mathrm{C}_{6} \mathrm{D}_{6}(0.5 \mathrm{~mL})$ for $10 \mathrm{mins}$. The NMR tube was sealed under hydrogen with a septum and the reaction was followed by ${ }^{1} \mathrm{H}$ and ${ }^{31} \mathrm{P}$ NMR spectroscopy. No reaction was observed after three days.

\section{1:1 reaction of $\left[\mathrm{Pt}(t-\mathrm{Bu}-\right.$ thixantphos $\left.)\left(\eta^{2}-\mathrm{O}_{2}\right)\right]$ with pta}

1,3,5-triaza-7-phosphaadamantane $(0.003 \mathrm{~g}, 0.020 \mathrm{mmol})$ was added to a solution of $\left[\mathrm{Pt}(t-\mathrm{Bu}\right.$-thixantphos $\left.)\left(\eta^{2}-\mathrm{O}_{2}\right)\right](0.015 \mathrm{~g}, 0.020 \mathrm{mmol})$ in $\mathrm{CDCl}_{3}$. The ${ }^{31} \mathrm{P}$ NMR spectrum showed peaks indicative of $75 \%$ starting material and uncoordinated $t$-Bu-thixantphos ( $25 \%$ of the $t$-Bu-thixantphos signals). There was also a complex at $-74.1 \mathrm{ppm}\left(\mathrm{JtP}_{\mathrm{Pt}}=3562 \mathrm{~Hz}\right)$ consistent with literature precedence for $\left[\mathrm{Pt}(\mathrm{pta})_{4}\right] . \underline{319}$

\section{1:4 reaction of $\left[\mathrm{Pt}(t-\mathrm{Bu}-\mathrm{thixantphos})\left(\eta^{2}-\mathrm{O}_{2}\right)\right]$ with pta}

1,3,5-triaza-7-phosphaadamantane $(0.019 \mathrm{~g}, 0.120 \mathrm{mmol})$ was added to a solution of $\left[\mathrm{Pt}(t\right.$-Bu-thixantphos $\left.)\left(\eta^{2}-\mathrm{O}_{2}\right)\right](0.022 \mathrm{~g}, 0.030 \mathrm{mmol})$ in $\mathrm{CDCl}_{3}$. The ${ }^{31} \mathrm{P}$ NMR spectrum showed the presence of uncoordinated $t$-Bu-thixantphos and $\left[\mathrm{Pt}(\mathrm{pta})_{4}\right] . \underline{319}$ 


\section{Reaction of $t$-Bu-thixantphos with $\left[\mathrm{Pt}\left(\mathrm{C}_{6} \mathrm{H}_{10}\right) \mathrm{Cl}_{2}\right]$ in $\mathrm{C}_{6} \mathrm{D}_{6}$}

$\left[\mathrm{Pt}\left(\mathrm{C}_{6} \mathrm{H}_{10}\right) \mathrm{Cl}_{2}\right](0.007 \mathrm{~g}, 0.020 \mathrm{mmol})$ was weighed into an NMR tube and placed under argon. A solution of $t$-Bu-thixantphos $(0.010 \mathrm{~g}, 0.019 \mathrm{mmol})$ in $\mathrm{C}_{6} \mathrm{D}_{6}(0.4$ $\mathrm{mL}$ ) was added to the NMR tube and the top was closed with a septum and parafilm. The reaction mixture was gently shaken to promote dissolution of the sparingly soluble $\left[\mathrm{Pt}\left(\mathrm{C}_{6} \mathrm{H}_{10}\right) \mathrm{Cl}_{2}\right]$. The reaction was kept at room temperature for four hours, after which time no change was observed by NMR spectroscopy. The reaction was then heated for 72 hours at $40{ }^{\circ} \mathrm{C}$, with intermediary monitoring by NMR spectroscopy, during which time the colourless solution became dark red. After 72 hours the reaction showed $75 \%$ conversion (by ${ }^{31} \mathrm{P}$ NMR spectroscopy) to trans-[Pt(t-Bu-xantphos $\left.) \mathrm{Cl}_{2}\right]$.

\section{Attempted reaction of $t$-Bu-thixantphos with $\left[\mathrm{Pt}\left(\mathrm{C}_{6} \mathrm{H}_{10}\right) \mathrm{Cl}_{2}\right]$ in $\mathrm{CH}_{2} \mathrm{Cl}_{2}$}

$\left[\mathrm{Pt}\left(\mathrm{C}_{6} \mathrm{H}_{10}\right) \mathrm{Cl}_{2}\right](0.136 \mathrm{~g}, 0.39 \mathrm{mmol})$ was added to a Schlenk tube containing a solution of $t$-Bu-thixantphos $(0.202 \mathrm{~g}, 0.39 \mathrm{mmol})$ in $\mathrm{CH}_{2} \mathrm{Cl}_{2}(10 \mathrm{~mL})$. The reaction was stirred overnight generating a yellow solution. The solvent was removed under reduced pressure leaving a yellow solid. NMR analysis of this solid in $\mathrm{C}_{6} \mathrm{D}_{6}$ showed a mixture of unreacted $t$-Bu-thixantphos $(43.5 \%), t$-Bu-thixantphosH+ $(32.5 \%)$ and trans-[Pt $(t-B u$-thixantphos $\left.) \mathrm{Cl}_{2}\right]\left(24.0 \%\right.$, yields based on ${ }^{31} \mathrm{P}$ NMR spectroscopy).

\section{Attempted reaction of $t$-Bu-thixantphos with $\left[\mathrm{PtCl}_{2}\left(\mathrm{SEt}_{2}\right)_{2}\right]$}

t-Bu-Thixantphos $(0.012 \mathrm{~g}, 0.023 \mathrm{mmol})$ in $\mathrm{C}_{6} \mathrm{D}_{6}(0.4 \mathrm{~mL})$ was added to an NMR tube containing $\left[\mathrm{PtCl}_{2}\left(\mathrm{SEt}_{2}\right)_{2}\right](0.012 \mathrm{~g}, 0.025 \mathrm{mmol})$. The top was sealed with a septum and wrapped with parafilm. The reaction was kept at room temperature for 24 hours then analysed by ${ }^{1} \mathrm{H}$ and ${ }^{31} \mathrm{P}$ NMR spectroscopy. No change was observed in either spectrum. The reaction was heated to $40{ }^{\circ} \mathrm{C}$ for 7 days then analysed again displaying unreacted $t$-Bu-thixantphos $(99.6 \%)$ and trans- $\left[\mathrm{PtCl}_{2}\left(t-\mathrm{Bu}^{-}\right.\right.$ thixantphos)] (0.4\% based on ${ }^{31} \mathrm{P}$ NMR spectroscopy). The reaction was heated to $60{ }^{\circ} \mathrm{C}$ for a further 4 days at which time the reaction had progressed to $3.1 \%$ 
trans-[ $\mathrm{PtCl}_{2}$ (t-Bu-thixantphos)]. Heating at $60{ }^{\circ} \mathrm{C}$ was continued to give a total of 28 days at this temperature. NMR spectroscopy showed $6.7 \%$ trans- $\left[\mathrm{PtCl}_{2}(t-\right.$ Bu-thixantphos)] with unreacted $t$-Bu-thixantphos accounting for the remaining $92.4 \%$.

\section{Attempted reaction of $t$-Bu-thixantphos with cis- $\left[\mathrm{PtCl}_{2}(\mathrm{NCMe})_{2}\right]$}

t-Bu-Thixantphos $(0.014 \mathrm{~g}, 0.027 \mathrm{mmol})$ was dissolved in $\mathrm{CD}_{2} \mathrm{Cl}_{2}(0.2 \mathrm{~mL})$ and added to a solution of cis- $\left[\mathrm{PtCl}_{2}(\mathrm{NCMe})_{2}\right](0.009,0.026 \mathrm{mmol})$ in $\mathrm{CD}_{2} \mathrm{Cl}_{2}(0.2 \mathrm{~mL})$ in an NMR tube. The tube was sealed with a septum wrapped in parafilm. The reaction was followed by ${ }^{1} \mathrm{H}$ and ${ }^{31} \mathrm{P}$ NMR spectroscopy. After 72 hours the reaction mixture contained unreacted $t$-Bu-thixantphos $(83.8 \%)$ and $t$-Bu-thixantphos $\mathrm{H}^{+}$ (16.2\%) by ${ }^{31} \mathrm{P}$ NMR spectroscopy.

\section{Attempted reaction of $t$-Bu-thixantphos with trans-[PtCl${ }_{2}\left(\mathrm{NCMe}_{2}\right]$}

t-Bu-Thixantphos $(0.012,0.023 \mathrm{mmol})$ was dissolved in $\mathrm{CDCl}_{3}(0.2 \mathrm{~mL})$ and added to an NMR tube containing a solution of trans-[PtCl $2\left(\mathrm{NCMe}_{2}\right](0.010 \mathrm{~g}, 0.029$ $\mathrm{mmol})$ in $\mathrm{CDCl}_{3}(0.2 \mathrm{~mL})$. The tube was sealed with a septum and the reaction was followed by ${ }^{1} \mathrm{H}$ and ${ }^{31} \mathrm{P}$ NMR spectroscopy. After 24 hours at room temperature no changes were observed in the NMR spectrum. The reaction was heated to $50{ }^{\circ} \mathrm{C}$ for 7 days. At this stage the reaction showed unreacted $t$-Bu-thixantphos $(66.7 \%)$ and $t$-Bu-thixantphos $\mathrm{H}^{+}(33.3 \%)$ based on the ${ }^{31} \mathrm{P}$ NMR spectrum. After a total of 21 days heating at $50{ }^{\circ} \mathrm{C}$ the reaction contained unreacted $t$-Buthixantphos $(43.0 \%), t$-Bu-thixantphos $\mathrm{H}^{+}(31.7 \%)$ and trans-[ $\mathrm{PtCl}_{2}(t$-Bu-thixantphos)] (25.3\%).

\section{Attempted reaction of $t$-Bu-thixantphos with trans- $\left[\mathrm{PtCl}_{2}(\mathrm{NCt}-\right.$ $\mathrm{Bu})_{2}$ ]}

t-Bu-Thixantphos $(0.024 \mathrm{~g}, 0.046 \mathrm{mmol})$ was dissolved in $\mathrm{CDCl}_{3}(0.4 \mathrm{~mL})$ and added to an NMR tube containing a solution of trans-[ $\left.\mathrm{PtCl}_{2}(\mathrm{NCt}-\mathrm{Bu})_{2}\right](0.020$ $\mathrm{g}, 0.046 \mathrm{mmol})$ in $\mathrm{CDCl}_{3}(0.4 \mathrm{~mL})$. The reaction was sealed with a septum and followed by ${ }^{1} \mathrm{H}$ and ${ }^{31} \mathrm{P}$ NMR spectroscopy. After 24 hours at room temperature 
the reaction showed unreacted $t$-Bu-thixantphos (91.1\%) and $t$-Bu-thixantphos $\mathrm{H}^{+}$ (8.9\%). The reaction was heated to $50{ }^{\circ} \mathrm{C}$ for 24 hours upon which the amount of $t$-Bu-thixantphos $\mathrm{H}^{+}$had increased to $24.4 \%$. After 7 days at $50{ }^{\circ} \mathrm{C}$ the reaction contained unreacted $t$-Bu-thixantphos (49.3\%), $t$-Bu-thixantphos $\mathrm{H}^{+}(32.5 \%)$ and trans-[PtCl 2 (t-Bu-thixantphos)] (18.2\%). After 21 days at $50{ }^{\circ} \mathrm{C}$ only $7.4 \%$ unreacted $t$-Bu-thixantphos remained, with $50.7 \% t$-Bu-thixantphos $\mathrm{H}^{+}$and $41.9 \%$ trans-[PtCl$\left.{ }_{2}(t-B u-t h i x a n t p h o s)\right]$.

\section{trans-[Pt(t-Bu-thixantphos $\left.) \mathrm{Cl}_{2}\right]$}

t-Bu-Thixantphos $(0.198 \mathrm{~g}, 0.38 \mathrm{mmol})$ and $\left[\mathrm{Pt}\left(\mathrm{C}_{6} \mathrm{H}_{10}\right) \mathrm{Cl}_{2}\right](0.133 \mathrm{~g}, 0.38 \mathrm{mmol})$ were dissolved in toluene $(10 \mathrm{~mL})$ and heated to $50{ }^{\circ} \mathrm{C}$ for three days, resulting in an orange solution. The solvent was removed in vacuo and the resulting solid was dissolved in a minimum of dichloromethane. Diethyl ether was added until a small amount of oily residue became evident. The sample was cooled to $-14{ }^{\circ} \mathrm{C}$, resulting the title compound as red crystals $(0.199 \mathrm{~g}, 66 \%)$.

${ }^{31} \mathrm{P}$ NMR $\left(121 \mathrm{MHz}, \mathrm{C}_{6} \mathrm{D}_{6}\right): \delta 32.9\left(\mathrm{~s}, J_{\mathrm{PtP}}=2700 \mathrm{~Hz}\right){ }^{1} \mathrm{H} \mathrm{NMR}\left(500 \mathrm{MHz}, \mathrm{C}_{6} \mathrm{D}_{6}\right)$ : $\delta 7.11(\mathrm{~s}, \mathrm{PC}(\mathrm{Ar}) \mathrm{CH}), 6.98(\mathrm{~s}, \mathrm{SCCH}), 1.86\left(\mathrm{~s}, \mathrm{C}(\mathrm{Ar}) \mathrm{CH}_{3}\right), 1.71\left(\mathrm{vt}, J_{\mathrm{PH}}=7.3 \mathrm{~Hz}\right.$, $\left.\mathrm{PCCH}_{3}\right), 1.56\left(\mathrm{bs}, \mathrm{PCCH}{ }_{3}\right),{ }^{13} \mathrm{C} \mathrm{NMR}\left(125 \mathrm{MHz}, \mathrm{C}_{6} \mathrm{D}_{6}\right): \delta 155.8\left(\mathrm{vt}, J_{\mathrm{PC}}=6.3 \mathrm{~Hz}\right.$, CO), 134.3 (s, PC(Ar)CH), $131.0\left(\mathrm{vt}, J_{\mathrm{PC}}=3.4 \mathrm{~Hz}, \mathrm{C}(\mathrm{Ar}) \mathrm{CH}_{3}\right), 129.9\left(\mathrm{~s}, \mathrm{SCCC}\left(\mathrm{CH}_{3}\right)\right.$ ), 124.0 (vt, $J=18.5 \mathrm{~Hz}, \mathrm{PC}(\mathrm{Ar})), 124.5$ (vt, $J=6.7 \mathrm{~Hz}, \mathrm{CS}), 20.3$ (s, C(Ar) $\mathrm{CH}_{3}$ ), 39.7 $\left(\mathrm{vt}, J_{\mathrm{PC}}=11.6 \mathrm{~Hz}, \mathrm{PCCH}_{3}\right), 38.8\left(\mathrm{vt}, J_{\mathrm{PC}}=11.1 \mathrm{~Hz}, \mathrm{PCCH}_{3}\right), 32.8\left(\mathrm{vt}, J_{\mathrm{PC}}=3.9 \mathrm{~Hz}\right.$, $\mathrm{PCCH}_{3}$ ), 30.2 (bs, $\mathrm{PCCH}_{3}$ ), HRMS calcd for $\mathrm{C}_{30} \mathrm{H}_{46} \mathrm{OP}_{2} \mathrm{SClPt}[\mathrm{M}-\mathrm{Cl}]^{+} \mathrm{m} / z=$ 745.2060; found $=745.2052$.

\section{trans-[Pt(t-Bu-sixantphos) $\left.\mathrm{Cl}_{2}\right]$}

A solution of $t$-Bu-sixantphos $(0.051 \mathrm{~g}, 0.10 \mathrm{mmol})$ in toluene $(5 \mathrm{~mL})$ was added to a suspension of $\left[\mathrm{Pt}\left(\mathrm{C}_{6} \mathrm{H}_{10}\right) \mathrm{Cl}_{2}\right](0.035 \mathrm{~g}, 0.10 \mathrm{mmol})$ in toluene $(5 \mathrm{~mL})$. The reaction mixture was stirred at $50{ }^{\circ} \mathrm{C}$ for 72 hours. The solvent was removed under reduced pressure and the solid was taken up in $\mathrm{C}_{6} \mathrm{D}_{6}$ for NMR analysis. The reaction showed a mixture of unreacted $t$-Bu-sixantphos (53.4\%), $t$-Bu-sixantphos $\mathrm{H}^{+}$ $(22.8 \%)$ and trans-[Pt $t$-Bu-sixantphos $\left.) \mathrm{Cl}_{2}\right]$ (23.8\%). The NMR sample was heated at $50{ }^{\circ} \mathrm{C}$ for a further 11 days. At this stage no $\left[\mathrm{Pt}\left(\mathrm{C}_{6} \mathrm{H}_{10}\right) \mathrm{Cl}_{2}\right]$ was observed in the 
${ }^{1} \mathrm{H}$ NMR spectrum and no change in the ratio of the compounds in the ${ }^{31} \mathrm{P} N M R$ spectrum was observed over a period of 24 hours. The final ratio of the compounds was $63.8 \% t$-Bu-sixantphos $\mathrm{H}^{+}$, and $36.2 \%$ trans-[Pt $(t-\mathrm{Bu}$-sixantphos $\left.) \mathrm{Cl}_{2}\right]$. Attempts to isolate trans-[Pt(t-Bu-sixantphos $\left.) \mathrm{Cl}_{2}\right]$ were unsuccessful. Due to the complex nature of the ${ }^{1} \mathrm{H}$ and ${ }^{13} \mathrm{C}$ NMR spectra for this system the product trans$\left[\mathrm{Pt}(t\right.$-Bu-sixantphos $\left.) \mathrm{Cl}_{2}\right]$ is proposed based on the similarities of the ${ }^{31} \mathrm{P} \mathrm{NMR}$ spectrum with those for trans-[Pt(t-Bu-thixantphos $\left.) \mathrm{Cl}_{2}\right]$ and trans-[Pt $(t-\mathrm{Bu}$-xantphos) $\mathrm{Cl}_{2}$ ] which were formed via the same reaction conditions.

${ }^{31} \mathrm{P}$ NMR (121 MHz, C $\left.{ }_{6} \mathrm{D}_{6}\right): \delta 34.7$ (s, J $\left.J_{\mathrm{PtP}}=2686 \mathrm{~Hz}\right)$. HRMS calcd for $\mathrm{C}_{30} \mathrm{H}_{48} \mathrm{ClOP}_{2} \mathrm{PtSi}[\mathrm{M}-\mathrm{Cl}]^{+} \mathrm{m} / \mathrm{z}=739.2237$; found $=739.2178$.

\section{Reaction of $t$-Bu-xantphos with $\left[\mathrm{Pt}\left(\mathrm{C}_{6} \mathrm{H}_{10}\right) \mathrm{Cl}_{2}\right]$}

A solution of $t$-Bu-xantphos $(0.063 \mathrm{~g}, 0.126 \mathrm{mmol})$ in toluene $(5 \mathrm{~mL})$ was added to a suspension of $\left[\mathrm{Pt}\left(\mathrm{C}_{6} \mathrm{H}_{10}\right) \mathrm{Cl}_{2}\right](0.044 \mathrm{~g}, 0.126)$ in toluene $(5 \mathrm{~mL})$. The reaction mixture was stirred at $50{ }^{\circ} \mathrm{C}$ for 72 hours. The solvent was removed in vacuo leaving an orange solid. The solid was recrystallised by inwards diffusion of diethyl ether into a dichloromethane solution of the complex, yielding the title complex as a yellow solid $(0.027 \mathrm{~g}, 28 \%)$. The complex was analysed by NMR spectroscopy in $\mathrm{C}_{6} \mathrm{D}_{6}, \mathrm{CDCl}_{3}, \mathrm{CD}_{2} \mathrm{Cl}_{2}$ and $\mathrm{d}_{6}$-acetone. In $\mathrm{C}_{6} \mathrm{D}_{6}$ the complex is formulated as trans- $\left[\mathrm{Pt}(t-\mathrm{Bu}\right.$-xantphos $\left.) \mathrm{Cl}_{2}\right]$ while in $\mathrm{CDCl}_{3}$ and $\mathrm{CD}_{2} \mathrm{Cl}_{2}$ the complex is thought to lose a chloride ligand resulting in $\left[\mathrm{Pt}\left(t\right.\right.$-Bu-xantphos- $\left.\left.\mathrm{P}, \mathrm{O}, \mathrm{P}^{\prime}\right) \mathrm{Cl}\right] \mathrm{Cl}$. In $\mathrm{d}_{6}$-acetone the ${ }^{31} \mathrm{P}$ NMR spectrum appears as a broad singlet at $46 \mathrm{ppm}$. The full NMR characterisation in the other solvents is given below.

\section{trans-[Pt(t-Bu-xantphos) $\left.\mathrm{Cl}_{2}\right]$}

${ }^{31} \mathrm{P}$ NMR $\left(121 \mathrm{MHz}, \mathrm{C}_{6} \mathrm{D}_{6}\right): \delta 32.4\left(\mathrm{~s}, J_{\mathrm{PtP}}=2721.4 \mathrm{~Hz}\right) .{ }^{1} \mathrm{H}$ NMR $(500 \mathrm{MHz}$, $\left.\mathrm{C}_{6} \mathrm{D}_{6}\right): \delta 7.36(\mathrm{~m}, \mathrm{PC}(\mathrm{Ar}) \mathrm{CH}), 7.14(\mathrm{~d}, J=7.8 \mathrm{~Hz}, \mathrm{C}($ bridge $) \mathrm{CCH}), 6.89$ (t, $J=7.0 \mathrm{~Hz}, \mathrm{PCCCH}), 1.67$ (bs, PC $\left.\left(\mathrm{CH}_{3}\right)_{3}\right), 1.37$ (s, C(bridge) $\left.\left(\mathrm{CH}_{3}\right)_{2}\right), 1.40$ (s, $\mathrm{C}$ (bridge) $\left.\left(\mathrm{CH}_{3}\right)_{2}\right) .{ }^{13} \mathrm{C} \mathrm{NMR}\left(125 \mathrm{MHz}, \mathrm{C}_{6} \mathrm{D}_{6}\right): \delta 157.1(\mathrm{vt}, J=6.3 \mathrm{~Hz}, \mathrm{PCCO}), 136.0$ (vt, $J=4.8 \mathrm{~Hz}, \mathrm{C}$ (bridge)CCH), 132.9 (s, PC(Ar)CH), 126.0 (s, C(bridge)CCH), $122.5(\mathrm{vt}, J=38.9 \mathrm{~Hz}, \mathrm{PC}(\mathrm{Ar})), 122.1(\mathrm{vt}, J=6.3 \mathrm{~Hz}, \mathrm{PCCCH}), 39.2\left(\mathrm{bs}, \mathrm{PC}\left(\mathrm{CH}_{3}\right)_{3}\right)$, $36.5\left(\mathrm{~m}, \mathrm{C}(\right.$ bridge $\left.)\left(\mathrm{CH}_{3}\right)_{2}\right), 32.8\left(\mathrm{bs}, \mathrm{PC}\left(\mathrm{CH}_{3}\right)_{3}\right), 27.5$ (s, $\mathrm{C}($ bridge $\left.)\left(\mathrm{CH}_{3}\right)_{2}\right)$. 


\section{$[\mathrm{Pt}(t-\mathrm{Bu}$-xantphos-кP,O,P')Cl]Cl}

${ }^{31} \mathrm{P}$ NMR $\left(121 \mathrm{MHz}, \mathrm{CDCl}_{3}\right): \delta 47.7\left(\mathrm{~s}, J_{\mathrm{PtP}}=2349.6 \mathrm{~Hz}\right){ }^{1} \mathrm{H}$ NMR $(500 \mathrm{MHz}$, $\left.\mathrm{CDCl}_{3}\right): \delta 7.96(\mathrm{~d}, J=7.9 \mathrm{~Hz}, \mathrm{C}($ bridge$) \mathrm{CCH}$ ), 7.93 (bs, $\mathrm{PC}(\mathrm{Ar}) \mathrm{CH}$ ), 7.70 $(\mathrm{t}, J=7.3 \mathrm{~Hz}, \mathrm{PCCCH}), 1.80\left(\mathrm{~s}, \mathrm{C}(\right.$ bridge $\left.)\left(\mathrm{CH}_{3}\right)_{2}\right), 1.57\left(\mathrm{vt}, J_{\mathrm{PH}}=15.6 \mathrm{~Hz}\right.$, $\left.\mathrm{PC}\left(\mathrm{CH}_{3}\right)_{3}\right) .{ }^{13} \mathrm{C} \mathrm{NMR}\left(125 \mathrm{MHz}, \mathrm{CDCl}_{3}\right): \delta 158.5\left(\mathrm{vt}, J_{\mathrm{PC}}=11.0 \mathrm{~Hz}, \mathrm{PCCO}\right)$, 134.7 (s, PC(Ar)CH), 133.8 (s, C(bridge)CCH), 132.5 (s, C(bridge)CCH), 128.6 (vt, $\left.J_{\mathrm{PC}}=6.8 \mathrm{~Hz}, \mathrm{PCCCH}\right), 117.2\left(\mathrm{vt}, J_{\mathrm{PC}}=33.2 \mathrm{~Hz}, \mathrm{PC}(\mathrm{Ar})\right), 39.6\left(\mathrm{vt}, J_{\mathrm{PC}}=21.1 \mathrm{~Hz}\right.$, $\left.\mathrm{PC}\left(\mathrm{CH}_{3}\right)_{3}\right), 34.1\left(\mathrm{~s}, \mathrm{C}(\right.$ bridge $\left.)\left(\mathrm{CH}_{3}\right)_{2}\right), 30.1\left(\mathrm{vt}, J_{\mathrm{PC}}=4.3 \mathrm{~Hz}, \mathrm{PC}\left(\mathrm{CH}_{3}\right)_{3}\right), 27.1(\mathrm{~s}$, $C$ (bridge)).

${ }^{31} \mathrm{P}$ NMR $\left(121 \mathrm{MHz}, \mathrm{CD}_{2} \mathrm{Cl}_{2}\right): \delta 47.8\left(\mathrm{~s}, J_{\mathrm{PtP}}=2345.3 \mathrm{~Hz}\right){ }^{1} \mathrm{H}$ NMR $(500 \mathrm{MHz}$, $\left.\mathrm{CD}_{2} \mathrm{Cl}_{2}\right): \delta 7.93(\mathrm{~m}, \mathrm{PC}(\mathrm{Ar}) \mathrm{CH}), 7.78(\mathrm{dd}, J=7.7,1.2 \mathrm{~Hz}, \mathrm{C}($ bridge $) \mathrm{CCH}), 7.60(\mathrm{t}$, $J=7.8 \mathrm{~Hz}, \mathrm{PCCCH}), 1.78\left(\mathrm{~s}, \mathrm{C}(\right.$ bridge $\left.)\left(\mathrm{CH}_{3}\right)_{2}\right), 1.57\left(\mathrm{vt}, J=16.1 \mathrm{~Hz}, \mathrm{PC}\left(\mathrm{CH}_{3}\right)_{3}\right)$. ${ }^{13} \mathrm{C} \mathrm{NMR}\left(125 \mathrm{MHz}, \mathrm{CD}_{2} \mathrm{Cl}_{2}\right): \delta 158.8(\mathrm{vt}, J=11.1 \mathrm{~Hz}, \mathrm{PCCO}), 135.0(\mathrm{~s}, \mathrm{PC}(\mathrm{Ar}) C \mathrm{H})$, 133.72 (s, C(bridge)CCH), 132.7 (s, C(bridge) CCH) $128.3(\mathrm{vt}, J=6.7 \mathrm{~Hz}, \mathrm{PCCCH})$, $117.8(\mathrm{vt}, J=33.1 \mathrm{~Hz}, \mathrm{PC}(\mathrm{Ar})), 39.8\left(\mathrm{vt}, J=21.1 \mathrm{~Hz}, \mathrm{PC}\left(\mathrm{CH}_{3}\right)_{3}\right), 34.5$ (s, $C$ (bridge)), 34.1 (s, C(bridge) $\left.\left(\mathrm{CH}_{3}\right)_{2}\right), 30.0$ (vt, $\left.J=4.8 \mathrm{~Hz}, \mathrm{PC}\left(\mathrm{CH}_{3}\right)_{3}\right)$.

HRMS calcd for $\mathrm{C}_{31} \mathrm{H}_{48} \mathrm{ClOP}_{2} \mathrm{Pt}[\mathrm{M}-\mathrm{Cl}]^{+} \mathrm{m} / \mathrm{z}=723.2468$; found $=723.2455$.

\section{$\left[\mathrm{Pt}\left(t-B u-t h i x a n t p h o s-\kappa P, O, P^{\prime}\right) \mathrm{Cl}\right] \mathrm{PF}_{6}$}

Dissolved (tert-butyl-Thixantphos)platinum dichloride $(0.035 \mathrm{~g}, 0.045 \mathrm{mmol})$ in dichloromethane $(2 \mathrm{~mL})$ and added ammonium hexafluorophosphate $(0.015 \mathrm{~g}$, $0.090 \mathrm{mmol}$ ). After 1 hour of stirring the red solution had become yellow with a white precipitate. The solution was filtered through a plug of alumina and the solvent removed in vacuo yielding the title compound as a yellow solid $(0.030 \mathrm{~g}$, $75 \%)$.

${ }^{31} \mathrm{P} \mathrm{NMR}\left(121 \mathrm{MHz}, \mathrm{CD}_{2} \mathrm{Cl}_{2}\right): \delta 46.4\left(\mathrm{~s}, J_{\mathrm{PtP}}=2347 \mathrm{~Hz}\right)-144.5$ (septet, $J_{\mathrm{PF}}=710.5 \mathrm{~Hz}$, $\left.\mathrm{PF}_{6}\right)^{1} \mathrm{H}$ NMR (500 MHz, $\mathrm{CD}_{2} \mathrm{Cl}_{2}$ ): $\delta 7.41$ (s, PC(Ar)CH), 7.11 (s, SCCH), 2.38 (s, $\left.\mathrm{C}(\mathrm{Ar}) \mathrm{CH}_{3}\right), 1.55\left(\mathrm{vt}, J_{\mathrm{PH}}=8.0 \mathrm{~Hz}, \mathrm{PC}\left(\mathrm{CH}_{3}\right)_{3}\right),{ }^{13} \mathrm{C} \mathrm{NMR}\left(125 \mathrm{MHz}, \mathrm{C}_{6} \mathrm{D}_{6}\right): \delta 157.4$ (m, PCCO), 138.9 (s, $\left.C(\mathrm{Ar}) \mathrm{CH}_{3}\right), 134.4(\mathrm{~s}, \mathrm{PC}(\mathrm{Ar}) \mathrm{CH}), 132.6(\mathrm{~s}, \mathrm{SCCH}), 119.8(\mathrm{~m}$, $\mathrm{PC}(\mathrm{Ar})), 119.1(\mathrm{~m}, \mathrm{SCCH}), 40.0\left(\mathrm{vt}, J_{\mathrm{PC}}=10.4 \mathrm{~Hz}, \mathrm{PC}\left(\mathrm{CH}_{3}\right)_{3}\right), 30.1\left(\mathrm{~s}, \mathrm{PC}\left(\mathrm{CH}_{3}\right)_{3}\right)$, $20.4\left(\mathrm{~s}, \mathrm{C}(\mathrm{Ar}) \mathrm{CH}_{3}\right),{ }^{19} \mathrm{~F} \mathrm{NMR}\left(282 \mathrm{MHz}, \mathrm{CD}_{2} \mathrm{Cl}_{2}\right): \delta-73.4\left(\mathrm{~d}, J_{\mathrm{PF}}=710.6 \mathrm{~Hz}, \mathrm{PF}_{6}\right)$ HRMS calcd for $\mathrm{C}_{30} \mathrm{H}_{46} \mathrm{ClOP}_{2} \mathrm{PtS}\left[\mathrm{M}-\mathrm{PF}_{6}\right]^{+} \mathrm{m} / \mathrm{z}=741.2032$; found $=741.2069$. 


\section{$\left[P t\left(t-B u-x a n t p h o s-\kappa P, O, P^{\prime}\right) C l\right] P_{6}$}

A solution of $t$-Bu-xantphos $(0.014 \mathrm{~g}, 0.018 \mathrm{mmol})$ in dichloromethane $(2.0 \mathrm{~mL})$ was added to solid ammonium hexafluorophosphate $(0.006 \mathrm{~g}, 0.037 \mathrm{mmol})$ in a Schlenk tube. The reaction mixture was stirred for 1 hour before filtering through a plug of alumina. The solvent was removed in vacuo yielding the title compound as a yellow solid $(0.008 \mathrm{~g}, 50 \%)$.

${ }^{31} \mathrm{P}$ NMR $\left(121 \mathrm{MHz}, \mathrm{CD}_{2} \mathrm{Cl}_{2}\right): \delta 47.8\left(\mathrm{~s}, J_{\mathrm{PtP}}=2350.3 \mathrm{~Hz}\right),-144.5$ (septet, $J_{\mathrm{PF}}$ $\left.=710.4 \mathrm{~Hz}, \mathrm{PF}_{6}\right) .{ }^{1} \mathrm{H} \mathrm{NMR}\left(600 \mathrm{MHz}, \mathrm{CD}_{2} \mathrm{Cl}_{2}\right): \delta 7.92(\mathrm{~m}, \mathrm{PC}(\mathrm{Ar}) \mathrm{CH}), 7.82$ $(\mathrm{dd}, J=7.7,1.5 \mathrm{~Hz}, \mathrm{C}($ bridge)CCH), $7.53(\mathrm{t}, J=7.7 \mathrm{~Hz}, \mathrm{PCCCH}), 1.75$ (s, $\mathrm{C}$ (bridge) $\left.\left(\mathrm{CH}_{3}\right)_{2}\right), 1.57\left(\mathrm{vt}, J=15.9 \mathrm{~Hz}, \mathrm{PC}\left(\mathrm{CH}_{3}\right)_{3}\right) .{ }^{13} \mathrm{C} \mathrm{NMR}\left(150 \mathrm{MHz}, \mathrm{CD}_{2} \mathrm{Cl}_{2}\right)$ : $\delta 158.8$ (vt, $J=9.4 \mathrm{~Hz}, \mathrm{PCCO}$ ), 135.0 (s, PC(Ar)CH), 133.4 (s, C(bridge)CCH), 132.6 (vt, $J=5.8 \mathrm{~Hz}, \mathrm{C}($ bridge)CCH), 128.1 (vt, $J=6.4 \mathrm{~Hz}, \mathrm{PCCCH}), 118.0$ (vt, $J=32.9 \mathrm{~Hz}, \mathrm{PC}(\mathrm{Ar})), 39.8\left(\mathrm{vt}, J=21.4 \mathrm{~Hz}, \mathrm{PC}\left(\mathrm{CH}_{3}\right)_{3}\right), 34.5$ (s, C(bridge)), 33.9 (s, $\mathrm{C}$ (bridge) $\left.\left(\mathrm{CH}_{3}\right)_{2}\right), 30.0$ (vt, $\left.J=4.6 \mathrm{~Hz}, \mathrm{PC}\left(\mathrm{CH}_{3}\right)_{3}\right)$. HRMS calcd for $\mathrm{C}_{31} \mathrm{H}_{48} \mathrm{OP}_{2} \mathrm{Pt}$ $\left[\mathrm{M}-\mathrm{PF}_{6}\right]^{+} \mathrm{m} / \mathrm{z}=723.2468$; found $=723.2513$.

\section{Attempted reaction of $t$-Bu-thixantphos with $\left[\mathrm{Pt}\left(\mathrm{C}_{6} \mathrm{H}_{10}\right) \mathrm{Me}_{2}\right]$}

A solution of $t$-Bu-thixantphos $(0.020 \mathrm{~g}, 0.039 \mathrm{mmol})$ in $\mathrm{C}_{6} \mathrm{D}_{6}(0.5 \mathrm{~mL})$ was added to an NMR tube containing $\left[\mathrm{Pt}\left(\mathrm{C}_{6} \mathrm{H}_{10}\right) \mathrm{Me}_{2}\right]$. No reaction was observed after 24 hours at room temperature. The reaction was heated to $60{ }^{\circ} \mathrm{C}$. After 28 days no conversion was evident by ${ }^{31} \mathrm{P}$ or ${ }^{1} \mathrm{H}$ NMR spectroscopy. $\mathrm{CH}_{2}\left(\mathrm{SO}_{2} \mathrm{CF}_{3}\right)_{2}$ (1 eq.) was added, resulting in the immediate formation of $[(t \text {-Bu-thixantphos }) \mathrm{H}]^{+}$.

\section{$\left[\operatorname{Pt}\left(t-B u-t h i x a n t p h o s-\kappa P, O, P^{\prime}\right) \mathrm{Me}\right] \mathrm{Cl}$}

A solution of $t$-Bu-thixantphos $(0.043 \mathrm{~g}, 0.083 \mathrm{mmol})$ in toluene $(1.0 \mathrm{~mL})$ was added to solid $\left[\mathrm{Pt}\left(\mathrm{C}_{6} \mathrm{H}_{10}\right) \mathrm{ClMe}\right](0.027 \mathrm{~g}, 0.083 \mathrm{mmol})$ in a Schlenk tube. The reaction mixture was stirred for 24 hours at room temperature, during which, an off-white precipitate formed. The solution was decanted and the solid dried under vacuum, giving $[\mathrm{Pt}(t-\mathrm{Bu}$-thixantphos $) \mathrm{Me}] \mathrm{Cl}$ as an off-white powder $(0.057$ g, 90\%). 
${ }^{31} \mathrm{P}$ NMR $\left(121 \mathrm{MHz}\right.$, acetone $\left.-\mathrm{d}_{6}\right): \delta 50.5\left(\mathrm{~s}, J_{\mathrm{PtP}}=2793.3 \mathrm{~Hz}\right){ }^{1} \mathrm{H}$ NMR $(600$ $\mathrm{MHz}$, acetone $\left.-\mathrm{d}_{6}\right): \delta 7.69\left(\mathrm{~d}, J_{\mathrm{PH}}=1.8 \mathrm{~Hz}, \mathrm{PC}(\mathrm{Ar}) \mathrm{CH}\right), 7.34\left(\mathrm{~d}, J_{\mathrm{PH}}=1.2 \mathrm{~Hz}\right.$, $\mathrm{SCCH}), 2.42\left(\mathrm{~s}, \mathrm{C}(\mathrm{Ar}) \mathrm{CH}_{3}\right), 1.56\left(\mathrm{vt}, J_{\mathrm{PH}}=15.6 \mathrm{~Hz}, \mathrm{PC}\left(\mathrm{CH}_{3}\right)_{3}\right), 1.94\left(\mathrm{t}, J_{\mathrm{PH}}=5.5\right.$, $\left.{ }^{2} J_{\mathrm{PtP}}=97.4 \mathrm{~Hz}, \mathrm{Pt}-\mathrm{CH}_{3}\right),{ }^{13} \mathrm{C} \mathrm{NMR}\left(150 \mathrm{MHz}\right.$, acetone- $\left.\mathrm{d}_{6}\right): \delta 153.8(\mathrm{vt}$, $\left.J_{\mathrm{PC}}=10.3 \mathrm{~Hz}, \mathrm{PCCO}\right), 137.1\left(\mathrm{vt}, J_{\mathrm{PC}}=6.3 \mathrm{~Hz}, C(\mathrm{Ar}) \mathrm{CH}_{3}\right), 134.4(\mathrm{~s}, \mathrm{PC}(\mathrm{Ar}) C \mathrm{H})$, $131.4(\mathrm{~s}, \mathrm{SCCH}), 120.8\left(\mathrm{vt}, J_{\mathrm{PC}}=31.8 \mathrm{~Hz}, \mathrm{PC}(\mathrm{Ar})\right), 118.2\left(\mathrm{vt}, J_{\mathrm{PC}}=7.2 \mathrm{~Hz}, \mathrm{SCCH}\right)$, $38.8\left(\mathrm{vt}, J_{\mathrm{PC}}=20.6 \mathrm{~Hz}, \mathrm{PC}\left(\mathrm{CH}_{3}\right)_{3}\right), 29.6$ (obscured, assigned by HSQC correlation, $\left.\mathrm{PC}\left(\mathrm{CH}_{3}\right)_{3}\right), 19.2\left(\mathrm{~s}, \mathrm{C}(\mathrm{Ar}) \mathrm{CH}_{3}\right),-23.8\left(\mathrm{t}, \mathrm{J}_{\mathrm{PC}}=5.6,{ }^{1} J_{\mathrm{PtC}}=777.2 \mathrm{~Hz}, \mathrm{Pt}-\mathrm{CH}_{3}\right) \mathrm{HRMS}$ calcd for $\mathrm{C}_{31} \mathrm{H}_{49} \mathrm{OP}_{2} \mathrm{PtS}[\mathrm{M}-\mathrm{Cl}]^{+} \mathrm{m} / z=721.2579$; found $=721.2602$.

\section{$[\mathrm{Pt}(t-B u-x a n t p h o s) \mathrm{Me}] \mathrm{Cl}$}

A solution of $t$-Bu-xantphos $(0.024 \mathrm{~g}, 0.048 \mathrm{mmol})$ in toluene $(0.5 \mathrm{~mL})$ was added to solid $\left[\mathrm{Pt}\left(\mathrm{C}_{6} \mathrm{H}_{10}\right) \mathrm{ClMe}\right](0.016 \mathrm{~g}, 0.048 \mathrm{mmol})$ in a Schlenk tube. The reaction mixture was stirred for 24 hours at room temperature, during which, an off-white precipitate formed. The solution was decanted and the solid dried under vacuum, giving the title compound as an off-white powder $(0.028 \mathrm{~g}, 78 \%)$.

${ }^{31} \mathrm{P}$ NMR $\left(121 \mathrm{MHz}\right.$, acetone $\left.-\mathrm{d}_{6}\right): \delta 51.0\left(\mathrm{~s}, J_{\mathrm{PtP}}=2788.2 \mathrm{~Hz}\right){ }^{1} \mathrm{H}$ NMR $(500$ $\mathrm{MHz}$, acetone $\left.-\mathrm{d}_{6}\right): \delta 8.15(\mathrm{dt}, J=7.5,3.7 \mathrm{~Hz}, \mathrm{PC}(\mathrm{Ar}) \mathrm{CH}), 8.07(\mathrm{~d}, J=7.8 \mathrm{~Hz}$, $\mathrm{C}($ bridge $) \mathrm{CCH}), 7.63(\mathrm{t}, J=7.9 \mathrm{~Hz}, \mathrm{PCCCH}), 1.92\left(\mathrm{t}, J_{\mathrm{PH}}=5.4{ }^{2} J_{\mathrm{PtP}}=97.4 \mathrm{~Hz}, \mathrm{Pt}-\right.$ $\left.\mathrm{CH}_{3}\right), 1.78\left(\mathrm{~s}, \mathrm{C}(\right.$ bridge $\left.)\left(\mathrm{CH}_{3}\right)_{2}\right) 1.55\left(\mathrm{vt}, J_{\mathrm{PH}}=15.4 \mathrm{~Hz}, \mathrm{PC}\left(\mathrm{CH}_{3}\right)_{3}\right){ }^{13} \mathrm{C} \mathrm{NMR}(125$ $\mathrm{MHz}$, acetone $\left.-\mathrm{d}_{6}\right): \delta 156.1\left(\mathrm{vt}, J_{\mathrm{PC}}=10.2 \mathrm{~Hz}, \mathrm{PCCO}\right), 135.9(\mathrm{~s}, \mathrm{PC}(\mathrm{Ar}) C H), 133.2$ (s, C(bridge)CCH), 132.7 (s, C(bridge)CCH), $127.4\left(\mathrm{vt}, J_{\mathrm{PC}}=6.6 \mathrm{~Hz}, \mathrm{PCCCH}\right)$, $119.7\left(\mathrm{vt}, J_{\mathrm{PC}}=34.1 \mathrm{~Hz}, \mathrm{PC}(\mathrm{Ar})\right), 39.5\left(\mathrm{vt}, J_{\mathrm{PC}}=21.4,{ }^{2} J_{\mathrm{PtC}}=43.3 \mathrm{~Hz}, \mathrm{PC}\left(\mathrm{CH}_{3}\right)_{3}\right)$, 35.0 (s, C(bridge) ), 33.7 (s, C(bridge) $\left.\left(\mathrm{CH}_{3}\right)_{2}\right), 30.4\left(\mathrm{vt}, \mathrm{J}_{\mathrm{PC}}=6.1 \mathrm{~Hz}, \mathrm{PC}\left(\mathrm{CH}_{3}\right)_{3}\right)$. $-23.9\left(\mathrm{t}, J_{\mathrm{PC}}=5.6,{ }^{1} J_{\mathrm{PtC}}=774.6 \mathrm{~Hz}, \mathrm{Pt}-\mathrm{CH}_{3}\right) \mathrm{HRMS}$ calcd for $\mathrm{C}_{32} \mathrm{H}_{51} \mathrm{OP}_{2} \mathrm{Pt}[\mathrm{M}-\mathrm{Cl}]^{+}$ $\mathrm{m} / \mathrm{z}=703.3014 ;$ found $=703.2987$.

\section{$[\mathrm{Pt}(t-\mathrm{Bu}$-sixantphos) $\mathrm{Me}] \mathrm{Cl}$}

A solution of $t$-Bu-sixantphos $(0.026 \mathrm{~g}, 0.051 \mathrm{mmol})$ in $\mathrm{C}_{6} \mathrm{D}_{6}$ was added to an NMR tube containing $\left[\mathrm{Pt}\left(\mathrm{C}_{6} \mathrm{H}_{10}\right) \mathrm{ClMe}\right](0.017 \mathrm{~g}, 0.051 \mathrm{mmol})$. After 72 hours at room temperature little reaction was observed, so the solution was heated to $50{ }^{\circ} \mathrm{C}$ for 24 hours during which time a white precipitate formed. NMR spectroscopy 
showed no unreacted $t$-Bu-sixantphos was evident in the ${ }^{1} \mathrm{H}$ or ${ }^{31} \mathrm{P}$ NMR spectra. The solution was decanted and the title compound was dried under vacuum (0.014 g, 36\%).

${ }^{31} \mathrm{P}$ NMR $\left(121 \mathrm{MHz}\right.$, acetone $\left.-\mathrm{d}_{6}\right): \delta 48.7\left(\mathrm{~s}, J_{\mathrm{PtP}}=2762.9 \mathrm{~Hz}\right){ }^{1} \mathrm{H} \mathrm{NMR}(500$ $\mathrm{MHz}$, acetone $\left.-\mathrm{d}_{6}\right): \delta 8.39-8.36(\mathrm{~m}, \mathrm{PC}(\mathrm{Ar}) \mathrm{CH}), 8.11(\mathrm{dd}, 7.1,1.7 \mathrm{~Hz}, \mathrm{SiCCH})$, $7.65(\mathrm{t}, J=7.4 \mathrm{~Hz}, \mathrm{PCCCH}), 2.01\left(\mathrm{t}, J=5.5^{2} J_{\mathrm{PtP}}=98.6 \mathrm{~Hz}, \mathrm{Pt}-\mathrm{CH}_{3}\right), 1.55(\mathrm{vt}$, $\left.J=15.2 \mathrm{~Hz}, \mathrm{PC}\left(\mathrm{CH}_{3}\right)_{3}\right), 0.59\left(\mathrm{~s}, \mathrm{Si}\left(\mathrm{CH}_{3}\right)_{2}\right) .{ }^{13} \mathrm{C} \mathrm{NMR}\left(125 \mathrm{MHz}\right.$, acetone $\left.-\mathrm{d}_{6}\right): \delta$ $167.3\left(\mathrm{vt}, J_{\mathrm{PC}}=8.2 \mathrm{~Hz}, \mathrm{PCCO}\right), 140.72$ (s, PC(Ar)CH), 140.67 (s, SiCCH), 126.1 (vt, $\left.J_{\mathrm{PC}}=6.1 \mathrm{~Hz}, \mathrm{PCCCH}\right), 123.5(\mathrm{~s}, \mathrm{SiCCH}), 121.2\left(\mathrm{vt}, J_{\mathrm{PC}}=32.4 \mathrm{~Hz}, \mathrm{PC}(\mathrm{Ar})\right), 39.7(\mathrm{vt}$, $\left.J_{\mathrm{PC}}=21.8 \mathrm{~Hz}, \mathrm{PC}\left(\mathrm{CH}_{3}\right)_{3}\right), 30.6\left(\mathrm{vt}, J_{\mathrm{PC}}=5.6 \mathrm{~Hz}, \mathrm{PC}\left(\mathrm{CH}_{3}\right)_{3}\right),-0.5\left(\mathrm{~s}, \mathrm{Si}\left(\mathrm{CH}_{3}\right)_{2}\right),-22.7$ $\left(\mathrm{t}, J_{\mathrm{PC}}=5.6,{ }^{1} J_{\mathrm{PtC}}=780.5 \mathrm{~Hz}, \mathrm{Pt}-\mathrm{CH}_{3}\right)$. HRMS calcd for $\mathrm{C}_{31} \mathrm{H}_{51} \mathrm{OP}_{2} \mathrm{PtSi}[\mathrm{M}-\mathrm{Cl}]^{+}$ $\mathrm{m} / \mathrm{z}=724.2829$; found $=724.2760$.

\subsection{Palladium Complexes}

\section{Reaction of $t$-Bu-thixantphos with $\left[\operatorname{Pd}(\mathrm{nb})_{3}\right]$}

A solution of $t$-Bu-thixantphos $(0.051 \mathrm{~g}, 0.10 \mathrm{mmol})$ in $\mathrm{C}_{6} \mathrm{D}_{6}(0.5 \mathrm{~mL})$ was added to an NMR tube containing $\left[\mathrm{Pd}(\mathrm{nb})_{3}\right](0.038 \mathrm{~g}, 0.10 \mathrm{mmol})$ and a small crystal of norbornene. The NMR tube was sealed with a J. Young's tap. After 72 hours at room temperature no $t$-Bu-thixantphos was present by ${ }^{31} \mathrm{P}$ NMR spectroscopy. The mixture contained $[\mathrm{Pd}(t-\mathrm{Bu}$-thixantphos $)](62.0 \%)$ and $[\mathrm{Pd}(t-\mathrm{Bu}-$ thixantphos)(nb)] (38.0\%).

[Pd(t-Bu-thixantphos)(nb)]

${ }^{31} \mathrm{P}$ NMR (121 MHz, $\left.\mathrm{C}_{6} \mathrm{D}_{6}\right): \delta 33.6(\mathrm{~s})$

\section{[Pd(t-Bu-thixantphos)]}

A solution of $t$-Bu-thixantphos $(0.056 \mathrm{~g}, 0.11 \mathrm{mmol})$ in toluene $(1.0 \mathrm{~mL})$ was added to a Schlenk tube containing $\left[\mathrm{Pd}(\mathrm{nb})_{3}\right](0.042 \mathrm{~g}, 0.11 \mathrm{mmol})$ and a small crystal of norbornene $(0.012 \mathrm{~g})$. The reaction was stirred overnight. The solvent 
was removed in vacuo leaving $[\mathrm{Pd}(t$-Bu-thixantphos $)]$ as a oily brown solid $(0.061$ g, 90\%).

${ }^{31} \mathrm{P}$ NMR (121 MHz, $\left.\mathrm{C}_{6} \mathrm{D}_{6}\right): \delta 42.9$ (s) ${ }^{1} \mathrm{H}$ NMR $\left(500 \mathrm{MHz}, \mathrm{C}_{6} \mathrm{D}_{6}\right): \delta 7.35$ (s, PC(Ar)CH), 6.83 (s, SCCH), $1.95\left(\mathrm{~s}, \mathrm{C}(\mathrm{Ar}) \mathrm{CH}_{3}\right), 1.46\left(\mathrm{vt}, J_{\mathrm{PH}}=13.7 \mathrm{~Hz}\right.$, $\left.\mathrm{PC}\left(\mathrm{CH}_{3}\right)_{3}\right),{ }^{13} \mathrm{C} \mathrm{NMR}\left(125 \mathrm{MHz}, \mathrm{C}_{6} \mathrm{D}_{6}\right): \delta 155.9\left(\mathrm{vt}, J_{\mathrm{PC}}=14.0 \mathrm{~Hz}, \mathrm{PCCO}\right), 133.6$ (s, PC(Ar)CH), 131.9 (s, $\left.C(\mathrm{Ar}) \mathrm{CH}_{3}\right), 128.8$ (s, SCCH) $127.8\left(\mathrm{vt}, J_{\mathrm{PC}}=8.2 \mathrm{~Hz}\right.$, $\mathrm{PC}(\mathrm{Ar})), 124.6\left(\mathrm{vt}, J_{\mathrm{PC}}=5.3 \mathrm{~Hz}, \mathrm{SCCH}\right), 35.7\left(\mathrm{vt}, J_{\mathrm{PC}}=3.8 \mathrm{~Hz}, \mathrm{PC}\left(\mathrm{CH}_{3}\right)_{3}\right), 31.9(\mathrm{~s}$, $\left.\mathrm{PC}\left(\mathrm{CH}_{3}\right)_{3}\right), 20.4\left(\mathrm{~s}, \mathrm{C}(\mathrm{Ar}) \mathrm{CH}_{3}\right)$

\section{Reaction of $t$-Bu-xantphos with $\left[\operatorname{Pd}(\mathrm{nb})_{3}\right]$}

A solution of $t$-Bu-xantphos $(0.046 \mathrm{~g}, 0.092 \mathrm{mmol})$ in $\mathrm{C}_{6} \mathrm{D}_{6}(0.5 \mathrm{~mL})$ was added to an NMR tube containing $\left[\mathrm{Pd}(\mathrm{nb})_{3}\right](0.036 \mathrm{~g}, 0.092 \mathrm{mmol})$ and a small crystal of norbornene. The NMR tube was sealed with a J. Young's tap. The NMR after one hour showed a mixture with no uncoordinated $t$-Bu-xantphos and multiplet products. The mixture contained $[\mathrm{Pd}(t$-Bu-xantphos) $)$ (73.1\%) and $[\mathrm{Pd}(t-B u-x a n t-$ phos)(nb)] (12.6\%), as well as several other unidentified phosphorus containing compounds.

\section{$[\operatorname{Pd}(t-B u-x a n t p h o s)(n b)]$}

${ }^{31} \mathrm{P}$ NMR (121 MHz, $\left.\mathrm{C}_{6} \mathrm{D}_{6}\right): \delta 32.6$ (s)

\section{$[\operatorname{Pd}(t-B u-x a n t p h o s)]$}

${ }^{31} \mathrm{P}$ NMR (121 MHz, $\left.\mathrm{C}_{6} \mathrm{D}_{6}\right): \delta 42.6(\mathrm{~s})$

\section{[Pd(t-Bu-sixantphos)]}

A solution of $t$-Bu-sixantphos $(0.040 \mathrm{~g}, 0.078 \mathrm{mmol})$ in $\mathrm{C}_{6} \mathrm{D}_{6}(0.5 \mathrm{~mL})$ was added to a J. Young's tap NMR tube containing $\left.[\mathrm{Pd}(\mathrm{nb}))_{3}\right](0.030 \mathrm{~g}, 0.078 \mathrm{mmol})$ and norbornene $(0.010 \mathrm{~g})$. The tube was sealed under argon and the reaction was followed by NMR spectroscopy. After 48 hours at room temperature a mixture 
of uncoordination $t$-Bu-sixantphos (56.9\%) and [Pd(t-Bu-sixantphos)] (43.1\%) is present by ${ }^{31} \mathrm{P}$ NMR. After 24 hours no progress was observed. However, after six months, the mixture contained $t$-Bu-sixantphos $(32.0 \%)$ and $[\mathrm{Pd}(t$-Bu-sixantphos)] (68\%).

${ }^{31} \mathrm{P}$ NMR (121 MHz, $\left.\mathrm{C}_{6} \mathrm{D}_{6}\right): \delta 41.9$ (s) ${ }^{1} \mathrm{H}$ NMR $\left(300 \mathrm{MHz}, \mathrm{C}_{6} \mathrm{D}_{6}\right): \delta 7.51(\mathrm{~m}$, PC(Ar)CH), 7.30 (dd, $J=7.1,1.5 \mathrm{~Hz}, \mathrm{SiCCH}), 6.92(\mathrm{t}, J=7.4 \mathrm{~Hz}, \mathrm{PCCCH}), 1.63$ (bs, $\left.\mathrm{PC}\left(\mathrm{CH}_{3}\right)_{3}\right), 0.23\left(\mathrm{~s}, \mathrm{Si}\left(\mathrm{CH}_{3}\right)_{2}\right)$.

\section{$\left[\operatorname{Pd}(t-B u-t h i x a n t p h o s)\left(\eta^{2}-\mathrm{O}_{2}\right)\right]$}

Air was bubbled through a solution of [Pd(t-Bu-thixantphos)] (0.058 g, 0.093 $\mathrm{mmol})$ in $\mathrm{C}_{6} \mathrm{D}_{6}(0.5 \mathrm{~mL})$ for 10 minutes. The solution turned green immediately. The sample was filtered through a plug of alumina and reduced to dryness giving the title compound as a green solid $(0.052 \mathrm{~g}, 90 \%)$.

${ }^{31} \mathrm{P}$ NMR (121 MHz, $\mathrm{CDCl}_{3}$ ): $\delta 52.0$ (s) ${ }^{1} \mathrm{H} \mathrm{NMR}\left(500 \mathrm{MHz}, \mathrm{CDCl}_{3}\right): \delta 7.49$ (s, 2H, $\mathrm{Ar}), 7.23(\mathrm{~s}, 2 \mathrm{H}, \mathrm{Ar}), 2.32\left(\mathrm{~s}, \mathrm{C}(\mathrm{Ar}) \mathrm{CH}_{3}\right), 1.50\left(\mathrm{~d}, J=13.7 \mathrm{~Hz}, \mathrm{PC}\left(\mathrm{CH}_{3}\right)_{3}\right)$.

\section{trans-[Pd(t-Bu-sixantphos $\left.) \mathrm{Cl}_{2}\right]$}

Combined $t$-Bu-sixantphos $(0.015 \mathrm{~g}, 0.029 \mathrm{mmol})$ and $\left[\mathrm{Pd}(\mathrm{cod}) \mathrm{Cl}_{2}\right](0.008 \mathrm{~g}, 0.029$ $\mathrm{mmol}$ ) in an sealed tube and stirred at $60{ }^{\circ} \mathrm{C}$ for hours then $35^{\circ} \mathrm{C}$. The solvent was removed in vacuo yielding the title compound as a red solid $(0.018 \mathrm{~g}, 89 \%)$.

${ }^{31} \mathrm{P}$ NMR (121 MHz, $\left.\mathrm{CDCl}_{3}\right): \delta 45.7$ (s) ${ }^{1} \mathrm{H}$ NMR (500 $\left.\mathrm{MHz}, \mathrm{CDCl}_{3}\right): \delta 7.71$ (m, PC(Ar)CH), 7.68 (d, $7.1 \mathrm{~Hz}, \mathrm{SiCCH}), 7.33$ (t, $7.3 \mathrm{~Hz}, \mathrm{PCCCH}), 1.60$ (vt, $\left.J_{\mathrm{PH}}=7.3 \mathrm{~Hz}, \mathrm{PC}\left(\mathrm{CH}_{3}\right)_{3}\right), 0.52\left(\mathrm{~s}, \mathrm{Si}\left(\mathrm{CH}_{3}\right)_{2}\right) .{ }^{13} \mathrm{C} \mathrm{NMR}\left(125 \mathrm{MHz}, \mathrm{CDCl}_{3}\right): \delta$ 165.1 (vt, $J_{\mathrm{PC}}=6.8 \mathrm{~Hz}, \mathrm{PCCO}$ ), 137.6 (s, PC(Ar)CH), 136.9 (s, SiCCH), 124.9 (s, $\mathrm{SiCCH}), 123.1(\mathrm{~s}, \mathrm{PCCCH}), 121.8\left(\mathrm{vt}, J_{\mathrm{PC}}=13.4 \mathrm{~Hz}, \mathrm{PC}(\mathrm{Ar})\right), 39.6\left(\mathrm{vt}, J_{\mathrm{PC}}=6.3 \mathrm{~Hz}\right.$, $\left.\mathrm{PC}\left(\mathrm{CH}_{3}\right)_{3}\right), 31.1\left(\mathrm{vt}, J_{\mathrm{PC}}=2.7 \mathrm{~Hz}, \mathrm{PC}\left(\mathrm{CH}_{3}\right)_{3}\right),-2.0\left(\mathrm{~s}, \mathrm{Si}\left(\mathrm{CH}_{3}\right)_{2}\right)$.

${ }^{31} \mathrm{P}$ NMR (121 MHz, $\left.\mathrm{CD}_{2} \mathrm{Cl}_{2}\right): \delta 46.7$ (s) ${ }^{1} \mathrm{H}$ NMR $\left(600 \mathrm{MHz}, \mathrm{CD}_{2} \mathrm{Cl}_{2}\right): \delta 7.72-7.74$ (m, 2H, SiCCH, PC(Ar)CH), 7.35 (t, J = 7.2 Hz, PCCCH), 1.59 (vt, $J=15.0 \mathrm{~Hz}$, $\left.\mathrm{PC}\left(\mathrm{CH}_{3}\right)_{3}\right), 0.53\left(\mathrm{~s}, \mathrm{Si}\left(\mathrm{CH}_{3}\right)_{2}\right) .{ }^{13} \mathrm{C} \mathrm{NMR}\left(150 \mathrm{MHz}, \mathrm{CD}_{2} \mathrm{Cl}_{2}\right): \delta 165.4$ (bs, PCCO), 137.9 (s, PC(Ar)CH), 137.2 (s, SiCCH), 125.2 (s, SiCCH), 123.2 (s, PCCCH), 122.0 
(vt, $J=25.4 \mathrm{~Hz}, \mathrm{PC}(\mathrm{Ar})), 39.7\left(\mathrm{vt}, J=12.8 \mathrm{~Hz}, \mathrm{PC}\left(\mathrm{CH}_{3}\right)_{3}\right), 31.1$ (bs, $\left.\mathrm{PC}\left(\mathrm{CH}_{3}\right)_{3}\right)$, $-2.2\left(\mathrm{~s}, \mathrm{Si}\left(\mathrm{CH}_{3}\right)_{2}\right)$.

${ }^{31} \mathrm{P}$ NMR (121 MHz, $\left.\mathrm{C}_{6} \mathrm{D}_{6}\right): \delta 42.0$ (s) ${ }^{1} \mathrm{H}$ NMR $\left(500 \mathrm{MHz}, \mathrm{C}_{6} \mathrm{D}_{6}\right): \delta 7.51$ (dtd, $J=7.7,3.9,1.8 \mathrm{~Hz}, \mathrm{PC}(\mathrm{Ar}) \mathrm{CH}), 7.30(\mathrm{dd}, J=7.0,1.6 \mathrm{~Hz}, \mathrm{SiCCH}), 6.92(\mathrm{t}, J=7.3 \mathrm{~Hz}$, $\mathrm{PCCCH}), 1.64$ (bs, PC $\left.\left(\mathrm{CH}_{3}\right)_{3}\right), 0.23\left(\mathrm{~s}, \mathrm{Si}\left(\mathrm{CH}_{3}\right)_{2}\right) .{ }^{13} \mathrm{C} \mathrm{NMR}\left(125 \mathrm{MHz}, \mathrm{C}_{6} \mathrm{D}_{6}\right): \delta$ 164.9 (vt, $J=6.2 \mathrm{~Hz}, \mathrm{PCCO}), 137.2$ (s, PC(Ar)CH), 135.5 (s, SiCCH), 125.4 (vt, $J=3.0 \mathrm{~Hz}, \mathrm{SiCCH}), 124.1$ (vt, $J=26.9 \mathrm{~Hz}, \mathrm{PC}(\mathrm{Ar})), 121.9$ (vt, $J=4.8 \mathrm{~Hz}, \mathrm{PCCCH})$, $39.4\left(\mathrm{vt}, J=11.5 \mathrm{~Hz}, \mathrm{PC}\left(\mathrm{CH}_{3}\right)_{3}\right), 31.6\left(\mathrm{bs}, \mathrm{PC}\left(\mathrm{CH}_{3}\right)_{3}\right),-3.1$ (bs, $\left.\mathrm{Si}\left(\mathrm{CH}_{3}\right)_{2}\right)$.

HRMS calcd for $\mathrm{C}_{30} \mathrm{H}_{48} \mathrm{OP}_{2} \mathrm{SiPdCl}[\mathrm{M}-\mathrm{Cl}]^{+} \mathrm{m} / \mathrm{z}=655.1676$; found = 655.1663.

\section{trans-[Pd(t-Bu-thixantphos $\left.) \mathrm{Cl}_{2}\right]$}

t-Bu-Thixantphos $(0.197 \mathrm{~g}, 0.38 \mathrm{mmol})$ and $\left[\mathrm{Pd}(\mathrm{cod}) \mathrm{Cl}_{2}\right](0.108 \mathrm{~g}, 0.38 \mathrm{mmol})$ were dissolved in toluene then heated to $40{ }^{\circ} \mathrm{C}$ for three days. The solvent was removed in vacuo yielding the title compound as an orange solid $(0.265 \mathrm{~g}, 100 \%)$.

${ }^{31} \mathrm{P}$ NMR (121 MHz, $\mathrm{CD}_{2} \mathrm{Cl}_{2}$ ): $\delta 40.0$ (s) ${ }^{1} \mathrm{H}$ NMR (600 MHz, $\mathrm{CD}_{2} \mathrm{Cl}_{2}$ ): $\delta 7.16$ (s, $\mathrm{SCCH}), 7.15$ (s, PC(Ar)CH), 2.33 (s, $\left.\mathrm{C}(\mathrm{Ar}) \mathrm{CH}_{3}\right), 1.59\left(\mathrm{vt}, J=13.6 \mathrm{~Hz}, \mathrm{PC}\left(\mathrm{CH}_{3}\right)_{3}\right)$, ${ }^{13} \mathrm{C}$ NMR (150 MHz, $\left.\mathrm{CD}_{2} \mathrm{Cl}_{2}\right): \delta 155.1(\mathrm{vt}, J=8.7 \mathrm{~Hz}, \mathrm{PCCO}), 134.4(\mathrm{~s}, \mathrm{SCCH})$, 133.1 (vt, $J=5.8 \mathrm{~Hz}, \mathrm{C}(\mathrm{Ar}) \mathrm{CH}_{3}$ ), 130.2 (s, PC(Ar)CH), 123.8 (vt, $J=26.1 \mathrm{~Hz}$, $\mathrm{PC}(\mathrm{Ar})), 123.3(\mathrm{vt}, J=6.3 \mathrm{~Hz}, \mathrm{SCCH}), 39.5\left(\mathrm{vt}, J=12.1 \mathrm{~Hz}, \mathrm{PC}\left(\mathrm{CH}_{3}\right)_{3}\right), 31.3(\mathrm{bs}$, $\left.\mathrm{PC}\left(\mathrm{CH}_{3}\right)_{3}\right), 20.7$ (s, $\left.\mathrm{C}(\mathrm{Ar}) \mathrm{CH}_{3}\right)$. HRMS calcd for $\mathrm{C}_{30} \mathrm{H}_{46} \mathrm{OP}_{2} \mathrm{SPdCl}[\mathrm{M}-\mathrm{Cl}]^{+} \mathrm{m} / z=$ 657.1468 ; found $=657.1446$.

\section{trans-[Pd(t-Bu-xantphos $\left.) \mathrm{Cl}_{2}\right]$}

t-Bu-Xantphos $(0.021 \mathrm{~g}, 0.042 \mathrm{mmol})$ and $\left[\mathrm{Pd}(\mathrm{cod}) \mathrm{Cl}_{2}\right](0.012 \mathrm{~g}, 0.042 \mathrm{mmol})$ were dissolved in $\mathrm{C}_{6} \mathrm{D}_{6}$ in an NMR tube then heated to $40{ }^{\circ} \mathrm{C}$ for three days. The solvent was removed in vacuo and the residue was washed with pentane yielding the title compound as an orange solid $(0.015 \mathrm{~g}$, 53\%).

${ }^{31} \mathrm{P}$ NMR (121 MHz, $\left.\mathrm{C}_{6} \mathrm{D}_{6}\right): \delta 40.0$ (s) ${ }^{1} \mathrm{H}$ NMR $\left(600 \mathrm{MHz}, \mathrm{C}_{6} \mathrm{D}_{6}\right): \delta 7.33$ (dtd, $J=8.1,3.4,1.5 \mathrm{~Hz}, \mathrm{PC}(\mathrm{Ar}) \mathrm{CH}), 7.13(\mathrm{dd}, J=7.7,1.2 \mathrm{~Hz}, \mathrm{C}($ bridge $) \mathrm{CCH})$, $6.87(\mathrm{t}, J=7.7 \mathrm{~Hz}, \mathrm{PCCCH}), 1.65$ (bs, PC $\left.\left(\mathrm{CH}_{3}\right)_{3}\right), 1.32$ (bs, C(bridge) $\left.\left(\mathrm{CH}_{3}\right)_{2}\right)$. 
${ }^{13} \mathrm{C}$ NMR (150 MHz, $\left.\mathrm{C}_{6} \mathrm{D}_{6}\right): \delta 156.9$ (vt, $\left.J=8.7 \mathrm{~Hz}, \mathrm{PCCO}\right), 135.4$ (vt, $J=4.6 \mathrm{~Hz}, \mathrm{C}($ bridge $) \mathrm{CCH}), 132.8$ (s, PC(Ar)CH), 126.2 (s, C(bridge)CCH), 123.0 (vt, $J=27.7 \mathrm{~Hz}, \mathrm{PC}(\mathrm{Ar})), 122.2$ (vt, $J=5.3 \mathrm{~Hz}, \mathrm{PCCCH}), 39.1$ (vt, $\left.J=12.1 \mathrm{~Hz}, \mathrm{PC}\left(\mathrm{CH}_{3}\right)_{3}\right), 36.2$ (s, $C$ (bridge)), 31.5 (bs, $\left.\mathrm{PC}\left(\mathrm{CH}_{3}\right)_{3}\right), 24.9$ (s, $\mathrm{C}$ (bridge $\left.)\left(\mathrm{CH}_{3}\right)_{2}\right)$.

${ }^{31} \mathrm{P}$ NMR (121 MHz, $\left.\mathrm{CD}_{2} \mathrm{Cl}_{2}\right): \delta 54.1$ (s) ${ }^{1} \mathrm{H} \mathrm{NMR}\left(600 \mathrm{MHz}, \mathrm{CD}_{2} \mathrm{Cl}_{2}\right): \delta 7.87$ (d, $J=7.6 \mathrm{~Hz}, \mathrm{C}$ (bridge)CCH), $7.76(\mathrm{~m}, \mathrm{PC}(\mathrm{Ar}) \mathrm{CH}), 7.52(\mathrm{t}, J=7.7 \mathrm{~Hz}, \mathrm{PCCCH})$, 1.75 (s, C(bridge) $\left.\left(\mathrm{CH}_{3}\right)_{2}\right), 1.59\left(\mathrm{vt}, J=15.5 \mathrm{~Hz}, \mathrm{PC}\left(\mathrm{CH}_{3}\right)_{3}\right),{ }^{13} \mathrm{C}$ NMR $(150 \mathrm{MHz}$, $\left.\mathrm{CD}_{2} \mathrm{Cl}_{2}\right): \delta 156.6(\mathrm{vt}, J=11.6 \mathrm{~Hz}, \mathrm{PCCO}$ ), 134.6 (s, C(bridge)CCH) 132.0 (s, PC(Ar)CH), 126.5 (s, C(bridge)CCH), 118.1 (vt, $J=24.3 \mathrm{~Hz}, \mathrm{PC}(\mathrm{Ar})), 117.6$ (s, PCCCH), $39.8\left(\mathrm{vt}, J=13.8 \mathrm{~Hz}, \mathrm{PC}\left(\mathrm{CH}_{3}\right)_{3}\right), 32.6$ (s, C(bridge)), 31.4 (s, $\mathrm{C}$ (bridge) $\left.\left(\mathrm{CH}_{3}\right)_{2}\right), 30.3\left(\mathrm{vt}, J=5.8 \mathrm{~Hz}, \mathrm{PC}\left(\mathrm{CH}_{3}\right)_{3}\right)$.

HRMS calcd for $\mathrm{C}_{31} \mathrm{H}_{48} \mathrm{OP}_{2} \mathrm{PdCl}[\mathrm{M}-\mathrm{Cl}]^{+} \mathrm{m} / \mathrm{z}=635.1925$; found = 635.1966.

\section{$\left[\mathrm{Pd}\left(t-\mathrm{Bu}-\right.\right.$ thixantphos- $\left.\left.\kappa P, O, P^{\prime}\right) \mathrm{Cl}\right] \mathrm{PF}_{6}$}

$\mathrm{NH}_{4} \mathrm{PF}_{6}(0.052 \mathrm{~g}, 0.32 \mathrm{mmol})$ and trans-[Pd(t-Bu-thixantphos $\left.) \mathrm{Cl}_{2}\right](0.050,0.061$ $\mathrm{mmol}$ ) were combined in an Schlenk tube and suspended in $\mathrm{CH}_{2} \mathrm{Cl}_{2}(10 \mathrm{~mL})$. The reaction mixture was stirred overnight and the volume was reduced by half. The solution was filtered through a plug of alumina and reduced to dryness giving the title compound as a yellow solid $(0.039 \mathrm{~g}, 68 \%)$.

${ }^{31} \mathrm{P} \mathrm{NMR}\left(121 \mathrm{MHz}, \mathrm{CD}_{2} \mathrm{Cl}_{2}\right): \delta 56.4$ (s) -144.5 (septet, $\left.J_{\mathrm{PF}}=710.5 \mathrm{~Hz}, P F_{6}\right){ }^{1} \mathrm{H} \mathrm{NMR}$ $\left(500 \mathrm{MHz}, \mathrm{CD}_{2} \mathrm{Cl}_{2}\right): \delta 7.36\left(\mathrm{~d}, J_{\mathrm{PH}}=2.0 \mathrm{~Hz}, \mathrm{PC}(\mathrm{Ar}) \mathrm{CH}\right), 7.15(\mathrm{~s}, \mathrm{SCCH}), 2.38(\mathrm{~s}$, $\left.\mathrm{C}(\mathrm{Ar}) \mathrm{CH}_{3}\right), 1.58\left(\mathrm{vt}, J_{\mathrm{PH}}=8.2 \mathrm{~Hz}, \mathrm{PC}\left(\mathrm{CH}_{3}\right)_{3}\right),{ }^{13} \mathrm{C} \mathrm{NMR}\left(125 \mathrm{MHz}, \mathrm{C}_{6} \mathrm{D}_{6}\right): \delta 154.86$ $\left(\mathrm{vt}, J_{\mathrm{PC}}=6.5 \mathrm{~Hz}, \mathrm{PCCO}\right), 138.3\left(\mathrm{vt}, J_{\mathrm{PC}}=5.8 \mathrm{~Hz}, C(\mathrm{Ar}) \mathrm{CH}_{3}\right), 134.6(\mathrm{~s}, \mathrm{PC}(\mathrm{Ar}) C \mathrm{H})$, 132.6 (s, SCCH), 119.1 (s, SCCH), 118.7 (vt, $\left.J_{\mathrm{PC}}=10.8 \mathrm{~Hz}, \mathrm{PC}(\mathrm{Ar})\right), 40.2$ (vt, $\left.J_{\mathrm{PC}}=7.2 \mathrm{~Hz}, \mathrm{PC}\left(\mathrm{CH}_{3}\right)_{3}\right), 30.1\left(\mathrm{~s}, \mathrm{PC}\left(\mathrm{CH}_{3}\right)_{3}\right), 20.4\left(\mathrm{~s}, \mathrm{C}(\mathrm{Ar}) \mathrm{CH}_{3}\right){ }^{19} \mathrm{~F} \mathrm{NMR}(282$ $\left.\mathrm{MHz}, \mathrm{CD}_{2} \mathrm{Cl}_{2}\right): \delta-73.4\left(\mathrm{~d}, J_{\mathrm{PF}}=710.6 \mathrm{~Hz}, \mathrm{PF}_{6}\right) \mathrm{HRMS}$ calcd for $\mathrm{C}_{30} \mathrm{H}_{46} \mathrm{ClOP}_{2} \mathrm{PdS}$ $\left[\mathrm{M}-\mathrm{PF}_{6}\right]^{+} \mathrm{m} / \mathrm{z}=653.1489 ;$ found $=653.1527$. 


\section{$\left[\mathrm{Pd}\left(t-B u-s i x a n t p h o s-\kappa P, O, P^{\prime}\right) \mathrm{Cl}\right] \mathrm{PF}_{6}$}

This compound was synthesised as for $\left[\mathrm{Pd}\left(t\right.\right.$-Bu-thixantphos- $\left.\left.\mathrm{k} P, \mathrm{O}, \mathrm{P}^{\prime}\right) \mathrm{Cl}\right] \mathrm{PF}_{6}$ using trans-[Pd(t-Bu-sixantphos $\left.) \mathrm{Cl}_{2}\right](0.018 \mathrm{~g}, 0.026 \mathrm{mmol})$ and $\mathrm{NH}_{4} \mathrm{PF}_{6}(0.005 \mathrm{~g}$, $0.030 \mathrm{mmol})$ giving the title compound as a yellow solid $(0.020 \mathrm{~g}, 96 \%)$.

${ }^{31} \mathrm{P}$ NMR (121 MHz, $\mathrm{CD}_{2} \mathrm{Cl}_{2}$ ): $\delta 52.4$ (s, t-Bu-sixantphos) -144.5 (septet, $\left.J_{\mathrm{PF}}=710.3 \mathrm{~Hz}, \mathrm{PF}_{6}\right){ }^{1} \mathrm{H} \mathrm{NMR}\left(500 \mathrm{MHz}, \mathrm{CD}_{2} \mathrm{Cl}_{2}\right): \delta 8.07(\mathrm{~m}, \mathrm{PC}(\mathrm{Ar}) \mathrm{CH}), 7.91$ $(\mathrm{dd}, J=7.0,1 / 9 \mathrm{~Hz}, \mathrm{SiCCH}), 7.56(\mathrm{t}, J=7.4 \mathrm{~Hz}, \mathrm{PCCCH}), 1.59(\mathrm{vt}, J=16.1 \mathrm{~Hz}$, $\left.\mathrm{PC}\left(\mathrm{CH}_{3}\right)_{3}\right), 0.60\left(\mathrm{~s}, \mathrm{Si}\left(\mathrm{CH}_{3}\right)_{2}\right) .{ }^{13} \mathrm{C} \mathrm{NMR}\left(125 \mathrm{MHz}, \mathrm{CD}_{2} \mathrm{Cl}_{2}\right): \delta 168.4$ (vt, $J=10.6 \mathrm{~Hz}, \mathrm{PCCO}), 140.9$ (s, SiCCH), 139.7 (s, PC(Ar)CH), 126.1 (vt, $J=5.3 \mathrm{~Hz}$, PCCCH), 124.1 (s, SiCCH) 118.0 (vt, $J=22.1 \mathrm{~Hz}, \mathrm{PC}(\mathrm{Ar})), 40.2(\mathrm{vt}, J=15.4 \mathrm{~Hz}$, $\left.\mathrm{PC}\left(\mathrm{CH}_{3}\right)_{3}\right), 30.2\left(\mathrm{vt}, J=5.3 \mathrm{~Hz}, \mathrm{PC}\left(\mathrm{CH}_{3}\right)_{3}\right),-0.4\left(\mathrm{~s}, \mathrm{Si}\left(\mathrm{CH}_{3}\right)_{2}\right) .{ }^{19} \mathrm{~F} \mathrm{NMR}(282 \mathrm{MHz}$, $\left.\mathrm{CD}_{2} \mathrm{Cl}_{2}\right): \delta-73.4\left(\mathrm{~d}, J=-706.6 \mathrm{~Hz}, \mathrm{PF}_{6}\right) \mathrm{HRMS}$ calcd for $\mathrm{C}_{30} \mathrm{H}_{48} \mathrm{ClOP}_{2} \mathrm{PdSi}\left[\mathrm{M}-\mathrm{PF}_{6}\right]^{+}$ $\mathrm{m} / \mathrm{z}=651.1694 ;$ found $=651.1717$.

\section{$\left[\mathrm{Pd}\left(t-\mathrm{Bu}-\mathrm{xantphos}-\kappa P, O, P^{\prime}\right) \mathrm{Cl}\right] \mathrm{PF}_{6}$}

This compound was synthesised as for $\left[\mathrm{Pd}\left(t-\mathrm{Bu}\right.\right.$-thixantphos- $\left.\left.\kappa P, O, P^{\prime}\right) \mathrm{Cl}\right] \mathrm{PF}_{6}$ using trans-[Pd(t-Bu-xantphos $\left.) \mathrm{Cl}_{2}\right](0.020 \mathrm{~g}, 0.030 \mathrm{mmol})$ and $\mathrm{NH}_{4} \mathrm{PF}_{6}(0.005 \mathrm{~g}$, $0.030 \mathrm{mmol})$ giving the title compound as a yellow solid $(0.020 \mathrm{~g}, 86 \%)$.

${ }^{31} \mathrm{P}$ NMR (121 MHz, $\mathrm{CD}_{2} \mathrm{Cl}_{2}$ ): $\delta 58.3$ (s, t-Bu-xantphos), -144.5 (septet, $\left.J_{\mathrm{PF}}=710.4 \mathrm{~Hz}, \mathrm{PF}_{6}\right){ }^{1} \mathrm{H} \mathrm{NMR}\left(500 \mathrm{MHz}, \mathrm{CD}_{2} \mathrm{Cl}_{2}\right): \delta 7.87(\mathrm{dd}, J=7.7,1.6 \mathrm{~Hz}$, $\mathrm{PC}(\mathrm{Ar}) \mathrm{CH}), 7.84(\mathrm{~m}, \mathrm{C}($ bridge $) \mathrm{CCH}), 7.53(\mathrm{t}, J=7.9 \mathrm{~Hz}, \mathrm{PCCCH}), 1.76$ (s, $\mathrm{C}$ (bridge) $\left.\left(\mathrm{CH}_{3}\right)_{2}\right), 1.59$ (vt, $J=16.1 \mathrm{~Hz}, \mathrm{C}$ (bridge) $\left.\left(\mathrm{CH}_{3}\right)_{2}\right){ }^{13} \mathrm{C} \mathrm{NMR}(125 \mathrm{MHz}$, $\mathrm{CD}_{2} \mathrm{Cl}_{2}$ ): $\delta 156.7$ (vt, $J=11.6 \mathrm{~Hz}, \mathrm{PCCO}$ ), 135.1 (s, PC(Ar)CH), 133.4 (s, $\mathrm{C}$ (bridge)CCH), 132.7 (bs, C(bridge)CCH), 127.4 (vt, $J=6.6 \mathrm{~Hz}, \mathrm{PCCCH}), 117.1$ (vt, $J=24.0 \mathrm{~Hz}, \mathrm{PC}(\mathrm{Ar})), 40.1\left(\mathrm{vt}, J=14.9 \mathrm{~Hz}, \mathrm{PC}\left(\mathrm{CH}_{3}\right)_{3}\right), 34.5$ (s, $C$ (bridge)), 34.0 (s, C(bridge) $\left.\left(\mathrm{CH}_{3}\right)_{2}\right), 30.0\left(\mathrm{vt}, J=7.3 \mathrm{~Hz}, \mathrm{PC}\left(\mathrm{CH}_{3}\right)_{3}\right) .{ }^{19} \mathrm{~F} \mathrm{NMR}(282 \mathrm{MHz}$, $\left.\mathrm{CD}_{2} \mathrm{Cl}_{2}\right): \delta-73.5\left(\mathrm{~d}, J=-709.6 \mathrm{~Hz}, \mathrm{PF}_{6}\right) \mathrm{HRMS}$ calcd for $\mathrm{C}_{31} \mathrm{H}_{48} \mathrm{ClOP}_{2} \mathrm{Pd}\left[\mathrm{M}-\mathrm{PF}_{6}\right]^{+}$ $\mathrm{m} / \mathrm{z}=635.1925 ;$ found $=635.1959$. 


\section{Bibliography}

[1] Lamansky, S.; Djurovich, P.; Murphy, D.; Abdel-Razzaq, F.; Lee, H.-E.; Adachi, C.; Burrows, P. E.; Forrest, S. R.; Thompson, M. E. J. Am. Chem. Soc. 2001, 123, 4304-4312.

[2] Rosenberg, B.; VanCamp, L.; Trosko, J. E.; Mansour, V. H. Nature 1969, 222, 385-386.

[3] Wilson, J. J.; Lippard, S. J. Chem. Rev. 2013, 114, 4470-4495.

[4] Orpen, A. G.; Connelly, N. G. Organometallics 2001, 9, 1206-1210.

[5] Noyori, R. Angew. Chem. Int. Ed. Engl. 2002, 41, 2008-2022.

[6] Nicolaou, K. C.; Bulger, P. G.; Sarlah, D. Angew. Chem. Int. Ed. 2005, 44, 4442-4489.

[7] Knowles, W. S. Angew. Chem. Int. Ed. 2002, 41, 1998-2007.

[8] Tolman, C. A. Chem. Rev. 1977, 77, 313-348.

[9] Banger, K. K.; Brisdon, A. K.; Herbert, C. J.; Ghaba, H. A.; Tidmarsh, I. S. J Fluorine Chem 2009, 130, 1117-1129.

[10] Dunne, B. J.; Morris, R. B.; Orpen, A. G. J. Chem. Soc., Dalton Trans. 1991, 653-661.

[11] Roodt, A.; Otto, S.; Steyl, G. Coord. Chem. Rev. 2003, 245, 121-137.

[12] Mann, B. E.; Musco, A. J. Chem. Soc., Dalton Trans. 1980, 776-785.

[13] Tiburcio, J.; Bernès, S.; Torrens, H. Polyhedron 2006, 25, 1549-1554.

[14] Tolman, C. A. J. Am. Chem. Soc. 1970, 92, 2953-2956. 
[15] Connelly, N. G.; Damhus, T.; Hartshorn, R. M.; Hutton, A. T. Nomenclature of Inorganic Chemistry - IUPAC Recommendations 2005; The Royal Society of Chemistry: Cambridge, 2005.

[16] Pregosin, P. S. NMR in Organometallic Chemistry; Wiley VCH GmbH \& Co. KGaA: Weinheim, 2012.

[17] Beckmann, U.; Süslüyan, D.; Kunz, P. C. Phosphorus, Sulfur Silicon Relat Elem 2011, 186, 2061-2070.

[18] Allman, T.; Goel, R. G. Can. J. Chem. 1982, 60, 716-722.

[19] Hillier, A. C.; Sommer, W. J.; Yong, B. S.; Petersen, J. L.; Cavallo, L.; Nolan, S. P. Organometallics 2003, 22, 4322-4326.

[20] Clavier, H.; Nolan, S. P. Chem. Commun. 2010, 46, 841.

[21] Agbossou, F.; Carpentier, J.-F.; Mortreux, A. Chem. Rev. 2001, 95, 2485-2506.

[22] Shimizu, H.; Nagasaki, I.; Matsumura, K.; Sayo, N.; Saito, T. Acc. Chem. Res. 2007, 40, 1385-1393.

[23] Housecroft, C. E.; Sharpe, A. G. Inorganic Chemistry, 2nd ed.; Pearson Education Limited: Madrid, 2005.

[24] Casey, C. P.; Whiteker, G. T. Israel J. Chem. 1990, 30, 299-304.

[25] Dierkes, P.; van Leeuwen, P. J. Chem. Soc., Dalton Trans. 1999, 1519-1529.

[26] Lai, T. W.; Sen, A. Organometallics 2001, 3, 866-870.

[27] Drent, E.; Van Broekhoven, J.; Doyle, M. J. J. Organomet. Chem. 2014, 417, 235-251.

[28] van Leeuwen, P. W. N. M., Claver, C., Eds. Rhodium Catalyzed Hydroformylation; Kluwer Academic Publishers: Dordrecht, 2000.

[29] Eastham, G. R.; Tooze, R. P.; Heaton, B. T.; Iggo, J. A.; Whyman, R.; Zacchini, S. Chem. Commun. 2000, 609-610.

[30] Kranenburg, M.; Vanderburgt, Y.; Kamer, P.; van Leeuwen, P.; Goubitz, K.; Frannje, J. Organometallics 1995, 14, 3081-3089. 
[31] Tsuji, J. Palladium Reagents and Catalysts - Innovations in Organic Synthesis; John Wiley \& Sons, Ltd.: Chichester, 1995.

[32] Freixa, Z.; van Leeuwen, P. Dalton Trans. 2003, 1890-1901.

[33] Freixa, Z.; Beentjes, M. S.; Batema, G. D.; Dieleman, C. B.; van Strijdonck, G. P. F.; Reek, J. N. H.; Kamer, P. C. J.; Fraanje, J.; Goubitz, K.; van Leeuwen, P. W. N. M. Angew. Chem. Int. Ed. 2003, 42, 1284-1287.

[34] Kamer, P.; van Leeuwen, P.; Reek, J. Acc. Chem. Res. 2001, 34, 895-904.

[35] Serrano-Becerra, J. M.; Morales-Morales, D. Curr. Org. Synth. 2009, 6, 169192.

[36] Choi, J.; MacArthur, A. H. R.; Brookhart, M.; Goldman, A. S. Chem. Rev. 2011, 111, 1761-1779.

[37] van der Vlugt, J. I.; Reek, J. N. H. Angew. Chem. Int. Ed. 2009, 48, 88328846.

[38] Kataoka, Y.; Tsuji, Y.; Matsumoto, O.; Ohashi, M.; Yamagata, T.; Tani, K. J. Chem. Soc., Chem. Commun. 1995, 2099-2100.

[39] Singleton, J. T. Tetrahedron 2003, 59, 1837-1857.

[40] Takenaka, K.; Minakawa, M.; Uozumi, Y. J. Am. Chem. Soc. 2005, 127, 12273-12281.

[41] Zim, D.; Gruber, A. S.; Ebeling, G.; Dupont, J.; Monteiro, A. L. Org. Lett. 2000, 2, 2881-2884.

[42] Hahn, F. E.; Jahnke, M. C.; Pape, T. Organometallics 2007, 26, 150-154.

[43] Moulton, C. J.; Shaw, B. L. J. Chem. Soc., Dalton Trans. 1976, 1020-1024.

[44] Alcock, N. W.; Brown, J. M.; Jeffery, J. C. J. Chem. Soc., Dalton Trans. 1976, $583-588$.

[45] Zhu, H. J.; Ziegler, T. Organometallics 2008, 27, 1743-1749.

[46] Albrecht, M.; Gossage, R. A.; Lutz, M.; Spek, A. L.; van Koten, G. Chem. Eur. J. 2000, 6, 1431-1445. 
[47] Albrecht, M.; Lutz, M.; Spek, A. L.; van Koten, G. Nature 2000, 406, 970974.

[48] Albrecht, M.; van Koten, G. Angew. Chem. Int. Ed. 2001, 40, 3750-3781.

[49] Bedford, R. B.; Draper, S. M.; Noelle Scully, P.; Welch, S. L. New J. Chem. 2000, 24, 745-747.

[50] Kimura, T.; Uozumi, Y. Organometallics 2006, 25, 4883-4887.

[51] Obora, Y.; Kimura, M.; Ohtake, T.; Tokunaga, M.; Tsuji, Y. Organometallics 2006, 25, 2097-2100.

[52] Sandhya, K. S.; Suresh, C. H. Organometallics 2011, 30, 3888-3891.

[53] Holscher, M.; Prechtl, M. H. G.; Leitner, W. Chem. Eur. J. 2007, 13, 66366643.

[54] Crabtree, R. H. J. Chem. Soc., Dalton Trans. 2001, 2951-2951.

[55] van der Veen, L. A.; Keeven, P. K.; Kamer, P. C. J.; van Leeuwen, P. W. N. M. J. Chem. Soc., Dalton Trans. 2000, 2105-2112.

[56] Malaise, G.; Sortais, J.-B.; Barloy, L.; Pfeffer, M.; Kyritsakas, N. Polyhedron 2006, 25, 3349-3365.

[57] Goertz, W.; Keim, W.; Vogt, D.; Englert, U.; Boele, M. D. K.; van der Veen, L. A.; Kamer, P. C. J.; van Leeuwen, P. W. N. M. J. Chem. Soc., Dalton Trans. 1998, 2981-2988.

[58] van Haaren, R. J.; Keeven, P. H.; van der Veen, L. A.; Goubitz, K.; van Strijdonck, G. P. F.; Oevering, H.; Reek, J. N. H.; Kamer, P. C. J.; van Leeuwen, P. W. N. M. Inorg. Chim. Acta 2002, 327, 108-115.

[59] Goedheijt, M. S.; Kamer, P.; van Leeuwen, P. J. Mol. Catal. A-Chem. 1998, $134,243-249$.

[60] Goedheijt, M. S.; Reek, J. N. H.; Kamer, P. C. J.; van Leeuwen, P. W. N. M. Chem. Commun. 1998, 2431-2432.

[61] van der Veen, L. A.; Kamer, P. C. J.; van Leeuwen, P. W. N. M. Organometallics 1999, 18, 4765-4777. 
[62] van der Veen, L. A.; Keeven, P. H.; Schoemaker, G. C.; Reek, J.; Kamer, P.; van Leeuwen, P.; Lutz, M.; Spek, A. L. Organometallics 2000, 19, 872-883.

[63] van der Veen, L. A.; Boele, M. D. K.; Bregman, F. R.; Kamer, P. C. J.; van Leeuwen, P. W. N. M.; Goubitz, K.; Fraanje, J.; Schenk, H.; Bo, C. J. Am. Chem. Soc. 1998, 120, 11616-11626.

[64] van Leeuwen, P. W. N. M.; Kamer, P. C. J.; Reek, J. N. H.; Dierkes, P. Chem. Rev. 2000, 100, 2741-2770.

[65] van der Slot, S. C.; Duran, J.; Luten, J.; Kamer, P. C. J.; van Leeuwen, P. W. N. M. Organometallics 2002, 21, 3873-3883.

[66] Kranenburg, M.; Kamer, P.; van Leeuwen, P.; Vogt, D.; Keim, W. J. Chem. Soc., Chem. Commun. 1995, 2177-2178.

[67] Birkholz née Gensow, M.-N.; Freixa, Z.; van Leeuwen, P. W. N. M. Chem. Soc. Rev. 2009, 38, 1099-1118.

[68] Allen, F. H. Acta Crystall. B-Stru. 2002, 58, 380-388.

[69] Escalle, A.; Mora, G.; Gagosz, F.; Mezailles, N.; Le Goff, X. F.; Jean, Y.; Le Floch, P. Inorg. Chem. 2009, 48, 8415-8422.

[70] Moxham, G. L.; Randell-Sly, H. E.; Brayshaw, S. K.; Woodward, R. L.; Weller, A. S.; Willis, M. C. Angew. Chem. Int. Ed. 2006, 45, 7618-7622.

[71] Moxham, G. L.; Randell-Sly, H.; Brayshaw, S. K.; Weller, A. S.; Willis, M. C. Chem. Eur. J. 2008, 14, 8383-8397.

[72] Pawley, R. J.; Moxham, G. L.; Dallanegra, R.; Chaplin, A. B.; Brayshaw, S. K.; Weller, A. S.; Willis, M. C. Organometallics 2010, 29, 1717-1728.

[73] Zuideveld, M. A.; Swennenhuis, B. H. G.; Boele, M. D. K.; Guari, Y.; van Strijdonck, G. P. F.; Reek, J. N. H.; Kamer, P. C. J.; Goubitz, K.; Fraanje, J.; Lutz, M.; Spek, A. L.; van Leeuwen, P. W. N. M. J. Chem. Soc., Dalton Trans. 2002, 2308-2317.

[74] Miedaner, A.; Raebiger, J. W.; Curtis, C. J.; Miller, S. M.; DuBois, D. L. Organometallics 2004, 23, 2670-2679. 
[75] Raebiger, J. W.; Miedaner, A.; Curtis, C. J.; Miller, S. M.; Anderson, O. P.; DuBois, D. L. J. Am. Chem. Soc. 2004, 126, 5502-5514.

[76] Asensio, G.; Cuenca, A. B.; Esteruelas, M. A.; Medio-Simón, M.; Oliván, M.; Valencia, M. Inorg. Chem. 2010, 49, 8665-8667.

[77] Alós, J.; Bolaño, T.; Esteruelas, M. A.; Oliván, M.; Oñate, E.; Valencia, M. Inorg. Chem. 2013, 52, 6199-6213.

[78] Alós, J.; Bolaño, T.; Esteruelas, M. A.; Oliván, M.; Oñate, E.; Valencia, M. Inorg. Chem. 2014, 53, 1195-1209.

[79] Bakhmutov, V. I.; Bozoglian, F.; Gómez, K.; González, G.; Grushin, V. V.; Macgregor, S. A.; Martin, E.; Miloserdov, F. M.; Novikov, M. A.; Panetier, J. A.; Romashov, L. V. Organometallics 2012, 31, 1315-1328.

[80] Jover, J.; Miloserdov, F. M.; Benet-Buchholz, J.; Grushin, V. V.; Maseras, F. Organometallics 2014, 33, 6531-6543.

[81] Esteruelas, M. A.; Oliván, M.; Vélez, A. Inorg. Chem. 2013, 52, 5339-5349.

[82] Haibach, M. C.; Wang, D. Y.; Emge, T. J.; Krogh-Jespersen, K.; Goldman, A. S. Chem. Sci. 2013, 4, 3683-3692.

[83] Esteruelas, M. A.; Oliván, M.; Vélez, A. Inorg. Chem. 2013, 52, 12108-12119.

[84] Mispelaere-Canivet, C.; Spindler, J. F.; Perrio, S.; Beslin, P. Tetrahedron 2005, 61, 5253-5259.

[85] Dongol, K. G.; Koh, H.; Sau, M.; Chai, C. L. L. Adv. Synth. Catal. 2007, 349, 1015-1018.

[86] Ohshima, T.; Miyamoto, Y.; Ipposhi, J.; Nakahara, Y.; Utsunomiya, M.; Mashima, K. J. Am. Chem. Soc. 2009, 131, 14317-14328.

[87] Cabello-Sanchez, N.; Jean, L.; Maddaluno, J.; Lasne, M. C.; Rouden, J. J. Org. Chem. 2007, 72, 2030-2039.

[88] Partyka, D. V.; Updegraff, J. B.; Zeller, M.; Hunter, A. D.; Gray, T. G. Dalton Trans. 2010, 39, 5388-5397. 
[89] Pintado-Alba, A.; de la Riva, H. c.; Nieuwhuyzen, M.; Bautista, D.; Raithby, P. R.; Sparkes, H. A.; Teat, S. J.; L pez-de Luzuriaga, J. M.; Lagunas, M. C. Dalton Trans. 2004, 3459-3467.

[90] Zhan, J.-H.; Lv, H.; Yu, Y.; Zhang, J.-L. Adv. Synth. Catal. 2012, 354, 15291541.

[91] Miyata, K.; Nakagawa, T.; Kawakami, R.; Kita, Y.; Sugimoto, K.; Nakashima, T.; Harada, T.; Kawai, T.; Hasegawa, Y. Chem. Eur. J. 2011, $17,521-528$.

[92] Miyata, K.; Nakanishi, T.; Fushimi, K.; Hasegawa, Y. J Photoch. Photobio. A 2012, 235, 35-39.

[93] Friis, S. D.; Skrydstrup, T.; Buchwald, S. L. Org. Lett. 2014, 16, 4296-4299.

[94] Dang, T. T.; Ramalingam, B.; Shan, S. P.; Seayad, A. M. ACS Catal. 2013, 3, 2536-2540.

[95] Liu, Y.-Y.; Yang, X.-H.; Huang, X.-C.; Wei, W.-T.; Song, R.-J.; Li, J.-H. J. Org. Chem. 2013, 78, 10421-10426.

[96] Raoufmoghaddam, S.; Drent, E.; Bouwman, E. Adv. Synth. Catal. 2013, 355, 717-733.

[97] Ashcroft, C. P.; Fussell, S. J.; Wilford, K. Tetrahedron Lett. 2013, 54, 45294532.

[98] Behr, A.; Vorholt, A. J.; Rentmeister, N. Chem. Eng. Sci. 2013, 99, 38-43.

[99] Raoufmoghaddam, S.; Drent, E.; Bouwman, E. ChemSusChem 2013, 6, 1759-1773.

[100] Shang, R.; Xu, Q.; Fu, Y.; Guo, Q.; Liu, L. Method for Preparing 2Benzylpyridine Compound in the Presence of Palladium Catalyst and Phosphine Ligand; CN 101863826, 2010.

[101] Shang, R.; Fu, Y.; Liu, L. Process for Preparation of Naproxen from 2-halo6-methoxynaphthalenes; CN 102001933, 2011. 
[102] Shang, R.; Huang, Z.; Fu, Y.; Guo, Q.; Liu, L. Method for Synthesizing 2,2'-(5-methyl-1,3-phenylene)di(2-methylpriopionitrile) as Intermediate for Preparing Anastrozole; CN 101973911, 2011.

[103] Shang, R.; Huang, Z.; Fu, Y.; Liu, L. Process for Preparation of Flurbiprofen; CN 101973869, 2011.

[104] Shang, R.; Ji, D.; Fu, Y.; Guo, Q.; Liu, L. Method for Preparing Arylactonitrile Compounds; CN 102452867, 2012.

[105] Brandstadt, K.; Cook, S.; Nguyen, B. T.; Surgenor, A.; Taylor, R.; Tzou, M.-s. Cobalt Containing Hydrosilylation Catalysts and Compositions Containing the Catalysts; PCT Int. Appl. WO 2013/043783 A2, 2013.

[106] Brandstadt, K.; Cook, S.; Nguyen, B. T.; Surgenor, A.; Taylor, R.; Tzou, M.-s. Nickel Containing Hydrosilylation Catalysts and Compositions Containing the Catalysts; PCT Int. Appl. WO 2013043785 A2 20130328, 2013.

[107] Brandstadt, K.; Cook, S.; Nguyen, B. T.; Surgenor, A.; Taylor, R.; Tzou, M.-s. Iron-Containing Hydrosilylation Catalysts and Compositions Containing the Catalysts; PCT Int. Appl. WO 2013043912 A2 20130328, 2013.

[108] Brandstadt, K.; Cook, S.; Nguyen, B. T.; Surgenor, A.; Taylor, R.; Tzou, M.-s. Ruthenium Containing Hydrosilylation Catalysts and Compositions Containing the Catalysts; PCT Int. Appl. WO 2013043787 A2 20130328, 2013.

[109] Surgenor, A.; Brandstadt, K.; Nguyen, B. T.; Cook, S.; Tzou, M.-s.; Taylor, R. Vanadium Containing Hydrosilylation Catalysts and Compositions Containing the Catalysts; PCT Int. Appl. WO 2013043825 A2 20130328, 2013.

[110] Kranenburg, M.; Kamer, P. C. J.; van Leeuwen, P. W. N. M. Eur. J. Inorg. Chem. 1998, 25-27.

[111] Meijboom, R.; Bowen, R. J.; Berners-Price, S. J. Coord. Chem. Rev. 2009, 253, $325-342$.

[112] van der Veen, L. A.; Kamer, P. C. J.; van Leeuwen, P. W. N. M. Angew. Chem. Int. Ed. 1999, 38, 336-338.

[113] van der Veen, L. A.; Kamer, P. C. J.; van Leeuwen, P. W. N. M. CATTECH 2002, 6, 116-120. 
[114] Zuidema, E.; Escorihuela, L.; Eichelsheim, T.; Carbo, J. J.; Bo, C.; Kamer, P. C. J.; van Leeuwen, P. W. N. M. Chem. Eur. J. 2008, 14, 1843-1853.

[115] Petocz, G.; Berente, Z.; Kegl, T.; Kollar, L. J. Organomet. Chem. 2004, 689, 1188-1193.

[116] Bronger, R. P. J.; Kamer, P. C. J.; van Leeuwen, P. W. N. M. Organometallics 2003, 22, 5358-5369.

[117] Marimuthu, T.; Friedrich, H. B. ChemCatChem 2012, 4, 2090-2095.

[118] Vivekananda Bhatt, M.; Kulkarni, S. U. Synthesis 1983, 1983, 249-282.

[119] Gilman, H.; Haubein, A. H.; Hartzfeld, H. J. Org. Chem. 1954, 19, 10341040.

[120] Gilman, H.; Bebb, R. L. J. Am. Chem. Soc. 1939, 61, 109-112.

[121] Wittig, G.; Fuhrman, B. Chem. Ber. 1940, 73, 1197.

[122] Wakefield, B. J. Organolithium Methods. 1988.

[123] Turck, A.; Ple, N.; Pollet, P.; Mojovic, L.; Duflos, J.; Queguiner, G. J. Heterocyclic Chem. 1997, 34, 621-627.

[124] Harris, R. K. Can. J. Chem. 1964, 42, 2275-2281.

[125] Abraham, R. J.; Bernstein, H. J. Can. J. Chem. 1961, 39, 216-230.

[126] Hierso, J.-C. Chem. Rev. 2014, 114, 4838-4867.

[127] van Haaren, R. J.; van Strijdonck, G. P. F.; Oevering, H.; Reek, J. N. H.; Kamer, P. C. J.; van Leeuwen, P. W. N. M. Eur. J. Inorg. Chem. 2001, 837843.

[128] Dudle, B.; Rajesh, K.; Blacque, O.; Berke, H. J. Am. Chem. Soc. 2011, 133, 8168-8178.

[129] Fanjul, T.; Eastham, G.; Floure, J.; Forrest, S. J. K.; Haddow, M. F.; Hamilton, A.; Pringle, P. G.; Orpen, A. G.; Waugh, M. Dalton Trans. 2012, 42, 100.

[130] Aguilà, D.; Escribano, E.; Speed, S.; Talancón, D.; Yermán, L.; Alvarez, S. Dalton Trans. 2009, 6610. 
[131] Becke, A. D. J. Chem. Phys. 1993, 98, 5648-5652.

[132] Lee, C,; Yang, W.; Parr, R. G. Phys. Rev. B 1988, 785-789.

[133] Vosko, S. H.; Wilk, L.; Nusair, M. Can. J. Chem. 1980, 1200-1211.

[134] Stephens, P. J.; Devlin, F. J.; Chabalowski, C. F.; J, F. M. The Journal of Physical Chemistry 1994, 98, 11623-11627.

[135] Andrae, D.; Haeussermann, U.; Dolg, M.; Stoll, H.; Preuss, H. Theoretica Chimica Acta 1990, 77, 123-141.

[136] Weigend, F.; Ahlrichs, R. Phys. Chem. Chem. Phys. 2005, 7, 3297.

[137] Freixa, Z.; van Leeuwen, P. W. N. M. Coord. Chem. Rev. 2008, 252, 17551786.

[138] Yano, T. B. Chem. Soc. JPN 2006, 50, 1272-1277.

[139] Netherton, M. R.; Fu, G. C. Org. Lett. 2001, 3, 4295-4298.

[140] Koppel, I. A.; Koppel, J.; Pihl, V.; Leito, I.; Mishima, M.; Vlasov, V. M.; Yagupolskii, L. M.; Taft, t. 1. R. W. J. Chem. Soc., Perkin Trans. 2 2000, 11251133.

[141] Muller, A.; Otto, S.; Roodt, A. Dalton Trans. 2008, 650-657.

[142] Andersen, N. G.; Keay, B. A. Chem. Rev. 2001, 101, 997-1030.

[143] Bungu, P. N.; Otto, S. J. Organomet. Chem. 2007, 692, 3370-3379.

[144] Bent, H. A. Chem. Rev. 2001, 61, 275-311.

[145] Jahromi, B. T.; Kharat, A. N.; Zamanian, S.; Bakhoda, A.; Mashayekh, K.; Khazaeli, S. Appl. Catal. A-Gen. 2012, 433-434, 188-196.

[146] Enghag, P. Encylcopedia of the Elements; Wiley VCH GmbH \& Co. KGaA, 2004.

[147] Berners-Price, S. J.; Johnson, R. K.; Giovenella, A. J.; Faucette, L. F.; Mirabelli, C. K.; Sadler, P. J. J. Inorg. Biochem. 1988, 33, 285-295. 
[148] Liu, J. J.; Galettis, P.; Farr, A.; Maharaj, L.; Samarasinha, H.; McGechan, A. C.; Baguley, B. C.; Bowen, R. J.; Berners-Price, S. J.; McKeage, M. J. J. Inorg. Biochem. 2008, 102, 303-310.

[149] Suzuki, A. J. Organomet. Chem. 1999, 576, 147-168.

[150] Iglesias, M. J.; Nicasio, M. C.; Caballero, A.; Pérez, P. J. Dalton Trans. 2012, 42, 1191-1195.

[151] Sawamura, M.; Hamashima, H.; Ito, Y. J. Org. Chem. 1990, 55, 5935-5936.

[152] Yanagisawa, A.; Kageyama, H.; Nakatsuka, Y.; Asakawa, K.; Matsumoto, Y.; Yamamoto, H. Angew. Chem. Int. Ed. 1999, 38, 3701-3703.

[153] Balakrishna, M. S.; Venkateswaran, R.; Mobin, S. M. Polyhedron 2008, 27, 899-904.

[154] Kobayashi, N.; Higashimura, H.; Suenobu, K. Luminescent Silver Complexes; PCT Int. Appl. WO 2010064601 A1 20100610, 2010.

[155] Kobayashi, N.; Higashimura, H. Preparation of Triphenylphosphine-Silver Complexes and Luminescent Membrane Containing Silver Complexes; PCT Int. Appl. WO2011152358 A1 20111208, 2011.

[156] Kobayashi, N.; Higashimura, H. Preparation of Amine-Containing Phosphine Compound and its Metal Complex; PCT Int. Appl. WO 2012056966 A1 20120503, 2012.

[157] Kaltzoglou, A.; Cox, P. J.; Aslanidis, P. Polyhedron 2007, 26, 1634-1642.

[158] Pingen, D.; Lebl, T.; Lutz, M.; Nichol, G. S.; Kamer, P. C. J.; Vogt, D. Organometallics 2014, 33, 2798-2805.

[159] Ainscough, E. W.; Bowmaker, G. A.; Brodie, A. M.; Freeman, G. H.; Hanna, J. V.; Jameson, G. B.; Otter, C. A. Polyhedron 2011, 30, 638-646.

[160] Bayler, A.; Schier, A.; Bowmaker, G. A.; Schmidbaur, H. J. Am. Chem. Soc. 1997, 118, 7006-7007.

[161] van der Vlugt, J. I.; Siegler, M. A.; Janssen, M.; Vogt, D.; Spek, A. L. Organometallics 2009, 28, 7025-7032. 
[162] Heuer, B.; Pope, S. J.; Reid, G. Polyhedron 2000, 19, 743-749.

[163] Joshi, B. Unpublished Work.

[164] Yam, V. W.-W.; Fung, W. K.-M.; Cheung, K.-K. Organometallics 1997, 16, 2032-2037.

[165] Wing-Wah Yam, V.; Kam-Wing Lo, K.; Kit-Mai Fung, W.; Wang, C.-R. Coordination Chemistry Reviews 2003, 171, 17-41.

[166] Xu, L.-J.; Wang, J.-Y.; Zhang, L.-Y.; Shi, L.-X.; Chen, Z.-N. Organometallics 2013, 32, 5402-5408.

[167] Enghag, P. Encyclopedia of the Elements; Wiley VCH GmbH \& Co. KGaA: Weinheim, 2004.

[168] Heck, R. M.; Farrauto, R. J. Applied Catalysis A: General 2001, 221, 443457.

[169] Cui, X. H.; Burgess, K. Chem. Rev. 2005, 105, 3272-3296.

[170] Pospech, J.; Fleischer, I.; Franke, R.; Buchholz, S.; Beller, M. Angew. Chem. Int. Ed. 2013, 52, 2852-2872.

[171] Osborn, J. A.; Jardine, F. H.; Young, J. F.; Wilkinson, G. J. Chem. Soc. A 1966, 1711-1732.

[172] Evans, D. A.; Fu, G. C.; Hoveyda, A. H. J. Am. Chem. Soc. 2001, 110, 69176918.

[173] Ojima, I.; Kogure, T. Organometallics 2001, 1, 1390-1399.

[174] Sharpless, K. B. Angew. Chem. Int. Ed. 2002, 41, 2024-2032.

[175] Knowles, W. S.; Sabacky, M. J. Chem. Commun. (London) 1968, 1445-1446.

[176] Knowles, W. S.; Noyori, R. Acc. Chem. Res. 2007, 40, 1238-1239.

[177] Dallanegra, R.; Chaplin, A. B.; Weller, A. S. Organometallics 2012, 31, 27202728.

[178] Pawley, R. J.; Huertos, M. A.; Lloyd-Jones, G. C.; Weller, A. S.; Willis, M. C. Organometallics 2012, 31, 5650-5659. 
[179] Canepa, G.; Brandt, C. D.; Ilg, K.; Wolf, J.; Werner, H. Chem. Eur. J. 2003, 9, 2502-2515.

[180] Bleeke, J. R.; Donaldson, A. J. Organometallics 1986, 5, 2401-2405.

[181] Fryzuk, M. D.; Piers, W. E.; Rettig, S. J.; Einstein, F. W.; Jones, T.; Albright, T. A. J. Am. Chem. Soc. 1989, 111, 5709-5721.

[182] Hashimoto, H.; Suzuki, T.; Tobita, H. Dalton Trans. 2010, 39, 9386-9400.

[183] Khan, M. M.; Khan, B. T.; Begum, S.; Aali, S. M. Journal of Molecular Catalysis 1988, 49, 43-58.

[184] Hermann, D.; Gandelman, M.; Rozenberg, H.; Shimon, L. J. W.; Milstein, D. Organometallics 2002, 21, 812-818.

[185] van der Boom, M. E.; Liou, S.-Y.; Ben-David, Y.; Shimon, L. J.; Milstein, D. J. Am. Chem. Soc. 1998, 120, 6531-6541.

[186] Winter, A. M.; Eichele, K.; Mack, H.-G.; Potuznik, S.; Mayer, H. A.; Kaska, W. C. J. Organomet. Chem. 2003, 682, 149-154.

[187] Salem, H.; Shimon, L. J. W.; Leitus, G.; Weiner, L.; Milstein, D. Organometallics 2008, 27, 2293-2299.

[188] Fryzuk, M. D.; MacNeil, P. A.; Rettig, S. J. Organometallics 1986, 5, 2469 2476.

[189] Hanson, S. K.; Heinekey, D. M.; Goldberg, K. I. Organometallics 2008, 27, 1454-1463.

[190] Bronger, R. P. J.; Silva, S. M.; Kamer, P. C. J.; van Leeuwen, P. W. N. M. Chem. Commun. 2002, 3044-3045.

[191] Bronger, R. P. J.; Bermon, J. P.; Herwig, J.; Kamer, P. C. J.; van Leeuwen, P. W. N. M. Adv. Synth. Catal. 2004, 346, 789-799.

[192] Bronger, R. P. J.; Bermon, J. P.; Reek, J. N. H.; Kamer, P. C. J.; van Leeuwen, P. W. N. M.; Carter, D. N.; Licence, P.; Poliakoff, M. J. Mol. Catal. A-Chem. 2004, 224, 145-152. 
[193] Bronger, R. P. J.; Silva, S. M.; Kamer, P. C. J.; van Leeuwen, P. W. N. M. Dalton Trans. 2004, 1590-1596.

[194] Buhling, A.; Kamer, P.; van Leeuwen, P.; Elgersma, J. W.; Goubitz, K.; Fraanje, J. Organometallics 1997, 16, 3027-3037.

[195] Buhling, A.; Kamer, P.; vanLeeuwen, P.; Elgersma, J. W. J. Mol. Catal. AChem. 1997, 116, 297-308.

[196] Dieleman, C. B.; Kamer, P. C. J.; Reek, J. N. H.; van Leeuwen, P. W. N. M. Helv. Chim. Acta 2001, 84, 3269-3280.

[197] Leclercq, L.; Hapiot, F.; Tilloy, S.; Ramkisoensing, K.; Reek, J. N. H.; van Leeuwen, P. W. N. M.; Monflier, E. Organometallics 2005, 24, 2070-2075.

[198] van Leeuwen, P. W. N. M.; Kamer, P. C. J.; Reek, J. N. H. Pure Appl. Chem. 1999, 71, 1443-1452.

[199] Mora, G.; Deschamps, B.; van Zutphen, S.; Le Goff, X. F.; Ricard, L.; Le Floch, P. Organometallics 2007, 26, 1846-1855.

[200] Sandee, A. J.; van der Veen, L. A.; Reek, J. N. H.; Kamer, P. C. J.; Lutz, M.; Spek, A. L.; van Leeuwen, P. W. N. M. Angew. Chem. Int. Ed. 1999, 38, 3231-3235.

[201] Silva, S. M.; Bronger, R. P. J.; Freixa, Z.; Dupont, J.; van Leeuwen, P. W. N. M. New J. Chem. 2003, 27, 1294-1296.

[202] van der Vlugt, J. I.; Sablong, R.; Magusin, P. C. M. M.,; Mills, A. M.; Spek, A. L.; Vogt, D. Organometallics 2004, 23, 3177-3183.

[203] Zuidema, E.; Daura-Oller, E.; Carbo, J. J.; Bo, C.; van Leeuwen, P. W. N. M. Organometallics 2007, 26, 2234-2242.

[204] Zuidema, E.; Goudriaan, P. E.; Swennenhuis, B. H. G.; Kamer, P. C. J.; van Leeuwen, P. W. N. M.; Lutz, M.; Spek, A. L. Organometallics 2010, 29, 1210 1221.

[205] Johnson, H. C.; McMullin, C. L.; Pike, S. D.; Macgregor, S. A.; Weller, A. S. Angew. Chem. Int. Ed. 2013, 52, 9776-9780. 
[206] Guiu, E.; Caporali, M.; Munoz, B.; Muller, C.; Lutz, M.; Spek, A. L.; Claver, C.; van Leeuwen, P. W. N. M. Organometallics 2006, 25, 3102-3104.

[207] Bertoux, F.; Tilloy, S.; Monflier, E.; Castanet, Y.; Mortreux, A. J. Mol. Catal. A-Chem. 1999, 138, 53-57.

[208] Kjonaas, R. A.; Williams, P. E.; Counce, D. A.; Crawley, L. R. J. Chem. Educ. 2011, 88, 825-828.

[209] Franke, R.; Selent, D.; Börner, A. Chem. Rev. 2012, 112, 5675-5732.

[210] Henderson, W.; Nickleson, B. K.; McCaffrey, L. J. Polyhedron 1998, 17, 4291-4313.

[211] Sanger, A. R. Canadian Journal of Chemistry 1984, 62, 2168-2169.

[212] Sanger, A. R. Canadian Journal of Chemistry 1985, 63, 571-575.

[213] Deb, B.; Dutta, D. K. J. Mol. Catal. A-Chem. 2010, 326, 21-28.

[214] Williams, G. L.; Parks, C. M.; Smith, C. R.; Adams, H.; Haynes, A.; Meijer, A. J.; Sunley, G. J.; Gaemers, S. Organometallics 2011, 30, 6166-6179.

[215] Valentine, J. S. Chem. Rev. 1973, 73, 235-245.

[216] Choy, V. J.; J O'Connor, C. Coordination Chemistry Reviews 1972, 9, 145170.

[217] Baird, M. C.; Lawson, D. N.; Mague, J. T.; Osborn, J. A.; Wilkinson, G. Chem. Commun. (London) 1966, 129-130.

[218] Atlay, M. T.; Gahan, L. R.; Kite, K.; Moss, K.; Read, G. J. Mol. Catal. 1980, 7, $31-42$.

[219] Atlay, M. T.; Carlton, L.; Read, G. Journal of Molecular Catalysis 2001, 19, $57-68$.

[220] Carlton, L.; Read, G.; Urgelles, M. J. Chem. Soc., Chem. Commun. 1983, $586-588$.

[221] Read, G.; Urgelles, M. J. Chem. Soc., Dalton Trans. 1985, 1591-1596. 
[222] Ahijado, M.; Braun, T.; Noveski, D.; Kocher, N.; Neumann, B.; Stalke, D.; Stammler, H.-G. Angew. Chem. Int. Ed. 2005, 44, 6947-6951.

[223] Aresta, M.; Quaranta, E.; Ciccarese, A. Journal of Molecular Catalysis 1987, $41,355-359$.

[224] Bennett, M. J.; Donaldson, P. B. Inorg. Chem. 1977, 16, 1581-1585.

[225] Busetto, C.; D'Alfonso, A.; Maspero, F.; Perego, G.; Zazzetta, A. J. Chem. Soc., Dalton Trans. 1977, 1828-1834.

[226] Van Gaal, H.; van den Bekerom, F. A. J. Organomet. Chem. 1977, 134, 237248.

[227] Richter, B.; Spek, A. L.; van Koten, G.; Deelman, B.-J. J. Am. Chem. Soc. 2000, 122, 3945-3951.

[228] Selke, M.; Foote, C. S.; Karney, W. L. Inorg. Chem. 1993, 32, 5425-5426.

[229] Selke, M.; Karney, W. L.; Khan, S. I.; Foote, C. S. Inorg. Chem. 2001, 34, 5715-5720.

[230] Teets, T. S.; Nocera, D. G. Inorg. Chem. 2012, 51, 7192-7201.

[231] Wakatsuki, Y.; Maniwa, M.; Yamazaki, H. Inorg. Chem. 1990, 29, 4208-4214.

[232] Banwell, M. G.; Coster, M. J.; Edwards, A. J.; Karunaratne, O. P.; Smith, J. A.; Welling, L. L.; Willis, A. C. Aust. J. Chem. 2003, 56, 585.

[233] James, B. R.; Mahajan, D. Canadian Journal of Chemistry 1980, 58, 9961004.

[234] Mague, J. T.; Davis, E. J. Inorg. Chem. 1977, 16, 131-137.

[235] McGinnety, J. A.; Payne, N. C.; Ibers, J. A. J. Am. Chem. Soc. 1969, 91, 63016310.

[236] Miller, J. S.; Caulton, K. G. J. Am. Chem. Soc. 1975, 97, 1067-1073.

[237] Morvillo, A.; Bressan, M. Inorganica Chimica Acta 1986, 121, 219-222.

[238] Pettinari, C.; Marchetti, F.; Cingolani, A.; Bianchini, G.; Drozdov, A.; Vertlib, V.; Troyanov, S. .. of organometallic ... 2002, 
[239] Slack, D. A.; Greveling, I.; Baird, M. C. Inorg. Chem. 1979, 18, 3125-3132.

[240] Kashiwabara, K.; Morikawa, A.; Suzuki, T.; Isobe, K.; Tatsumi, K. J. Chem. Soc., Dalton Trans. 1997, 1075-1082.

[241] Lindner, E.; Wang, Q.; Mayer, H. A.; Bader, A.; Kuehbauch, H.; Wegner, P. Organometallics 1993, 12, 3291-3296.

[242] Perera, S. D.; Shaw, B. L. J. Chem. Soc., Dalton Trans. 1995, 633-639.

[243] Yu, X.-Y.; Patrick, B. O.; James, B. R. Organometallics 2006, 25, 4870-4877.

[244] Doux, M.; Mézailles, N.; Ricard, L.; Le Floch, P. Organometallics 2003, 22, $4624-4626$.

[245] Frech, C. M.; Shimon, L. J.; Milstein, D. Helv. Chim. Acta 2006, 89, 17301739.

[246] Hayashi, Y.; Szalda, D. J.; Grills, D. C.; Hanson, J. C.; Huang, K.-W.; Muckerman, J. T.; Fujita, E. Polyhedron 2013, 58, 106-114.

[247] Lanci, M. P.; Roth, J. P. J. Am. Chem. Soc. 2006, 128, 16006-16007.

[248] Vasapollo, G.; Giannoccaro, P.; Nobile, C. F.; Sacco, A. Inorganica Chimica Acta 2000, 48, 125-128.

[249] Verat, A. Y.; Fan, H.; Pink, M.; Chen, Y. S.; Caulton, K. G. Chem. Eur. J. 2008, $14,7680-7686$.

[250] Vigalok, A.; Shimon, L. J. W.; Milstein, D. Chem. Commun. 1996, 1673-1674.

[251] Aylward, T.; Findlay, G. SI Chemical Data; Wiley, 2002.

[252] Appleton, T. G.; Bennett, M. A. Inorg. Chem. 2001, 17, 738-747.

[253] Rigamonti, L.; Rusconi, M.; Manassero, C.; Manassero, M.; Pasini, A. Inorg. Chim. Acta 2010, 363, 3498-3505.

[254] Lindner, E.; Wang, Q.; Mayer, H. A.; Fawzi, R. J. Organomet. Chem. 1993, 453, 289-293.

[255] Penner, A.; Braun, T. Eur. J. Inorg. Chem. 2011, 2011, 2579-2587. 
[256] Cipot-Wechsler, J.; Covelli, D.; Praetorius, J. M.; Hearns, N.; Zenkina, O. V.; Keske, E. C.; Wang, R.; Kennepohl, P.; Crudden, C. M. Organometallics 2012, 31, 7306-7315.

[257] Ledger, A. E. W.; Moreno, A.; Ellul, C. E.; Mahon, M. F.; Pregosin, P. S.; Whittlesey, M. K.; Williams, J. M. J. Inorg. Chem. 2010, 49, 7244-7256.

[258] Gangopadhyay, S.; Basak, P.; Drew, M.; Gangopadhyay, P. K. Chem. Commun. 2010, 46, 7436-7438.

[259] Lipscomb, J. D. Annu. Rev. Microbiol. 1994, 48, 371-399.

[260] Merkx, M.; Kopp, D. A.; Sazinsky, M. H.; Blazyk, J. L.; Muller, J.; Lippard, S. J. Angew. Chem. Int. Ed. 2001, 40, 2782-2807.

[261] Tinberg, C. E.; Lippard, S. J. Acc. Chem. Res. 2011, 44, 280-288.

[262] de Montellano, P. R. O. Chem. Rev. 2010, 110, 932-948.

[263] Balcells, D.; Clot, E.; Eisenstein, O. Chem. Rev. 2010, 110, 749-823.

[264] Borovik, A. S. Chem. Soc. Rev. 2011, 40, 1870-1874.

[265] Holm, R. H. Chem. Rev. 1987, 87, 1401-1449.

[266] Poverenov, E.; Efremenko, I.; Frenkel, A. I.; Ben-David, Y.; Shimon, L. J. W.; Leitus, G.; Konstantinovski, L.; Martin, J. M. L.; Milstein, D. Nature 2008, 455, 1093-1096.

[267] Anderson, T. M.; Neiwert, W. A.; Kirk, M. L.; Piccoli, P. M. B.; Schultz, A. J.; Koetzle, T. F.; Musaev, D. G.; Morokuma, K.; Cao, R.; Hill, C. L. Science 2004, 306, 2074-2077.

[268] Winkler, J. R.; Gray, H. B. Electronic Structures of Oxo-Metal Ions; Springer Berlin Heidelberg: Berlin, Heidelberg, 2011; pp 17-28.

[269] Betley, T. A.; Wu, Q.; Van Voorhis, T.; Nocera, D. G. Inorg. Chem. 2008, 47, 1849-1861.

[270] Hay-Motherwell, R. S.; Wilkinson, G.; Hussain-Bates, B.; Hursthouse, M. B. Polyhedron 1993, 12, 2009-2012. 
[271] Tsvetkov, N. P.; Andino, J. G.; Fan, H.; Verat, A. Y.; Caulton, K. G. Dalton Trans. 2013, 42, 6745-6755.

[272] Di Pasqua, A. J.; Goodisman, J.; Kerwood, D. J.; Toms, B. B.; Dubowy, R. L.; Dabrowiak, J. C. Chem. Res. Toxicol. 2007, 20, 896-904.

[273] Dierkes, P.; Ramdeehul, S.; Barloy, L.; De Cian, A.; Fischer, J.; Kamer, P. C. J.; van Leeuwen, P. W. N. M.; Osborn, J. A. Angew. Chem. Int. Ed. 1998, $37,3116-3118$.

[274] Peris, E.; Crabtree, R. H. Coord. Chem. Rev. 2004, 248, 2239-2246.

[275] Shen, Q.; Ogata, T.; Hartwig, J. F. J. Am. Chem. Soc. 2008, 130, 6586-6596.

[276] Wu, X.-F.; Neumann, H.; Beller, M. Chem. Rev. 2013, 113, 1-35.

[277] Simeone, J. P.; Braun, M. P.; Liu, L.; Natarajan, S. R. J. Labelled Compd. Radiopharm. 2010, 53, 511-516.

[278] Trost, B. M.; Thaisrivongs, D. A.; Donckele, E. J. Angew. Chem. Int. Ed. 2012, 52, 1523-1526.

[279] Wagaw, S.; Yang, B. H.; Buchwald, S. L. J. Am. Chem. Soc. 1999, 121, 1025110263.

[280] Chianese, A. R.; Lee, S. J.; Gagne, M. R. Angew. Chem. Int. Ed. 2007, 46, 4042-4059.

[281] Johns, A. M.; Utsunomiya, M.; Incarvito, C. D.; Hartwig, J. F. J. Am. Chem. Soc. 2006, 128, 1828-1839.

[282] Klingensmith, L. M.; Strieter, E. R.; Barder, T. E.; Buchwald, S. L. Organometallics 2006, 25, 82-91.

[283] Yin, J. J.; Buchwald, S. L. J. Am. Chem. Soc. 2002, 124, 6043-6048.

[284] Carr, N.; Dunne, B. J.; Mole, L.; Orpen, A. G.; Spencer, J. L. J. Chem. Soc., Dalton Trans. 1991, 863-871.

[285] Grushin, V. V.; Marshall, W. J. J. Am. Chem. Soc. 2006, 128, 12644-12645.

[286] Harrison, N. C.; Murray, M.; Spencer, J. L.; Stone, F. G. A. J. Chem. Soc., Dalton Trans. 1978, 1337-1342. 
[287] Howard, J. A.; L, S. J.; Mason, S. A. Proceedings of the Royal Society of London. A. Mathematical and Physical Sciences 1983, 386, 145-161.

[288] Yoneda, N.; Kusano, S.; Yasui, M.; Pujado, P.; Wilcher, S. Applied Catalysis A: General 2001, 221, 253-265.

[289] Degnan, T. F., Jr; Smith, C. M.; Venkat, C. R. Applied Catalysis A: General 2001, 221, 283-294.

[290] Ugo, R.; Cariati, F.; La Monica, G. Chem. Commun. (London) 1966, 868869.

[291] Green, M.; Howard, J. A.; Spencer, J. L.; Stone, F. G. A. J. Chem. Soc., Chem. Commun. 1975, 3-4.

[292] Otsuka, S.; Yoshida, T.; Matsumoto, M.; Nakatsu, K. J. Am. Chem. Soc. 1976, $98,5850-5858$.

[293] Clark, H. C.; Smith, M. H. J. Am. Chem. Soc. 2001, 108, 3829-3830.

[294] Hackett, M.; Whitesides, G. M. J. Am. Chem. Soc. 2001, 110, 1449-1462.

[295] Nicolas, E.; le Goff, X.-F.; Bouchonnet, S.; Mézailles, N. Chem. Commun. 2012, 48, 8350-8352.

[296] Williams, D. B. G.; Lawton, M. J. Org. Chem. 2010, 75, 8351-8354.

[297] Allan, K. M. Hybrid P,E Ligands. Synthesis, Coordination Chemistry and Catalysis; Ph.D thesis, Victoria University of Wellington, 2014.

[298] Somerville, R. Unpublished Work.

[299] Green, M.; Grove, D. M.; Spencer, J. L.; Stone, F. G. A. J. Chem. Soc., Dalton Trans. 1977, 2228-2234.

[300] Fulmer, G. R.; Miller, A. J. M.; Sherden, N. H.; Gottlieb, H. E.; Nudelman, A.; Stoltz, B. M.; Bercaw, J. E.; Goldberg, K. I. Organometallics 2010, 29, 21762179.

[301] Tolman, C. A. J. Am. Chem. Soc. 2001, 96, 2780-2789.

[302] Goel, A. B.; Goel, Sarla, Inorg. Chim. Acta 1983, 77, L5-L6. 
[303] Yoshida, T.; Otsuka, S. J. Am. Chem. Soc. 1977, 99, 2134-2140.

[304] Kashiwagi, T.; Yasuoka, N.; Kasai, N.; Kakudo, M.; Takahashi, S.; Hagihara, N. J. Chem. Soc. D 1969, 743.

[305] Cheng, P. T.; Cook, C. D.; Nyburg, S. C.; Wan, K. Y. Canadian Journal of Chemistry 1971, 49, 3772-3777.

[306] Cook, C. D.; Cheng, P.-T.; Nyburg, S. C. J. Am. Chem. Soc. 2001, 91, 21232123.

[307] Yoshida, T.; Tatsumi, K.; Matsumoto, M.; Nakatsu, K.; Nakamura, A.; Fueno, T.; Otsuka, S. Nouveau Journal De Chimie 1979, 3, 761-774.

[308] Cramer, C. J.; Tolman, W. B.; Theopold, K. H.; Rheingold, A. L. Proc. Natl. Acad. Sci. U.S.A. 2003, 100, 3635-3640.

[309] Clark, H. C.; Goel, A. B.; Wong, C. S. J. Am. Chem. Soc. 1978, 100, 62416243.

[310] Mimoun, H. Journal of Molecular Catalysis 1980, 7, 1-29.

[311] Qu, F.; Khusnutdinova, J. R.; Rath, N. P.; Mirica, L. M. Chem. Commun. 2014, 50, 3036-3039.

[312] Sugimoto, R.; Suzuki, H.; Moro-Oka, Y.; Ikawa, T. Chem. Lett. 1982, 18631866.

[313] Sergeev, A. G.; Neumann, H.; Spannenberg, A.; Beller, M. Organometallics 2010, 29, 3368-3373.

[314] Garrou, P. E. Chem. Rev. 1981, 81, 229-266.

[315] Zayya, A. I.; Spencer, J. L. Organometallics 2012, 31, 2841-2853.

[316] Zayya, A. I.; Vagana, R.; Nelson, M. R. M.; Spencer, J. L. Tetrahedron Lett. 2012, 53, 923-926.

[317] Hoyte, S. A. Platinum Complexes of Bicyclopropylidene and Related Ligands; Ph.D thesis, Victoria University of Wellington, 2014.

[318] Phillips, A. D.; Gonsalvi, L.; Rornerosa, A.; Vizza, F.; Peruzzini, M. Coord. Chem. Rev. 2004, 248, 955-993. 
[319] Darensbourg, D. J.; Decuir, T. J.; Stafford, N. W.; Robertson, J. B.; Draper, J. D.; Reibenspies, J. H.; Kathó, A.; Joó, F. Inorg. Chem. 1997, 36, $4218-4226$.

[320] Spencer, J. L. Inorg. Synth. 1979, 19, 213-218.

[321] Green, M.; Howard, J. A.; Spencer, J. L.; Stone, F. G. A. J. Chem. Soc., Dalton Trans. 1977, 271-277.

[322] Pregosin, P. S.; Favez, R.; Roulet, R.; Boschi, T.; Michelin, R. A.; Ros, R. Inorg. Chim. Acta 1980, 45, L7-L9.

[323] Addison, A. W.; Rao, T. N.; Reedijk, J.; van Rijn, J.; Verschoor, G. C. J. Chem. Soc., Dalton Trans. 1984, 1349.

[324] Yang, L.; Powell, D. R.; Houser, R. P. Dalton Trans. 2007, 955.

[325] Bachechi, F.; Zambonelli, L. Acta Crystallogr., Sect. C: Cryst. Struct. 1992, $1-5$.

[326] Engeldinger, E.; Armspach, D.; Matt, D.; Jones, P. G. Chem. Eur. J. 2003, 9, 3091-3105.

[327] Newman, P. D.; Cavell, K. J.; Kariuki, B. M. Dalton Trans. 2012, 41, 1239512407.

[328] Owens, S. B., Jr.; Smith, D. C., Jr.; Lake, C. H.; Gray, G. M. Eur. J. Inorg. Chem. 2008, 2008, 4710-4718.

[329] van den Beuken, E. K.; Meetsma, A.; Kooijman, H.; Spek, A. L.; Feringa, B. L. Inorg. Chim. Acta 1997, 264, 171-183.

[330] Ruhland, K.; Brück, A.; Herdtweck, E. Eur. J. Inorg. Chem. 2007, 2007, 944964.

[331] van der Vlugt, J. I.; Sablong, R.; Mills, A. M.; Kooijman, H.; Spek, A. L.; Meetsma, A.; Vogt, D. Dalton Trans. 2003, 4690-4699.

[332] Mora, G.; Piechaczyk, O.; Houdard, R.; Mézailles, N.; le Goff, X.-F.; Le Floch, P. Chem. Eur. J. 2008, 14, 10047-10057. 
[333] van Duren, R.; Cornelissen, L. L.; van der Vlugt, J. I.; Huijbers, J. P.; Mills, A. M.; Spek, A. L.; Müller, C.; Vogt, D. Helvetica chimica acta 2006, $89,1547-1558$.

[334] van Duren, R.; van der Vlugt, J. I.; Kooijman, H.; Spek, A. L.; Vogt, D. Dalton Trans. 2007, 1053-1059.

[335] Niksch, T.; Görls, H.; Friedrich, M.; Oilunkaniemi, R.; Laitinen, R.; Weigand, W. Eur. J. Inorg. Chem. 2010, 2010, 74-94.

[336] Armarego, W. L. F.; Chai, C. L. L. Purification of Laboratory Chemicals, 5th ed.; Butterworth-Heinemann: Burlington MA, 2003.

[337] Kranenburg, M.; Kamer, P. C. J.; van Leeuwen, P. W. N. M. Eur. J. Inorg. Chem. 1998, 155-157.

[338] Doherty, S.; Eastham, G. R.; Tooze, R. P.; Scanlan, T. H.; Williams, D.; Elsegood, M. R. J.; Clegg, W. Organometallics 1999, 18, 3558-3560.

[339] Moss, J. R. Journal of Molecular Catalysis A: Chemical 2003, 107, 169-174.

[340] Bernskoetter, W. H.; Schauer, C. K.; Goldberg, K. I.; Brookhart, M. Science 2009, 326, 553-556.

[341] Silverstein, R. M.; Webster, F. X.; Kiemle, D. J. Spectrometric Identification of Organic Compounds, 7th ed.; John Wiley \& Sons, Ltd., 2005.

[342] Scollard, J. D.; Day, M.; Labinger, J. A.; Bercaw, J. E. Helvetica chimica acta 2001, 84, 3247-3268.

[343] Ingleson, M. J.; Mahon, M. F.; Weller, A. S. Chem. Commun. 2004, 23982399.

[344] Walter, M. D.; White, P. S.; Brookhart, M. New J. Chem. 2013, 37, 1128-1133.

[345] Daigle, D. J.; Decuir, T. J.; Robertson, J. B.; Darensbourg, D. J. Inorg. Synth. 1998, 32, 40-45.

[346] Dieter, R. K.; Topping, C. M.; Nice, L. E. J. Org. Chem. 2001, 66, 2302-2311.

[347] Suter, C. M.; Maxwell, C. E. Org. Synth. 1938, 18, 64-65. 
[348] Koshar, R. J.; Mitsch, R. A. The Journal of Organic Chemistry 2001, 38, 33583363.

[349] Vaughan, T. F.; Koedyk, D. J.; Spencer, J. L. Organometallics 2011, 30, 51705180.

[350] Giordano, G.; Crabtree, R. H.; Heintz, R. M.; Forster, D.; Morris, D. E. Inorg. Synth. 1979, 19, 218-220.

[351] Hartley, F. R.; Murray, S. G.; McAuliffe, C. A. Inorg. Chem. 1979, 18, 13941397.

[352] Crascall, L. E.; Spencer, J. L.; Doyle, R. A.; Angelici, R. J. Inorg. Synth. 1990, $28,126-132$.

[353] Jensen, C. M. Acta Chem. Scand. 1953, 7, 866-868.

[354] Cramer, R. D.; Jones, F. N. US Pat., US3356748. 1967.

[355] Anderson, B. G. Late transition metal complexes of pentafluorophenylphosphino-pincer ligands.; Ph.D thesis, Victoria University of Wellington, 2012.

[356] Palatinus, L.; Chapuis, G. J. Appl. Crystallogr. 2009, 50, 834-835.

[357] Sheldrick, G. M. Acta Crystallogr., Sect. A: Found. Crystallogr. 2008, 64, 112-122.

[358] Dolomanov, O. V.; Bourhis, L. J.; Gildea, R. J.; Howard, J. A. K.; Puschmann, H. J. J. Appl. Crystallogr. 2009, 42, 339-341.

[359] Frisch, M. J.; al, e. Gaussian 09 Revision B.01. Wallingford CT, 2009.

[360] Glendening, E. D.; Reed, A. E.; Carpenter, J. E.; Weinhold, F. NBO Version 3.1 E. D. 